Michael Broer

\title{
Der kommunale \\ Finanzausgleich in \\ Hessen
}

Historische Darstellung und ökonomische Analyse unter besonderer Berücksichtigung der Schlüsselzuweisungen 


\section{Michael Broer}

\section{Der kommunale Finanzausgleich in Hessen}

In Deutschland sind die Bundesländer nach Art. 106 Abs. 7 GG verpflichtet, ihre Gemeinden und Gemeindeverbände im Rahmen eines kommunalen Finanzausgleichs an den eigenen Steuereinnahmen zu beteiligen. Daim Grundgesetz und den jeweiligen Landesverfassungen ausschließlich allgemeine Ausführungen zu diesem Themenkomplex zu finden sind, kommt es häufig zum Verteilungsstreit zwischen Land und Kommunen einerseits und Kommunen andererseits. Der Verfasser beschreibt und analysiert die Bestimmungen des hessischen kommunalen Finanzausgleichs bezüglich ihrer ökonomischen Zielführung und vergleicht diese mit Regelungen anderer Bundesländer. Anschließend legt er eigene Reformvorschläge dar, mit deren Hilfe die angesprochenen Probleme gelöst werden können.

Michael Broer wurde 1969 in Hannover geboren. Von 1990 bis 1995 studierte er Wirtschaftswissenschaften an der Universität Hannover mit dem Abschluß als Diplom-Ökonom. Promotionsstudium an der Universität Frankfurt am Main. Von 1996 bis 2001 war er als Wissenschaftlicher Mitarbeiter am Lehrstuhl für Finanzwissenschaft des Fachbereichs Wirtschaftswissenschaften der Universität Frankfurt tätig. Seit 2001 arbeitet er im Bundesfinanzministerium. 
Der kommunale Finanzausgleich

in Hessen 


\section{FINANZWISSENSCHAFTLICHE SCHRIFTEN}

Herausgegeben von den Professoren

Albers ( $\dagger$ ), Krause-Junk, Littmann, Oberhauser, Pohmer, Schmidt

Band 105

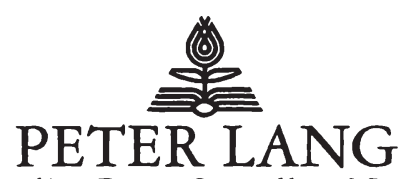

Frankfurt am Main · Berlin · Bern · Bruxelles - New York · Oxford · Wien 


\section{Michael Broer}

\section{Der kommunale Finanzausgleich in Hessen}

Historische Darstellung und ökonomische Analyse

unter besonderer Berücksichtigung der Schlüsselzuweisungen

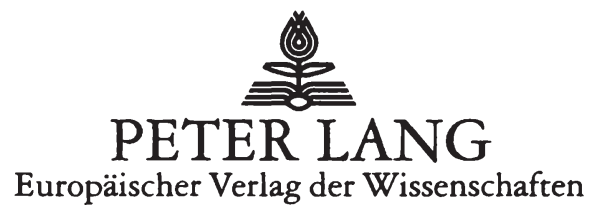


Die Deutsche Bibliothek - CIP-Einheitsaufnahme

Broer, Michael:

Der kommunale Finanzausgleich in Hessen : historische Darstellung und ökonomische Analyse unter besonderer Berücksichtigung der Schlüsselzuweisungen / Michael Broer. Frankfurt am Main ; Berlin ; Bern ; Bruxelles ; New York ; Oxford ; Wien : Lang, 2001

(Finanzwissenschaftliche Schriften; Bd. 105)

Zugl.: Frankfurt (Main), Univ., Diss., 2000

ISBN3-631-37686-3

Open Access: The online version of this publication is published on www.peterlang.com and www.econstor.eu under the international Creative Commons License CC-BY 4.0. Learn more on how you can use and share this work: http://creativecommons. org/licenses/by/4.0.

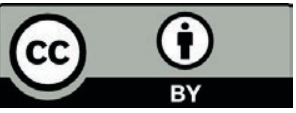

This book is available Open Access thanks to the kind support of ZBW - Leibniz-Informationszentrum Wirtschaft.

Gedruckt auf alterungsbeständigem, säurefreiem Papier.

\author{
D30 \\ ISSN 0170-8252 \\ ISBN3-631-37686-3 \\ ISBN 978-3-631-75180-0 (eBook) \\ (C) Peter Lang $\mathrm{GmbH}$ \\ Europäischer Verlag der Wissenschaften \\ Frankfurt am Main 2001 \\ Alle Rechte vorbehalten.
}

Das Werk einschließlich aller seiner Teile ist urheberrechtlich geschützt. Jede Verwertung außerhalb der engen Grenzen des

Urheberrechtsgesetzes ist ohne Zustimmung des Verlages unzulässig und strafbar. Das gilt insbesondere für

Vervielfältigungen, Übersetzungen, Mikroverfilmungen und die Einspeicherung und Verarbeitung in elektronischen Systemen.

\title{
Printed in Germany 124567
}

www.peterlang.de 


\section{Vorwort}

Die vorliegende Arbeit wurde im Wintersemester 2000/01 vom Fachbereich Wirtschaftswissenschaften der Johann Wolfgang Goethe-Universität in Frankfurt am Main als Dissertation angenommen.

Mein besonderer Dank gilt Herrn Prof. Dr. Norbert Andel für seine kritischen Hinweise und für die Möglichkeit, meine Forschung an seinem Lehrstuhl durchführen zu können. Herrn Prof. Dr. Paul B. Spahn danke ich für die zügige Zweitkorrektur. Herrn Professor Dr. Kurt Schmidt bin ich für die freundliche Unterstützung bei der Aufnahme meiner Arbeit in die Reihe Finanzwissenschaftliche Schriften sehr verbunden.

Bei all meinen ehemaligen und derzeitigen Kollegen am Lehrstuhl möchte ich mich für die langjährige freundschaftliche Zusammenarbeit und herzliche Arbeitsatmosphäre bedanken. Für sehr hilfreiche redaktionelle Hinweise und Kommentare danke ich Herrn Dipl.-Volkswirt Wolfgang Knoke, Herrn Dipl.-Volkswirt Martin Gasche für die fachliche Diskussion.

Diese Arbeit widme ich meinen Großeltern Irma und Wilhelm Grages. 

Inhaltsverzeichnis

Abbildungsverzeichnis

XIII

Tabellenverzeichnis

$\mathrm{XV}$

Abkürzungsverzeichnis

XVII

1. Problemstellung

2. Die kommunale Ebene in der Bundesrepublik Deutschland

2.1. Kommunale Selbstverwaltung im Grundgesetz und der Hessischen Verfassung

2.1.1. Verhältnis zwischen Staat und kommunaler Ebene 5

2.1.2. Verhältnis zwischen Gemeinden und Gemeindeverbänden 8

2.1.3. Struktur und historische Entwicklung des Bundeslandes Hessen $\quad 10$

2.1.3.1. Staatliche Ebene $\quad 10$

2.1.3.2. Kommunale Ebene $\quad 12$

2.2. Kommunale Aufgaben und Einnnahmen 18

2.2.1. Arten kommunaler Aufgaben - ein Überblick 18

2.2.1.1. Freiwillige Aufgaben 18

$\begin{array}{ll}\text { 2.2.1.2. Weisungsfreie Pflichtaufgaben } & 19\end{array}$

2.2.1.3. Weisungsgebundene Pflichtaufgaben 20

2.2.2. Kommunale Einnahmen - ein Überblick 20

2.3. Der kommunale Finanzausgleich - ein Überblick 23

2.3.1. Grundgesetzliche und landesrechtliche Vorschriften zum KFA 23

2.3.2. Ermittlung der heutigen Verbundmasse des KFA 25

3. Ausgestaltung eines kommunalen Finanzausgleichs 29

3.1. Aufgaben eines kommunalen Finanzausgleichs 29

3.1.1. Verteilungspolitik $\quad 29$

3.1.1.1. Vertikale Verteilung $\quad 29$

3.1.1.2. Horizontale Verteilung $\quad 29$

3.1.2. Allokationspolitik 31

3.1.3. Stabilitätspolitik $\quad 31$ 
3.2. Anforderungskriterien eines kommunalen Finanzausgleichs 32

3.2.1. Vertikale Anforderungskriterien $\quad 34$

3.2.1.1. Angemessenheit der Verbundmasse $\quad 34$

3.2.1.2. Geringe Konjunkturreagibilität der Verbundmasse 35

3.2.1.3. Selbstbestimmung der Mittelverwendung 36

3.2.2. Horizontale Anforderungskriterien $\quad 37$

3.2.2.1. Angemessenheit der Teil-Verbundmassen $\quad 37$

3.2.2.2. Ausgleichsrelevante Bedarfsermittlung $\quad 38$

3.2.2.3. Realitätsnahe Finanzkraftermittlung $\quad 40$

3.2.2.4. Angemessene Ausgleichsintensität $\quad 41$

4. Kommunaler Finanzausgleich in Hessen - Darstellung, Analyse und Reform 43

4.1. Verteilung der Finanzmasse zwischen Land und Kommunen 43

4.1.1. Strukturelle Entwicklung der Verbundmasse 43

4.1.2. Entwicklung des Volumen der Verbundmasse 50

4.1.3. Angemessenheit der Verbundmasse 53

4.1.3.1. Entwicklung der Einnahmenanteile von staatlicher und kommunaler Ebene $\quad 55$

4.1.3.2. Beachtung des Kommunalisierungsgrads der Aufgabenwahrnehmung $\quad 57$

4.1.3.3. Ein Ansatz zur Sicherung einer angemessenen $\quad 59$ Finanzausstattung

4.1.4. Konjunkturelle Entwicklung der Verbundmasse $\quad 60$

4.1.5. Ansätze zur Verstetigung der Einnahmen durch den KFA 63

4.2. Verteilung der Finanzmasse auf die Zuweisungskategorien 68

4.2.1. Charakterisierung der Zuweisungskategorien 68

4.2.2. Dotierung der Zuweisungskategorien im Zeitablauf 72

4.2.2.1. Zeitliche Entwicklung $\quad 72$

4.2.2.2. Analyse und Reform 74

4.2.2.2.1. Entwicklungsvergleich zwischen Hessen und westdeutschen Bundesländern

4.2.2.2.2. Anstehende Veränderungen für Hessen - Erhöhung der Autonomie bei der Mittelverwendung durch Abbau von Sonderlastenausgleichen 
4.3. Verteilung der Schlüsselmasse zwischen den kommunalen Gruppen 79

4.3.1. Strukturelle Veränderungen der Schlüsselzuweisungen 79

$\begin{array}{ll}\text { 4.3.1.1. Historische Entwicklung } & 79\end{array}$

4.3.1.2. Separierung der drei kommunalen Gruppen $\quad 81$

4.3.1. Strukturelle Veränderungen der Schlüsselzuweisungen 79

4.3.1.1. Historische Entwicklung $\quad 79$

4.3.1.2. Separierung der drei kommunalen Gruppen $\quad 81$

4.3.2. Dotierung der Teilschlüsselmassen - Analyse und Reform 83

4.3.2.1. Vergleich kreisangehöriger und kreisfreier Raum 84

4.3.2.2. Vergleich kreisangehörige Gemeinden und Landkreise $\quad 88$

4.4. Verteilung der Schlüsselmasse innerhalb der kommunalen Gruppen 90

4.4.1. Allgemeine Grundlagen der Bedarfsermittlung 90

4.4.1.1. Funktion des Hauptansatzes 91

4.4.1.2. Funktion der Neben- oder Ergänzungsansätze 92

4.4.1.3. Funktion des Grundbetrags 93

4.4.2. Ermittlung des Finanzbedarfs von Gemeinden und Städten 94

4.4.2.1. Verwendung des Hauptansatzes in Hessen 94

4.4.2.1.1. Darstellung der bisherigen Regelungen 94

4.4.2.1.2. Analyse und Reform 95

4.4.2.2. Verwendung von Nebenansätzen in Hessen 103

4.4.2.2.1. Darstellung der bisherigen Regelungen 103

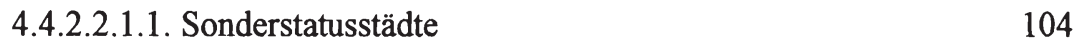

4.4.2.2.1.2. Stationierungsstreitkräfte 104

$\begin{array}{ll}\text { 4.4.2.2.1.3. Kurorte } & 105\end{array}$

4.4.2.2.1.4. Bevölkerungswachstum 105

4.4.2.2.1.5. Schulträgerschaft 106

$\begin{array}{ll}\text { 4.4.2.2.2. Analyse und Reform } & 107\end{array}$

$\begin{array}{ll}4.4 .2 .2 .2 .1 \text {. Sonderstatusstädte } & 108\end{array}$

$\begin{array}{ll}4.4 .2 .2 .2 .2 \text {. Stationierungsstreitkräfte } & 110\end{array}$

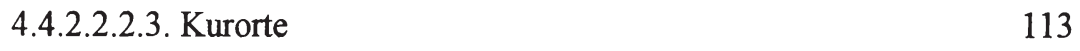

4.4.2.2.2.4. Bevölkerungswachstum $\quad 115$

4.4.2.2.2.5. Schulträgerschaft 118

4.4.2.2.2.6. Notwendigkeit weiterer Nebenansätze? 119 
4.4.2.3. Folgerungen für die zukünftige Bedarfsermittlung 121

4.4.3. Ermittlung des Finanzbedarfs der Landkreise 122

4.4.3.1. Verwendung des Hauptansatzes in Hessen 122

4.4.3.1.1. Darstellung der bisherigen Regelungen 122

4.4.3.1.2. Analyse und Reform 123

4.4.3.2. Verwendung eines Nebenansatzes in Hessen 125

4.4.3.2.1. Darstellung der bisherigen Regelungen 125

4.4.3.2.2. Analyse und Reform 126

4.4.3.2.3. Notwendigkeit eines weiteren Nebenansatzes? 127

4.4.3.3. Folgerungen für die zukünftige Bedarfsermittlung 129

4.4.4. Allgemeine Grundlagen der Finanzkraftermittlung 129

4.4.5. Ermittlung der Finanzkraft von Gemeinden und Städten 131

4.4.5.1. Darstellung der bisherigen Regelungen 131

4.4.5.2. Analyse und Reform 136

4.4.5.2.1. Abgrenzung der ausgleichsrelevanten Einnahmen 136

4.4.5.2.1.1. Vermögenserträge und vergleichbare Einnahmen 136

$\begin{array}{ll}\text { 4.4.5.2.1.2. Zuweisungen } & 137\end{array}$

$\begin{array}{ll}\text { 4.4.5.2.1.3. Leistungsentgelte } & 137\end{array}$

4.4.5.2.1.4. Weitere kommunale Steuern 138

4.4.5.2.1.5. Krediteinnahmen 141

4.4.5.2.2. Erfassungsgrad der ausgleichsrelevanten Einnahmen $\quad 141$

4.4.5.2.2.1. Normierung des Realsteueraufkommens 142

4.4.5.2.2.1.1. Einheitlicher oder differenzierter Nivellierungshebesatz 142

4.4.5.2.2.1.2. Niveaubestimmung des Nivellierungshebesatzes $\quad 146$

4.4.5.2.2.1.3. Dynamisierung des Erfassungsgrades der Steuerkraft 148

4.4.5.2.2.2. Normienung des Konzessionsabgabenaufkommens $\quad 149$

4.4.5.3. Folgerungen für die zukünftige Steuerkraftberechnung 153

4.4.6. Ermittlung der Umlagekraft der Landkreise $\quad 154$

4.4.6.1. Darstellung der bisherigen Regelungen 154

4.4.6.1.1. Finanzkraft der Landkreise $\quad 154$

4.4.6.1.2. Berechnung der Umlagekraft der Landkreise 158

4.4.6.2. Analyse und Reform 160

4.4.6.2.1. Abgrenzung der ausgleichrelevanten Einnahmen 160 
4.4.6.2.2. Erfassungsgrad der ausgleichsrelevanten Einnahmen

4.4.6.2.3. Probleme der Sonderstatusstädte - Interdependenzen zwischen Umlagen und Gemeindeschlüsselzuweisungen

4.4.6.2.3.1. Behandlung der Sonderstatusstädte bei an den Landkreis $\mathrm{zu}$ entrichtende Umlagen

4.4.6.2.3.2. Behandlung der Sonderstatusstädte bei vom Landkreis zu entrichtende Umlagen

4.4.6.2.3.2.1. Auswirkungen auf die vom Landkreis zu entrichtenden Umlagen

4.4.6.2.3.2.2. Reformansätze der Sonderstatusstadtbehandlung

4.4.6.2.3.3. Interdependenzen mit den Gemeindeschlüsselzuweisungen

4.4.7. Allgemeine Grundlagen zum Ausgleich von Finanzbedarf und Finanzkraft

4.4.8. Ausgleich von Finanzbedarf und Finanzkraft in Hessen

4.4.8.1. Entwicklung des Ausgleichsverfahrens im Zeitablauf

4.4.8.2. Regelungen für abundante Gemeinden im Zeitablauf

4.4.8.3. Funktionsweise des hessischen Ausgleichsmechanismus $\quad 176$

4.4.9. Ausgleichsmechanismus bei Gemeinden und Städten 178

$\begin{array}{ll}\text { 4.4.9.1. Wirkungen der bisherigen Regelungen } & 178\end{array}$

4.4.9.2. Problemlösungsvorschlag

4.4.10. Ausgleichsmechanismus bei Landkreisen $\quad 190$

4.4.10.1. Wirkungen der bisherigen Regelungen 190

4.4.10.2. Problemlösungsvorschlag 191

4.4.11. Behandlung abundanter Kommunen 194

4.4.11.1. Höhe der Mindestzuweisungen 194

4.4.11.2. Finanzausgleichsumlage 196

4.4.11.2.1. Zur Notwendigkeit einer Abschöpfungskomponente 196

4.4.11.2.2. Ausgestaltungsformen der Abschöpfungskomponente 197

4.4.11.2.3. Kompatibilität zwischen Abschöpfungskomponente und den übrigen Umlagen 



\section{Abbildungsverzeichnis}

Abbildung 1: Anteil der Steuerverbund- und der Finanzausgleichsmasse an den gesamten Landessteuereinnahmen nach LFA

Abbildung 2: Steuereinnahmenentwicklung des Landes nach LFA und der Kommunen

Abbildung 3: Anteil des Landes und der Kommunen an den hessischen Einnahmen aus Steuern nach LFA und Leistungen des KFA

Abbildung 4: Entwicklung der Einnahmen und Ausgaben im Zeitablauf 61

Abbildung 5: Entwicklung von BIP sowie Einnahmen aus Steuern und KFA 62

Abbildung 6: Verstetigung der KFA-Zuweisungen durch Fondsbildung $\quad 67$

Abbildung 7: Typisierung der Zuweisungen des KFA in Hessen 68

Abbildung 8: Anteile der Zuweisungsarten an der Finanzausgleichsmasse im Zeitablauf

Abbildung 9: Entwicklung finanzkraft-/-bedarfbezogener Schlüsselzuweisungen an den gesamten Leistungen im Rahmen des KFA im Zeitablauf

Abbildung 10: Finanzkraftvergleich von kreisfreiem und kreisangehörigem Raum

Abbildung 11: Finanzkraft kreisfreier und kreisangehöriger Oberzentren

Abbildung 12: Finanzkraftvergleich einzelner Gebietskörperschaften

Abbildung 13: Berechnung des Grundbetrags

Abbildung 14: Vergleich von bestehender und einer möglichen neuen Hauptansatzstaffel der kreisangehörigen Gemeinden in Hessen

Abbildung 15: Hauptansatzstaffel bei einheitlicher Gemeindeschlüsselmasse

Abbildung 16: Hessische kreisfreie Städte im Hauptansatzregime anderer Länder

Abbildung 17: Finanzkraftmindernde Wirkung der Gewerbesteuerumlage

Abbildung 18: Entwicklung der Erfassung der kommunalen Finanzkraft in Hessen

Abbildung 19: Gemeindegruppenspezifische gewogene Realsteuerhebesätze 1997

Abbildung 20: Gewerbesteuergrundbetrag pro Kopf

Abbildung 21: Berechnung des KA-Anrechnungsfaktors zur Steuerkraftermittlung bei Gemeinden 
Abbildung 22: Berechnung des KA-Anrechnungsfaktors zur Umlagekraftermittlung bei Landkreisen

Abbildung 23: Entwicklung der Mindestzuweisungen im Zeitablauf

Abbildung 24: Finanzkraft kreisangehöriger Gemeinden vor und nach KFA 180

Abbildung 25: Zusammenhang zwischen Finanzkraft und Sockelgarantie sowie Ausgleichsquote bei einem Anreiz zur Einnahmensteigerung

Abbildung 26: Finanzkraft kreisfreier Städte vor und nach KFA

Abbildung 27: Vergleich der Relation von Finanzkraft zur Bedarfsmeßzahl in Abhängigkeit vom gewählten Ausgleichsmechanismus

Abbildung 28: Vergleich der Relation von Finanzkraft zum Gesamtansatz in Abhängigkeit vom gewählten Ausgleichsmechanismus; in DM

Abbildung 29: Kreisfinanzkraft vor und nach KFA

Abbildung 30: Auswirkung einer unterschiedlichen Umlagekraftanrechnung bei der Verteilung der Schlüsselzuweisungen mit einem Ausgleichsgrad von 50 v.H. und einer Sockelgarantie von 79 v.H.

Abbildung 31: Wirkungen einer Ausgleichsumlage bei einem Ausgleichsgrad von 75 v.H. auf die Finanzkraft der umlagepflichtigen abundanten Kommunen

Abbildung 32: Wirkungen einer Ausgleichsumlage bei einem Ausgleichsgrad von 75 v.H. auf die Finanzkraft der umlageempfangenden Kommunen 


\section{Tabellenverzeichnis}

Tabelle 1: Kommunale Ebene nach Gruppen und Größenklassen im $\begin{array}{ll}\text { Zeitablauf } & 18\end{array}$

Tabelle 2: Einnahmen der kommunalen Ebene in Hessen 1996

Tabelle 3: Von der Steuerverbund- zur Finanzausgleichsmasse (1996) 27

Tabelle 4: Volumenmäßige Auswirkungen der strukturellen Veränderungen 51

Tabelle 5: Einnahmen und Ausgaben staatlicher und kommunaler Ebene in Hessen und den übrigen westlichen Flächenländern 1996 (DM/Kopf) 58

Tabelle 6: Wirkungen eines Fonds zur Glättung der Zuweisungen im KFA (in Mio. DM)

Tabelle 7: Verteilungswirkungen der Substitution eines Schülersonderlastenausgleichs durch einen Schülernebenansatz $\quad 77$

Tabelle 8: Gruppenneutrale Mittelumschichtung im kreisangehörigen Raum 78

Tabelle 9: Veränderungen der Anteile der drei Gebietskörperschaftsgruppen an den Schlüsselzuweisungen seit Ende der Gebietsreform

Tabelle 10: Einfluß der Kreisumlage auf die kreisinterne Finanzkraftrelation 90

Tabelle 11: Unterschiede bei der Hauptansatzermittlung 92

Tabelle 12: Relevanz des Zentralitätsfaktors im KFA 99

Tabelle 13: Auswirkungen der Zentralität auf den Hauptansatz der kreisangehörigen Gemeinden 101

Tabelle 14: Umverteilung bei Abschaffung des Zentralitätsansatzes $\quad 102$

Tabelle 15: Berücksichtigte Bedarfsdeterminanten seit 1949

Tabelle 16: Wirkungen der Nebenansätze auf den gemeindlichen $\begin{array}{ll}\text { Gesamtansatz } & 107\end{array}$

Tabelle 17: Umverteilung bei Abschaffung des Sonderstatusstadtansatzes 108

Tabelle 18: Umverteilung bei Abschaffung des Stationierungsansatzes $\quad 110$

Tabelle 19: Umverteilung bei Abschaffung des Heilkurorteansatzes 113

Tabelle 20: Umverteilung bei Abschaffung des gemeindlichen Ansatzes für Bevölkerungszuwachstum 115

Tabelle 21: Umverteilung bei Abschaffung des Schulträgeransatzes $\quad 118$

Tabelle 22: Umverteilungswirkungen der Kreisschlüsselzuweisungen bei $\begin{array}{ll}\text { Abschaffung der Einwohnerveredelung } & 124\end{array}$

Tabelle 23: Umverteilung bei Abschaffung des Bevölkerungszuwachsansatzes für Landkreise 
Tabelle 24: Wirkungen des Normierungssatzes auf die Schlüsselzuweisungen (in Mio. DM)

Tabelle 25: Nettoertragsvergleich zwischen Konzessionsabgabenerhebung und Gewinnausschüttung ohne KFA-Einfluß bei Gemeinden

Tabelle 26: Ermittlung der gemeindlichen Finanzkraft für die Kreisumlage

Tabelle 27: Umverteilung bei Abschaffung der ermäßigten Finanzkraftberücksichtigung der Sonderstatusstädte bei den Kreisschlüsselzuweisungen

Tabelle 28: Die Behandlung der Sonderstatusstädte und ihre Auswirkungen auf die Umlagegrundlagen der Landesumlagen

Tabelle 29: Effekte der Behandlung der Sonderstatusstädte auf die Umlagenhöhe für das Jahr 1998; in Mio. DM und in v.H.

Tabelle 30: Grundbetragsermittlung unter Berücksichtigung von Abundanz und Sockelkraftgarantie einzelner Gemeinden

Tabelle 31: Nivellierungswirkung der Sockelgarantie

Tabelle 32: Nettoeffekt einer Steuerkrafterhöhung kreisangehöriger Gemeinden um 0,5 Mio. DM bei einem Ausgleichsgrad von 50 v.H. und einer Sockelgarantie von 80 v.H. (nach KFA); in DM und in v.H. der Steuerkrafterhöhung

Tabelle 33: Nettoeffekt einer Steuerkrafterhöhung kreisfreier Städte um 0,5 Mio. DM bei einem Ausgleichsgrad von 50 v.H. und einer Sockelgarantie von 76 v.H. (nach KFA); in DM und in v.H. der Steuerkrafterhöhung

Tabelle 34: Nettoeffekt einer Steuerkrafterhöhung kreisangehöriger Gemeinden um 0,5 Mio. DM (nach KFA) bei einem Ausgleichsgrad von 75 v.H.; in DM und in v.H. der Steuerkrafterhöhung

Tabelle 35: Keine Nivellierungswirkung bei erhöhtem Ausgleichsgrad

Tabelle 36: Keine Nivellierung bei Umlagekraftberücksichtigung unter 100 v.H. bei einem Ausgleichsgrad von 50 v.H. und einer Sockelgarantie von 79 v.H.

Tabelle 37: Wirkung verschiedener Ausgleichsumlagen auf die Finanzkraftdifferenzen; in Finanzkraft pro Bedarfseinheit

Tabelle 38: Auswirkungen verschiedener Umlagen auf die Steuerkraft nach Umlagezahlung in v.H. der originären Steuerkraft 


\section{Abkürzungsverzeichnis}

\begin{tabular}{|c|c|}
\hline Abs. & Absatz \\
\hline AfK & Archiv für Kommunalwissenschaften \\
\hline Art. & Artikel \\
\hline Aufl. & Auflage \\
\hline Bd. & Band \\
\hline $\mathrm{BEZ}$ & Bundesergänzungszuweisungen \\
\hline BIP & Bruttoinlandsprodukt \\
\hline BGBl. & Bundesgesetzblatt \\
\hline BVerfGG & Bundesverfassungsgerichtsgesetz \\
\hline bzw. & beziehungsweise \\
\hline dass. & dasselbe \\
\hline ders. & derselbe \\
\hline d.h. & das heißt \\
\hline dies. & dieselbe \\
\hline DÖV & Die öffenliche Verwaltung \\
\hline DVBl. & Deutsches Verwaltungsblatt \\
\hline ESt & Einkommensteuer \\
\hline EU & Europäische Union \\
\hline FAG & Finanzausgleichsgesetz \\
\hline f. & folgende \\
\hline $\mathrm{FN}$ & Fußnote \\
\hline GewSt. & Gewerbesteuer \\
\hline GFRG & Gemeindefinanzreformgesetz \\
\hline GG & Grundgesetz \\
\hline GrESt & Grunderwerbsteuer \\
\hline GV & Gemeindeverbände \\
\hline GVBl. & Gesetz- und Verordnungsblatt \\
\hline HGO & Hessische Gemeindeordnung \\
\hline $\mathrm{HKO}$ & Hessische Kreisordnung \\
\hline Hrsg. & Herausgeber \\
\hline HV & Hessische Verfassung \\
\hline $\mathrm{Jg}$ & Jahrgang \\
\hline
\end{tabular}




\section{XVIII}

KAG

Gesetz über kommunale Abgaben

KFA

kommunaler Finanzausgleich

$\mathrm{KSt}$

Körperschaftsteuer

LFA

Länderfinanzausgleich

Nds.

niedersächsisches; niedersächsischer

NRW

Nordrhein-Westfalen

NV

Niedersächsische Verfassung

o.J.

ohne Jahr

o.O.

ohne Ort

o.V.

ohne Verfasser

Rdnr.

Randnummer

Tz.

Textziffer

$\mathrm{S}$.

Seite

Sp.

Spalte

u.U.

unter Umständen

vgl.

vergleiche

v.H.

vom Hundert

ZKF

Zeitschrift für Kommunalfinanzen 


\section{Problemstellung}

Die kommunale Ebene in Deutschland sieht sich ihren finanziellen Verpflichtungen nicht mehr gewachsen. Ihr werden immer größere Bürden auf der Ausgabenseite aufgelastet (z. B. gesetzlicher Kindergartenplatzanspruch). Auf der anderen Seite entwickeln sich die kommunalen Einnahmen weniger positiv als die der staatlichen Ebene. Eine an sich längst überfällige grundlegende Reform des kommunalen Steuersystems und vor allem der Gewerbesteuer ist nicht in Sicht; die Beteiligung der Gemeinden an der Umsatzsteuer als Ausgleich für die Abschaffung der Gewerbekapitalsteuer kann in diesem Zusammenhang nicht als der groBe Wurf angesehen werden. Berücksichtigt man die Zeitdimension und die politischen Verhältnisse in Bundestag und -rat, so ist in absehbarer Zeit nicht mit den erforderlichen Maßnahmen zu rechnen. Dies führt dazu, daß der kommunale Finanzausgleich (KFA) ins Blickfeld rückt.

Die Zuweisungen aus dem Finanzausgleich machen in Hessen etwa einen Anteil von 26,5 v.H. an den kommunalen Einnahmen aus; die Schlüsselzuweisungen sind nach der Gewerbe- und Einkommensteuer die wichtigste Quelle für allgemeine ungebundene Deckungsmittel. Daß das Land sowohl das Volumen als auch die Verteilung der bereitgestellten Mittel bestimmen kann, hat nicht nur positive Auswirkungen. Dies kann an einer Vielzahl von kommunalen Verfassungsklagen in verschiedenen Bundesländern gegen einzelne Regelungen oder das gesamte System des Finanzausgleichs abgelesen werden, wenn es auch bis jetzt noch keine kommunalen Verfassungsklagen in Hessen gegen den Finanzausgleich gegeben hat. ${ }^{1}$

Vor diesem Hintergnund wird in dieser Untersuchung der Finanzausgleich und vor allem das System der Schlüsselzuweisungen in Hessen nach einer historischen Darstellung seiner maßgeblichen Entwicklungslinien ökonomisch analysiert, um mögliche Schwachstellen aufzudecken und Reformvorschläge zu unterbreiten. $\mathrm{Zu}$ diesem Zweck wird im zweiten Kapitel in einem ersten Schritt eine Eingruppierung der kommunalen Ebene in das verfassungspolitische Geflecht der Bundesrepublik Deutschland vorgenommen. Nach der Darstellung des internen Staatsaufbaus des Landes Hessen und seiner Entwicklung erfolgt im Überblick eine Betrachtung der verschiedenen von dieser Ebene wahrzunehmenden Aufgaben. Dem

Besonders hervorzuheben sind dabei die Klagen in Niedersachsen, da diese - im Gegensatz zu NRW - auch erfolgreich waren, so daß die Finanzausgleichsgesetze 1996 und 1998 jeweils für verfassungswidrig erklärt worden sind. Vgl.: Niedersächsischer Staatsgerichtshof: Beschluß vom 15.08.1995, in: DVBl., 110. Jg., 1995, S. 1175-1179; Ders.: Urteil vom 27.11.1997, in: DVBl., 113. Jg., 1998, S. 185-189. Für das Jahr 2000 steht die nächste Klage an. Stüer, B.; Ehebrecht-Stüer, E.-M.: Normenkontrollantrag des Vorsitzenden der CDU-Fraktion im Niedersächsischen Landtag, Christian Wulff, sowie der weiteren Mitglieder der CDU-Fraktion im Niedersächsischen Landtag, Gutachten im Auftrag der CDUFraktion des Niedersächsischen Landtags, Münster, o.J. 
schließt sich eine kurze Darstellung der tatsächlichen Einnahmen der Gemeinden und Gemeindeverbände (GV) an. Zuletzt werden die rechtlichen Grundlagen eines kommunalen Finanzausgleichs geschildert, und anschließend die Ermittlung der Finanzausgleichsmasse, die der kommunalen Ebene in Hessen zur Verfügung gestellt wird, beschrieben.

In Kapitel 3 werden die Aufgaben eines Finanzausgleichssystems auf kommunaler Ebene dargestellt und die von einem solchen System zu erfüllenden Kriterien entwickelt. Dieses Kriterienraster soll im weiteren Verlauf der Untersuchung helfen, mögliche Schwachpunkte in der derzeitigen Ausgestaltung zu erkennen.

Kapitel 4 stellt den Hauptteil der Arbeit dar. In ihm wird der kommunale Finanzausgleich in seiner historischen Entwicklung bis heute dargestellt und auf Verbesserungsmöglichkeiten hin untersucht, die dann auch aufgezeigt werden. Das Kapitel ist in vier Teile untergliedert. In jedem dieser Abschnitte findet zuerst eine kurze historische Darstellung bzw. die Erläuterung der grundlegenden systematischen Zusammenhänge statt. Dem schließt sich dann eine Beschreibung der derzeit geltenden Regelungen an. Abgeschlossen wird jeder dieser vier Bereiche mit einer Analyse der derzeitigen Situation und der Schilderung möglicher Reformvorschläge.

Im ersten dieser vier Abschnitte geht es um die Frage, ob das Volumen der vom Land den Kommunen bereitgestellten Finanzausgleichsmasse angemessen ist. Es geht also um die vertikale Verteilung der in Hessen verbleibenden gesamten Steuereinnahmen auf die staatliche und die kommunale Ebene mit Hilfe des kommunalen Finanzausgleichs. Da keine allgemeingültigen Indikatoren zur Beurteilung des Aufteilungsverhältnisses zur Verfügung stehen, wird als Vergleichsmaßstab der Durchschnitt der übrigen westdeutschen Flächenländer herangezogen und anschließend ein Vorschlag vorgestellt, um ein als angemessen empfundenes vertikales Aufteilungsverhältnis auch für die Zukunft zu sichern. Im engen $\mathrm{Zu}$ sammenhang mit der vertikalen Aufteilung der Mittel steht auch der Aspekt der konjunkturpolitischen Instrumentalisierung des KFA im Sinne einer antizyklischen Finanzpolitik. Denn die derzeitige der Stabilitätspolitik zuwiderlaufende Haushaltspolitik der kommunalen Ebene ist als nicht unproblematisch einzustufen. Sie beruht auf der stark konjunkturabhängigen Aufkommenselastizität der kommunalen Steuern. Zudem entwickelt sich auch die Verbundmasse des KFA aufgrund ihrer konjunkturellen Abhängigkeit von den staatlichen Steuereinnahmen prozyklisch, so daß das ebenfalls prozyklische Ausgabeverhalten der Kommunen noch verstärkt wird. Es ist deshalb zu überlegen, ob die der kommunalen Ebene zustehende Finanzausgleichsmasse auch in jedem Jahr in voller Höhe ausgeschüttet werden sollte oder ob nicht ein Anteil in einen Fonds einzustellen ist, um im Zeitablauf eine Glättung der kommunalen Einnahmenentwicklung 
anzustreben. Dabei ist der Anspruch der Kommunen auf die Mittel der Finanzausgleichsmasse, die aus konjunkturpolitischen Erwägungen in den Fonds eingezahlt werden, gesetzlich zu sichern, um sie dem Zugriff des Landes zu entziehen.

Im zweiten Abschnitt ist zu klären, wie die Verbundmasse auf die verschiedenen Zuweisungskategorien aufzuteilen ist. Hierbei geht es vor allem darum, die Anteile von zweckgebundenen und von autonomiefreundlichen ungebundenen Zuweisungen, hier vor allem der Schlüsselzuweisungen, zu bestimmen. Zum besseren Verständnis dieses Problems werden zuerst einmal die verschiedenen Zuweisungstypen und ihr jeweiliger Anteil im Zeitablauf dargestellt. Auch hier gibt es keine allgemeingültige Norm, die zur Aufteilung der Mittel genutzt werden kann. Deshalb wird als Vergleichsmaßstab die Entwicklung der Anteile in anderen westdeutschen Flächenländern herangezogen. An diesem Vergleich schließt sich ein Vorschlag an, den Anteil der ungebundenen Zuweisungen zu erhöhen, ohne die mit der Zweckbindung verfolgten Zielsetzungen außer acht zu lassen.

Im dritten Abschnitt gilt es zu klären, wie die für Schlüsselzuweisungen zur Verfügung stehenden Mittel auf die drei kommunalen Gruppen, kreisfreie Städte, Landkreise und kreisangehörige Gemeinden, verteilt werden sollen bzw. ob Argumente für eine Veränderung der bisherigen Aufteilung gefunden werden können. $\mathrm{Zu}$ diesem $\mathrm{Zweck}$ werden die Landkreise und ihre kreisangehörigen Gemeinden zum Gesamtkreis aggregiert und mit den kreisfreien Städte verglichen. Dem schließt sich dann noch eine Untersuchung darüber an, wie die dem kreisangehörigen Raum zur Verfügung gestellten Mittel auf die Landkreise und die kreisangehörigen Gemeinden zu verteilen sind.

Im letzten Abschnitt von Kapitel 4 wird untersucht, wie die auf die Teilschlüsselmassen der einzelnen Gruppen entfallenden Mittel intern zu verteilen sind. $\mathrm{Zu}$ diesem Zweck werden die für die gruppeninterne Verteilung relevanten Größen, Finanzbedarf, Finanzkraft und Ausgleichsmechanismus, der Reihenfolge nach dargestellt. Dabei werden jeweils allgemeine Überlegungen zum betreffenden Sachverhalt vorangestellt. Daran schließen sich Schilderungen der derzeitigen Regelungen an, die dann dahingehend geprüft werden, ob sie zielführend sind. Abschließend werden jeweils Reformvorschläge unterbreitet. Entsprechend werden zuerst die derzeit zur Bedarfsermittlung verwendeten Faktoren dargestellt und überprüft; ein besonderes Augenmerk gilt dabei der Behandlung der sog. Sonderstatusstädte. ${ }^{1}$ Diese Untersuchung erfolgt getrennt für das Bedarfsermittlungsverfahren der kreisfreien Städte und kreisangehörigen Gemeinden einerseits und jenem der Landkreise andererseits. Genauso ist die Vorgehensweise bei der

1 Den kreisangehörigen Gemeinden mit mehr als 50.000 Einwohnern wird bei der Aufgabenwahrnehmung eine besondere Stellung eingeräumt; sie werden deshalb als Sonderstatusstädte bezeichnet. 
Überprüfung der bisherigen Steuerkrafterfassung der kommunalen Ebene, die unter zwei Gesichtspunkten analysiert wird, nämlich ob der derzeit eingeschränkte Umfang der Berücksichtigung der Steuerkraft weiterhin beizubehalten ist oder ob Erweiterungen angebracht erscheinen. Unabhängig davon ist zu klären, wie die grundlegenden Unterschiede der Einnahmenkategorien - solche mit und solche ohne kommunaler Einflußmöglichkeit auf die jeweilige Aufkommenshöhe - besser zu berücksichtigen sind, um einen möglichst identischen Anrechnungsgrad zu erreichen. Ähnliche Aspekte sind auch bei der Umlagekraftberechnung der Landkreise zu beachten; allerdings wird dort die Situation durch das Vorhandensein der Sonderstatusstädte und der damit verbundenen Problematik bei der Bedarfsund der Umlagekraftberechnung verschärft. Um dieses Problem zu lösen, wird eine neue Herangehensweise vorgeschlagen, durch welche die bisherige durch die Sonderstatusstädte hervorgerufene Komplexität bzw. die verminderte Transparenz des Systems aufgehoben werden könnte. Zum Schluß wird noch das bisherige Ausgleichssystem von Finanzbedarf und Steuer- bzw. Umlagekraft betrachtet und - nachdem die Nachteile der derzeitigen Regelung angeführt wurden - ein modifizierter Ansatz vorgestellt, der um eine Abschöpfungskomponente erweitert ist.

Im abschließenden fünften Kapitel werden die Reformvorschläge noch einmal kurz zusammengefaßt.

Die der empirischen Untersuchung zugrunde liegenden Daten, vor allem bei der Betrachtung des hessischen kommunalen Finanzausgleichs, und rechtlichen Gegebenheiten beziehen sich auf das Jahr 1998. Später vorgenommene Gesetzesänderungen wurden bis zum 01.01 .2000 berücksichtigt. 


\section{Die kommunale Ebene in der Bundesrepublik Deutschland}

\subsection{Kommunale Selbstverwaltung im Grundgesetz und der Hessischen Verfassung}

\subsubsection{Verhältnis zwischen Staat und kommunaler Ebene}

Nach Art. 20 Abs. 1 GG ist die Bundesrepublik Deutschland ein Bundesstaat, dessen konstituierenden Ebenen der Bund und die Länder sind. Die Gemeinden und Gemeindeverbände (GV) stellen keine dritte staatliche Ebene neben dem Bund und den Ländern dar, sondern sind integraler Bestandteil der letzteren. ${ }^{1}$ Die Länder tragen somit die Verantwortung für ihre Gemeinden und vertreten deren Interessen gegenüber den übrigen Ländern und dem Bund im Bundesrat. Daraus ist abzuleiten, daß der Bund keine direkte Beziehung zu der kommunalen Ebene eingehen darf. ${ }^{2}$ Für die Bundesrepublik Deutschland ergibt sich somit ein zweistufiger Staatsaufbau. ${ }^{3}$ Davon zu unterscheiden ist die Verwaltungsgliederung. Diese ist vierstufig und verteilt sich auf Bund, Länder, Kreise und Gemeinden. Sie ermöglicht durch Dezentralisierung eine ortsnahe Ausführung der Aufgaben und unterstützt die Gewaltenteilung im Bundesstaat. ${ }^{4}$

Die kommunale Ebene - bestehend aus kreisfreien und kreisangehörigen Städten sowie den Landkreisen - nimmt dennoch aufgrund der expliziten Nennung und Sicherung ihrer Selbstverwaltungsrechte in der Verfassung eine besondere Stellung ein; ${ }^{5}$ sie wird noch dadurch betont, daß ihre Aufgaben als gleichwertig mit denen von Bund und Ländern anzusehen sind. ${ }^{6}$ Den Gemeinden ist nach Art. 28 Abs. 2

1 Vgl: Bundesverfassungsgericht: Urteil vom 27.05.1992, in: Entscheidungen des Bundesverfassungsgerichts, Bd. 86, Tübingen 1993, S. 215.

2 Vgl.: Dass.: Urteil vom 10.06.1969, in: Entscheidungen des Bundesverfassungsgerichts, Bd. 26, Tübingen 1970, S. 181. Eine Ausnahme stellt Art. 106 Abs. 8 GG dar, nach dem der Bund direkte Finanzbeziehungen zu Gemeinden in Form eines Sonderlastenausgleichs eingehen kann.

3 Vgl.: Maunz, T.: Kommentar zu Art. 28 GG, in: Maunz, T. u.a. (Hrsg.): Kommentar zum Grundgesetz (Loseblattsammlung), Stand 1998, Rdnr. 79.

4 Vgl.: Maurer, H.: Verfassungsrechtliche Grundlagen der kommunalen Selbstverwaltung, in: DVB1., 110. Jg., 1995, S. 1040.

5 Schutz für die Kommunen gegenüber dem Staat bietet auch noch Art. 115c Abs. 3 GG Dort wird bestimmt, daß auch im Verteidigungsfall "die Lebensfähigkeit der Länder, Gemeinden und Gemeindeverbände, insbesondere auch in finanzieller Sicht, zu wahren ist", woraus entnommen werden kann, daß die Selbstverwaltung der kommunalen Gebietskörperschaftsebene dem Verfassungsgeber ein wichtiges Anliegen gewesen ist.

" Vgl.: Deutscher Bundestag: Entwurf eines Gesetzes zur Änderung und Ergänzung der Finanzverfassung (Finanzverfassungsgesetz), eines Gesetzes zur Anpassung der Finanzbeziehungen zwischen Bund und Ländern an die Finanzverfassung (Finanzanpassungsgesetz) und eines Gesetzes über den Finanzausgleich unter den Ländern (Länderfinanzausgleichsgesetz) vom 29.04.1954, Drucksache II/480, Nr. 36. 
GG das Recht zugestanden worden, "alle Angelegenheiten der örtlichen Gemeinschaft im Rahmen der Gesetze in eigener Verantwortung zu regeln"; für die GV gilt dies im Rahmen der ihnen per Gesetz zugewiesenen Aufgabenbereiche. Durch die Betonung der räumlichen (hier: lokalen) Komponente bei der Aufgabenzuständigkeit wird verhindert, daß staatliche Aufgaben wie innere und äußere Sicherheit unter Bezugnahme auf die Allzuständigkeit von den Gemeinden übernommen werden. ${ }^{1}$ Die kommunale Selbstverwaltung wurde durch zwei Verfassungsreformen in den 90er Jahren gestärkt. 1994 wurde der Zusatz eingefügt, nach dem die finanzielle Eigenverantwortung zur Selbstverwaltung gehört, wobei es sich allerdings nicht um eine konstitutive Erweiterung sondern nur um eine $\mathrm{Be}$ stätigung der bestehenden Rechte handelt. ${ }^{3}$ Durch die Ändenung 1997 wurde dieser Passus präzisiert, in dem eine "wirtschaftskraftbezogene und mit Hebesatzrecht ausgestattete Steuerquelle" ${ }^{4}$ für die finanzielle Eigenverantwortung als notwendig angesehen wird. Damit entfiel die in der Vergangenheit geführte Diskussion, ob nur eigene Steuern oder auch schon Finanzzuweisungen zur Sicherung der kommunalen Selbstverwaltung genügen. ${ }^{5}$

Eine positive Abgrenzung der kommunalen Aufgaben wird weder im Grundgesetz noch in den Länderverfassungen mit Ausnahme Bayerns vorgenommen. Dort werden in Art. 83 Abs. 1 Bay. Verf. die lokale Verkehrs- und Wegeplanung, der Feuerschutz, das Kulturwesen usw. als gemeindliche Aufgaben aufgeführt. Nach Art. 137 Abs. 1 Hessische Verfassung (HV) gilt die Allverantwortlichkeit der örtlichen öffentlichen Verwaltung den Gemeinden prinzipiell als unbegrenzt übertragen, doch kann der Gesetzgeber durch Gesetze die Rechte der kommunalen Ebene einschränken, wobei diese aber nicht zu einer leeren Hülle verkommen dürfen. ${ }^{6}$ Folglich sind Zuständigkeitsverlagerungen von der kommunalen auf die staatliche Ebene nur "im dringenden öffentlichen Interesse" (Art. 137 Abs. 1 Satz

1 Vgl.: Bundesverfassungsgericht: Urteil vom 23.11.1988, in: Entscheidungen des Bundesverfassungsgerichts, Bd. 79, Tübingen 1989, S. 147; vgl. auch: Schnur, R.: Zur Abgrenzung der gemeindlichen Aufgaben von den Aufgaben der Kreise gemäss Art. 28 Abs. 2 GG, in: Die Verwaltung, Bd. 19, 1986, S. 46.

2 Vgl.: Gesetz zur Änderung des Grundgesetzes (Artikel 3, 20a, 28, 29, 72, 74, 75, 76, 77, 80, 87, 93, 118a und 125a) vom 27.10.1994, BGBl. I, S. 3146 und Gesetz zur Änderung des Grundgesetzes (Artikel 28 und 106) vom 24.10.1997, BGBI. I, S. 2470.

3 Vgl.: Maunz, T.; Scholz, R.: Kommentar zu Art. 28 GG, in: Maunz, T. u.a. (Hrsg.): Kommentar zum Grundgesetz (Loseblattsammlung), Stand 1997, Rdnr. 84a.

4 Vgl.: Art. 28 Abs. 2 Satz 3 GG.

5 Vgl.: Deutscher Bundestag: Entwurf eines ... Gesetzes zur Änderung und Ergänzung des Grundgesetzes (Finanzreformgesetz) vom 30.04.1968, Drucksache V/2861, Nr. 183; Bundesverfassungsgericht: Urteil vom 10.06.1969, in: Entscheidungen des Bundesverfassungsgerichts, Bd. 26, Tübingen 1970, S. 181.

6 Vgl.: Bundesverfassungsgericht: Urteil vom 24.07.1979, in: Entscheidungen des Bundesverfassungsgerichts, Bd. 52, Tübingen 1980, S. 117. 
$2 \mathrm{HV}$ ) und nicht schon bei Vorliegen rein zweckmäßiger Überlegungen möglich.' Um dies beurteilen zu können, müssen bestimmte Kriterien ${ }^{2}$ beachtet werden. Eine Kosteneinsparung im Verwaltungsbereich genügt nicht als Begründung für eine Verlagerung der Kompetenzen auf eine übergeordnete Gebietskörperschaftsebene, denn der Kostenminderung ist der gewichtigere Tatbestand der daraus resultierenden verringerten politischen Einflußnahme der Gemeindebürger entgegenzustellen. ${ }^{3}$ Ausdrücklich betont das Bundesverfassungsgericht, daß für die $\mathrm{Zu}$ weisung gemeindlicher Aufgaben auf höhere Ebenen die (fehlende) "Verwaltungskraft der Gemeinde" ${ }^{\prime 4}$ keine Rolle spielt. Denn durch Kooperation einzelner Gemeinden auch auf lokaler Ebene kann eine kosteneffiziente Erfüllung der Aufgaben erreicht werden. ${ }^{5}$

Das Recht auf Wahrnehmung der kommunalen Interessen wird durch die Möglichkeiten der kommunalen Verfassungsbeschwerde nach Art. 93 Abs. 1 Nr. 4b GG und § 91 BVerfGG verstärkt, denn Begriffe wie "örtliche Gemeinschaft" bzw. "eigene Verantwortung" sind auslegungsbedürftig. ${ }^{6}$ Im Falle der Verletzung der auf landesrechtlichen Vorschriften beruhenden Selbstverwaltung ist, wenn diese Möglichkeit besteht, bei den Landesverfassungsgerichten die Klage einzureichen. ${ }^{7}$ In diesem Fall gilt für das Bundesverfassungsgericht nach $\S 91$ Satz 2 BVerfGG eine Subsidiaritätsklausel ${ }^{8}$ gegenüber den Verfassungsgerichten der Länder.

1 Vgl.: Zezschwitz, F. v.: Kommentar zu Art. 137 HV, in: Zinn, G. A.; Stein, E.: Verfassung des Landes Hessen - Kommentar (Loseblattsammlung), Bad Homburg v. d. Höhe 1990, Erl. V 3 c).

2 Ein solches Kriterienraster geben sowohl das Bundesverfassungsgericht als auch der Niedersächsische Staatsgerichtshof vor. Siehe hierzu: Bundesverfassungsgericht: Urteil vom 23.11.1988, in: Entscheidungen des Bundesverfassungsgerichts, Bd. 79, Tübingen 1989, S. 153f. und Niedersächsischer Staatsgerichtshof vom 23.01.1974, in: Entscheidungen der Oberverwaltungsgerichte für das Land Nordrhein-Westfalen in Münster und für das Land Niedersachsen in Lüneburg mit Entscheidungen des Verfassungsgerichtshofes NordrheinWestfalen und des Niedersächsischen Staatsgerichtshofes (OVGE), Bd. 29, Münster 1976, S. 507f.

3 Vgl.: Bundesverfassungsgericht: Urteil vom 23.11.1988, in: Entscheidungen des Bundesverfassungsgerichts, Bd. 79, Tübingen 1989, S. 153.

4 Dass.: Urteil vom 23.11.1988, in: Entscheidungen des Bundesverfassungsgerichts, Bd. 79, Tübingen 1989, S. 152.

5 Vgl.: Püttner, G.: Kommunale Selbstverwaltung, in: Isensee, J.; Kirchhof, P. (Hrsg.): Handbuch des Staatsrechts, Bd. 4, Heidelberg 1990, S. 1177 f.

6 Vgl.: Stern, K.: Das Staatsrecht der Bundesrepublik Deutschland, Bd. 1, 2. Aufl., München 1984, S. 408.

7 Vgl: Bundesverfassungsgericht: Urteil 07.05.1957, in: Entscheidungen des Bundesverfassungsgerichts, Bd. 6, Tübingen 1957, S. 380f. Dies ist in Hessen erst seit der Änderung des Gesetzes über den Staatsgerichtshof aus dem Jahr 1994 möglich.

8 Vgl.: Stern, K.: Die Verfassungsgarantie der kommunalen Selbstverwaltung, in: Püttner, G. (Hrsg.): Handbuch der kommunalen Wissenschaft und Praxis, Bd. 1, 2. Aufl., Berlin u.a.O 


\subsubsection{Verhältnis zwischen Gemeinden und Gemeindeverbänden}

Neben der Abgrenzung der Befugnisse der kommunalen gegenüber der staatlichen Ebene spielt auch die Aufgabenverteilung innerhalb der kommunalen Ebene eine Rolle. Als Gemeinden im Sinne des Grundgesetzes gelten kreisangehörige und kreisfreie Städte. Unter dem verfassungsrechtlichen Begriff der Gemeindeverbände werden die Landkreise, Samtgemeinden, aber nicht Zweckverbände wie der Umlandverband Frankfurt subsumiert, da letztere Bundkörperschaften ${ }^{1}$ darstellen. ${ }^{2}$ Die Gemeinden stellen die unterste Stufe der Verwaltung dar. Sie befassen sich mit den örtlichen Aufgaben der Selbstverwaltung ( $\$ 2$ HGO). Die zweite Stufe besteht aus den Landkreisen und den kreisfreien Städten. ${ }^{3}$ Letztere sind aufgrund ihrer Größe und Finanzkraft in der Lage, für ihr Gebiet sowohl die örtlichen als auch die überörtlichen Selbstverwaltungsaufgaben zu erfüllen, während die Kreise überörtliche Funktionen für ihre Gemeinden ausüben. Hierunter fallen alle Aufgaben, die sich aufgrund ihrer Raumbezogenheit nicht nur auf das jeweilige Gebiet einer Gemeinde beschränken. ${ }^{4}$

Da nach Art. 28 Abs. 2 Satz 1 GG alle Gemeinden - im Rahmen der Gesetze - für die Erfüllung der örtlichen Selbstverwaltungsaufgaben zuständig sind, dürften keine Abgrenzungsprobleme zu den Kreisen und deren Aufgaben bestehen. Allerdings ist auch von Seiten der Kreise die Gefahr der Aneignung von Kompetenzen des gemeindlichen Bereichs gegeben, da in den Landkreisordnungen der Länder neben der Erfüllung überörtlicher Aufgaben noch ergänzende und ausgleichende Funktionen aufgeführt sind, durch die die eingeschränkte Leistungsfähigkeit der Gemeinden kompensiert werden soll. ${ }^{5}$ So sollen die Kreise nach $\S 2$ der Hessischen Landkreisordnung (HKO) auch die örtlichen Aufgaben erfüllen, "die über die Leistungsfähigkeit der kreisangehörigen Gemeinden hinausgehen" und zudem einen "gerechten Ausgleich der unterschiedlichen Belastung" innerhalb des Kreises ermöglichen. Bei den ergänzenden Funktionen geht es darum, daß wegen fehlender Leistungsfähigkeit einzelne Gemeinden Aufgaben nicht ausführen können und diese deshalb vom Kreis zu übernehmen sind.

Durch die Ausgleichsfunktion soll eine akzeptable Verteilung der Lasten zwischen den Gemeinden erreicht und gleichzeitig auch eine gleichmäßige

1981, S. 227.

1 Bundkörperschaften sind dadurch gekennzeichnet, daß sie - im Gegensatz zu Gebietskörperschaften - von juristischen Personen (Mitgliedsstädte des Umlandverbandes Frankfurt) und nicht von den Bewohnern des Gebietes (Kreiseinwohner) getragen werden.

2 Vgl.: Stern, K.: Das Staatsrecht der Bundesrepublik Deutschland, Bd. 1, 2. Aufl., München 1984, S. 417.

3 Vgl.: Ellwein, R; Hesse, J.J.: Staatsreform in Deutschland - das Beispiel Hessen, hrsg. vom Bund der Steuerzahler Hessen e.V., Wiesbaden 1997, S. 35.

4 Vgl.: Pagenkopf, H.: Kommunalrecht, Köln u.a.O. 1971, S. 261.

5 Vgl.: Ebenda, S. 260. 
Versorgung der Einwohner angestrebt werden. Dazu wird vom Kreis ein einnahmen- und ein ausgabenseitiger Ausgleich vorgenommen. Dies geschieht dadurch, da $\beta$ auf der einen Seite die Kreisumlage an die gemeindliche Finanzkraft als Bemessungsgrundlage anknüpft und somit die absoluten Finanzkraftunterschiede mindert. ${ }^{1}$ Auf der anderen Seite werden einmal Aufgaben durch den Kreis wahrgenommen, die finanzschwache Gemeinden nicht hätten ausüben können, und es werden zusätzlich noch Zuweisungen gewährt. ${ }^{2}$ So müssen die hessischen Kreise in jedem Jahr einen Betrag dem Kreisausgleichsstock (§ $42 \mathrm{FAG}$ ) zuführen, dessen Volumen mindestens 1 v.H. der Höhe der den Gemeinden zufließenden Schlüsselzuweisungen zu entsprechen hat. Diese Mittel dienen den Kreisen "zum Ausgleich außergewöhnlicher Belastungen ihrer Gemeinden"3 4

Es besteht nun die Gefahr, daß die Kreise mit Verweis auf ihre ergänzende Funktion gemeindliche Aufgaben an sich ziehen, obwohl nicht alle Gemeinden des Kreises eine ungenügende Leistungsfähigkeit bei der Erfüllung dieser Aufgabe aufweisen. Dieser Vorgang widerspricht aber der subsidiären Stellung der Kreise zu ihren Gemeinden, da die Priorität der gemeindlichen Aufgabenerfüllung "zugunsten kreisangehöriger Gemeinden auch gegenüber den Kreisen"s und nicht nur gegenüber dem Staat gilt. ${ }^{6}$ Trotz der Ausgleichs- und Ergänzungsfunktionen der Kreise handelt es sich um örtliche Aufgaben, die somit den Gemeinden vorbehalten bleiben und nur aus Ermangelung der gemeindlichen Erfüllung durch den Kreis ausgeübt werden, denn bei Veränderung der gemeindlichen Situation ist eine Rückübertragung denkbar und sinnvoll. ${ }^{7}$ Sind einige Gemeinden in der Lage, die entsprechenden Aufgaben selbständig auszuführen, so sind sie für diese Orte nicht vom Kreis wahrzunehmen, es sei denn, daß eine einheitliche Ausführung unumgänglich ist. So haben die Kreise nach $\S 19$ HGO die Möglichkeit, durch Beschluß des Kreistages und Zustimmung der Aufsichtsbehörde Aufgaben an sich

1 Die Kreisumlage dient der Finanzierung der Kreise, da ihnen keine originären Steuereinnahmen im ausreichenden Umfang zustehen. Jeder Kreis erhebt einen individuellen Umlagesatz auf die gemeindliche Finanzkraft.

2 Vgl.: Schnur, R.: Zur Abgrenzung der gemeindlichen Aufgaben von den Aufgaben der Kreise gemäss Art. 28 Abs. 2 GG, in: Die Verwaltung, Bd. 19, 1986, S. 51-53.

3 Vgl.: Käss, W.: Der kommunale Finanzausgleich und finanzielle Zuweisungen des Landes zur Erfüllung öffentlicher Aufgaben, Erläuterungen zu § 42 FAG, in: Praxis der Gemeindeverwaltung, Hessen, Bd. E1, (Loseblattsammlung), Stand 1999.

4 Die bis 1992 für die Kreise geltende Verpflichtung, die Summe des Ausgleichstocks jährlich an die Gemeinden zu verteilen ( $\$ 42$ Satz 3 FAG), wurde abgeschafft, damit Vorsorge fur zukünftige Belastungen getroffen werden kann.

5 Bundesverfassungsgericht: Urteil vom 23.11.1988, in: Entscheidungen des Bundesverfassungsgerichts, Bd. 79, Tübingen 1989, S. 150.

6 Vgl.: Stern, K.: Das Staatsrecht der Bundesrepublik Deutschland, Bd. 1, 2. Aufl., München 1984, S. 418.

7 Vgl:: Schoch, F.: Aufgaben und Funktionen der Landkreise, in: DVBl., 110. Jg., 1995, S. 1050. 
zu ziehen. Da die Mandatsträger in den Kreistagen mit ihren Gemeinden verbunden sind, wird das Verfahren auf diese Art demokratisch legitimiert. ${ }^{1}$

Eine andere Vorgehensweise besteht darin, auf eine gruppenspezifische Behandlung der Gemeinden zurückzugreifen, indem einer Klasse von Großgemeinden Aufgaben des Kreises übertragen werden ${ }^{2}$; so unterteilt die HGO die kreisangehörigen Gemeinden entsprechend ihrer Leistungskraft und weist ihnen im unterschiedlichen Umfang Aufgaben zu. Den kreisangehörigen Gemeinden mit mehr als 50.000 Einwohnern (Sonderstatusstädte) gilt die Zuständigkeit für die örtliche Sozialhilfe als vollständig übertragen. Dies wird Gemeinden über 7.500 Einwohnern nur auf Antrag zugestanden. ${ }^{3}$ Festzuhalten ist, daß Art. 28 Abs. 1 GG eine Abwehrfunktion sowohl gegen den Staat als auch gegen die Kreise enthält. ${ }^{4}$

\subsubsection{Struktur und historische Entwicklung des Bundeslandes Hessen}

\subsubsection{Staatliche Ebene}

Das Bundesland Hessen wurde 1946 gegründet $^{5}$, wobei die Rücksichtnahme auf vorhandene politische, wirtschaftliche und geographische Belange nur gering ausgeprägt war. So wurde das um das linksrheinische Rheinhessen verkleinerte Gebiet des früheren Volksstaates Hessen mit Teilen der ehemaligen preußischen Provinz Hessen-Nassau zum Land Hessen vereinigt. ${ }^{6}$ Die sich durch die Übernahme der historischen Verwaltungsstrukturen ergebende Situation wurde als sehr reformbedürftig angesehen, da ein unterschiedliches Rechts- und Verwaltungssystem in den beiden Landesteilen gegeben war und der hessische Regierungsbezirk Darmstadt aus zwei räumlich getrennten Teilen bestand. ${ }^{7}$ So gehörte die Stadt Offenbach zum Regierungsbezirk Darmstadt, während Frankfurt und Hanau dem Regierungsbezirk Wiesbaden zugeteilt waren; aufgrund der engen Verflechtung dieses Raums mußten für notwendige gemeinsame Planungen mehrere staatliche

1 Vgl.: Henneke, H.-G.: Möglichkeiten zur Stärkung der kommunalen Selbstverwaltung, in: DÖV, 47. Jg., 1994,S. 712.

2 Vgl.: Püttner, G.: Kommunale Selbstverwaltung, in: Isensee, J.; Kirchhof, P. (Hrsg.): Handbuch des Staatsrechts, Bd. 4, Heidelberg 1990, S. $1181 \mathrm{f}$.

3 Vgl: $\S 4$ Hessisches Ausführungsgesetz zum Bundesozialhilfegesetz (HAG/BSHG) vom 28.05.1962 in der Fassung vom 16.09.1970.

4 Vgl.: Knemeyer, F.-L.: Das verfassungsrechtliche Verhältnis der Kommunen zueinander und zum Staat, in: DVBl., 99. Jg., 1984, S. 23.

5 Verfassung des Landes Hessen vom 01.12.1946, Hess. GVBI. I, S. 229.

6 Vgl.: Pittermann, W.: Entwicklung in der Organisation der Landesverwaltung - Grundlinien, Schnittstellen, Funktionalreform, in: Stein, E. (Hrsg.): 30 Jahre Hessische Verfassung 1946-1976, Wiesbaden 1976, S. 322.

7 Vgl: Püttner, G.: Stufenweise Reform. Stand und Methode der Verwaltungsreform in Hessen, in: AfK, 8. Jg., 1969, S. 270; Berger, H.: Verwaltungsreform in Hessen, in: DÖV, 22 Jg., 1969, S. 449; Pünder, T.: Der Landeswohlfahrtsverband Hessen und seine Probleme, in: Hessische Städte- und Gemeinde-Zeitung, 35. Jg., 1985, S. 462. 
Instanzen beteiligt werden. Zur Vorbereitung der anstehenden Reformen wurde 1947 eine Kommission gebildet, die u.a. eine starke Verringerung der Zahl der Landkreise und Städte vorschlug. ${ }^{1}$ Als erstes Resultat der Reformbemühungen ist die Neuordnung der Mittelstufe der Verwaltung aus dem Jahre $1953 \mathrm{zu}$ nennen. ${ }^{2}$ Durch sie wurde der Landeswohlfahrtsverband (LWV) gegründet, der die Aufgabenerfüllung der überörtlichen Daseinsvorsorge bündelte und vereinheitlichte. ${ }^{3} \mathrm{Er}$ finanziert sich über eine von den Kommunen zu zahlende Umlage.

Weitere Reformbemühungen wurden durch Vorschläge der CDU/F.D.P.-Opposition zur Reform der Regierungsbezirke bzw. zu deren Abschaffung eingeleitet. ${ }^{4}$ Die von der SPD-Regierung daraufhin einberufene Sachverständigenkommission plädierte aber für die Beibehaltung der Regierungsbezirke als Mittelinstanz der Verwaltung. ${ }^{5}$ Unklar war allerdings noch, ob neben dem Regierungsbezirk Kassel in Nordhessen die Schaffung eines - den zweigeteilten Regierungsbezirk Darmstadt und den Regierungsbezirk Wiesbaden umfassenden - Bezirks oder jeweils für Süd- und Mittelhessen eine eigenständige Mittelstufe angestrebt werden sollte. Aufgrund der vorhandenen Vernetzung wesentlicher Teile Mittelhessens mit der Rhein-Main-Region wurde eine separate Verwaltungsinstanz für Mittelhessen nicht als sinnvoll angesehen, da "eine verwaltungsmäßige Trennung unnatürlich wäre und in Zukunft erneut Schwierigkeiten bereiten würde"6. Somit bestanden nach Gesetzesverabschiedung ${ }^{7}$ zwei sehr unterschiedliche Regierungsbezirke, nämlich Darmstadt mit 24 Landkreisen, 6 kreisfreien Städten, fast 4 Mio. Einwohnern und einer Fläche von 11,9 Mio. $\mathrm{km}^{2}$ und Kassel mit 15 Landkreisen, 3 kreisfreien Städten mit insgesamt mehr als 1,3 Mio. Einwohnern auf ungefähr 9,2 Mio. $\mathrm{km}^{2}{ }^{8}$ Letzterem wurde im Zuge der kommunalen Kreisreform 1974 noch

1 Vgl.: Kabinetts-Kommission zur Vorbereitung der Verwaltungsreform: Die Verwaltungsreform in Hessen, Bd. I, Wiesbaden 1947, S. 11-15.

2 Gesetz über die Mittelstufe der Verwaltung und den Landeswohlfahrtsverband vom 07.05.1953, Hess. GVB1. I, S. 93.

3 Vgl.: Pünder, T.: Der Landeswohlfahrtsverband Hessen und seine Probleme, in: Hessische Städte- und Gemeinde-Zeitung, 35. Jg., 1985, S. 462.

4 Vgl: Hessischer Landtag: Antrag der Fraktion der CDU betreffend Verwaltungsreform in Hessen vom 04.05.1965, Drucksache 5/1361, gleichlautend vom 21.01.1966 Drucksache 6/681; Initiativantrag der Fraktion der F.D.P. betreffend den Entwurf eines Gesetzes über die Bildung von Regionen vom 24.01.1966, Drucksache 5/1681, gleichlautend vom 30.01.1968 Drucksache 6/939.

5 Vgl.: Hessischer Minister des Innern (Hrsg.): Verwaltungsreform in Hessen. Bestandsaufnahme, Maßnahmen, Überlegungen, Vorausschau, Wiesbaden 1968, S. 21-24.

6 Vgl: Hessischer Landtag: Gesetz über die Grenzen der Regierungsbezirke und den Dienstsitz der Regierungspräsidenten vom 23.11.1967, Drucksache 6/857, S. 3.

7 Vgl: Gesetz über die Grenzen der Regierungsbezirke und den Dienstsitz der Regierungspräsidenten vom 29.04.1968, Hess. GVBI. I, S. 119.

8 Vgl: Statistisches Bundesamt: Statistisches Jahrbuch der Bundesrepublik Deutschland 1969, Stuttgart - Mainz 1969, S. 27 f. 
das Gebiet des vormaligen Landkreises Biedenkopf zugeordnet. ${ }^{1}$ Im Jahr 1981 wurde das geographische "Mittelhessen ${ }^{2}$ aus seiner organisatorischen Randlage" 3 befreit, indem dieses Gebiet vom Regierungsbezirk Darmstadt abgespalten wurde und den Regierungsbezirk Gießen bildete. Dies sollte zudem der Schaffung neuer Arbeitsplätze in der öffentlichen Verwaltung in dieser Region dienen, allerdings nicht auf Kosten der übrigen Mittelstufenverwaltung, da dort eine personelle Mindestausstattung gesichert bleiben sollte. ${ }^{4}$ Seitdem wurde die Struktur der hessischen Mittelinstanz nicht mehr verändert.

\subsubsection{Kommunale Ebene}

Nachdem die Bezirksreform abgeschlossen war, hat Hessen - wie auch die übrigen Flächenländer der Bundesrepublik ${ }^{5}$ - Ende der 60er Jahre mit einer Verwaltungs- und Territorialreform auf kommunaler Ebene begonnen. Sie wurde durch die Regierungserklärung vom 18.01.1967 eingeleitet, in der es hieß, daß eine groBe Anzahl von Gemeinden "nicht mehr in der Lage ist, ihre Aufgaben verwaltungsmäßig zu bewältigen"6. Ziel war es, die kommunale Ebene wieder in die Lage zu versetzen, die ihr zustehenden und zugewiesenen Aufgaben zu erfüllen, um somit gleichzeitig eine (präferenzorientierte) bürgernahe und effiziente Bereitstellung und Erfüllung der öffentlichen Leistungen zu ermöglichen. ${ }^{7}$

Die gestiegenen Ansprüche der Bürger an ihre örtliche Verwaltung konnte ein Großteil der Orte aufgrund der nach dem Ende des Krieges unveränderten Gemeindestruktur nicht erfüllen, da (31.12.1969) von den 2.642 Gemeinden 1.835 (69,5 v.H.) weniger als 1.000 Einwohner hatten, 1.133 (42,9 v.H.) Gemeinden sogar weniger als 500 Einwohner. $^{8}$ Die von einer einberufenen

1 Vgl.: Gesetz zur Neugliederung der Landkreise Biedenkopf und Marburg und der Stadt Marburg (Lahn) vom 12.03.1974, Hess. GVBl. I, S. 154.

2 "Eine solche Region hat es in der geschichtlichen Entwicklung des Landes Hessen nie gegeben", Pletsch, A.: Bausteine der hessischen Identität, in: Heidenreich, B.; Schacht, K. (Hrsg.): Hessen: eine politische Landeskunde, Stuttgart 1993, S. 46-51.

3 Hessischer Landtag: Regierungserklärung des Hessischen Ministerpräsidenten Börner, 9. Wahlperiode, Plenarprotokolle, 2. Sitzung vom 13.12.1978, S. 21.

4 Vgl.: Ders.: Gesetzentwurf der Landesregierung für ein Gesetz zur Neuorganisation der Regierungsbezirke und der Landesplanung vom 23.06.1980, Drucksache 9/3135, S. 13-16.

5 Vgl.: Mattenklodt, H.-F.: Territoriale Gliederung - Gemeinden und Kreise vor und nach der Gebietsreform, in: Püttner, G. (Hrsg.): Handbuch der kommunalen Wissenschaft und Praxis, Bd. 2, 2. Aufl., Berlin u.a.O. 1981, S.154-182.

6 Hessischer Landtag: Regierungserklärung des Hessischen Ministerpräsidenten Dr. Zinn, 6. Wahlperiode, Stenographische Berichte, 3. Sitzung vom 27.01.1967, S. 19.

7 Vgl.: Hessischer Minister des Innern (Hrsg.): Verwaltungsreform in Hessen. Bestandsaufnahme, Maßnahmen, Überlegungen, Vorausschau, Wiesbaden 1968, S. 29; Hessischer Landtag: Vorlage der Landesregierung betreffend den Entwurf furr ein Gesetz zur Neugliederung der Landkreise Alsfeld und Lauterbach vom 01.03.1972, Drucksache 7/1370, S. 7f.

8 Vgl: : Ebenda. 
Sachverständigenkommission vorgetragenen Vorschläge sahen eine pflichtige $\mathrm{Zu}$ sammenlegung von Kleinstgemeinden sowie die Bildung von Verwaltungsgemeinschaften (Zusammenlegung von verwaltungstechnischen Aufgaben und Kassengeschäften unter Beibehaltung der politischen Selbständigkeit) vor. ${ }^{1}$ Sie dienten als Grundlage für die weiteren Reformschritte. ${ }^{2}$ Darauf aufbauend führte das Innenministerium für die Gemeinden und die Landkreise eine Modellplanung durch, bei der unterschiedliche Neuordnungsmöglichkeiten dargelegt wurden. ${ }^{3}$ Diese sollten in zwei Stufen, einer ersten freiwilligen und einer zweiten gesetzlich geregelten, ablaufen. Gegen die zweite Stufe wurde von kommunaler Ebene unter Berufung auf die nach Art. 28 GG und Art. 137 HV besonders geschützte kommunale Selbstverwaltung vor dem Bundesverfassungsgericht vergeblich geklagt. ${ }^{4}$ Denn durch das GG ist nur die Institution der kommunalen Gebietskörperschaft als solche geschützt, nicht aber der Bestand einzelner Gemeinden oder einzelner Kreise. $^{5}$

In der ersten Phase sollten freiwillige Zusammenschlüsse von Gemeinden bzw. gemeinschaftliche Verwaltungen die größten Probleme vermindern. Dazu wurden einerseits entsprechende finanzielle Unterstützungen in Form erhöhter Schlüsselzuweisungen für die den Gemeindezusammenschlüssen folgenden 10 Jahre gewährt. ${ }^{6}$ Dies konnte zu zusätzlichen Einnahmen zwischen 100.000 und 500.000 DM pro Jahr führen. ${ }^{7}$ Weiterhin wurden mit dem Gesetz über kommunale Gemeinschaftsarbeit (KGG) die rechtlichen Voraussetzungen geschaffen, um selbständigen Gemeinden die Möglichkeit für die gemeinschaftliche Aufgabenerfüllung zu geben. ${ }^{8}$ Allerdings wurde diese Möglichkeit nur in geringem Umfang genutzt. ${ }^{9}$ Dies kann wohl als Indiz dafür angesehen werden, daß das langfristige

1 Vgl:: Sachverständigenkommission für Verwaltungsreform und Verwaltungsvereinfachung in Hessen: Zur Stärkung der Verwaltungskraft der Gemeinden, Veröffentlichungen 2, Wiesbaden 1968, S. 7f.

2 Vgl.: Püttner, G.: Stufenweise Reform. Stand und Methode der Verwaltungsreform in Hessen, in: AfK, 8. Jg., 1969, S. 268.

3 Vgl: Hessischer Minister des Innern (Hrsg.): Zur Planung der gebietlichen Neuordnung auf Gemeindeebene in Hessen, o.0. 1969; ders.: Vorschläge zur gebietlichen Neugliederung auf der Kreisebene in Hessen, 0.0. 1971.

4 Ders.: Hessen - Gemeinden und Landkreise nach der Gebietsreform, Melsungen 1977, S. 60f.

$5 \mathrm{Vgl}$ : Maunz, T.: Kommentar zu Art 28 GG, in: Maunz, T. u.a. (Hrsg.): Kommentar zum Grundgesetz (Loseblattsammlung), Stand 1998, Rdnr. 45.

6 Vgl.: $§ 9$ Abs. 2 Nr. 3 Satz 3 des Gesetzes zur Regelung des Finanzausgleichs (Finanzausgleichsgesetz - FAG -) vom 17.01.1966, Hess. GVBI. I, S. 1.

7 Vgl: Herbel, A.: Verwaltungs- und Gebietsreform in Hessen, in: Kommunalwirtschaft, 1971, S. 322.

8 Vgl.: Gesetz über kommunale Gemeinschaftsarbeit (KGG) vom 16.12.1969, Hess. GVBl. I, S. 307.

9 Vgl.: Muntzke, H.: Wie soll die Verwaltungsreform auf der kommunalen Ebene in Hessen fortgefuhrt werden?, in: Der Gemeindetag, 23. Jg., 1970, S. 392. 
Ziel - nämlich die Bildung großer Gemeinden - erkannt wurde. Für diese Sichtweise spricht auch die Entwicklung der Gemeindezahlen in diesen Jahren; so hat sich die Zahl der Gemeinden zwischen dem 31.12.1969 und dem 31.12.1971 aufgrund freiwilliger Zusammenschlüsse und Eingemeindungen von 2.642 um 1.409 (53,3 v.H.) auf 1.233 verringert. ${ }^{1}$

In einer zweiten Phase wurden - getrennt nach Landesteilen - Gemeinde- und Kreisreform aufgrund von Interdependenzen simultan vorgenommen. Denn es hatte sich herausgestellt, daß sinnvolle Gemeindezusammenschlüsse nicht unter Beibehaltung der bestehenden Kreisgrenzen erfolgen konnten. Dabei wurde die Schaffung von Gemeinden angestrebt, die in der Lage sind, alle ihnen übertragenen Aufgaben eigenständig zu erfüllen. ${ }^{2}$ Ein Problem stellten allerdings die kreisfreien Städte mit weniger als 100.000 Einwohnern dar ${ }^{3}$, weil ihre Leistungsfähigkeit zur alleinigen Erfüllung aller Aufgaben der kommunalen Ebene als zu gering angesehen wurde. Zudem erfordert die enge Verflechtung der Stadt mit ihrem Umland eine einheitliche Verwaltung, denn regionale Planungsgemeinschaften erweisen sich als unbrauchbar. ${ }^{4}$ "Nur eine Gebietskörperschaft mit eigener parlamentarischer Legitimation, eigenem Haushaltsrecht und eigenen Exekutivorganen ist in der Lage"s, eine zukunftsorientierte und einheitliche Planung, Finanzierung und Ausführung für das gesamte Gebiet zu erreichen. Aus diesen Gründen verloren diese Städte ${ }^{6}$ den Status einer kreisfreien Stadt. ${ }^{7}$ Dies betraf Hanau, Fulda und Marburg, denen vorübergehend ein finanzieller Ausgleich durch Schlüsselzuweisungen bzw. Verringerung der Kreisumlagen gewährt wurde. ${ }^{8}$

1 Vgl.: Voit, H.: Die kommunale Gebietsreform in Hessen, in: 30 Jahre Hessische Verfassung, hrsg. von Stein, E., Wiesbaden 1976, S. 370.

2 Vgl.: o.V.: Vereinbarung zur Bildung einer Koalitionsregierung im Land Hessen, in: Der Gemeindetag, 23. Jg. 1970, S. 396; Hessischer Landtag: Vorlage der Landesregierung betreffend den Entwurf für ein Gesetz zur Neugliederung der Landkreise Alsfeld und Lauterbach vom 01.03.1972, Drucksache 7/1370, S. 9.

3 In Hessen erhält heute eine kreisangehörige Gemeinde mit mehr als 100.000 Einwohnern den Status einer kreisfreien Stadt.

4 Vgl.: Hessischer Landtag: Vorlage der Landesregierung betreffend den Entwurf für ein Gesetz zur Neugliederung der Landkreise Fulda und Hünfeld und der Stadt Fulda vom 30.03.1972, Drucksache 7/1499, S. 9f.

5 Ebenda, S. 47.

6 Ähnliche Begründungen wurden auch bei der Rückkreisung der Städte Hanau (Drucksache 7/3837 vom 17.08.1973) und Marburg (Drucksache 7/3836 vom 17.08.1973) angeführt.

7 Vgl: Voit, H.: Die kommunale Gebietsreform in Hessen, in: 30 Jahre Hessische Verfassung, hrsg. von Stein, E., Wiesbaden 1976, S. 375; Hessischer Landtag: Vorlage der Landesregierung betreffend den Entwurf für ein Gesetz zur Neugliederung der Landkreise Fulda und Hünfeld und der Stadt Fulda vom 30.03.1972, Drucksache 7/1499, S. 47.

8 Vgl.: Finanzausgleichsgesetze; § 42a vom 19.03.1974, Hess. GVBl. I, S. 153; § 42a vom 21.12.1976, Hess. GVBI. I, S. 530; $\$ 47$ vom 28.12.1977, Hess. GVBl. I, S. 491; Art. 4a vom 16.05.1979, Hess. GVBI. I, S. 88 und Art. 3 vom 20.12.1979, Hess. GVB1. I, S. 14. 
Bei der ebenfalls weniger als 100.000 Einwohner zählenden Stadt Gießen wurde ein anderer Ansatz gewählt. In den einzelnen Regionen sollten kreisfreie Städte als Oberzentren zentralörtliche Aufgaben wahrnehmen und somit für das Umland entsprechende Leistungen vorhalten. Für die Region Südhessen waren dies die Städte Frankfurt sowie Wiesbaden, Darmstadt und Offenbach und für Nordhessen Kassel. Im Bereich Mittelhessen sollte eine neu gegründete kreisfreie Stadt mit einer entsprechenden Leistungsfähigkeit und Größe diese oberzentralen Funktionen übernehmen und als "Innovationskern"1 dienen, wodurch für diese Region verbesserte Entwicklungschancen erwartet wurden. Zu diesem Zweck wurden die Städte Gießen und Wetzlar sowie weitere kleinere Umlandgemeinden zur Stadt Lahn zusammengeschlossen. Für diesen Zusammenschluß sprach einmal die unterschiedliche Struktur der beiden Städte (Wetzlar industriell geprägt, Gießen dienstleistungsorientiert), so daß jeweils eine gegenseitige Ergänzungsfunktion gegeben war. ${ }^{2}$ Zum anderen sollte durch eine gelenkte Entwicklung mittels einer einheitlichen Verwaltung die unkoordinierte Vorgehensweise des Rhein-MainGebietes vermieden werden. ${ }^{3}$ Am Ende der Reformaßnahmen sollten 20 (vorher 39) Landkreise und 6 (vorher 9) kreisfreie Städte bestehen. ${ }^{4}$

Aufgrund politischer Widerstände ${ }^{5}$ wurde die Stadt Lahn aufgelöst und wieder die kreisangehörigen Städte Gießen und Wetzlar gebildet. ${ }^{6}$ Anschließend wurde für kreisangehörige Gemeinden mit mehr als 50.000 Einwohnern, hierzu gehören neben den ehemals kreisfreien Städten Fulda, Gießen, Hanau und Marburg noch Bad Homburg und Rüsselsheim sowie Wetzlar, ein Sonderstatus geschaffen, indem ihnen zusätzliche Aufgaben wie beispielsweise aus den Bereichen der örtlichen Sozialhilfe, der unteren Bauaufsichtsbehörden und der Schulträgerschaft

1 Hessischer Minister des Innern (Hrsg.): Hessen - Gemeinden und Landkreise nach der Gebietsreform, Melsungen 1977, S. 50.

2 Vgl: Hessischer Landtag: Gesetzentwurf der Landesregierung für ein Gesetz zur Neugliederung des Dillkreises, der Landkreise Gießen und Wetzlar und der Stadt Gießen vom 15.01.1974, Drucksache 7/4685, S. 11-15.

3 Vgl: Borchmann, M.: Stadt Lahn - Gebietsreform ohne Abschluß?, in: DVB1., 93. Jg., 1978, S. 789.

4 Vgl.: Hessischer Minister des Innern (Hrsg.): Hessen - Gemeinden und Landkreise nach der Gebietsreform, Melsungen 1977, S. 38.

5 Vgl.: Hessischer Landtag: Ausführungen des Abgeordneten Wilke (F.D.P.), 9. Wahlperiode, Stenographische Berichte, 6. Sitzung vom 21.03.1979, S. 276. Die CDU-Opposition konnte ihren Stimmenanteil in der Stadt Lahn bei den Kommunalwahlen 1977 um mehr als 20 Prozentpunkte erhöhen, während die Regierungsparteien SPD und F.D.P. zusammen 16 Prozentpunkte ihrer Stimmen einbüßten; vgl.: Häßler, G.: Die Kommunalwahlen in Hessen am 20. März 1977 - Teil 1: Gemeindewahlen in den kreisfreien Städten und Kreiswahlen, in: Hessisches Statistisches Landesamt Wiesbaden: Staat und Wirtschaft in Hessen, 32. Jg., 1977, S. 93.

6 Vgl.: Thieme, W.; Prillwitz, G.: Durchführung und Ergebnisse der kommunalen Gebietsreform, Baden-Baden 1981, S. 236. 
übertragen wurden. ${ }^{1}$ Dadurch wird "diesen Städten praktisch der Status einer kreisfreien Stadt" ${ }^{\prime 2}$ bei den weisungsfreien Pflichtaufgaben (siehe Abschnitt 2.2.1.2.) gewährt. Sie werden somit aus der Gruppe der kreisangehörigen $\mathrm{Ge}-$ meinden herausgehoben. ${ }^{3}$ Bei den übrigen kreisangehörigen Gemeinden wird durch die Übertragung von vorher durch die Landreise wahrgenommenen Weisungsaufgaben nach $\S 59$ Abs. $1 \mathrm{HKO}$ die Aufgabenbefugnis erweitert, etwa in den Bereichen des Gaststätten-, Gewerbe-, Straßenverkehrs- und Paßrechts. ${ }^{4}$

Da durch die vorgestellten Maßnahmen nicht alle Stadt-Umland-Probleme gelöst werden konnten, wurde in der Rhein-Main-Region ein anderer Ansatz als im übrigen Hessen realisiert. ${ }^{5}$ Mit der Gründung des Umlandverbands Frankfurt (UVF) wurde eine demokratisch legitimierte Organisation geschaffen, deren Mitglieder neben den kreisfreien Städten Frankfurt und Offenbach die drei Landkreise Offenbach, Main-Taunus- und Hochtaunuskreis mit den ihnen angehörigen Gemeinden sowie die kreisangehörigen Gemeinden Kelsterbach (Landkreis GroßGerau), Bad Vilbel (Wetteraukreis) und Maintal (Main-Kinzig-Kreis) sind. ${ }^{6}$ Ziel ist es, durch eine institutionalisierte Zusammenarbeit kommunaler Gebietskörperschaften auftretende Probleme, die über die jeweiligen Gebietsgrenzen hinausgehen, dezentral kooperativ zu lösen, anstatt sie einer übergeordneten Instanz zu zuweisen. ${ }^{7} \mathrm{Zu}$ diesem Zweck wurden dem UVF u.a. die ausschließlichen Kompetenzen für den Flächennutzungs- und den Generalverkehrsplan, der Trinkund Brauchwasserbeschaffung und -entsorgung sowie Abfallbeseitigung zugesprochen. ${ }^{8}$ Allerdings wurde ihm keine Kompetenz-Kompetenz übertragen, so $\mathrm{da} ß$ er nicht selbständig Aufgaben an sich ziehen, sondern sie nur von den Mitgliedskörperschaften übertragen bekommen kann. Da der UVF nicht in der Lage

1 Vgl: : Gesetz zur Neugliederung des Lahn-Dill-Gebiets und der Übertragung von weiteren Aufgaben auf kreisangehörige Gemeinden mit mehr als 50000 Einwohnern sowie zur Regelung sonstiger Fragen der Verwaltungsreform vom 10.07.1979, Hess. GVBl. I, S. 179.

2 Vgl.: Ausführungen des Abgeordneten Wilke (F.D.P.), Hessischer Landtag, 9. Wahlperiode, Stenographische Berichte, 6. Sitzung vom 21.03.1979, S. 278.

3 So ist nach $\S 136$ HGO für alle Städte mit mehr als 50.000 Einwohnern der Regierungspräsident die Aufsichtsbehörde (für Frankfurt und Wiesbaden der Innenminister); bei den übrigen (kreisangehörigen) Gemeinden fällt diese Zuständigkeit dem jeweiligen Landrat zu.

4 Vgl:: Gesetz zur Änderung kommunalrechtlicher Vorschriften vom 15.05.1974, Hess. GVB1. I, S. 241 und Verordnung zur Übertragung von Aufgaben auf Gemeinden mit 7500 und mehr Einwohnern vom 24.10.1974, Hess. GVB1. I, S. 551. Diese Aufgaben gelten den Gemeinden mit mehr als 7.500 Einwohnern als übertragen, solchen mit weniger als 7.500 Einwohnern können sie übertragen werden.

5 Vgl: Hessischer Landtag: Gesetzentwurf der Landesregierung für ein Gesetz über die Bildung des Umlandverbandes Frankfurt vom 20.05.1974, Drucksache 7/5321, S. $11 \mathrm{f}$.

6 Vgl.: Gesetz über den Umlandverband Frankfurt vom 11.09.1974, Hess. GVB1. I, S. 427.

7 Vgl: Hessischer Minister des Innern (Hrsg.): Hessen - Gemeinden und Landkreise nach der Gebietsreform, Melsungen 1977, S. 55f.

8 Vgl: $§ 3$ des Gesetzes über den Umlandverband Frankfurt vom 11.09.1974, Hess. GVBl. I, S. 427; zuletzt geändert am 17.12.1998, Hess. GVBl. I, S. 584. 
war, die Abfallwirtschaftsproblematik zu lösen und zudem Kritik an den starken Kostensteigerungen von Seiten der kommunalen Gebietskörperschaften geübt wurde, entschloß sich der Landtag zu einer Reform der Aufgabenverteilung. ${ }^{1}$ Als Ergebnis wurde die Müllentsorgung den Kreisen und kreisfreien Städten zum 01.01.1999 rückübertragen. ${ }^{2}$

Nach Abschluß der Gebietsreform verteilen sich die 5 kreisfreien Städte und 21 Landkreise mit 421 kreisangẹörigen Gemeinden auf die drei bestehenden Regierungsbezirke wie folgt:

-Darmstadt: Auf einer Fläche von 7,4 Mio. $\mathrm{km}^{2}$ mit ca. 3,7 Mio. Einwohnern (1995) befinden sich 4 kreisfreie Städte und 5 Landkreise mit 3 Sonderstatusstädten sowie 180 sonstigen kreisangehörigen Gemeinden mit einer Bruttowertschöpfung von insgesamt 232.965 Mio. DM oder 63.359 DM je Einwohner.

- Gießen: Auf einer Fläche von fast 5,4 Mio. $\mathrm{km}^{2}$ mit ca. 1,0 Mio. Einwohnern (1995) befinden sich 5 Landkreise mit 3 Sonderstatusstädten und 98 sonstigen kreisangehörigen Gemeinden mit einer Bruttowertschöpfung von 39.602 Mio. DM oder 37.651 DM je Einwohner.

-Kassel: Auf einer Fläche von ungefähr 5,4 Mio. $\mathrm{km}^{2}$ mit ca. 1,3 Mio. Einwohnern (1995) befinden sich eine kreisfreie Stadt und 6 Landkreise mit einer Sonderstatusstadt sowie 136 sonstigen kreisangehörigen Gemeinden mit einer Bruttowertschöpfung von 52.207 Mio. DM oder 41.289 DM je Einwohner. ${ }^{3}$

Die Entwicklung der drei kommunalen Gruppen nach Größenklassen sind in Tabelle 1 dargestellt.

1 Vgl: Hessischer Landtag: Gesetzentwurf der Fraktionen der CDU, der SPD, BÜNDNIS 90/ DIE GRÜNEN und der F.D.P. für ein Gesetz zur Aufgabenänderung des Umlandverbandes Frankfurt vom 28.04.1998, Drucksache 14/3850 sowie Änderungsantrag vom 10.12.1998, Drucksache 14/4421.

2 Vgl:: Gesetz zur Aufgabenänderung des Umlandverbandes Frankfurt vom 17.12.1998, Hess. GVB1. I, S. 584. Den kreisangehörigen Gemeinenden Bad Vilbel, Kelsterbach und Maintal wird die Möglichkeit gegeben, selbst die Abfallentsorgung zu übernehmen.

3 Vgl:: Statistisches Bundesamt: Statistisches Jahrbuch der Bundesrepublik Deutschland 1997, Stuttgart 1997, S. 51 und Emmel, W.: Die Entwicklung der Bruttowertschöpfung in den kreisfreien Städten und Landkreisen Hessens 1980 und 1995, in: Hessisches Statistisches Landesamt Wiesbaden: Staat und Wirtschaft in Hessen, 53. Jg., 1998, S. 235. 
Tabelle 1:Kommunale Ebene nach Gruppen und GröBenklassen im Zeitablauf

\begin{tabular}{|c|c|c|c|c|}
\hline Gruppe & Einwohnergröße $\mathrm{e}^{1}$ & 1969 & 1979 & 1996 \\
\hline \multirow[t]{5}{*}{ Kreisfreie Städte } & insgesamt & 9 & 6 & 5 \\
\hline & 400.000 und mehr & 1 & 1 & 1 \\
\hline & 200.000 - unter 400.000 & 2 & 1 & 2 \\
\hline & 100.000 - unter 200.000 & 2 & 4 & 2 \\
\hline & unter 100.000 & 4 & 0 & 0 \\
\hline \multirow[t]{6}{*}{ Kreisangehörige Städte } & insgesamt & 2.653 & 417 & 421 \\
\hline & 50.000 bis unter 100.000 & 1 & 5 & 7 \\
\hline & 10.000 bis unter 50.000 & 50 & 134 & 158 \\
\hline & 5.000 bis unter 10.000 & 89 & 138 & 148 \\
\hline & 1.000 bis unter 5.000 & 710 & 138 & 107 \\
\hline & unter 1.000 & 1.803 & 2 & 1 \\
\hline \multirow[t]{5}{*}{ Landkreise } & insgesamt & 39 & 20 & 21 \\
\hline & 300.000 und mehr & 0 & 2 & 2 \\
\hline & 200.000 - unter 300.000 & 3 & 8 & 11 \\
\hline & 100.000 - unter 200.000 & 11 & 9 & 7 \\
\hline & unter 100.000 & 25 & 1 & 1 \\
\hline
\end{tabular}

Quelle: Statistisches Bundesamt: Statistisches Jahrbuch der Bundesrepublik Deutschland der Jahre 1970/ 1980 und 1998; Stuttgart - Mainz 1970/ 1980 und 1998, S. 27, 28 und 34/ S. 53 und $58 /$ S. 50 und 56 .

\subsection{Kommunale Aufgaben und Einnnahmen}

\subsubsection{Arten kommunaler Aufgaben - ein Überblick}

\subsubsection{Freiwillige Aufgaben}

Es können freiwillige und pflichtige Aufgaben unterschieden werden. Der Bereich der freiwilligen Selbstverwaltungsaufgaben ist im Prinzip unbeschränkt; der Gestaltungsspielraum der kommunalen Ebene ist hier aus rechtlicher Perspektive am größten, da es kein staatliches Weisungsrecht gibt. ${ }^{1}$ Es besteht sowohl die Entscheidungsfreiheit bezüglich des "Ob" als auch des "Wie" der Durchführung

1 Vgl.: Wolff, H. J.; Bachof, O.; Stober, R.: Verwaltungsrecht II, 5. Aufl., München 1987, S. 56. 
bestimmter Aufgaben. Dazu gehören die finanzielle Unterstützung von Kulturund Sportveranstaltungen, aber auch die gemeindliche Daseinsvorsorge wie Strom- und Wasserversorgung. Allerdings kann es durch die zivilisatorische Entwicklung und die damit einhergehenden gestiegenen Ansprüche an die Leistungserstellung der Kommunen dazu kommen, daß die rein rechtlich vorhandenen Ermessens- und Entscheidungsspielräume bei der Aufgabenwahrnehmung durch faktische Zwänge eingegrenzt sind. ${ }^{1}$ Von staatlicher Seite dürfen keine Vorschriften bezüglich der Aufgabenerfüllung gemacht, sondern nur die Rechtstaatlichkeit des Handelns überprüft werden. ${ }^{2}$ Es wird durch die Aufsichtsbehörden nur überwacht, daß die Gemeinden und GV "ihre Verwaltung im Einklang mit den Gesetzen" ${ }^{\prime 3}$ führen. Somit besteht bei diesen Aufgaben die größte Möglichkeit der politischen Gestaltung auf der gemeindlichen Ebene. Wird das Recht auf Selbstverwaltung der kommunalen Ebene durch den Staat beeinträchtigt, besteht die Möglichkeit der Verfassungsbeschwerde. Ebenso kann gegen Eingriffe der Aufsichtsbehörden die Verwaltungsgerichtsbarkeit angerufen werden. ${ }^{4}$

\subsubsection{Weisungsfreie Pflichtaufgaben}

Bei diesem Typ der Selbstverwaltungsaufgaben sind die Entscheidungsbefugnisse der kommunalen Ebene im Vergleich zu den freien Selbstverwaltungsaufgaben eingeschränkt. Zur Durchführung dieser Aufgaben sind die Gemeinden gesetzlich angehalten, wobei eine weitgehende Freiheit in der Form der Ausführung besteht, da den Aufsichtsbehörden keine Weisungskompetenzen zugebilligt werden. ${ }^{5}$ Das Land überträgt in diesem Fall bestimmte Aufgaben zur pflichtigen Erfüllung, da es deren Notwendigkeit als gegeben sieht und die Entscheidung darüber nicht der kommunalen Ebene überlassen möchte. Als Grund für diese pflichtige Übertragung kann die Sicherung einer Mindestausstattung auf kommunaler Ebene angesehen werden. ${ }^{6} \mathrm{Zu}$ nennen wären hier Bauverwaltung, Abfallentsorgung, Bestattungswesen (Friedhöfe) usw. Die Eigenverantwortlichkeit besteht nur noch in der Art der Umsetzung, nicht mehr in der Entscheidung über die Aufgabenerfüllung selbst, wobei die staatlichen Aufsichtsbehörden wie bei den freiwilligen Aufgaben der Selbstverwaltung nur darauf achten, daß Gesetze nicht verletzt werden,

1 Vgl.: Schmidt-Jortzig, E.: Gemeinde- und Kreisaufgaben. Funktionsordnung des Kommunalbereiches nach "Rastede", in: DÖV, 46. Jg., 1993, S. $975 f$.

2 Vgl.: Knemeyer, F.-L.: Das verfassungsrechtliche Verhältnis der Kommunen zueinander und zum Staat, in: DVBl., 99. Jg., 1984, S. 28.

3 Art. 137 Abs. 3 Satz 2 HV.

4 Vgl:: Schneider, G; Dreßler, U.; Lüll, J.: Kommentar zu §§ 1,2 HGO, in: Schneider, G; Dreßler, U.; Lüll, J. (Hrsg.): Hessische Gemeindeordnung, Kommentar (Loseblattsammlung), Mainz, Stand 1999, S. 3f.

5 Vgl: Haverkamp, F.: Die Finanzbeziehungen zwischen Ländern und Gemeinden, in: Arnold, V.; Geske, O.-E. (Hrsg.): Öffentliche Finanzwirtschaft, München 1988, S. 57.

6 Vgl.: Schmidt-Jortzig, E.: Gemeinde- und Kreisaufgaben. Funktionsordnung des Kommunalbereiches nach "Rastede", in: DÖV, 46. Jg., 1993, S. 975. 
aber keinerlei Entscheidungsbefugnisse bezüglich Fach- oder Zweckmäßigkeitsüberlegungen haben. ${ }^{1}$ Die staatliche Ebene greift allerdings häufig mittels entsprechender Regelungsdichte so in den Entscheidungsspielraum der Aufgabenträger ein, daß diese kaum mehr über die Art und Weise der Ausführung beschließen können. ${ }^{2}$ Bei diesen Aufgaben bestehen die gleichen Möglichkeiten, gegen Eingriffe des Staates gerichtlich vorzugehen, wie bei den freiwilligen Aufgaben.

\subsubsection{Weisungsgebundene Pflichtaufgaben}

Bei den Weisungsaufgaben oder Auftragsangelegenheiten nutzt der Staat - also Bund und Länder - die Verwaltungskraft der kommunalen Ebene, um durch Gesetz übertragene eigene Aufgaben von dieser durchführen zu lassen, da so daß Wissen über die örtlichen Gegebenheiten genutzt werden kann, um den Aufgabenvollzug effektiver zu gestalten. ${ }^{3}$ Die Koordination staatlicher und kommunaler Aufgaben durch die Fokussierung auf eine ausführende Instanz ist effizienzsteigernd, da auf eine parallele staatliche Verwaltung verzichtet bzw. diese gering gehalten werden kann. ${ }^{4}$ Die Entscheidungsbefugnisse der Kommunen sind bei diesen Aufgaben weitgehend eingeschränkt, da die staatlichen Aufsichtsbehörden nicht nur die Gesetzmäßigkeit, sondern auch die Zweckmäßigkeit der kommunalen Aufgabenerfüllung kontrollieren. ${ }^{5}$ Dem Staat steht ein unbegrenztes Weisungsrecht gegenüber den ausführenden Verwaltungen zu. Bei diesen Aufgaben können prinzipiell die Verwaltungsgerichte nicht gegen Anordnungen der Aufsichtsbehörde angerufen werden, da es sich um verwaltungsinterne Anweisungen handelt. ${ }^{6}$

\subsubsection{Kommunale Einnahmen - ein Überblick}

Nach Art 137 Abs. 5 HV ist das Land verpflichtet, der kommunalen Ebene eine entsprechende Finanzausstattung zur Verfügung zu stellen, um die eigenen und

1 Vgl.: Hendler, R.: Das Prinzip der Selbstverwaltung, in: Isensee, J.; Kirchhof, P. (Hrsg.): Handbuch des Staatsrechts, Bd. 4, Heidelberg 1990, S. 1149.

2 Vgl.: Schoch, F.: Finanzierungsverantwortung für gesetzgeberisch veranlaßte Ausgaben, in: Der Landkreis, 64. Jg., 1994, S. 256.

3 Vgl: Stern K.: Gemeinden I: Rechtsstellung in der Bundesrepublik Deutschland, in: Albers, W. u.a. (Hrsg.): Handbuch der Wirtschaftswissenschaft, Bd. 3, Stuttgart - New York 1981, S. 491

4 Vgl: Schnur, R.: Zur Abgrenzung der gemeindlichen Aufgaben von den Aufgaben der Kreise gemäss Art. 28 Abs. 2 GG, in: Die Verwaltung, Bd. 19, 1986, S. 49.

5 Vgl.: Püttner, G.: Kommunale Selbstverwaltung, in: Isensee, J.; Kirchhof, P. (Hrsg.): Handbuch des Staatsrechts, Bd. 4, Heidelberg 1990, S. 1187

6 Vgl.: Hessischer Verwaltungsgerichtshof: Urteil vom 23.09.1970, in: Entscheidungen des Hessischen Verwaltungsgerichtshofs und des Verwaltungsgerichtshofs Baden-Württemberg mit Entscheidungen der Staatsgerichtshöfe beider Länder - ESVGH -, Bd. 21, Karlsruhe 1971, S. 78 oder Bundesverwaltungsgericht: Urteil vom 09.07.1964, in: Entscheidungen des Bundesverwaltungsgerichts, Bd. 19, Berlin 1965, S. 123. 
die übertragenen Aufgaben zu finanzieren. Eine Doppelsicherung enthalten $\S \S 3$, 4 HGO für die Zuweisung neuer Aufgaben auf die kommunale Ebene; denn die Übertragung bedarf eines formalen Gesetzes und einer gleichzeitigen Regelung für die Aufbringung der notwendigen finanziellen Mittel. ${ }^{1}$ Dabei ist dem Staat die Art der Mittelaufbringung freigestellt, so daß dies durch Erschließung neuer Steuerquellen oder durch Zuweisungen geschehen kann. ${ }^{2}$ Bei einer Lösung im Rahmen des KFA wird allerdings, im Gegensatz zu Regelungen in anderen Bundesländern, die Entgeltung der Kosten der übertragenen Aufgaben nicht separat ausgewiesen. ${ }^{3}$ Dies birgt die Gefahr einer Unterfinanzierung, da die getrennte Ausweisung der Zuweisungen sowohl zur allgemeinen Finanzkraftstärkung als auch zur Abgeltung der übertragenen Aufgaben höher ausfallen dürfte, als dies bei einer integrierten Zahlungsverpflichtung der Fall ist. ${ }^{4}$ Daraus kann sich ein Problem bei der Finanzierung der freiwilligen Aufgaben ergeben, die im Vergleich zu den übertragenen Aufgaben subsidiären Charakter haben, da fast 80 v.H. der Bundesgesetze bzw. 90 v.H. der Landesgesetze von der kommunalen Ebene vollzogen und somit auch die Verwaltungskosten getragen werden. ${ }^{5}$ Für diese stehen nur die nicht zur Finanzierung der Pflichtaufgaben benötigten Mittel bereit.

Die Gemeinden sind nach $\S 93$ Abs. 2 HGO angehalten, prioritär Gebühren und Beiträge als Finanzierungsinstrument ihrer Aufgaben zu nutzen, um somit den Begünstigten und nicht der Allgemeinheit die Zahllast aufzubürden. ${ }^{6}$ Nach Art. 106 GG stehen den Gemeinden die Einnahmen aus der Grundsteuer, der Gewerbesteuer nach Abzug der Gewerbesteuerumlage sowie die lokalen Aufwand- und Verbrauchsteuern zu, letztere auch den Landkreisen. Weiterhin ist dort die Beteiligung an der Einkommen- und Umsatzsteuer kodifiziert. In Tabelle 2 sind die Gesamteinnahmen inklusive Zuweisungen von anderen Ebenen und Kreditaufnahmen der Gemeinden und Gemeindeverbände in Hessen aufgeteilt auf die verschiedenen Einnahmenarten für das Jahr 1996 dargestellt.

1 Vgl.: Schlempp, H.; Schlempp, D.: Kommentar zur Hessischen Gemeindeordnung (HGO) (Loseblattsammlung), Stand 1996, S. 30.

2 Vgl.: Schneider, G; Dreßler, U.; Lüll, J.: Kommentar zu $\S \S 1,2$ HGO, in: Schneider, G; Dreßler, U.; Lüll, J. (Hrsg.): Hessische Gemeindeordnung, Kommentar (Loseblattsammlung), Mainz, Stand 1999, S. 13f.

3 Vgl.: Zezschwitz, F. v.: Kommentar zu Art. 137 HV, in: Zinn, G. A.; Stein, E.: Verfassung des Landes Hessen - Kommentar (Loseblattsammlung), Bad Homburg v. d. Höhe 1990, Erl. IX 3 a).

4 Vgl.: Schmidt-Jortzig, E.: Gemeinde- und Kreisaufgaben. Funktionsordnung des Kommunalbereiches nach "Rastede", in: DÖV, 46. Jg., 1993, S. 978

5 Vgl: Ellwein, T.: Perspektiven der kommunalen Selbstverwaltung in Deutschland, in: AfK, 36. Jg., 1997, S. 12.

6 Vgl.: Schneider, G; Dreßler, U.; Lüll, J.: Kommentar zu § 93 HGO, in: Schneider, G; Dreßler, U.; Lüll, J. (Hrsg.): Hessische Gemeindeordnung, Kommentar (Loseblattsammlung), Mainz, Stand 1999, S. 2. 
Tabelle 2:Einnahmen der kommunalen Ebene in Hessen 1996

\begin{tabular}{|l|c|c|c|}
\hline Einnahmenart & in Mio. & in v.H. & $\begin{array}{c}\text { davon in } \\
\text { v.H. }\end{array}$ \\
\hline Steuern und steuerähnliche Abgaben & $9.905,70$ & 40,76 & 40,54 \\
\hline -kommunaler Einkommensteueranteil & $-4.016,10$ & & 38,10 \\
\hline - Gewerbesteuer (netto) & $-3.773,70$ & & 10,98 \\
\hline - Grundsteuer & $-1.088,00$ & & 2,84 \\
\hline - lokale Aufwand- und Verbrauchsteuern & $-281,80$ & & 1,02 \\
\hline - Konzessionsabgaben & 101,20 & & 6,09 \\
\hline - sonstige Abgaben (z.B. Spielbankabgabe) & $-603,30$ & & 0,42 \\
\hline Leistungsentgelte & 41,60 & & \\
\hline Zuweisungen & $6.870,20$ & 28,27 & \\
\hline - allgemeine Zuweisungen durch KFA & $6.447,40$ & 26,53 & \\
\hline - sonstige Zuweisungen durch KFA & $2.738,30$ & & 42,47 \\
\hline sonstige Landeszuweisungen außerhalb KFA & $-1.618,40$ & & 25,10 \\
\hline - Bundeszuweisungen & $-59,00$ & & 0,92 \\
\hline Krediteinnahmen & $1.077,30$ & 4,43 & \\
\hline Gesamteinnahmen & $24.300,60$ & 100,00 & \\
\hline
\end{tabular}

Quelle: Eigene Berechnungen nach: Statistisches Bundesamt: Fachserie 14, Finanzen und Steuern, Reihe 3.3, Rechnungslegung der kommunalen Haushalte 1996, Stuttgart 1997; S. 22, 62; Dass: Fachserie 14, Finanzen und Steuern, Reihe 4, Steuerhaushalt - 4. Vierteljahr und Jahr 1996, Stuttgart 1997, S. 53; Dass.: Fachserie 14, Finanzen und Steuern: Reihe 3.1, Rechnungsergebnisse des öffentlichen Gesamthaushalts 1996, Stuttgart 1998, S. 272; Staatsanzeiger für das Land Hessen, 1996, S. 1291; Auskünfte des Hessischen Ministeriums der Finanzen.

Die Einnahmen betrugen etwa 24,3 Mrd. DM, wobei auf die Leistungsentgelte, d.h. die Bereiche wirtschaftliche Tätigkeit, spezielle Entgelte und Vermögensnutzung, ungefähr 28,3 v.H. (6,9 Mrd. DM) entfielen. Der Anteil der Steuereinnahmen (inklusive dem kommunalen Grunderwerbsteueranteil ${ }^{1}$, der

1 Die kreisfreien Städte und Landkreise sind seit dem Wegfall des kommunalen Grunderwerbsteuerzuschlags aufgrund des hessischen Grunderwerbsteuerzuweisungsgesetzes (Grunderwerbsteuerzuweisungsgesetz vom 24.03.1983, Hess. GVBl. I, S. 31, geändert am 
Spielbankabgabe ${ }^{1}$ sowie den $\left.\mathrm{KA}^{2}\right)$ betrug ca. 40,8 v.H. (9,9 Mrd. DM), wovon etwa fast 78,6 v.H. (7,8 Mrd. DM) auf die Einkommen- und die Gewerbesteuer entfielen. Die Quote der Zuweisungen belief sich auf 26,5 v.H. der Gesamteinnahmen. Davon entfielen 2,7 Mrd. DM auf allgemeine Zuweisungen.

\subsection{Der kommunale Finanzausgleich - ein Überblick}

\subsubsection{Grundgesetzliche und landesrechtliche Vorschriften zum KFA}

In Art. 106 GG sind neben der grundlegenden Verteilung der Steuereinnahmen zwischen den verschiedenen Gebietskörperschaftsebenen auch die Grundsätze des staatlichen und des kommunalen Finanzausgleichs geregelt. Es werden allerdings im Grundgesetz keinerlei spezifische Regelungen über den KFA getroffen, sondern es gibt nur allgemeine Ausführungen, die auch nur qualitativen und keinen quantitativen Bezug haben. Nach Art. 106 Abs. 7 GG ist jedes Bundesland dazu verpflichtet, seine Kommunen am Aufkommen aus den Gemeinschaftsteuern $\mathrm{zu}$ beteiligen (obligatorischer Steuerverbund). Inwieweit die übrigen (Landes-)Steuern freiwillig in den Steuerverbund einbezogen werden, ist den Ländern selbst überlassen (fakultativer Steuerverbund). ${ }^{3}$ Eine Aussage über die Beteiligungsquote beim obligatorischen Steuerverbund wird im GG nicht vorgenommen, so daß dem Landesgesetzgeber hier ein großer Entscheidungsspielraum gegeben ist ${ }^{4}$, was auch das Bundesverfassungsgericht bestätigt hat. ${ }^{5}$ Dies kann darauf zurückgeführt werden, daß die kommunale Ebene Bestandteil des Landes ist,

25.03.1997, Hess. GVB1. I, S. 50 sowie Verordnung zur Durchführung des Grunderwerbsteuerzuweisungsgesetzes vom 10.10.1983, Hess. GVBl. I, S. 141) direkt am Grunderwerbsteueraufkommen des Landes beteiligt, derzeit mit einem Drittel. Der auf die einzelne Kommune entfallende Betrag richtet sich nach dem örtlichen Aufkommen.

1 Aufgrund der Steuerfreiheit der deutschen Spielbanken (z.B. Gewerbe- und Einkommensteuer) müssen diese eine Abgabe an die Länder entrichten. Die Spielbankgemeinden werden wiederum mit 29,375 v.H. am örtlichen Aufkommen beteiligt ( 33 Abs. 2 Hessisches Spielbankgesetz (Hess. SpielbG) vom 21.12.1988, Hess. GVBl. I, 1989, S. 1 und $\S 1$ der Verordnung über den Anteil der Spielbankgemeinden an der Spielbankabgabe und die Verwendung des Troncs der öffentlichen Spielbanken in Hessen vom 15.11.1989, Hess. GVBl. I, S. 431).

2 Nach der Konzessionsabgabenverordnung (Verordnung über Konzessionsabgaben für Strom und Gas (Konzessionsabgabenverordnung KAV) vom 09.01.1992, BGBl. I, S. 12-14) haben die Kommunen das Recht, von den Versorgungsunternehmen fur die Zubilligung des Rechts der Leitungsverlegung auf kommunalen Grundstücken und Straßen sowie des ausschließlichen Rechts der Versorgung der örtlichen Bevölkerung und Unternehmen Konzessionsabgaben zu erheben.

3 In Hessen ist dies in $\S 1$ Abs. 2 des Hessischen Gesetz zur Regelung des Finanzausgleichs in der jeweils gültigen Fassung geregelt.

4 Vgl.: Kirchhof, P.: Rechtliche Rahmenbedingungen des kommunalen Finanzausgleichs, in: Hoppe, W. (Hrsg.): Reform des kommunalen Finanzausgleichs, Köln u.a.O. 1985, S. 4.

5 Vgl.: Bundesverfassungsgericht: Urteil vom 21.05.1968, in: Entscheidungen des Bundesverfassungsgerichts, Bd. 23, Tübingen 1968, S. 369. 
welches die Aufteilung der Aufgaben zwischen staatlicher und kommunaler Ebene festlegen kann. Von dem so bestimmten Kommunalisierungsgrad der Aufgaben im jeweiligen Land ist das Finanzausgleichsvolumen abhängig. ${ }^{1}$ Darüber hinaus können im Zeitablauf auch ohne die Einwirkung der Länder auf den Kommunalisierungsgrad Veränderungen bei den Einnahmen und Aufgaben bzw. Ausgaben auftreten, etwa durch Bundesgesetze. Diese Umstände sind dann von den Ländern im KFA zu berücksichtigen. ${ }^{2}$ Allerdings ist diese Freiheit bei der Gestaltung des Finanzausgleichs auf Seiten der Länder durch den Art. 28 GG insoweit eingeschränkt, als dort eine Garantie für die kommunale Selbstverwaltung abgegeben wird. ${ }^{3}$ Diese umfaßt unter anderem eine Finanzautonomie, worunter auch eine "Befugnis zu einer eigenverantwortlichen Einnahmen- und Ausgabenwirtschaft" ${ }^{4} \mathrm{zu}$ verstehen ist.

Nach Art. 137 Abs. 5 HV hat der Staat der gemeindlichen Ebene die notwendigen Finanzmittel im Rahmen eines "Lasten- und Finanzausgleichs" bereitzustellen, um ihr die Erfüllung ihrer Aufgaben zu ermöglichen. Die genaue Ausgestaltung dieses Ausgleichssystems ist im Finanzausgleichsgesetz geregelt. In $\S 1$ Abs. 1 des Hessischen Finanzausgleichsgesetzes wird festgelegt, daß den "Gemeinden und Gemeindeverbänden (...) im Wege des Lasten- und Finanzausgleichs Geldmittel zur Verfügung gestellt" werden, "die erforderlich sind, um ihre eigenen und die ihnen übertragenen Aufgaben" zu erfüllen. ${ }^{5}$

Weiterhin besteht, wie in $\S 1$ Abs. 3 FAG vorgesehen, noch die Möglichkeit, separate Zuweisungen laut Landeshaushalt oder anderen Gesetzen zur Verfügung zu stellen. Diese außerhalb des Steuerverbundes erfolgten Leistungen können u.a. komplementäre Zahlungen zu den über den Landeshaushalt verteilten Bundeszuweisungen sein oder die Realisierung vom Land erwünschter Projekte unterstützen. ${ }^{6} \mathrm{Da}$ für jedes Förderprojekt die dafür vorgesehene Summe festgelegt wird (sog. "Töpfchenwirtschaft"), kommt es aufgrund der Förderungsvielfalt zwangsläufig zu einer Aufsplitterung des Gesamtbetrages des KFA. Dabei entsteht

1 Vgl.: Steinherr, M.: Kommunaler Finanzausgleich: Balanceakt zwischen Verteilungs- und Allokationszielen - Das Beispiel Rheinland-Pfalz, in: ifo-Schnelldienst, 51. Jg., Nr. 8, 1998, S. 15 .

2 Vgl.: Bundesverfassungsgericht: Beschlu $\beta$ vom 10.06.1969, in: Entscheidungen des Bundesverfassungsgerichts, Bd. 26, Tübingen 1970, S. 181, 185.

$3 \mathrm{Vgl}$ : Kirchhof, P.: Der Finanzausgleich als Grundlage kommunaler Selbstverwaltung, in: DVBl., 95. Jg., 1980, S. 712.

4 Vgl: Bundesverfassungsgericht: Urteil vom 24.06.1969, in: Entscheidungen des Bundesverfassungsgerichts, Bd. 26, Tübingen 1970, S. 244.

5 So werden den Kommunen zur Unterhaltung ihrer Straßen Zuweisungen gewährt, deren Höhe sich u.a. nach der Straßenlänge richtet.

6 Vgl:: Käss, W.: Der kommunale Finanzausgleich und finanzielle Zuweisungen des Landes zur Erfüllung öffentlicher Aufgaben, Erläuterungen zu § $1 \mathrm{FAG}$, in: Praxis der Gemeindeverwaltung, Hessen, Bd. E1, (Loseblattsammlung), Stand 1999, S. 22. 
häufig für die kommunale Ebene ein weiteres Problem, weil oft nach wenigen Jahren die Finanzierung durch allgemeine Haushaltsmittel der Länder beendet und die weiterhin notwendige Summe nun durch den Steuerverbund und somit kommunale Mittel ersetzt wird. ${ }^{1}$ Dieser als Befrachtung des KFA bezeichnete Vorgang mindert dann das für die übrigen Zuweisungen an die Kommunen vorhandene Volumen.

\subsubsection{Ermittlung der heutigen Verbundmasse des KFA}

Als Basis für die Bestimmung des Volumens der Zuweisungen im Rahmen des KFA dient die Finanzausgleichsmasse. In Hessen setzt sie sich aus der Steuerverbundmasse, deren Anteil an der gesamten Ausgleichsmasse bei etwa 94 v.H. liegt, und weiteren Finanzmitteln - etwa Zuführungen aus der Grundwasserabgabe - zusammen. ${ }^{2}$ Durch die Steuerverbundmasse wird festgelegt, an welchen Steuereinnahmen des Landes die Gemeinden über die Gemeinschaftsteuern (obligatorischer Verbund) hinaus zusätzlich und freiwillig (fakultativer Verbund) beteiligt werden. In Hessen enthält der fakultative Steuerverbund nach $\S 2$ Abs. 2 FAG folgende Einnahmen:

- Vermögensteuer,

- Kraftfahrzeugsteuer,

- Grunderwerbsteuer,

- Gewerbesteuerumlage.

An der Summe der aufgeführten Einnahmen wird die hessische kommunale Ebene mit 22,9 v.H. beteiligt. Allerdings partizipieren die Gemeinden nur an die dem Land - nach Abzug von gesetzlich zu leistenden Beträgen - verbleibenden Einnahmen. Da Hessen im Länderfinanzausgleich ein Geberland ist, sinkt die Verbundmasse um die entsprechenden Zahlungen. Ebenso verhält es sich bei der Grunderwerbsteuer, von der ein Drittel vorab den Landkreisen und kreisfreien Städten zugewiesen wird $^{3}$, und einem Teil der Umsatzsteuereinnahmen, den die Gemeinden in Form eines Vorababzugs als Ausgleich für die aus der Neuregelung des

1 Vgl:: Patzig, W.: Der kommunale Finanzausgleich im Zeichen der Konsolidierung der Länderhaushalte, in: DVB1., 100. Jg., 1985, S. 140; Haverkamp, F.: Die Finanzbeziehungen zwischen Ländern und Gemeinden, in: Arnold, V.; Geske, O.-E. (Hrsg.): Öffentliche Finanzwirtschaft, München 1988, S. 96; Kommunaler Finanzausgleich 1997, in: Hessischer Städtetag Informationen, 1996, S. 110 .

2 Vgl:: Hessisches Ministerium der Finanzen: Veröffentlichung nach $\S 49$ Abs. 3 des Finanzausgleichsgesetzes furr das Ausgleichsjahr 1997 vom 31.03.1997, in: Staatsanzeiger furr das Land Hessen, S. 1023.

3 Vgl: : 11 des Gesetzes über die Zuweisung von Grunderwerbsteueranteilen an die Landkreise und kreisfreien Städte (Grunderwerbsteuerzuweisungsgesetz) vom 25.03.1997, Hess. GVBI. I, S. 50. Dort ist auch geregelt, daß die kreisfreien Städte und Landkreise mit vier Siebtel an grunderwerbsteuerpflichtigen Rechtsvorgängen beteiligt werden, die noch im Jahr 1996 getätigt und deshalb nach altem Grunderwerbsteuergesetz (BGBl. I, S. 1777) mit 2 v.H. besteuert wurden. 
Familienleistungsausgleichs entstandenen Belastungen erhalten. ${ }^{1}$ Die Beteiligung an der Gewerbesteuerumlage bezieht sich allerdings der Höhe nach nur auf den Landesvervielfältiger ${ }^{2}$ der neuen Länder. ${ }^{3}$ Dieser beträgt ebenso wie der Bundesvervielfältiger 19 v.H., während der entsprechende Vervielfältiger für die alten Länder 1998 insgesamt 39 Prozentpunkte über dem der neuen Länder liegt; 10 Prozentpunkte sind auf die Beteiligung der Gemeinden an den Finanzierungskosten der Länder für den "Fonds Deutsche Einheit" zurückzuführen, während 29 Prozentpunkte im Gesetz zur Umsetzung des Föderalen Konsolidierungsprogramms begründet liegen. ${ }^{4}$ Somit werden von den Einnahmen des Landes Hessen aus der Gewerbesteuerumlage nur etwa 32,8 v.H. in die Steuerverbundmasse eingeführt.

Die sich durch Multiplikation der Steuerverbundmasse mit dem Verbundsatz ergebende Summe wird nun noch um die sich aus der Endabrechnung des Finanzausgleichs der Vorjahre ergebenden Differenz und um sonstige Zuführungen korrigiert. Erstere ist darauf zurückzuführen, daß es sich bei den Ansätzen um Schätzwerte handelt, die erst nach Abschluß des Haushaltsjahres endgültig feststehen. Für die sonstigen Zuführungen können Einnahmen aus der Grundwasserabgabe oder der Krankenhausumlage ${ }^{5}$ angeführt werden. In Tabelle 3 ist die Ermittlung der Finanzausgleichsmasse dargestellt.

1 Durch die Reform des Kinderlastenausgleichs und der damit verbundenen verstärkten Inanspruchnahme der Kinderfreibeträge kommt es im Rahmen der Einkommensteuer zu Mindereinnahmen bei den Gemeinden, die auszugleichen sich die Länder verpflichtet haben. Das Land Hessen beteiligt die Gemeinden deshalb nach § 46a des FAG mit 26 v.H. des Mehraufkommens der Umsatzsteuer.

2 Der Vervielfältiger ist der Umlagesatz der Gewerbesteuerumlage.

3 Vgl: $§ 2$ Abs. 2 des Gesetzes zur Regelung des Finanzausgleichs (Finanzausgleichgesetz FAG-) in der neuesten Fassung.

4 Vgl: $§ 6$ Abs. 3, 5 des Gesetzes zur Neuordnung der Gemeindefinanzen (Gemeindefinanzreformgesetz) vom 06.02.1995, BGB1. I, S. 189.

5 Umlagepflichtig sind die kreisfreien Städte und die Landkreise. 
Tabelle 3:Von der Steuerverbund- zur Finanzausgleichsmasse (1996)

\begin{tabular}{|l|r|}
\hline Verbundmasse & \multicolumn{1}{|c|}{ Mio. DM } \\
\hline ESt- und KSt-Einnahmen des Landes & $13.628,70$ \\
\hline USt-Einnahmen des Landes & $6.667,78$ \\
\hline Vermögensteuer & 890,00 \\
\hline Kraftfahrzeugsteuer & $1.075,00$ \\
\hline Grunderwerbsteuer & 493,06 \\
\hline Gewerbesteuerumlage & 717,10 \\
\hline Ausgangssumme & $\mathbf{2 3 . 4 7 1 , 6 4}$ \\
\hline Minderungen durch den LFA & $-2.950,00$ \\
\hline Grunderwerbsteueranteil der Städte und Kreise (§ 1 GrESt-ZuwG) & $-281,75$ \\
\hline Erhöhte Gewerbesteuerumlage & $-486,29$ \\
\hline Ausgleichsleistungen für den Familienlastenausgleich (§ 46a FAG) & $-212,40$ \\
\hline Steuerverbundmasse & $\mathbf{1 9 . 5 4 1 , 2 0}$ \\
\hline 22,9 v.H. der Steuerverbundmasse & $4.474,94$ \\
\hline Finanzausgleichsabrechnung (§ 4 FAG) & $\mathbf{8 3 , 2 0}$ \\
\hline Sonstige Zuführungen & 216,00 \\
\hline Finanzausgleichsmasse & $\mathbf{4 . 7 7 4 , 1 4}$ \\
\hline
\end{tabular}

Quelle: Eigene Berechnungen nach: Statistisches Bundesamt: Fachserie 14, Finanzen und Steuern, Reihe 4, Steuerhaushalt - 4. Vierteljahr und Jahr 1996, Stuttgart 1997, S. 53, 57; Dass.: Fachserie 14, Finanzen und Steuern, Reihe 3.3, Rechnungsergebnisse der kommunalen Haushalte 1996, Stuttgart 1997, S. 62; Dass.: Fachserie 14, Finanzen und Steuern, Reihe 4, Steuerhaushalt - 4. Vierteljahr und Jahr 1997, Stuttgart 1998, S. 53, 57; Staatsanzeiger für das Land Hessen, 1996, S. 1291; 1997, S. 1023. 



\section{Ausgestaltung eines kommunalen Finanzausgleichs}

\subsection{Aufgaben eines kommunalen Finanzausgleichs}

\subsubsection{Verteilungspolitik}

Als Aufgabe des kommunalen Finanzausgleichs kann allgemein die Stärkung der kommunalen Finanzkraft bei gleichzeitiger Verringerung der interkommunalen Finanzkraftunterschiede genannt werden. ${ }^{1}$ Es kann somit zwischen einer vertikalen und einer horizontalen Orientierung differenziert werden. Erstere bezieht sich auf das Verhältnis aller Gemeinden und GV zum Staat, also dem Land, während die letztere auf das interkommunale Problem der Finanzmittelverteilung fokussiert ist.

\subsubsection{Vertikale Verteilung}

Die fiskalische Aufgabe besteht darin, allgemein die Finanzkraft aller Kommunen zu stärken, um die Selbstverwaltung zu sichern. ${ }^{2}$ Die Verteilung der Aufgaben und Einnahmen auf die Gebietskörperschaftsebenen ergibt sich aus den entsprechenden Regelungen des Grundgesetzes (Art. 106 Abs. 5-7) und den landesrechtlichen Regelungen (HGO, Hess. Grunderwerbsteuerzuweisungsgesetz). Die den Gemeinden dort zugewiesenen Steuern ermöglichen allerdings nicht die Erfüllung der gemeindlichen Aufgaben. KIRCHHOF spricht in diesem Zusammenhang davon, daß die "Unfähigkeit zur Selbstfinanzierung gegenwärtig der gesetzlich veranlaßte Regelfall" ${ }^{3}$ für die kommunale Gebietskörperschaftsebene ist. Diese Aussage läßt sich auch durch einen Vergleich des Stellenwerts der Steuereinnahmen der drei Gebietskörperschaftsebenen der Bundesrepublik darlegen. So ist der Anteil der Steuereinnahmen an den kommunalen Gesamteinnahmen weitaus geringer als beim Bund und den Ländern; er belief sich 1996 auf 26 v.H. bei den Gemeinden, während die Quote beim Bund und den Ländern bei 76 v.H. bzw. 62 v.H. lag. ${ }^{4}$

\subsubsection{Horizontale Verteilung}

Als weiteres Problem des kommunalen Finanzsystems ist neben der zu geringen Steuerkraft der Kommunen noch die ungleiche Streuung der Steuereinnahmen vor allem bei der Gewerbesteuer - im Raum zu nennen. ${ }^{5}$ Zumal diese Steuer die Eigenschaft hat, die regionalen Aufkommensdifferenzen selbst zu verstärken. ${ }^{6}$

1 Vgl.: Littmann, K. u.a.: Die Gestaltung des kommunalen Finanzsystems unter raumordnungspolitischen Gesichtspunkten, Hannover 1968, S. 13.

2 Vgl:: Kirchhof, P.: Der Finanzausgleich als Grundlage kommunaler Selbstverwaltung, in: DVB1., 95. Jg., 1980, S. 711.

3 Ders.: Rechtliche Rahmenbedingungen des kommunalen Finanzausgleichs, in: Hoppe, W. (Hrsg.): Die Reform des kommunalen Finanzausgleichs, Köln u.a.O. 1985, S. 11.

4 Errechnet nach Bundesministerium der Finanzen (Hrsg.): Finanzbericht 1998, Bonn 1997, S. 319,324 .

5 Vgl:: Strauß, W. Ökonomische Wirkungen einer Substitution der Gewerbesteuern (Realsteuern) durch eine Wertschöpfungsteuer, Frankfurt u.a. 1988

6 Gemeinden mit hoher Bemessungsgrundlage erzielen beim gleichen Hebesatz höhere Ein- 
Auch die Gemeindefinanzreform von 1969 - die u.a. dem Ziel diente, diese ungleiche Verteilung zu verringern ${ }^{1}$ - hat keine Abhilfe gebracht. Zum Ausgleich dieser Unterschiede stehen sowohl Zahlungen auf horizontaler Ebene als auch horizontal differenzierte vertikale Landeszuweisungen zur Verfügung. ${ }^{2}$

Der Ausgleich der Finanzkraft zwischen den Gemeinden wird allerdings - im Gegensatz zum Länderfinanzausgleich - nicht durch direkte Transfers zwischen finanzstarken und -schwachen Kommunen angestrebt. ${ }^{3}$ Statt dessen kommt es in Abhängigkeit von der jeweiligen kommunalen Finanzkraft zu Differenzierungen der Landeszuweisungen (vertikaler Finanzausgleich mit horizontalem Effekt), daneben dient sie als Bemessungsgrundlage für von der kommunalen Ebene abzuführende Umlagen (beispielsweise bei der Krankenhausumlage), die ein horizontales Ausgleichssystem darstellen. Weiterhin führt auch die Ausgestaltung der Gewerbesteuerumlage - die Höhe der zu zahlenden Umlage richtet sich nach der jeweiligen Finanzkraft - und ihre Zuführung zur Finanzausgleichsmasse zu einer Umverteilung, da die finanzstarken Gebietskörperschaften stärker zur Umlage herangezogen werden und relativ weniger Zuweisungen erhalten als die finanzschwachen Gebietskörperschaften. Im Bereich der kreisangehörigen Gemeinden kommt es darüber hinaus noch durch die Kreisumlage und ihre Verausgabung ${ }^{4}$ ansatzweise zu einem interkommunalen Finanzausgleich. ${ }^{5}$ Ziel der Finanzbeziehungen zwischen Land und Kommunen ist es somit, allen Gemeinden und GV eine Mindestausstattung an Finanzmitteln zu sichern, um eigene und ihnen übertragene Aufgaben erfüllen zu können. ${ }^{6}$

nahmen als solche mit niedriger Bemessungsgrundlage. Sie können dann über die Verbesserung der lokalen Infrastruktur zusätzliche Unternehmen attrahieren, was wiederum Bemessungsgrundlage und Steuereinnahmen steigert.

1 Vgl.: Deutscher Bundestag: Entwurf eines ... Gesetzes zur Änderung und Ergänzung des Grundgesetzes (Finanzreformgesetz) vom 30.04.1968, Drucksache V/2861, S. 95.

2 Vgl:: Fischer-Menshausen, H.: Finanzausgleich II: Grundzüge des Finanzausgleichsrechts, in: Albers, W. u.a. (Hrsg.): Handbuch der Wirtschaftswissenschaften, Bd. 2, Stuttgart New York 1980, S. 657f.

3 Vorschläge dafür gibt es bei: Albers, W.: Möglichkeiten und Grenzen eines interkommunalen Finanzausgleichs, in: Friedrich-Ebert-Stiftung (Hrsg.): Kommunale Finanzreform, Schriftenreihe der Forschungsstelle der Friedrich-Ebert-Stiftung, Hannover 1962, S. 63-83; Hansmeyer, K.-H.: Interkommunaler Finanzausgleich, in: AfK, 5. Jg., 1966, S. 261-284

4 Siehe Abschnitt 2.1.2.

5 Vgl: Voigt, R.: Finanzierung kommunaler Aufgaben in Nordrhein-Westfalen, Diskussionsbeiträge der Universität/Gesamthochschule Siegen, HiMoN DB 88/86, Siegen 1986, S. $17 f$

6 Vgl.: Eckey, H.-F.: Möglichkeiten der Anpassung und Veränderungen des Kommunalen Finanzausgleichssystems für raumordnerische Zielsetzungen, in: Informationen zur Raumentwicklung: Die Reform der Kommunalfinanzen, Heft 8/9, hrsg. von der Bundesforschungsanstalt für Landeskunde und Raumentwicklung, Bonn 1995, S. 584 


\subsubsection{Allokationspolitik}

Nachdem in den vorherigen Ausführungen die Notwendigkeit einer gleichmäßigeren Verteilung der Finanzmittel begründet wurde, geht es nun darum zu erklären, weshalb eine gewisse Ungleichheit bestehen bleiben sollte. Die allokative (auch raumordnungspolitische) Zielsetzung steht in einem Zielkonflikt mit der distributiven Notwendigkeit, nach der die Gemeinden die Möglichkeit haben sollten, vergleichbare Leistungen für die Bürger anzubieten, wäre dies doch ökonomisch ineffizient. Denn die nach Art. 106 Abs. 3 Ziff. 2 GG zu wahrende "Einheitlichkeit der Lebensverhältnisse" ist insoweit zu relativieren, als zu berücksichtigen ist, $\mathrm{da} ß$ nicht in jeder Gemeinde ein Theater oder Opernhaus wirtschaftlich betrieben werden kann, da für eine effiziente Bereitstellung auch eine Mindestauslastung notwendig ist; "Gleichwertigkeit der Lebensverhältnisse kann aber nicht Gleichartigkeit der Versorgung mit öffentlichen Gütern bedeuten."1

Statt dessen ist darauf zu achten, da $B$ ein entsprechendes Angebot von Leistungen in einer angemessenen Entfernung vom jeweiligen Wohnort bereitgestellt wird. Als theoretische Grundlage kann hier das Konzept der zentralen Orte genannt werden, dem zufolge ausgewählte Orte Leistungen nicht nur für die eigene Bevölkerung, sondern auch für die des Umlandes anbieten. ${ }^{2}$ Diese zusätzlichen Kapazitäten an öffentlich bereitgestellten Gütern müssen den Kommunen entgolten werden. Sie stellen erwünschte Spillover-Effekte dar, die es zu internalisieren gilt, da die räumliche Grenze des Bereitstellers eines öffentlichen Gutes nicht identisch mit der räumlichen Grenze der Empfänger ist. ${ }^{3}$ Hierbei bieten sich in erster Linie spezielle Nutzungsentgelte oder direkte horizontale Zahlungen zwischen den Gemeinden an; können diese Ansätze nicht realisiert werden oder ist der Einsatz ökonomisch nicht sinnvoll, so sollten entstehende Spillover-Effekte durch horizontal differenzierte Landeszuweisungen ausgeglichen werden. ${ }^{4}$

\subsubsection{Stabilitätspolitik}

Neben dem Bund und den Ländern sind nach Art. 109 GG und § 1 und 16 StWG auch die Gemeinden und GV als dritte Gebietskörperschaftsebene dem Stabilitätsziel verpflichtet. Da die Steuereinnahmen der Gemeinden aufgrund des hohen Anteils der Gewerbeertragsteuer aber konjunkturreagibler sind als die von Bund

1 Parsche, R.; Steinherr, M.: Reformvorschläge zum kommunalen Finanzausgleich des Landes Nordrhein-Westfalen, in: ifo-Schnelldienst, 48. Jg., Nr. 28, 1995, S. 11.

2 Vgl: Hansmeyer, K.-H.: Der kommunale Finanzausgleich als Instrument zur Förderung Zentraler Orte, in: Pohmer, D. (Hrsg.): Probleme des Finanzausgleichs II, Schriften des Vereins für Socialpolitik, N.F. Bd. 96/II, Berlin 1980, S. 90f.

3 Vgl:: Münstermann, E.: Die Berücksichtigung zentralörtlicher Funktionen im kommunalen Finanzausgleich, Opladen 1975, S. 122 f.

4 Vgl:: Smekal, C.: Transfers zwischen Gebietskörperschaften (TG) - Ziele und Ausgestaltungsprobleme, in: Pohmer, D. (Hrsg.): Probleme des Finanzausgleichs II, Schriften des Vereins für Socialpolitik, N.F. Bd. 96/II, Berlin 1980, S. $187 f$. 
und Ländern und zudem der Verschuldungspolitik dieser Ebene durch die Gemeinde- und Kreisordnungen enge Grenzen gesetzt sind ( $\$ 103$ HGO und $\S 52$ HKO), wird bei den Kommunen das daraus resultierende Abweichen vom Stabilitätsziel aufgrund der faktischen Situation überwiegend akzeptiert. ${ }^{1}$ Allerdings ist erstrebenswert - wenn schon ein antizyklisches Verhalten der Gemeinden nicht zu erreichen ist -, eine nichtprozyklische Ausgabenpolitik, die somit der "Intention staatlicher Konjunkturpolitik nicht ausgeprägt zuwiderlaufen" ${ }^{2}$ würde, zu realisieren. Dies könnte durch eine Verstetigung der Einnahmen geschehen. Da das Volumen des KFA etwa 48,2 v.H. der kommunalen Steuereinnahmen erreicht, könnten durch eine Verstetigung der Zuweisungen die durch das Steueraufkommen hervorgerufenen Einnahmeschwankungen gemindert werden. ${ }^{3}$

\subsection{Anforderungskriterien eines kommunalen Finanzausgleichs}

Um ein in der Praxis verwendetes Finanzausgleichssystem beurteilen zu können, sind vorab Kriterien zu bestimmen, mit deren Hilfe eine Bewertung vorgenommen werden kann, d.h., die vorher genannten Aufgaben und Ziele müssen operationalisiert werden. Zu diesem Zweck werden die Beurteilungskriterien in Gruppen eingeteilt. Eine Kategorie bezieht sich auf das Volumen und die Verteilung der bereitgestellten Finanzmittel auf verschiedene Zuweisungstypen. Es geht hier somit vor allem um die Beziehungen zwischen dem Land und der kommunalen Ebene, also um eine vertikale Betrachtung. Das nächste Kriterienraster bezieht sich auf die horizontale Mittelverteilung. Dabei geht es sowohl um die Dotierung der drei kommunalen Gruppen als auch um die Verteilung der Mittel innerhalb einer Gebietskörperschaftsklasse. Weiterhin gibt es allgemeine Anforderungen, die generellen Geltungscharakter haben, die deshalb vorab aufgeführt werden:

- Transparenz: Transparenz ist nicht nur für ein Finanzausgleichssystems wünschenswert, sie ist auch in anderen Bereichen zweckdienlich und sinnvoll. ${ }^{4} \mathrm{Sie}$ ist erforderlich, um den Zuweisungsempfängern die Berechnung der ihnen zustehenden Gelder zu erleichtern und somit auch eine prospektive Finanzpolitik auf kommunaler Ebene zu ermöglichen. ${ }^{5}$ Eine weitaus wichtigere Begründung für

1 Vgl.: Zimmermann, H.: Allgemeine Probleme und Methoden des Finanzausgleichs, in: F. Neumark, N. Andel und H. Haller (Hrsg.): Handbuch der Finanzwissenschaft, Bd. 4, Tübingen 1983, S. 30f.

2 Schwarting, G.: Grundsätze für die Kreditaufnahme der Gemeinden, in: Püttner, G. (Hrsg.): Handbuch der kommunalen Wissenschaft und Praxis, Bd. 6, 2. Aufl., Berlin u.a.O. 1985, S 634.

3 Vgl.: Klein, R. R.; Münstermann, E.: Kommunen und Konjunkturpolitik, in: AfK, 17. Jg., 1978, S. 220, 223 und Kock, H.: Vorschläge zu einer Verstetigung der Gemeindefinanzen, in: Konjunkturpolitik, 21. Jg., 1975, S. 334.

4 Z.B. für ein Steuersystem. Vgl: Neumark, F.: Steuern I: Grundlagen, in: Albers, W (Hrsg.): Handbuch der Wirtschaftswissenschaft, Bd. 2, Stuttgart-New York 1977, S. 305.

5 Vgl.: Ehrlicher, W. u.a.: Kommunaler Finanzausgleich und Raumordnung, Hannover 1967, S. 43. 
Transparenz ergibt sich aus der ökonomischen Theorie der Politik. Denn für die einzelnen Länder - und somit auch die Regierungen und Parteien - besteht "angesichts des dem Gesetzgeber belassenen Spielraums für die Ausgestaltung der kommunalen Steuerbeteiligung"' die Möglichkeit, entsprechend ihren eigenen Zielsetzungen den KFA relativ frei zu gestalten ${ }^{2}$; zumal es in einigen Ländern kein kommunales Klagerecht vor dem Landesverfassungsgericht gibt und der Bewertungsmaßstab des Bundesverfassungsgerichts weniger streng ist. ${ }^{3} \mathrm{Zu}-$ dem hat die Einschränkung, daß der KFA "nicht allein nach Gründen politischer Zweckmäßigkeit gestaltet werden"4 darf, nur deklaratorischen Charakter, da eine entsprechende Überprüfung in der Realität kaum möglich ist. Dieser Sachverhalt induziert die Gefahr von nicht rein unter ökonomischen Gesichtspunkten getroffenen Entscheidungen. Wird ein System entsprechend kompliziert aufgebaut, indem unterschiedliche Größen miteinander verwoben werden, so geht die Übersichtlichkeit verloren, was eine effiziente Problemlösung erschwert. SEILER bezeichnet die derzeitigen Finanzausgleichssysteme als Geheimwissenschaft und legt in einigen Beispielen dar, wie die Einflußnahme von Interessenvertretern zu Resultaten führten, die ihren Überlegungen und Zielsetzungen zuwiderlaufen. ${ }^{5}$ In diesem Zusammenhang wäre beispielsweise für den KFA zu fordern, daß die Ermittlung von Finanzbedarf und -kraft isoliert durchgeführt und eine Vermischung dieser beiden Aspekte vermieden wird. ${ }^{6}$ Wird eine bestimmte Verteilungssituation aus politischen Gründen angestrebt, so kann dies dann durch die Wahl des Ausgleichsmechanismus realisiert werden, ohne daß die Übersichtlichkeit des Systems beeinträchtigt wird. ${ }^{7}$

- Innere Geschlossenheit: Eng mit der Transparenz ist die Forderung nach innerer Geschlossenheit des Systems verbunden. Denn nur bei einem transparenten System ist erkennbar, ob es Ungereimtheiten in der Ausgestaltung gibt. So ist darauf zu achten, daß die relevanten Größen auch einen Bezug zum Bedarf oder zur Finanzkraft haben. Die verschiedenen Regelungen zur Bedarfsmessung

1 Bundesverfassungsgericht: Beschluß vom 15.10.1985, in: Entscheidungen des Bundesverfassungsgerichts, Bd. 71, Tübingen 1986, S. 38.

2 Vgl.: Henneke, H. G.: Die beabsichtigte Erweiterung des Art. 28 Abs. 2 GG - ein Danaergeschenk für Kreise und Gemeinden?, in: Der Landkreis, 63, Jg., 1993, S. 215.

3 In Hessen gibt es erst seit 1994 ein kommunales Klagerecht vor dem Staatsgerichtshof: Gesetz über den Staatsgerichtshof vom 30.11.1994, Hess. GVBI. S. 684.

4 Verfassungsgerichtshof Nordrhein-Westfalen: Urteil vom 19.07.1985, in: OVGE, Bd. 38, Münster 1989, S. 315.

5 Vgl: Seiler, G.: Ziele und Mittel des kommunalen Finanzausgleichs - Ein Rahmenkonzept für einen aufgabenbezogenen kommunalen Finanzausgleich, in: Pohmer, D. (Hrsg.): Probleme des Finanzausgleichs II, Schriften des Vereins für Socialpolitik, N.F. Bd. 96/II, Berlin 1980, S. $63 f$.

6 Vgl.: Deubel, I.: Der kommunale Finanzausgleich in Nordrhein-Westfalen, Köln 1984, S. $122 \mathrm{f}$.

7 Vgl.: Micosatt, G.: Raumwirksamkeit einer Reform des kommunalen Finanzausgleichs, Bochum 1990, S. 73. 
müssen einer nachvollziehbaren Gewichtung unterliegen, und es sind Sprünge bei der Aufstellung der Bedarfsgrößen zwischen Gemeinden zu vermeiden; ebenso ist bei der Finanzkraft zu verfahren. Sie muß möglichst vollständig erfaßt werden, indem alle relevanten Einnahmenkategorien Berücksichtigung finden. Diese müssen dann wiederum gleichmäßig und kontinuierlich herangezogen werden, da Differenzierungen nur dann statthaft sind, wenn die Verfahrensweise als sachgerecht anzusehen ist, ansonsten ist darauf zu verzichten. ${ }^{1}$

- Ausführungsbilligkeit: Die Ausführungsbilligkeit impliziert ein System, das den gewünschten Zielen möglichst nahe kommt, dabei aber trotzdem einfach ausgestaltet ist, um die Verwaltungskosten möglichst gering zu halten. Für Regelungen des KFA ist zu klären, ob es nicht vergleichbare Mechanismen gibt, die mit geringeren Kosten $\mathrm{zu}$ einem dem bisherigen Verteilungsergebnis ähnlichen Resultat führen. So wäre zu prüfen, ob sich die verschiedenen Regelungen nicht gegenseitig kompensieren und somit eine unnötige Verkomplizierung hervorrufen. ${ }^{2}$ Denn die dafür notwendigen Daten müssen zuerst einmal erhoben und ausgewertet werden, so daß der zusätzlich induzierte Verwaltungsaufwand ins Verhältnis $\mathrm{zu}$ den dadurch generierten Umverteilungswirkungen zu setzen ist. ${ }^{3}$

- Geringe Sensitivität des Zuweisungssystems: Bei Verminderung der Finanzkraftunterschiede ist darauf zu achten, daß die Sensitivität des Anpassungsprozesses nicht $\mathrm{zu}$ groß ist, um $\mathrm{zu}$ vermeiden, daß kleine Veränderungen große Auswirkungen hervorrufen. ${ }^{4}$ Ein geringer Anstieg der Finanzkraft führte dann z.B. zu einem fast vollständigen Verlust der Zuweisungen. Dies hätte zur Folge, daß die Planbarkeit der Einnahmen und Ausgaben im großen Maße erschwert würde.

\subsubsection{Vertikale Anforderungskriterien}

\subsubsection{Angemessenheit der Verbundmasse}

Aussagen über die Höhe der KFA-Verbundmasse können direkt weder aus dem GG noch aus der HV abgeleitet werden. Als Anhaltspunkt kann aber angeführt werden, daß dem Land die Aufgabe zufällt, die zur Aufgabenwahrnehmung und zum politischen Handeln notwendige Finanzausstattung sicherzustellen. ${ }^{5}$ Dazu gehört auch, die Entwicklung der Ausgabenbelastung regelmäßig zu überprüfen.

1 Vgl. dazu die Ausführungen des Verfassungsgerichtshofs in Nordrhein-Westfalen zum gespaltenen Tarif bei der Erfassung der Realsteuerkraft. Verfassungsgerichtshof für das Land Nordrhein-Westfalen: Urteil vom 06.07.1993, in: OVGE, Bd. 43, Münster 1996, 261, 263.

2 Vgl.: Littmann, K. u.a.: Die Gestaltung des kommunalen Finanzsystems unter raumordnungspolitischen Gesichtspunkten, Hannover 1968, S. 62.

3 Vgl:: Münstermann, E.; Becker, H.: Finanzausgleichsleistungen an Kommunen. Ein Vergleich der Finanzausgleichssysteme in den Bundesländern (Reihe G; DST-Beiträge zur Finanzpolitik, Heft 7), Köln 1978, S. 52.

4 Vgl.: Michalk, J.: Die Garantieklauseln im Länderfinanzausgleich, in: Wirtschaftsdienst, 69. Jg., 1989, S. 453

5 Vgl.: Hoppe, W.: Der Anspruch der Kommunen auf aufgabengerechte Finanzausstattung, in: DVBl., 107. Jg., 1992, S. $118 \mathrm{f}$. 
Denn durch die kontinuierliche Übertragung von weiteren Pflichtaufgaben mit und ohne staatlicher Weisungsbefugnis werden die kommunalen Finanzmittel immer stärker in Anspruch genommen. Da gleichzeitig mit der Entwicklung zum Wohlfahrtsstaat die Ansprüche und damit auch die Ausgaben der allgemeinen Daseinsfürsorge gestiegen sind, besteht die Gefahr einer allgemeinen Unterfinanzierung. Daher ist der Finanzausgleich im Zeitablauf dahingehend zu prüfen, ob die Bemessung der Verbundmasse noch ausreichend ist, um die mit der Aufgabenerfüllung verbundenen Ausgaben tragen zu können. Aus diesem Grund wird häufig als nicht zu unterschreitende verfassungsrechtliche Grenze die Summe der originären und zugewiesenen Mittel angesehen, mit der die unabweisbaren Aufgaben gerade noch finanziert werden können. ${ }^{1}$ Darüber hinaus ist es noch erforderlich, $\mathrm{da} ß$ ein genügend großer finanzieller Spielraum zur Wahrnehmung der freiwilligen Aufgaben zur Verfügung steht, denn ansonsten ist die kommunale Selbstverwaltung eine leere Hülle. ${ }^{2}$

\subsubsection{Geringe Konjunkturreagibilität der Verbundmasse}

Durch $\S 1$ StWG ist die staatliche Ebene zu einer antizyklischen Wirtschafts- und Finanzpolitik verpflichtet. Zwar sind auch die Gemeinden nach § $16 \mathrm{StWG}$ zu einer entsprechenden Haushaltswirtschaft aufgerufen, doch wird diese Forderung durch die $\mathrm{HGO}^{3}$ eingeschränkt, da dort die Führung einer stetigen Haushaltswirtschaft im Vordergrund steht und das gesamtwirtschaftliche Gleichgewicht eine untergeordnete Rolle spielt. ${ }^{4}$ Denn die Gemeinden haben vor allem einen Versorgungsauftrag in Form der Bereitstellung staatlicher Vorleistungen furr private Haushalte und Unternehmen zu erfüllen. Dieses Leistungsangebot, wie z.B. Schulen und Straßen, ist unabhängig von der jeweiligen konjunkturellen Situation bereitzustellen und eignet sich somit wenig als konjunkturpolitische Manövriermasse. Zudem wird vor dem Hintergrund des Umfangs kommunaler Investitionen ein stetiges Ausgabenverhalten der Gemeinden als notwendig erachtet; die Gemeinden tätigen etwa 64 v.H. der gesamten öffentlichen Sachinvestitionen. ${ }^{5}$ Die Verstetigung der Ausgaben führt zu einer Mindestnachfrage durch die kommunale

1 Vgl.: Mutius, A. v.; Henneke, H. G.: Verfassungsrechtliche Anforderungen an die Durchfưhrung des kommunalen Finanzausgleichs - dargestellt am Beispiel Nordrhein-Westfalens, in: AfK, 24. Jg., 1985, S. 269.

2 Vgl:: Mutius, A. v.; Henneke, H. G.: Grenzen der verfassungsrechtlichen Zulässigkeit bei der Festsetzung von Schlüsselzuweisungen, in: Der Gemeindehaushalt, 85. Jg., 1984, S. 150.

3 "Die Gemeinde hat ihre Haushaltswirtschaft so zu planen und zu führen, daß die stetige Erfüllung ihrer Aufgaben gesichert ist. Dabei ist den Erfordernissen des gesamtwirtschaftlichen Gleichgewichts Rechnung zu tragen", § 92 Abs. 1 HGO.

4 Vgl.: Schneider, G; Dreßler, U.; Lüll, J.: Kommentar zu $\S 92$ HGO, in: Schneider, G; Dreßler, U.; Lüll, J. (Hrsg.): Hessische Gemeindeordnung, Kommentar (Loseblattsammlung), Mainz, Stand 1999, S. 5.

5 Vgl:: Sachverständigenrat zur Begutachtung der gesamtwirtschaftlichen Entwicklung: "Wachstum, Beschäftigung, Währungsunion - Orientierungen für die Zukunft" Jahresgutachten 1998/99, Stuttgart 1998, S. 372f. 
Ebene, so daß ein Mindestauslastungsgrad der Wirtschaft gesichert und eine Stabilisierung auf diesem Niveau erreicht wird. Treten hingegen prozyklische Einnahmenschwankungen auf, so verschärft sich die konjunkturelle Lage unter der Annahme, daß die Gemeinden die in der Periode anfallenden Einnahmen im vollen Umfang ausgeben und somit eine Parallelpolitik betreiben. ${ }^{1}$

\subsubsection{Selbstbestimmung der Mittelverwendung}

Ein weiterer aus kommunalpolitischer Sicht wichtiger Aspekt ist die Frage, inwieweit die zufließenden Mittel zweckgebunden oder frei verwendbar sind. Denn diesem Punkt kommt eine sehr wichtige Rolle in der Beurteilung der vertikalen Finanzbeziehung zu, da nur ungebundene Zuweisungen als ein Steuersubstitut angesehen werden können. ${ }^{2}$ Der Unterschied besteht dann vor allem darin, daß eine Bemessungsgrundlagen-Politik nicht betrieben werden kann und die rechtliche Absicherung meist geringer ist als bei einer gesetzlich kodifizierten Steuerzuteilung. Bei zweckgebundenen Zuweisungen ist hingegen der Entscheidungsspielraum der Gemeinden eingeschränkt, da mit ihnen i.d.R. ein Eingriff in die Entscheidungsautonomie der kommunalen Gebietskörperschaften verbunden ist. Denn die Mittelverwendung ist vorgegeben und häufig wird auch noch eine finanzielle Selbstbeteiligung gefordert; Ausnahmen gibt es bei 100\%iger Kostenübernahme (auch der Folgekosten) oder bei einem vollständigen Mitnahmeeffekt. ${ }^{3}$ Dieser Zuweisungstyp wird weiterhin von der kommunalen Ebene kritisiert, weil die damit verbundene Vergabepraxis häufig nicht bekannt bzw. nicht nachvollziehbar ist. ${ }^{4}$ Zudem werden die mit der Antragstellung verbundenen Verwaltungskosten kritisiert. ${ }^{5}$ Auch die Vereinbarkeit mit der Selbstverwaltungsgarantie des Art. $28 \mathrm{GG}$ ist nicht unumstritten. ${ }^{6}$ Häufig verleitet dieses Instrument die kommunale Ebene dazu, vermehrt die entsprechend geförderten Aufgaben anzugehen, ohne dabei den tatsächlichen Dringlichkeitsaspekt im Auge zu behalten. ${ }^{7}$

1 Vgl.: Zwilling, E.: Untersuchungen zu einem rationalen Steuersystem der Gemeinden, Meisenheim am Glan 1971, S. $72 \mathrm{f}$.

2 Vgl.: Voigt, R.: Das System des kommunalen Finanzausgleichs in der Bundesrepublik Deutschland, Stuttgart u.a.O. 1980, S. 65.

3 Vgl.: Zimmermann, H.: Stärkung der kommunalen Finanzautonomie, in: Staatswissenschaft und Staatspraxis, 6. Jg., 1995, S. 663f.

4 Vgl.: Münstermann, E.: Möglichkeiten und Grenzen einer "Kommunalisierung" von Zweckzuweisungen, in: Räumliche Aspekte des kommunalen Finanzausgleichs (Veröffentlichungen der Akademie für Raumforschung und Landesplanung: Forschungs- und Sitzungsberichte, Bd. 159), Hannover 1985, S. 240-242.

5 Vgl.: Fuchs, M.: Zweckgebundene Zuweisungen - Hilfe oder Last fur die Gemeinden?, in: Der Gemeindehaushalt, 70. Jg., 1969, S. 168-171.

6 Vgl: Petri, W.: Die staatlichen Zweckzuweisungen im kommunalen Finanzsystem. Dargestellt am Beispiel des Landes Niedersachsen, Berlin 1977, S. 55-57.

7 Vgl: Sachverständigenrat zur Begutachtung der gesamtwirtschaftlichen Entwicklung: "Arbeitsplätze im Wettbewerb"; Jahresgutachten 1988/89, Stuttgart 1988, S. 201, Tz. 431. 
Für die Länder sind die zweckgebundenen Zuweisungen aus mehreren Gründen attraktiv. Es können damit eigene Interessen verfolgt werden, indem die Zuweisungen für Aufgaben bereitgestellt werden, für die es in der Landesregierung entsprechende Präferenzen gibt. ${ }^{1}$ Diese Verfahrensweise wird als "goldener Zügel" bezeichnet, mit deren Hilfe die Gemeinden in die gewünschte Richtung gelenkt werden können. ${ }^{2}$ Ein anderer Aspekt ist vor dem Hintergrund der zunehmenden Verschuldung der öffentlichen Gebietskörperschaften und verfassungsrechtlicher Verschuldungsgrenzen wie Art. 115 Abs. 1 GG oder Art. 71 NV zu sehen. Werden nämlich die ungebundenen Zuweisungen durch für investive Zwecke zu verwendende Zuweisungen ersetzt, so erhöht dieser Vorgang die Investitionen des Landes und ermöglicht somit eine zusätzliche Kreditaufnahme. ${ }^{3}$ Diese Verfahrens praktizierte das Land Niedersachsen 1996, indem es einen Teil der Schlüsselzuweisungen einer Zweckbindung für Investitionen unterwarf. ${ }^{4}$ Der Niedersächsische Staatsgerichtshof erklärte diese Zweckbindung allerdings für verfassungswidrig, da die Gemeinden selbst über den für Investitionen verwendeten Anteil der Schlüsselzuweisungen entscheiden dürfen. ${ }^{5}$

\subsubsection{Horizontale Anforderungskriterien}

\subsubsection{Angemessenheit der Teil-Verbundmassen}

Wenn unterstellt wird, daß die Aufteilung der Finanzmasse zwischen der staatlichen und der kommunalen Gebietskörperschaftsebene aufgabenadäquat erfolgt ist, muß ferner gesichert werden, daß diese Finanzmittel so auf die drei verschiedenen kommunalen Gruppen - kreisfreie Städte, kreisangehörige Gemeinden und Landkreise - verteilt werden, daß eine angemessene Finanzausstattung zur Erfüllung der jeweiligen Aufgaben gegeben ist. Bei dieser Verteilung können drei in Deutschland angewendete Verfahren unterschieden werden. Im Ein-Säulen-Modell werden kreisangehöriger und kreisfreier Raum gegenübergestellt und aus einer Schlüsselmasse dotiert, wobei die kreisinterne Verteilung mittels eines fixierten Schlüssels und der Kreisumlage erfolgt. Im Zwei-Säulen-Modell werden die

1 Münstermann, E.: Möglichkeiten und Grenzen einer "Kommunalisierung" von Zweckzuweisungen, in: Räumliche Aspekte des kommunalen Finanzausgleichs (Veröffentlichungen der Akademie für Raumforschung und Landesplanung: Forschungs- und Sitzungsberichte, Bd. 159), Hannover 1985, S. 241. Hierunter fallen auch Zuweisungen zur Internalisierung externer Effekte.

2 Vgl.: Smekal, C.: Transfers zwischen Gebietskörperschaften (TG) - Ziele und Ausgestaltungsprobleme, in: Pohmer, D. (Hrsg.): Probleme des Finanzausgleichs II, Schriften des Vereins für Socialpolitik, N.F. Bd. 96/I, Berlin 1980, S. 189f.

3 Vgl.: o.V.: Das Land benachteiligt die Kommunen, in: Hannoversche Allgemeine Zeitung vom 26.11.1997, S. 1.

4 Vgl: Gesetz zur Änderung des Haushaltsgesetzes 1995/1996 (Nachtragshaushaltsgesetz) vom 20.12.1995, Nds. GVB1., S. 472.

5 Vgl: Niedersächsischer Staatsgerichtshof: Urteil vom 25.11.1997, in: DVBl., 113. Jg., 1998, S. 189. 
Gemeinden und Städte aus einer Schlüsselmasse finanziert, die Landkreise erhalten eine separate Schlüsselmasse. Im Drei-Säulen-Modell wird jeder Gebietskörperschaftsgruppe eine eigene Schlüsselmasse zugewiesen. Jedes Verfahren weist Vor- und Nachteile auf, so daß keine generellen Aussagen bezüglich der Vorteilhaftigkeit gemacht werden können. ${ }^{1}$

Um Anhaltspunkte für die Verteilung innerhalb der kommunalen Ebene zu bekommen, sind die Steuerkraft und der Finanzbedarf heranzuziehen. Dabei bietet es sich an, zuerst den kreisfreien und den kreisangehörigen Raum zu vergleichen, da diese gleiche Aufgaben erfüllen. Zu diesem Zweck werden die Kreise und die Gemeinden zu Gesamtkreisen aggregierte und dann der Gruppe der kreisfreien Städte gegenübergestellt. ${ }^{2}$ Weiterhin ist noch die Verteilung der Zuweisungen innerhalb des kreisangehörigen Raumes zu überprüfen. Es geht dabei um das Volumen der Schlüsselmassen und die Wirkungen der Kreisumlage.

\subsubsection{Ausgleichsrelevante Bedarfsermittlung}

Der Finanzbedarf einer Gebietskörperschaft ist neben der noch zu ermittelnden Finanzkraft für die Verteilung der Zuweisungen relevant, denn nur der Vergleich dieser beiden Größen ermöglicht es, die relative Stellung jeder einzelnen Gebietskörperschaft im KFA abzuleiten und damit die notwendige Zuweisungshöhe $\mathrm{zu}$ bestimmen. Denn ein reiner Finanzkraftvergleich hat aufgrund der unterschiedlichen ausgabenbedingten Belastungssituationen nur geringe Aussagekraft.

Der Finanzbedarf wird von zwei Einflußfaktoren bestimmt, nämlich einerseits von autonomen bzw. internen und andererseits von vorgefundenen bzw. externen Faktoren. ${ }^{3}$ Ist der jeweilige Finanzbedarf auf autonom getroffene Entscheidungen zurückzufuihren, so dürfen diese im Rahmen des KFA nicht als ausgleichsfähig gelten. Als Ursachen für eine entsprechende Entwicklung kommt eine sparsame oder ausgabenfreudige Mittelverwendung in Betracht. ${ }^{4}$ Würden diese Verhaltensweisen bei der Zuweisung der Schlüsselzuweisungen berücksichtigt, hätte dies anreizfeindliche Wirkungen. Zudem widerspricht eine solche Vorgehensweise

1 Vgl:: Steinherr, M.; u.a.: Das Schlüsselzuweisungssystem im kommunalen Finanzausgleich des Freistaates Sachsen, ifo-Studien zur Finanzpolitik, Bd. 63, München 1997, S. 67-70, 75f. Das Ein- bzw. das Drei-Säulen-Modell wird in Westdeutschland nur in Rheinland-Pfalz bzw. Hessen realisiert, die übrigen Westländer wenden das Zwei-Säulen-Modell an.

2 Vgl:: Steinherr, M.: Kommunaler Finanzausgleich: Balanceakt zwischen Verteilungs- und Allokationszielen - Das Beispiel Rheinland-Pfalz, in: ifo-Schnelldienst, 51. Jg., Nr. 8, 1998, S. 18.

3 Vgl: Junkernheinrich, M.: Sonderbedarfe im kommunalen Finanzausgleich, Berlin 1992, S. 31.

4 Stehen zur Straßenbeleutung bei gleicher Leistung Lampen unterschiedlicher Preiskategorie zur Wahl, sollte eine Kommune die Mehrkosten tragen, die beim Kauf luxuriöser Lampen anfallen. 
auch dem Postulat der eigenverantwortlichen Aufgabenerfüllung der kommunalen Ebene, nach der die Bürger die von ihnen sanktionierten politischen Entscheidungen auch selbst zu tragen haben und diese nicht externalisieren sollen. ${ }^{1}$ Entsprechend sollen sie von einer wirtschaftlichen Mittelverwendung profitieren bzw. durch die Folgen einer ineffizienten Verwendung belastet werden. Bei der zweiten Art der Beeinflussung - durch vorgefundene oder externe Faktoren - sind die Gegebenheiten, die auf die Selbstverwaltungskörperschaft wirken, von dieser nicht $\mathrm{zu}$ beeinflussen. $\mathrm{Zu}$ nennen sind hier etwa topographische (Gebirgslage) oder politische (ehemaliger Zonenrand) Faktoren. ${ }^{2}$ Diese Einwirkungen treffen die Gebietskörperschaften der kommunalen Ebene unverschuldet und sind deshalb von der Solidargemeinschaft innerhalb des jeweiligen Bundeslandes über den KFA mitzutragen. ${ }^{3}$

Zur Bedarfsermittlung stehen theoretisch zwei Verfahrensweisen zur Verfügung. Entweder wird aus den vergangenen Ist-Ausgaben mittels statistischer und ökonometrischer Verfahren oder mittels eines Aufgabenkatalogs auf den tatsächlichen Bedarf geschlossen. ${ }^{4}$ Die Erstellung eines solchen gemeindeindividuellen Aufgabenkatalogs gilt aufgrund des damit verbundenen Verwaltungsaufwands als utopisch. Da auch eine Klassifizierung nach Gemeindegruppen kaum möglich erscheint - es müßte Einigung über die jeweils der Gemeindeklasse zuzuordnenden Aufgaben, deren Ausführung sowie vor allem deren Monetarisierung erzielt werden, wobei jeweils vorgegebene Größen wie Siedlungsstruktur und geographische Besonderheiten zu beachten wären -, wird diese Art der Bedarfsermittlung verworfen. Als weiteres Hindernis wird die verfassungsrechtliche Selbstverwaltungsautonomie angeführt, da die Aufzählung und Normierung der kommunalen Aufgaben die Gefahr der teilweisen Beschränkung der Wahlfreiheit bei der Aufgabenwahmehmung ${ }^{5}$ birgt, auch wenn dies nicht zwangsläufig folgen muß. ${ }^{6}$ Denn eine Aufgabenbeschränkung ist im Grundgesetz nicht vorgesehen, und eine

1 Vgl.: Kirchhof, P.: Der Finanzausgleich als Grundlage kommunaler Selbstverwaltung, in: DVB1., 95. Jg., 1980, S. 714.

2 Vgl.: Achatz, M.: Finanzausgleichstheoretische Überlegungen zum Finanzbedarf, in: Smekal, C.; Theurl, E. (Hrsg.): Finanzkraft und Finanzbedarf von Gebietskörperschaften, Wien u.a.O. 1990 , S. 176-182.

3 Vgl.: Kirchhof, P.: Die kommunale Finanzhoheit, in: Püttner, G. (Hrsg.): Handbuch der kommunalen Wissenschaft und Praxis, Bd. 6, 2. Aufl., Berlin u.a.O. 1985, S. $15 f$.

4 Vgl: Zimmermann, H.; Postlep, R.-D.: Probleme des kommunalen Finanzausgleichs - Überblick und Einordnung der Untersuchungsergebnisse, in: Räumliche Aspekte des kommunalen Finanzausgleichs (Veröffentlichungen der Akademie für Raumforschung und Landesplanung: Forschungs- und Sitzungsberichte, Bd. 159), Hannover 1985, S. 4-6.

5 Vgl: Haverkamp, F.: Die Finanzbeziehungen zwischen Ländern und Gemeinden, in: Arnold, V.; Geske, O.-E. (Hrsg.): Öffentliche Finanzwirtschaft, München 1988, S. 78.

6 Vgl.: Henneke, H.-G.: Probleme des kommunalen Finanzausgleichs, in: Ipsen, J. (Hrsg.) Kommunale Aufgabenerfüllung im Zeichen der Finanzkrise, Baden-Baden, 1995, S. 110. 
Bestimmung des Finanzbedarfs für den fast unendlichen Bereich der freiwilligen Aufgaben ist unmöglich.

Deshalb wird ein Ansatz von PoPIrz ${ }^{1}$ verwendet, nach dem der Bedarf einer Kommune aus ihrem Ausgabenniveau abgeleitet wird. Dabei wird mit Hilfe der Regressionsanalyse aus dem sog. gemeindlichen Zuschußbedarf ${ }^{2}$ ein einwohnerbezogener Bedarfsindikator ermittelt. Zwar ist dem Bundesverfassungsgericht zuzustimmen, daß die Einwohnerzahl als einziges bedarfsinduzierendes Kriterium wohl seine Gültigkeit verloren hat und deshalb weitere Indikatoren zu berücksichtigen sind. ${ }^{3}$ Doch daraus auf die Verfassungswidrigkeit der bisherigen Bedarfsermittlung zu schließen, dürfte etwas voreilig sein. Denn im Gegensatz zum beanstandeten ausschließlich einwohnerbezogenen Bedarfsermittlungsverfahren bei der kommunalen Ebene im LFA werden in fast allen Landesgesetzen zum KFA neben der Einwohnerzahl noch andere bedarfsinduzierende Größen wie die Schülerzahl oder die zentralörtliche Aufgabenerfüllung berücksichtigt.

\subsubsection{Realitätsnahe Finanzkraftermittlung}

Neben der möglichst genauen Erfassung des Finanzbedarfs ist auch die exakte Bestimmung der Finanzkraft anzustreben, denn nur dann ist die Ermittlung einer Deckungslücke möglich. Allerdings können auch hier Probleme auftreten; diese liegen in der Abgrenzung der zu berücksichtigenden Einnahmenkategorien sowie in der Festlegung des Anrechnungsumfangs bzw. der -höhe der heranzuziehenden Einnahmenart begründet. ${ }^{4}$ Zwar sollten prinzipiell alle Einnahmen bei der Finanzkraftermittlung einbezogen werden, doch kann darauf verzichtet werden, wenn ihr Aufkommen gleichmäßig im Raum streut, es sich nur um sehr geringe Beträge handelt oder der Verwaltungsaufwand zu deren Erfassung unverhältnismäßig hoch ist. ${ }^{5}$ Grundsätzlich sollten wie beim für natürliche Personen geltenden Leistungsfähigkeitsprinzip alle Einnahmenerzielungsmöglichkeiten berücksichtigt werden; Abweichungen davon müssen wohlbegründet sein.

1 Vgl:: Popitz, J.: Der künftige Finanzausgleich zwischen Reich, Ländern und Gemeinden, Berlin 1932, S. 262-285.

2 Der Zuschußbedarf wird definiert als: Ausgaben des Verwaltungshaushalts vermindert um Einnahmen des Verwaltungshaushalts, Zuführungen an den Vermögenshaushalt und Abwicklung von Fehlbeträgen und erhöht um Zuführungen aus dem Vermögenshaushalt, Aufkommen der Realsteuern (netto), der Einkommensteuer, Schlüssel- sowie Bedarfszuweisungen. Die Hauptansatzstaffelung dient der Bedarfsermittlung, wobei für Gemeindegrößenklassen unterschiedliche Multiplikatoren verwendet werden.

3 Bundesverfassungsgericht: Urteil vom 27.05.1992, in: Entscheidungen des Bundesverfassungsgerichts, Bd. 86, Tübingen 1993, S. 236.

4 Vgl: Hardt, U.: Kommunale Finanzkraft. Die Problematik einer objektiven Bestimmung kommunaler Einnahmemöglichkeiten in der gemeindlichen Haushaltsplanung und im kommunalen Finanzausgleich, Frankfurt u.a. 1988, S, 46.

5 Vgl: Bundesverfassungsgericht: Urteil vom 24.06.1986, in: Entscheidungen des Bundesverfassungsgerichts, Bd. 72, Tübingen 1987, S. 400. 
Nachdem eine Abgrenzung der ausgleichsrelevanten Größen stattgefunden hat, gilt es zu klären, in welchem Umfang einzelne Einnahmenkategorien einzubeziehen sind. Es ist dabei anzustreben, alle Arten im gleichen Umfang zu berücksichtigen, da ansonsten schon durch den Erfassungsgrad unerwünschte Verteilungswirkungen entstehen. ${ }^{1}$ Dabei kann eine Klassifizierung nach dem Grad der Einnahmenautonomie vorgenommen werden, indem zwischen solchen ohne und solchen mit Möglichkeiten der direkten Einflußnahme auf das Aufkommen unterschieden wird. Bei der ersten Kategorie werden die tatsächlich erzielten Einnahmen als Finanzkraft angerechnet. Unter der zweiten Kategorie werden dann die Arten subsumiert, bei denen eine Variationsmöglichkeit des Aufkommens gegeben ist, da zwar die Bemessungsgrundlage einheitlich geregelt ist, die Höhe der jeweils zu leistenden Zahlungen aber durch die Selbstverwaltungskörperschaften direkt beeinflußt werden kann. Bei diesen Einnahmekategorien soll nicht die tatsächliche Einnahmenhöhe, sondern nur die normierte Einnahmenfähigkeit zur Finanzkraftberechnung herangezogen werden, wodurch erreicht wird, daß die durch autonome Entscheidungen induzierten zusätzlichen (geringeren) Einnahmen aufgrund der stärkeren (schwächeren) Belastung der Zahler auch bei der jeweiligen Gebietskörperschaft verbleiben und nicht externalisiert werden. $\mathrm{Zu}$ diesem Zweck muß eine Normierung derart vorgenommen werden, daß die potentielle Finanzkraft der Gebietskörperschaften möglichst genau abgebildet wird, ohne dabei der einzelnen Gemeinde die Möglichkeit zu geben, etwa durch Hebesatzvariationen ihre Finanzkraft für den Finanzausgleich zu verringern und damit zu Lasten Dritter die eigenen Schlüsselzuweisungen zu erhöhen.

\subsubsection{Angemessene Ausgleichsintensität}

Das Ziel des Finanzausgleichssystems ist eine Minderung der Unterschiede der vorgefundenen Finanzausstattung, ohne die Anreize zur Pflege der eigenen Steuerquellen zu verringern. Zu diesem Zweck wird ein Vergleich der beiden ermittelten Größen vorgenommen. Um die Transparenz des Systems zu wahren, sind die Finanzkraft und der -bedarf möglichst genau zu erfassen. Der Ausgleichsgrad legt fest, mit welchem v.H.-Satz die Differenz zwischen Finanzbedarf und -kraft durch Zuweisungen ausgeglichen werden soll. Dabei ist der Ausgleichsmechanismus so zu gestalten, daß er einerseits den aus distributiven Aspekten notwendigen Ausgleich von Bedarf und Einnahmen ermöglicht, andererseits aber auch noch einen aus allokativer Sicht genügend großen Anreiz zur Pflege der eigenen Steuerquellen zum Zwecke der Einnahmensteigerung induziert.

Um dies zu erreichen, darf weder die Rangfolgeneutralität gestört werden, die Finanzkraftreihenfolge vor und nach KFA muß identisch sein, noch eine (Über-)Nivellierung zustande kommen, d.h. eine vorgefundene unterschiedliche Ausstattung

1 Vgl.: Gläser, M.: Die staatlichen Finanzzuweisungen an die Gemeinden, Frankfurt - Thun 1981, S. 294. 
mit Finanzmitteln muß nach KFA fortbestehen. Die Finanzkraft je Bedarfseinheit ${ }^{1}$ gilt dabei als Beurteilungskriterium. ${ }^{2}$ Zwar könnte der Begriff "Finanzausgleich" nahelegen, daß bei enger Betrachtung - nur Einnahmen unter Vernachlässigung der Aufgaben und Ausgaben - die Identität nach Verteilung der Zuweisungen bei der Finanzausstattung angestrebt werden soll, doch diese Interpretation muß aus rechtlichen und allokativen Gründen abgelehnt werden ${ }^{3}$; zumal auch die nach dem Grundgesetz zu wahrende Einheitlichkeit der Lebensverhältnisse keine Nivellierung verlangt. ${ }^{4}$ Statt dessen ist eine angemessene Verringerung der vorgefundenen Unterschiede anzustreben. ${ }^{5}$

Zudem muß das Kriterium der strikten Monotonie gelten. Es besagt, daß "Grenzzuweisungs- und Grenzentzugssatz" im Finanzausgleichssystem unter 100 v.H. liegen müssen, damit eine Steigerung der Steuerkraft eines Zuweisungsempfängers vor KFA sich auch in einem erhöhten Einnahmevolumen nach Finanzausgleich niederschlägt. ${ }^{6}$ Andernfalls besteht kein unmittelbares fiskalisches Interesse daran, die Steuereinnahmen zu erhöhen, da bei einem denkbaren Anrechnungssatz von 100 v.H. im gleichen Maße die Zuweisungen gemindert werden. ${ }^{7}$ Es könnten höchstens qualitative Überlegungen dergestalt eine Rolle spielen, daß die Ausgestaltung der Finanzzuweisungen der Höhe und der Art nach stärker von den Entscheidungen der übergeordneten aber auch der Gebietskörperschaften der gleichen Ebene abhängig sind, als dies bei den originären Einnahmequellen der Fall ist. ${ }^{8}$

1 Mit Hilfe dieses Quotienten kann die nivellierende Wirkung des KFA gemessen werden. Die Einwohnerzahl eignet sich aufgrund der Veredelung und der Nebenansätze genauso wenig, wie der ermittelte Finanzbedarf, der sich wiederum durch den Grundbetrag verändern kann.

2 Vgl.: Verfassungsgerichtshof für das Land Nordrhein-Westfalen: Urteil vom 19.07.1985, in: OVGE, Bd. 38, Münster 1989, S. 315, 317; Niedersächsischer Staatsgerichtshof: Beschluß vom 15.08.1995, in: DVBl., 110. Jg., 1995, S. 1175.

3 Vgl.: Henneke, H.-G.: Der kommunale Finanzausgleich. Einer der verfassungsgerichtlich am sorgfältigsten behauenen Ecksteine der Grundlagen kommunaler Selbstverwaltung, in: DÖV, 47. Jg., 1994, S. 5.

4 Vgl: Neumark, F.: Bemerkungen zu einigen ökonomischen Aspekten der grundgesetzlichen Vorschriften über die Einheitlichkeit der Lebensverhältnisse in der Bundesrepublik Deutschland, in: Dreißig, W. (Hrsg.): Probleme des Finanzausgleichs I, Schriften des Vereins für Socialpolitik, N.F. Bd. 96/I, Berlin 1978, S. 165-175.

5 Vgl: Bundesverfassungsgericht: Urteil vom 27.05.1992, in: Entscheidungen des Bundesverfassungsgerichts, Bd. 86, Tübingen 1993, S. 220.

6 Vgl: : Sachverständigenrat zur Begutachtung der gesamtwirtschaftlichen Entwicklung: "Arbeitsplätze im Wettbewerb"; Jahresgutachten 1988/89, Stuttgart 1988, S. 201, Tz. 432.

7 Unbeachtet bleibt hier, daß die Gemeinden aus anderen Gründen - etwa der Schaffung neuer Arbeitsplätze - an steigenden Steuereinnahmen interessiert sein können, da es nur um die Betrachtung von Anreizeffekten geht.

8 Vgl:: Wissenschaftlicher Beirat beim Bundesministerium der Finanzen: Gutachten zum Gemeindesteuersystem und zur Gemeindesteuerreform in der Bundesrepublik Deutschland, Schriftenreihe des Bundesministeriums der Finanzen, Heft 10, Bonn 1968, S. 26-28. 


\section{Kommunaler Finanzausgleich in Hessen - Darstellung, Analyse und Reform}

\subsection{Verteilung der Finanzmasse zwischen Land und Kommunen}

\subsubsection{Strukturelle Entwicklung der Verbundmasse}

In der Verfassung des 1946 gegründeten Landes Hessen wurde in Art. 137 Abs. 5 festgelegt, daß das Land seinen Gemeinden und Gemeindeverbänden Finanzmittel zur Erfüllung der eigenen und der übertragenen Aufgaben bereitstellen muß. ${ }^{1} \mathrm{Zu}$ dem bestimmen $\S \S 3,4 \mathrm{HGO}$, daß bei Übertragung zusätzlicher Aufgaben auf die kommunale Ebene auch die entsprechende Finanzierung zu regeln ist.

Die der kommunalen Ebene insgesamt zugewiesenen finanziellen Mittel wurden im jeweiligen Jahr im Haushaltsplan festgelegt und orientierten sich anfänglich sowohl der Höhe als auch der Verteilung nach an der Verordnung über die einstweilige Regelung des Finanz- und Lastenausgleichs aus dem Jahr 1944. ${ }^{2}$ Dabei wurden für verschiedene Bedarfsindikatoren feste Beträge gezahlt, die nur vergleichsweise gering erhöht wurden. Die beschränkte Steigerung der bereitgestellten Mittel hatte zur Folge, daß die Rückkehr zur "verbundenen Steuerwirtschaft"3 gefordert wurde, wie sie in Deutschland bis 1938 durch eine Beteiligung der Kommunen an der Einkommen-, Körperschaft- und Umsatzsteuer Bestand hatte. ${ }^{4}$ Zudem gab es keinen rechtlichen Anspruch auf die Höhe der Zahlungen, da diese von der Exekutive im Haushalt und nicht durch die Legislative per Gesetz bestimmt wurde, so daß die Gefahr der Konsolidierung des Landes auf Kosten der Kommunen bei entsprechend schlechter wirtschaftlicher Entwicklung gegeben war. ${ }^{5}$

Diese Probleme sollten durch ein neues Verfahren gelöst werden, nach dem die betragsmäßigen Einzeldotationen durch eine gesetzlich fixierte prozentuale Beteiligung an den Steuereinnahmen des Landes abzulösen waren. Dadurch wurde die Möglichkeit diskretionärer Eingriffe der Regierung auf Kosten der Kommunen im

1 Vgl.: Verfassung des Landes Hessen (HV) vom 18.12.1946, Hess. GVBl. I, S. 229

2 Vgl.: $§ 1$ des Gesetzes über die Regelung des Finanzausgleichs für das Haushaltsjahr 1946 vom 08.04.1947, Hess. GVB1. I, S. 24. Verordnung über die einstweilige Regelung des Finanz- und Lastenausgleichs (Finanzausgleichs-Verordnung) vom 30.10.1944, RGB1. I, S. 282.

3 Vgl.: o.V.: Rückkehr zur verbundenen Steuerwirtschaft, in: Der Städtetag, 6. Jg., 1953, S. 185; Deutscher Städtetag: Rückkehr zur verbundenen Steuerwirtschaft, Köln 1953; Deutscher Bundestag: Antrag der Fraktionen der CDU/CSU, SPD, FDP, GB/BHE, DP über den Entwurf eines Gesetzes zur Ergänzung des Artikels 106 Grundgesetz vom 07.12.1954, Drucksache 2/1050, S. 1 .

4 Deutscher Städtetag: Rückkehr zur verbundenen Steuerwirtschaft, Köln 1953, S. 5.

5 Vgl:: Storck, H.: Der Krebsschaden der Finanzzuweisungen, in: Der Städtetag, 2. Jg., 1949, S. $217 f$. 
Vergleich zu individuell festzulegenden Summen eingeschränkt, da bei der alten Regelung eine Variation im Haushaltsplan durch die Regierung genügte, während nun auch das Finanzausgleichsgesetz mit Zustimmung des Parlamentes modifiziert werden mußte. Zudem gewährte eine prozentuale Beteiligung an den Steuereinnahmen des Landes, in erster Linie der Einkommen- und Körperschaftsteuer, der kommunalen Ebene die Chance, an den steigenden Einnahmen im Umfang der Beteiligungsquote automatisch zu partizipieren. Da sich die jeweilige Steuereinnahmenentwicklung gleichmäßig auf alle Beteiligten auswirkt, kommt es "zu einer engen Schicksalsverbundenheit des Bundes, der Länder und der Gemeinden"1. Angestrebt wurde eine grundgesetzliche Regelung, denn nach der Verfassung sollte die endgültige Aufteilung der Steuereinnahmen auf die einzelnen Gebietskörperschaftsebenen durch ein Ausführungsgesetz zu Art. 107 GG bis zum 31.12.1952 getroffen werden. ${ }^{2}$ Es wurde dabei auch an eine Berücksichtigung der kommunalen Ebene gedacht; da sich Bundestag und Bundesrat nicht einigen konnten, wurde diese Reform verschoben. Daraufhin strebte die hessische Regierung eine landesrechtliche Lösung dieser Fragestellung durch den Ausbau des Finanzausgleichsystems zu einer Steuerverbundwirtschaft an. ${ }^{3}$

Mit dem FAG vom 30.05.1956 $6^{4}$ wurden die Gemeinden und GV noch vor der entsprechenden Regelung im Grundgesetz vom 24.12.1956 - welche bis 1958 in Kraft treten mußte - an der Einkommen- und Körperschaftsteuer beteiligt. ${ }^{5}$ Allerdings verzichtete Hessen darauf, noch weitere Steuern in den Verbund aufzunehmen, im Gegensatz zu anderen Ländern, die durch zusätzliche Steuern eine Verbreiterung der Bemessungsgrundlage und somit eine Verringerung der Konjunkturreagibilität der Verbundmasse erreichen wollten. ${ }^{6}$ Dieser Lösungsansatz wurde mit der überragenden Stellung der beiden Steuern begründet, die 1955 zusammen fast 81 v.H. der Steuereinnahmen des Landes ausmachten. ${ }^{7}$ Aufgrund dieses Anteils konnte von einer "Schicksalsgemeinschaft" gesprochen werden, die das Verhältnis zwischen beiden Gebietskörperschaftsebenen in Hessen auf der Einnahmenseite stabilisierte. In den Steuerverbund flossen 18,5 v.H. der dem Land nach Länderfinanzausgleich verbleibenden Einnahmen.

o.V.: Rückkehr zur verbundenen Steuerwirtschaft, in: Der Städtetag, 6. Jg., 1953, S. 185.

Vgl: Art. 107 GG vom 23.05.1949, BGBl. I, S. 1.

Vgl.: Hessischer Landtag: Regierungserklärung des Hessischen Ministerpräsidenten Dr. Zinn, 3. Wahlperiode, Stenographische Berichte, 3. Sitzung vom 19.01.1955, S. 19.

4 Vgl: Gesetz zur Regelung des Finanzausgleichs (Finanzausgleichsgesetz - FAG -), Hess. GVB1. I, S. 107.

5 Gesetz zur Änderung und Ergänzung des Artikels 106 des Grundgesetzes vom 24.12.1956, BGBI. I, S. 1077.

6 Vgl.: Patzig, W.: Der kommunale Finanzausgleich im Zeichen des Steuerverbundes, in: DVB1., 74. Jg., 1959, S. 2.

7 Vgl.: Hessischer Landtag: Vorlage der Landesregierung für ein Gesetz zur Regelung des Finanzausgleichs vom 15.02.1956, Drucksachen Abteilung I, 3/373, S. 995. 
In den Anfangsjahren nach Einführung des Einkommensteuerverbundes gab es aber auch Befürchtungen, daß bei einer konjunkturbedingten starken Verringerung der Steuereinnahmen die dadurch gesunkenen Zuweisungen von der kommunalen Ebene nicht zu verkraften wären. ${ }^{1}$ Deshalb wurden für die Schlüsselzuweisungen in den Jahren 1956-1962 Mindestsummen bestimmt. ${ }^{2}$ Als Berechnungszeitraum für die Finanzausgleichsmasse wurde aus verwaltungstechnischen Gründen das vorangegangene Jahr gewählt, da diese Daten vorab bekannt sind und der kommunalen Ebene somit "eine ordnungsgemäße Finanzplanung in Übereinstimmung mit den Terminen der Hessischen Gemeindeordnung ermöglicht" ${ }^{3}$. Zudem konnten die Kommunen somit im vorhinein auf sich verändernde Einnahmen reagieren. Seit dem Finanzausgleichsgesetz aus dem Jahr 1960 ${ }^{4}$ wird auf die SollEinnahmen des Ausgleichsjahres als Berechnungsgrundlage abgestellt, da ansonsten die Leistungen des Landes an seine kommunale Ebene im nationalen Vergleich aufgrund der Phasenverschiebung geringer sind. ${ }^{5}$

Die nächste einschneidende Veränderung im Volumen der bereitgestellten Mittel trat mit der Einführung des separaten Kraftfahrzeugsteuerverbundes zum 01.01.1961 ein. ${ }^{6}$ Damit wurde eine Forderung der kommunalen Spitzenverbände erfüllt, diese Steuereinnahmen auch für den Ausbau und den Erhalt der kommunalen Straßen zu nutzen. Bis dahin wurden die Mittel zur Erhaltung und zum Ausbau der Straßen, die der hessischen kommunalen Ebene zugeordnet waren, sowohl aus dem allgemeinen Steuerverbund als auch aus übertragenen Landeshaushaltsmitteln bestritten. Aufgrund der Zunahme des Kraftverkehrs und der zentralen Lage Hessens wurden diese Mittel als nicht mehr ausreichend angesehen, so daß sich das Land zu einer Beteiligung der Gemeinden und GV mit 25 v.H. am Aufkommen der Kraftfahrzeugsteuer entschloB. ${ }^{7}$ Auch hier sollte nun das SollAufkommen des Ausgleichsjahres als Berechnungsgrundlage dienen. Allerdings

1 Vgl.: Seidel, J.: Kommunaler Finanzausgleich 1958, in: Der Gemeindetag, 11. Jg., 1958, S. 50 .

2 Vgl.: Hessischer Landtag: Vorlage der Landesregierung fur ein Gesetz zur Regelung des Finanzausgleichs vom 15.02.1956, Drucksachen Abteilung I, 3/373, S. 1002f. und ders.: Vorlage der Landesregierung über ein Drittes Gesetz zur Änderung des Finanzausgleichsgesetzes vom 12.02.1963, Drucksachen Abteilung I, 5/45, S. 6.

3 Ders.: Vorlage der Landesregierung für ein Gesetz zur Regelung des Finanzausgleichs vom 15.02.1956, Drucksachen Abteilung I, 3/373, S. 1000.

4 Vgl.: $\S 1$ Abs. 2 des Gesetzes zur Regelung des Finanzausgleichs (Finanzausgleichsgesetz FAG -) vom 06.04.1960, S. 36.

5 Vgl.: Hessischer Landtag: Ausführungen des Abgeordneten Krämer (SPD), 4. Wahlperiode, Stenographische Berichte, 23. Sitzung vom 09.03.1960, S. 864.

6 Vgl: Gesetz über die Beteiligung der Gemeinden und Landkreise am Aufkommen der Kraftfahrzeugsteuer (Kraftfahrzeugsteuerverbund) vom 19.12.1969, Hess. GVBI. I, S. 233.

7 Vgl: : Hessischer Landtag: Vorlage der Landesregierung für ein Gesetz über die Beteiligung der Gemeinden und Landkreise am Aufkommen der Kraftfahrzeugsteuer (Kraftahrzeugsteuerverbundgesetz) vom 31.08.1960, Drucksachen Abteilung I, 4/698, S. 1942. 
ist darauf hinzuweisen, daß das Land den kommunalen Gebietskörperschaften vor Einführung dieses Verbundes schon finanzielle Mittel für diesen Bereich zugewiesen hatte, die sogar den Betrag der Kraftfahrzeugsteuereinnahmen übertrafen. ${ }^{1}$ Die gesetzliche Regelung in Form des Steuerverbundes führte jedoch zu einer größeren Sicherheit bei der Einnahmenplanung im Vergleich zu den durch die Exekutive veränderbaren Haushaltsansätzen. Im Jahr darauf wurde die Quote für den Einkommensteuerverbund von 18,5 v.H. auf 21,0 v.H. erhöht, um einen Ausgleich für die wegen der Anhebung der Freibeträge verminderten Gewerbesteuereinnahmen zu schaffen. ${ }^{2}$

In den Jahren 1963 bzw. 1965 wurde die Bemessungsgrundlage des KFA durch die Einführung eines Vermögensteuerverbunds ${ }^{3}$ bzw. durch die direkte Beteiligung der kreisfreien Städte und der Landkreise am Landesanteil der Grunderwerbsteuer ${ }^{4}$ nach dem örtlichen Aufkommen verbreitert. Mit der Zuweisung der Vermögensteuer sollte den Kommunen eine stetige und wachsende Einnahmequelle erschlossen werden, wobei sie 100 v.H. der dem Land nach Abzug der Lastenausgleichsleistungen verbleibenden Summe zugesprochen bekamen. Diese Einnahmen sollten aber nur zur Finanzierung von Investitionen verwendet werden und somit die aufgrund des hohen Investitionsbedarfs sonst notwendige Zunahme der kommunalen Verschuldung vermindern. Es wurde darauf hingewiesen, daß es sich bei diesem Verbund nur um ein Provisorium bis zu einer Änderung der Finanzverfassung handeln sollte, die kommunalen Investitionsausgaben aber schon vorher durch Zuweisungen finanziell abgesichert werden sollten. ${ }^{5}$ Von einer Beteiligung am Grunderwerbsteueranteil des Landes wurde bei den kreisangehörigen Gemeinden im Gegensatz zu den Landkreisen und kreisfreien Städten aufgrund der großen Zahl abgesehen. Diese Einnahmen waren nicht zweckgebunden, sondern wurden als allgemeine Deckungsmittel veranschlagt. ${ }^{6}$ In den

1 Vgl: Seidel, J.: Kommunaler Finanzausgleich 1958, in: Der Gemeindetag, 11. Jg., 1958, S. 51f.

2 Vgl.: Hessischer Landtag: Vorlage der Landesregierung über ein Zweites Gesetz zur Änderung des Finanzausgleichsgesetzes vom 17.08.1961, Drucksachen Abteilung I, 4/1131, S. 3416 .

3 Vgl: Gesetz über die Verwendung der Vermögensteuer zugunsten der Gemeinden und Gemeindeverbände (Vermögensteuerverbundgesetz) vom 09.05.1963, Hess. GVBI. I, S. 60.

4 Vgl: $§ 6$ des Gesetzes zur Regelung des Finanzausgleichs (Finanzausgleichsgesetz -FAG-) vom 21.12.1964, Hess. GVBl. I, S. 235; mit diesem Gesetz wurden die drei separat geregelten Verbünde (Einkommen-, Kraftfahrzeug- und Vermögensteuer) in einem Gesetz zusammengefaßt, um die Transparenz bezüglich der Leistungen des Landes zu erhöhen.

5 Vgl.: Hessischer Landtag: Vorlage der Landesregierung für ein Gesetz über die Verwendung der Vermögensteuer zugunsten der Gemeinden und Gemeindeverbände (Vermögensteuerverbundgesetz) vom 12.02.1963, Drucksachen Abteilung I, 5/44, S. 2.

6 Vgl.: Ders.: Vorlage der Landesregierung für ein Gesetz zur Regelung des Finanzausgleichs (Finanzausgleichsgesetz - FAG -) vom 31.08.1964, Drucksachen Abteilung I, 5/989, S. 39, 41 . 
Ausführungen des hessischen Finanzministers zur Grunderwerbsteuerbeteiligung heißt es, "daß die systematische Durchforstung der Steuerarten hinsichtlich ihrer Tauglichkeit zur Verbesserung der kommunalen Finanzsituation im Rahmen der Länderkompetenz an ihrem Ende angelangt ist.(...) Dies heißt nichts anderes, als $\mathrm{da} B$ der Punkt erreicht ist, an dem endgültig die Diskussion über die Verbesserung der kommunalen Finanzsituation auf Bundesebene entschieden werden muß"1. Die Anhebung des Einkommensteuerverbundes 1966 um 2 Prozentpunkte auf 23,0 v.H. diente dazu, die investiven Zuweisungen zu erhöhen, ohne gleichzeitig die Schlüsselzuweisungen senken zu müssen. ${ }^{2}$

1970 wurde eine strukturelle Modifikation mit der Bildung des allgemeinen Steuerverbundes im Gefolge des Finanzrefomgesetzes ${ }^{3}$ vorgenommen, durch das die Gemeinden direkt an der Einkommensteuer auf Kosten der Gewerbesteuer beteiligt wurden. Entsprechend Art. 106 Abs. 7 GG wurde neben der Einkommen- und Körperschaft- auch die Umsatzsteuer in die Bemessungsgrundlage der Verbundmasse einbezogen. Diese wurde noch um die von den Gemeinden an das Land abzuführende Gewerbesteuerumlage erhöht, was damit begründet wurde, daß beim Land die Umlage als Substitut für den an die Gemeinden abgetretenen Einkommensteueranteil anzusehen und deshalb wie die Einkommensteuer in den Verbund einzubeziehen ist. ${ }^{4}$ 1973/74 wurden dann beim Vermögensteuer- und 1976 beim Kraftfahrzeugsteuer- sowie beim allgemeinen Verbund die Verbundquoten gesenkt. ${ }^{5}$ Als Begründung für die Verringerung beim Vermögensteuerverbund wurde die angespannte Haushaltssituation des Landes und die vergleichsweise stärker wachsenden Einnahmen der kommunalen Ebene genannt, wobei die Minderung im parlamentarischen Prozeß noch von 15 auf 39 bzw. 38,5 Prozentpunkte (1973 bzw. ab 1974) erhöht wurde. ${ }^{6}$ Da sich die finanzielle Situation des Landes in den Folgejahren nicht verbesserte, sollte die kommunale Ebene weiterhin zum Sparen angehalten werden, weshalb die Quote 1976 beim allgemeinen Verbund von 23,0 auf 20,6 v.H. vermindert wurde. Gleichzeitig wurden die

1 Vgl.: Ders.: Haushaltsrede des Hessischen Finanzministers Dr. Conrad, 5. Wahlperiode, Stenographische Berichte, 29. Sitzung vom 09.09.1964, S. 1135.

2 Vgl.: Ders.: Vorlage der Landesregierung für ein Erstes Gesetz zur Änderung des Gesetzes zur Regelung des Finanzausgleichs (Finanzausgleichsgesetz - FAG -) vom 26.08.1965, Drucksachen Abteilung I, 5/1464, S. 5, 7.

3 Vgl: Einundzwanzigstes Gesetz zur Änderung des Grundgesetzes (Finanzreformgesetz) vom 12.05.1969, BGBI. I, S. 359.

4 Vgl: Hessischer Landtag: Vorlage der Landesregierung furr ein Fünftes Gesetz zur Änderung des Finanzausgleichsgesetzes vom 07.07.1969, Drucksache 6/2194, S. 8.

5 Vgl:: Art. $1 \mathrm{Nr} .1$ und 17 des Achten Gesetzes zur Änderung des Finanzausgleichsgesetzes vom 18.12.1972, Hess. GVBl. I, S. 432, 434; Art. 1 Nr. 1 des Elften Gesetzes zur Änderung des Finanzausgleichsgesetzes vom 15.12.1975, Hess. GVB1. I, S. 299.

6 Vgl: Hessischer Landtag: Vorlage der Landesregierung betreffend den Entwurf für ein Achtes Gesetz zur Änderung des Finanzausgleichsgesetzes vom 07.08.1972, Drucksache 7/2030, S. 8 f. 
Landeseinnahmen aus der Kraftfahrzeugsteuer nur noch zu 90 v.H. zweckgebunden für den Straßenbau verwendet, so daß die Verbundquote um 2,5 Prozentpunkte auf 22,5 v.H. gesenkt werden mußte, um bei Bezugnahme auf das Gesamtaufkommen eine unveränderte Beteiligung am zweckgebundenen Aufkommen $\mathrm{zu}$ sichern. ${ }^{1}$

Im Jahre 1977 wurden mit der Verabschiedung des FAG 1978 der allgemeine, der Kraftfahrzeugsteuer- und der Vermögensteuerverbund durch einen einheitlichen Steuerverbund mit einer Quote von 23,0 v.H. ersetzt. ${ }^{2}$ Dies geschah mit dem Ziel, flexibler als bisher auf sich ändernde gesamtwirtschaftliche Entwicklungen eingehen zu können, was durch die Aufteilung in nur für Investitionen (Vermögensteuer- und Kraftfahrzeugsteuerverbund) und in nur für Schlüsselzuweisungen und Sonderlastenausgleiche (allgemeiner Steuerverbund) zu verwendende Mittel nur eingeschränkt möglich war. Gleichzeitig wurde durch die damit einhergehende Verringerung der zweckgebundenen Investitionsmittel zugunsten allgemeiner Zuweisungen auch dem Anliegen der kommunalen Spitzenverbände Rechnung getragen. ${ }^{3}$ Zudem wurde durch den einheitlichen Steuerverbund die Schicksalsgemeinschaft zwischen Land und kommunaler Ebene gestärkt, da sich nun die Finanzausgleichsverbundmasse im Gleichklang mit über 97,25 v.H. statt vorher ca. 86,14 v.H. der dem Land verbleibenden Steuereinnahmen entwickelte. ${ }^{4} 1981$ wurde eine Verminderung der Verbundquote auf $22,0 \mathrm{v} . \mathrm{H}$. angestrebt, womit die Gemeinden einen Beitrag an den notwendigen Kürzungen im Landeshaushalt leisten sollten. ${ }^{5}$ Allerdings wurde dann im parlamentarischen Prozeß nur eine Minderung der Verbundmasse um 0,3 Prozentpunkte auf 22,7 v.H. vorgenommen. ${ }^{6}$

Der Gleichklang der Einnahmen erhöhte sich noch dadurch, daß ab dem Jahr 1982 die Verteilung des Landesanteils am Grunderwerbsteueraufkommen im Finanzausgleich neu geregelt wurde. Ohne Begründung wurde nun abweichend von der vorherigen Bestimmung das Aufkommen in die auch den

1 Vgl.: Ders.: Gesetzentwurf der Landesregierung für ein Elftes Gesetz zur Änderung des Finanzausgleichsgesetzes vom 04.09.1975, Drucksache 8/1439, S. 5, 8.

2 Vgl: Gesetz zur Regelung des Finanzausgleichs und zur Änderung anderer Vorschriften vom 28.12.1977, Hess. GVB1. I, S. 481.

3 Vgl: Hessischer Landtag: Gesetzentwurf der Landesregierung für ein Gesetz zur Regelung des Finanzausgleichs und zur Änderung anderer Vorschriften vom 11.10.1977, Drucksache 8/4877, S. 25-27.

4 Eigene Berechnungen nach: Statistisches Bundesamt: Fachserie 14, Finanzen und Steuern. Reihe 4.S.1, Kassenmäßige Steuereinnahmen 1977 bis 1987, Stuttgart 1989, S. 72-76, 100, 103f; dass.: Arbeitsunterlage über die Rechnungsergebnisse der staatlichen Haushalte von 1970-1989, S. 25.

5 Vgl.: Hessischer Landtag: Gesetzentwurf der Landesregierung für ein Gesetz zur Änderung des Finanzausgleichsgesetzes vom 29.09.1980, Drucksachen 9/3593, S. 1, 5.

6 Vgl.: $§ 2$ des Gesetzes zur Änderung des Finanzausgleichsgesetzes und des Schulverwaltungsgesetzes vom 17.12.1980, Hess. GVBI. I, S. 506. 
kreisangehörigen Gemeinden und dem Landeswohlfahrtsverband zustehende Steuerverbundmasse eingestellt, allerdings nicht im vollen Umfang, sondern nur entsprechend der Verbundquote. Diese Maßnahme führte trotz der gleichzeitigen Anhebung der Verbundquote auf 22,9 v.H. insgesamt zu Mindereinnahmen für die kommunale Ebene. ${ }^{1}$ Dies liegt darin begründet, daß der dem Land verbliebene Anteil an der Grunderwerbsteuer, der vorher vollständig der kommunalen Ebene wenn auch nur Landkreisen und kreisfreien Städten - zur Verfügung stand, nun aber nur noch nach der Verbundquote, größer war als der durch Verbreiterung der Bemessungsgrundlage und gleichzeitiger Anhebung der Verbundmasse zuflieBende Betrag. Somit traten Belastungsverschiebungen zwischen Land und Kommunen sowie zwischen und innerhalb der kommunalen Gruppen auf. Denn sowohl der Landeswohlfahrtsverband als auch die kreisangehörigen Gemeinden gewannen entsprechend ihrer prozentualen Beteiligung an der Schlüsselmasse, da sie im vollen Umfang von der Erhöhung der Verbundquote und -masse profitieren konnten, vorher aber nicht am Grunderwerbsteueraufkommen partizipierten. Die Gruppe der kreisfreien Städte und Landkreise erlitt hingegen Verluste, da bei diesen der Zugewinn aufgrund der erhöhten Verbundmasse und -quote geringer ausfiel als der Verlust des nun in die Verbundmasse eingestellten Landesanteils an der Grunderwerbsteuer. Innerhalb dieser Gruppe kam es ebenfalls zu Verschiebungen, da nun nicht mehr das örtliche Aufkommen, sondern die mangelnde Finanzkraft als Verteilungsmaßstab verwendet wurde.

Zum 01.01.1983 wurde als Folge der Grunderwerbsteuerreform des Bundesgesetzgebers und der damit verbundenen Abschaffung des kommunalen Steueranteils eine aufkommensneutrale Änderung vorgenommen, indem nach dem Grunderwerbsteuerzuweisungsgeset $z^{2}$ das jetzt ausschließlich dem Land zukommende Grunderwerbsteueraufkommen um vier Siebtel vermindert und dieser Betrag zur Kompensation den kreisfreien Städten und Kreisen direkt zugewiesen wurde. ${ }^{3}$

Die bislang letzte Reform wurde im Zuge der Finanzierung der Deutschen Einheit vorgenommen. Um die Gemeinden im angemessenen Rahmen finanziell an der Aufbringung der Mittel zu beteiligen, wurde im GFRG der Landesvervielfältiger ${ }^{4}$

1 Vgl.: Hessischer Landtag: Begründung zu Art. 5 des Gesetzentwurfs der Landesregierung für ein Gesetz zur Verbesserung der Haushaltsstruktur (Haushaltsstrukturgesetz) vom 12.11.1981, Drucksache 9/5583, S. $6 f$.

2 Vgl: Gesetz über die Zuweisung von Grunderwerbsteueranteilen an die Landkreise und kreisfreien Städte (Grunderwerbsteuerzuweisungsgesetz) vom 23.03.1983, Hess. GVBI. I, S. 31 .

3 Vgl:: Hessischer Landtag: Gesetzentwurf der Landesregierung für ein Gesetz über die $\mathrm{Zu-}$ weisung von Grunderwerbsteueranteilen und über die Verwendung der Gesamtschlüsselmasse 1983 vom 20.01.1983, Drucksache 10/169, S. 4. Im Jahr 1997 wurde dieser Anteil aufkommensneutral auf ein Drittel gesenkt (siehe Abschnitt 2.2.2.).

4 Der Umlagesatz (Vervielfältiger) setzt sich aus einem Landes- und einem Bundesvervielfätiger zusammen. 
der Gewerbesteuerumlage für die alten Länder mehrmals erhöht. Die aus dieser Erhöhung resultierende Summe wird nicht mehr der Verbundmasse des KFA zugeführt, da ansonsten die Erreichung des genannten Ziels konterkariert würde. ${ }^{1}$

\subsubsection{Entwicklung des Volumen der Verbundmasse}

Während es im vorherigen Abschnitt nur um die qualitative Zusammensetzung der Verbundmasse ging, werden nun die Auswirkungen dieser strukturellen Veränderungen auf die Höhe der Verbundmasse dargestellt. Dies geschieht dadurch, daß die Veränderung der Verbundquoten auf die Finanzausgleichsmasse des vorherigen Jahres bezogen wird. Dabei wird aus erfassungstechnischen Gründen darauf verzichtet, die Auswirkungen über das Entstehungsjahr hinaus zu betrachten. Denn aufgrund zusätzlicher Änderungen zu späteren Zeitpunkten ist eine Isolierung der einzelnen sich kumulierenden Effekte nicht möglich. Ferner werden nur die direkten Folgen ermittelt, während die indirekten Auswirkungen, wie Veränderungen etwa des Länderfinanzausgleichs i.e.S. oder der Finanzverfassung - z.B. Modifikationen bei der Verteilung der Umsatzsteuer auf Bund und Länder - und deren Resultate für das Volumen des der kommunalen Ebene zustehenden Ländersteueranteils, nicht bestimmt werden können.

Aus der Tabelle 4 ist zu entnehmen, daß im ersten Jahrzehnt des Steuerverbundes eine kontinuierliche Verbesserung der finanziellen Ausstattung der kommunalen Ebene erfolgte. Dabei erhöhte sich die Steuerverbundmasse im Jahr 1963 um 112,5 Mio. DM dies sind mehr als 21 v.H. der gesamten bereitgestellten Summe. Die Aufstockung des KFA geschah mittels zweierlei Maßnahmen. Einerseits durch die Erhöhung der Beteiligungsquote bei den schon bestehenden Verbünden, hier ist der Einkommensteuerverbund zu nennen, andererseits durch die Gründung neuer Verbünde, wie Kraftfahrzeugsteuer- und Vermögensteuerverbund. Die Verschlechterung der Haushaltslage des Landes in den 70er und zu Beginn der 80er Jahre führte zur Verringerung der Beteiligungsquoten der kommunalen Ebene, wobei der größte Beitrag zur Konsolidierung des Landeshaushalts im Jahr 1975 mit 177,3 Mio. DM oder 8,75 v.H. geleistet wurde. Seit 1982 befindet sich der KFA in Hessen "in ruhigem Fahrwasser"2, da kaum mehr strukturelle Veränderungen vorgenommen wurden.

1 Vgl.: Hessischer Landtag: Gesetzentwurf der Landesregierung für ein Gesetz zur Änderung des Finanzausgleichsgesetzes vom 25.07.1994, Drucksache 13/6401, S. 8f. und ders.: Gesetzentwurf der Landesregienung zur Änderung des Gesetzes über die Feststellung des Haushaltsplans des Landes Hessen für die Jahre 1990 und 1991 (Drittes Nachtragshaushaltsgesetz 1990/91) und zur Änderung des Finanzausgleichsgesetzes vom 11.10.1990, Drucksache 12/7470, S. 7.

2 Karrenberg, H.; Münstermann, E.: Der Gemeindefinanzbericht 1999, in: Der Städtetag, 52. Jg., 1999, S. 179. 
Tabelle 4:Volumenmäßige Auswirkungen der strukturellen Veränderungen ${ }^{1}$

\begin{tabular}{|c|c|c|c|c|c|c|c|}
\hline \multirow{2}{*}{$\begin{array}{c}\text { Aus- } \\
\text { gleichs- } \\
\text { jahr }\end{array}$} & \multirow{2}{*}{$\begin{array}{c}\text { allge- } \\
\text { meiner } \\
\text { Verbund; } \\
\text { v.H. }\end{array}$} & \multirow{2}{*}{$\begin{array}{c}\text { Kfz- } \\
\text { Steuer- } \\
\text { verbund; } \\
\text { v.H. }\end{array}$} & \multirow{2}{*}{$\begin{array}{c}\text { VSt- } \\
\text { Ver- } \\
\text { bund; } \\
\text { v.H. }\end{array}$} & \multirow{2}{*}{$\begin{array}{l}\text { GrESt- } \\
\text { Landes- } \\
\text { anteil; } \\
\text { v.H. }\end{array}$} & \multirow{2}{*}{$\begin{array}{l}\text { einheitlich- } \\
\text { er Steuer- } \\
\text { verbund; } \\
\text { v.H. }\end{array}$} & \multicolumn{2}{|c|}{$\begin{array}{l}\text { Verbundänderung im } \\
\text { 1. Jahr; DM }\end{array}$} \\
\hline & & & & & & absolut & in v.H. \\
\hline 1956 & 18,50 & - & - & - & - & + 8,6 Mio. & 7,25 \\
\hline 1961 & 18,50 & 25,00 & - & - & - & + 40,0 Mio. & 13,68 \\
\hline 1962 & 21,00 & 25,00 & - & - & - & + 38,9 Mio. & 8,41 \\
\hline 1963 & 21,00 & 25,00 & 100,00 & - & - & $+112,5$ Mio. & 21,09 \\
\hline 1965 & 21,00 & 25,00 & 100,00 & 100,00 & - & $+20,0$ Mio. & 2,64 \\
\hline 1966 & 23,00 & 25,00 & 100,00 & 100,00 & - & $+49,2$ Mio. & 6,01 \\
\hline 1973 & 23,00 & 25,00 & 61,00 & 100,00 & - & - 108,2 Mio. & $-6,06$ \\
\hline 1974 & 23,00 & 25,00 & 61,50 & 100,00 & - & + 1,7 Mio. & 0,08 \\
\hline 1976 & 20,60 & 22,50 & 61,50 & 100,00 & - & - 177,3 Mio. & $-8,75$ \\
\hline 1978 & - & - & - & 100,00 & 23,00 & $+43,3$ Mio. & 1,97 \\
\hline 1981 & - & - & - & 100,00 & 22,70 & - 33,8 Mio. & $-1,14$ \\
\hline 1982 & - & - & - & - & 22,90 & - 65,5 Mio. & $-2,32$ \\
\hline
\end{tabular}

Quelle: Eigene Berechnungen nach Staatsanzeiger des Landes Hessen: Abrechnungen über den

Finanzausgleich der jeweiligen Jahre.

In der folgenden Abbildung 1 sind sowohl für den Steuerverbund- als auch für die Finanzausgleichsmasse die Anteile an den gesamten Steuereinnahmen des Landes nach LFA dargestellt. Dieser Wert liegt bei den durch den Steuerverbund bereitgestellten Geldern im betrachteten Zeitraum zwischen 26,73 (1975) und 18,76 (1996) v.H. und sinkt seit Anfang der 80er Jahre fast kontinuierlich, so daß das Land die Kommunen an einem immer geringeren Anteil seiner Steuereinnahmen beteiligt, obwohl seit der Einfuihrung des einheitlichen Steuerverbundes im Jahr 1982 die Verbundquote unverändert bei 22,9 v.H. liegt. Die Quote lag bei Berücksichtigung aller - auch der nicht in den Verbund fließenden - Steuereinnahmen des Landes höchstens bei 22,38 v.H. (1982). Diese Entwicklung ist darauf zurückzuführen, daß das Aufkommen der nicht in den Verbund eingehenden Steuern - z.B. die Erbschaftsteuer - relativ stärker gestiegen ist. Die Quote der tatsächlich bereitgestellten Finanzausgleichsmasse liegt in allen Jahren über der tatsächlichen Steuerverbundquote, da daß Land noch zusätzliche Mittel zur

1 Bis einschließlich 1969 bestand der allgemeine Verbund aus einer Beteiligung am Einkommen- und Körperschaftsteueraufkommen, seit 1970 enthält er zusätzlich noch die Umsatzsteuer und die Gewerbesteuerumlage. In den einheitlichen Steuerverbund fließen seit 1978 bis auf den GrESt-Landesanteil - seit 1982 - die übrigen genannten Einnahmen mit der gleichen Quote ein. 
Verfügung stellt (z.B. Einnahmen aus der Grundabwasserabgabe und der Krankenhausumlage).

\section{Abbildung 1:Anteil der Steuerverbund- und der Finanzausgleichsmasse an den gesamten Landessteuereinnahmen nach LFA}

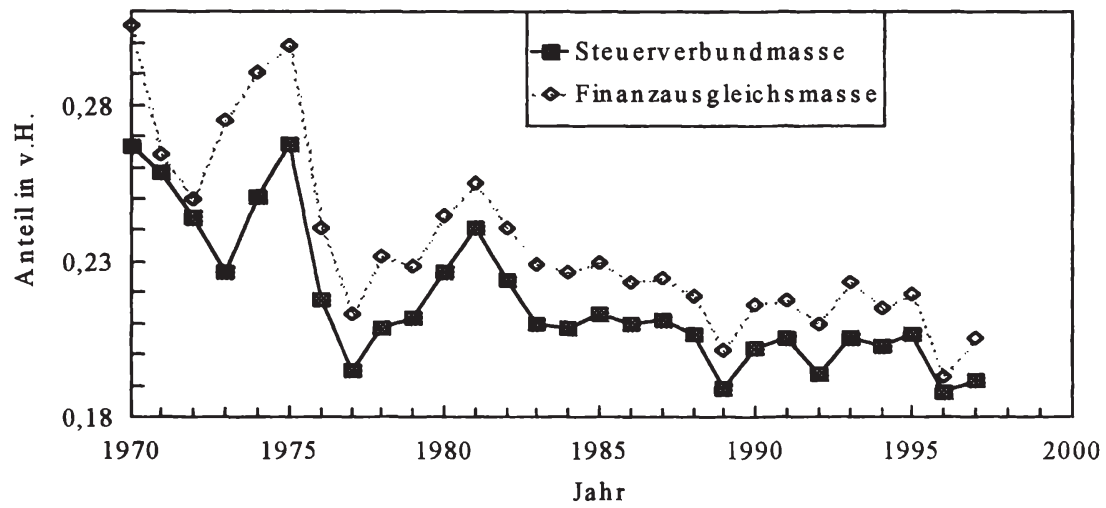

Quelle: Eigene Berechnungen nach Daten des Finanzministeriums.

Insgesamt ist aus diesen Ausführungen ersichtlich, daß die von den Landesregierungen so hoch gepriesene "Schicksalsgemeinschaft" in der Realität häufig genug durch die tatsächliche finanzielle Situation in den Hintergrund gedrängt wird. So wurde in den 60er Jahren die Verbundmasse durch strukturelle Veränderungen erhöht, da dem Land aufgrund der prosperierenden Wirtschaft die dazu notwendigen Geldmittel zur Verfügung standen, obwohl die kommunale Ebene aufgrund der Verbundquote schon an dieser Entwicklung beteiligt war. Entsprechend war die Reaktion des Landes, als in den 70er und 80er Jahren die Steuereinnahmen nicht mehr im gleichen Umfang wuchsen bzw. stagnierten oder gar sanken. Denn auch in diesem Fall wurde die kommunale Ebene über die Verbundquote an den Einnahmeminderungen automatisch beteiligt, was aber vom Land mit dem Hinweis darauf, daß sich die Steuereinnahmen der Kommunen besser als die eigenen entwickelt haben, als nicht ausreichend angesehen wurde; "der mit der Steuerentwicklung dynamisch verbundene kommunale Finanzausgleich kann von der Ausgabenkürzung nicht unberührt bleiben" ${ }^{\prime \prime}$. Aus Abbildung 2 kann aber entnommen werden, daß zumindest bis Mitte der 80er Jahre die Entwicklung der Steuereinnahmen der Kommunen und des Landes nach Abzug der Leistungen im LFA vergleichbar gewesen ist.

Hessischer Landtag: Gesetzentwurf der Landesregierung für ein Elftes Gesetz zur Änderung des Finanzausgleichsgesetzes vom 04.09.1975, Drucksache 8/1439, S. 1. 


\section{Abbildung 2: Steuereinnahmenentwicklung des Landes nach LFA und der Kommunen}

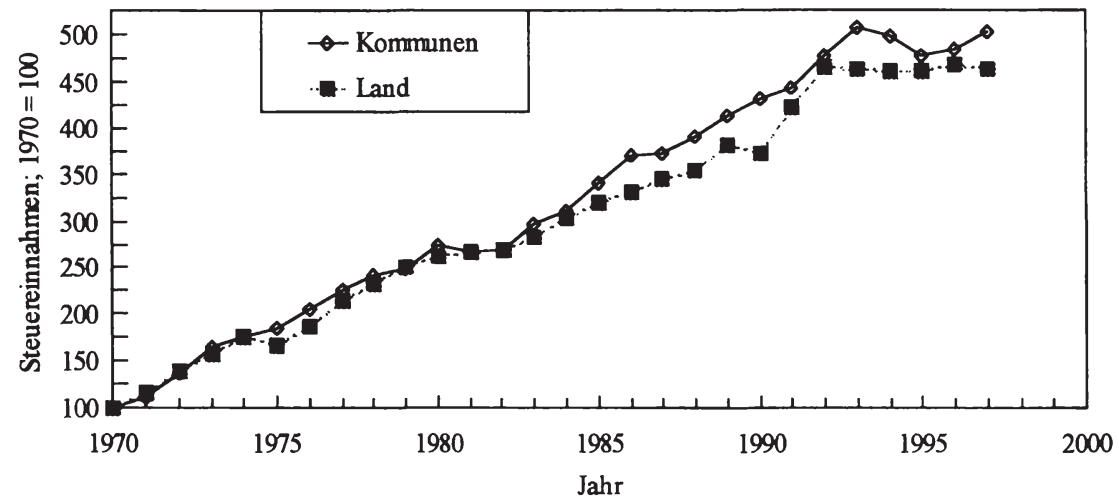

Quelle: Eigene Berechnungen nach: Statistisches Bundesamt: Fachserie 14, Finanzen und Steuern, Reihe 4.S.1, Kassenmäßige Steuereinnahmen 1967 bis 1976 und 1977 bis 1987, Dass.: Fachserie 14, Finanzen und Steuern, Reihe 4, Steuerhaushalt, verschiedene Jahrgänge.

\subsubsection{Angemessenheit der Verbundmasse}

Die im Grundgesetz getroffenen Regelungen über den KFA beziehen sich nur auf die Mindest-Qualität der Steuerverbundmasse, nicht aber auf die Quantität. Dies hat sich in der Vergangenheit als problematisch erwiesen. Die kommunale Ebene fordert meist eine Aufstockung des Anteils. ${ }^{1}$ Dies wird mit dem im Zeitablauf gestiegenen Finanzbedarf der Aufgabenwahrnehmung begründet, der sowohl durch zusätzliche Aufgabenübertragungen - etwa Kindergartenplatzgarantie - als auch durch eine höhere Ausgabenintensität bei unverändertem Aufgabenvolumen - gestiegene Sozialhilfeausgaben - hervorgerufen wird. ${ }^{2}$

Es ist schwierig, Aussagen über die Angemessenheit der Verbundmasse zu treffen, denn die Entwicklung hinreichender Kennziffern ist nicht einfach. Zwar hat GrosseKETTLER einen dezidierten Kriterienkatalog zur Lösung dieses Problems aufgestellt, doch sind die dazu notwendigen Daten entweder derzeit nicht vorhanden, oder der Aussagegehalt der so gewonnenen ist eher zweifelhaft. ${ }^{3}$

1 Vgl: Hessischer Landtag: Gesetzentwurf der Landesregierung für ein Gesetz zur Änderung des Finanzausgleichsgesetzes - Finanzausgleichsänderungsgesetz 1985 vom 29.10.1984, Drucksache 11/2228, S. 6.

$2 \mathrm{Vgl}$ : Schoch, F.: Finanzierungsverantwortung fur gesetzgeberisch veranlaßte Ausgaben, in: Der Landkreis, 64. Jg., 1994, S. 253-255; ders.: "...Wer anschaffen will, der soll gefälligst bezahlen...", in: Der Landkreis, 64. Jg., 1994, S. 531f.

3 So wird die sog. "freie Spitze" als ein Indikator genannt. Diese kann aber durch das Ausga- 
Auch in der HV sind keine expliziten Vorgaben für einen Finanzausgleich bzw. die Ausgleichsmasse zu finden. Allerdings wird dort dem Land aufgetragen, den Kommunen "die zur Durchführung ihrer eigenen und der übertragenen Aufgaben erforderlichen Geldmittel im Wege des Lasten- und Finanzausgleichs zu sichern"1. Dabei darf einerseits nicht die finanzielle Leistungsfähigkeit des Landes aus den Augen verloren werden; ${ }^{2}$ andererseits ist es nicht zulässig, den KFA als Reservekasse der Länder zu nutzen. ${ }^{3}$ Es ist aber zu bezweifeln, daß die Leistungsfähigkeit des Landes durch die aktuelle Haushaltssituation richtig widergespiegelt wird, da diese auch durch autonome Entscheidungen beeinflußt worden ist. Angebracht wäre als Indikator deshalb eher ein zu ermittelnder finanzieller Bedarf des Landes. ${ }^{4}$

Auch ein Blick auf die Finanzbeziehungen zwischen Bund und Ländern hilft nicht weiter. In Art. 106 Abs. 4 GG heißt es zwar, "die Anteile von Bund und Ländern an der Umsatzsteuer sind neu festzusetzen, wenn sich das Verhältnis zwischen Einnahmen und Ausgaben des Bundes und der Länder wesentlich anders entwickelt", doch konnte kein angemessenes Verfahren für diese Festsetzung bestimmt werden. ${ }^{5}$ Zur Lösung dieses Problems wurde eine Sachverständigenkommission beauftragt, die sich aber bei der erforderlichen Bestimmung des Vergleichskriteriums "notwendige Ausgaben" nicht auf ein einvernehmliches Ergebnis einigen konnte. ${ }^{6}$ Doch auch bei den Aspekten, die einstimmig gebilligt

beverhalten der jeweiligen Gebietskörperschaft beeinflußt werden, so daß sie ärmer erscheint. Vgl.: Grossekettler, H.: Die Bestimmung der Schlüsselmasse im kommunalen Finanzausgleich. Eine ordnungspolitische Analyse der Entstehung von Finanzausgleichsbedarfen und der Möglichkeiten ihrer Quantifizierung, in: Finanzarchiv N.F., Bd. 45, 1987, S. 393-440.

1 Verfassung des Landes Hessen (HV) vom 18.12.1946, Hess. GVBI. I, S. 229.

2 Vgl:: Niedersächsischer Staatsgerichtshof: Beschluß vom 15.08.1995, in: DVBl., 110. Jg., 1995, S. 1177.

3 Vgl.: Henneke, H.-G.: Jenseits von Bückeburg. Gesetzgeberische Gestaltungsspielräume für den kommunalen Finanzausgleich, in: Niedersächsische Verwaltungsblätter, 3. Jg., 1996, S. 11 .

4 Vgl.: Ders.: Der kommunale Finanzausgleich. Einer der verfassungsgerichtlich am sorgfältigsten behauenen Ecksteine der Grundlagen kommunaler Selbstverwaltung, in: DÖV, 47. Jg., 1994, S. 2.

5 Das in der Praxis verwendete Kriterium des Deckungsquotenvergleichs ist abzulehnen, da diese Kennziffer keinerlei Aussagen über die Finanzschwäche einer Gebietskörperschaft zuläßt und sich zudem noch kontraproduktiv auf Konsolidierungsmaßnahmen auswirkt. Vgl.: Peffekoven, R.: Zur Problematik der Umsatzsteuerverteilung, in: Cansier, D.; Kath, D.: Öffentliche Finanzen, Kredit und Kapital, Berlin 1985, S. 59-61.

6 Vgl: Sachverständigenkommission zur Vorklärung finanzverfassungsrechtlicher Fragen für künttige Neufestlegungen der Umsatzsteueranteile: Verteilung der Umsatzsteuer - Maßstäbe und Verfahren, in: Schriftenreihe des Bundesministeriums der Finanzen, Heft 30, Bonn 1981, Tz. 93, 99. Vgl. auch: Wissenschafticher Beirat beim Bundesministerium der Finanzen: Einnahmenverteilung zwischen Bund und Ländern, Schriftenreihe des Bundesministeri- 
wurden, ergab sich keine praktische Relevanz, da sie von Bund und Ländern nicht für die weiteren Gespräche akzeptiert wurden. ${ }^{1}$ Das jeweilige Aufteilungsverhältnis resultiert somit aus einem im Verhandlungsprozeß gefundenen Kompromiß. Da bei der Bestimmung der Verbundquote im KFA das Land die stärkere Position inne hat, ergibt sich auch hieraus kein Lösungsansatz für das geschilderte Problem.

Das Bundesverfassungsgericht gesteht den Ländern einen weiten Gestaltungsspielraum bei der Regelung des KFA zu. ${ }^{2}$ Jedoch wurden diese Freiheit in den letzten Jahren durch Verfassungsgerichts- bzw. Staatsgerichtshofurteile in den Ländern eingeschränkt. ${ }^{3}$ Minderungen der Verbundmasse wurden nun kritischer bewertet als in der Vergangenheit. Begründet werden kann diese Betrachtungsweise mit der grundsätzlichen Gleichwertigkeit von kommunalen und staatlichen Aufgaben ${ }^{4}$ Deshalb ist für die Höhe der Verbundmasse die Entwicklung der Einnahmen und Aufgaben bzw. Ausgaben der beiden Ebenen zu betrachten.

\subsubsection{Entwicklung der Einnahmenanteile von staatlicher und kommunaler Ebene}

Aufgrund der oben angezeigten Indikatorproblematik wird in einem ersten Schritt die Verfahrensweise des Niedersächsischen Staatsgerichtshofs in seinem letzten Urteil zum KFA übernommen. ${ }^{5}$ Ein Indiz zur Beurteilung wäre somit der Vergleich der Entwicklung der Einnahmenverteilungen zwischen staatlicher und

um der Finanzen, Heft 56, Bonn 1995 und Ders.: Stellungnahme zum Finanzausgleichsurteil des Bundesverfassungsgerichts vom 11. November 1999, Schriftenreihe des Bundesministerium der Finanzen, Heft 68, Bonn 2000, S. 12-24.

1 Vgl:: Wolf, G.: Zur Umsatzsteuerverteilung zwischen Bund und Ländern, in: Bundesministerium der Finanzen (Hrsg.): Die Finanzbeziehungen zwischen Bund, Ländern und Gemeinden aus finanzverfassungsrechtlicher und finanzwissenschaftlicher Sicht, Bonn 1982, S. 300.

2 Bundesverfassungsgericht: Urteil vom 15.10.1985, in: Entscheidungen des Bundesverfassungsgerichts, Bd. 71, Tübingen 1986, S. 38.

3 Vgl:: Niedersächsischer Staatsgerichtshof: Beschluß vom 15.08.1995, in: DVB1., 110. Jg., 1995, S. 1175-1179, ders.: Urteil vom 25.11.1997, in DVB1., 113. Jg., 1998, S. 185-189.

4 Vgl:: Niedersächsischer Staatsgerichtshof: Leitsatz 4 des Urteils des Niedersächsischen Staatsgerichtshofs vom 27.11.1997 (StGH 14/95), in: DVBl., 113. Jg., 1998, S. 185; § 24 Abs. 1 des Gesetz zur Förderung der Stabilität und des Wachstums der Wirtschaft vom 08.06.1967, BGBI. I, S. 582; Deutscher Bundestag: Entwurf eines Gesetzes zur Änderung und Ergänzung der Finanzverfassung (Finanzverfassungsgesetz), eines Gesetzes zur Anpassung der Finanzbeziehungen zwischen Bund und Ländern an die Finanzverfassung (Finanzanpassungsgesetz) und eines Gesetzes über den Finanzausgleich unter den Ländern (Länderfinanzausgleichsgesetz) vom 29.04.1954, Drucksache II/480 Nr. 36.

5 Dort wurde die Einnahmenentwicklung von Land und Kommunen als Beurteilungsmaßstab herangezogen und wegen starker Divergenzen das Volumen der KFA-Verbundmasse fur verfassungswidrig erklärt, da die finanzielle Mindestausstattung der kommunalen Ebene nicht als gesichert gelten konnte. Vgl.: Niedersächsischer Staatsgerichtshof: Urteil vom 25.11.1997, in: DVB1., 113. Jg., 1998, S. 188. 
kommunaler Ebene in Hessen mit Niedersachsen. Würden die Verschiebungen auf der Einnahmenseite zwischen der kommunalen und der staatlichen Ebene in Hessen eine vergleichbare Größenordnung wie in Niedersachsen annehmen, so könnte vermutet werden, daß auch der Hessische Staatsgerichtshof verfassungsrechtliche Bedenken hinsichtlich des Finanzierungsvolumens äußern würde.

Aus der Abbildung 3 ist ersichtlich, daß im Durchschnitt seit Mitte der 70er Jahre das Land zu etwa 53 v.H. und die kommunalen Gebietskörperschaften zu ungefähr 47 v.H. an den gesamten in Hessen verbleibenden Einnahmen aus Steuern und LFA beteiligt sind. Im Zeitablauf ist ein Niveaueffekt zugunsten des Landes festzustellen gewesen. So lag bis 1975 der Landesanteil noch im Durchschnitt bei etwa 50 v.H., doch seit der Verminderung der Verbundquote 1976 beträgt er immer über 52 v.H. und erreicht 1997 den Wert 52,8 v.H. Der niedrigste Wert wurde 1975 mit 47,2 v.H., der höchste 1977 mit 54,6 v.H. erreicht; entsprechend spiegelbildlich ist die Entwicklung der kommunalen Ebene verlaufen.

\section{Abbildung 3:Anteil des Landes und der Kommunen an den hessischen Ein- nahmen aus Steuern nach LFA und Leistungen des KFA}

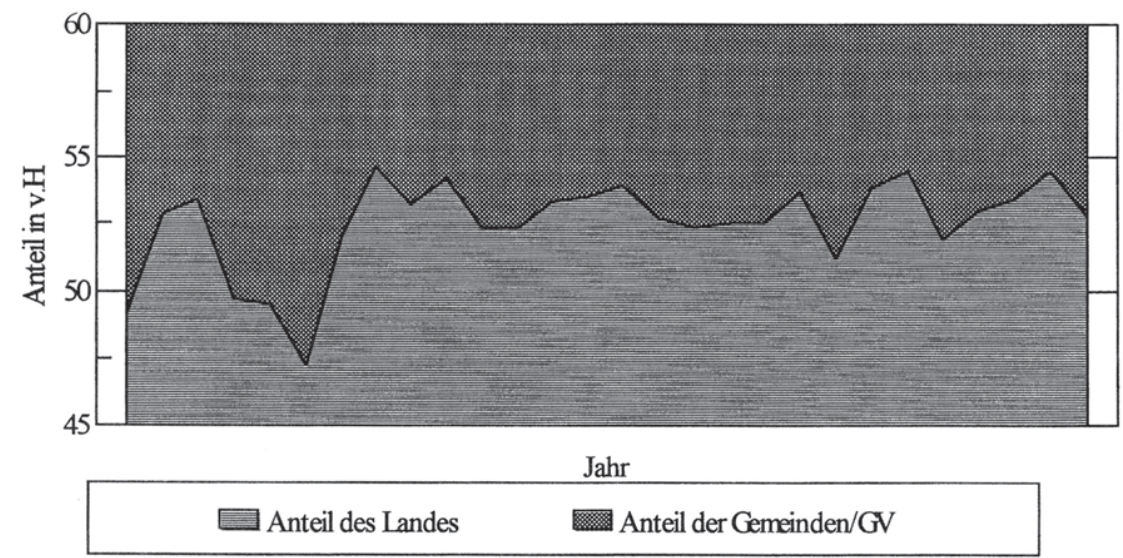

Quelle: Eigene Darstellung nach Daten des Hessischen Finanzministeriums.

In dem vom Niedersächsischen Staatsgerichtshof gewählten Zeitraum von 1985 bis 1996 erhöhte sich der Anteil des Landes Niedersachsen an den betrachteten Einnahmen von 59,8 auf 65,2 v.H., während er in Hessen von 53,5 auf 55,4 v.H. stieg. Da die tatsächliche Entwicklung nicht ohne Schwankungen verlaufen ist, wird zur besseren Vergleichbarkeit mittels einer Regressionsanalyse der lineare Trend ermittelt. Bei Betrachtung des trendmäßigen Wachstums ergibt sich, daß sich der Anteil des Landes Niedersachsen jährlich um 0,272 Prozentpunkte erhöhte, wodurch dieser Anstieg doppelt so hoch ausfiel als in Hessen mit 0,136 
Prozentpunkten pro Jahr. ${ }^{1}$ Somit ist zumindest auf der Einnahmenseite die Verschiebung zugunsten der staatlichen Ebene in Hessen im Gegensatz zu Niedersachsen als nicht so problematisch anzusehen. ${ }^{2}$

\subsubsection{Beachtung des Kommunalisierungsgrads der Aufgabenwahrnehmung}

Der reine Vergleich des bereitgestellten Finanzvolumens mit den übrigen Bundesländern ist aufgrund der unterschiedlichen Aufgabenteilung zwischen kommunaler und staatlicher Ebene nicht unproblematisch. Es ergibt sich ein Anhaltspunkt, wenn der Kommunalisierungsgrad der Aufgaben zwischen den Ländern verglichen wird. Dies erfolgt mit Hilfe der jeweiligen Anteile an den Ausgaben, wobei auch die Finanzausstattung Berücksichtigung findet. ${ }^{3}$ Mit Hilfe derartiger Untersuchungen können keine genauen Aussagen über das "richtige" Volumen der Verbundmasse getätigt werden. Es ergibt sich aber durch den Ländervergleich ein Hinweis darauf, wie gut die finanzielle Ausstattung der Kommunen in Hessen in Relation zum Durchschnitt der übrigen Länder ist. Der Schwachpunkt dieses Vergleichs besteht aber in der Bewertung der Ausgaben, denn die Ist-Ausgaben werden als die durch die Aufgabenerfüllung induzierten notwendigen Ausgaben angesehen. Ineffizientes Verhalten würde also den Finanzbedarf erhöhen. ${ }^{4}$

In der Tabelle 5 ist nun für 1996 dargestellt, wie sich die Einnahmen und Ausgaben des Landes Hessen auf die staatliche und die kommunale Ebene aufteilen, wobei zu Vergleichszwecken auch das durchschnittliche Aufteilungsverhältnis innerhalb der übrigen westlichen Flächenländer angegeben ist. Ausgangspunkt sind die unmittelbar von jeder Ebene zu finanzierenden Ausgaben und ihre originären Einnahmen, ohne Berücksichtigung der finanziellen Verknüpfungen mit dem übrigen öffentlichen Bereich. ${ }^{5}$ Die Einnahmen müssen zur weiteren

1 Auf die Betrachtung prozentualer Veränderungen wird hier verzichtet, da der Landesanteil in Niedersachsen höher als in Hessen ist, so daß in beiden Ländern absolut gleiche Änderungen in Niedersachsen einer relativ geringeren prozentualen Veränderung entsprechen.

2 In Niedersachsen haben die Einnahmen des Landes aus Steuern und LFA abzüglich KFA zwischen 1985 und 1996 eine Steigerungsquote von 72,8 v.H. erreicht, während diese bei den Gemeinden und GV bei 31,9 v.H. lag. Im Vergleich dazu lagen die Werte in Hessen bei 49,4 und 39,3 v.H. beim Land bzw. bei den Gemeinden und GV. Vgl.: Niedersächsischer Staatsgerichtshof: Urteil vom 27.11.1997, in: DVBl. 113. Jg., 1998, S. 188; eigene Berechnungen.

3 Die letzte Untersuchung zu dieser Thematik, die sowohl die Steuereinnahmen und Finanzausgleichsleistungen als auch eine detailierte Analyse der Aufgabenübertragungen enthält, wurde von MónstermanN und BECKER 1978 vorgenommen. Vgl.: Münstermann, E.; Bekker, H.: Finanzausgleichsleistungen an Kommunen. Ein Vergleich der Finanzausgleichssysteme in den Bundesländern (Reihe G; DST-Beiträge zur Finanzpolitik, Heft 7), Köln 1978, S. 15-29.

4 Da vorab die Verwendung dieser Daten aber nicht bekannt gewesen ist, dürfen die Vergangenheitsdaten als annähernd genauer Indikator angesehen werden.

5 Bei den unmittelbaren Einnahmen handelt es sich um die im jeweiligen Aufgabengebiet ver- 
Betrachtung jeweils noch um den Saldo der Zahlungen innerhalb des Landes und zum übrigen öffentlichen Bereich korrigiert werden.

Tabelle 5:Einnahmen und Ausgaben staatlicher und kommunaler Ebene in Hessen und den übrigen westlichen Flächenländern 1996 (DM/Kopf)

\begin{tabular}{|c|c|c|c|c|}
\hline \multirow{2}{*}{} & \multicolumn{2}{|c|}{ Hessen } & \multicolumn{2}{c|}{ übrige westl. Flächenländer } \\
\cline { 2 - 5 } & Land & Kommunen & Land & Kommunen \\
\hline unmittelbare Ausgaben (a) & $3.976,54$ & $4.386,36$ & $3.947,74$ & $4.062,52$ \\
\hline + unmittelbare Einnahmen & $4.791,85$ & $3.480,70$ & $4.299,01$ & $3.035,97$ \\
\hline = originärer Saldo & 815,31 & $-905,66$ & 351,27 & $-1.026,55$ \\
\hline + Saldo Land/Kommunen ${ }^{1}$ & $-948,17$ & 948,17 & $-974,21$ & 974,21 \\
\hline + Saldo übriger öff. Bereich & $-232,61$ & $-62,73$ & 269,46 & $-49,95$ \\
\hline = Saldo Einnahmen/Ausgaben & $-365,47$ & $-20,22$ & $-353,48$ & $-102,30$ \\
\hline verfugbare Einnahmen (b) & $3.611,07$ & $4.366,36$ & $3.594,26$ & $3.960,22$ \\
\hline Verhältnis (b) zu (a) & 90,81 v.H. & 99,54 v.H. & 91,05 v.H. & 97,48 v.H. \\
\hline
\end{tabular}

1 Da wegen statistischer Abweichungen geleistete und empfangene Zahlungen divergieren, wurde das arithmetische Mittel der beiden Werte verwendet.

Quelle: Eigene Berechnungen nach: Statistisches Bundesamt: Fachserie 14, Finanzen und Steuern, Reihe 3.1, Rechnungsergebnisse des öffentlichen Gesamthaushalts 1996, Stuttgart 1998, S. 126-257; 266-273.

Es zeigt sich nun, daß bei einem Vergleich der mit den unmittelbaren Einnahmen zu finanzierenden unmittelbaren Ausgaben bei den Kommunen in allen westlichen Flächenländern ein Defizit auftritt, während die jeweilige staatliche Ebene einen Überschuß aufweist. Dabei ist die Situation in Hessen jeweils besser, was mit der vergleichsweise starken Steuerkraft sowohl des Landes als auch der Gemeinden und GV erklärt werden kann. So liegt der originäre Saldo bei den hessischen Kommunen nur bei -905,66 DM pro Kopf gegenüber -1.026,55 DM pro Kopf in den übrigen westlichen Flächenländern. Die staatliche Ebene erzielt in Hessen mit 815,31 gegenüber 351,27 DM pro Kopf in den übrigen Flächenländern einen höheren Überschuß. ${ }^{1}$

einnahmten Beträge ohne Zahlungen vom öffentlichen Bereich. Die unmittelbaren Ausgaben werden im Zuge der Aufgabenerfüllung getätigt, wobei keine Zahlungen an den öffentlichen Bereich berücksichtigt werden. Vgl.: Statistisches Bundesamt: Fachserie 14, Finanzen und Steuern, Reihe 3.1, Rechnungsergebnisse des öffentlichen Gesamthaushalts 1996, Stuttgart 1998, S. 6-8.

1 Diese Ergebnisse könnten ganz allgemein als Indiz dafür angesehen werden, daß die Steuerverteilung zwischen der staatlichen und der kommunalen Ebene bei den derzeitig durch die Aufgabenwahrnehmung induzierten Ausgaben zugunsten letzterer zu ändern ist. Denn durch Erhöhung der originären Einnahmen würde der notwendige Umfang der durch den Finanzausgleich umverteilten Mittel sinken. 
Da jedes Land durch Art. 106 Abs. 7 GG dazu verpflichtet ist, die kommunalen Gebietskörperschaften an den Steuereinnahmen partizipieren zu lassen, andererseits die Gemeinden Finanzmittel in Form der Gewerbesteuerumlage an das Land abzuführen haben, ist dieser Saldo zu den unmittelbaren Einnahmen hinzuzufügen. Gleiches gilt für die finanziellen Verbindungen zum übrigen öffentlichen Bereich. ${ }^{1}$ Im Rahmen der Finanzbeziehungen innerhalb der Landesgrenzen werden den hessischen Kommunen saldiert weniger Mittel zur Verfügung gestellt, als dies bei den übrigen kommunalen Gebietskörperschaften der Fall ist, nämlich nur 948,17 im Vergleich zu 974,21 DM pro Kopf. Dies kann mit der relativen Steuerstärke der hessischen Kommunen begründet werden, weshalb weniger Zuweisungen notwendig sind. Beim Saldo mit dem übrigen öffentlichen Bereich ergeben sich abgesehen von den sonstigen westlichen Flächenländern aufgrund der Zahlungen aus dem LFA und den Bundesergänzungszuweisungen nur Defizite. Diese sind für die Kommunen in Hessen mit 62,73 gegenüber 49,95 DM pro Kopf bei den übrigen Kommunen deshalb höher, weil die an den Bund abzuführende Gewerbesteuerumlage den größten Anteil ausmacht. Diese ist von der Gewerbesteuerkraft abhängig, die wiederum in Hessen überdurchschnittlich hoch ist.

Bei der Gesamtbetrachtung erweist sich die Ausgabendeckung durch die angeführten Einnahmen bei den hessischen Kommunen mit 99,54 v.H. am höchsten, während das Land Hessen mit 90,81 v.H. den niedrigsten Wert aufweist. Wird die Quote jeweils auf die staatliche Ebene normiert, um Aussagen über das jeweilige Land-Kommunen-Verhältnis treffen zu können, so ergibt sich für die Kommunen in Hessen eine Relation von 1,10: 1 und für die übrigen kommunalen Gebietskörperschaften in Westdeutschland ein Verhältnis zum Land von 1,07 : 1 . Somit kann festgestellt werden, daß im Vergleich zu den übrigen Kommunen in den westlichen Flächenländern die Kommunen in Hessen finanziell zumindest nicht schlechter, sondern wohl eher besser gestellt werden.

\subsubsection{Ein Ansatz zur Sicherung einer angemessenen Finanzausstattung}

Damit zukünftig die Verteilungssymmetrie zwischen Land und Gemeinden/GV in Hessen gewahrt wird, könnte der in Sachsen und seit 1999 auch in Niedersachsen praktizierte "Milbradtsche Gleichmäßigkeitsgrundsatz"2 genutzt werden. Dieser soll gewährleisten, daß sich die Einnahmen von Land und Kommunen gleich entwickeln. ${ }^{3}$ Dazu ist eine Endabrechnung des KFA dergestalt notwendig, daß die

1 Hier sind für die Länder der LFA und die BEZ zu nennen, bei den Kommunen kann die Gewerbesteuerumlage und Fördermittel nach Art. 104a Abs. 4 GG angeführt werden.

2 Dieser Ansatz ist auf den sächsischen Staatsminister für Finanzen Milbradt zurückzuführen.

3 "Die Entwicklung der Gesamteinnahmen der sächsischen Kommunen aus Steuern sowie aus den Zuweisungen aus dem kommunalen Finanzausgleich soll sich ab 1996 gleichmäßig zur Entwicklung der dem Freistaat verbleibenden Finanzmasse aus Steuern sowie dem Länderfinanzausgleich einschließlich Bundesergänzungszuweisungen, abzüglich der den Kommunen zufließenden Steuerverbundmasse im kommunalen Finanzausgleich, also zu seinen Gesamt- 
Verbundquote je nach Entwicklung der staatlichen und kommunalen Steuereinnahmen so verändert wird, daß durch die dann bereitgestellte Verbundmasse die erwünschte gleichmäßige Entwicklung erreicht ist. ${ }^{1}$ Dabei wird für eine Ausgangssituation ein Aufteilungsverhältnis der betrachteten Einnahmen festgelegt, welches als Zielgröße gilt und für zukünftige Zeiträume fortgeschrieben wird. In Sachsen wurden z.B. für das Land 63,4 und für die Kommunen 35,6 v.H. der Einnahmen bestimmt, für Niedersachsen wurden 61,6 und 38,4 v.H. angenommen. ${ }^{2}$

Dieser Lösungsansatz vermeidet Konflikte bei der Verteilung der Einnahmen, solange die durch die Aufgabenwahrnehmung induzierte Ausgabenverteilung konstant bleibt. Erst wenn zusätzliche Aufgaben übertragen werden oder sich durch Modifikation von Standards die Kosten der Wahrnehmung von Weisungsaufgaben verändern, ist eine Überarbeitung des Aufteilungsverhältnisses notwendig. Deshalb ist eine Erweiterung derart vorzunehmen, daß das Volumen der Finanzausgleichsmasse "auf der Grundlage einer umfassenden Bewertung der Einnahmen und der aufgabenbedingten Ausgaben der Gemeinden und des Landes zu erfolgen" ${ }^{13}$ hat, wobei eine Untersuchung der Aufgabenverteilung regelmäßig erfolgen sollte. ${ }^{4}$ Für die kommunale Ebene ergibt sich aus dieser Regelung neben der institutionellen Garantie solange ein finanzieller Vorteil, wie ihre eigenen Steuereinnahmen im geringeren Umfang steigen als die Einnahmen des Landes.

\subsubsection{Konjunkturelle Entwicklung der Verbundmasse}

Problematisch am Verhalten der Kommunen ist aus konjunkturpolitischer Sicht, daß sich ihre Ausgaben der Einnahmenentwicklung anpassen, welche wiederum unstetig verläuft. Dies ist $u$. a. auf die eingeschränkte Verschuldungsmöglichkeit zurückzuführen. Das Kreditaufnahmevolumen ist abhängig von den laufenden Einnahmen, die u.a. der Schuldentilgung dienen. ${ }^{5}$ Dadurch können besonders in

einnahmen netto verhalten." Vgl:: Gesetz über einen Finanzausgleich mit den Gemeinden und Landkreisen 1998 im Freistaat Sachsen (Finanzausgleichsgesetz 1998 - FAG 1998) vom 09.12.1997, Sächsisches GVB1., S. 663.

1 Vgl.: Karrenberg, H.; Münstermann, E.: Der Gemeindefinanzbericht 1998, in: Der Städtetag, 51. Jg., 1998, S. 189 und Niedersächsischer Landtag: Entwurf eines Gesetzes zur Regelung der Niedersächsischen Finanzverteilung (Niedersächsisches Finanzverteilungsgesetz NFVG -) vom 02.02.1999, Drucksache 14/500, S. 4, 8.

2 Vgl: Sächsischer Landtag: Gesetzentwurf der Staatsregierung über ein Gesetz über einen Finanzausgleich mit den Gemeinden und Landkreisen 1998 im Freistaat Sachsen (Finanzausgleichsgesetz 1998 - FAG 1998) vom 04.08.1997, Drucksache 2/6535, S. 4 und Niedersächsischer Landtag: Entwurf eines Zweiten Gesetzes zur Änderung des Niedersächsischen Gesetzes über den Finanzausgleich vom 12.01.1998, Drucksache 14/440, S. 101.

3 Steinherr, M.; u.a.: Das Schlüsselzuweisungssystem im kommunalen Finanzausgleich des Freistaates Sachsen, ifo-Studien zur Finanzpolitik, Bd. 63, München 1997, S. 52.

4 Vgl:: Ebenda, S. 53-55.

5 Vgl: $§ 22$ Abs. 1 Verordnung über die Aufstellung und Ausführung des Haushaltsplans der Gemeinden (Gemeindehaushaltsverordnung - GemHVO) vom 13.07.1973, Hess. GVBl. I, 
Boomzeiten die Ausgaben durch zusätzliche Kredite noch weiter gesteigert werden, was aber in der Rezession, wenn es notwendig wäre, nicht möglich ist. In Abbildung 4 ist der Zusammenhang zwischen den kommunalen Einnahmen (ohne Kreditaufnahme) und den Ausgaben wiedergegeben. ${ }^{1}$

\section{Abbildung 4:Entwicklung der Einnahmen und Ausgaben im Zeitablauf}

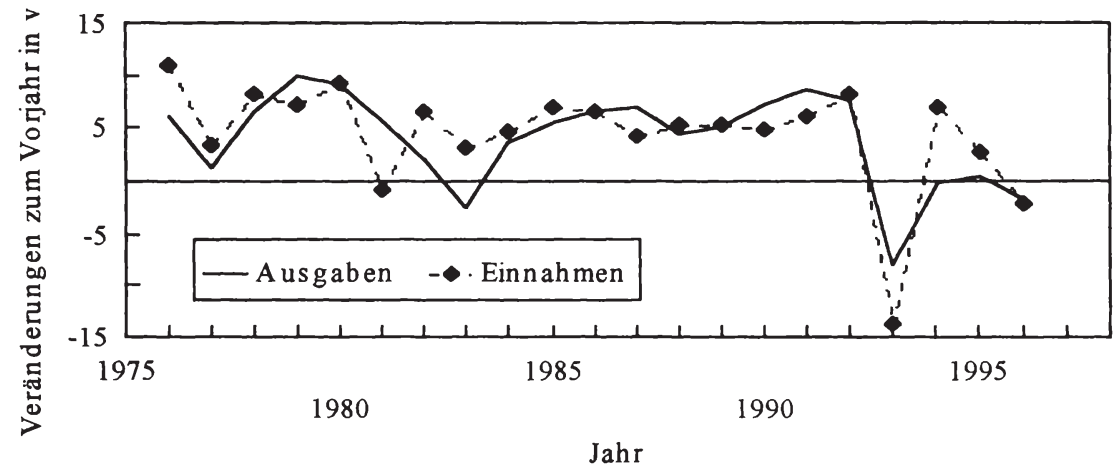

Quelle: Eigene Berechnungen nach: Daten des Statistischen Bundesamtes.

Es ist eine starke positive Korrelation zwischen diesen beiden Größen festzustellen. So steigen die Einnahmen 1978 (1993) um 8,36 v.H. (13,65 v.H.) und die Ausgaben 6,52 v.H. (12,93 v.H.) gegenüber dem Vorjahreswert. Eine ähnliche Entwicklung ist auch bei Betrachtung der Investitionen und der Investitionszuweisungen festzustellen. ${ }^{2}$ Das Ziel muß es deshalb sein, die Konjunkturreagibilität der kommunalen Einnahmen und Ausgaben zu verringern, damit zumindest nicht die Maßnahmen der staatlichen Konjunkturpolitik konterkariert werden. ${ }^{3}$ Dies wird erreicht, wenn die Einnahmen unabhängig vom Konjunkturverlauf relativ gleichmäßig anfallen, was aber bei Betrachtung der kommunalen Steuern nur bedingt der Fall ist. Die örtlichen Verbrauch- und Aufwandsteuern, die Grundsteuer sowie die abgeschaffte Gewerbekapitalsteuer erfüllen das Kriterium der geringen Konjunkturabhängigkeit, doch liegt ihr Anteil an den gesamten Steuereinnahmen bei unter 15 v.H. Vor allem die Gewerbeertragsteuer erweist sich als sehr konjunkturreagibel. So schwankten die Gewerbesteuereinnahmen der Stadt Rüsselsheim innerhalb eines Zehnjahreszeitraums zwischen 6 und 100 Mio. DM. ${ }^{4}$

S. 275 und ÄndVO v. 08.07.1996, Hess. GVBl. I, S. 334.

1 Da seit 1993 die Krankenhäuser mit kaufmännischen Rechnungswesen nicht mehr berücksichtigt werden, deren Anteil an den Ausgaben im Jahr 1992 etwa 12,90 v.H. ausmachten, kann es zu Differenzen kommen.

2 Für Gesamtdeutschland vgl.: Deutsche Bundesbank: Monatsbericht, April 1999, S. $35 f$.

3 Vgl:: Tettinger, P. J.: Konjunkturpolitik und Gemeinden, Finanzplanung, in: Püttner, G. (Hrsg.): Handbuch der kommunalen Wissenschaft und Praxis, Bd. 6, 2. Aufl., Berlin u.a.O. 1985, S. 445.

4 Vgl.: Littmann, K.: Ergebnisse und Empfehlungen des Gutachtens des Wissenschaftlichen 
Aufgrund der beschriebenen Einnahmenentwicklung der kommunalen Steuern wäre es von Vorteil, wenn die Verbundmasse des KFA und somit auch die $\mathrm{Zu}$ weisungen eine entgegengerichtete Entwicklung nähmen. Denn somit bestünde die Möglichkeit einer konjunkturpolitischen Instrumentalisierung des KFA, wofür keine Verfassungsänderung notwendig wäre. Die gewünschte Entwicklung tritt aber nicht ein (siehe Abbildung 5). Denn die Verbundgrundlagen verändern sich wie die kommunalen Steuern, wobei sich der hohe Anteil der konjunkturreagiblen Einkommensteuer an der Verbundmasse als problematisch erweist. So ist abgesehen von wenigen Jahren, z.B. 1976 mit einer Verringerung der Leistungen des KFA um 11,1 v.H. und Steigerung der kommunalen Steuereinnahmen um 11,0 v.H., die Entwicklung sowohl vom Vorzeichen als auch von der Größenordnung her gleich, so daß der KFA keinerlei kompensierende Wirkungen auf die Entwicklung der Steuereinnahmen ausübt. Das Gegenteil ist eher der Normalfall.

\section{Abbildung 5:Entwicklung von BIP sowie Einnahmen aus Steuern und KFA}

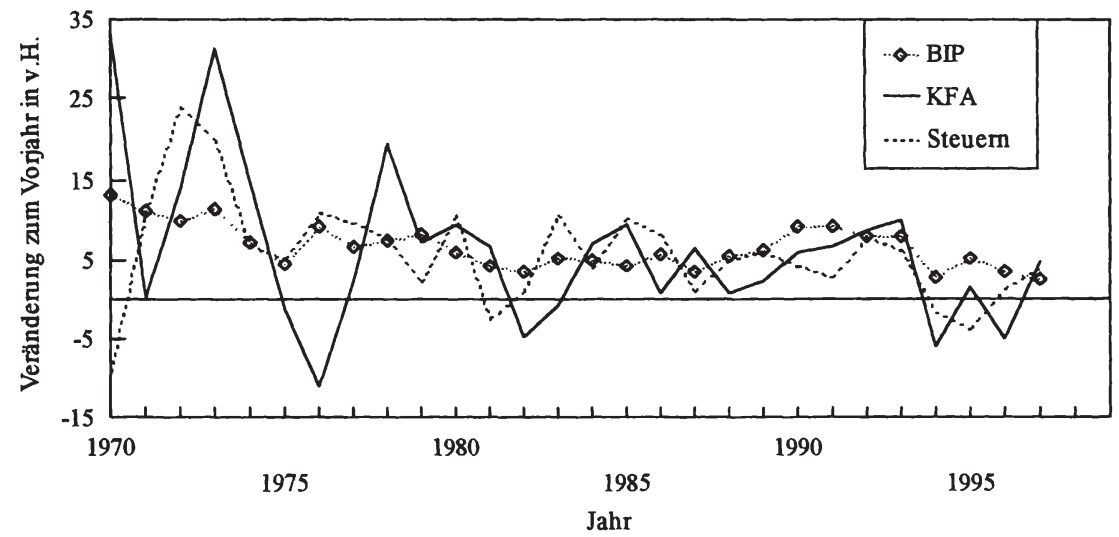

Quelle: Eigene Berechnung nach Daten des Finanzministeriums zum KFA 1998.; Sachverständigenrat zur Begutachtung der gesamtwirtschaftlichen Entwicklung: Jahresgutachten 1998/99, Stuttgart 1998, Tabelle A 1; Daten des Statistischen Bundesamtes.

So stiegen 1972 (1973) die Einnahmen aus kommunalen Steuern und KFA gegenüber dem Vorjahr um 24,1 und 13,9 v.H. (20,0 und 31,4 v.H.), 1985 um 10,1 und 9,4 v.H. und 1993 um 6,2 und 9,9 v.H. Da die gleichlaufende Entwicklung dieser Einnahmen nicht antizyklisch zur Wirtschaftsentwicklung erfolgte, verstärkte der KFA die prozyklische Ausgabenpolitik der Kommunen. Dies konstatiert auch der SACHVERSTÄNDIGENRAT, der darauf hinweist, daß die Zuweisungen "wie die Erfahrung lehrt, eher prozyklische Schwankungen zeigen"1. Zu beachten ist noch,

Beirats beim Bundesministerium der Finanzen zur Reform der Gemeindesteuern - Sollte die Gewerbesteuer durch eine Wertschöpfungsteuer ersetzt werden?, in: Der Gemeindehaushalt, 84. Jg., 1983, S. 179f.

1 Sachverständigenrat zur Begutachtung der gesamtwirtschaftlichen Entwicklung: "Unter An- 
daß die Amplitude der Einnahmenschwankungen größer sind als jene des BIP, so daß dessen zyklische Entwicklung weiter verstärkt wird.

Hinzu kommen noch diskretionäre Eingriffe der Länder, die in Boomphasen den Verbundsatz erhöhen und in der Rezession verringern, wodurch die prozyklische Wirkung noch gesteigert wird. ${ }^{1}$ So wurde in Hessen 1966 der Anteil am Einkommensteuerverbund von 21 auf 23 v.H. erhöht, um den Gemeinden zusätzliche Einnahmen zu verschaffen; 1981 wurde dann die Quote des einheitlichen Verbundes von 23 auf 22,7 v.H. gemindert, um so eine Beteiligung der Kommunen an den notwendigen Kürzungen im Landeshaushalt zu erreichen.

\subsubsection{Ansätze zur Verstetigung der Einnahmen durch den KFA}

Naheliegend wäre es, Finanzzuweisungen antizyklisch auszugestalten, um einen Ausgleich für die prozyklische Einnahmenentwicklung bei den Steuereinnahmen zu erreichen. ${ }^{2} \mathrm{Da}$ es aber keine Steuerarten gibt, die ein antizyklisches Aufkommen aufweisen, wäre dies kaum mit einem Steuerverbund zu bewerkstelligen. In vorherigen Ausführungen wurde schon darauf hingewiesen, welche Gefahr die diskretionäre Bestimmung der Verbundmasse bergen kann. Deshalb ist grundsätzlich die Regelung des quotalen Steuerverbunds nicht anzutasten.

Ziel sollte es sein, daß zumindest die Zuweisungen stetig fließen. Dadurch induziert der KFA selbst kein zyklisches Ausgabeverhalten und verringert gleichzeitig die Schwankungen der Gesamteinnahmen, was zur derzeitigen Parallelpolitik schon einen Fortschritt darstellen würde. ${ }^{3}$ Dies könnte durch eine Veränderung des Steuerverbundes erreicht werden. Derzeitig werden die hessischen Kommunen mit 22,9 v.H. an allen in den Steuerverbund eingehenden Steuern beteiligt. Dieser macht wiederum über 90 v.H. der Finanzausgleichsmasse aus. ${ }^{4} \mathrm{Da}$ das Volumen der konjunkturreagiblen Einkommen- und Körperschaftsteuer aber bei über 70 v.H. der Verbundmasse liegt, ändert sich die Steuerverbundmasse stärker als die gesamten Steuereinahmen des Landes, da dort die Quote der genannten Steuern nur 57,4 v.H. beträgt. ${ }^{5}$ Die prozentuale Gleichgewichtung aller in den Verbund einbezogenen Steuern, die eine möglichst stetige Entwicklung der

passungszwang"; Jahresgutachten 1980/81, Stuttgart 1980, S. 162, Tz. 361; ähnlich ders.: "Im Standortwettbewerb", Jahresgutachten 1995/96, Stuttgart 1995, S. 212, Tz. 342.

1 Vgl.: Massat, D.: Die ökonomische Problematik von Zuweisungen an Kommunen, Thun Frankfurt 1984, S. 88, 114.

2 Vgl:: Kock, H.: Vorschläge zur Verstetigung der Gemeindefinanzen, in: Konjunkturpolitik, 21. Jg., 1975, S. 334.

3 Vgl.: Sachverständigenrat zur Begutachtung der gesamtwirtschaftlichen Entwicklung: "Unter Anpassungszwang"; Jahresgutachten 1980/81, Stuttgart 1980, S. 162, Tz. 361.

4 In den Jahren 1998 bzw. 1997 betrug für Hessen der Anteil der Steuerverbund- an der gesamten Finanzausgleichsmasse 91,5 bzw. 93,6 v.H.

5 Errechnet aus dem Steueraufkommen des Landes Hessen in 1997. 
Verbundmasse verhindert, wird damit begründet, daß die "Aufgabengemeinschaft von Staat und Kommunen durch eine finanzwirtschaftliche Schicksalsgemeinschaft ergänzt"1 wird.

Als erstes sollte die Verbundgrundlage um die bisher nicht berücksichtigten weniger konjunkturabhängigen Steuern erweitert werden, und zweitens sollten die prozentualen Beteiligungen nach der Konjunkturreagibilität differenziert werden. So ist der Anteil der Einkommensteuer zu mindern, da deren Aufkommen aufgrund des progressiven Tarifs und der wirtschaftspolitischen Instrumentalisierung im Konjunkturverlauf starken Schwankungen unterliegt. Dabei ist aber darauf zu achten, daß die Einnahmenentwicklung weiterhin mit dem Wirtschaftswachstum positiv verknüpft bleibt. Allerdings erweist sich das angestrebte Ziel einer Einnahmenverstetigung auf diese Weise als kaum erreichbar. Denn auch bei einer Erweiterung der Steuerverbundmasse und einer veränderten Gewichtung einzelner Komponenten zeigt sich, daß die Schwankungen der kommunalen Einnahmen aus Steuern und Leistungen des KFA kaum gemindert werden können. Das erste Problem besteht dabei in der Identifizierung der Steuern, die eine geringe Konjunkturreagibilität aufweisen. Denn es ist derzeit kaum möglich, Aussagen über die Aufkommenselastizität der einzelnen Steuern zu treffen. Dies liegt in der Vielzahl von steuerpolitischen Maßnahmen der letzten Jahre begründet, deren Auswirkungen sich teilweise auch überlagern und deshalb nicht zu trennen sind, was sich auch in der Prognosesicherheit der jährlichen Steuerschätzungen niederschlägt. ${ }^{2}$ Zudem erweist es sich, daß die Steuern, denen eine geringe Aufkommenselastizität zugesprochen werden könnte, Kfz- und Vermögen- und Umsatzsteuer, entweder - selbst bei vollständiger Einbeziehung in die Verbundmasse - nur ein ungenügendes Gesamtaufkommen erreichen oder häufigen Steueränderungen unterworfen sind, so daß sie sich aus diesem Grund nur wenig für eine Verstetigung der Zuweisungen eignen. ${ }^{3}$ Entsprechend kann der Anteil der konjunkturreagiblen und gleichzeitig fiskalisch ergiebigen Steuern (Einkommensteuer) nur bedingt gemindert werden. Eine starke Einengung der Verbundbasis induziert zudem die Gefahr, daß die staatliche Ebene - soweit ihr dies möglich ist - eher bei den Verbundsteuern Steuersenkungen durchführt, da der Einnahmenverlust besonders die kommunale Ebene trifft, und sich vor allem um die eigenen Steuern bemüht. ${ }^{4}$

1 Patzig, W.: Der kommunale Finanzausgleich im Zeichen des Steuerverbundes, in: DVB1. 74. $\mathrm{Jg}, 1959, \mathrm{~S} .1$.

2 Vgl:: Sachverständigenrat zur Begutachtung der gesamtwirtschaftlichen Entwicklung: "Wachstum, Beschäftigung, Währungsunion - Orientierungen für die Zukunft"; Jahresgutachten 1997/98, Stuttgart 1997, S. 116.

3 So beträgt das Aufkommen der Kfz-Steuer 1998 1,10 Mrd. DM und auch die Vermögensteuer führte 1996 nur zu Einnahmen von 0,98 Mrd. DM, während 1998 das Aufkommen des Steuerverbundes bei 4,58 Mrd. DM lag.

4 Vgl.: Spahn, P. B.: Dezentralisierte Haushaltspolitik und makroökonomische Steuerung, in: Galler, H. P.; Wagner, G. (Hrsg.): Empirische Forschung und wirtschaftspolitische Bera- 
Ein anderer Ansatz zur Verstetigung der Zuweisungen führt dazu, daß zwischen der ermittelten und der ausgeschütteten Ausgleichsmasse unterschieden wird. In Boomphasen wird ein Teil der Ausgleichsmasse der Kommunen in einen Fonds eingezahlt, so daß sich die Summe der zur Verfügung stehenden Einnahmen mindert. In der Rezession bei Verringerung von Steuerverbundmasse und kommunalen Steuereinnahmen erhöht sich die Ausgleichsmasse durch Zuführungen aus dem Fonds. ${ }^{1}$ Die Bildung eines separaten Fonds soll sichern, daß das Land nicht die einbehaltenen Mittel selbst verwendet und den Kommunen verdeutlichen, daß die Bereitstellung der Mittel nur zeitlich verschoben wird. ${ }^{2}$ Die Vorgehensweise bei dieser Methode soll nun für das Land Hessen in der folgenden Tabelle 6 demonstriert werden. Als Betrachtungszeitraum werden die Jahre von 1982 bis 1999 verwendet, da seit 1982 mit der Einbeziehung des Landesanteils an der Grunderwerbsteuer in den einheitlichen Steuerverbund keine Veränderungen der Verbundgrundlagen mehr vorgenommen wurden.

Tabelle 6:Wirkungen eines Fonds zur Glättung der Zuweisungen im KFA (in Mio. DM)

\begin{tabular}{|c|c|c|c|c|c|}
\hline Jahr & Ist-KFA & Änderung & Prognose-KFA & Änderung & Differenz (Fonds) \\
\hline 1989 & 3.804 .470 & - & 3.904 .475 & - & -100.005 \\
\hline 1990 & 4.032 .702 & 6,00 v.H. & 4.046 .369 & 3,63 v.H. & -13.667 \\
\hline 1991 & 4.296 .818 & 6,55 v.H. & 4.188 .262 & 3,51 v.H. & 108.556 \\
\hline 1992 & 4.671 .900 & 8,73 v.H. & 4.330 .155 & 3,39 v.H. & 341.745 \\
\hline 1993 & 5.134 .125 & 9,89 v.H. & 4.472 .048 & 3,28 v.H. & 662.077 \\
\hline 1994 & 4.823 .300 & $-6,05$ v.H. & 4.613 .941 & 3,17 v.H. & 209.359 \\
\hline 1995 & 4.887 .850 & 1,34 v.H. & 4.755 .834 & 3,08 v.H. & 132.016 \\
\hline 1996 & 4.638 .100 & $-5,11$ v.H. & 4.897 .727 & 2,98 v.H. & -259.627 \\
\hline 1997 & 4.850 .100 & 4,57 v.H. & 5.039 .620 & 2,90 v.H. & -189.520 \\
\hline 1998 & 4.824 .300 & $-0,53$ v.H. & 5.181 .514 & 2,82 v.H. & -357.214 \\
\hline 1999 & 5.064 .900 & 4,99 v.H. & 5.323 .407 & 2,74 v.H. & -258.507 \\
\hline Summe & 51.028 .565 & & 50.753 .352 & & 275.213 \\
\hline
\end{tabular}

Quelle: Eigene Berechnungen nach Abrechnungen des KFA im Staatsanzeiger verschiedener Jahre.

tung. Festschrift für Hans-Jürgen Krupp zum 65. Geburtstag, Frankfurt, New York 1998, S. $331,335$.

1 Vgl.: Ebenda, S. 335.

2 Vgl:: Sachverständigenrat zur Begutachtung der gesamtwirtschaftlichen Entwicklung: "Unter Anpassungszwang"; Jahresgutachten 1980/81, Stuttgart 1980, S. 163, Tz. 363. 
Aus den ersten sieben Jahren dieses Zeitraums wird mit Hilfe einer linearen Regression eine Funktion ermittelt, mit der die folgenden elf Jahre prognostiziert werden sollen. Zu diesem Zweck erfolgt eine Normierung der Werte auf das Ausgangsjahr 1982, um die Veränderungen zum Basisjahr zu ermitteln. ${ }^{1}$ Die Differenz zwischen der prognostizierten und der tatsächlichen Ausgleichsmasse wird über den Fonds ausgeglichen, indem die ausgeschüttete Finanzausgleichsmasse in den Jahren aufgestockt wird, in denen die tatsächlichen geringer als die prognostizierten Finanzmittel sind bzw. in den Jahren gesenkt wird, in denen die tatsächlichen über den prognostizierten Mittel liegen. Die negativen Werte in der Tabelle zeigen den Abbau von Fondsmitteln an, da in diesem Fall die tatsächlichen unter den prognostizierten Zahlungen liegen, positive den Aufbau. Werden die Veränderungen zum Vorjahr herangezogen, so mindern sich die Änderungsraten, da die Bezugsgrößen sich erhöhen. ${ }^{2}$

Als Ausgangswert für die jährlichen Veränderungen des Zuweisungsvolumens wird nun jeweils der Wert aus dem Jahr 1989 verwendet, so daß die unterschiedliche Intensität und Richtung der jeweiligen Veränderungen für zehn Jahre betrachtet werden können. In den Jahren 1989 und 1990 sind die prognostizierten Werte höher als die tatsächlich durch den Steuerverbund bereitgestellten Mittel, so daß die Auszahlungen durch den Fonds größer als die vorhandenen Mittel wären. Das Defizit der ersten zwei Jahre summiert sich auf 113,67 Mio. DM. Da sich somit der Fonds zu Beginn nicht selbst finanziert, müßte das Land die notwendigen Mittel zur Verfügung stellen, da es auch ein Interesse an einem stetigen Ausgabeverhalten hat. Am Ende der betrachteten elf Jahre wurden tatsächlich 51.028,57 Mio. DM an die Kommunen ausgezahlt, bei Realisierung des Fonds wären es 50.753,35 Mio. DM gewesen, der Differenzbetrag von 275,21 Mio. DM befindet sich noch im Fonds. Insgesamt werden durch den Fonds Mittel in Höhe von 1.453,75 Mio. DM im betrachteten Zeitraum umverteilt, wodurch eine Verstetigung der Zuweisungen erfolgt. So verändert sich das Zuweisungsvolumen im Vergleich zum Vorjahr nach der Bildung des Fonds nur mit geringen Schwankungen zwischen den Jahren; der Maximalwert beträgt 3,63 v.H. (1990), der Minimalwert 2,74 v.H. (1999). Hingegen haben sich die Zuweisungen in der Realität sehr stark von Jahr zu Jahr geändert. So stiegen die Zuweisungen von 1992 auf 1993 um 9,89 v.H., um im folgenden Jahr um 6,05 v.H. zu sinken. Die

Die Regressionsanalyse ergibt gerundet für die Konstante den Wert 97,920 und für den XKoeffizienten 4,874. Bei Verwendung dieser gerundeten Zahlen würden sich die Abweichungen zum Ergebnis in der Tabelle kumulieren, so daß der Fonds 1999 einen um 0,12 Mio. DM geringeren Überschuß ausweisen würde.

2 Werden die Änderungsraten aufs jeweilige Ausgangsjahr bezogen, das auf 100 normiert wird, so ergibt sich für die Fondslösung eine gleichbleibende Änderung von 3,634 v.H., während sich für den tatsächlichen KFA die Schwankungen erhöhen und zwischen 12,15 und $-8,17$ v.H. liegen. 
Verstetigung der Zuweisungen durch die Bildung des Fonds ist Abbildung $6 \mathrm{zu}$ entnehmen.

Abbildung 6:Verstetigung der KFA-Zuweisungen durch Fondsbildung

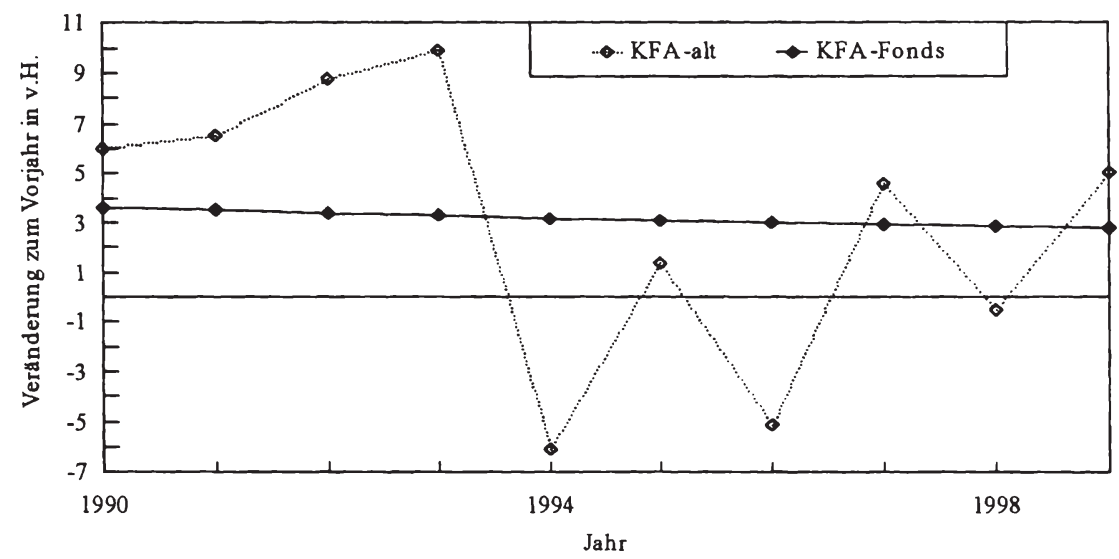

Quelle: Eigene Berechnungen nach: Abrechnungen des KFA im Staatsanzeiger verschiedener Jahre.

$\mathrm{Zu}$ klären ist noch, wie die Fondsmittel verwendet bzw. aufgebracht werden sollen, also welche Zuweisungskategorien zu erhöhen bzw. zu verringern sind. Die geringsten politischen Durchsetzungsschwierigkeiten gäbe es wohl, wenn alle Kategorien um den gleichen v.H.-Satz gemindert würden, bis die in den Fonds einzuzahlende Summe aufgebracht ist bzw. umgekehrt bei Auszahlung der Mittel. Allerdings dürte eine solche Vorgehensweise nicht angemessen sein. In der Rezession sollten eher Zuweisungen für kommunale Investitionen erhöht werden, da sie besonders nachfragewirksam sind und auch die zukünftigen Wachstumschancen verbessern; sie wären entsprechend im Boom zu kürzen. ${ }^{1}$

Reicht die Variation dieser Mittel nicht aus, so ist abzuwägen, ob die besonderen oder die Schlüsselzuweisungen zu mindern sind. Gegen die Minderung der besonderen Zuweisungen spricht auf den ersten Blick, daß mit ihnen Aufgaben finanziell abgegolten werden sollen, deren Wahrnehmung im Konjunkturverlauf kaum - wie Ausgaben für Schulen - oder sogar antizyklisch - etwa Ausgaben für Sozialhilfe - schwanken. Da diese Mittel aber ohne Berücksichtigung von Finanzkraft und Finanzbedarf verteilt werden, kann dies dazu führen, daß an sich schon finanzschwache Kommunen einen weiteren Einnahmenrückgang bei Kürzung der

1 Vgl.: Fischer, H.: Finanzzuweisungen. Theoretische Grundlegung und praktische Ausgestaltung im bundesstaatlichen Finanzausgleich Australiens und der Bundesrepublik Deutschland, Berlin 1988, S. 123. Fischer weist dort aber auf einen möglichen Konflikt mit dem Wachstumsziel hin, welches stetige Investitionen erfordert. 
Schlüsselmasse erleiden, während abundante ${ }^{1}$ Gemeinden weiterhin besondere Zuweisungen erhalten, so daß die Ausgleichfunktion beeinträchtigt wäre. ${ }^{2}$ Hinzu kommt, daß der Freiheitsgrad der Schlüsselzuweisungen größer ist als jener der besonderen Zuweisungen, so daß um die Ausgabenautonomie der Kommunen nicht zu mindern, die Schlüsselmasse nicht verringert werden sollte. Somit ist eher das Volumen der besonderen Zuweisungen zu senken und das Niveau der Schlüsselmasse beizubehalten.

\subsection{Verteilung der Finanzmasse auf die Zuweisungskategorien}

\subsubsection{Charakterisierung der Zuweisungskategorien}

Die Zuweisungen des Landes Hessen im Rahmen des kommunalen Finanzausgleichs können in drei Gruppen unterschieden werden, wobei sich die Abgrenzung auf $\S 3$ Abs. 1 der neuesten Fassung des FAG bezieht:

- allgemeine ungebundene Zuweisungen,

- zweckgebundene besondere Zuweisungen oder Sonderlastenausgleiche,

- Zuweisungen zur Finanzierung von Investitionen.

Die beiden erstgenannten Zuweisungsgruppen wiederum werden im Verwaltungshaushalt verbucht und stehen von der Idee her den Gemeinden zur Finanzierung laufender Ausgaben zur Verfügung und können als "Schlüsselzuweisungen im engeren Sinn und (...) (als) die gleichfalls schlüsselmäßig zu berechnenden Sonderlastenausgleiche" ${ }^{3}$ bezeichnet werden. Die letzte Gruppe ist im Vermögenshaushalt $\mathrm{zu}$ veranschlagen und dient der Finanzierung kommunaler Investitionen, wobei zwischen zweckgebundenen projektbezogenen und ungebundenen allgemeinen Investitionszuweisungen zu unterscheiden ist. Siehe dazu Abbildung 7.

Abbildung 7:Typisierung der Zuweisungen des KFA in Hessen

\begin{tabular}{|c|c|c|c|}
\cline { 2 - 4 } \multicolumn{1}{l|}{} & Verwaltungshaushalt & Vermögenshaushalt \\
\hline Zweckbindung & ja & $\begin{array}{c}\text { Besondere Zuweisungen } \\
\text { (Sonderlastenausgleiche) }\end{array}$ & $\begin{array}{c}\text { projektbezogene } \\
\text { Zuweisungen }\end{array}$ \\
\cline { 2 - 4 } & nein & $\begin{array}{c}\text { Schlüsselzuweisungen und } \\
\text { Landesausgleichsstockmittel }\end{array}$ & $\begin{array}{c}\text { Investitionspauschale } \\
\text { (derzeit nicht dotiert) }\end{array}$ \\
\hline
\end{tabular}

Abundanz ist so definiert, daß die ermittelte Finanzkraft größer ist als der ermittelte Finanzbedarf. Die abundanten Kommunen erhalten keine Schlüsselzuweisungen.

2 Vgl.: Voigtländer, H.: Die Finanzzuweisungen an die Gemeinden und Gemeindeverbände in konjunkturpolitischer Sicht, in: AfK, 9. Jg., 1970, S. 308; Klein, R. R.; Münstermann, E.: Kommunen und Konjunkturpolitik, in: AfK, 17. Jg., 1978, S. $223 \mathrm{f}$.

3 Hessischer Landtag: Vorlage der Landesregierung betreffend den Entwurf für ein Siebentes Gesetz zur Änderung des Finanzausgleichsgesetzes vom 14.02.1972, Drucksache 7/1322, S. 8. 
Die Schlüsselzuweisungen sollen allgemein die Finanzkraft der kommunalen Ebene stärken; gleichzeitig wird durch die Berücksichtigung der Steuerkraft und die daraus folgende horizontale Differenzierung des jeweils auf die einzelne Kommune entfallenden Zuweisungsbetrags die unterschiedliche kommunale Leistungsfähigkeit berücksichtigt und somit eine Minderung der Einnahmenungleichverteilung angestrebt. Neben den Einnahmen sind auch die Aufgaben und die durch sie induzierten Ausgaben - also der Finanzbedarf - der jeweiligen Körperschaft zu beachten. Diese unterscheiden sich in Abhängigkeit von der Stellung in der Verfassung bzw. der Gemeindeordnung. Nur so kann die Situation einer Gemeinde beurteilt werden. Das Verteilungsverfahren der Zuweisungen ist stark schematisiert, wobei vor allem die Steuerkraft und der einwohnerabhängige Finanzbedarf als Zuweisungsindikatoren genutzt werden, indem gemeindespezifische in gruppenmäßige Bedarfe transformiert und berücksichtigt werden. ${ }^{1}$ Die Verwendung zusätzlicher Indikatoren ermöglicht eine weitere Differenzierung der Belastungen, geschieht aber auch ausschließlich finanzkraftbezogen. ${ }^{2}$ Auf die Höhe der Zuweisungen wirken sich neben Finanzkraft und Finanzbedarf noch der Ausgleichsgrad aus; alle drei Größen bilden ein interdependentes System. ${ }^{3}$ Die Schlüsselzuweisungen stellen in Hessen die am stärksten dotierte Zuweisungskategorie dar. $\mathrm{Thr}$ Volumen an der Finanzausgleichsmasse belief sich 1998 auf 2.737 Mio. DM; der Anteil betrug somit etwa 56,7 v.H. der Zuweisungen im Rahmen des KFA.

Die besonderen Zuweisungen bzw. Sonderlastenausgleiche sollen die von bestimmten Gebietskörperschaftsgruppen zu tragenden speziellen Lasten entgelten und somit "dem Ausgleich eines regional unterschiedlichen und weitgehend einwohnerunabhängigen Bedarfs bei den großen Ausgabenblöcken" ${ }^{4}$ dienen. Diese Ausgaben werden durch die Übertragung von Aufgaben des Bundes und der Länder induziert und können aufgrund der unterschiedlichen Lastverteilung nicht mittels Schlüsselzuweisungen kompensiert werden. ${ }^{5}$ Die Mittel werden wie die Schlüsselzuweisungen "nach allgemeinen Kriterien berechnet und ausgezahlt,

I Vgl.: Münstermann, E.; Becker, H.: Finanzausgleichsleistungen an Kommunen. Ein Vergleich der Finanzausgleichssysteme in den Bundesländern (Reihe G; DST-Beiträge zur Finanzpolitik, Heft 7), Köln 1978, S. 59. Dies geschieht z.B. bei der Ermittlung des Zuschußbedarfs einer Gemeindeklasse, der aus dem Durchschnitt der Daten der einzelnen Gemeinden errechnet wird.

2 Vgl.: Katz, A.: Der kommunale Finanzausgleich, in: Püttner, G. (Hrsg.): Handbuch der kommunalen Wissenschaft und Praxis, Bd. 6, 2. Aufl., Berlin u.a.O. 1985, S. 324f.

3 Vgl:: Deubel, I.: Mängel im Finanzausgleichssystem des Landes Nordrhein-Westfalen, in: Hoppe, W. (Hrsg.): Reform des kommunalen Finanzausgleichs, Köln u.a.O. 1985, S. 72.

4 Hessischer Landtag: Vorlage der Landesregierung betreffend den Entwurf für ein Siebentes Gesetz zur Änderung des Finanzausgleichsgesetzes vom 14.02.1972, Drucksache 7/1322, S. 8.

5 Vgl:: Hessischer Landtag: Gesetzentwurf der Landesregierung für ein Gesetz zur Regelung des Finanzausgleichs und zur Änderung anderer Vorschriften vom 11.10.1977, Drucksache $8 / 4877$, S. 26. 
ohne daß ihre Verwendung im einzelnen überwacht wird"'; es steht der einzelnen Empfängergemeinde somit frei, "ob und inwieweit sie diese Mittel im Ergebnis auch tatsächlich für diese Aufgaben einsetzt" ${ }^{2}$. Bei der Bestimmung der Zuweisungshöhe wird im Gegensatz zu den allgemeinen Zuweisungen nicht die Leistungsfähigkeit, sondern der jeweilige zweckbezogene Aufwand berücksichtigt oder Indikatoren, welche mit diesem korrelieren. ${ }^{3}$ In Hessen verteilen sich die für besondere Finanzzuweisungen bereitstehenden Mittel 1998 auf 14 verschiedene Bereiche. ${ }^{4}$ Dabei werden im FAG neben den Sonderlastenausgleichen nach den $\S \S 22-28$ jedes Jahr aufgrund der Generalnorm des $\S 21$ Abs. 1 besondere Aufgabenbereiche als förderungswürdig deklariert und dotiert. Die Sonderlasten können danach unterschieden werden, ob ein rechtlicher Anspruch (z.B. § 22 FAG) oder aber nur die Möglichkeit (etwa § 24 FAG) finanzieller Zuweisungen nach Ermessen der Landesregierung besteht. Neben den im Gesetz aufgeführten ausgleichsfähigen Sonderlasten können bei akuten Notsituationen auch Zuweisungen aus dem Landesausgleichsstock nach $\S 28 \mathrm{FAG}$ erfolgen. Der Anteil der besonderen Finanzzuweisungen lag 1998 bei ungefähr 23,6 v.H. der Finanzausgleichsmasse; die absolute Höhe betrug dabei 1.140 Mio. DM, wobei die aufgabenbezogenen Zuweisungssummen zwischen 3,4 Mio. DM für kommunale Bibliotheken u.ä. und 304 Millionen DM zum Ausgleich der Kosten der örtlichen Sozialhilfe schwankten. $^{5}$

Als dritte Kategorie sind noch Zuweisungen zur Finanzierung von Investitionen der kommunalen Ebene zu nennen, ihr Anteil lag 1998 bei ca. 19,7 v.H. Mit ihnen soll ein vergleichbares Infrastrukturniveau in allen Landesteilen erreicht werden. ${ }^{6}$

1 Hessischer Landtag: Antwort der Landesregierung auf die Große Anfrage der Fraktion der CDU betreffend Finanzielle Abhängigkeit der hessischen Kommunen von Land und Bund Drucksache 9/3470 vom 01.07.1981, Drucksache 9/4951, S. 3.

2 Hessischer Minister der Finanzen (Hrsg.): Kommunaler Finanzausgleich 1956-1988, Wiesbaden 1989, S. 12.

3 Nur bei den auch als besondere Zuweisungen klassifizierten Bedarfszuweisungen, die aus dem Landesausgleichsstock ( $§ 28$ FAG) zur Linderung außergewöhnlicher Notlagen der Kommunen gewährt und üblicherweise den freien Zuweisungen zugeteilt werden, da ihre Verausgabung nicht zweckgebunden ist, hängt die Höhe des Betrags von der Finanzkraft der jeweiligen Kommune ab. Die Mittel des Landesausgleichsstocks ergeben sich aus der nach § 4 FAG jährlich stattfindenden Abrechnung des Finanzausgleichs. Die wegen der Differenzen zwischen dem Soll und dem Ist der Verbundmasse notwendigen Verrechnungen werden mit dem Landesausgleichsstock vorgenommen.

4 Museen ( $(21)$, Aussiedler ( $(21)$, Programm "Arbeit statt Sozialhilfe" (§21), Schulen ( 22), Grundschulen ( $(22 a)$, Sozialhilfeausgaben ( $(23)$, Arbeitslosigkeit ( $§ 23 a)$, Jugendhilfe (§ 23b), Kinderbetreuungseinrichtungen ( $(23 \mathrm{c})$, ÖPNV ( $\S 24,25)$, Theater $(\S 26)$, StraBen $(\S 27)$ und den Landesausgleichsstock ( $§ 28)$.

5 Vgl:: Hessisches Ministerium der Finanzen: Veröffentlichung nach $\S 49$ Abs. 3 des Finanzausgleichsgesetzes für das Ausgleichsjahr 1997 vom 31.03.1997, in: Staatsanzeiger für das Land Hessen, S. 1023f.

6 Vgl.: Hessischer Landtag: Gesetzentwurf der Landesregierung für ein Zwölftes Gesetz zur 
Aufgrund des stark schwankenden Investitionsbedarfs einzelner Gemeinden ist es nicht möglich, mit Hilfe eines Steuersystems ausreichend Finanzmittel für die anstehenden Investitionen zur Verfügung zu stellen. Würde auf ein Zuweisungssystem zu Gunsten von Steuereinnahmen verzichtet, so müßten diese auch den Spitzenbedarf abdecken, während in Zeiten ohne Investitionen entsprechende Mittel übrig wären. Die Steuern haben deshalb einen Normalbedarf zu finanzieren, die Spitzenbedarfe werden dann durch Zuweisungen gedeckt. ${ }^{1}$ Hierbei ist zwischen pauschalierten Investitionszuweisungen und Projektförderungen von Einzelobjekten zu unterscheiden. Die Einführung pauschalierter Zuweisungen wurde mit den zukünftigen Herausforderungen der Daseinsfürsorge (demographische Entwicklung) begründet, welche erfordert, "die Investitionskraft der Kommunen generell zu stärken, wobei finanzschwächere oder strukturell benachteiligte Gebiete besonders zu begünstigen sind"2. Ziel war es, die Verwaltungskosten der Überprüfung der rechtmäßigen Verwendung der Mittel, die bei den projektbezogenen Investitionszuweisungen üblicherweise auftreten, zu mindern und gleichzeitig die kommunale Planungsautonomie zu stärken; nur noch $25 \mathrm{v} . \mathrm{H}$. der normalerweise zu bearbeitenden Zahlungsfälle sollten nach der aufwendigeren Methode ausgezahlt werden. ${ }^{3} 1988$ wurde eine allgemeine Investitionspauschale als Pflichtleistung in den KFA eingeführt, wobei sich die Höhe der auf die einzelne Gebietskörperschaft entfallenden Mittel nach der Finanzkraft richtete. ${ }^{4} 1996$ wurde diese Regelung aufgrund der angespannten Haushaltssituation in eine "Kann-Bestimmung"s geändert; seit 1997 wird sie nicht mehr dotiert. Somit werden seit 1998 nur noch für Investitionen im Schulbereich pauschalierte Zahlungen in Höhe von 80 Mio. DM oder 8,45 v.H. der investiven Zuweisungen gewährt. Folglich entfallen mit 867 Mio. DM 91,55 v.H. der Mittel auf zweckgebundene Investitionszuweisungen, bei denen ein Einzelverwendungsnachweis für die erhaltenen Mittel notwendig ist, wobei die Dotierungen der förderungsfähigen Projekte zwischen 0,5 Mio. DM für Biotopvernetzung und -sicherung ( $\$ 33$ Abs. 1 Nr. 8 FAG) und 415 Mio. DM zur Krankenhausfinanzierung ( $\$ 33$ Abs. 1 Nr. 1) liegen. ${ }^{6}$

Änderung des Finanzausgleichsgesetzes vom 13.09.1976, Drucksache 8/3054, S. 7, Nr. 2.

1 Vgl: Gräf, H.: Investitionszuweisungen zwischen Schlüssel- und Zweckzuweisungen, in: Räumliche Aspekte des kommunalen Finanzausgleichs (Veröffentlichungen der Akademie für Raumforschung und Landesplanung: Forschungs- und Sitzungsberichte, Bd. 159), Hannover 1985, S.276.

2 Hessischer Landtag: Gesetzentwurf der Landesregierung für ein Gesetz zur Änderung des Finanzausgleichsgesetzes und anderer Rechtsvorschriften - Finanzausgleichsänderungsgesetz 1988 - vom 25.11.1987, Drucksache 12/1178, S. 12.

3 Vgl: Ebenda, S. 12-14.

4 Später wurden noch Investitionspauschalen für Straßen- und Schulbau eingeführt.

5 Hessischer Landtag: Änderungsantrag der Landesregierung zu dem Gesetzentwurf der Landesregienung für ein Gesetz zur Änderung des Finanzausgleichsgesetzes Drucksache 14/312 vom 22.12.1995, Drucksache 14/946, S. 1, 5.

6 Vgl: Hessisches Ministerium der Finanzen: Veröffentlichung nach $\S 49$ Abs. 3 des Finanzausgleichsgesetzes für das Ausgleichsjahr 1997 vom 31.03.1997, in: Staatsanzeiger für das 


\subsubsection{Dotierung der Zuweisungskategorien im Zeitablauf}

\subsubsection{Zeitliche Entwicklung}

Für die Verteilung der gesamten Finanzmasse auf die einzelnen Kategorien stehen verschiedene Systeme zur Wahl. So können im FAG Quoten für die einzelnen Zuweisungsarten vorab bestimmt sein. ${ }^{1}$ Dieses Verfahren führt aber zu einer Starrheit bei der Verteilung der Mittel, beispielsweise unter veränderten wirtschaftlichen Situationen. In Hessen wird deshalb die Verteilung auf die einzelnen Kategorien nicht im FAG, sondern nach $\S 3$ Abs. 2 FAG im Landeshaushalt festgelegt. Diese Regelung "stellt sicher, daß der Finanzausgleich veränderten kommunalen Finanzbedürfnissen im Rahmen der gesetzlich bestimmten Finanzausgleichsmasse flexibel angepaßt werden kann"2. Denn der für allgemeine Zuweisungen vorgesehene Teil ergibt sich als Restgröße, nachdem von der Finanzausgleichsmasse die besonderen und die investiven sowie die allgemeinen Zuweisungen des Landeswohlfahrtsverbandes abgezogen worden sind. Somit kann eine gezielte Einflußnahme durch unterschiedliche Ansätze bei der Vorabdotierung aus der Verbundmasse realisiert werden. Abbildung 8 gibt die Entwicklung der Anteile der einzelnen Zuweisungsarten an den gesamten den Gemeinden und GV im Rahmen des KFA zur Verfügung gestellten Mitteln wieder.

\section{Abbildung 8:Anteile der Zuweisungsarten an der Finanzausgleichsmasse im Zeitablauf}

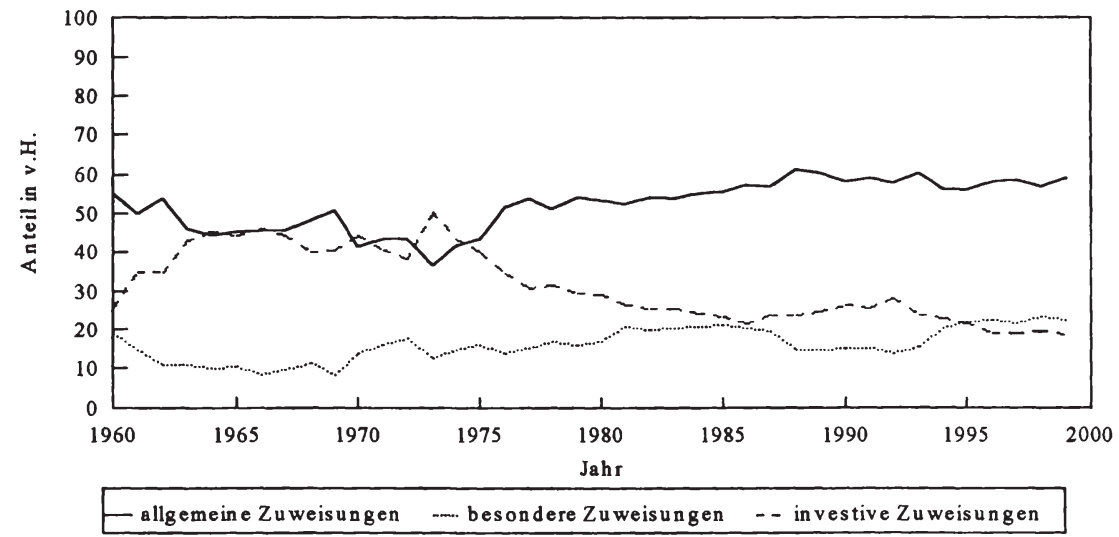

Quelle: Eigene Berechnung nach: Daten des Hessischen Finanzministeriums.

Land Hessen, S. 1024.

1 Dies ist etwa im Niedersächsischen FAG der Fall gewesen ist. Vgl.: $§ 2$ Niedersächsisches Gesetz über den Finanzausgleich (NFAG) vom 19.12.1995, Nds. GVB1., S. 463.

2 Hessischer Landtag: Gesetzentwurf der Landesregierung für ein Gesetz zur Regelung des Finanzausgleichs und zur Änderung anderer Vorschriften vom 11.10.1977, Drucksache 8/4877, S. 28 , Nr. 3. 
Dabei ist die Entwicklung weg von dem investitionsdominierten Zuweisungssystem der 60er und ersten Hälfte der 70er Jahre hin zu einer Entlastung der kommunalen Ebene von den Kosten der laufenden Aufgaben durch Erhöhung der allgemeinen, vor allem aber der besonderen Zuweisungen zu erkennen. Damit verbunden ist auch der wachsende Anstieg der nicht zweckgebundenen allgemeinen Zuweisungen, die sich aufgrund der freien Verfügbarkeit positiv auf die kommunale Selbstverwaltung auswirken. So stieg der Anteil der investiven Zuweisungen von 25,88 v.H. 1960 mit der Begründung des Kraftfahrzeugsteuerverbundes 1961 auf 35,00 v.H. und erreichte die Höchstmarke von 50,49 v.H. 1973, als 1.028,2 Mio. DM bereitstanden; damit einher ging die Verminderung der allgemeinen und besonderen Zuweisungen. Die investive Ausrichtung des KFA bis Mitte der 70er Jahre tritt deutlich hervor, wenn berücksichtigt wird, daß sich das Volumen der investiven Zuweisungen in diesem Zeitraum um den Faktor 21 erhöhte, während die Verbundmasse im gleichen Zeitraum nur um den Faktor 11 anstieg. Selbst bei unverändertem Anteil von 25,88 v.H. aus dem Jahr 1960 hätten sich die Zuweisungen bis 1973 von 49,60 Mio. auf 266,11 Mio. DM erhöht. In den folgenden Jahren wurde der Anteil auf 18,73 v.H. im Jahr 1999 oder 948,6 Mio. DM gesenkt. Diese Verringerung wurde damit erklärt, daß nach Erstellung eines angemessenen Infrastrukturniveaus ab Mitte der 70er Jahre die Belastung durch laufende Verwaltungskosten (Folgekosten der Investitionen) immer größer geworden ist. $^{1}$

Bis 1973 verringerte sich auch entsprechend der prozentuale Anteil der allgemeinen Zuweisungen von 55 v.H. auf knapp 37 v.H., allerdings konnte das Wachstum der Verbundmasse diese Entwicklung überkompensieren, so daß es trotzdem zu einem kontinuierlichen absoluten Anstieg von 105,50 Mio. auf 746 Mio. DM kam. In den nächsten Jahren nahm der Anteil dieser Zuweisungskategorie fast stetig zu und erreichte 1988 mit über 61 v.H. und 2.272 Mio. DM sein relatives Maximum, während die absolute Höhe auch in den folgenden Jahren stieg. Diese Entwicklung ist aus kommunaler Sicht positiv zu beurteilen, da die allgemeinen Zuweisungen vom Autonomiegrad den eigenen Steuern sehr nahe kommen.

Der Anstieg der besonderen Zuweisungen von 8,33 v.H. (81 Mio. DM) 1966, was als Endpunkt einer kontinuierlichen Verringerung dieser Zuweisungskategorie anzusehen ist, über 14,10 v.H. (192 Mio. DM) 1970 - durch die Einführung des Schullastenausgleiches ${ }^{2}$ - auf 22,44 v.H. (1.137 Mio. DM) 1999 liegt hauptsächlich in den im Zeitablauf zusätzlich entstandenen Belastungen durch erhöhte laufende Ausgaben der kommunalen Ebene begründet. Neben dem schon genannten Schullastenausgleich sind hier vor allem die steigenden Ausgaben für die

1 Vgl: Hessischer Landtag: Gesetzentwurf der Landesregierung für ein Zwölftes Gesetz zur Änderung des Finanzausgleichsgesetzes vom 13.09.1976, Drucksache 8/3054, S. 7.

2 Vgl.: Hessischer Landtag: Vorlage der Landesregierung für ein Fünftes Gesetz zur Änderung des Finanzausgleichsgesetzes vom 07.07.1969, Drucksache 6/2194, S. 11, $14 f$. 
Sozialhilfe zu nennen, die durch den Sozialhilfelastenausgleich ${ }^{1}$ seit 1978 besonders dotiert werden. Der Anteil betrug bei seiner Einführung mit 30 Mio. DM ungefähr 7 v.H. dieser Zuweisungskategorie und stieg im Zeitablauf mit 295 Mio. DM im Jahr 1998 auf fast 26 v.H.

\subsubsection{Analyse und Reform}

\subsection{Entwicklungsvergleich zwischen Hessen und westdeutschen Bundesländern}

In Kapitel 3 wurde angeführt, daß aus kommunaler Sicht zweckgebundene $\mathrm{Zu}$ weisungen problematisch sind, da die Selbstverwaltungsautonomie eingeschränkt wird. Es ist zu erwarten, daß den Entscheidungsträgern vor Ort die lokalen Präferenzen der Wirtschaftssubjekte eher und besser bekannt sind als der Landesregierung. ${ }^{2} \mathrm{Da}$ nur normativ Entscheidungen über die Aufteilung der Finanzmittel zwischen gebundenen und ungebundenen getroffen werden können, bieten sich als Beurteilungskriterien neben der Untersuchung der Anteilswerte der jeweiligen Zuweisungskategorien im Zeitablauf (Längsschnittbetrachtung) noch der Vergleich mit der Situation in den übrigen westdeutschen Flächenländern (Querschnittsbetrachtung) an. Der erste Aspekt gibt Aufschluß darüber, inwieweit die Vergabepraxis der Finanzzuweisungen kommunalfreundlicher geworden ist, während die zweite Größe einen Anhaltspunkt dafür gibt, ob die hessische Verfahrensweise einem bundesdeutschen Trend folgt und wie sie dort einzuordnen ist.

Aus dem vorherigen Abschnitt ist bekannt, daß sich im KFA von Hessen der Anteil der allgemeinen Zuweisungen im Zeitablauf von knapp 37 v.H. 1973 auf über 58 v.H. Jahr 1999 erhöht hat. Dies spricht für eine kommunalfreundliche Veränderung der Zuweisungspraxis. Die Längsschnittbetrachtung sagt aber nichts über die allgemeinen Entwicklunglinien aus, denen ein Finanzausgleichssystem im Zeitablauf unterworfen ist. Möglicherweise hat sich in den übrigen Ländern aufgrund eines Paradigmenwandels etwas vergleichbares ergeben, so daß Hessen trotz der positiv zu beurteilenden feststellbaren Verschiebungen der Anteile der einzelnen Zuweisungen im Ländervergleich schlecht dasteht. Die Entwicklung in ausgewählten westdeutschen Flächenländern ist in Abbildung 9 wiedergegeben. ${ }^{3}$

1 Vgl:: Ders.: Gesetzentwurf der Landesregierung furr ein Gesetz zur Regelung des Finanzausgleichs und zur Änderung anderer Vorschriften vom 11.10.1977, Drucksache 8/4877, S. 29,41 .

2 Anders ist die Situation zu beurteilen, wenn die Zuweisungen der Internalisierung externer Effekte dienen sollen. Für diesen Fall sind zweckgebundene Zuweisungen sehr wohl geeignet.

3 Für die übrigen Länder gab es weder bei den statistischen Landesämtern noch bei den Landesregierungen die gewünschten Daten bzw. es bestand keine Kooperationsbereitschaft. 
Abbildung 9:Entwicklung finanzkraft-/-bedarfbezogener Schlüsselzuweisungen an den gesamten Leistungen im Rahmen des KFA im Zeitablauf

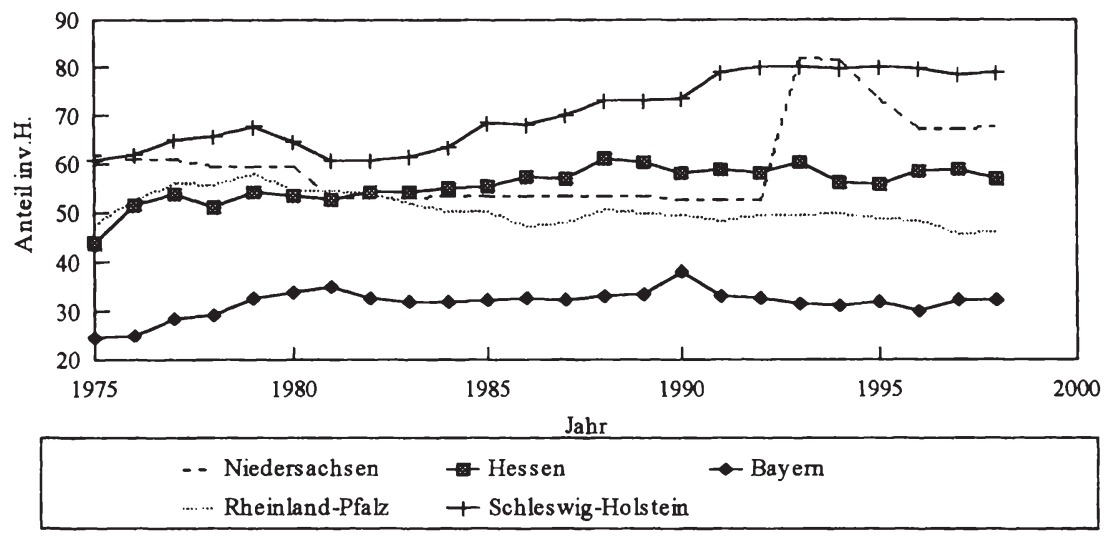

Quelle: Eigene Berechnung nach: Daten der Finanz-/Innenministerien der jeweiligen Länder.

Es ist zu erkennen, daß sich in fast allen Ländern der Anteil der finanzkraft-/-bedarfsbezogenen Schlüsselzuweisungen im Zeitablauf erhöht hat. Zwar liegen die Wachstumsraten in Bayern vor denen in Hessen und in Schleswig-Holstein mit fast 30 v.H. sowie in Niedersachsen ${ }^{1}$ mit 8,9 v.H., nur in Rheinland-Pfalz sank der Anteil um 3,5 v.H. Da aber in der Ausgangssituation die prozentualen Anteile der Schlüsselzuweisungen an den Leistungen des KFA stark divergierten - sie lagen zwischen 23,8 v.H. in Bayern und 52,0 v.H. in Schleswig-Holstein - ist die Betrachtung der Veränderung in Prozentpunkten sinnvoller. Es fällt auf, daß Schleswig-Holstein, welches 1975 schon den höchsten Anteil an Schlüsselzuweisungen aufwies, eine Erhöhung um 18,2 Prozentpunkte vorgenommen hat, während es in Hessen 13,0 und in Bayern bzw. Niedersachsen nur 7,8 bzw. 5,5 Prozentpunkte gewesen sind auch hier ergibt sich für Rheinland-Pfalz der schlechteste Wert mit $-1,69$. Wichtiger als die betrachteten Veränderungsraten ist aber für die Kommunen der tatsächliche Anteil an den gesamten Zuweisungen. Dabei kann festgestellt werden, daß Schleswig-Holstein fast über den gesamten betrachteten Zeitraum den höchsten Schlüsselzuweisungsanteil aufweist. Er liegt in der Ausgangssituation bei 60 v.H. und steigt auf 78,8 v.H. an. Es folgen Niedersachsen, Hessen und Rheinland-Pfalz mit aktuellen Schlüsselzuweisungsanteilen von 67,6, 56,7 und 46,1 v.H. Bayern hat trotz einer starken Erhöhung mit 32,3 v.H. immer noch den geringsten Anteil, der fast 15 Prozentpunkte unter dem Wert des Landes Rheinland-Pfalz liegt. Als Ergebnis kann somit festgehalten werden, daß

1 Die Werte für Niedersachsen in den Jahren 1993-1996 sind für den Vergleich problematisch, da in diesen Jahren die Zuweisungen für Aufgaben des übertragenen Wirkungskreises sowie alle übrigen Zuweisungen zur Erhöhung der Ausgleichsfunktion in die Schlüsselmasse eingestellt wurden, was vom Staatsgerichtshof für verfassungswidrig erklärt worden ist. 
auf der einen Seite Schleswig-Holstein das kommunalfreundlichste Verhalten zeigt, während auf der anderen Seite Bayern seinen Kommunen nur einen vergleichsweise geringen Grad an Autonomie bei der Mittelverwendung gewährt. Die drei übrigen Länder befinden sich zwischen diesen beiden Extremen.

\subsection{Anstehende Veränderungen für Hessen - Erhöhung der Autonomie bei der Mittelverwendung durch Abbau von Sonderlastenausgleichen}

Als Folge dieser Analyse bietet sich für Hessen an, den Anteil der finanzkraftunabhängig verteilten Mittel der Sonderlastenausgleiche zurückzuführen und im Gegenzug die Schlüsselzuweisungen zu erhöhen. Durch Gewährung geeigneter Nebenansätze - wie beispielsweise bei kreisangehörigen Gemeinden mit Schulträgerschaft - werden die Belastungen weiterhin finanziell im gleichen Umfang berücksichtigt, doch aufgrund der nun einbezogenen Finanzkraft käme es zu einer Erhöhung der Verteilungsgerechtigkeit. Damit verbunden wäre zudem eine Steigerung des Freiheitsgrads der Mittelverwendung, ${ }^{1}$ da es sich bei Schlüsselzuweisungen um ungebundene Zuweisungen handelt, und auch eine Stärkung der Ausgleichsgerechtigkeit zwischen finanzstarken und -schwachen Kommunen.

Es wäre deshalb zu überlegen, ob nicht die Finanzmittel der beiden größten Sonderlastenausgleiche - für Schulen und für Sozialhilfe - in die Schlüsselmassen integriert werden sollten. Für diese Vorgehensweise spricht auch, daß eine Integration in das Schlüsselzuweisungssystem, ohne dabei die vorher berücksichtigten Belastungen außer acht zu lassen, relativ unproblematisch sein dürfte, da die relevanten Daten gut abgrenzbar und kaum manipulierbar sind. ${ }^{2}$ Zudem wurde 1988 beim Schullastenausgleich schon ein erster Schritt in diese Richtung getan. ${ }^{3}$ Diese Maßnahme wäre beim Soziallastenausgleich aufgrund der drei separaten Schlüsselmassen problemlos, da die Mittel den schon vorher bezuschußten Gruppen zufließen würden, nämlich den kreisfreien Städten und Landkreisen als Trägern der örtlichen Sozialhilfe.

Beim Schullastenausgleich entstehen zwei Probleme. So müßte erst eine volumenmäßige Aufteilung zwischen kreisfreien Städten und kreisangehörigen Gemeinden vorgenommen werden, da die Schlüsselmassen im Gegensatz zur Finanzierung im Sonderlastenausgleich separiert sind. Weiterhin ist zu beachten, daß von der Erhöhung der Schlüsselmasse der kreisangehörigen Gemeinden neben

1 Dies gilt, wenn die Zuweisungen über den tatsächlichen Ausgaben liegen. Da sich die Sonderlastenzuweisungen für Schulen und für Straßen nicht nach den Kosten, sondern nach der Schülerzahl bzw. der Straßenlänge richten, ist für einzelne Kommunen eine Überdeckung der Kosten und eine damit verbundene Einschränkung der Ausgabenautonomie denkbar.

2 Vgl:: Steinherr, M.; u.a.: Das Schlüsselzuweisungssystem im kommunalen Finanzausgleich des Freistaates Sachsen, ifo-Studien zur Finanzpolitik, Bd. 63, München 1997, S. 132 f Auch die Paxis anderer Länder zeigt, daß dies ohne große Probleme möglich ist.

3 Siehe Abschnitt 4.4.2.2.1.5. 
den sechs Schulträgern auch die 415 übrigen Gemeinden sowie die Landkreise über die Kreisumlage bei unveränderter Bedarfsermittlung profitieren würden. Durch einen entsprechenden Nebenansatz kann der schulinduzierte Bedarf so berücksichtigt werden, daß sich für die Schulträgergemeinden in ihrer Gesamtheit im Zeitpunkt der Systemumstellung keine Verminderung der Zuweisungen ergibt. ${ }^{1}$ Es würde somit nur zu einer Veränderung der Mittelverteilung innerhalb der Gruppe kommen, wobei finanzstarke Schulträger trotz der bedarfssteigernden $\mathrm{Be}$ rücksichtigung der Schullasten als so reich gelten, daß sie relativ geringere Mittel zugewiesen bekommen als finanzschwache Schulträger, denn bei den Schlüsselzuweisungen wird im Gegensatz zu den Sonderlastenausgleichen die Finanzkraft-/-bedarfsrelation berücksichtigt. Dies soll an einem Beispiel verdeutlicht werden (siehe Tabelle 7). Es wird von drei Orten (A, B, und C) mit der Finanzkraft und dem Hauptansatz wie angegeben ausgegangen; die Schlüsselmasse beträgt $120.000 \mathrm{DM}$, die Mindestzuweisung für abundante Gemeinden $10 \mathrm{DM}$ je Einwohner und die Ausschüttungsquote 50 v.H. B und C sind Schulträger und erhalten für jeden Schüler 50 DM, insgesamt bei 350 Schülern 17.500 DM.

Tabelle 7:Verteilungswirkungen der Substitution eines Schülersonderlastenausgleichs durch einen Schülernebenansatz

\begin{tabular}{|l|c|c|c|c|c|c|c|c|}
\hline Ort & $\begin{array}{c}\text { Finanz- } \\
\text { kraft }\end{array}$ & $\begin{array}{c}\text { Haupt- } \\
\text { ansatz }\end{array}$ & $\begin{array}{c}\text { Schüler- } \\
\text { neben- } \\
\text { ansatz }\end{array}$ & $\begin{array}{c}\text { Grund- } \\
\text { betrag }\end{array}$ & $\begin{array}{c}\text { Finanz- } \\
\text { bedarf }\end{array}$ & $\begin{array}{c}\text { Schlüssel- } \\
\text { zuweisung }\end{array}$ & $\begin{array}{c}\text { Sonder- } \\
\text { lasten- } \\
\text { ausgleich }\end{array}$ & $\begin{array}{c}\text { Gesamt- } \\
\text { ein- } \\
\text { nahmen }\end{array}$ \\
\hline \multicolumn{8}{|c|}{ Separater Schülerlastenausgleich } \\
\hline A & 200.000 & 1.200 & - & 249,6 & 299.520 & 49.760 & 0 & 249.760 \\
\hline B & 200.000 & 1.300 & - & 249,6 & 324.480 & 62.240 & 10.000 & 272.240 \\
\hline C & 200.000 & 800 & - & 249,6 & 199.680 & 8.000 & 7.500 & 215.500 \\
\hline \multicolumn{8}{|c|}{ Schülernebenansatz } \\
\hline A & 200.000 & 1.200 & 0 & 249,6 & 299.520 & 49.760 & - & 249.760 \\
\hline B & 200.000 & 1.300 & 116 & 249,6 & 353.434 & 76.717 & - & 276.717 \\
\hline C & 200.000 & 800 & 87 & 249,6 & 221.395 & 10.698 & - & 210.698 \\
\hline
\end{tabular}

In der Ausgangssituation ist Ort $\mathrm{C}$ abundant und erhält als Mindestzuweisung 8.000 DM sowie zusätzlich noch 7.500 DM aus dem Sonderlastenausgleich, da

1 Eine ähnliche Vorgehensweise wurde schon einmal 1994 praktiziert, als ein Teil der für den Schullastenausgleich vorgesehenen Mittel in die Schlüsselmassen integriert wurde und den Schulträgergemeinden ein Nebenansatz gewährt wurde. Vgl.: Artikel 2 des Gesetzes zur Änderung des Finanzausgleichsgesetzes und anderer Rechtsvorschriften vom 30.12.1988, Hess. GVB1. I, S. 433. Verändert sich später die Finanzkraftrelation zwischen den sechs Schulträgern und den übrigen 415 Gemeinden, kommt es auch zu einer Mittelumverteilung zwischen den Gruppen, nämlich zugunsten der finanzschwächeren Kommunen. 
dieser finanzkraftunabhängig gewährt wird. Nun sollen zur Erhöhung des Freiheitsgrads der Mittelverwendung und der Verteilungsgerechtigkeit die Schlüsselzuweisungen auf Kosten des Sonderlastenausgleich auf 137.500 DM erhöht werden. Wird jeder Schüler im gewählten Beispiel mit 58 v.H. bedarfssteigernd angerechnet, ${ }^{1}$ so zeigt sich, daß eine Umverteilung nur innerhalb der Schulträgergemeinden auftritt. Die finanzschwächere Gemeinde B erhält auf Kosten der finanzstärkeren Gemeinde C zusätzliche Zuweisungen, ${ }^{2}$ für Gemeinde A tritt keine Veränderung auf.

Ziel der hessischen Reform der Berücksichtigung der Schülerlasten ist es gewesen, nur den Modus der Mittelverteilung innerhalb einer jeden Gruppe zu verändern, nicht aber Mittel zwischen den drei kommunalen Gruppen umzuschichten. ${ }^{3}$ Die Berücksichtigung dieses Sachverhalts ist für den kreisangehörigen Raum wichtig, da die Kreise über die Kreisumlage an der erhöhten Schlüsselmasse der Gemeinden partizipieren. Um die Gruppenneutralität der Maßnahme zu gewährleisten, ist deshalb vorab eine ausgleichende Umverteilung der Mittel von den Landkreisen zugunsten ihrer Gemeinden notwendig, wobei die Höhe wie folgt zu ermitteln ist (Tabelle 8).

Tabelle 8:Gruppenneutrale Mittelumschichtung im kreisangehörigen Raum

\begin{tabular}{|c|c|c|c|}
\hline Gebietskörperschaft & Finanzkraft & Sonderlastenausgleich & Gesamt \\
\hline kreisangehörige Gemeinden & $50-0,4 * 50=30$ & 5,00 & 35,00 \\
\hline Kreise; Umlagesatz 40 v.H. & $50 * 0,4+5=25$ & 10,00 & 35,00 \\
\hline
\end{tabular}

$+\Delta$ Schlüsselmasse: $55+\mathrm{y}-0,4 \cdot(55+\mathrm{y})=35 \Leftrightarrow \mathrm{y}=\frac{10}{3}$

$+\Delta$ Schlüsselmasse $=\frac{-(1-\text { Umlagesatz }) \cdot \text { anvisierte Brutto- }+ \text { Nettozuweisung }}{(1-\text { Umlagesatz })}$

In der Ausgangslage haben die Gemeinden eine Finanzkraft von 50, die sich durch die Kreisumlage bei einem durchschnittlichen Umlagesatz von 40 v.H. auf 30 vermindert, zusätzlich erhalten sie einen nicht der Umlage unterliegenden Sonderlastenausgleich in Höhe von 5. Die Landkreise besitzen eine Umlagekraft von 20 , erhalten Schlüsselzuweisungen von 5 und einen Sonderlastenausgleich von 10. Beiden Gruppen stehen somit Finanzmittel in Höhe von 35 zur Verfügung.

Haupt- und Schülernebenansatz werden addiert und mit dem Grundbetrag multipliziert.

2 Da die Schlüsselmasse nicht vollständig verteilt wird, differieren Gewinn und Verlust.

3 Vgl.: Hessischer Landtag: Gesetzentwurf der Landesregierung für ein Gesetz zur Änderung des Finanzausgleichsgesetzes und anderer Rechtsvorschriften - Finanzausgleichsänderungsgesetz 1988 - vom 25.11.1987, Drucksache 12/1178, S.17. 
Würde die Schlüsselmasse der Gemeinden nur um das Volumen des Sonderlastenausgleichs erhöht (5), so würden die Kreise entsprechend des Umlagesatzes an den Mehreinnahmen partizipieren und die für die Gemeinden angestrebte neue Zuweisungshöhe nicht erreicht werden. Bei der Erhöhung der Gemeindeschlüsselmasse ist dieser Zusammenhang zu berücksichtigen, damit trotz Kreisumlage der Status quo erhalten bleibt.

Allgemein gilt, daß der Erhöhungsbetrag für die Schlüsselmasse der Gemeinden errechnet wird, indem die Differenz zwischen erwünschter Nettozuweisung (hier 35) und der - mit einer vom Umlagesatz abhängigen Komponente (hier 0,6) gewichteten - Bruttozuweisung (hier 55) durch eben diese Komponente dividiert wird. Um diesen Betrag (hier 3,33) ist die Zuführung der Kreissonderlastenausgleichsmittel zur Kreisschlüsselmasse zu mindern und die Gemeindeschlüsselmasse entsprechend zu erhöhen.

\subsection{Verteilung der Schlüsselmasse zwischen den kommunalen Gruppen}

\subsubsection{Strukturelle Veränderungen der Schlüsselzuweisungen}

\subsubsection{Historische Entwicklung}

Einen Anspruch auf Schlüsselzuweisungen haben die kreisfreien Städte, die Landkreise und ihre kreisangehörigen Gemeinden sowie seit 1956 der LWV. Bei letzterem wurden besondere und allgemeine Zuweisungen im Jahr 1988 zusammengefaßt und als allgemeine Deckungsmittel ausgestaltet. ${ }^{1}$ Dies wurde mit der Vereinfachung des Zuweisungsverfahrens und einer Erhöhung der Transparenz bei den Landeszuweisungen an den LWV begründet. Gleichzeitig wurde eine finanzielle Mindestausstattung mit Hilfe von Mindestquoten - bezogen auf die Finanzausgleichsmasse bzw. die Steuerverbundmasse - erreicht. Diese betragen 4,8 v.H. der an die kommunale Ebene ausgeschütteten Zuweisungen; mindestens aber 2,7 v.H. der Steuerverbundmasse, so daß bei - wegen konjunktureller Entwicklungen - steigender Verbundmasse eine entsprechende Partizipation an den Mehreinnahmen ermöglicht wird. ${ }^{2}$ Es ist aber auch gesichert, daß die Einnahmen des LWV ebenfalls dann steigen, wenn etwa nur die besonderen Zuweisungen erhöht werden. ${ }^{3}$ Die Mittel werden der Finanzausgleichsmasse vorab entnommen. Für die übrigen drei Gebietskörperschaftsgruppen werden drei separate Schlüsselmassen in Form einer prozentualen Beteiligung an der verbliebenen Finanzmasse bestimmt.

1 Vgl.: Gesetz zur Änderung des Finanzausgleichsgesetzes und anderer Rechtsvorschriften Finanzausgleichsänderungsgesetz 1988 - vom 16.12.1987, Hess. GVBI. I, S. 226.

2 Vgl:: $§ 20$ Hess. Finanzausgleichsgesetz.

3 Vgl:: Hessischer Landtag: Gesetzentwurf der Landesregierung für ein Gesetz zur Änderung des Finanzausgleichsgesetzes und anderer Rechtsvorschriften - Finanzausgleichsänderungsgesetz 1988 - vom 25.11.1987, Drucksache 12/1178, S. 18. 
In den ersten Jahren des hessischen Finanzausgleichs gab es aufgrund der anstehenden Probleme der unmittelbaren Nachkriegszeit einen häufigen Wechsel beim Verteilungsmodus der allgemeinen Zuweisungen auf die Kommunen. Zuerst wurde an die Verteilungsregeln der Finanzausgleichsverordnung des Jahr 1944 angeknüpft, so daß den Gemeinden und GV Schlüsselzuweisungen in der damaligen Höhe zuflossen, um ihre Finanzkraft anzuheben und anzugleichen. Weiterhin standen ihnen noch zuerst das volle Soll, später nur noch prozentuale Anteile der Bürgersteuerausgleichsbeträge ${ }^{1} \mathrm{zu}$. Mit dem FAG von 1947 wurde geregelt, daß von den Landkreisen abgesehen - keine Schlüsselzuweisungen mehr nach der Verordnung des Jahres $1944 \mathrm{zu}$ gewähren sind; die entsprechende Begründung lautete: "aus Gründen der Verwaltungsvereinfachung erhalten die Gemeinden keine Schlüsselzuweisungen und leisten dafür keine Schulstellenbeiträge"2. Als weitere Begründung wurde hierfür einerseits die angespannte Haushaltssituation des Landes und andererseits die allgemein relativ gute Finanzsituation der kommunalen Ebene genannt, die es nicht rechtfertigte, jeder - also auch der finanziell besser gestellten - Gebietskörperschaft Schlüsselzuweisungen zu gewähren; den finanziell Schwächsten sollte über den (Landes-)Ausgleichsstock direkt geholfen werden. ${ }^{3}$ Für die Wahrnehmung ihrer eigenen und der ihnen zugewiesenen Aufgaben wurden 1947 und 1948 den Stadt- und Landkreisen jeweils 3 bzw. 2,5 RM pro Einwohner zugewiesen, den letzteren zusätzlich noch Zuweisungen in Höhe der Verordnung des Jahres 1944 ; die kreisangehörigen Gemeinden bekamen erst bei mehr als 5.000 Einwohnern einwohnerabhängige Kopfbeträge zwischen 1 und 1,5 RM. Die Landkreise erhielten als einzige Gruppe 1947 und 1948 Schlüsselzuweisungen. Allerdings wurde erst 1948 der veraltete Verteilungsschlüssel durch einen neuen ersetzt, wobei dann die Schlüsselmasse auf 10 Mio. RM und ein Mindestbetrag von 2 RM je Einwohner festgelegt wurden. ${ }^{5}$ Erst mit dem Finanzausgleichsgesetz von 1949 wurden dann wieder an alle kommunalen Gruppen Schlüsselzuweisungen nach einem Finanzkraft-Finanzbedarf-Ausgleich gezahlt.

Hierbei handelte es sich um die schon im Dritten Reich gezahlten Ausgleichsbeträge für die Abschaffung der kommunalen Personen- oder Bürgersteuer, die dem Volumen aus dem Jahr 1944 entsprachen. Vgl.: Deutscher Städtetag: Rückkehr zur verbundenen Steuerwirtschaft, Köln 1953, S. 5; Hessischer Landtag: Entwurf des Ministers der Finanzen für ein Gesetz zur Regelung des Finanzausgleichs für das Haushaltsjahr 1946 vom 09.03.1947, Drucksachen Abteilung I, 1/122, S. 157. Sie wurden 1951 abgeschafft und der entsprechende Betrag in die Schlüsselmasse eingestellt. Vgl.: Begründung zu dem Gesetz zur Regelung des Finanzausgleichs vom 17.07.1951, in: Staatsanzeiger für das Land Hessen, 1951, S. 521.

2 Hessischer Landtag: Entwurf eines Gesetz zur Regelung des Finanzausgleichs für das Haushaltsjahr 1947 vom 12.07.1947, Drucksachen Abteilung I, 1/340, S. 408.

3 Vgl.: Ebenda, S. 406f.

41948 erhielt jede kreisfreie Stadt einen spezifischen Kopfbetrag, der zwischen 3 RM für Marburg und 12 RM fur Hanau schwankte.

5 Vgl.: $\S \S 3,5$ und 6 Gesetz zur Regelung des Finanzausgleichs für das Haushaltsjahr 1947 vom 01.08.1947, Hess. GVBI. I, S 6; $\S \S 4$ und 5 Gesetz zur Regelung des Finanzausgleichs vom 10.06.1948, Hess. GVBI. I, S. 83. 
Für die kreisfreien Städte galt zwischen 1950 und 1972 eine Besonderheit. In diesem Zeitraum gab es eine einheitliche Schlüsselmasse für kreisangehörige $\mathrm{Ge}$ meinden und kreisfreie Städte, wie sie in den meisten Ländern heute noch besteht. Darüber hinaus partizipierten sie zuerst auch noch an der Kreisschlüsselmasse, die zwischen den beiden Gruppen nach der Einwohnerzahl aufgeteilt wurde. 1953 wurde der auf die kreisfreien Städte entfallende Betrag in eine separate dritte Schlüsselmasse eingestellt, da die Aufgaben- und Ausgabenentwicklung der beiden Gebietskörperschaftsgruppen nur noch in geringem Umfang mit der Einwohnerzahl korrelierte. ${ }^{1}$ Dieses Verfahren - Dotierung der kreisfreien Städte aus zwei Schlüsselmassen - erwies sich als umständlich und zeitaufwendig, wobei sich diese Probleme noch durch die Auswirkungen der Gebietsreform und hier vor allem die Bildung der Stadt Lahn verstärkt hätten. Zudem ist die angestrebte Minderung der Steuerkraftunterschiede im kreisangehörigen Raum nur dann realisierbar gewesen, wenn die kreisfreien Städte eine separate Schlüsselmasse erhalten, da ihre Werte stark von denen der Gemeinden abweichen. ${ }^{2}$ Deshalb wurde die ausschließlich den kreisfreien Städten vorbehaltene Schlüsselmasse um den auf sie entfallenden Betrag der einheitlichen Schlüsselmasse erhöht, so daß es nun für alle drei Gruppen separate Schlüsselmassen gibt.

\subsubsection{Separierung der drei kommunalen Gruppen}

Seit 1973 wurden die Anteile der drei Gebietskörperschaftsgruppen an der ihnen zustehenden Schlüsselmasse mehrmals verändert. Dabei dürfen allerdings nicht ausschließlich die jeweiligen Schlüsselmassen und ihr Volumen betrachtet werden. Denn aufgrund der Kreisumlagen gibt es einen engen Zusammenhang zwischen den Zuweisungen an die kreisangehörigen Gemeinden und die Landkreise. So führt eine Erhöhung der Schlüsselzuweisungen der kreisangehörigen Gemeinden c.p. automatisch zu einer Verbesserung der finanziellen Ausstattung der Kreise über gestiegene Einnahmen aus der Kreisumlage. Aufgrund der strukturellen Veränderung der drei Gebietskörperschaftsgruppen durch die Gebiets- und Verwaltungsreform werden nur die quotalen Änderungen seit 1981 berücksichtigt. Tabelle 9 ist zu entnehmen, daß im Zeitablauf der Anteil der kreisfreien Städte stetig von 12,75 v.H. 1981 auf 19,5 v.H. im Jahr 1999 erhöht wurde.

Vgl.: Hessischer Landtag: Vorlage der Landesregierung über den Entwurf eines Gesetzes zur Regelung des Finanzausgleichs vom 23.02.1953, Drucksachen Abteilung I, 2/588, S. 1146.

2 Vgl.: Ders.: Vorlage der Landesregierung betreffend den Entwurf für ein Sechstes Gesetz zur Änderung des Finanzausgleichsgesetzes vom 09.03.1971, Drucksache 7/151, S. 11; Sechstes Gesetz zur Änderung des Finanzausgleichsgesetzes vom 15.06.1971, Hess. GVBl. I, S. 154. 
Tabelle 9:Veränderungen der Anteile der drei Gebietskörperschaftsgruppen an den Schlüsselzuweisungen seit Ende der Gebietsreform ${ }^{1}$

\begin{tabular}{|c|c|c|c|c|}
\hline \multirow[t]{2}{*}{ Jahr } & \multirow{2}{*}{$\begin{array}{c}\text { Gesamtschlüsselmasse } \\
\text { (DM) }\end{array}$} & \multicolumn{2}{|c|}{ Kreisangehöriger Raum (v.H.) } & \multirow{2}{*}{$\begin{array}{c}\text { Kreisfreie Städte } \\
\text { (v.H.) }\end{array}$} \\
\hline & & Kreise & Gemeinden & \\
\hline 1981 & 1.346 .746 .000 & 42,21 & 45,03 & 12,75 \\
\hline 1984 & 1.546 .909 .000 & 44,62 & 41,70 & 13,68 \\
\hline 1985 & 1.711 .012 .000 & 43,35 & 40,45 & 16,20 \\
\hline 1988 & 1.976 .045 .000 & 44,80 & 38,50 & 16,70 \\
\hline 1992 & 2.343 .632 .000 & 44,60 & 37,50 & 17,90 \\
\hline 1993 & 2.594 .496 .000 & 42,40 & 39,50 & 17,90 \\
\hline 1997 & 2.716 .901 .000 & 40,40 & 41,20 & 18,40 \\
\hline 1998 & 2.611 .856 .000 & 38,20 & 42,80 & 19,00 \\
\hline 1999 & 2.843 .034 .000 & 36,00 & 44,50 & 19,50 \\
\hline
\end{tabular}

Quelle: Eigene Berechnungen nach: Finanzausgleichsabrechnung, in: Staatsanzeiger des Landes Hessen, jeweilige Jahre.

Begründet wird diese Verschiebung mit zwei Argumenten. Neben dem Ausgleich für die stark gestiegenen Sozialhilfeausgaben ${ }^{2}$ sollen teilweise auch die Verluste der kreisfreien Städte, die durch die Neufestlegung der Schlüsselzahlen für den kommunalen Einkommensteueranteil entstanden sind, kompensiert werden. ${ }^{3}$ Letzteres ist notwendig, da aufgrund der separaten Schlüsselmassen auf Gemeindeebene kein Ausgleich über den KFA erfolgt. ${ }^{4}$ Diese Trennung - die es so in keinem anderen westdeutschen Land mehr gibt - ruft ein gewichtiges Problem hervor. Sie führt dazu, daß der sonst bei einheitlicher gemeindlicher Schlüsselmasse automatisch gegebene Ausgleich der Einnahmeverluste bei der Einkommensteuer, der durch die Abwanderung von Einwohnern aus den Kernstädten (kreisfreie Städte) in das Umland (kreisangehörige Gemeinden) verursacht wird, ausbleibt. ${ }^{5}$

1 Die Rückkreisung kreisfreier Städte macht einen Vergleich erst nach Abschluß der Gebietsreform möglich. Seit 1988 erhält der LWV keine Schlüsselzuweisungen mehr. Zur besseren Vergleichbarkeit wurde fur die vorangegangenen Jahre eine Bereinigung um seine Anteile vorgenommen und die Gesamtschlüsselmasse entsprechend verringert.

2 Vgl.: Ders.: Gesetzentwurf der Landesregierung für ein Gesetz zur Änderung des Finanzausgleichsgesetzes vom 21.10.1996, Drucksache 14/2259, S. 7. Diese Kosten werden aber schon im Sozialhilfelastenausgleich berücksichtigt.

3 Vgl:: Ders.: Gesetzentwurf der Landesregierung für ein Gesetz zur Änderung des Finanzausgleichsgesetzes - Finanzausgleichsänderungsgesetz 1985 vom 29.10.1984, Drucksache 11/2228, S. 5; ders.: Gesetzentwurf der Landesregierung fur ein Gesetz zur Änderung des Finanzausgleichsgesetzes und anderer Rechtsvorschriften - Finanzausgleichsänderungsgesetz 1992 - vom 01.11.1991, Drucksache 13/841, S. 7, 10 f.

4 Vgl.: Ders.: Gesetzentwurf der Landesregierung für ein Gesetz zur Änderung des Finanzausgleichsgesetzes vom 21.10.1996, Drucksache 14/2259, S. 7-9.

$5 \mathrm{Vgl}$.: Recker, E.: Räumliche Verteilung des Gemeindeanteils an der Einkommensteuer, in: 
Es kommt somit nur zu Umverteilungen innerhalb der jeweiligen Gruppe. Verliert im Zeitablauf immer die gleiche Gruppe (z.B. die kreisfreien Städte), so ist eine diskretionäre Anpassung der Schlüsselmassen notwendig. ${ }^{1}$ Die Verschiebung innerhalb des kreisangehörigen Raums zugunsten der Gemeinden dient zur Kompensation für die - durch exaktere und damit auch höhere Erfassung der gemeindlichen Steuerkraft - steigenden Belastungen durch die Kreisumlage.

\subsubsection{Dotierung der Teilschlüsselmassen - Analyse und Reform}

Bei der Verteilung der Verbundmasse auf die drei kommunalen Gruppen sind für Hessen mehrere Aspekte zu beachten. Die Separierung der Schlüsselmassen für die kreisfreien Städte und kreisangehörigen Gemeinden führt zum Problem der fehlenden automatischen Umverteilung vom kreisangehörigen zum kreisfreien Raum. Dies wird andererseits auch positiv gesehen, da horizontale (intergemeindliche) nicht mit vertikalen (Kreis-Gemeinde-Ebene) Verteilungswirkungen vermischt werden. ${ }^{2}$ Zudem können als Begründung für drei separate Schlüsselmassen noch weitere Vorteile angeführt werden. Zum einen kann somit die Spreizung bei der Hauptansatzstaffelung geringer ausfallen, was aus finanzpsychologischen Gründen wichtig ist, da die Dimension der Spreizung häufig kritisiert wird. ${ }^{3}$ Zudem dürften sich bei der Bestimmung dieser Werte geringere Probleme ergeben, da die Unterschiede bei den Aufgaben und somit auch der Bedarfsstruktur innerhalb der Klasse der kreisfreien bzw. kreisangehörigen Gemeinden geringer sind als zwischen den beiden Gruppen. In diesem Zusammenhang hat auch der Verfassungsgerichtshof in NRW dem Gesetzgeber den Auftrag gegeben, zu prüfen, "ob die Gleichstellung kreisfreier und kreisangehöriger Gemeinden bei der Bemessung des Finanzbedarfs (...) vertretbar ist" ${ }^{\prime 4}$. Außerdem behält somit die Regierung weiterhin die Möglichkeit, ohne große Mühe diskretionär in die Finanzverteilung der beiden Gemeindegruppen einzugreifen. Denn eine Umverteilung zwischen diesen beiden bzw. allen drei Schlüsselmassen ist leichter und mit weniger Aufwand zu realisieren als eine völlige Überarbeitung aller Hauptansatzwerte.

Räumliche Aspekte des kommunalen Finanzausgleichs (Veröffentlichungen der Akademie für Raumforschung und Landesplanung: Forschungs- und Sitzungsberichte, Bd. 159), Hannover 1985 , S. 346.

1 Vgl.: Karrenberg, H.; Münstermann, E.: Der Gemeindefinanzbericht 1997, in: Der Städtetag, Heft 3, 1997, S. 156.

2 Vgl: Henneke, H.-G.: Die Landkreise im kommunalen Finanzausgleich 1997, in: Der Landkreis, 67. Jg., 1997, S. 126.

3 Vgl.: Steinherr, M.; u.a.: Das Schlüsselzuweisungssystem im kommunalen Finanzausgleich des Freistaates Sachsen, ifo-Studien zur Finanzpolitik, Bd. 63, München 1997, S. 75f. Es könnten zwar auch durch die Hervorhebung der kreisangehörigen Gemeinden über 50.000 Einwohner - Sonderstatusstädte - Probleme induziert werden, doch dürften diese geringer sein, als bei der Einbeziehung der kreisfreien Städte.

4 Verfassungsgerichtshof für das Land Nordrhein-Westfalen: Urteil vom 06.07.1993, in: OVGE, Bd. 43, Münster 1996, S. 260. 
Aus den genannten Gründen sollten deshalb die getrennten Schlüsselmassen beibehalten werden. Um aber im Zeitablauf einen finanziellen Ausgleich zwischen den drei Gruppen zu sichern, bietet sich als Lösungsansatz in Hessen die für Sachsen vorgeschlagene Regelung an. Dort soll der Gleichmäßigkeitsgrundsatz nicht nur für die Aufteilung der vorhandenen Mittel zwischen Land und Kommunen Geltung haben, sondern auch zwischen den drei kommunalen Gebietskörperschaftsgruppen. Durch eine solche Vorgehensweise würde das Verteilungssystem auf die drei kommunalen Gruppen transparenter werden, da offengelegt werden $\mathrm{mu}$, inwieweit es zu einer Abweichung vom ursprünglichen Aufteilungsverhältnis gekommen ist, ob sich also die Finanzkraft und/oder die Bedarfssituation verändert hat. ${ }^{1}$ Falls aber keine Aufgabenverschiebung zwischen dem kreisfreien und dem kreisangehörigen Raum eintritt, kann mit Hilfe des Gleichmäßigkeitsgrundsatzes die Dotierung der Teilschlüsselmassen an sich verändernde Entwicklungen bei den Steuereinnahmen, wie sie regelmäßig nach der Neufestlegung der Schlüsselzahlen und des Sockelbetrages zur Verteilung des Einkommensteueranteils kommt, automatisch da regelgebunden angepaßt werden. Ein Konflikt zwischen den verschiedenen kommunalen Gruppen kann somit vermieden werden. Zu klären ist in diesem Fall nur einmal, nämlich bei der erstmaligen Aufteilung der Finanzmittel auf die drei Gruppen, ob diese als adäquat angesehen werden kann und somit als zu konservierende Ausgangsverteilung gelten soll. Andernfalls käme es bei jeder Verschiebung der Einnahmenanteile zu Verteilungskonflikten.

\subsubsection{Vergleich kreisangehöriger und kreisfreier Raum}

Zur Beurteilung der finanziellen Ausstattung werden Landkreise und kreisangehörige Gemeinden als eine Gruppe betrachtet und mit kreisfreien Städten verglichen. In Hessen gibt es seit Ende der Gebietsreform fünf kreisfreie Städte und 21 Landkreise. 1998 erhalten die kreisfreien Städte, in denen mit ca. 1,37 Mio. Einwohnern etwa 22,87 v.H. der hessischen Bevölkerung leben, 19,0 v.H. der Schlüsselzuweisungen und 1999 19,5 v.H.; ab dem Jahr 2000 ist ein Anteil von 20,1 v.H. vorgesehen. Die kreisfreien Städte fordern allerdings eine Beteiligung entsprechend der tatsächlichen Quote an der Gesamtbevölkerung des Landes. ${ }^{2}$

Diese Forderung wird damit begründet, da $\beta$ die kreisfreien Städte als zentrale Orte Leistungen für das Umland zur Verfügung stellen, die, da nicht durch Zahlungen des empfangenden Umlands entgolten, im Rahmen des KFA zu berücksichtigen sind. ${ }^{3}$ Entsprechend ist auch die Formulierung im FAG, wonach "die zentralörtlichen Funktionen der kreisfreien Städte (...) bei der Verwendung der

1 Vgl.: Steinherr, M.; u.a.: Das Schlüsselzuweisungssystem im kommunalen Finanzausgleich des Freistaates Sachsen, ifo-Studien zur Finanzpolitik, Bd. 63, München 1997, S. 76-78.

2 Vgl: Karrenberg, H.; Münstermann, E.: Der Gemeindefinanzbericht 1997, in: Der Städtetag, 50. Jg., 1997, S. 156.

3 Nach den regionalen Raumordnungsplänen sind alle fünf kreisfreien Städte Hessens Oberzentren. 
Gesamtschlüsselmasse"1, also ihre Aufteilung auf die drei Gruppen, berücksichtigt werden. Ihr können aber die bedarfssteigernden Besonderheiten des kreisangehörigen Raums entgegengehalten werden, daß nämlich "im Gegensatz zum kreisfreien Raum die Verwaltungsorganisation im kreisangehörigen Raum zwei selbständige Ebenen umfaßt, die einen höheren Aufwand bedingt". Zudem sind "teilweise höhere Kosten, die sich in den Kreisen bei gleichgearteten Aufgaben aufgrund der gegenüber den kreisfreien Städten unterschiedlichen Raumverhältnisse - insbesondere der regelmäßig großräumigeren Flächen bei geringerer Einwohnerdichte - ergeben können"2 zu berücksichtigen.

Somit sind vor Betrachtung der Finanzkraftverteilung kaum Aussagen darüber möglich, ob die Dotierung der Schlüsselmasse des kreisfreien im Vergleich zum kreisangehörigen Raum als angemessen anzusehen ist. Deshalb wird die Steuerkraft der beiden Gruppen - Gesamtkreis und kreisfreie Städte - vor mit der Finanzkraft nach Finanzausgleich verglichen. Die Finanzkraft des Gesamtkreises ergibt sich dabei aus der Steuerkraft der Gemeinden, deren Schlüsselzuweisungen sowie denjenigen der Kreise. ${ }^{3}$ Dieses Verfahren wird im rheinland-pfälzischen KFA angewendet, um eine Gleichstellung des kreisangehörigen und des kreisfreien Raumes zu sichern. Abbildung 10 zeigt die Finanzkraft der beiden Räume vor und nach KFA.

\section{Abbildung 10:Finanzkraftvergleich von kreisfreiem und kreisangehörigem Raum}

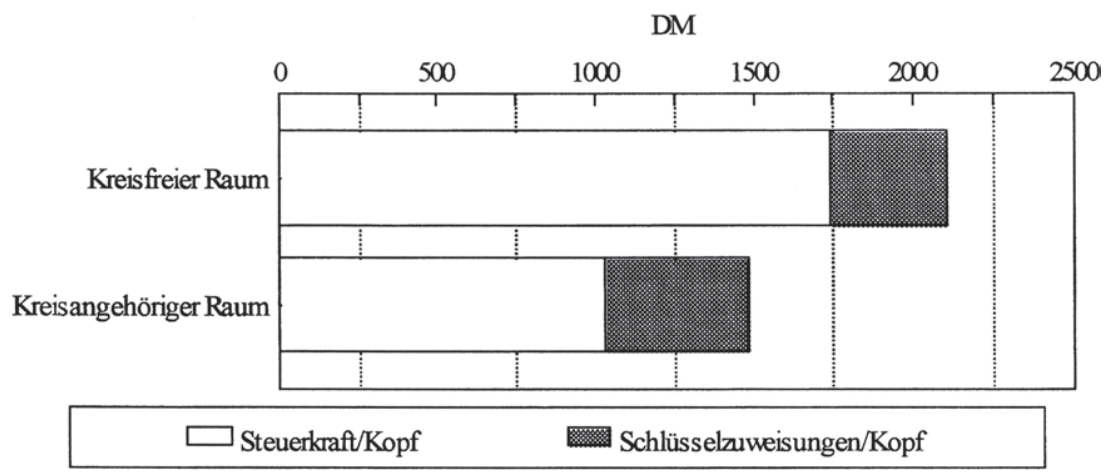

Quelle: Eigene Berechnung nach Daten des Finanzministeriums zum KFA 1998.

1 Gesetz zur Regelung des Finanzausgleichs (Finanzausgleichsgesetz - FAG -) in der jeweils gültigen Fassung.

2 Verfassungsgerichtshof für das Land Nordrhein-Westfalen: Urteil vom 06.07.1993, in: OVGE, Bd. 43, Münster 1996, S. $259 f$.

3 Prinzipiell kann auf die Betrachtung des Finanzbedarfs verzichtet werden, da die Aufgabenerfullung in beiden Gruppen als gleichwertig anzusehen ist. 
Im kreisfreien Raum beträgt die im KFA berücksichtigte Steuerkraft pro Kopf 1.741 DM gegenüber nur 1.031 DM für den Gesamtkreis. Trotz dieser Unterschiede bei der originären Steuerkraft - der kreisangehörige Raum erzielt gerade einmal 59,2 v.H. der Steuerkraft der kreisfreien Städte - erhalten letztere noch 79,7 v.H. der Pro-Kopf-Schlüsselzuweisungen der Gesamtkreise, nämlich 362 DM im Vergleich zu 454 DM. Nach den Schlüsselzuweisungen erreicht der kreisangehörige somit 70,6 v.H. des Niveaus des kreisfreien Raumes.

Ein Vergleich ist auch derart möglich, daß kreisangehörige Gemeinden fiktiv den Status einer kreisfreien Stadt erhalten. Dadurch kann berücksichtigt werden, daß die fünf kreisfreien Städte sowohl Gemeinde- als auch Kreisaufgaben wahrnehmen und ihnen somit auch erhöhte Zuweisungen und ein größerer Finanzbedarf zugestanden werden muß. $\mathrm{Zu}$ diesem Vergleich werden die kreisangehörigen Oberzentren herangezogen, da sie die gleichen Gemeindeaufgaben zu erfüllen haben wie die kreisfreien Städte. Die Übernahme der Kreisaufgaben wird dadurch entgolten, daß sie die Verbundmasse der Kreise zugeschlüsselt bekommen, wodurch eine theoretische Finanzkraft bei den kreisangehörigen Oberzentren - bestehend aus Gemeindeschlüsselzuweisungen, gemeindlicher Steuerkraft und anteiligen Kreisschlüsselzuweisungen - gewonnen werden kann. ${ }^{1}$ Abbildung 11 zeigt das Ergebnis dieser Überlegung, indem die kleineren kreisfreien (Darmstadt und Offenbach) mit den kreisangehörigen (Fulda, Gießen und Marburg) Oberzentren verglichen werden.

\section{Abbildung 11:Finanzkraft kreisfreier und kreisangehöriger Oberzentren}

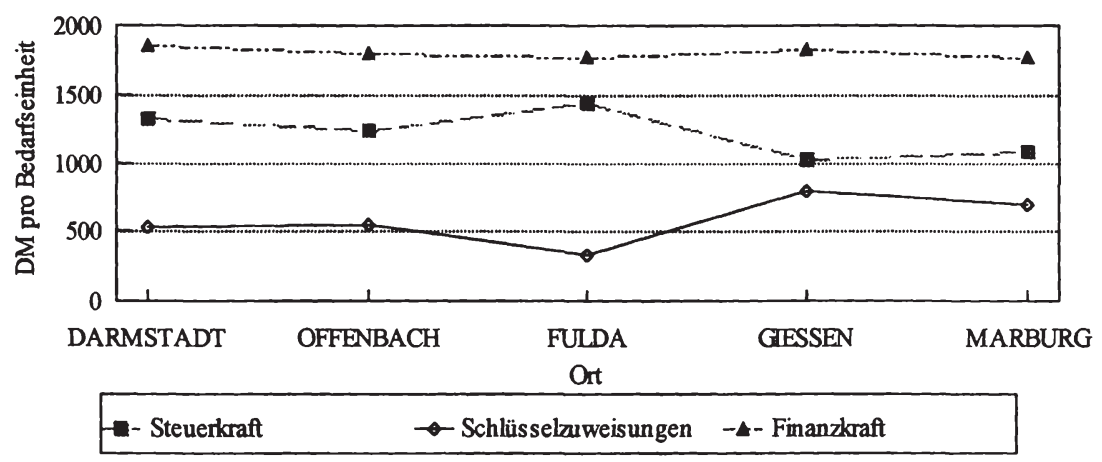

Quelle: Eigene Berechnung nach Daten des Finanzministeriums zum KFA 1998.

Die Summe der Schlüsselzuweisungen der kreisangehörigen Gemeinden aus der Kreisschlüsselmasse wird durch Multiplikation des Anteilswertes an der Gemeinde- mit der Kreisschlüsselmasse ermittelt. Es wird also eine gleiche Relation unterstellt. Ähnlich geht STENHERR bei der Beurteilung des KFA in Sachsen vor. Vgl.: Steinherr, M.; u.a.: Das Schlüsselzuweisungssystem im kommunalen Finanzausgleich des Freistaates Sachsen, ifoStudien zur Finanzpolitik, Bd. 63, München 1997, S. 84. 
Es zeigt sich, daß die Finanzkraft der kreisfreien Oberzentren relativ stärker angehoben wird, als dies bei den kreisangehörigen Oberzentren der Fall ist, obwohl letzteren die Kreisschlüsselzuweisungen angerechnet werden und ihnen zusätzliche Schlüsselzuweisungen aufgrund des Sonderstatusstadtansatzes zuflieBen. ${ }^{1}$ Darmstadt und Offenbach, mit einer Steuerkraft von 91,92 bzw. 86,34 v.H. der Steuerkraft von Fulda, erhalten 159,49 bzw. 164,25 v.H. v.H. der Schlüsselzuweisungen dieser Stadt. Dies führt zur unerwünschten Nivellierung, da Darmstadt und Offenbach nach Zuteilung der Schlüsselzuweisungen über eine höhere Finanzkraft verfügen als der steuerstärkste Ort Fulda. ${ }^{2}$ Weiterhin ist noch zu bedenken, daß die beiden genannten kreisfreien Städte sich im Rhein-MainBallungsgebiet befinden, sich selbst gegenseitig bei der zentralörtlichen Aufgabenerfüllung entlasten können und auch noch durch die Oberzentren Frankfurt und Wiesbaden unterstützt werden, was bei den drei kreisangehörigen Oberzentren nicht der Fall ist. So stellt Fulda für Osthessen das einzige Oberzentrum dar, und auch Gießen und Marburg üben als einzige Gemeinden oberzentale Aufgaben im Regierungsbezirk Gießen aus. Auch hier deutet nichts auf eine zu geringe Dotierung der Schlüsselmasse der kreisfreien Städte hin.

Bei Betrachtung der 26 einzelnen Gebietskörperschaften der beiden Gruppen (siehe Abbildung 12) zeigt sich, daß die ersten beiden Positionen bei einer ProKopf-Betrachtung sowohl vor als auch nach Verteilung der Schlüsselzuweisungen von den Städten Frankfurt und Wiesbaden gehalten werden. Fünf Gesamtkreise weisen eine höhere Steuerkraft auf als die "ärmste" kreisfreie Stadt, nämlich Kassel, doch ändert sich diese Reihenfolge nach KFA. Die Stadt Kassel rückt von Position zehn auf Rang vier vor. Es kommt somit teilweise durch die Schlüsselzuweisungen zur Übernivellierung. Bei Interpretation dieses Sachverhalts ist zwar zu berücksichtigen, daß die Pro-Kopf-Betrachtung nur ein Hilfsmaßstab ist, da die Bedarfssysteme der jeweiligen Gruppen untereinander nicht kompatibel sind und deshalb die Einnahmen-je-Bedarfseinheit als Indikator ausscheiden. ${ }^{3}$ Doch können die ermittelten Werte einen Anhaltspunkt für die Beurteilung der Dotierung des kreisfreien im Vergleich zum kreisangehörigen Raum geben, zumal der Finanzbedarf für beide Gruppen wegen der Aufgabenerfüllung etwa gleich gro $\beta$

Als Bezugsgröße wird der Gesamtansatz der Orte genommen. Es werden ausschließlich die bedarfssteigernden Merkmale der kreisfreien Städte verwendet. Als Veredelungsfaktor werden wie bei Darmstadt und Offenbach 100 v.H. angesetzt.

2 Auch Gießen weist eine höhere Finanzkraft als Fulda auf. Dies ist auf die hohen Schlüsselzuweisungen des Landkreises Gießen zurückzuführen, die anteilig der Stadt Gießen zugerechnet werden.

3 Bei gruppeninterner Betrachtung treten andere Ergebnisse auf, da dann der die Einnahmenje-Bedarfseinheit als Indikator für die Finanzkraft verwendet werden können. So gibt es z.B. keine Änderung der Finanzkraftreihenfolge zwischen den Städten Offenbach und Kassel, wenn als Vergleichsmaßstab die gruppeninterne Bedarfsmeßzahl bzw. der Gesamtansatz statt einer reinen Pro-Kopf-Betrachtung verwendet wird. 
ist. Aus diesen Daten kann also nicht abgeleitet werden, daß die Gruppe der kreisfreien Städte im Vergleich zum kreisangehörigen Raum eine viel zu gering dotierte Schlüsselmasse erhält. Folglich wäre die geplante Erhöhung des Anteils der kreisfreien Städte zu überdenken.

\section{Abbildung 12:Finanzkraftvergleich einzelner Gebietskörperschaften}

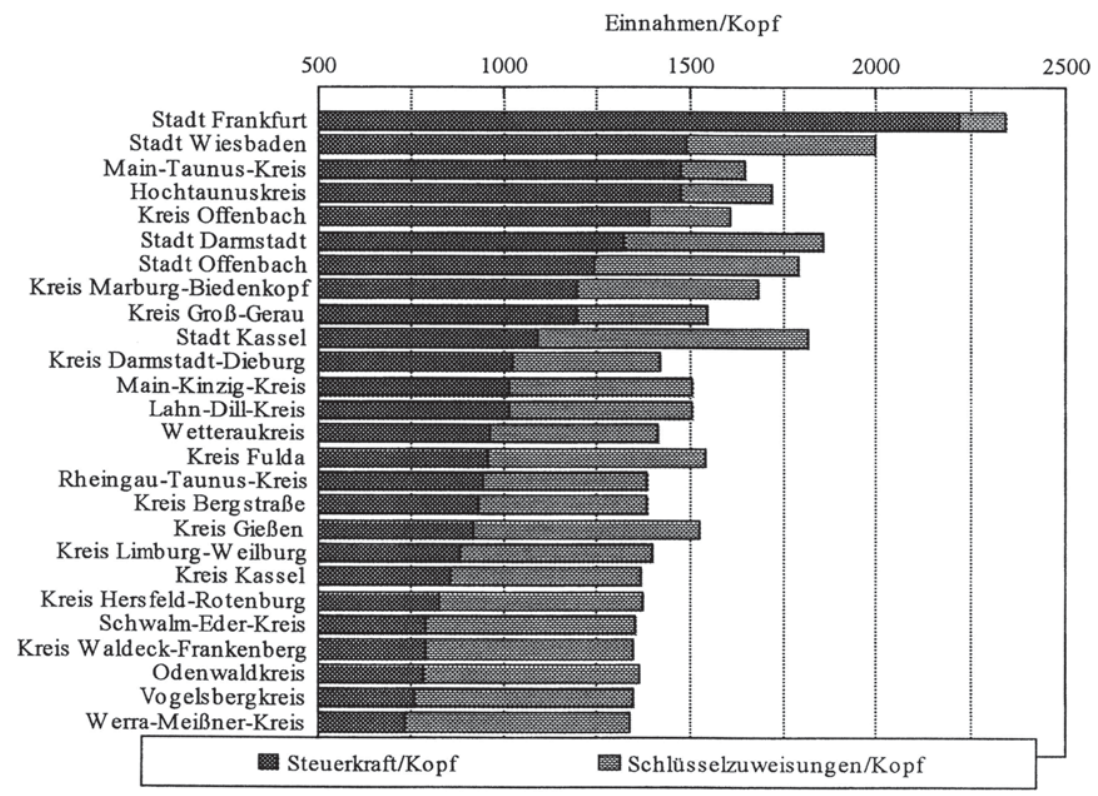

Quelle: Eigene Berechnung nach Daten des Finanzministeriums zum KFA 1998.

\subsubsection{Vergleich kreisangehörige Gemeinden und Landkreise}

Nachdem im vorherigen Abschnitt die Verteilung der Schlüsselmasse zwischen kreisfreiem und kreisangehörigen Raum im Mittelpunkt stand, stellt sich nun die Frage, wie die Aufteilung der dem Gesamtkreis zur Verfügung stehenden Schlüsselmasse auf den Landkreis und seine kreisangehörigen Gemeinden vollzogen werden soll. Es handelt sich hier aber nur um ein eher nachrangiges Problem, denn durch die Kreisumlage besteht die Möglichkeit, die Einnahmenverteilung zwischen den beiden Gruppen zu beeinflussen. Ist die Schlüsselmasse der Landkreise zu gering, so wird die Kreisumlage entsprechend stark angespannt und umgekehrt, so daß eine als notwendig angesehene Mittelverteilung zwischen den beiden Gruppen immer erreicht werden kann. ${ }^{1}$ Allerdings kommt es in

1 Vgl.: Verfassungsgerichtshof Rheinland-Pfalz: Entscheidung vom 30.01.1998, in: DÖV, 51. 
Abhängigkeit von der volumenmäßigen Dotierung der Schlüsselmassen zu Umverteilungseffekten innerhalb der Gruppe der kreisangehörigen Gemeinden. Ist die Finanzierung der Kreise über die Schlüsselzuweisungen sehr hoch, so müssen die Umlagesätze nur noch gering angespannt werden, was bei einem kreiseinheitlichen Umlagesatz und bei Verzicht auf Splitting und Progression zu einer proportionalen Verringerung der Steuerkraft führt. ${ }^{1}$ Bei einer geringeren Kreisschlüsselmasse müssen sich die Landkreise im stärkeren Umfang über die Umlage finanzieren. Entsprechend steigt der kreiseinheitliche Satz an. Durch die gestiegene Gemeindeschlüsselmasse werden nun die kreisinternen Finanzkraftdifferenzen stärker gemindert.

Mit Hilfe von Tabelle 10 sollen nun beispielhaft anhand eines Landkreises und zweier Gemeinden die Verteilungswirkungen dargelegt werden. In der Ausgangssituation A ergibt sich aufgrund der Dotierung der beiden Schlüsselmassen - nämlich $200 \mathrm{DM}$ für den Landkreis und 400 DM für die Gemeinden - bei allen drei Gebietskörperschaften ein Finanzbedarf von 1.200 DM. Die Relation der Steuerkraft von 60 v.H. zwischen Ort 2 und Ort 1 wird durch die Schlüsselzuweisungen auf 81,82 v.H. vermindert. Diese wird auch nicht mehr durch die Kreisumlage verändert. Es wird dabei unterstellt, daß der zur Berechnung der Kreisumlagekraft verwendete landesdurchschnittliche Umlagesatz von 40 v.H. auch im betrachteten Kreis gilt. In der Situation B wurde nun die zur Verfügung stehende Schlüsselmasse von $600 \mathrm{DM}$ hälftig auf die beiden Gruppen verteilt. Dies führt zu einer Verringerung des Grundbetrags und somit auch des Finanzbedarfs bei den Gemeinden und umgekehrt beim Landkreis. Da sich die finanzielle Ausstattung des Kreises durch die Schlüsselzuweisungen zur Ausgangssituation relativ verbessert hat, kann der Umlagesatz auf 36,84 v.H. gemindert werden. Entsprechend werden die Steuerkraftunterschiede der Orte nun nur noch in geringerem Umfang ausgeglichen, da sowohl die finanzielle Aufstockung durch die Schlüsselzuweisungen als auch die Abschöpfung der Finanzkraft durch die Kreisumlage geringer sind. Der Ort 2, der vor KFA nur 60 v.H. der Steuerkraft des Ortes 1 hatte, erreicht nun 80,95 v.H. statt 81,82 wie in Situation A. Als Ergebnis zeigt sich, daß unabhängig vom Volumen der Schlüsselmassen eine gewünschte Verteilung zwischen den beiden Gebietskörperschaftsgruppen im kreisangehörigen Raum über die Kreisumlage möglich ist, denn in beiden Fällen liegt nach der Kreisumlage die Finanzkraft des Kreises bei 1.000 und die der Gemeinden zusammen bei 1.200. Es treten aber sehr wohl Umverteilungseffekte innerhalb der Gruppe der kreisangehörigen Gemeinden auf.

Jg., 1998, S. 507.

1 Mit Splitting wird die Möglichkeit bezeichnet, für einzelne Umlagegrundlagen separate Umlagesätze zu bestimmen. Die Progression erlaubt es, für steuerstarke (abundante) Gemeinden die Umlagegrundlagen oder die Umlagesätze zu erhöhen. Beide Verfahren sind im Hessischen FAG nicht (mehr) vorgesehen. 
Tabelle 10:Einfluß der Kreisumlage auf die kreisinterne Finanzkraftrelation

\begin{tabular}{|l|r|r|r|r|}
\hline \multirow{2}{*}{ Situation A: } & \multicolumn{3}{|c|}{ Gebietskörperschaften } & Relation Ort \\
\cline { 2 - 4 } & Landkreis & Ort 1 & \multicolumn{1}{c|}{ Ort 2 } & 2u Ort 1 \\
\hline Steuer-/Umlagekraft & 800,00 & $1.000,00$ & 600,00 & 60,00 v.H. \\
\hline Finanzbedarf & $1.200,00$ & $1.200,00$ & $1.200,00$ & \\
\hline Schlüsselzuweisung & 200,00 & 100,00 & 300,00 & 81,82 v.H. \\
\hline Finanzkraft & $1.000,00$ & $1.100,00$ & 900,00 & \\
\hline Kreisumlage; Umlagesatz 40 v.H. & 800,00 & 440,00 & 360,00 & \\
\hline Finanzkraft nach Kreisumlage & $1.000,00$ & 660,00 & 540,00 & 81,82 v.H. \\
\hline Situation B: & \multicolumn{3}{|l|}{} \\
\hline Steuer-/Umlagekraft & 760,00 & $1.000,00$ & 600,00 & 60,00 v.H. \\
\hline Finanzbedarf & $1.360,00$ & $1.100,00$ & $1.100,00$ & \\
\hline Schlüsselzuweisung & 300,00 & 50,00 & 250,00 & \\
\hline Finanzkraft & $1.000,00$ & $1.050,00$ & 850,00 & 80,95 v.H. \\
\hline Kreisumlage; Umlagesatz 36,84 v.H. & 700,00 & 386,84 & 313,16 & \\
\hline Finanzkraft nach Kreisumlage & $1.000,00$ & 663,16 & 536,84 & 80,95 v.H. \\
\hline
\end{tabular}

Quelle: Eigene Berechnung in Anlehnung an: Scherf, W.: Schlüsselzuweisungen und Kreisumlage, Frankfurt u.a.O. 1998, S. 50.

Der Vorteil einer relativ gering dotierten Kreisschlüsselmasse liegt somit darin, daß die Angleichung der Gemeindefinanzkraft innerhalb des Kreises verstärkt wird. Das gestiegene Niveau der Umlagesätze kann aber dazu führen, daß das schon jetzt angespannte Verhältnis zwischen Kreisen und Gemeinden sich weiter verschlechtert. Zudem wird die Minderung der Finanzkraftunterschiede auf der Kreisebene, welche hier nicht betrachtet wird, aufgrund der geringeren Mittel eingeschränkt. Andererseits wird bei höheren Umlagesätzen die Bedarfssituation beider Gruppen genauer untersucht, als wenn den Kreisen schon vorab eine überdimensionierte Schlüsselmasse zugeteilt wurde. ${ }^{1}$ Somit kann über die Aufteilung der dem kreisangehörigen Raum zur Verfügung stehenden Schlüsselmasse keine eindeutige Aussage getroffen werden.

\subsection{Verteilung der Schlüsselmasse innerhalb der kommunalen Gruppen}

\subsubsection{Allgemeine Grundlagen der Bedarfsermittlung}

Im Rahmen des KFA sollen die Unterschiede zwischen Finanzkraft und -bedarf teilweise ausgeglichen werden. Um aber zu verhindern, daß die Gemeinden durch selbstverantwortliche expansive Ausgabepolitik einen erhöhten Finanzbedarf

1 Vgl.: Scherf, W.: Schlüsselzuweisungen und Kreisumlage, Frankfurt u.a.O. 1998, S. 85f. 
geltend machen können, der im Rahmen des KFA durch Zuweisungen auszugleichen ist, wird dieser normiert. ${ }^{1}$ Der Finanzbedarf wird als Bedarfsmeßzahl bezeichnet und ist keine exogen vorgegebene Größe, sondern ergibt sich aus der modifizierten Einwohnerzahl und einem von der Schlüsselmasse abhängigen Pro-Kopf-Bedarf, dem sog. Grundbetrag. Als Einwohnerzahl wird allerdings nicht die Wohnbevölkerung herangezogen, sondern ein modifizierter Wert, der als Gesamtansatz (Gesamtansatz = rechnerische Einwohnerzahl) bezeichnet wird. Dieser Gesamtansatz setzt sich wiederum aus einem Hauptansatz und Neben- oder Ergänzungsansätzen zusammen, auf die im folgenden eingegangen wird. Der Bedarf wird nun durch Multiplikation des Gesamtansatzes mit dem Grundbetrag ermittelt.

\subsubsection{Funktion des Hauptansatzes}

Beim Finanzbedarf der Gemeinden wird angenommen, daß dieser mehr oder minder von einwohnerbezogenen Größen abhängig ist, da der größte Teil der öffentlichen Leistungen den Einwohnern zugute kommt. Entsprechend dem BRECHTschen Gesetz wird dabei unterstellt, daß "sich die Ausgaben auf den Kopf der Bevölkerung stark steigern mit der größeren Bevölkerungsmassierung"2. Dieser von BRECHT empirisch belegte Zusammenhang der beiden Größen Bevölkerungsdichte und Pro-Kopf-Ausgaben läßt sich auch heute noch für die Gemeinden feststellen. ${ }^{3}$ Aus diesem Ergebnis wurde dann durch PopITZ ein Verfahren zur Bedarfsermittlung auf der kommunalen Ebene erstellt, nach dem den Gemeinden ein mit der Einwohnerzahl wachsender Pro-Kopf-Bedarf zugestanden wird. ${ }^{4}$

$\mathrm{Da}$ es problematisch ist, für jeden Ort einen solchen Faktor zu ermitteln, werden Gemeinden in Größenklassen eingeteilt, für die jeweils ein entsprechender Gewichtungsfaktor - Hauptansatz in v.H. - ermittelt wird. ${ }^{5}$ Das Produkt aus tatsächlicher Einwohnerzahl und dem Gewichtungsfaktor wird als Hauptansatz (absolute Größe) bezeichnet. Dieser gibt die für den KFA relevante "tatsächliche" Einwohnerzahl wieder. Letztere wird in Deutschland auf zwei Arten ermittelt, deren Unterschiede an einem Beispiel verdeutlicht werden sollen (Tabelle 11). Die GröBenklassen betragen bis und über 5.000 Einwohner. Die dazugehörigen Hauptansatzwerte lauten 107 v.H. und 114 v.H. Für drei Gemeinden (A, B und C) mit 4.900, 5.100 und 9.500 Einwohnern soll der Hauptansatz ermittelt

1 Vgl.: Kirchhof, P.: Rechtliche Rahmenbedingungen des kommunalen Finanzausgleichs, in: Hoppe, W. (Hrsg.): Reform des kommunalen Finanzausgleichs, Köln u.a.O. 1985, S. 12.

2 Brecht, A.: Internationaler Vergleich der öffentlichen Ausgaben, Leipzig - Berlin 1932, S. 6.

3 Vgl.: Deutscher Städtetag (Hrsg.): Statistisches Jahrbuch Deutscher Gemeinden, 85. Jg., Köln 1998, S. $394 \mathrm{f}$ und $428 \mathrm{f}$.

4 Vgl.: Popitz, J.: Der künftige Finanzausgleich zwischen Reich, Ländern und Gemeinden; Gutachten, erstattet der Studiengesellschaft für den Finanzausgleich, Berlin 1932, S. 266.

5 Die Differenz zwischen den gemeindegruppenspezifischen Veredelungsfaktoren wird als Spreizung der Hauptansatzstaffel bezeichnet. 
werden. ${ }^{1}$ Entweder werden alle Gemeinden einer Größenklasse mit dem gleichen Faktor veredelt, was zu Sprüngen zwischen den Klassen führt, oder es wird, um dies zu verhindern, für jede Gemeinde ein individueller Hauptansatz mit Hilfe einer lineare Interpolation errechnet. ${ }^{2} \mathrm{Zu}$ diesem Zweck wird die Differenz sowohl der beiden aufeinander folgenden Größenklassen als auch der beiden - ihnen entsprechenden - Faktoren gebildet, durcheinander dividiert und mit der oberhalb der unteren Klassengrenze liegenden Einwohnerzahl des Ortes multipliziert.

Tabelle 11:Unterschiede bei der Hauptansatzermittlung

\begin{tabular}{|c|c|c|c|}
\hline \multirow{2}{*}{ Ort } & \multirow{2}{*}{$\begin{array}{c}\text { Ein- } \\
\text { wohner }\end{array}$} & \multicolumn{2}{|c|}{ Hauptansatz in v.H.; mit/ohne lineare Interpolation } \\
\cline { 3 - 4 } & ohne & mit \\
\hline $\mathrm{A}$ & 4.900 & 107 & $100+\frac{107-100}{5.000-2.500} \bullet(4.900-2.500)=106,72$ \\
\hline $\mathrm{B}$ & 5.100 & 114 & $107+\frac{114-107}{10.000-5.000} \bullet(5.100-5.000)=107,14$ \\
\hline $\mathrm{C}$ & 9.500 & 114 & $107+\frac{114-107}{10.000-5.000} \bullet(9.500-5.000)=113,30$ \\
\hline
\end{tabular}

Quelle: Eigene Darstellung in Anlehnung an: Haverkamp, F: Die Finanzbeziehungen zwischen Ländern und Gemeinden, in: Arnold. V.; Geske, O.-E. (Hrsg.): Öffentliche Finanzwirtschaft, München 1988, S. 115.

\subsubsection{Funktion der Neben- oder Ergänzungsansätze}

$\mathrm{Da}$ aber nicht der gesamte Bedarf als einwohnerdeterminiert angesehen werden kann, werden noch andere Größen - Ergänzungs- oder Nebenansätze - berücksichtigt. ${ }^{3}$ Auch PoPITZ wies schon in seinem Gutachten darauf hin: "Die Gliederung nach Einwohnerzahlen stellt allein einen zutreffenden Ausdruck für die $\mathrm{Zu}-$ schußbedürftigkeit der Gemeinden noch nicht dar" ${ }^{\text {. }}$. Die weiteren Bedarfsunterschiede können etwa in speziellen geographischen (Gebirgslage) oder politischen (ehemaliger Zonenrand) Faktoren begründet liegen. ${ }^{5}$ Es ist somit Aufgabe der Ergänzungsansätze, die Bedarfsberechnung zu verfeinern. ${ }^{6}$ Die zusätzlich $\mathrm{zu}$

1 Auf die Multiplikation der tatsächlichen Einwohnerzahl mit dem Hauptansatzfaktor wird verzichtet, da die Unterschiede schon an den Hauptansatzfaktoren zu erkennen ist.

2 Vgl.: Beispielsweise $\S 5$ des Niedersächsischen Gesetz über den Finanzausgleich (NFAG) vom 19.12.1995, Nds. GVBI., S. 463 und die übrigen Finanzausgleichsgesetze.

3 Für Nordrhein-Westfalen wurde beispielsweise ermittelt, daß nur ca. 50 v.H. des kommunalen Finanzbedarfs durch die Einwohnerzahl induziert werden. Vgl.: Micosatt, G.: Raumwirksamkeit einer Reform des kommunalen Finanzausgleichs, Bochum 1990, S. 31.

4 Popitz, J.: Der künttige Finanzausgleich zwischen Reich, Ländern und Gemeinden; Gutachten, erstattet der Studiengesellschaft furr den Finanzausgleich, Berlin 1932, S. 282.

5 Vgl.: Fischer-Menshausen, H.: Finanzausgleich II - Grundzüge des Finanzausgleichsrechts, in: Albers, W. u.a. (Hrsg.): Handbuch der Wirtschaftswissenschaften, Bd. 2, Stuttgart New York 1980, S. 661.

6 Vgl: Voigt, R.: Das System des kommunalen Finanzausgleichs in der Bundesrepublik 
beachtenden Einflußfaktoren werden dann in Einwohneräquivalente umgerechnet, um durch Verknüpfung mit dem Hauptansatz einen Gesamtbedarfsindikator in Form des Gesamtansatzes zu erhalten. Diese Einflußfaktoren können grundsätzlich auf zwei Arten berücksichtigt werden. Beim ersten werden die Ergänzungsansätze in vom-Hundert-Beträgen des Hauptansatzes festgelegt und somit ebenfalls veredelt. Es wird also unterstellt, daß diese zusätzlichen Bedarfe, welche weitere Ausgaben induzieren, in Abhängigkeit von der Einwohnerzahl steigen. ${ }^{1}$ Bei der zweiten Methode wird keine Korrelation zwischen Gemeindegröße und Zusatzbedarfen unterstellt, so daß es zu gleichen absoluten Veränderungen des Gesamtansatzes unabhängig vom jeweiligen Hauptansatz kommt.

\subsubsection{Funktion des Grundbetrags}

Der Grundbetrag ist eine Variable in Abhängigkeit vom Volumen der Schlüsselmasse. Er stellt nur eine Rechengröße dar, die aufgrund des im KFA verwendeten Repartitionsprinzips notwendig ist, um die vorhandene Ausgleichsmasse möglichst vollständig auf die Gemeinden und Kreise zu verteilen. ${ }^{2}$ Im Gegensatz zum Länderfinanzausgleich ist beim KFA der Finanzbedarf und nicht die Ausgleichssumme die Resultierende. Der Grundbetrag wiederum wird von den Ausgleichsregeln bestimmt, welche die Ausgleichsintensität, also den Umfang, in dem die Differenz zwischen Finanzkraft und -bedarf ausgeglichen werden soll, beeinflussen. Er ergibt sich dadurch, daß die vorhandene Schlüsselmasse durch die Ausgleichsquote dividiert und um die Summe der Finanzkraft aller Gemeinden erhöht wird. Dieser Betrag wird wiederum durch den aufaddierten Gesamtansatz aller Gemeinden dividiert. Der formale Zusammenhang ist in Abbildung 13 dargelegt.

\section{Abbildung 13:Berechnung des Grundbetrags

$$
\text { Grundbetrag }=\frac{\frac{\text { Schlüsselmasse }}{\text { Ausgleichsquote }}+\Sigma \text { Finanzkraft aller Empfänger }}{\Sigma \text { Gesamtansatz aller Empfänger }}
$$

Sinken z.B. aufgrund konjunktureller Entwicklungen die Steuereinnahmen des Landes und somit auch die Verbund- sowie die Schlüsselmasse, so führt der angezeigte Mechanismus dazu, daß der Grundbetrag sich mindert. Denn nur so ist es möglich, daß bei Konstanz der übrigen Größen die geminderte Schlüsselmasse vollständig auf die Kommunen verteilt werden kann. Durch die Minderung des Grundbetrags verringert sich der Finanzbedarf aller Kommunen - einige werden abundant - und somit auch ihre Schlüsselzuweisungen.

Deutschland, Stuttgart u.a.O., 1980, S. 77.

1 Vgl.: Ehrlicher, W. u.a.: Kommunaler Finanzausgleich und Raumordnung, Hannover 1967, S. 26.

2 Vgl:: Münstermann, E.: Der kommunale Finanzausgleich in den Bundesländern - Teil III -, in: ZKF, 30. Jg., 1980, S. 120. 


\subsubsection{Ermittlung des Finanzbedarfs von Gemeinden und Städten}

\subsubsection{Verwendung des Hauptansatzes in Hessen}

\subsection{Darstellung der bisherigen Regelungen}

Der Finanzbedarf wird im hessischen KFA durch den Gesamtansatz wiedergegeben, der sich additiv aus dem Hauptansatz und diversen Nebenansätzen zusammensetzt. Das Verfahren zur Bestimmung des Hauptansatzes wurde im Zeitablauf mehrmals geändert. So wurden bis 195910 Größenklassen gebildet, wobei sich innerhalb der Gruppen durch lineare Interpolation allerdings eine Glättung der einzelnen Hauptansatzwerte ergab (siehe Tabelle 11). Auf dieses Verfahren wird in Hessen seit 1960 verzichtet, da nach einer Erhöhung der Gemeindeklassenzahl die Einwohnerdifferenzen innerhalb einer Klasse als vernachlässigbar angesehen wurden. ${ }^{1}$ Mittlerweile wurde die Klassenzahl von 91 auf 8 verringert.

Die auf Gemeindegrößenklassen bezogene Hauptansatzstaffelung wird in Hessen nur bei den kreisangehörigen Gemeinden praktiziert; für die fünf kreisfreien Städte werden die Hauptansätze individuell bestimmt. Üben kreisangehörige Gemeinden zentralörtliche Funktionen aus, indem sie für das Umland Leistungen wie etwa Museen und Theater bereitstellen, so wird dies bei der Ermittlung des Hauptansatzes beachtet. Unterschieden wird zwischen drei Zentralitätsgruppen, nämlich Oberzentren, Mittelzentren mit Teilfunktion eines Oberzentrums und Mittelzentren, wobei die Einteilung durch das Ministerium für Wirtschaft, Verkehr und Landesentwicklung nach dem Landesentwicklungsplan erfolgt. Kreisfreie Städte gelten als Oberzentren, ihre Funktion ist schon durch die Höhe der Schlüsselmasse berücksichtigt ( $§ 15$ Abs. 1 FAG). Im kreisangehörigen Raum ist den Gemeindegrößenklassen "eine Staffelung des Hauptansatzes zugeordnet, in welche die Bewertung der Zentralität bereits eingerechnet ist."2 Deshalb wird eine gesonderte Berücksichtigung der Raumordnungsfunktion als nicht notwendig angesehen. Auch ein Sonderlastenausgleich wird abgelehnt, da dieser nicht die Steuerkraft berücksichtigt, zentrale Orte aber meist eine hohe Steuerkraft besitzen und damit auch ihren Mehrbedarf aufgrund der Leistungsvorhaltung für das Umland finanzieren können. Um aber auch den einwohnerschwachen Zentren in dünn besiedelten Regionen die notwendigen Zuweisungen zuzuteilen, wird deshalb für den Hauptansatz ein Mindestwert festgelegt; Er beträgt z.B. für Oberzentren unabhängig von der Einwohnerzahl - 140 v.H. ${ }^{3}$

1 Vgl.: Hessischer Landtag: Vorlage der Landesregierung für ein Gesetz zur Regelung des Finanzausgleichs vom 08.12.1959, Drucksachen Abteilung I, 4/405, S. 1178.

2 Ders.: Gesetzentwurf der Landesregierung für ein Gesetz zur Regelung des Finanzausgleichs und zur Änderung anderer Vorschriften vom 11.10.1977, Drucksache 8/4877, S. 36. Weiter heißt es dort: "Allerdings werden im südhessischen Raum insgesamt sieben Gemeinden ebenfalls begünstigt, die nicht zentrale Orte sind." Somit ist diese Regelung nicht differenziert genug, um mögliche Bedarfsunterschiede genau abzubilden.

3 Vgl.: Ebenda, S. 29. Nach $\S 10$ Abs. 2 Hess. FAG beträgt der Hauptansatz für Mittelzen- 
Um bei Schwankungen der Einwohnerzahl den Hauptansatz nicht immer neu berechnen zu müssen, wirkt sich eine Minderung auf den ermittelten Hauptansatz erst aus, wenn die Einwohnerzahl - im Vergleich zum vorherigen Ausgleichsjahr um mehr als 10 v.H. unter die nächstniedrigere Gemeindegrößenklasse des FAG gesunken ist. Ansonsten bleibt auch für die folgenden Jahre der frühere Ansatz gültig, soweit sich nicht Zuständigkeiten nach $\S 148 \mathrm{HGO}$ verändern.

\subsection{Analyse und Reform}

Abbildung 14 zeigt die derzeitige Spreizung der Hauptansatzstaffel für die kreisangehörigen Gemeinden, also die Werte für die einzelnen gemeindegruppenspezifischen Veredelungsfaktoren, und die Ergebnisse eines aktuellen Gutachtens, bei dem diese Faktoren mit Hilfe einer multiplen Regressionsanalyse ${ }^{1}$ aus dem gemeindlichen Zuschußbedarf abgeleitet wurden.

\section{Abbildung 14:Vergleich von bestehender und einer möglichen neuen Haupt- ansatzstaffel der kreisangehörigen Gemeinden in Hessen}

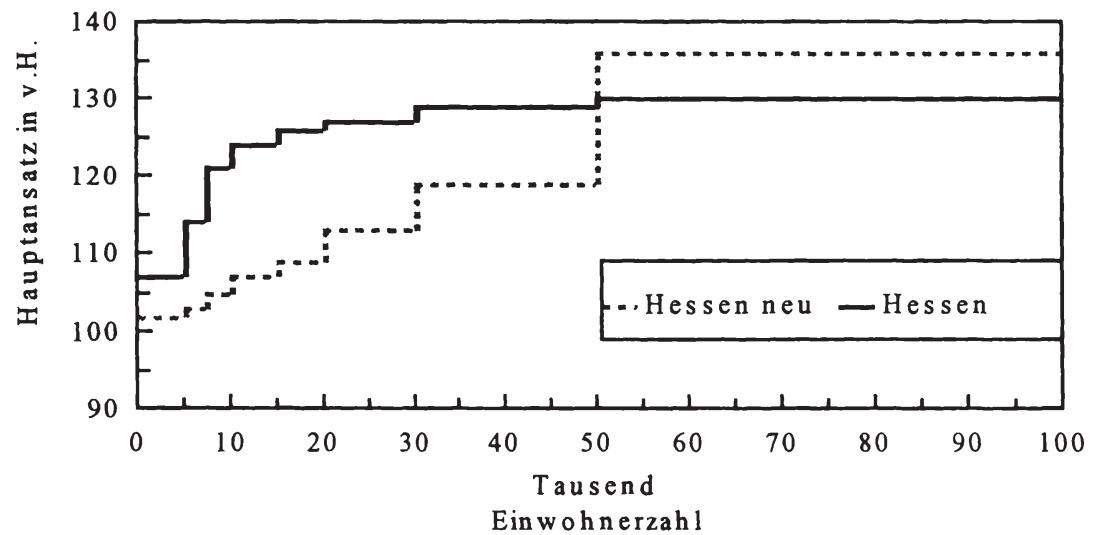

Quelle: Eigene Darstellung nach FAG und Kisseler, W.; Gretz, W.: Hauptansatzstaffel im Kommunalen Finanzausgleich. Eine empirische Untersuchung fur Hessen, in: HLT Gesellschaft fur Forschung, Planung und Entwicklung: HLT-Report Nr. 574, Wiesbaden 1999, S. 33.

Es ist zu erkennen, daß sich die Veredelungsfaktoren der einzelnen Gemeindeklassen teilweise sehr stark unterscheiden. ${ }^{2}$ Wobei nach der

tren mindestens 125 v.H. und für Mittelzentren mit Teilfunktionen eines Oberzentrums mindestens 130 v.H.

1 Damit kann festgestellt werden, wie groß der Erklärungswert verschiedener Variablen für den ermittelten Zuschußbedarf ist, also auch der Einfluß der Einwohnerzahl auf den $\mathrm{Zu}$ schußbedarf je Einwohner. Aus dem ermittelten Zuschußbedarf werden dann die Veredelungsfaktoren ermittelt.

2 Vgl:: Kisseler, W.; Gretz, W.: Hauptansatzstaffel im Kommunalen Finanzausgleich. Eine 
bestehenden Regelung der Anstieg bis zur Ortsgröße mit 10.000 Einwohnern besonders stark ist; danach sind nur noch geringe Steigerungen zu verzeichnen, während dies nach den Werten der Regressionsanalyse umgekehrt ist. Aus diesen Ergebnissen ist abzuleiten, daß ein Handlungsbedarf bestehen dürfte

Für die kreisfreien Städte kann dieses Verfahren aufgrund der geringen Anzahl und der somit durch das individuelle Ausgabeverhalten stark beeinflußbaren Durchschnittswerte nicht angewendet werden. ${ }^{1}$ Ein Vergleich mit den Regelungen anderer Bundesländer könnte bei der Beurteilung helfen, denn in den übrigen westdeutschen Flächenländern haben kreisfreie und kreisangehörige Städte eine gemeinsame Schlüsselmasse, so daß dort aufgrund der Anzahl der Kommunen eine Berechnung der Hauptansatzstaffel aus dem gemeindlichen Zuschußbedarf mit Hilfe einer Regressionsanalyse erfolgen kann. ${ }^{2}$ Um einen Vergleich zu ermöglichen, müssen für Hessen die Schlüsselmassen der kreisfreien Städte und der kreisangehörigen Gemeinden zusammengefaßt werden. Wegen dieser Maßnahme müssen auch die Hauptansätze der kreisfreien Städte, die der Bedarfsermittlung dienen, angepaßt werden. Dies geschieht so, daß die Abweichungen zur Ausgangsverteilung der Schlüsselzuweisungen für die einzelnen kreisfreien Städte möglichst gering gehalten werden. Aus Abbildung 15 kann entnommen werden, wie stark sich das Niveau der Hauptansatzstaffelung bei Realisation einer einheitlichen Gemeindeschlüsselmasse verändern würde, wenn bei den kreisfreien Städten der Status-quo-ante erhalten bleiben soll. Der Ansatz für Darmstadt hätte sich z.B. von 100 auf 214 v.H. erhöht. ${ }^{3}$ Da es für die kreisangehörigen Schulträger in Hessen einen entsprechenden Nebenansatz gibt, müßte dieser nun auch für die kreisfreien Städte gelten, auch wurde die zentralörtliche Funktion der kreisfreien Städte durch die Höhe der Schlüsselmasse berücksichtigt, was nun - wie in Bayern - durch einen Zuschlag von 10 v.H. des Hauptansatzwertes erfolgen soll. Duch diese Modifikationen bei der Bedarfsermittlung hätte sich der Hauptansatz weiter gesenkt, erliegt aber noch immer mindestens 85-95 Prozentpunkt über der Ausgangssituation bei getrennten Schlüsselmassen.

empirische Untersuchung für Hessen, in: HLT Gesellschaft für Forschung, Planung und Entwicklung: HLT-Report Nr. 574, Wiesbaden 1999, S. 25-36.

1 Vgl.: Steinherr, M.; u.a.: Das Schlüsselzuweisungssystem im kommunalen Finanzausgleich des Freistaates Sachsen, ifo-Studien zur Finanzpolitik, Bd. 63, München 1997, S. 115; in Sachsen gibt es sieben statt fünf kreisfreie Städte wie in Hessen.

2 Dies ist zuletzt in Niedersachsen geschehen. Vgl.: Hardt, U.; Schmidt, J.: Neuordnung des kommunalen Finanzausgleichs in Niedersachsen. Eine Untersuchungen im Auftrag des Niedersächsischen Innenministeriums, hrsg. vom Niedersächsischen Innenministerium, Hannover 1998 , S. 125-134.

3 Dieser Faktor beträgt 1998 bei Darmstadt und Offenbach 100, bei Kassel und Wiesbaden 102 und bei Frankfurt 105 v.H. Bei den genannten Werten wurde trotz Integration der Schlüsselmassen für die kreisfreien Städte die geringere Sockelgarantie von 76 v.H. der Bedarfsmeßzahl beibehalten. Läge sie ebenfalls bei 80 v.H. - wie bei den kreisangehörigen Gemeinden -, so wäre das Niveau der Hauptansatzwerte noch weiter erhöht worden. 


\section{Abbildung 15:Hauptansatzstaffel bei einheitlicher Gemeindeschlüsselmasse}

Hauptansatzstaffelung in v.H.

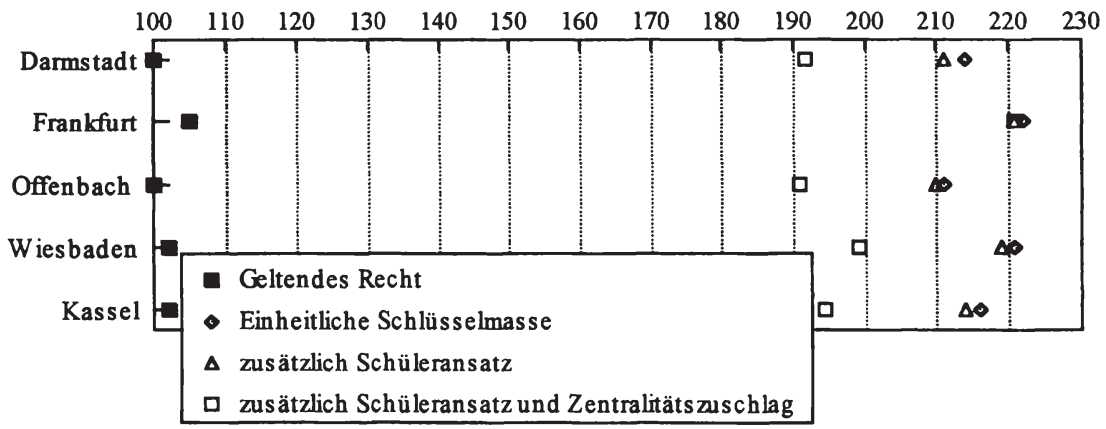

Quelle: Eigene Berechnung nach Daten des Finanzministeriums zum KFA 1998.

In Abbildung 16 wird nun ein Vergleich dieser Ergebnisse mit den Regelungen der Länder Baden-Württemberg, Bayern, Niedersachsen und Nordrhein-Westfalen vorgenommen, um ein Indiz zu erhalten, ob die Spreizung des Hauptansatzes bei den kreisfreien Städten in Hessen angemessen gewählt ist. Nur in diesen Ländern gibt es Städte und Gemeinden ähnlicher Größenordnung. In der Graphik sind nun das Niveau und die Spreizung des Hauptansatzes der fünf hessischen kreisfreien Städte für den Fall dargestellt, daß die Regelungen anderer Bundesländer gelten würden. Dabei werden für Hessen die Variante mit der größten und die mit der geringsten Spreizung des Hauptansatzes aus Abbildung 15 als Vergleichsgrundlage gewählt.

\section{Abbildung 16:Hessische kreisfreie Städte im Hauptansatzregime anderer Länder}

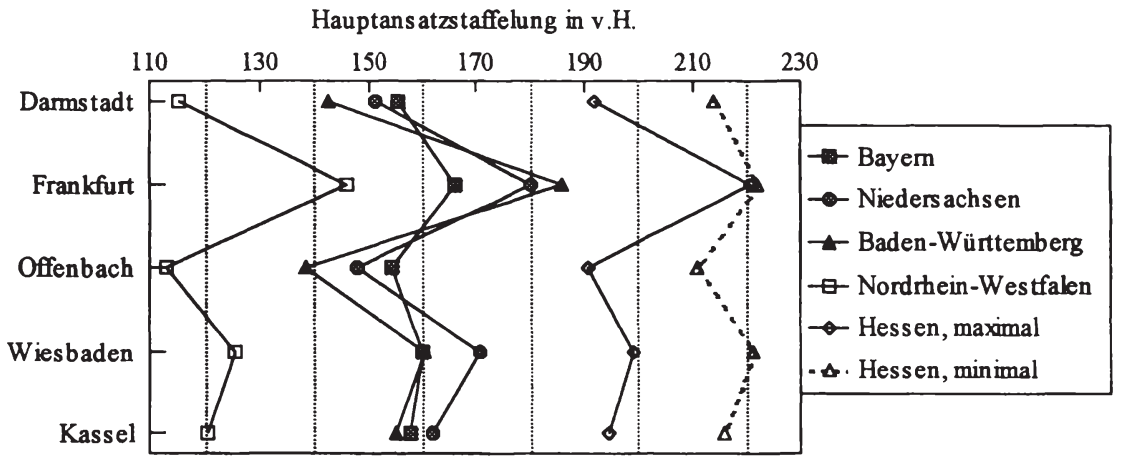

Quelle: Eigene Berechnung nach Daten des Finanzministeriums zum KFA 1998. 
Wichtig ist hier nur die Spreizung der Werte zwischen den einzelnen Städten, nicht aber das Niveau. ${ }^{1}$ Es ist zu erkennen, daß die Spreizung je nach Variante geringer ausfallen kann als in den übrigen Ländern. Dort beträgt sie bei Orten dieser verschiedenen Größenklassen zwischen 12 und 48 Prozentpunkte, während sie im Vergleich dazu für Hessen je nach verwendeter Variante 11 oder 30 Prozentpunkte ausmacht. Aus diesen Werten kann somit nicht gefolgert werden, daß die kleineren kreisfreien Städte von der derzeitigen Regelung in Hessen bevorzugt werden. Die Spreizung der Hauptansatzstaffel, die als ein Indiz herangezogen werden könnte, ist in den anderen Bundesländern nicht sehr viel größer als in Hessen, so daß eine offensichtliche Notwendigkeit zur Anpassung nicht zu erkennen ist. Weiterhin ist der Aspekt sehr wichtig, daß in Hessen alle kreisfreien Städte als Oberzentren vergleichbare Aufgaben erfüllen und somit auch annähernd gleiche Bedarfe haben dürften, so daß eine vergleichsweise geringere Spreizung nicht unangemessen sein muß. Zudem kann die Aufgabenwahrnehmung, die sich auf die Spreizung des Hauptansatzes auswirkt, zwischen den Kommunen verschiedener Länder nicht ohne weiteres verglichen werden. ${ }^{2}$ Folglich ist eine Änderung der Hauptansatzspreizung zwar denkbar, aber nicht zwingend notwendig.

Der hessische Gesetzgeber hat nun im Rahmen einer Reform des KFA vorgeschlagen, ausschließlich den Hauptansatzwert der Stadt Frankfurt zu verändern und in mehreren Stufen auf 109 v.H. anzuheben. Begründet wurde diese Vorgehensweise nicht nur mit einem steigenden Bedarf, sondern u.a. auch mit der Substitution der Gewerbekapital- durch den kommunalen Umsatzsteueranteil und den dadurch verminderten Steuereinnahmen der Stadt Frankfurt. ${ }^{3}$ Diese Begründung ist aber wegen der unzulässigen Vermischung von Bedarfs- und Steuerkraftbemessung abzulehnen. Falls Frankfurt nach der Gemeindesteuerreform eine geringere Steuerkraft aufweist, steigen automatisch die Schlüsselzuweisungen, eine Bedarfsanpassung ist unnötig.

1 Das Niveau der Hauptansätze, welches in Hessen im Bereich zwischen 211 und 222 v.H. bzw. 191 und 221 v.H. liegt und damit sehr viel höher ist als in den übrigen Bundesländern, kann als Hinweis für die Bevorzugung der kreisfreien Städte in Hessen gegenüber den kreisangehörigen Gemeinden gelten. Zudem liegt der Hauptansatz für die kreisangehörigen Oberzentren Fulda, Gießen und Marburg, die als Sonderstatusstädte auch Kreisaufgaben wahrnehmen, nur bei 161 v.H. im Vergleich zu ca. 190 v.H. bei Offenbach und Darmstadt.

2 In Hessen ist z.B. der Status einer kreisfreien Stadt und die damit verbundene Aufgabenwahrnehmung von der Einwohnerzahl abhängig, so daß alle Gemeinden mit mehr als 100.000 Einwohnern kreisfrei sind. In Niedersachsen gibt es z.B. kreisfreie Städte mit 50.000 Einwohnern und kreisangehörigen Gemeinden mit 110.000 Einwohnern

3 Vgl.: Hessischer Landtag: Gesetzentwurf der Landesregierung für ein Gesetz zur Änderung des Finanzausgleichsgesetzes vom 21.10.1996, Drucksache 14/2259, S. 11f. Ders.: Gesetzentwurf der Landesregierung für ein Finanzausgleichsänderungsgesetz 1999 vom 23.06.1998, Drucksache 14/4013, S. 6 f. 
Eine Aufgabe, die heute der Hauptansatzstaffel zugesprochen wird, ist es, zentralörtliche Leistungen von Gemeinden zu berücksichtigen. ${ }^{1}$ Beim Vergleich der GröBenordnung der Gemeinden und der zentralörtlichen Aufgabenerfüllung kann festgestellt werden, daß Orte gleicher Größe unterschiedliche Aufgaben für ihre Umgebung ausüben. Bei der Hauptansatzstaffelung werden aber nur die absoluten Einwohnerzahlen betrachtet, während für die raumordnungspolitischen Aufgaben die relative Größe - nämlich im Vergleich zu den umgebenden Gemeinden - ausschlaggebend ist. ${ }^{2}$ Somit führt dieses Verfahren nur dann zu einer Förderung der zentralen Orte, daß die Zentralität eine Funktion der Größe ist. ${ }^{3}$ Die Bedarfe kreisangehöriger und -freier zentraler Orte werden im hessischen KFA gesondert berücksichtigt. Im kreisfreien Raum geschieht dies nach $\S 15$ Abs. 1 FAG durch die Höhe der Schlüsselmasse, im kreisangehörigen Raum nach $\S 10$ Abs. 2 FAG durch die Gewährung eines Mindesthauptansatzes unabhängig von der tatsächlichen Einwohnerzahl. ${ }^{4}$ Im kreisangehörigen Raum gibt es drei Oberzentren, sieben Mittelzentren mit Teilfunktion eines Oberzentrums und 87 Mittelzentren. ${ }^{5}$ Interessant ist nun, für wieviele dieser kreisangehörigen Gemeinden sich die Berücksichtigung der Zentralörtlichkeit im KFA bedarfsteigernd auswirkt (Tabelle 12).

\section{Tabelle 12:Relevanz des Zentralitätsfaktors im KFA}

\begin{tabular}{|c|c|c|c|c|}
\hline Zentralität & Anzahl & Vorteil & Neutral & Nachteil \\
\hline Oberzentrum & 3 & 3 & - & - \\
\hline Teilfunktion Oberzentrum & 7 & 4 & 3 & - \\
\hline Mittelzentrum & 87 & 23 & - & 64 \\
\hline Gesamt & 97 & 30 & 3 & 64 \\
\hline
\end{tabular}

Quelle: Regionale Raumordnungspläne Nord-, Mittel-, Osthessen, Starkenburg, Rhein-MainTaunus und Untermain, in: Staatsanzeiger fur das Landes Hessen, 1979, S. 737 f., 822 , $398,671,445$, und 1287 .

1 Vgl.: Karrenberg, H.; Münstermann, E.: Der Gemeindefinanzbericht 1993, in: Der Städtetag, 46. Jg., 1993, S. 142.

2 Vgl.: Grimme, L.: Neuberechnung der Steuerkraft im kommunalen Finanzausgleich - dargestellt am Beispiel des Landes Bayern, in: Räumliche Aspekte des kommunalen Finanzausgleichs, Hannover 1985, S. 162.

3 Vgl:: Mäding, H.: Überlegungen zur Eignung des kommunalen Finanzausgleichs zur Förderung raumordnungspolitischer Konzepte, in: Informationen zur Raumentwicklung: Die Reform der Kommunalfinanzen, Heft 8/9. 1995, hrsg. von der Bundesforschungsanstalt für Landeskunde und Raumentwicklung, Bonn 1995, S. 609.

4 Der Mindestansatz für kreisangehörige zentrale Orte wird nur dann zur Bedarfsermittlung genutzt, wenn er größer als der einwohnerabhängige Wert der zentralen Orte ist. Ansonsten gilt der einwohnerabhängige Wert bei der Ermittlung des Hauptansatzes.

5 Regionale Raumordnungspläne Nord-, Mittel-, Osthessen, Starkenburg, Rhein-Main-Taunus und Untermain, in: Staatsanzeiger für das Landes Hessen, 1979, S. 734, 819, 397, 667, 444 und 1286. 
Es kann festgestellt werden, daß die praktische Relevanz dieses Ansatzes sehr begrenzt ist, da gerade bei 30 von 97 Gemeinden eine Erhöhung des Hauptansatzes im Vergleich zur normalen Einwohnerstaffelung auftritt, dies sind gerade 30,93 v.H. der möglichen Förderungsorte. Die übrigen Gemeinden haben aufgrund ihrer Einwohnerzahl einen gleichhohen oder vielfach sogar einen höheren Wert als bei zentalörtlicher Betrachtung. So würde sich bei 64 von 87 Mittelzentren der Hauptansatz vermindern, wenn der Zentralitätsansatz verbindlich wäre; demgegenüber kann die Aufgabenstellung bei den beiden anderen Gruppen als jedenfalls was die Richtung betrifft - erfüllt angesehen werden, da alle Oberzentren und auch vier von sieben Gemeinden mit Teilfunktion eines Oberzentrums von diesem Ansatz profitieren.

Weiterhin ist noch zu beachten, daß zehn Gemeinden ohne jegliche raumordnungspolitische Funktion einen höheren "einwohnerveredelten" Hauptansatz aufweisen als solche, denen die Funktion eines Mittelzentrums zugewiesen ist. Aus diesen Zahlen kann abgeleitet werden, daß die Zentralörtlichkeit im derzeitigen KFA nur im geringem Maße berücksichtigt wird. Diese Schlußfolgerung kann noch durch die Ergebnisse aus Tabelle 13 unterstützt werden. Dort erfolgt die Bedarfsermittling nur auf Basis des Hauptansatzes, also Einwohnerveredelung inklusive Zentralörtlichkeit, die Bedarfserhöhung durch Nebenansätze bleibt also unberücksichtigt. Dabei werden vier Möglichkeiten unterschieden:

-1. tatsächlicher Hauptansatz; Einwohnerveredelung unter Berücksichtigung der Zentralität, wenn diese zu einem höheren Bedarf führt

-2. Veredelung; normale Veredelung entsprechend der Hauptansatzstaffel

-3. verbindliche Zentralität + Veredelung; Einwohnerveredelung unter Berücksichtigung der Zentralität, auch wenn diese zu einem verringerten Bedarf führt -4. nur Zentralitätsansatz; bis auf die drei genannten Zentralitätsgruppen werden alle Gemeinden mit 100 v.H. veredelt, ähnlich wie in Rheinland-Pfalz

Es kann nun festgestellt werden, daß der bedarfsbestimmende Hauptansatz um 1.096.655 Einwohner über der tatsächlichen Einwohnerzahl liegt, was einer Erhöhung um 23,55 v.H. entspricht. Der Einfluß des Zentralitätsfaktors auf diese Erhöhung beträgt mit 27.062 Einwohnern gerade einmal 2,47 v.H. oder nur 0,47 v.H. des gesamten Hauptansatzes. Der Großteil mit 1.069.593 Einwohnern entfält auf die einfache Einwohnerveredelung, dies entspricht 97,53 v.H. der Erhöhung. Die Differenz von 9.507 Einwohnern zwischen der Hauptansatzveredelung und der verbindlichen Berücksichtigung der Zentralität liegt darin begründet, daß sich die Zentralität bei einem Teil der Gemeinden aufgrund deren EinwohnergröBe gar nicht positiv auswirken würde und deshalb auch nicht zur Anwendung kommt. Von einer theoretisch höheren Auswirkung der Zentralität - nämlich 36.569 Einwohner ${ }^{1}$ - sind nur 27.062 aufgrund dieses Größenverhältnisses von

1 Der Wert ergibt sich durch die Addition der tatsächlichen Erhöhung von 27.062 Einwoh- 
Hauptansatzstaffel zum Mindestansatz für Zentralität relevant. Käme nur ein Zentralitätsansatz ohne Einwohnerveredelung des Hauptansatzes zur Geltung, so würde sich der Bedarfsansatz zwar sehr viel geringer erhöhen, doch der Einfluß der raumordnungspolitischen Aufgabenwahrnehmung betrüge dann immerhin 13,71 v.H. auf den Hauptansatz.

Durch den Ansatz für zentrale Orte werden bei den kreisangehörigen Gemeinden etwa 17,77 Mio. DM umverteilt, daß sind 1,59 v.H. der Schlüsselmasse. Bei einer ersatzlosen Streichung käme es aber nur zu einer Minderung der Zuweisungen bei den zuvor begünstigten Gemeinden um ca. 15,20 Mio. DM, wobei die Verteilungswirkungen in der Tabelle 14 dargestellt sind. ${ }^{1}$ Die Differenz von 2,56 Mio. DM kann auf die Erhöhung des Grundbetrags zurückgeführt werden, an der alle Gemeinden partizipieren; ${ }^{2}$ sie ist gleichzeitig ein Indiz dafür, daß sich der Zentrale-Orte-Ansatz nicht sonderlich stark erhöhend auf die Bedarfsmeßzahl auswirkt, da diese bei den meisten Städten nur geringfügig steigt.

Tabelle 13:Auswirkungen der Zentralität auf den Hauptansatz der kreisangehörigen Gemeinden

\begin{tabular}{|l|r|r|r|}
\hline \multicolumn{1}{|c|}{ Art der Hauptansatzberechnung } & Hauptansatz & \multicolumn{2}{|c|}{ Hauptansatzdifferenz } \\
\cline { 3 - 4 } & (absolut) & (absolut) & (in v.H.) \\
\hline tatsächliche Einwohnerzahl & 4.656 .332 & 0 & 0,00 \\
\hline 1. tatsächlicher Hauptansatz & 5.752 .987 & 1.096 .655 & 23,55 \\
\hline - davon nach Einwohnerveredelung & -1.069 .593 & - & - \\
\hline - nach zusätzlicher Zentralität & 27.062 & - & 97,53 \\
\hline 2. veredelter Hauptansatz & 5.725 .925 & 1.069 .593 & 2,47 \\
\hline 3. verbindliche Zentralität + Veredelung & 5.716 .418 & 1.060 .086 & 22,97 \\
\hline 4. nur Zentralitätsansatz & 5.294 .507 & 638.175 & 13,71 \\
\hline
\end{tabular}

Quelle: Eigene Berechnung nach Daten des Finanzministeriums zum KFA 1998.

Von den 30 Gemeinden, deren Finanzbedarf sich aufgrund des Zentralitätsansatzes erhöht, ergeben sich bei einer Gemeinde wegen der Abundanz keine Auswirkungen auf die Schlüsselzuweisungen. Für die übrigen läßt sich die Vermutung der geringen Auswirkungen dieses Ansatzes auf die Verteilung der

nern und der um 9.507 verminderten Einwohnerzahl bei verbindlicher Berücksichtigung der Zentralität.

1 Der Unterschied zwischen den Gewinnen und den Verlusten liegt hier und bei allen anderen Nebenansätzen darin begründet, daß sich im Gegensatz zur Ausgangssituation nun bei Verzicht auf den jeweiligen Ansatz zur Bedarfsermittlung die ursprünglich verteilte Summe nicht mehr restlos den Kommunen zuweisen läßt. Entscheidend hierfür ist, daß der zur Verteilung relevante Grundbetrag nur auf zwei Stellen hinter dem Komma ermittelt wird.

2 Dies ist auch in den folgenden Tabelle berücksichtigt. 
Schlüsselzuweisungen verfestigen, wenn berücksichtigt wird, daß von den 15,20 Mio. DM, dies sind etwa 8,65 v.H. der auf diese Gruppe entfallenden Schlüsselzuweisungen, allein 13,11 Mio. DM (86,25 v.H.) den drei Oberzentren Fulda, Gießen und Marburg zustehen. Der durchschnittliche Pro-Kopf-Verlust von 24,84 DM setzt sich aus 61,79 DM für die drei Oberzentren und 0,47 DM für die übrigen 26 Gemeinden zusammen, wodurch wiederum die geringe Bedeutung der zentralen Orte im KFA abgesehen von den Oberzentren hervortritt. Von den nicht unter diesen Ansatz fallenden 390 Gemeinden profitieren 367 bei Abschaffung des Ansatzes aufgrund des steigenden Grundbetrags, während sich für $24 \mathrm{Ge}-$ meinden aufgrund ibrer Finanzkraft nichts ändert.

Tabelle 14:Umverteilung bei Abschaffung des Zentralitätsansatzes

\begin{tabular}{|c|r|r|r|}
\hline Kreisangehörige Gemeinden & \multicolumn{2}{|c|}{ Gemeinden mit Ansatz } & \multirow{2}{*}{$\begin{array}{c}\text { ohne Ansatz } \\
\text { Gewinner }\end{array}$} \\
\cline { 2 - 3 } & Gewinner & Verlierer & 29 \\
\hline Anzahl & 0 & 29 & 367 \\
\hline Gewinn/Verlust in DM & 0,00 & -15.201 .774 & 15.173 .996 \\
\hline Gewinn/Verlust in v.H. & 0,00 & $-8,65$ & 1,62 \\
\hline Gewinn/Verlust in pro DM/Kopf & 0,00 & $-24,84$ & 4,17 \\
\hline
\end{tabular}

Quelle: Eigene Berechnung nach Daten des Finanzministeriums zum KFA 1998.

Da seit der Einführung des Mindestansatzes für Orte mit zentralörtlicher Aufgabenerfüllung 1978 ein Bevölkerungsanstieg von 8,2 v.H. in Hessen zu verzeichnen ist, besteht die Möglichkeit, daß die zentralen Orte - entsprechend der gewünschten Entwicklung - aufgrund der Bevölkerungszunahme aus diesem gesonderten Ansatz "herausgewachsen" sind und dieser nur aus diesem Grund heute eine geringe Relevanz hat. ${ }^{1}$ Bei einer näheren Betrachtung ergibt sich allerdings, da $\beta$ diese Vermutung nur für sieben Gemeinden mit der Funktion eines Mittelzentrums gilt. ${ }^{2}$ Diese sind durch den Bevölkerungsanstieg im Zeitraum seit 1979/80 in eine andere Gemeindeklasse gewachsen und erhalten nun einen über dem Mindestansatz für zentrale Orte liegenden Hauptansatz. Für die übrigen 57 Mittelzentren (65,5 v.H. dieses Typs) löste der Ansatz also schon bei seiner Einführung keine Bedarfsteigerung aus. Somit war dieser Ansatz von Anfang an bezogen auf das verfolgte Ziel unterdimensioniert.

1 Ein anderer Grund könnte noch in einer allgemeinen Veränderung der Hauptansatzstaffel gesehen werden. Im betrachteten Zeitraum wurde aber nur die Gruppe der Orte unter 5000 Einwohnern in einer Hauptansatzstaffel zusammengefaßt, von denen aber keiner in die geförderten zentralen Ortegruppen gehörte.

2 Es handelt sich dabei um die Gemeinden Bürstadt, Groß-Umstadt, Gelnhausen, Bad Soden a.T., Idstein, Büdingen sowie Haiger. 


\subsubsection{Verwendung von Nebenansätzen in Hessen}

\subsection{Darstellung der bisherigen Regelungen}

Mittels Nebenansätzen soll, wie angeführt, das recht grobe Schema der reinen einwohnerabhängigen Bedarfsmessung modifiziert werden. Im Zeitablauf wurden Ergänzungsansätze eingeführt und zum Teil wieder abgeschafft. Tabelle 15 gibt einen Überblick über die bedarfssteigernd berücksichtigten Tatbestände und nennt dazu auch die vom Landesgesetzgeber angeführten Gründe. Nachfolgend werden die derzeit noch geltenden Nebenansätze kurz erläutert und im folgenden Abschnitt kritisch analysiert, wobei sich die Reihenfolge an $\S 11$ FAG orientiert. ${ }^{1}$

\section{Tabelle 15:Berücksichtigte Bedarfsdeterminanten seit 1949}

\begin{tabular}{|c|c|}
\hline Zeitraum & Bedarfsdeterminante \\
\hline $1949-1977$ & $\begin{array}{l}\text { Bevölkerungszusammensetzung; hierbei wurden Lohnempfänger, } \\
\text { Kinder und Erwerbslose berücksichtigt, um einen Ausgleich für ei- } \\
\text { ne fehlende Bürgersteuer zu gewähren }\end{array}$ \\
\hline seit 1949 & $\begin{array}{l}\text { Flüchtlinge und Evakuierte, später Bevölkerungszuwachs; soll die } \\
\text { Lasten, die mit überdurchschnittlichem Bevölkerungssteigerung ein- } \\
\text { hergehen, kompensieren }\end{array}$ \\
\hline $1949-1963$ & Kriegszerstörungen; Ausgleich für Grundsteuermindereinnahmen \\
\hline $1951-1963$ & Bevölkerungsrückgang; Erleichterung der Fixkostendeckung \\
\hline seit 1960 & $\begin{array}{l}\text { Stationierungsstreitkräfte; den Kosten für diese Gruppe stehen kei- } \\
\text { ne Steuereinnahmen gegenüber }\end{array}$ \\
\hline seit 1962 & $\begin{array}{l}\text { Bädergemeinden; die durch die Kurgäste induzierten Kosten kön- } \\
\text { nen nicht vollständig durch Kurabgaben finanziert werden }\end{array}$ \\
\hline $1963-1990$ & Zonenrandgemeinden; Ausgleich für geopolitische Nachteile \\
\hline $1966-1977$ & $\begin{array}{l}\text { Gemeindeeingliederungen/-zusammenlegungen; es sollte ein Anreiz } \\
\text { für freiwillige Zusammenlegungen gewährt und später Benachteili- } \\
\text { gungen aufgrund verordneter Eingliederungen vermieden werden }\end{array}$ \\
\hline seit 1978 & $\begin{array}{l}\text { zentralörtliche Aufgabenerfüllung; die Leistungserstellung für das } \\
\text { Umland soll finanziell entgolten werden }\end{array}$ \\
\hline seit 1980 & $\begin{array}{l}\text { Sonderstatusstädte, finanzieller Ausgleich für die Kosten der - den } \\
\text { Gemeinden über 50.000 Einwohner - übertragenen Aufgaben }\end{array}$ \\
\hline se & $\begin{array}{l}\text { Schulträgerschaft; Folgemaßnahme nach der Verminderung des } \\
\text { Schulsonderlastenausgleichs zugunsten der Schlüsselzuweisungen }\end{array}$ \\
\hline
\end{tabular}

Quelle: Finanzausgleichsgesetze und Landtagsdrucksachen der jeweiligen Jahre.

Abgesehen vom Ansatz für Sonderstatusstädte (Gemeinden über 50.000 Einwohner) und für Schulträgerschaft führen diese Aspekte nicht nur bei den kreisangehörigen Gemeinden sondern auch bei den kreisfreien Städten zu einer Erhöhung der relevanten Bedarfszahlen. 


\subsection{Sonderstatusstädte}

Der Ansatz für die Sonderstatusstädte wird aufgrund der Auflösung der kreisfreien Stadt Lahn und der Wiedergründung der nun kreisangehörigen Städte Gießen und Wetzlar im Zuge der Gebietsreform in Hessen seit 1980 allen Orten über 50.000 Einwohnern gewährt. ${ }^{1}$ Er ist ein Ausgleich für die Lasten, die damit verbunden sind, daß diese Städte für ihre Einwohner Kreisaufgaben wahrnehmen; gleichzeitig wurde auch die Minderung der Kreisumlage für diese Orte bestimmt. ${ }^{2}$ Sie sind in den Bereichen Bau-, Schul- und Sozialwesen den kreisfreien Städten gleichgestellt und nehmen deshalb einen Status zwischen den kreisfreien und den sonstigen kreisangehörigen Gemeinden an. ${ }^{3}$ Bei diesen Städten handelt es sich neben den beiden schon genannten um Bad Homburg und Rüsselsheim sowie um die vormals kreisfreien Städte Fulda, Hanau und Marburg. Der Ansatz führt zur Erhöhung des Hauptansatzes bei den betroffenen Städten um 15 v.H. ${ }^{4}(\S 11$ Abs. 1 FAG) und könnte deshalb prinzipiell auch in diesen eingerechnet werden, zumal alle sieben Sonderstatusstädte in der gleichen Größenklasse liegen. ${ }^{5}$ Auch hier gilt $\S 10$ Abs. 3 FAG, so daß eine Verringerung der Einwohnerzahl keine Änderung des maßgebenden Hauptsatzes nach sich zieht, solange die Bevölkerung mindestens noch 90 v.H. der nächstniedrigeren Gemeindegrößenklasse ausmacht. Da Fulda, Gießen und Marburg kreisangehörige Oberzentren sind und ihnen somit ein Hauptansatz von 140 v.H. zugewiesen wird, ergibt sich für sie eine Erhöhung um 21 Prozentpunkte auf 161 v.H.; für die übrigen vier Städte gilt ein Hauptansatz von 130 v.H., so daß die Erhöhung 19,5 Prozentpunkte beträgt.

\subsection{Stationierungsstreitkräfte}

Lasten, die einzelnen Gemeinden durch die Soldaten ausländischer Stationierungsstreitkräfte und deren Angehörigen entstehen, etwa durch den Besuch deutscher Schulen, sollen mit Hilfe dieses Ansatzes ( $\S 11$ Abs. 2 FAG) berücksichtigt werden. ${ }^{6}$ Denn den durch diese Personen hervorgerufenen

1 Vgl.: Gesetz zur Neugliederung des Lahn-Dill-Gebiets und zur Übertragung von weiteren Aufgaben auf kreisangehörigen Gemeinden mit mehr als 50000 Einwohnern sowie zur Regelung sonstiger Fragen der Verwaltungsreform vom 10.07.1979, Hess. GVB1. I, S. 179.

2 Vgl:: Hessischer Landtag: Gesetzentwurf der Landesregierung für ein Gesetz zur Änderung des Finanzausgleichsgesetzes und anderer Vorschriften vom 26.09.1979, Drucksache 9/1522, S. 10.

3 Vgl.: Schneider, G; Dreßler, U.; Lüll, J.: Kommentar zu § 4a HGO, in: Schneider, G; Dreßler, U.; Lüll, J. (Hrsg.): Hessische Gemeindeordnung, Kommentar (Loseblattsammlung), Mainz, Stand 1999, S. If.

4 Hat eine Sonderstatusstadt z.B. 60.000 Einwohner und einen Hauptansatzwert von 135 v.H., so ergibt sich die für die Bedarfsmeßzahl zu berücksichtigende Einwohnerzahl nach folgender Berechnung: 60.000 * 135 v.H. + 60.000 * 135 v.H. * 15 v.H. $=93.150$.

5 Vgl.: Kisseler, W.; Gretz, W.: Hauptansatzstaffel im Kommunalen Finanzausgleich. Eine empirische Untersuchung für Hessen, in: HLT Gesellschaft für Forschung, Planung und Entwicklung: HLT-Report Nr. 574, Wiesbaden 1999, S. 37.

6 Vgl.: Hessischer Landtag: Gesetzentwurf der Landesregierung für ein Gesetz zur Regelung 
Ausgaben stehen keine Steuereinnahmen gegenüber, da sie im Rahmen der Verteilung des kommunalen Einkommensteueranteils nicht berücksichtigt werden und zudem nicht die Möglichkeit gegeben ist, die Kosten wie bei Personen mit einem Zweitwohnsitz in der betreffenden Gemeinde über eine Zweitwohnungsteuer abzugelten. Dabei wird der Hauptansatz der jeweiligen Stationierungsorte um die Anzahl der Armeeangehörigen und ihrer Familien erhöht, wobei zur Verwaltungsvereinfachung die Mindestzahl von 50 Personen überschritten werden muß.

\subsection{Kurorte}

Die Einführung des Ergänzungsansatz für Heilkurorte wurde 1962 damit begründet, daß die Aufwendungen der Heilkurorte für die Infrastrukur (Polizei- und Feuerwehrfahrzeuge, Straßen und Straßenbeleuchtung) nicht im vollem Umfang durch die Kurabgaben gedeckt werden können und die Kurgäste deshalb als bedarfserhöhend anzusehen sind. ${ }^{1}$ Gemeinden, die nach dem hessischen Beihilfenrecht ${ }^{2}$ als Heilkurorte aufgeführt werden, können für die ihnen durch den Kurgasttourismus entstandenen Aufwendungen einen Ausgleich durch Schlüsselzuweisungen im Rahmen des Finanzausgleichs erhalten. $\mathrm{Zu}$ diesem Zweck wird nach $\S 11$ Abs. 3 FAG ein Ergänzungsansatz in Höhe der Anzahl der Übernachtungen gewährt, wobei 250 Übernachtungen einem Einwohner entsprechen. Es werden nur kurbeitragspflichtige Personen berücksichtigt, nicht aber Übernachtungen in Landschulheimen und Jugendherbergen sowie in Asylbewerber- und Aussiedlerheimen. ${ }^{3}$

\subsection{Bevölkerungswachstum}

Der Nebenansatz für ein starkes Bevölkerungswachstum wurde 1949 eingeführt, um die durch Flüchtlinge entstehenden zusätzlichen Belastungen zu berücksichtigen, und später in einen Ansatz umgeformt, der das allgemeine Bevölkerungswachstum berücksichtigt. ${ }^{4}$ Gemeinden mit stark steigender Bevölkerungszahl erhalten unter gewissen Umständen einen zusätzlichen Bedarf ( $\$ 11$ Abs. 4 FAG) zugesprochen, um den mit dem Bevölkerungsanstieg verbundenen zusätzlichen Ausgaben Rechnung zu tragen. Als Voraussetzung muß in den letzten zehn Jahren die tatsächliche Einwohnerzahl um mehr als 10 v.H. gestiegen sein. Für die

des Finanzausgleichs und zur Änderung anderer Vorschriften vom 11.10.1977, Drucksache 8/4877, S. 36.

1 Vgl: Hessischer Landtag: Zweites Gesetz zur Änderung des Finanzausgleichsgesetzes vom 17.08.1961, Drucksachen Abteilung I, 4/1131, S. 3419

2 Vgl.: Änderung des Hessischen Beihilferechts vom 14.12.1994, in: Staatsanzeiger für das Land Hessen, 1995, S. 1.

3 Vgl: Hessisches Ministerium der Finanzen: Ausführungsbestimmungen zum Finanzausgleichsgesetz vom 17.04.1995, in: Staatsanzeiger für das Land Hessen, 1995, S. 1239f.

4 Vgl: Vorlage der Landesregierung für ein Zweites Gesetz zu Änderung des Gesetzes zur Regelung des Finanzausgleichs vom 10.061948 (Hess. GVB1. S. 83) vom 20.05.1949, Drucksachen Abteilung I, 1/1124, Allgemeine Begründung. 
Berechnung der Schlüsselzuweisungen wird nur die Hälfte des über 10 v.H. hinausgehenden Bevölkerungswachstums berücksichtigt; mit diesem Prozentsatz wird die durch den Veredelungsfaktor gewichtete tatsächliche aktuelle Einwohnerzahl multipliziert und somit der Finanzbedarf erhöht.

\subsection{Schulträgerschaft}

Nach dem hessischen Schulgesetz liegt grundsätzlich die Schulträgerschaft - abgesehen von den Volkshochschulen - bei den kreisfreien Städten und den Landkreisen. Die kreisangehörigen Kommunen mit Sonderstatus - Gemeinden mit mehr als 50.000 Einwohnern - wurden, abgesehen von Wetzlar und Bad Homburg, im Rahmen der Gebietsreform als Schulträger eingesetzt. Für die übrigen kreisangehörigen Gemeinden ist die Möglichkeit geschaffen worden, in Absprache mit den Landkreisen als Träger der Schulen im eigenen Gebiet aufzutreten. ${ }^{1}$ Die Gemeinden haben die mit der Schulträgerschaft verbundenen Sachkosten wie Unterhaltung des Schulgeländes und der -gebäude zu tragen. Weiterhin müssen sie für die Personalkosten im Sinne des $§ 156$ Hessisches Schulgesetz aufkommen wie etwa Ausgaben für das Verwaltungs- und Reinigungspersonal, da diese nicht vom Land übernommen werden.

Beim Nebenansatz für die kreisangehörige Schulträgerschaft handelt es sich um den jüngsten Ansatz. Die Begründung für seine Einführung zeigt, wie kompliziert das System des KFA ist. Im Finanzausgleichsänderungsgesetz 1988 wurden aus kommunalpolitischen Erwägungen die besonderen Zuweisungen für den Bereich der Schullasten zugunsten einer Erhöhung der drei Schlüsselmassen aufkommensund gruppenneutral verringert, um somit den Anteil der ungebundenen Zuweisungen zu erhöhen und den Ausgleichsgrad zugunsten der finanzschwachen Gebietskörperschaften zu verstärken. ${ }^{2}$ Dies stellte bei den Landkreisen und kreisfreien Städten kein Problem dar, da in diesen Gruppen alle Gebietskörperschaften Schulträger sind. Bei den kreisangehörigen Gemeinden sind hingegen nur sechs Schulträger, so daß von der Erhöhung der Schlüsselmasse auch die übrigen 415 Gemeinden profitiert hätten. Um diese nicht gewünschte Umverteilung einzuschränken, wurde der entsprechende bedarfserhöhende Nebenansatz eingeführt ( $\S$ 11 Abs. 5 FAG), der bei den Schulträgern zu zusätzlichen Schlüsselzuweisungen führen soll. ${ }^{3}$ Als Bemessungsgröße für den Bedarf wird die Schülerzahl

1 Vgl.: § 138 Abs. 3 Hessisches Schulgesetz vom 17.06.1992, Hess. GVBl. I., S. 233. Nur die Gemeinde Kelsterbach hat diese Möglichkeit der Übertragung der Schulträgerschaft genutzt.

2 Vgl.: Hessischer Landtag: Gesetzentwurf der Landesregierung für ein Gesetz zur Änderung des Finanzausgleichsgesetzes und anderer Rechtsvorschriften - Finanzausgleichsgesetz 1988 - vom 25.11.1987, Drucksache 12/1178, S. 17.

3 Vgl.: Hessischer Landtag: Gesetzentwurf der Landesregierung für ein Gesetz über die Feststellung des Haushaltsplans des Landes Hessen für das Haushaltsjahr 1989 und zur Änderung des Finanzausgleichsgesetzes und anderer Rechtsvorschriften vom 19.09.1988, Druck- 
verwendet, wobei auf eine Differenzierung nach Schularten verzichtet wird. Berücksichtigung finden die Schüler, die sich auf den Schulen befinden, deren Träger die jeweiligen ausgleichsberechtigten Gemeinden sind. Der Hauptansatz wird dabei absolut um 15 v.H. der berücksichtigungsfähigen Schülerzahlen erhöht.

\subsection{Analyse und Reform}

Um einen Überblick über die Dimensionierung der Nebenansätze zu erhalten, wird ihr Anteil an dem bedarfsbestimmenden Gesamtansatz, der sich als Summe des Hauptansatzes und den Ergänzungsansätzen ergibt, in der folgenden Tabelle 16 dargestellt.

Tabelle 16:Wirkungen der Nebenansätze auf den gemeindlichen Gesamtansatz

\begin{tabular}{|c|r|r|r|r|}
\hline \multirow{2}{*}{$\begin{array}{c}\text { Art der } \\
\text { Gesamtansatzberechnung }\end{array}$} & \multicolumn{4}{|c|}{ Gesamtansatzdifferenz } \\
\cline { 2 - 5 } & kreisangehöriger Raum & \multicolumn{2}{c|}{ kreisfreier Raum } \\
\cline { 2 - 5 } & (absolut) & (in v.H.) & (absolut) & (in v.H.) \\
\hline $\begin{array}{c}\text { Erhöhung der Einwohnerzahl } \\
\text { durch den Gesamtansatz }\end{array}$ & 1.328 .505 & 28,53 & 58.702 & 4,28 \\
\hline Sonderstatusstadt & 94.123 & 7,08 & - & - \\
\hline Stationierungsstreitkräfte & 16.123 & 1,21 & 15.679 & 26,71 \\
\hline Kurgastübernachtungen & 39.478 & 2,97 & 1.286 & 2,19 \\
\hline Bevölkerungszuwachs & 71.972 & 5,42 & & 0,00 \\
\hline Schulträgerschaft & 10.154 & 0,76 & - & - \\
\hline Hauptansatzveredelung & 1.069 .593 & 80,51 & 41.737 & 71,10 \\
\hline Zentralitätsansatz & 27.062 & 2,04 & - & - \\
\hline
\end{tabular}

Quelle: Eigene Berechnung nach Daten des Finanzministeriums zum KFA 1998.

Es ist ersichtlich, daß für beide Gebietskörperschaftgruppen die Hauptansatzveredelung mit 80,51 bzw. 71,10 v.H. den Großteil der Erhöhung der ursprünglichen Bevölkerungszahl ausmacht. Entsprechend spielen die anderen Ansätze kaum eine Rolle. Allerdings ist die Erhöhung der tatsächlichen Einwohnerzahl durch das Verfahren des KFA bei den beiden Gruppen sehr unterschiedlich, da der bedarfsdeterminierende Gesamtansatz bei den kreisangehörigen Gemeinden um fast 30 v.H. steigt, während dies bei den kreisfreien Städten etwas über 4 v.H. sind. Entsprechend wirken sich selbst die 71,10 v.H. der Einwohnerveredelung bei den kreisfreien Städten nur mit 3,04 v.H. auf den Gesamtansatz aus, während diese Größe für den kreisangehörigen Raum bei 22,96 v.H. liegt. Weit wichtiger als diese Werte ist aber, wievielen Gemeinden diese Ergänzungssätze zugute 
kommen. So wirkt sich die Steigerung des Hauptansatz auf alle kreisangehörigen Gemeinden aus, während dies beim Ansatz für die Schulträgerschaft nur sechs Gemeinden sind. ${ }^{1}$ Deshalb werden im folgenden die auftretenden distributiven Effekte der Abschaffung einzelner Nebenansätze auf die Verteilung der Schlüsselzuweisungen untersucht, wobei abgesehen vom jeweils betrachten Nebenansatz alle übrigen exogenen Daten konstant gehalten werden, so daß sich nur der Grundbetrag verändern kann. Weiterhin wird geprüft, inwieweit die derzeitigen Ergänzungsansätze überhaupt gerechtfertigt sind, also ob sie tatsächlich als Indikatoren für einen zusätzlichen Bedarf geeignet sind.

\subsection{Sonderstatusstädte}

Die durch den Ansatz für Sonderstatusstädte induzierten Umverteilungssummen sind in der Tabelle 17 dargestellt. ${ }^{2}$ Von den sieben Sonderstatusstädten profitieren abgesehen von Bad Homburg ${ }^{3}$ alle vom Nebenansatz. Es handelt sich hierbei um den höchst dotierten Ergänzungsansatz, so daß es aufgrund der geringen Anzahl der betroffenen Orte sowohl relativ (38,05 v.H.) als auch absolut $(107,14$ $\mathrm{DM}) \mathrm{zu}$ den höchsten Einbußen kommt. Während bei 22 von 421 Gemeinden keine Veränderung auftritt, profitieren 392 Gemeinden von der grundbetragserhöhende Abschaffung des Ansatzes.

Tabelle 17:Umverteilung bei Abschaffung des Sonderstatusstadtansatzes

\begin{tabular}{|c|r|r|r|}
\hline Kreisangehörige Gemeinden & \multicolumn{2}{|c|}{ Gemeinden mit Ansatz } & \multirow{2}{*}{$\begin{array}{c}\text { ohne Ansatz } \\
\end{array}$} \\
\cline { 2 - 3 } & Gewinner & Verlierer & \multicolumn{1}{c|}{ Gewinner } \\
\hline Anzahl & 0 & 6 & 392 \\
\hline Gewinn/Verlust in DM & 0,00 & -44.453 .305 & 44.452 .482 \\
\hline Gewinn/Verlust in v.H. & 0,00 & $-38,05$ & 4,47 \\
\hline Gewinn/Verlust in pro DM/Kopf & 0,00 & $-107,14$ & 11,52 \\
\hline
\end{tabular}

Quelle: Eigene Berechnung nach Daten des Finanzministeriums zum KFA 1998.

Der Nebenansatz für die Sonderstatusstädte kann dem Grunde nach - Übertragung von Kreisaufgaben - als nicht gerechtfertigt angesehen werden. Denn diese Orte übernehmen für ihre eigene Bevölkerung Aufgaben, die sonst ihre Landkreise wahrgenommen hätten, und müßten deshalb als finanziellen Ausgleich für die Kosten der Aufgabenwahrnehmung von den Kreisen Mittel zugewiesen

1 Der Hauptansatz für Gemeinden unter 5.000 Einwohner beträgt statt 100 v.H. 107 v.H. Bei den kreisangehörigen Schulträgern handelt es sich bis auf Wetzlar und Bad Homburg um alle Sonderstatusstädte und zusätzlich noch um die kreisangehörige Gemeinde Kelsterbach.

2 Auch hier zu beachten ist, daß die Gesamtsumme der über diesen Ansatz verteilten Schlüsselzuweisungen mit 48,76 Mio. DM um 4,31 Mio. DM höher ausfällt, als der auftretende Verlust, da eine Teilkompensation über den gestiegenen Grundbetrag erfolgt.

3 Diese Stadt ist abundant. 
bekommen bzw. an diese eine geringere Kreisumlage entrichten, da letztere zur Finanzierung der kreisinternen Aufgaben dient. Durch den Nebenansatz finanzieren aber noch andere Gemeinden über einen Verzicht auf Schlüsselzuweisungen die Sonderstatusstädte mit. Gleiches gilt für deren Kreise, da sich deren Bemessungsgrundlage für die Kreisumlage mindert, ${ }^{1}$ es über die Kreisschlüsselzuweisungen aber nur einen Ausgleich von 50 v.H. für diesen Verlust gibt. Mit den Kosten der kreisinternen Aufgabenverteilung in den Kreisen mit Sonderstatusstädten (Sonderkreise) werden somit die übrigen Gemeinden und deren Kreise (Normalkreise) belastet. Nur wenn mit dem Sonderstatus zusätzliche zentralörtliche Aufgaben für andere Gemeinden wahrgenommen würden, wäre eine Berücksichtigung bei den gemeindlichen Schlüsselzuweisungen denkbar. Folglich ist dieser Ansatz in der bisherigen Form nicht mehr beizubehalten. Bei seiner Abschaffung ist aber auch das "Lahn-Dill-Gesetz" zu ändern, nach dem für Gemeinden über 50.000 Einwohnern "insbesondere die Gemeindeschlüsselzuweisungen und die Kreisumlage den im Vergleich zu anderen kreisangehörigen Gemeinden geänderten Aufgabenzuständigkeiten anzupassen" ${ }^{2}$ sind. Aufgrund der Interdependenzen zwischen diesem Ansatz und der Berücksichtigung der Sonderstatusstädte bei den kommunalen Umlagen wird auf die Bedarfsmessung der Kreise verwiesen. ${ }^{3}$

Aus politischen Gründen könnte für Orte dieser Größe weiterhin ein vergleichbar hoher Bedarf vorgesehen sein, wobei dann als Argument z.B, das schon angesprochene Gutachten zur Hauptansatzstaffel der kreisangehörigen Gemeinden anzuführen wäre, wonach sich für diese Gruppe ein Hauptansatz von 136 v.H. der Einwohnerzahl ergeben würde. ${ }^{4}$ Dieser Wert könnte zwar wieder in einen Ansatz für Sonderstatusstädte umgewandelt werden, wodurch die Spreizung des Hauptansatzes und somit auch der Widerstand anderer Gemeinden verringert würde, aber auch die Transparenz, wie schon bei der derzeitigen Regelung festgestellt werden kann. ${ }^{5}$ Der Bedarf wäre deshalb aufgrund der höheren Transparenz, aber auch um die Begründung dieser Veredelung - eben nicht wegen der Übernahme

1 Denn die Schlüsselzuweisungen gehen in die Bemessungsgrundlage der Kreisumlage ein. Da die übrigen Gemeinden aufgrund des Nebenansatzes für Sonderstatusstädte geringere Schlüsselzuweisungen erhalten, mindert sich c.p. die Einnahme aus der Kreisumlage.

2 Artikel 3, § 10 des Gesetzes zur Neugliederung des Lahn-Dill-Gebiets und zur Übertragung von weiteren Aufgaben auf kreisangehörigen Gemeinden mit mehr als 50000 Einwohnern sowie zur Regelung sonstiger Fragen der Verwaltungsreform vom 10.07.1979, Hess. GVBl. I, S. 183.

3 Siehe Abschnitt 4.4.6.2.3.

4 Vgl:: Kisseler, W.; Gretz, W.: Hauptansatzstaffel im Kommunalen Finanzausgleich. Eine empirische Untersuchung für Hessen, in: HLT Gesellschaft für Forschung, Planung und Entwicklung: HLT-Report Nr. 574, Wiesbaden 1999, S. 33.

5 So spricht BRỚCKMANN davon, daß sich bei Sonderstatusstädten der Hauptansatz um 15 Prozentpunkte erhöht, statt um 15 v.H. Vgl.: Brückmann, F.: Die Zentralität - Die vernachlässigte Bedarfsgröße im hessischen kommunalen Finanzausgleich, in: Kommunale SteuerZeitschrift, 39. Jg., 1990, S. 162. 
von Kreisaufgaben - darzulegen, im Hauptansatz zu belassen. Soll also trotz gegenteiliger Hinweise die Höhe der derzeitigen Bedarfssteigerung für die Sonderstatusstädte beibehalten werden, so ergibt sich nach Integration in die Hauptansatzstaffel für Gemeinden über 50.000 Einwohnern ein Wert von 149,5 v.H. Allerdings ist dann als Folge eine Anhebung des Mindestansatzes für die kreisangehörigen Oberzentren notwendig, da sich bei diesen derzeitig durch den Ergänzungsansatz der Hauptansatz auf 161 v.H. erhöht. ${ }^{1}$

\subsection{Stationierungsstreitkräfte}

Der Nebenansatz für die Stationierungsstreitkräfte wurde 1960 eingeführt. Im Jahr 1998 fielen unter diesen Ansatz noch 27 Gemeinden mit über 663.000 Einwohnern und drei kreisfreie Städte mit ca. 1.053.000 Einwohnern. ${ }^{2}$ Die Stationierungsstreitkräfte hatten mit 16.123 bzw. 15.679 Personen einen Anteil von 2,43 bzw. 1,49 v.H. an der Einwohnerzahl. Durch diesen Ansatz werden bei den kreisfreien Städten 18,4 Mio. bei den kreisangehörigen Gemeinden 10,2 Mio. DM verteilt; dies entspricht etwa 3,70 bzw. 0,92 v.H. der vorhandenen Schlüsselmasse. Bei Abschaffung des Ansatzes entstehen die in Tabelle 18 angeführten Umverteilungswirkungen, die aber geringer sind als die verteilten Summen, da sich gleichzeitig der Grundbetrag erhöht hat.

Tabelle 18:Umverteilung bei Abschaffung des Stationierungsansatzes

\begin{tabular}{|c|c|c|c|}
\hline \multirow[b]{2}{*}{ Kreisfreie Städte } & \multicolumn{2}{|c|}{ Gemeinden mit Ansatz } & \multirow{2}{*}{$\begin{array}{c}\text { ohne Ansatz } \\
\text { Gewinner }\end{array}$} \\
\hline & Gewinner & Verlierer & \\
\hline Anzahl & 1 & 2 & 2 \\
\hline Gewinn/Verlust in DM & 6.331 .416 & -11.506 .258 & 5.171 .430 \\
\hline Gewinn/Verlust in v.H. & 8,10 & $-5,54$ & 2,46 \\
\hline Gewinn/Verlust in pro DM/Kopf & 9,78 & $-28,33$ & 16,29 \\
\hline \multicolumn{4}{|l|}{ Kreisangehörige Gemeinden } \\
\hline Anzahl & 1 & 25 & 370 \\
\hline Gewinn/Verlust in DM & 54.497 & -8.685 .541 & 8.627 .893 \\
\hline Gewinn/Verlust in v.H. & 0,37 & $-5,61$ & 0,92 \\
\hline Gewinn/Verlust in pro DM/Kopf & 1,01 & $-14,42$ & 2,40 \\
\hline
\end{tabular}

Quelle: Eigene Berechnung nach Daten des Finanzministeriums zum KFA 1998.

1 Die 149,5 v.H. ergeben sich durch Multiplikation des Hauptansatzes für Orte über 50.000 Einwohner (130 v.H.) mit dem Nebenansatz für Sonderstatusstädte (1,15 v.H.) Bei den kreisangehörigen Oberzentren handelt sich um die Sonderstatusstädte Marburg, Fulda und Gießen. Diesen wird ein Zentralitätsansatz von 140 v.H. gewährt. 140 v.H. ${ }^{*} 1,15=161$ v.H.

2 Die kreisangehörige Gemeinde Großkrotzenburg profitiert nicht vom Stationierungsansatz, da sie abundant ist. 
- Von den drei kreisfreien Städten mit Stationierungsstreitkräften vermindern sich bei zweien die Schlüsselzuweisungen um 11,5 Mio. DM, da sich ihr Anteil am Gesamtbedarf von 29,841 auf 29,792 vermindert, während die dritte den theoretischen Schlüsselzuweisungsverlust mit einem Plus von 6,3 Mio. DM überkompensieren kann, da ihr Anteil von 47,653 auf 47,696 v.H. steigt. Die beiden übrigen Städte profitieren durch den höheren Grundbetrag und einem ebenfalls steigenden Anteil am Gesamtbedarf (von 22,507 auf 22,512 v.H.).

- Von 26 kreisangehörigen Gemeinden, die vom Stationierungsansatz profitieren, erleiden 25 einen Schlüsselzuweisungsverlust von insgesamt 8,7 Mio. DM, während eine Gemeinde trotz Abschaffung des Nebenansatzes ibre Schlüsselzuweisungen um 54.497 DM steigern kann. ${ }^{1}$ Von den 394 Gemeinden ohne Standorte ändert sich für 24 von ihnen nichts, da sie Schlüsselzuweisungen nur nach der Einwohnerzahl erhalten die übrigen steigern ihre Zuweisungen um etwa 1 v.H.

Dieser Ergänzungsansatz wird abgesehen von Nordrhein-Westfalen und Schleswig-Holstein auch in anderen Bundesländer in vergleichbarer Form praktiziert, doch nur im Saarland gehen die Stationierungsstreitkräfte zu 100 v.H., ansonsten mit 15 v.H. ein, so daß der hessische Ansatz als zu hoch erscheint. ${ }^{2}$ Es stellt sich aber die Frage, ob dieser Ansatz grundsätzlich gerechtfertigt ist, denn die ausländischen Streitkräfte haben für ihre Angehörigen eigene Anlagen bereitgestellt, so $\mathrm{da} B$ die Belastung der Kommunen durch zusätzliche Nachfrager in Form von Armeeangehörigen als sehr gering anzusehen ist. ${ }^{3}$ Weiterhin haben sich diese theoretischen Lasten aufgnund des massiven Abbaus der stationierten Truppen stark verringert. Der Umstand, daß von der EU finanzielle Mittel zur Verringerung der mit der Konversion einhergehenden Lasten bereitgestellt wurden, ${ }^{4}$ zeigt, daß es sich bei den Stationierungsstreitkräften weniger um eine lasten-, sondern eher um wohlstandsinduzierende Gruppe handelt. Denn durch die Standorte werden

1 Es handelt sich dabei um die Sonderstatusstadt Wetzlar, deren Anteil an den Stationierungsstreitkräften 0,76 v.H. betrug, am modifizierten Gesamtbedarf aber 1,35 v.H.

2 Für Rheinland-Pfalz ist ebenfalls die Abschaffung gefordert worden. Vgl.: Steinherr, M; Parsche, R.: Der Ausgleich zwischen Finanzbedarf und Finanzkraft im kommunalen Finanzausgleich des Landes Rheinland-Pfalz, ifo-Studien zur Finanzpolitik, Bd. 66, München 1998, S. 46.

3 Ursprünglich wurden die Stationierungsstreitkräfte aufgrund der geringeren Beanspruchung der öffentlichen Leistungen auch nur zu 50 v.H. berücksichtigt, 1977 wurde dann eine Anrechnung zu 100 v.H. eingefürt. Vgl.: Hessischer Landtag: Vorlage der Landesregierung für ein Gesetz zur Regelung des Finanzausgleichs vom 08.12.1959, Drucksachen Abteilung I, 4/405, S. 1178, ders.: Gesetzentwurf der Landesregierung für ein Gesetz zur Regelung des Finanzausgleichs und zur Änderung anderer Vorschriften vom 11.10.1977, Drucksache 8/4877, S. 36.

4 Vgl.: Hardt, U.; Schmidt, J.: Neuordnung des kommunalen Finanzausgleichs in Niedersachsen. Eine Untersuchung im Auftrag des Niedersächsischen Innenministeriums, hrsg. vom Niedersächsischen Innenministerium, Hannover 1998, S. 121. 
zusätzliche Steuereinnahmen in den Gemeinden erzielt. ${ }^{1}$ Somit ist fraglich, ob die Stationierungsstreitkräfte noch bedarfssteigernd zu berücksichtigten sind.

Soll dies noch für eine Übergangszeit geschehen, um mögliche Friktionen zu mildern, so ist die Dimensionierung zu prüfen, wobei der zu ermittelnde Zuschußbedarf als Indikator für den Bedarf genutzt werden kann. ${ }^{2} \mathrm{Zu}$ diesem Zweck werden die Ausgaben der Aufgabenbereiche betrachtet, die keinerlei Verbindungen zu den Stationierungsstreitkräften aufweisen. $\mathrm{Zu}$ nennen sind etwa Soziale Sicherung, sowie etwa 70 v.H. der Ausgaben der übrigen Bereiche, was hier sicherlich zu einer Unterschätzung des durch die Einwohner induzierten Bedarfs führt. Wird vom Zuschußbedarf der auf diese Aufgaben entfallende Anteil abgezogen, so ergibt sich ein Indiz für die maximale Bedarfsverursachung der Stationierungsstreitkräfte. ${ }^{3}$ Allerdings sind auch die Landkreise in dieser Größe enthalten, denn in den Statistiken wird keine weitergehende Trennung für den kreisangehörigen Raum vorgenommen. Wird deren Anteil auf 40 v.H. geschätzt - der durchschnittliche Kreisumlagesatz als Indiz für die Aufteilung auf der Einnahmenseite liegt bei 42,49 v.H. -, so ergäbe sich als Obergrenze für die durch Stationierungsstreitkräfte induzierten Bedarfe ein Wert von 48 v.H. des Betrags, der auf einen Einwohner entfällt. Aufgrund der getroffenen Annahmen dürfte der Wert noch darunter liegen. So wird für Rheinland-Pfalz - einem Bundesland mit einem höheren Anteil an Stationierungsstreitkräften an der Bevölkerung - die Berücksichtigung mit 30 v.H. als angemessen angesehen. ${ }^{4}$

Würden die Stationierungsstreitkräfte nun bei der Bedarfsermittlung mit 50 v.H. berücksichtigt, wie in Rheinland-Pfalz derzeit der Fall, so würden die höchsten Verluste in einer Größenordnung von 0,6 (Butzbach), 1,4 (Hanau) bzw. 4,9 Mio. DM (Wiesbaden), also bei maximal 7,18 v.H., 5,93 v.H. bzw. 3,6 v.H. der gesamten ursprünglichen Schlüsselzuweisungen liegen. Daran sieht man, daß dieser Ansatz abgebaut werden kann, gegebenenfalls mit vorübergehenden Sonderzuweisungen an die überdurchschnittlich stark betroffenen Gemeinden.

Für diese Vermutung spricht auch, daß es massive Proteste gegen Standortschließungen bei der Bundeswehr von Seiten der betroffenen Gemeinden gegeben hat.

2 Vgl.: Deubel, I.; Münstermann, E.: Reiche Städte - Arme Landkreise? - Zur aktuellen Verteilungsproblematik im kommunalen Finanzausgleich am Beispiel des Landes NordrheinWestfalen -, in: ZKF, 38. Jg., 1988, S. 247.

3 In diesem Fall muß vom Gesamtzuschußbetrag der ermittelte Wert abgezogen werden, um den maximalen Anteilswert der Stationierungsstreitkräfte zu erhalten. Dieser wird noch darunter liegen, da nur die Beträge abgezogen wurden, die ausschließlich durch die Einwohner verursacht wurden, im Residuum werden die Anteile der Einwohner immer noch sehr hoch sein.

4 Vgl.: Steinherr, M; Parsche, R.: Der Ausgleich zwischen Finanzbedarf und Finanzkraft im kommunalen Finanzausgleich des Landes Rheinland-Pfalz, ifo-Studien zur Finanzpolitik, Bd. 66, München 1998, S. 44. 


\subsection{Kurorte}

Aufgrund des Ergänzungsansatzes für Heilkurorte wurden 199829 Gemeinden $^{1}$ und zwei kreisfreie Städte ein erhöhter Bedarf zugerechnet, der zu zusätzlichen Schlüsselzuweisungen in Höhe von 32,3 Mio. DM geführt hat. Die Verteilungswirkungen einer Streichung dieses Ansatzes sind aus Tabelle $19 \mathrm{zu}$ entnehmen.

Tabelle 19:Umverteilung bei Abschaffung des Heilkurorteansatzes

\begin{tabular}{|c|c|c|c|}
\hline \multirow[b]{2}{*}{ Kreisfreie Städte } & \multicolumn{2}{|c|}{ Gemeinden mit Ansatz } & \multirow{2}{*}{$\begin{array}{l}\text { ohne Ansatz } \\
\text { Gewinner }\end{array}$} \\
\hline & Gewinner & Verlierer & \\
\hline Anzahl & 0 & 2 & 3 \\
\hline Gewinn/Verlust in DM & 0,00 & -1.001 .531 & 1.000 .236 \\
\hline Gewinn/Verlust in v.H. & 0,00 & $-0,36$ & 0,46 \\
\hline Gewinn/Verlust in pro DM/Kopf & 0,00 & $-2,14$ & 1,11 \\
\hline \multicolumn{4}{|l|}{ Kreisangehörige Gemeinden } \\
\hline Anzahl & 2 & 26 & 369 \\
\hline Gewinn/Verlust in DM & 139.341 & -28.464 .715 & 28.304 .981 \\
\hline Gewinn/Verlust in v.H. & 1,96 & $-25,27$ & 2,85 \\
\hline Gewinn/Verlust in pro DM/Kopf & 4,15 & $-99,42$ & 7,17 \\
\hline
\end{tabular}

Quelle: Eigene Berechnung nach Daten des Finanzministeriums zum KFA 1998.

- Bei den zwei kreisfreien Städten, die als Heilkurorte klassifiziert sind, verringern sich die Schlüsselzuweisungen um knapp 1,0 Mio. DM, was einem Pro-KopfBetrag von 2,14 DM entspricht. Die übrigen drei Städte verbuchen zusätzliche Schlüsselzuweisungen von etwas mehr als 1,0 Mio. DM, dies sind 1,11 DM pro Kopf.

- Von den 28 kreisangehörigen Gemeinden, die durch diesen Nebenansatz zusätzliche Schlüsselzuweisungen erhalten, steigen bei zweien trotz Abschaffung die Schlüsselzuweisungen um 139.341 DM (1,96 v.H.), da ihr Anteil am Nebenansatz gering gewesen ist und sich nach dessen Abschaffung ihr Anteil am Gesamtbedarf aller Gemeinden erhöht hat. Die restlichen 26 Gemeinden verlieren insgesamt fast 28,5 Mio. DM (25,27 v.H. ihrer Schlüsselzuweisungen). Da sich der übrige Gewinn auf 369 Gemeinden verteilt, erhöhen sich deren Schlüsselzuweisungen nur um $2,85 \mathrm{v} . \mathrm{H}$.

Die grundsätzliche Ausgestaltung des Kurorteansatzes ist zu kritisieren. So ist nicht nachvollziehbar, warum nur die Aufwendungen in Heilkurorten berücksichtigt werden sollen, während dies in anderen anerkannten Fremdenverkehrsorten nicht der Fall ist, obwohl dort die Aufwendungen in vergleichbarer Höhe liegen

1 Bad Homburg erhält aufgrund der Abundanz keinen Vorteil aus diesem Ansatz. 
dürften. Weiterhin bedeutet die Gleichsetzung von 250 Übernachtungen mit einem Einwohner, daß ein Dauergast über das Jahr gesehen mit einem Faktor von 1,46, also fast 50 v.H. stärker als ein tatsächlicher Einwohner gewichtet wird, was durch die Beanspruchung der öffentlichen Leistungen kaum gerechtfertigt werden kann. ${ }^{1}$

Auch ist zweifelhaft, ob der Status eines Heilkurortes als eine Last anzusehen ist, da die Gemeinden diesen erst auf Antrag erhalten und der Tourismus somit insgesamt positiv von den Kommunen beurteilt wird. ${ }^{2}$ Denn der Fremdenverkehr induziert zusätzliche Nachfrage und erhöht dadurch die Einnahmen bei Gewerbeund Einkommensteuer und schafft auch in der Regel zusätzliche Arbeitsplätze. ${ }^{3}$ Es ist somit wahrscheinlicher, daß es höchstens zu einer falschen Verteilung der durch den Tourismus induzierten Gewinne und Lasten zwischen öffentlichen und privaten Haushalten kommt, nämlich zu ungunsten des Gemeindefiskus. Aber selbst für den Fall, daß der Fremdenverkehr negative externe Effekt bei den betroffenen Kommunen auslöst, ist eine Internalisierung innerhalb der Orte und nicht über die Solidargemeinschaft der kommunalen Ebene anzustreben. ${ }^{4}$

Für eine innerkommunale Lösung des Problems stehen den Gemeinden verschiedene Instrumente zur Verfügung. Touristen können direkt durch Kurbeiträge - zumindest in Kurorten - oder indirekt über - auf sie abgewälzte, von den begünstigten Unternehmen zu zahlende - Fremdenverkehrsbeiträge belastet werden. ${ }^{5}$ Aus den vorhergehenden Überlegungen kann abgeleitet werden, daß dieser Ansatz überflüssig zu sein scheint, da es adäquate Finanzierungsinstrumente gibt, die auch in anderen Bundesländern angewendet werden. So betragen z.B. in BadenWürttemberg die Einnahmen aus Fremdenverkehrsabgaben 16,0 Mio. DM, während es in Hessen keinerlei Einnahmen gibt. ${ }^{6}$ Sie machen in Baden-Württemberg

1 Der Wert ergibt sich aus 365 (Jahrestage) dividiert durch 250 (Kurgastübernachtungen) = 1,46. In Rheinland-Pfalz etwa werden 600 Übernachtungen als ein Einwohner gewertet, Faktor 0,608.

2 Vgl.: Steinherr, M.: Kommunaler Finanzausgleich: Balanceakt zwischen Verteilungs- und Allokationszielen - Das Beispiel Rheinland-Pfalz, in: ifo-Schnelldienst, 51. Jg., Nr. 8, 1998, S. 23

3 Vgl.: Bienert, F.: Zur Bestimmung der ökonomischen Auswirkungen des Fremdenverkehrs unter Berücksichtigung seines Einflusses auf den Haushalt einer Gemeinde - Eine empirische Fallstudie. Dargestellt am Beispiel der Stadt Westerland auf Sylt, Berlin 1974.

4 Vgl.: Hardt, U.; Schmidt, J.: Neuordnung des kommunalen Finanzausgleichs in Niedersachsen. Eine Untersuchung im Auftrag des Niedersächsischen Innenministeriums, hrsg. vom Niedersächsischen Innenministerium, Hannover 1998, S. 122.

$5 \mathrm{Zu}$ den unterschiedlichen Instrumenten zur Internalisierung der Kosten des Fremdenverkehrs vgl.: Bayer, H.-W.; Elemenhorst, R.: Das System des gemeindlichen Fremdenverkehrsrechts, in: Kommunale Steuer-Zeitschrift, 44. Jg., 1995, S. 143-147.

6 Vgl.: Statistisches Bundesamt: Fachserie 14, Finanzen und Steuern, Reihe 3.3, Rechnungsergebnisse der kommunalen Haushalte 1996, Stuttgart 1997, S. 62. 
mehr als die Hälfte des Betrages aus, der in Hessen über den Kurortenebenansatz verteilt wird.

Es ist aber zu berücksichtigen, daß für einige wenige Gemeinden mit der vollständigen Streichung des Ansatzes kurzfristig kaum verkraftbare finanzielle Einbußen einhergehen. Würde beispielsweise eine Anpassung des Heilkurorte Ansatzes derart erfolgen, daß 730 Übernachtungen einem Einwohner entsprechen ${ }^{1}$, so würden sich die Schlüsselzuweisungen für Bad Wildungen um 3,25 Mio. (23,2 v.H.), für Bad Salzschlirf um 0,6 Mio. (28,2 v.H.) und für Willingen um 1,9 Mio. DM (36,37 v.H.) verringern. Für einen Übergangszeitraum sollten deshalb Zuweisungen gewährt werden, um die größten Friktionen zu mildern.

\subsection{Bevölkerungswachstum}

Der Ergänzungsansatz für Bevölkerungswachstum wirkt sich derzeit nur bei den kreisangehörigen Gemeinden bedarfserhöhend aus, da nur dort der zur Gewährung des Ansatzes notwendige Einwohneranstieg zu verzeichnen ist. Die Auswirkungen auf die Verteilung der Schlüsselzuweisungen ist in Tabelle 20 abgebildet.

Tabelle 20:Umverteilung bei Abschaffung des gemeindlichen Ansatzes für Bevölkerungszuwachstum

\begin{tabular}{|c|r|r|r|}
\hline Kreisangehörige Gemeinden & \multicolumn{2}{|c|}{ Gemeinden mit Ansatz } & \multirow{2}{*}{$\begin{array}{c}\text { ohne Ansatz } \\
\text { Gewinner }\end{array}$} \\
\cline { 2 - 3 } & Gewinner & Verlierer & \multicolumn{1}{c|}{197} \\
\hline Anzahl & 66 & 135 & 25.378 .333 \\
\hline Gewinn/Verlust in DM & 4.387 .335 & -29.781 .331 & 4,85 \\
\hline Gewinn/Verlust in v.H. & 2,58 & $-7,12$ & 11,44 \\
\hline Gewinn/Verlust in pro DM/Kopf & 6,91 & $-20,88$ & \\
\hline
\end{tabular}

Quelle: Eigene Berechnung nach Daten des Finanzministeriums zum KFA 1998.

Insgesamt wurden über diesen Ansatz, der für 208 Gemeinden relevant ist, 49,29 Mio. DM umverteilt. Es handelt sich somit um den Ergänzungsansatz, von dem die größte Gemeindezahl profitiert. Wird auf diesen Ansatz verzichtet, so steigen bei 66 dieser 208 Gemeinden die Schlüsselzuweisungen um zusammen 4,37 Mio. DM (2,58 v.H.), während 135 Orte 29,78 Mio. DM (7,12 v.H.) verlieren und bei den übrigen sieben keinerlei Veränderungen auftreten. Von den 213 Gemeinden ohne entsprechendes Bevölkerungswachstum erhöhen sich abgesehen von den 16 abundanten Orten bei 197 Gemeinden die Zuweisungen um insgesamt 25,38 Mio. $\operatorname{DM}(4,85$ v.H.).

1 Bei diesem Wert wird ein Dauerübernachtungsgast - wie die Stationierungsstreitkräfte - einem halben Einwohner gleichgesetzt. 
Gegen den beschriebenen Ergänzungsansatz für das Bevölkerungswachstum wird angeführt, daß die ungewünschte Suburbanisierung finanziell durch Zuweisungen unterstützt wird. ${ }^{1}$ Denn die kleineren Umlandgemeinden haben aufgrund der zusätzlichen Schlüsselzuweisungen die Möglichkeit, durch Baumaßnahmen wie etwa Kindergärten o.ä. verstärkt Einwohner aus den Kernstädten anzulocken, was die unerwünschte Zersiedlung der Landschaft verstärkt und zu einer Entleerung der Großstädte führt. ${ }^{2}$

Da die finanzielle Förderung der zentralen Orte ein wichtiges Anliegen des KFA ist, gilt es zu prüfen, ob zwischen den beiden genannten Ansätzen Zielkomplementarität oder -dichotomie besteht. Die 208 Orte mit einem Bevölkerungswachstum von mehr als 10 v.H., die somit vom Nebenansatz profitieren, weisen im Durchschnitt eine Steigerung von 15,80 v.H. auf. Bei den zentralen Orten ist hingegen nur ein Bevölkerungsanstieg von durchschnittlich 14,69 v.H. festzustellen, wobei es keine Rolle spielt, ob der Zentraleorteansatz zur Geltung kommt oder nicht. ${ }^{3}$ Die übrigen 169 Orte mit hohem Bevölkerungswachstum ohne raumordnungspolitische Funktion verzeichnen im Durchschnitt ein Wachstum vom 16,05 v.H. Somit tritt das Problem auf, daß durch den Nebenansatz für Bevölkerungswachstum in der Tat durch zusätzliche Zuweisungen die Zersiedlung gefördert bzw. zumindest nicht verringert wird, da er dem Ziel der finanziellen Förderung zentraler Orte entgegenwirkt. Denn nichtzentale Orte werden durch diesen Ansatz stärker mit zusätzlichen Schlüsselzuweisungen gefördert als zentrale. Weiterhin verringert sich die Gewichtung des Zentraleorteansatzes am Gesamtansatz. ${ }^{4}$ Dieser Zusammenhang spricht eher für eine Abschaffung des Nebenansatzes für Bevölkerungswachstum.

Nachdem festgestellt wurde, daß der Ergänzungsansatz für Bevölkerungswachstum eher das raumordnungspolitische Ziel der finanziellen Förderung zentraler Orte konterkariert, ist nun die Notwendigkeit des genannten Ansatzes zu prüfen. Sie wird damit begründet, daß mit einer stark steigenden Bevölkerungszahl auch ein Wachstum der öffentlichen Leistungen einhergeht, welches aber nicht

1 Vgl.: Eckey, H.-F.: Möglichkeiten der Anpassung und Veränderungen des Kommunalen Finanzausgleichssystems für raumordnerische Zielsetzungen, in: Informationen zur Raumentwicklung: Die Reform der Kommunalfinanzen, Heft 8/9, hrsg. von der Bundesforschungsanstalt für Landeskunde und Raumentwicklung, Bonn 1995, S. 594.

2 Vgl.: Postlep, R.-D.: Zur Berücksichtigung raumordnerischer Belange im Kommunalen Finanzausgleich, in: Informationen zur Raumentwicklung: Die Reform der Kommunalfinanzen, Heft 8/9. 1995, hrsg. von der Bundesforschungsanstalt für Landeskunde und Raumentwicklung, Bonn 1995, S. 601.

3 Wie angeführt, ergibt sich aufgrund der Unterdimensionierung dieses Ansatzes nur für etwa 30,93 v.H. der Gemeinden mit zentralörtlicher Aufgabenerfüllung eine Bedarfssteigerung.

4 Denn durch den Ansatz für Bevölkerungswachstum erhöht sich der Gesamtansatz aller Gemeinden, wodurch der Grundbetrag sinkt und den zentralen Orten weniger Schlüsselzuweisungen zufließen. 
ausschließlich über direkte Entgelte finanziert werden kann. Dabei ist zu beachten, daß möglicherweise auch Gemeinden mit sinkender Bevölkerungszahl zu berücksichtigen sind, da dort die Einnahmen schneller als die Ausgaben - aufgrund "sprungfixer" Kosten - sinken. Sollte die Notwendigkeit nicht gegeben sein, wäre der Ansatz wegen des genannten Zielkonflikts abzuschaffen, ansonsten wäre eine Modifikation anzustreben, um zumindest Zielneutralität zu erreichen.

Zwar wirkt sich ein Bevölkerungsanstieg erst mit zeitlicher Verzögerung in höheren Einkommensteuereinnahmen aus, denn die zu seiner Verteilung relevanten Schlüsselzahlen werden erst mit mehrjähriger Verspätung angepaßt, ${ }^{1}$ doch die Schlüsselzuweisungen erhöhen sich bei steigender Bevölkerung schon im folgenden Jahr. Zudem werden noch zweckgebundene Zuweisungen zur Straßenunterhaltung und auch Investitionszuweisungen gewährt. Insofern erscheint ein Nebenansatz für Bevölkerungswachstum nicht notwendig, da während starker Wachstumsphasen relativ mehr Zuweisungen zufließen. Bei stagnierender Einwohnerzahl ergeben sich dann folglich automatisch relativ geringere Schlüsselzuweisungen. Ein Problem stellen deshalb Orte mit stark und schnell schrumpfender Bevölkerung aufgrund der Problematik der "sprungfixen" Kosten dar. Allerdings erhalten diese wegen des Verteilungsverfahrens des kommunalen Einkommensteueranteils über Jahre hinweg höhere Steuereinnahmen als ihnen bei einem zeitnahen Verteilungsschlüssel zuständen. Dieser Umstand führt dann auch dazu, daß für Orte mit großen Nettowanderungsverlusten höchstens in Extremsituationen Zuweisungen zu gewähren sind. Folglich ist der Ansatz abzubauen.

Werden nun die tatsächlichen Einwohnerverluste bei hessischen Gemeinden betrachtet, so ist festzustellen, daß nur bei 32 Orten - überraschenderweise bei keiner kreisfreien Stadt - im letzten Jahrzehnt ein Wanderungsverlust festzustellen gewesen ist. Dies läßt sich auch mit der Einwohnerentwicklung für ganz Hessen erklären, die im Vergleich zwischen 1986 und 1996 um 8,72 v.H. gestiegen ist. Nur insgesamt zwei Gemeinden weisen eine Minderung der Einwohnerzahl um mehr als 10 v.H. auf, nämlich die Gemeinden Haina (Kreis Waldeck-Frankenberg) und Wahlsburg (Kreis Kassel) mit insgesamt 13,33 v.H. bzw. 12,09 v.H. Aufgrund dieses Sachverhalts würden sich statt eines entsprechenden Nebenansatzes im System der Schlüsselzuweisungen eher Zuweisungen aus dem Kreisausgleichsstock nach § 42 FAG oder notfalls aus dem Landesausgleichsstock nach $\S$ 28 FAG anbieten, um möglicherweise auftretende Probleme finanziell zu mindern.

1 So gilt für die Jahre 1997-1999 die Lohn- und Einkommensteuerstatistik von 1992. Vgl: Karrenberg, H.; Münstermann, E.: Der Gemeindefinanzbericht 1997, in: Der Städtetag, 50. Jg., 1997, S. 145. 


\subsection{Schulträgerschaft}

Vom Nebenansatz für die kreisangehörigen Schulträgergemeinden profitieren derzeit fünf der sechs Schulträger dieser Art. ${ }^{1}$ Insgesamt werden über diesen Ansatz 1998 6,38 Mio. DM verteilt (siehe Tabelle 21).

Tabelle 21:Umverteilung bei Abschaffung des Schulträgeransatzes

\begin{tabular}{|c|r|r|r|}
\hline \multirow{2}{*}{ Kreisangehörige Gemeinden } & \multicolumn{2}{|c|}{ Gemeinden mit Ansatz } & \multirow{2}{*}{$\begin{array}{c}\text { ohne Ansatz } \\
\end{array}$} \\
\cline { 2 - 3 } Gewinner
\end{tabular}

Quelle: Eigene Berechnung nach Daten des Finanzministeriums zum KFA 1998.

$\mathrm{Da}$ die Mittel, die über diesen Nebenansatz verteilt werden, bei dessen Abschaffung allen übrigen Gemeinden ebenfalls zugute kämen, ergibt sich für diese nur ein sehr geringer finanzieller Vorteil von 1,47 DM pro Kopf, während die fünf Schulträgergemeinden, die auch von diesem Ansatz profitieren, fast 16 DM pro Kopf an Schlüsselzuweisungen verlören, was etwa 5,7 v.H. ihrer Schlüsselzuweisungen ausmacht.

Prinzipiell ist die Gewährung eines Schulträgeransatzes gerechtfertigt, um die damit verbundenen Belastungen der Gemeinden zu berücksichtigen. Die parallele Realisierung von Sonderlastenausgleich und Ergänzungsansatz ist aber nicht unproblematisch. Denn aufgrund der zweifachen Berücksichtigung der Kosten der Schulträgerschaft wird der Kostenausgleichsgrad nicht offengelegt, so daß es teilweise zu einer ungewünschten Überdeckung der tatsächlichen Kosten kommen kann. Aus diesem Grund ist die Berücksichtigung dieser Lasten auf ein System zu fokussieren. Für einen Sonderlastenausgleich spricht, daß die Gebietskörperschaften kaum Einfluß auf diesen Aufgabenblock haben. Andererseits spricht für den Nebenansatz, daß dieser zur Erhöhung der Verteilungsgerechtigkeit und des Anteils zweckungebundener Zuweisungen führt, was als gewichtiger anzusehen ist. Bei Beibehaltung des Nebenansatzes wäre noch zu klären, ob weiterhin die Schülerzahlen einheitlich oder in Abhängigkeit von der Schulart unterschiedlich gewichtet werden sollen, wie dies z.B. in Nordrhein-Westfalen der Fall ist. ${ }^{2} \mathrm{Zu}$ diesem Zweck sollte bei allen Schulträgern untersucht werden, inwieweit es zu Belastungsdifferenzen kommt. Erst wenn die möglicherweise auftretenden

Die Gemeinde Kelsterbach erhält Mindestzuweisungen, so daß keine Änderungen auftreten.

2 Vgl.: Storck, F.: Der Schüleransatz im Finanzausgleich des Landes NW, in: Städte- und Gemeinderat, 38. Jg., 1984, S. 15; Junkernheinrich, M.: Sonderbedarfe im kommunalen Finanzausgleich, Berlin 1992, S. 55. 
Unterschiede nicht mehr akzeptabel sein sollten, wäre eine schulformabhängige Gewichtung einzuführen. Denn dem Vorteil der genaueren Belastungserfassung stehen die Kosten der dafür jährlich notwendigen Differenzierung gegenüber.

\subsection{Notwendigkeit weiterer Nebenansätze?}

In anderen Bundesländern gibt es noch weitere Ansätze, mit deren Hilfe Bedarfe berücksichtigt werden sollen, die nicht ausreichend mit der tatsächlichen Einwohnerzahl korrelieren. Denn nur für diesen Fall wäre ein zusätzlicher Nebenansatz sinnvoll, da zu beachten ist, daß die "genauere" Bedarfserfassung mit einer Komplizierung des Systems einhergeht. Bei der Auswahl anderer Ansätze ist zu beachten, daß die bedarfsinduzierende Gruppe gut abgrenzbar sein muß, um Manipulationen zu vermeiden. Man könnte dabei etwa etwa an Studenten und Wehrpflichtige denken; ${ }^{1}$ aber auch die Zahl von (nicht schulpflichtigen) Kindern kann sich erhöhend auf den Finanzbedarf auswirken, besonders nach Einführung der Kindergartenplatzgarantie.

So bestand in Hessen bis zur Finanzausgleichsreform 1977 ein Nebenansatz für die Bevölkerungszusammensetzung. Er berücksichtigte den Anteil der unter 18jährigen an der jeweiligen Gemeindebevölkerung, wobei nur bei Überschreitung einer Mindestquote von einer Bedarfssteigerung ausgegangen wurde. Die besondere Berücksichtigung dieser Bevölkerungsgruppe wurde damit begründet, daß "ein hoher Anteil von $\mathrm{K}$ i n d e r $\mathrm{n}$ u n d J u g e $\mathrm{n} \mathrm{d} \mathbf{l}$ i c h e $\mathrm{n}$ an der Einwohnerzahl einen besonderen Mehrbedarf (Kinderkrippen, Kindergärten, Kinderhorte (...))" begründen, "ohne zu den Einnahmen der Gemeinden nennenswert beizutragen"2. Er wurde mit einer lapidaren Begründung abgeschafft: "Nach der Gebietsreform ist jedoch davon auszugehen, daß die Bevölkerungsentwicklung in allen Landesteilen in etwa gleich verläuft, soweit die Bevölkerungszusammensetzung betroffen ist." ${ }^{3}$ Dies kann allerdings angezweifelt werden. So schwankt z.B. der Anteil der unter 6jährigen an der Gemeindebevölkerung 1995 zwischen 5,2 und 9,5 v.H., wobei gerade im strukturschwächeren Norden Hessens der Kinderanteil höher ist. ${ }^{4} \mathrm{Da}$ die nicht volljährige Bevölkenung nicht nur einen überdurchschnittlichen Bedarf induziert, sondern auch nur unterdurchschnittlich bzw. gar nicht $\mathrm{zu}$ den Steuereinnahmen der Gemeinden beiträgt, wäre eine gesonderte

Diese wurden z.B. in Niedersachsen bis 1998 berücksichtigt.

2 Hessischer Landtag: Vorlage der Landesregienung betreffend den Entwurf für ein Siebentes Gesetz zur Änderung des Finanzausgleichsgesetzes vom 14.02.1972, Drucksache 7/1322, S. 10f. Ähnlich schon früher ders.: Vorlage der Landesregierung über ein Gesetz zur Regelung des Finanzausgleichs vom 02.05.1950, Drucksachen Abteilung I, 1/1482, S. 2021.

3 Hessischer Landtag: Gesetzentwurf der Landesregierung für ein Gesetz zur Regelung des Finanzausgleichs und zur Änderung anderer Vorschriften vom 11.10.1977, Drucksache 8/4877, S. 37.

4 Vgl:: Hessisches Statistisches Landesamt: Hessische Gemeindestatistik 1996, 17. Aufl. 1997. 
Berücksichtigung angebracht. ${ }^{1}$ Hinzu kommt noch, daß auch die Möglichkeit der Finanzierung über Gebühren geringer ist, da diese für Kinder meist niedriger sind. ${ }^{2}$ Grundsätzlich könnte auf den früheren Ansatz für Bevölkerungszusammensetzung in modifizierter Form zurückgegriffen werden, so daß seine Implementierung in die derzeitige Bedarfsermittlung nur geringe Probleme hervorrufen dürfte.

Studenten können deshalb als eine zuschußbedarfsinduzierende Gruppe angesehen werden, da sie sich zwar sehr häufig und lange in ihrem Studienort aufhalten, doch vielfach nicht dort ihren Erstwohnsitz angemeldet haben, so daß sie nicht im KFA berücksichtigt werden. ${ }^{3}$ Sie stellen zudem noch eine einfach abzugrenzende und gleichzeitig große Gruppe dar, da sie sich auf die wenigen Studienstandorte konzentriert. Allerdings besteht innerhalb der Gruppe ein Klassifizierungsproblem, da es nicht möglich ist, Studenten mit Erst- und Zweitwohnsitz am Studienstandort zu trennen. Dadurch würde aber das Problem entstehen, daß bei einer Berücksichtigung aller Studenten diejenigen mit Erstwohnsitz doppelt gezählt würden. Als Lösung bieten sich zwei Ansätze an. Einmal könnte sich durch die Erhebung einer Zweitwohnungsteuer ein finanzieller Anreiz für die Studenten ergeben, den Erstwohnsitz in die Hochschulstadt zu verlegen. Zum anderen gehören alle Orte mit Hochschulen zu den Oberzentren, da diese ein Kriterium zur raumordnungspolitischen Klassifizierung darstellen. ${ }^{4}$ Bei einer entsprechenden Dimensionierung des Zentralitätsansatzes könnte somit auf einen zusätzlichen Studentenansatz verzichtet werden.

$\mathrm{Zu}$ der Gruppe der Wehrpflichtigen ist anzuführen, das sich diese nur in der Woche am Stationierungsort aufhalten, sofern dieser nicht ihr Wohnort und Erstwohnsitz ist. In diesem Zeitraum kann unterstellt werden, daß die Nutzungsintensität der lokalen Infrastruktur nicht über der von Tagesurlaubern liegt, ${ }^{5}$ denen aber

1 Vgl: Henneke, H.-G.: Der Finanzbedarf kommunaler Gebietskörperschaften, in: Niedersächsische Verwaltungsblätter, 1. Jg., 1994, S. 60. Da die Bevölkerungsgruppe am Ende der Alterspyramide (Rentner) zwar auch einen hohen Bedarf induzieren, gleichzeitig aber auch zum Steueraufkommen der Gemeinden beitragen, sind sie nicht gesondert zu berücksichtigen.

2 Vgl.: Kirchhof, P.: Der Finanzausgleich als Grundlage kommunaler Selbstverwaltung, in: DVBl., 95. Jg., 1980, S. 716.

3 Andererseits mußte nach der Volkszählung 1987 in Hochschulstädten die Einwohnerzahl nach unten korrigiert werden, was auf die fehlende Abmeldung nach Studienende zurückgeführt wurde. Vgl.: Hardt, U.; Schmidt, J.: Neuordnung des kommunalen Finanzausgleichs in Niedersachsen. Eine Untersuchung im Auftrag des Niedersächsischen Innenministeriums, hrsg. vom Niedersächsischen Innenministerium, Hannover 1998, S. 120.

4 Vgl:: Hessischer Minister für Wirtschaft, Verkehr und Landesentwicklung: Vorlage zum Landesentwicklungsplan 2000, o.O., o.J., S. 57.

5 Vgl.: Steinherr, M; Parsche, R.: Der Ausgleich zwischen Finanzbedarf und Finanzkraft im kommunalen Finanzausgleich des Landes Rheinland-Pfalz, ifo-Studien zur Finanzpolitik, Bd. 66, München 1998, S. 54. 
keine Bedarfssteigerung zugerechnet wird, weil sie nicht zu ermitteln ist. Zudem gilt auch für die Wehrpflichtigen, daß es wie bei den kasernierten Stationierungsstreitkräften bundeswehreigene Einrichtungen gibt, wodurch die Frequentierung gemeindlicher Anlagen beschränkt bleibt.

\subsubsection{Folgerungen für die zukünftige Bedarfsermittlung}

Aus der Untersuchung des bisherigen Systems der Bedarfsermittlung kann abgeleitet werden, daß die Nebenansätze bis auf die Ansätze für zentrale Orte und für Schulträgergemeinden abgeschafft werden sollten, da ihre Notwendigkeit nicht gegeben sein dürfte und zumindest der Ansatz für Bevölkerungswachstum dem Raumordnungsziel entgegengerichtet ist. Der Nebenansatz für Sonderstatusstädte sollte abgebaut werden, da die Berücksichtigung des besonderen Verhältnisses dieser Gemeinden zu ihren Kreisen nur durch die Kreisumlage, nicht aber über Gemeindeschlüsselzuweisungen zu erfolgen hat, was als systemfremd anzusehen ist. Sollte aufgrund großer politischer Widerstände eine Abschaffung der Ansätze für Kurorte und Stationierungsstreitkräfte nicht möglich sein, so sollte ihre Dimensionierung - wie schon angeführt - vermindert werden.

Der bisherige Mindestansatz für kreisangehörige zentrale Orte wird aber dem dahinter stehenden Raumordnungsziel derzeit nicht gerecht, da er in der Praxis nicht die erwünschte bedarfserhöhende Wirkung zeigt. So ergibt sich für die meisten Orte dieser Kategorie durch die größenbezogene Einwohnerveredelung ein höherer Bedarf als durch den Zentralitätsansatz. Deshalb sollte der Mindestansatz für zentrale Orte so erhöht werden, daß er in der Praxis auch seine Aufgabenstellung erfüllen kann. Auf die Berücksichtigung in Form eines prozentualen Zuschlags zum Hauptansatz sollte verzichtet werden, denn dies würde unterstellen, daß "die Zentralörtlichkeit von der Einwohnerzahl der Kommunen abhängig"1 ist. Als Folge führte dies zu einer Ungleichbehandlung von zentralen Orten in Abhängigkeit von ihrer jeweiligen Größe, letztere wird aber schon durch den Hauptansatz berücksichtigt. ${ }^{2}$ Die Ortsgröße sagt zudem selbst nicht viel über die Leistungserbringung für das Umland aus, wenn - wie in Hessen - die Bevölkerungsdichte und Gemeindegröße zwischen den Regionen oder Regierungsbezirken stark abweicht, so daß Gemeinden gleicher Größe unterschiedliche zentrale Aufgaben wahrnehmen. ${ }^{3}$ Relevant ist nämlich die Relation der eigenen zu den umgebenden Einwohnern.

1 Wissenschaftlicher Beirat beim Bundesministerium der Finanzen: Gutachten zur Reform der Gemeindesteuern, Schriftenreihe des Bundesministeriums der Finanzen, Heft 31, Bonn 1982, S. 15.

2 Vgl:: Schelpmeier, H.: Finanzausgleich furr zentrale Orte?, in: Raumforschung und Raumordnung, 56. Jg., 1998, S. 303f.

3 So beträgt die durchschnittliche Einwohnergröße kreisangehöriger Gemeinden in Südhessen 13.795 Einwohner, in Mittelhessen 10.499 und in Nordhessen 7.821. Von den Mittelzentren haben in Mittelhessen 25 v.H. eine Einwohnerzahl unter 13.795 Personen. Vgl:: Brück- 
Die Hauptansatzstaffel der kreisangehörigen Gemeinden konnte auf Basis empirischer Daten grundsätzlich für Hessen bestätigt werden, wenn auch mit einer veränderten Spreizung zwischen den Gemeindegrößenklassen. Hier deutet sich also ein Reformbedarf an. Es wäre zu überlegen, ob nicht die Anzahl der Gemeindegruppen vermindert werden könnte, da die Unterschiede zwischen den Verdelungsfaktoren teilweise gering sind und somit die Wahrscheinlichkeit eines Wechsels in eine andere Gemeindegrößenklasse und die damit eintretenden Veränderungen vermindert werden könnten. Dies könnte aber aufgrund des politischen Widerstands problematisch sein, der sowohl von den negativ betroffenen Kommunen ausgeht als auch von den Landtagsabgeordneten aus den jeweiligen Gemeinden. Zumindest sollten alle Gemeinden bis 7.500 Einwohner mit dem gleichen Ansatz veredelt werden, da Gemeinden bis zu dieser Größe auch die gleichen Aufgaben wahrnehmen und es somit eigentlich keinen Grund für eine unterschiedliche Behandlung gibt.

$\mathrm{Zu}$ den fünf kreisfreien Städte ist anzumerken, daß aufgrund ihrer geringen Anzahl eine Untersuchung der Hauptansatzstaffel auf empirischer Basis nicht möglich ist, da die zur Ermittlung des Zuschußbedarfs notwendige Durchschnittsbildung zu stark von der Entwicklung in einzelnen Städte beeinflußt wird. ${ }^{1}$ Im Jahr 1998 betrug der Veredelungsfaktor für Frankfurt 105 v.H., für Kassel und Wiesbaden 102 v.H. und für Darmstadt und Offenbach 100 v.H. Aus dem Vergleich dieser Werte mit den Systemen anderer Länder hat sich keine offensichtliche Notwendigkeit zur Veränderung ergeben, da die Veredelungsfaktoren eine vergleichbare Spreizung aufweisen. Deshalb wurden zur zukünftigen Bedarfsermittlung bei den kreisfreien Städten keine neuen Hauptanssätze vorgeschlagen.

\subsubsection{Ermittlung des Finanzbedarfs der Landkreise}

\subsubsection{Verwendung des Hauptansatzes in Hessen}

\subsection{Darstellung der bisherigen Regelungen}

Die Ermittlung der Bedarfsmeßzahl bei den Landkreisen läuft nach dem gleichen Muster wie bei den Gemeinden ab. Auch hier ergibt sich die Bedarfsmeßzahl aus dem Produkt von Grundbetrag und Gesamtansatz. Letzterer setzt sich aus dem Hauptansatz und nur einem Nebenansatz zusammen. Der Hauptansatz des Landkreises ( $\$ 17 \mathrm{Abs}$. $3 \mathrm{FAG}$ ) wird aus der zu veredelnden Einwohnerzahl unter Berücksichtigung der Gemeindegrößenstruktur des Kreises ermittelt. Diese wird dadurch berücksichtigt, daß statt der Kreiseinwohnerzahl die Einwohnerzahlen der kreisangehörigen Gemeinden veredelt werden, wobei im Gegensatz zur

mann, F; Dette, W.: Stadt-Umland-Beziehungen und kommunaler Finanzausgleich nicht nur ein Problem der kreisfreien Städte, in: Kommunale Steuer-Zeitschrift, 45. Jg., 1996, S. $45 f$.

1 Für die 421 kreisangehörigen Gemeinden kann unterstellt werden, daß die Ausgabenentwicklung einer einzelnen Gemeinde keinen merklichen Einfluß auf die Höhe des Zuschußbedarfs hat. 
gemeindlichen Bedarfsermittlung die Veredelungsfaktoren mit zunehmender Gemeindegröße sinken. Diese Vorgehensweise führt dazu, daß sich bei gleicher Kreiseinwohnerzahl für Kreise mit vielen kleinen Gemeinden ein höherer Bedarf ergib. Als Begründung wird angeführt, daß bei gleicher Bevölkerungszahl die Lasten für einen Landkreis mit wenigen großen Orten geringer sind, da die Gemeinden viele Aufgaben selbst übernehmen können, als bei einem Kreis mit vielen kleinen Gemeinden, die aufgrund ihrer geringeren Verwaltungskraft Aufgaben an den Landkreis abgeben oder nur mit seiner Unterstützung erfüllen können. ${ }^{1}$

Derzeit werden aber nur noch zwei Größenklassen unterschieden; so werden die Einwohner aus Gemeinden mit weniger als 7.500 Einwohnern mit 105 v.H. angesetzt, während für die übrigen Gemeinden 100 v.H. für die Einwohnerzahl gilt. Als Begründung wird angeführt, daß durch die Gebiets- und Verwaltungsreform in den 70er Jahren die bis dahin zwischen den Gemeinden bestehenden Differenzen in der Verwaltungskraft abgebaut wurden. Da sich somit auch die unterschiedlichen Belastungen der Kreise durch ihre Gemeinden verringerte, hat die stärkere Differenzierung "mit dem fortschreitenden Zusammenschluß zu größeren Gemeinden im Zug der Gebietsreform weitgehend ihren Sinn"2 verloren. Zudem wurde das Problem gesehen, daß bei unverändert starker Spreizung der Hauptansatzstaffelung die Kreise wenig Interesse für Gemeindezusammenschlüsse verspüren, da sich ihre Schlüsselzuweisungen ansonsten vermindert hätten. ${ }^{3}$ Die Ortsgröße von 7.500 Einwohnern wurde gewählt, weil ab dieser Einwohnerzahl Aufgaben der Landesverwaltung von den Landkreisen auf deren Gemeinden nach $\S 59$ Abs. 1 Hessische Landkreisordnung als übertragen gelten. ${ }^{4}$

\subsection{Analyse und Reform}

Von der mit der Gemeindeeinwohnerzahl sinkenden Veredelung profitierten 1998 19 der 21 Landkreise; nur in den beiden finanzstärksten Kreisen Offenbach und Main-Taunus befinden sich keine Gemeinden unter 7.500 Einwohnern. Die zu berücksichtigende Bevölkerung der Landkreise wird um 46.784 auf 4.703.116 Personen angehoben, dies entspricht etwa einem Anstieg von 1,0 v.H. Das mittels der Veredelung umverteilte Volumen belief sich auf 29,50 Mio. DM, was etwa 2,96 v.H. der gesamten Kreisschlüsselmasse entspricht. Der Verzicht auf die Einwohnerveredelung hätte zur Folge, daß sieben der 19 profitierenden Kreise sich bei den Schlüsselzuweisungen besser stellen würden, da bei ihnen der Anteil der

1 Vgl.: Münstermann, E.: Der kommunale Finanzausgleich in den Bundesländern - Teil V-, in: ZKF, 30. Jg., 1980, S. 153.

2 Hessischer Landtag: Gesetzentwurf der Landesregierung furr ein Neuntes Gesetz zur Änderung des Finanzausgleichsgesetzes vom 05.10.1973, Drucksache 7/4135, S. 7.

3 Vgl.: Ders.: Vorlage der Landesregierung betreffend den Entwurf für ein Sechstes Gesetz zur Änderung des Finanzausgleichsgesetzes vom 09.03.1971, Drucksache 7/151, S. 12.

4 Vgl.: Hessischer Landtag: Gesetzentwurf der Landesregierung furr ein Zehntes Gesetz zur Änderung des Finanzausgleichsgesetzes vom 27.03.1975, Drucksache 8/457, S. 11. 
Einwohner in Orten unter 7.500 Einwohnern unter dem Durchschnitt liegt bzw. bei einem von ihnen trotz etwas überdurchschnittlichem Wert der steigende Grundbetrag aufgrund der hohen Gesamteinwohnerzahl zur Überkompensierung ausreicht. Die Verteilungswirkungen sind in Tabelle 22 wiedergegeben.

Tabelle 22: Umverteilungswirkungen der Kreisschlüsselzuweisungen bei Abschaffung der Einwohnerveredelung

\begin{tabular}{|c|r|r|r|}
\hline \multirow{2}{*}{ Landkreise } & \multicolumn{2}{|c|}{ Landkreise mit Ansatz } & \multirow{2}{*}{$\begin{array}{c}\text { ohne Ansatz } \\
\text { Gewinner }\end{array}$} \\
\cline { 2 - 3 } & \multicolumn{1}{|c|}{ Gewinner } & \multicolumn{1}{c|}{ Verlierer } & 2 \\
\hline Anzahl & 7 & 12 & 2.364 .739 \\
\hline Gewinn/Verlust in DM & 5.428 .761 & -7.797 .166 & 3,59 \\
\hline Gewinn/Verlust in v.H. & 1,30 & $-1,52$ & 4,34 \\
\hline Gewinn/Verlust in pro DM/Kopf & 2,78 & $-3,61$ & \\
\hline
\end{tabular}

Quelle: Eigene Berechnung nach Daten des Finanzministeriums zum KFA 1998.

Aufgrund des geringen Umverteilungsvolumens wird klar, daß im Durchschnitt sowohl die relativen als auch die Umverteilungsbeträge pro Einwohner sehr gering ausfallen, sie liegen zwischen $-1,52$ und 3,59 v.H. bzw. zwischen $-3,61$ und 4,34 DM je Einwohner. Doch selbst die Maximalwerte streuen nicht stark um den Durchschnitt. So liegt die maximale Steigerung der Schlüsselzuweisungen bei 3,81 v.H. (Main-Taunus-Kreis), der maximale Verlust bei 3,84 v.H. (Kreis Waldeck-Frankenberg, da fast 50 v.H. der Einwohner in Orten unter 7.500 Einwohnern leben). Den größten Verlust pro Einwohner mit 9,62 DM erleidet ebenfalls der Kreis Waldeck-Frankenberg, während der Kreis Groß-Gerau den höchsten Gewinn mit 5,24 DM je Einwohner zu verzeichnen hat.

Grundsätzlich ist die Vorgehensweise bei der Ermittlung des Hauptansatzes für die Landkreise nachvollziehbar, denn die Größenstruktur der Gemeinden eines Kreises dürfte sich auf dessen Kosten auswirken. Sie ist auch einsichtiger als die z.B. in Niedersachsen praktizierte Methode, nach der zwar auch die Veredelungsfaktoren mit steigender Einwohnerzahl gesunken ist, allerdings in Abhängigkeit von der Kreiseinwohnerzahl. ${ }^{1}$ Somit fand in Niedersachsen die Struktur der Orte keine Berücksichtigung. Dies war auch ein Grund dafür, daß das Gesetz vom Niedersächsischen Staatsgerichtshof für verfassungswidrig erklärt wurde. ${ }^{2}$ Die in Hessen vorgenommene Zweiteilung der Gemeinden ist begründbar mit der Aufgabenverteilung im kreisangehörigen Raum nach $\S 59$ HKO. Denn für die

1 Vgl.: $§ 7$ NFAG vom 19.12.1995, Nds. GVBl., S. 463; Kritik daran von HENNEKE. Vgl.: Henneke, H.-G.: Der Finanzbedarf kommunaler Gebietskörperschaften, in: Niedersächsische Verwaltungsblätter, 1. Jg., 1994, S. 58f.

2 Vgl: Urteil des Niedersächsischen Staatsgerichtshofs vom 27.11.1997 (StGH 14/95), in: DVB1., 113. Jg., 1998, S. 185, Leitsatz 5. 
Gemeinden unter 7.500 Einwohner muß der Kreis mehr Aufgaben wahrnehmen als für größere Orte, denen verschiedene Aufgaben übertragen worden sind. Eine besondere Berücksichtigung der Sonderstatusstädte, beispielsweise durch eine geringere Veredelung, ist abzulehnen, da dadurch nur bestimmte Kreise betroffen sind, die eine von der Mehrheit abweichende kreisinterne Aufgabeverteilung vorgenommen haben, ohne daß sich der Bedarf des Gesamtkreises dadurch ändert.

Anders ist die Situation zu beurteilen, wenn entgegen den vorgebrachten Argumenten am Ansatz für Sonderstatusstädte bei der Verteilung der Gemeindeschlüsselzuweisungen festgehalten werden soll. Dann wäre in der Tat die besondere Stellung der Sonderstatusstädte bei der Kreisbedarfsermittlung zu berücksichtigen, und für diese Kreiseinwohnergruppe müßte der Kreisbedarf geringer ausfallen als bei den übrigen; denn der Bedarf des Gesamtkreises ergibt sich als Summe aus Gemeinde- und Kreisbedarf. Wird den Sonderstatusstädten ein erhöhter Bedarf bei der Verteilung der Gemeindeschlüsselzuweisungen zugesprochen, so ist entsprechend der Bedarf des Kreises zu mindern, da ansonsten diesem Gesamtkreis der Bedarf der Einwohner in Sonderstatusstädten zweifach angerechnet wird. Als Lösungsansatz könnte auf frühere rechtliche Regelungen bei der Bedarfsermittlung der Kreise zurückgegriffen werden, als die Hauptansatzstaffel noch mehr Gemeindegrößenklassen unterschied. Entsprechend wäre der Ansatz für Einwohner der Sonderstatusstädte unter 100 v.H. festzulegen.

\subsubsection{Verwendung eines Nebenansatzes in Hessen}

\subsection{Darstellung der bisherigen Regelungen}

Als Ergänzungsansatz wird bei den Kreisen ausschließlich das Bevölkerungswachstum der letzten zehn Jahre im Kreisgebiet berücksichtigt, wobei die Gewährung an die gleichen Voraussetzungen wie bei den Gemeinden gebunden sind. Die Bevölkerungszahl muß also um mehr als $10 \mathrm{v} . \mathrm{H}$. gestiegen sein, wovon dann wiederum nur die Hälfte des über den 10 v.H. liegenden Betrags berücksichtigt wird. Bei den Kreisen wird unterstellt, daß "die Aufgaben mit steigender Bevölkerungszahl überproportional zunehmen"1. Deshalb findet auch der Ansatz auf die veredelte und nicht auf die tatsächliche Einwohnerzahl Anwendung. Auf die übrigen Ergänzungssätze für Kurorte, Stationierungsstreitkräfte und Schulträgerschaft wird hingegen verzichtet. Für den letztgenannten ist die Erklärung einfach, da er auf Gemeindeebene nur aufgrund der Umgestaltungsproblematik des Schullastensonderausgleichs bei den kreisangehörigen Gemeinden eingeführt worden ist. Für die beiden anderen Ansätze wird angenommen, daß den Kreisen aus diesen gemeindlichen Bedarfsträgern keinerlei weitere Lasten entstehen.

Gretz-Roth, V.: Der kommunale Finanzausgleich und finanzielle Zuweisungen des Landes zur Erfüllung öffentlicher Aufgaben, Erläuterungen zu $§ 17$ FAG, in: Praxis der Gemeindeverwaltung, Hessen, Bd. E1, (Loseblattsammlung), Stand 1999, S 53. 


\subsection{Analyse und Reform}

In neun der 21 Landkreise lag 1996 die Bevölkerungszahl um mehr als 10 v.H. über dem Stand von 1986, so daß für sie über den Ergänzungsansatz eine Bedarfssteigerung erfolgt. Auch hier fällt die Erhöhung der tatsächlichen Kreisbevölkerung mit 0,4 v.H. oder 18.717 Einwohnern sehr niedrig aus. Der durch den Ergänzungsansatz für Bevölkerungswachstum zwischen den Kreisen umverteilte Betrag liegt mit 12,18 Mio. DM bzw. 1,22 v.H. der gesamten Kreisschlüsselmasse noch unter jenem Wert bei der Hauptansatzstaffel. ${ }^{1}$ Sollte der Ansatz für Bevölkerungswachstum nicht weiter beibehalten werden, so würden von dieser Maßnahme neben den zwölf Landkreisen, die kein entsprechend hohes Bevölkerungswachstum im letzten Jahrzehnt aufwiesen, noch zwei weitere Kreise, die selbst von der heutigen Regelung profitieren, zu den Gewinnern zählen. Die damit induzierten Verteilungen sind in Tabelle 23 dargestellt.

Tabelle 23:Umverteilung bei Abschaffung des Bevölkerungszuwachsansatzes für Landkreise

\begin{tabular}{|c|r|r|r|}
\hline \multirow{2}{*}{ Landkreise } & \multicolumn{2}{|c|}{ Landkreise mit Ansatz } & \multirow{2}{*}{$\begin{array}{c}\text { ohne Ansatz } \\
\end{array}$} \\
\cline { 2 - 3 } & Gewinner & Verlierer & \multicolumn{1}{c|}{ Gewinner } \\
\hline Anzahl & 2 & 7 & 12 \\
\hline Gewinn/Verlust in DM & 1.251 .414 & -7.336 .477 & 6.081 .352 \\
\hline Gewinn/Verlust in v.H. & 0,78 & $-2,11$ & 1,24 \\
\hline Gewinn/Verlust in pro DM/Kopf & 1,91 & $-4,93$ & 2,42 \\
\hline
\end{tabular}

Quelle: Eigene Berechnung nach Daten des Finanzministeriums zum KFA 1998.

Auch hier sind die Umverteilungsgewinne und Verluste sehr gering. Sie belaufen sich bei den sieben Verlierern im Durchschnitt auf 4,93 DM pro Kopf bzw. 2,11 v.H. der Schlüsselzuweisungen, wobei der Odenwaldkreis die Spitzenposition mit 10,48 DM pro Kopf und 3,92 v.H. hält. Die Werte bei den Gewinnern fallen geringer aus, da die Bezugsgröße größer ist. So beträgt der maximale Gewinn pro Einwohner bei 2,88 DM (Kreis Hersfeld-Rotenburg), die stärkste relative Zunahme 1,59 v.H. (Main-Taunus-Kreis).

Wie bei den Gemeinden ist bei den Kreisen nach der Notwendigkeit des angeführten Ergänzungsansatzes und die Beziehung zum raumordnungspolitischen Ziel zu fragen. Eine konfliktäre Beziehung ist bei dieser Gruppe nicht zu vermuten, da die Attraktivität des Umlands gegenüber den (kreisfreien) Kernstädten, die $\mathrm{zu}$ Wanderungsbewegungen führt, weniger durch die Aufgabenwahrnehmung der Kreise als durch die der Gemeinden induziert wird. Insofern wird die Verteilung der Kreisschlüsselzuweisungen kaum Relevanz haben.

1 Letztlich spielt somit weder die derzeitige Einwohnerveredelung noch der Nebenansatz bei der Verteilung der Schlüsselzuweisungen der Landkreise eine gewichtige Rolle. 
Um Aussagen darüber machen zu können, inwieweit ein solcher Ansatz aufgrund der gestiegenen Ausgaben notwendig sein kann, ist auf die für die Gemeinden angestellten Überlegungen zurückzugreifen. Da die Bevölkerungsentwicklung eines Kreises sich als Saldo der Entwicklung seiner Gemeinden ergibt, sind die Unterschiede zwischen den Kreisen geringer als zwischen den Gemeinden, da kreisinterne Wanderungsbewegungen keinerlei Einfluß auf die Kreisbevölkerung haben. ${ }^{1}$ Dies kann auch an der im Vergleich zu den Gemeinden geringeren Umverteilung bei den Kreisen abgelesen werden. Da ein großer Teil der Wanderungsbewegungen innerhalb der Kreise abläuft, induzieren diese auch keinen erhöhten Bedarf, da z.B. das zu beseitigende Müllaufkommen unverändert bleibt. Doch selbst bei Erhöhung der Bevölkerungszahl dürfte der Bedarf beispielsweise an zusätzlichen (Kreis-)Straßen gering sein, da etwa Neubauten durch Gemeindestraßen an die Kreisstraßen angeschlossen werden. Zudem werden die mit dem Straßenbau verbundenen Kosten durch einen Sonderlastenausgleich abgegolten. Gleiches gilt für möglicherweise steigende Kosten beim Schülertransport.

Es ist somit nicht einsichtig, weshalb der Bedarf von Kreisen überproportional mit dem Bevölkerungswachstum zunehmen soll. Zudem reagiert wie bei den Gemeinden auch die Einnahmenseite positiv auf steigenden Bevölkerungszahlen, denn der Kreis erhält zusätzliche Schlüsselzuweisungen. Weiterhin erhöht sich die Umlagekraft der Kreise, da die Schlüsselzuweisungen der Gemeinden, die Bestandteil der Umlagebemessungsgnundlage sind, ebenfalls schon im folgenden Jahr auf Veränderungen der Bevölkerungzahl reagieren. Auch ein Ländervergleich zeigt, daß in keinem anderen Land der Bevölkerungsanstieg bedarfssteigernd berücksichtigt wird. Somit erscheint ein Ergänzungsansatz zur Berücksichtigung des Bevölkenungswachstums nicht notwendig.

\subsection{Notwendigkeit eines weiteren Nebenansatzes?}

Häufig wird noch gefordert, daß für Landkreise ein Ergänzungsansatz eingeführt wird, der die mit der Besiedlungsdichte verbundenen Kostendifferenzen berücksichtigt. Rheinland-Pfalz berücksichtigt als einziges Bundesland die mit der Fläche verbundenen Kosten im Schlüsselzuweisungssystem. In Hessen bestand von 1954 bis 1976 ein vergleichbarer Ansatz, mit dem eine geringe Besiedlungsdichte berücksichtigt werden sollte, was tatsächlich aber nur anhand der Bevölkerungszahl ohne Betrachtung der Fläche geschah. ${ }^{2}$ Als Begründung für diese Forderung wird vorgebracht, "daß bei abnehmender Besiedlungsdichte, sowohl der Verwaltungs- als auch der Sachaufwand steigt, die kommunale 'Infrastruktur'

1 So beträgt der Variationskoeffizient als Maß für die Streuung der Werte und somit auch für die Wanderungsbewegungen nach eigenen Berechnungen aus den Daten des KFA 1998 bei den Gemeinden 0,70, bei den Kreisen aber nur 0,33.

2 Hessischer Landtag: Erstes Gesetz zur Änderung und Ergänzung des Gesetzes zur Regelung des Finanzausgleichs vom 11.05.1954, Drucksachenabteilung I, 2/904, S. 1941. 
somit teurer wird"1. Als Beispiel für diese höheren Kosten in überdurchschnittlich großen und dünn besiedelten Kreisen können die Bereiche Abfallwirtschaft, StraBenunterhaltung und Schülertransport angeführt werden. ${ }^{2}$

Generell kann gegen diese Argumentation vorgebracht werden, daß die Besiedlungsdichte selbst nur eine geringe Aussagekraft für die mit der Gebietsfläche verbundenen Kosten hat. Denn nicht der Quotient von Einwohnerzahl und Gebietsfläche ist relevant, sondern eher die Verteilung der Einwohner im Raum (Siedlungsstruktur) und die Gemeindegröße. Ein Kostenunterschied besteht nämlich zwischen Gemeinden mit vielen räumlich getrennten Ortsteilen, wie sie vielfach durch die Gebiets- und Verwaltungsreform entstanden sind, und solchen mit einer Kerngemeinde, die baulich geschlossen ist. Um Unterschiede dieser Art zu identifizieren, ist aber die Einwohnerdichte eher ein unzweckmäßiger Indikator, da dieser keine Aussagen über die entscheidungsrelevante Siedlungsstruktur zuläßt. Für den Bereich der in der Fläche teuren öffentlichen Leistungen im Straßenbau und Schülertransport gibt es in Hessen zweckgebundene Zuweisungen für laufende und investive Zwecke, so daß dieser Bedarf zielgenau abgedeckt sein dürfte. ${ }^{3}$ Im Rahmen der Müllbeseitigung sind kostendeckende Gebühren zu verlangen. Verzichtet eine Kommune darauf, ist dies ihre autonome Entscheidung, und sie muß nun zur Finanzierung des Defizits andere Einnahmequellen stärker anspannen, so daß hier eigentlich kein Handlungsbedarf besteht.

Wird hingegen vom Land eine kostendeckende Gebührenhöhe als zu große Härte angesehen, so könnten zweckgebundene Mittel fließen. ${ }^{4}$ Werden diese Gelder wie in NRW der Finanzausgleichsmasse entnommen, wodurch das Defizit einer Kommune von den übrigen in Form geringerer anderer Zuweisungen mitfinanziert wird, ist eine angemessen Gebührenhöhe vom Empfänger zu verlangen, was

Landtag Rheinland-Pfalz: Gesetzentwurf der Landesregierung für ein Landesgesetz über den Finanzausgleich in Rheinland-Pfalz (Finanzausgleichsgesetz - FAG -) vom 16.05.1977, Drucksache 8/2106, S. 29.

2 Für Rheinland-Pfalz ergab eine Untersuchung, daß die Korrelation zwischen Fläche und Kosten in diesen Aufgabenbereichen recht gering waren. Vgl.: Steinherr, M; Parsche, R.: Der Ausgleich zwischen Finanzbedarf und Finanzkraft im kommunalen Finanzausgleich des Landes Rheinland-Pfalz, ifo-Studien zur Finanzpolitik, Bd. 66, München 1998, S. 122-128.

3 Die Mittel für den Schülertransport werden innerhalb der Gruppe der Landkreise (kreisfreien Städte und kreisangehörigen Gemeinden) zu 85 v.H. (95 v.H.) nach der Schülerzahl und zu 15 v.H. (5 v.H.) nach der Fläche auf den einzelnen Schulträger verteilt. Die Verteilung der Gelder für die Unterhaltung der bestehenden Straßen erfolgt nach der Länge der Straßen in der jeweiligen Gebietskörperschaft.

4 Vgl: $§ 20$ Abs. 1 Nr. 5 des Gesetzes zur Regelung der Zuweisungen des Landes NordrheinWestfalen an die Gemeinden und Gemeindeverbände im Haushaltsjahr 1999 und zur Regelung des interkommunalen Ausgleichs der finanziellen Beteiligung der Gemeinden am Solidarbeitrag zur Deutschen Einheit im Haushaltsjahr 1999 und zur Änderung anderer Vorschriften vom 17.12.1998, GVB1. für das Land Nordrhein-Westfalen, S. 765. 
durch einen interkommunalen Vergleich geprüft werden kann. ${ }^{1}$ Allerdings sollten bestehende Finanzierungslücken nicht zu 100 v.H. durch Zuweisungen abgedeckt werden, da es ansonsten für den betroffenen Kreis keinen Anreiz zur Steigerung der Wirtschaftlichkeit gibt.

Somit spricht wenig für einen neuen Nebenansatz, zumal in einer Untersuchung über die Bedarfsstruktur der niedersächsischen Kreise festgestellt wurde, daß "die absolute Kreisfläche (...) gar keinen Einfluß auf die Zuschußbedarfe je Einwoh-

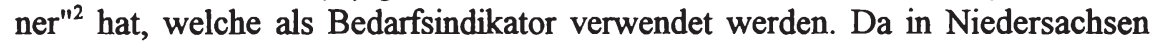
die Kreise eine größere Fläche aufweisen als in Hessen, dürfte dieses Ergebnis auch für Hessen gelten. ${ }^{3}$ Die Einführung eines Ansatzes zur Berücksichtigung möglicher flächeninduzierter Bedarfe ist somit abzulehnen.

\subsubsection{Folgerungen für die zukünftige Bedarfsermittlung}

Aus den bisherigen Argumenten kann gefolgert werden, daß auch bei den Kreisen eine Reform der Bedarfsermittlung angebracht ist. Der Nebenansatz für Bevölkerungswachstum sollte abgeschafft werden, da seine Notwendigkeit eher zweifelhaft ist. Die Einwohnerveredelung kann in der derzeitigen Form beibehalten werden, da die unterschiedliche Aufgabenteilung im Kreis berücksichtigt wird. Gemeinden mit über 7.500 Einwohner erhalten bei der Verteilung der Gemeindeschlüsselzuweisungen aufgrund ihres erweiterten Aufgabenspektrums einen höheren Bedarf zugesprochen, folglich vermindert sich der Kreisbedarf. Die besondere Stellung der Sonderstatusstädte soll aber nur über die Kreisumlage berücksichtigt werden. Erst wenn dieser Gemeindegruppe wegen der Wahrnehmung von Kreisaufgaben ein höherer Bedarf bei den Gemeindeschlüsselzuweisungen zugesprochen wird, müßte als Ausgleich dafür den Sonderkreisen für die Einwohner dieser Orte ein geringerer Bedarf angerechnet werden.

\subsubsection{Allgemeine Grundlagen der Finanzkraftermittlung}

Die Einnahmen, die zur Ermittlung der Finanzkraft herangezogen werden, können in zwei Gruppen eingeteilt werden. Zur ersten Kategorie gehören die Einnahmen, bei denen die Kommunen eine direkte Möglichkeit zur Beeinflussung des jeweiligen Aufkommens haben. Dies ist etwa bei den Realsteuern und der Kreisumlage

So hat der Bund der Steuerzahler in Rheinland-Pfalz festgestellt, daß bei vergleichbarem Müllaufkommen die durchschnittlichen Gebühren in den Landkreisen geringer sind als in den kreisfreien Städten, was bei den vermuteten höheren Kosten der Müllbeseitigung im großflächigeren Landkreis überrascht. Vgl: Bund der Steuerzahler Rheinland-Pfalz: Abfallgebühren 1999, o. O., o.J., S. 9f.

2 Hardt, U.; Schmidt, J.: Neuordnung des kommunalen Finanzausgleichs in Niedersachsen. Eine Untersuchung im Auftrag des Niedersächsischen Innenministeriums, hrsg. vom Niedersächsischen Innenministerium, Hannover 1998, S. 141.

3 Die Durchschnittsfläche eines Landkreises ist in Hessen mit $971 \mathrm{~km}^{2}$ um 22,25 v.H. geringer als in Niedersachsen mit $1187 \mathrm{~km}^{2}$. 
der Fall, da dort mit Hilfe eines autonom festzusetzenden (Umlage-)Hebesatzes das Einnahmevolumen bestimmt wird. ${ }^{1}$ Zur zweiten Gruppe gehören die Einnahmen, bei denen die Kommunen keinen direkten Einfluß auf das ihnen zustehende Volumen haben, wie etwa der gemeindliche Einkommensteueranteil. Bei dieser Gruppe werden die Ist-Einnahmen zur Finanzkraftberechnung herangezogen. Bei der ersten Kategorie, werden nicht die tatsächlich erzielten Steuereinnahmen herangezogen, sondern eine normierte Größe, denn "Vor- und Nachteile einer autonomen Entscheidung über die Nutzung einer Steuerquelle müssen den einzelnen Gemeinden bleiben"2. Ansonsten könnten die Gemeinden durch Veränderung des Hebesatzes auch die Höhe der Finanzzuweisungen variieren. Würden die tatsächlichen Einnahmen als adäquater Maßstab für die kommunale Finanzkraft angesehen, so könnten die Kommunen sich strategisch verhalten und durch die Senkung der Hebesätze und damit der Steuereinnahmen zusätzliche Unternehmen attrahieren, da sich deren Steuerlast verringerte, und gleichzeitig zur Kompensation für die kurzfristigen Realsteuermindereinnahmen zusätzliche Schlüsselzuweisungen erhalten. Dies führt bei einer plafondierten Finanzausgleichsmasse zu einer Verringerung der Zuweisungen zu Lasten der übrigen Gemeinden. Somit ist klar, daß die tatsächlichen Steuereinnahmen nur dann verwendet werden können, wenn die Steuersätze ein gleiches Niveau besitzen. ${ }^{3}$

Um das angeführte strategische Verhalten zu verhindern, muß ein System gefunden werden, welches einerseits das Einnahmepotential möglichst genau abbildet, andererseits aber nicht zusätzliche Schlüsselzuweisungen bei Verminderung der tatsächlichen Hebesätze induziert. Zu diesem Zweck wird ein Aufkommen verwendet, welches sich unter Verwendung eines normierten Hebesatzes ${ }^{4}$ ergibt. Für das Jahr 1998 lagen die Normierungssätze für kreisangehörige Gemeinden und kreisfreie Städte bei der Gewerbesteuer bei 295 v.H., bei der Grundsteuer A bei 175 v.H. und bei der Grundsteuer B bei 200 und 260 v.H. ${ }^{5}$

1 Die nachfolgend angeführte Begründung für das bei den Realsteuern verwendete Normierungsverfahren gilt analog auch für die Umlagesätze der Kreisumlage.

2 Vgl.: Kirchhof, P.: Der Finanzausgleich als Grundlage kommunaler Selbstverwaltung, in: DVB1., 95. Jg., 1980, S. 718.

3 Vgl: Fischer, H.: Finanzzuweisungen. Theoretische Grundlegung und praktische Ausgestaltung im bundesstaatlichen Finanzausgleich Australiens und der Bundesrepublik Deutschland, Berlin 1988, S. 100.

4 Der Nivellierungshebesatz kann sich beispielsweise am gewichteten Landesdurchschnitt orientieren, um einfach und doch möglichst genau das Einnahmenpotential zu erfassen.

5 Bis einschließlich 1998 wurde der Hebesatz bei der Grundsteuer B in Abhängigkeit von der Bemessungsgrundlage (Grundbetrag) differenziert; er betrug 200 v.H. für die erste Million des Grundbetrages und 260 v.H. für die darüber liegenden Beträge, so daß nur im rein theoretischen Fall eine Annäherung an den Wert 260 v.H. möglich ist. Der zur Normierung der Kreisumlagekraft verwendete Umlagesatz lag bei 39 v.H. 
Die Berücksichtigung der finanzkraftmindernden Wirkung der Gewerbesteuerumlage, deren Höhe mittels eines bundeseinheitlichen Normierungssatzes bestimmt wird, ist 1993 neu geregelt worden. Bis dahin wurde ein Betrag, der sich aufgrund der Division des tatsächlichen Aufkommens der Gewerbesteuerumlage durch den individuellen Hebesatz ergab, mit einem landeseinheitlichen Normierungssatz multipliziert und diese Größe mindernd auf die Gewerbesteuerkraft angerechnet. Dieses Berechnungsweise wurde ausschließlich in Hessen praktiziert. ${ }^{1}$ Bei dem neuen Verfahren wird die fiktive Gewerbesteuerkraft um das tatsächliche Aufkommen (Umlagesoll) gemindert. Die Unterschiede sollen kurz in Abbildung 17 dargestellt werden.

\section{Abbildung 17:Finanzkraftmindernde Wirkung der Gewerbesteuerumlage ${ }^{2}$}

\section{alt: Bemessungsgrundlage - GFRG-Umlagesatz gemeindeindividueller Hebesatz \\ FAG-Anrechnungssatz}

neu: Bemessungsgrundlage • GFRG-Umlagesatz

Beide Verfahren führen nur dann zum identischen Ergebnis, wenn der FAGAnrechnungssatz und der individuelle Hebesatz übereinstimmen. Ist dies nicht der Fall, so profitieren bei gleichem Grundbetrag diejenigen Gemeinden von der alten Regelung, deren Hebesatz geringer als der FAG-Anrechnungssatz ist, da die zu berücksichtigende Gewerbesteuerumlage mit einem Faktor über eins vervielfältigt wird, während dies für Gemeinden mit höheren Hebesätzen die umgekehrte Wirkung hervorruft. Durch die Modifikation des Anrechnungsverfahrens wurde die ungerechtfertigte Diskriminierung von Gemeinden mit hohen Gewerbesteuerhebesätzen abgeschafft, die das alte Berechnungssystem enthielt, denn zwischen Höhe der Gewerbesteuerumlage und individuellem Hebesatz besteht kein Bezug.

\subsubsection{Ermittlung der Finanzkraft von Gemeinden und Städten}

\subsubsection{Darstellung der bisherigen Regelungen}

Im Rahmen des KFA in Hessen werden als Indikator für die gemeindliche Finanzkraft die Steuereinnahmen herangezogen, und zwar die Gewerbesteuer unter Berücksichtigung der Gewerbesteuerausgleichszuschüsse bis $1969^{3}$ und seit 1970

1 Vgl.: Leibfritz, W.; Teschner, S.: Der Einfluß des Steuersystems und des kommunalen Finanzsystems auf die Landesentwicklung, Schriftenreihe des Ifo-Instituts für Wirtschaftsforschung, Nr. 108, Berlin 1981, S. 125.

2 Das Ist-Aufkommens dividiert durch den individuellen Hebesatz ergibt den Grundbetrag.

3 Vor Beteiligung der Gemeinden an der Einkommensteuer gab es in den Bundesländern einen sog. Gewerbesteuerausgleich. Betriebsgemeinden mußten einen Anteil der Gewerbesteuereinnahmen an die umliegenden Wohngemeinden nach Maßgabe der von dort einpendelnden Mitarbeiter leisten. Die Gewerbesteuerausgleichszuschüsse wurden 1949 und 1950 nur zu 50 v.H. in die Finanzkraftberechnung bei den Empfängern und Zahlern einbezogen, 
der Gewerbesteuerumlage, die Grundsteuern A und B, bis 1950 die Bürgersteuerausgleichsbeträge ${ }^{1}$ und seit 1970 der kommunale Anteil an der Einkommensteuer sowie seit dem KFA von 1999 jener an der Umsatzsteuer. Andere Steuereinnahmen wie die örtlichen Aufwand- und Verbrauchsteuern oder der Grunderwerbsteueranteil finden ebensowenig Berücksichtigung wie die übrigen Einnahmeninstrumente der Gemeinden, etwa Einnahmen aus Vermögensnutzung und -veräußerung, Krediten, Gebühren und Beiträgen usw. Somit reflektierten im Jahr 1996 in Hessen 89,62 v.H. der kommunalen Steuerkraft die Finanzkraft, dies sind ca. 38,23 v.H. der kommunalen Einnahmen ohne Kredite.

Die Finanzkraft ergibt sich durch Addition der mittels Nivellierungssatz normierten Realsteuern unter Berücksichtigung der Gewerbesteuerumlage und dem mit einem Anrechnungsfaktor ${ }^{2}$ multiplizierten Ist-Aufkommen von Einkommen- und Umsatzsteuerbeteiligung. In Abbildung 18 ist der jeweils gültige Anrechnungssatz für den Einkommensteueranteil angegeben, für die Gewerbesteuer und die Grundsteuer A wird er als Verhältnis von Nivellierungssatz und dem gewogenen Durchschnittshebesatz errechnet. Für die Grundsteuer B ist eine solche Darstellung nicht möglich, da es erst seit 1999 einen einheitlichen Normierungssatz gibt. ${ }^{3}$ Diese Größen können als Erfassungsgrad der kommunalen Steuer- oder Finanzkraft interpretiert werden. Es ist ersichtlich, daß die im KFA verwendeten Nivellierungshebesätze zu keiner Zeit das Niveau der tatsächlichen gewichteten durchschnittlichen Hebesätze bei den Realsteuern erreichten. ${ }^{4}$ Seit fast 20 Jahren liegt der Normierungssatz der Grundsteuer A etwa bei 70 v.H. des gewichteten

ab 1951 jeweils zu 100 v.H.; seit 1955 bzw. 1959 wurden sie bei den Wohngemeinden nur noch zu 50 bzw. 1/3 v.H. angerechnet, um bei unveränderten Ausgleichszuschußbetrag über die zu leistenden Umlagen im KFA einen indirekten Ausgleich zu erhalten. Aufgrund des Finanzreformgesetz und des GFRG wurde in Hessen das Gesetz über den Gewerbesteuerausgleich abgeschafft. Statt dessen wurde nun die an die staatliche Ebene zu leistende Gewerbesteuerumlage mindernd berücksichtigt.

1 Zugeflossene Bürgersteuerausgleichsbeträge gingen voll in die Finanzkraftberechnung ein.

2 Die Verwendung eines Anrechnungsfaktors wird damit begründet, daß durch das Normierungsverfahren bei den Realsteuern nicht das gesamte tatsächliche Aufkommen bei der Finanzkraftberechnung erfaßt wird. Deshalb sollen die nicht direkt beeinflußbaren Einnahmen nur im gleichen Umfang berücksichtigt werden. Für 1998 betrug der Anrechnungsfaktor 93 v.H.

3 Bis 1998 wurde ein gespaltener Normierungssatz furr die Grundsteuer B verwendet, so daß zur Ermittlung des Erfassungsgrades die Bemessungsgrundlagen jeder einzelnen Gemeinde für die entsprechenden Jahre bekannt sein müßte.

4 Auf die Darstellung der Werte vor 1970 wird verzichtet, da dies nur die Gewerbesteuer und die Grundsteuer A betrifft. Im ersten Jahrzehnt des 1956 gegründeten Steuerverbundes wurde der maximale Erfassungsgrad erreicht, als die Normierungssätze den gewichteten durchschnittlichen Hebesätzen der Gewerbesteuer zu mehr als 90 und der Grundsteuer A zu mehr als 80 v.H. entsprachen. In den folgenden Jahren verringerte sich der Erfassungsgrad trotz diskretionärer Anpassungen an die zeitliche Entwicklung kontinuierlich und betrug bei der Grundsteuer A 1976 nur noch 56,68 v.H. 
Mittelwertes, bei der Gewerbesteuer schwankt er zwischen 65 und 75 v.H. Diese Entwicklung führt zu einer entsprechend starken Untererfassung der kommunalen Finanzkraft, von der wiederum die finanzstarken Gebietskörperschaften absolut im größeren Maße profitieren, da ihre - durch die größere Bemessungsgrundlage und höheren Hebesätze gegebenen - Mehreinnahmen nicht eingerechnet werden.

\section{Abbildung 18:Entwicklung der Erfassung der kommunalen Finanzkraft in Hessen $^{1}$}

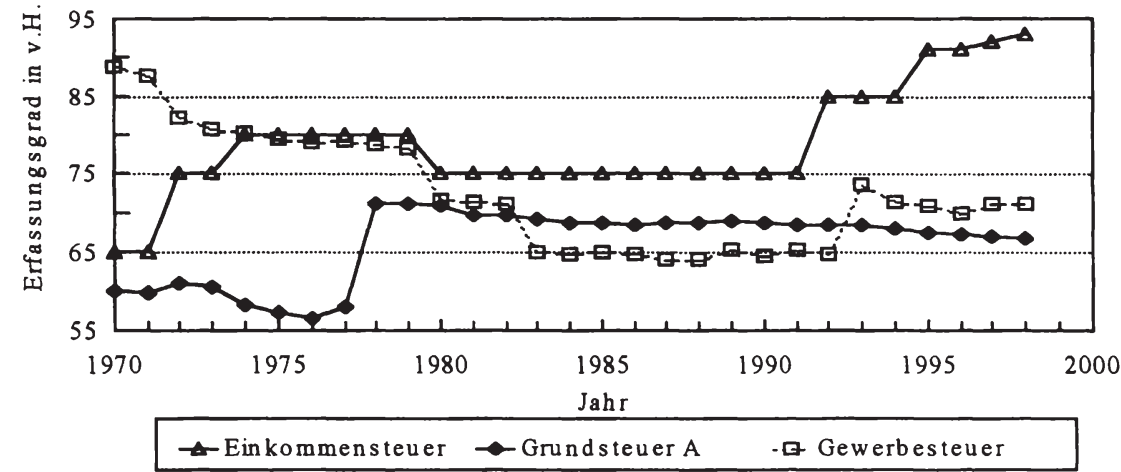

Quelle: Finanzausgleichsgesetze und Statistisches Bundesamt: Finanzen und Steuern, Reihe 9, Realsteuern, I. Realsteuervergleich; Fachserie 14, Finanzen und Steuern, Reihe 10.1, Realsteuervergleich; Fachserie 14, Finanzen und Steuern, Reihe 10.1., Realsteuervergleich Realsteuern und kommunale Einkommensteuerbeteiligung; verschiedene Jahre.

Weiterhin ist festzustellen, daß sich der Grad der Finanzkrafterfassung zwischen den Realsteuern einerseits und dem kommunalen Einkommensteueranteil andererseits, bei dem kein Hebesatzproblem gegeben ist, unterscheidet. So wurde der kommunale Einkommensteueranteil in den ersten Jahren in einem geringerem Umfang zur Finanzkraftermittlung herangezogen als etwa die ähnlich aufkommenstarke Gewerbesteuer, nämlich mit 65 im Vergleich zu über 80 v.H., während dieser Satz bei der eher unwichtigen Grundsteuer A 60 v.H. betrug. Diese Verfahrensweise wurde damit erklärt, daß der Einkommensteueranteil bei den Wohngemeinden auch noch "den Wegfall der (...) Gewerbesteuerausgleichsbeträge ausgleichen soll"2 . Als Begründung für die in zwei Stufen vorgenommene weitere Erhöhung des Erfassungsgrads der Einkommensteuer wurde angeführt, daß beide Steuern als gleichwertig anzusehen und deshalb auch gleich anzurechnen sind. ${ }^{3}$ Doch erst 1974 wurde - aufgrund der diskretionären Erhöhung des

1 Auf die Berücksichtigung der Bürgersteuerausgleichsbeträge und der Vergnügungsteuer wurde verzichtet, da diese nur wenige Jahre zur Finankraftermittlung verwendet wurden.

2 Hessischer Landtag: Vorlage der Landesregierung für ein Fünftes Gesetz zur Änderung des Finanzausgleichsgesetzes vom 07.07.1969, Drucksache 6/2194, S. 15.

3 Vgl:: Ders.: Vorlage der Landesregierung betreffend den Entwurf furr ein Siebentes Gesetz 
Anrechnungsfaktors der Einkommensteuer auf 80 v.H. - etwa das Niveau der Gewerbesteuer mit 80,36 v.H. erreicht.

Dieses Bild hat sich in späteren Jahren geändert, denn der Erfassungsgrad des Einkommensteueranteils wurde immer weiter angehoben, während dies bei den Realsteuern kaum und dann auch nur geringfügig geschah, so daß die vergleichsweise geringere Erfassung beibehalten wurde. Im Jahr 1980 wurde als Folge bundesgesetzlicher Regelungen - Abschaffung der Lohnsummensteuer bei gleichzeitiger Erhöhung des kommunalen Einkommensteueranteils und der Senkung der Gewerbesteuerumlage - die Verminderung der Anrechnung bei der Einkommen- und der Gewerbesteuer veranlaßt. Da die Lohnsummensteuer im Gegensatz zur Einkommensteuer weder bei der Verteilung der gemeindlichen Schlüsselzuweisungen noch bei der Kreisumlagebemessung berücksichtigt worden war, hätte sich ohne Veränderung der Anrechnungsfaktoren die Bemessungsgrundlage der von den Gemeinden zu entrichtenden Umlagen erhöht. Dadurch wäre ein Teil der Kompensationsmittel unerwünschter Weise an die Kreise abgeflossen. Die späteren Anhebungen des Anrechnungssatzes der Einkommensteuer 1992 und 1995 wurden mit dem Ziel der stärkeren Heranziehung einkommensteuerstarker Gemeinden zur Finanzierung der Deutschen Einheit - über verringerte Schlüsselzuweisungen - begründet, da gewerbesteuerstarke Gemeinden durch die Erhöhung der Gewerbesteuerumlage schon entsprechend belastet waren. ${ }^{1}$

Aus der Sicht des Landes waren nach der Neufestlegung des Gewerbesteuernormierungssatzes 1993 die beiden wichtigsten Steuerquellen im gleichen Umfang zu 85 v.H. - berücksichtigt, allerdings wurde dabei nur Bezug auf den kreisangehörigen Raum genommen und die weitaus höheren Hebesätze der kreisfreien Städte vernachlässigt. ${ }^{2}$ Als Folge der fehlenden Dynamisierung der Hebesätze hat sich der auf den gewichteten Mittelwert vom kreisfreien und kreisangehörigen Raum bezogene Erfassungsgrad bei der Gewerbesteuer fast stetig auf 71,26 v.H. und bei der Grundsteuer A auf 66,79 v.H. 1998 verringert, während der Anrechnungsfaktor bei der Einkommensteuer bei 93 v.H. angelangt ist (Abbildung 18).

zur Änderung des Finanzausgleichsgesetzes vom 14.02.1972, Drucksache 7/1322, S. 11; ders.: Gesetzentwurf der Landesregierung für ein Neuntes Gesetz zur Änderung des Finanzausgleichsgesetzes vom 05.10.1973, Drucksache 7/4135, S. $6 f$.

1 Vgl: Ders.: Gesetzentwurf der Landesregierung für ein Gesetz zur Änderung des Finanzausgleichsgesetzes und anderer Rechtsvorschriften - Finanzausgleichsänderungsgesetz 1992 - vom 01.11.1991, Drucksache 13/841, S. 2; ders.: Gesetzentwurf der Landesregierung für ein Gesetz zur Änderung des Finanzausgleichsgesetzes vom 25.07.1994, Drucksache 13/6401, S. 2.

2 Vgl:: Ders.: Art 2 des Gesetzentwurf der Landesregierung für ein Gesetz zur Überleitung der Aufgaben der Hilfe zur Pflege vom 23.10.1992, Drucksache 13/2984, S. 7. So liegt für die kreisfreien Städte der Erfassungsgrad bei der Gewerbesteuer seit Anfang der 80er Jahre etwa bei 57 v.H., bei den kreisangehörigen Gemeinden hingegen zwischen 75 und 85 v.H. 
Im Jahr 1996 wurde dann eine Strukturreform mit dem Ziel begonnen, "durch eine höhere Erfassung der tatsächlichen Einnahmen der Kommunen bei der Steuerkraftmeßzahl und bei den Umlagegrundlagen die Ausgleichsfunktion des Kommunalen Finanzausgleichs zu stärken", wobei "grundsätzlich (...) die kommunalen Einnahmen im Finanzausgleich zu 95 v.H. angerechnet werden"1 sollen. Eine Übergangsbestimmung sorgt dafür, daß die Erhöhung des Anrechnungsgrades von 95 v.H. schrittweise bis zum Jahr 2000 erreicht wird. ${ }^{2}$ Berücksichtigt wurden von den Hebesätzen des Jahres 1995 zur Ermittlung des Normierungssatzes aber nur diejenigen der kreisangehörigen Gemeinden, ${ }^{3}$ obwohl beide Gemeindegruppen separate Schlüsselmassen haben und deshalb auch die Durchschnittswerte separat berechnet werden können. Im Gegensatz zur Einkommensteuer und den Schlüsselzuweisungen erreicht der Erfassungsgrad bei der Gewerbesteuer selbst im kreisangehörigen Raum im Jahr 2000 - bezogen auf die Hebesätze von 1995 nur 93,7 v.H. statt der angestrebten 95 v.H., da der Normierungssatz nur 310 und nicht 314 v.H. beträgt. ${ }^{4}$

Für 1999 hat man auch noch die Grundsteuer in die Reform einbezogen, wobei nochmals angeführt wurde, daß ausschließlich "die ausgleichsrelevanten Einnahmen der kreisangehörigen Gemeinden zu 95 v.H. bei der Finanzkraft berücksichtigt werden sollen"s. Aber auch hier wurde der Anspruch nicht Wirklichkeit, denn im Gesetzgebungsverfahren wurde der Normierungssatz von den ermittelten 228 auf 220 v.H. verringert. Weiterhin ist noch ein inkonsistentes Vorgehen bei der Berechnung der Normierungssätze zu konstatieren. Denn als Berechnungsgröße wurden nicht die Werte von 1995 wie zu Beginn der Reform bei der Gewerbesteuer, sondern jene von 1997 verwendet. Dies hätte zu einer vergleichsweise stärkeren Erfassung bei der Grundsteuer geführt, wenn jeweils ein Erfassungsgrad von 95 v.H. erreicht worden wären, da sich in der Zwischenzeit das gewichtete

1 Ders.: Gesetzentwurf der Landesregierung für ein Gesetz zur Änderung des Finanzausgleichsgesetz vom 21.10.1996, Drucksache 14/2259, S. 1.

2 Für den Einkommensteueranteil beträgt die Berücksichtigung der Steuerkraft für die Jahre 1997 bis 1999 92, 93 und 94 v.H.; ab 2000 zu 95 v.H. Für die Jahre 1997 bis 1999 wird der Nivellierungshebesatz der Gewerbesteuer mit 287, 295 und 302 v.H. festgelegt, ab 2000 beträgt er dann 310 v.H. Vgl: Gesetz zur Regelung des Finanzausgleichs (Finanzausgleichsgesetz - FAG -) vom 18.03.1997, Hess. GVB1. I, S. 61, FN 4f.

3 Vgl.: Ebenda, S. 10; ders.: Änderung des Finanzausgleichsgesetzes, Art. 2 des Gesetzentwurf der Landesregierung für ein Gesetz zur Überleitung der Aufgaben der Hilfe zur Pflege vom 23.10.1992, S. 7; ders.: Gesetzentwurf der Landesregierung für ein Neuntes Gesetz zur Änderung des Finanzausgleichsgesetzes vom 05.10.1973, Drucksache 7/4135, S. 6.

$4 \mathrm{Daß}$ der tatsächliche dann unter dem vorher propagierten Erfassungsgrad von 95 v.H. liegen würde, wird sogar explizit im Gesetzentwurf aufgeführt. Vgl: Ders.: Gesetzentwurf der Landesregierung für ein Gesetz zur Änderung des Finanzausgleichsgesetzes vom 21.10.1996, Drucksache 14/2259, S. 10.

5 Hessischer Landtag: Gesetzentwurf der Landesregierung für ein Finanzausgleichsänderungsgesetz 1999 vom 23.06.1998, Drucksache 14/4013, S. 1. 
Mittel bei allen Steuern erhöht hatte. ${ }^{1}$ Als Ergebnis ergibt sich für die kreisangehörigen Gemeinden bezogen auf 1995 (1997) ein Erfassungsgrad bei der Grundsteuer A von 84,9 (84,3) v.H. und bei der Grundsteuer B von $92,8(91,7)$ v.H.

Für die kreisfreien Städte wird der angestrebte Anrechnungsgrad von 95 v.H. bei den Realsteuern im Gegensatz zur Einkommensteuer nie erreicht. So betrug z.B. 1997 der gewichtete durchschnittliche Hebesatz der Gewerbesteuer für diese Gruppe 492 v.H. ${ }^{2}$, was einer Finanzkrafterfassung von 58,3 v.H. gegenüber 85,2 v.H. bei den kreisangehörigen Gemeinden und 92 v.H. bei der Einkommensteuer entsprach. Auch bei der Grundsteuer B liegt nach der Reform 1999 der Erfassungsgrad mit 42,9 v.H. weit unter den zu Beginn der Strukturreform propagierten 95 v.H. bzw. dem bei den kreisangehörigen Gemeinden erreichten Wert. Nur bei der für die kreisfreien Städte verhältnismäßig unwichtigen Grundsteuer A liegt der Erfassungsgrad mit 87,6 v.H. über jenem der kreisangehörigen Gemeinden.

\subsubsection{Analyse und Reform}

\subsection{Abgrenzung der ausgleichsrelevanten Einnahmen}

Im Kapitel 3 wurde gefordert, zur Finanzkraftermittlung die kommunalen Einnahmen möglichst umfassend einzubeziehen. In Hessen werden aber - wie in allen anderen Systemen des KFA und im LFA - ausschließlich die Steuereinnahmen und auch diese nur zu einem Teil - nämlich nur die Realsteuern und der kommunale Anteil an der Einkommen- und Umsatzsteuer - berücksichtigt. Es ist nun zu klären, ob nicht auch auf andere Einnahmenarten zurückgegriffen werden sollte.

\subsection{Vermögenserträge und vergleichbare Einnahmen}

Den Grundsätzen der kommunalen Einnahmenerzielung folgend stehen nach $\S 93 \mathrm{HGO}$ an erster Stelle alle Einnahmen, die nicht unter die Kategorie Steuern, Gebühren und Beiträge sowie Kredite - außer Kassenkredite - fallen. $\mathrm{Zu}$ nennen sind etwa Erträge aus Vermögensnutzung und -veräußerung sowie Zuweisungen von Dritten. Da aber Veräußerungsgewinne und Darlehensrückflüsse nicht die Finanzkraft erhöhen, es sich in beiden Fällen vielmehr um einen Aktivtausch handelt, sind diese Größen nicht bei der Bemessung der Finanzkraft zu berücksichtigen, denn sie stellen keine Erhöhung der Leistungsfähigkeit dar. ${ }^{3} \mathrm{Zu}$ veräußernde

1 Für die Grundsteuer beträgt der Nivellierungssatz seit 1999 einheitlich 220 v.H. Vgl.: Finanzausgleichsänderungsgesetz 1999 vom 25.11.1998 Hess. GVB1. I, S. 496. Er hätte aber für die Grundsteuer A 246 v.H. (1995) bzw. 248 v.H. (1997) und für die Grundsteuer B 225 v.H. (1995) bzw. 228 v.H. (1997) betragen müssen.

2 Vgl.: Statistisches Bundesamt: Fachserie 14, Finanzen und Steuern, Reihe 10.1., Realsteuervergleich - Realsteuern und kommunale Einkommensteuerbeteiligung 1997, Stuttgart 1998, S. 62 .

3 Vgl.: Kirchhof, P.: Der Finanzausgleich als Grundlage kommunaler Selbstverwaltung, in: DVB1., 95. Jg., 1980, S. $716 f$. 
Vermögensgegenstände können nur durch eigene Einnahmen etwa Steuern erworben worden sein, diese wurden aber schon einmal bei der Finanzkraftmessung im KFA berücksichtigt (s.u.). Ebenso verhält es sich bei Darlehensrückflüssen, da zur vorherigen Kreditgewährung ebenfalls Einnahmen vorhanden gewesen sein mußten. Damit die unterschiedliche Verwendung von Finanzmitteln keinerlei Auswirkungen auf die Finanzkrafterfassung hat, dürfen nach der Übertragung des für natürliche Personen bei der Besteuerung geltenden Korrespondenzprinzips die Einnahmen nur einmal erfaßt werden. ${ }^{1}$ Dies ist aber schon bei der Steuerkrafterfassung der Fall gewesen.

\subsection{Zuweisungen}

Die Zuweisungen erhöhen hingegen die Leistungsfähigkeit der Gemeinden und GV, wobei aus der Berechnungsweise klar wird, daß die Schlüsselzuweisungen nur für die im Finanzausgleichsgesetz geregelten Umlagen Berücksichtigung finden können. Es stellt sich allerdings die Frage, ob auf die übrigen Zuweisungen zurückzugreifen ist, da sie ohne explizite Berücksichtigung der Finanzkraft verteilt werden. ${ }^{2}$ Dem ist aber entgegenzuhalten, daß mit Hilfe der zweckgebundenen Mittel spezifische Belastungen der Gemeinden (etwa Ausgaben für Sozialhilfe) kompensiert werden sollen und diese Gelder somit keine Erhöhung der Leistungsfähigkeit darstellen. Bei dieser Vorgehensweise würden Gemeinden versuchen, auf Zweckzuweisungen zu verzichten, da durch diese Schlüsselzuweisungen, welche einen höheren Freiheitsgrad haben, substituiert würden. ${ }^{3}$ Das Ziel der Zweckzuweisungen, gerade den benachteiligten Gemeinden und Regionen gezielt zu helfen, wäre somit konterkariert. ${ }^{4}$

\subsection{Leistungsentgelte}

Als nächste Einnahmenart sind die Leistungsentgelte zu betrachten. Ob Gebühren und Beiträge bei der Ermittlung der Finanzkraft zu berücksichtigen sind, ist umstritten. ${ }^{5}$ Von einer Finanzkrafterhöhung könnte aber nur beim rechtlich nicht

Vgl.: Andel, N.: Finanzwissenschaft, 4. Aufl., Tübingen 1998, S. 318.

2 Vgl:: Hansmeyer, K.-H; Kops, M.: Finanzwissenschaftliche Grundsätze für die Ausgestaltung des kommunalen Finanzausgleichs, in: Hoppe, W. (Hrsg.): Reform des kommunalen Finanzausgleichs, Köln u.a.O. 1985, S. 48f.

3 Das Substitutionsverhältnis zwischen zweckgebundenen und Schlüsselzuweisungen liegt allerdings nicht immer bei eins zu eins. So würde sich für eine abundante Kommune nichts ändern, da sie schon jetzt wegen ihrer Finanzkraft nur die Mindestzuweisungen erhält.

4 Vgl.: Deubel. I.: Der kommunale Finanzausgleich in Nordrhein-Westfalen, Köln u.a.O 1984, S. 120.

5 KIRCHHOF sieht Gebühren und Beiträge als ein Entgelt für eine staatliche Leistung an, während Fischer einen Einfluß auf die kommunale Finanzkraft unterstellt. Vgl.: Kirchhof, P.: Die kommunale Finanzhoheit, in: Püttner, G. (Hrsg.): Handbuch der kommunalen Wissenschaft und Praxis, Bd. 6, 2. Aufl., Berlin u.a.O. 1985, S. 25; Fischer, H.: Finanzzuweisungen. Theoretische Grundlegung und praktische Ausgestaltung im bundesstaatlichen Finanz- 
zulässigen Fall einer Kostenüberdeckung ${ }^{1}$ gesprochen werden, nicht aber bei den beiden verbleibenden Möglichkeiten, nämlich einer Kostendeckung zu 100 v.H. oder einer Kostenunterdeckung. ${ }^{2}$ Der erste Fall wirkt sich nicht auf die Leistungsfähigkeit aus, da die Gebühren und Beiträge ein Entgelt für die öffentlich bereitgestellten Leistungen und den damit verbundenen Kosten darstellen. ${ }^{3}$ Im Falle der Kostenunterdeckung müssen aber entweder die zur Finanzierung notwendigen Einnahmen aus anderen Quellen wie Steuern oder durch Minderausgaben erwirtschaftet werden. Da substantielle Steuermehreinnahmen nur mittels Hebesatzänderungen bei den Realsteuern möglich sind, kommt es zu einer Umverteilung innerhalb der Gemeinde zugunsten der Nutzer einer Anlage auf Kosten der Gewerbetreibenden bei einer unterstellten Finanzierung mittels einer erhöhten Gewerbesteuer. Diese erhöhten Einnahmen haben aber aufgrund der Art der Steuerkraftermittlung keinerlei Auswirkungen auf den KFA. Zudem dürfte eine annähernd gleichmäßige Streuung der Einnahmen aus Leistungsentgelten zwischen den Gemeinden und GV unterstellt werden. Aus diesen Gründen ist auf eine Berücksichtigung dieser Einnahmenkategorie bei der Finanzkraftberechnung zu verzichten.

\subsection{Weitere kommunale Steuern}

Steuern und Abgaben erhöhen allgemein die Leistungsfähigkeit und sind deshalb prinzipiell bei der Ermittlung der Finanzkraft zu berücksichtigen. ${ }^{4}$ Deshalb sollte der kommunale Anteil an der Grunderwerbsteuer neben den schon berücksichtigten Steuern in die Finanzkraftbemessung einbezogen werden, ${ }^{5}$ denn diese Beteiligungsform kann mit jener der Einkommensteuer verglichen werden. Das Aufkommen des kommunalen Grunderwerbsteueranteils lag 1996 mit etwa 282 Mio. DM mehr als achtmal höher als das Aufkommen aus der Grundsteuer A. Die Grunderwerbsteuer geht aber bisher nur in Rheinland-Pfalz in die Berechnung der kommunalen Finanzkraft ein.

ausgleich Australiens und der Bundesrepublik Deutschland, Berlin 1988, S. 108-111.

1 Allerdings bietet der Ansatz von Wiederbeschaffungs- statt von Anschaffungskosten die Chance einer teilweisen - aus handelsrechtlicher Perspektive - Überdeckung der Kosten.

2 Vgl.: $\S 10$ Abs. 2 Gesetz über kommunale Abgaben (KAG) vom 17.03.1970, Hess. GVBl. I, S. 225.

3 Vgl: Henneke, H.-G.: Finanzausgleich: Die Verteilung der Schlüsselzuweisungen als DreiKomponenten-System, in: Die Gemeindekasse, 1995, S. 5. Es handelt sich um einen Aktivtausch, da daß vorhandene Vermögen (z.B. Abwasserkanäle) durch Zahlungen der Nutzer ersetzt wird.

4 Vgl: Bundesverfassungsgericht: Urteil vom 24.06.1986, in: Entscheidungen des Bundesverfassungsgerichts, Bd. 72, Tübingen 1987, S. 400.

5 Vgl:: Kirchhof, P.: Rechtliche Rahmenbedingungen des kommunalen Finanzausgleichs, in: Hoppe, W. (Hrsg.): Reform des kommunalen Finanzausgleichs, Köln u.a.O. 1985, S. 19; Hardt, U.: Kommunale Finanzkraft. Die Problematik einer objektiven Bestimmung kommunaler Einnahmemöglichkeiten in der gemeindlichen Haushaltsplanung und im kommunalen Finanzausgleich, Frankfurt u.a.O. 1988,S. 39. 
Als Besonderheit ist bei den örtlichen Aufwand- und Verbrauchsteuern, die den Kommunen laut Art. 106 Abs. 6 GG zugeteilt sind, die sehr weitgehende kommunale Gestaltungsfreiheit bei gleichzeitig geringem Einzelaufkommen anzuführen. Die Autonomie äußert sich in der grundsätzlichen Frage der Steuererhebung an sich, in der unterschiedlichen Ausgestaltung der Bemessungsgrundlage und des Tarifs. Dies führt dazu, daß sich die interkommunale Normierung des jeweiligen Steueraufkommens sehr viel aufwendiger gestalten würde als bei den Realsteuern, bei denen dies mit Hilfe der Grundbeträge und fiktiver Normierungssätze vergleichsweise einfach ist. Weit wichtiger ist aber das geringe Aufkommen, welches als nicht ausgleichsrelevant gilt. ${ }^{1}$ So liegen die Einnahmen der Vergnügungsteuer als aufkommenstärkste Steuer 1996 mit 39,7 Mio. DM sehr viel niedriger als die neu zu berücksichtigende Grunderwerbsteuer mit 281,8 Mio. DM. Auch das Argument der ungleichen räumlichen Steuerkraftverteilung dürfte - im Gegensatz zur Gewerbesteuer - kaum Relevanz haben; die Anzahl der Hunde je Einwohner dürfte im Raum relativ gleichmäßig streuen. Somit kann auf die Erfassung verzichtet werden, ohne ins Gewicht fallende Ungerechtigkeiten hervorzurufen.

Anders ist die Situation bei den Konzessionsabgaben (KA) zu beurteilen, die Versorgungsunternehmen für die Zubilligung des Rechts der Leitungsverlegung auf kommunalen Grundstücken und Straßen an die Kommunen entrichten. ${ }^{2}$ Sie erhöhen die Leistungsfähigkeit der Kommune, und auch vom Volumen her sind sie als ausgleichsrelevant anzusehen; das Aufkommen betrug 1996 in Hessen etwa 603 Mio. DM. ${ }^{3}$ Davon zu sprechen, daß die Einnahmen verhältnismäßig gleich in den Kommunen anfallen, ist kaum möglich. ${ }^{4}$ Somit ist die derzeitige fehlende Berücksichtigung nicht unproblematisch. ${ }^{5}$ Auch nach Auffassung des Bundesverfassungsgerichts könnten die KA vom Prinzip her berücksichtigt werden, doch aufgrund der Situation im Jahr 1992 entschied das Gericht gegen ihre Verwendung bei der Finanzkraftermittlung. ${ }^{6}$ Denn die aus dem Jahr 1941 stammende Regelung ${ }^{7}$ war gerade erst unter Gewährung von mehrjährigen Übergangsfristen

Vgl.: Hidien, J. W.: Handbuch Länderfinanzausgleich, 1. Aufl., Baden-Baden 1999, S. 454.

Vgl.: Gern, A.: Deutsches Kommunalrecht, 1. Aufl., Baden-Baden 1995, S. $361 f$.

Von dieser Summe entfielen etwa 53,9 v.H. auf die kreisfreien Städte, 46 v.H. auf die kreisangehörigen Gemeinden und 0,1 v.H. auf die Landkreise.

4 Vgl.: Eschenbach, J.: Die Neukonzeption des kommunalen Finanzausgleichs in Niedersachsen - Neuanfang oder fortgesetzter Verfassungsbruch?, in: ZKF, 49. Jg., 1999, S. 60.

5 Vgl:: Albers, H.: Die Ausgleichsregelungen des kommunalen Finanzausgleichs in Niedersachsen im Spannungsfeld von verfassungsrechtlichen Vorgaben und politischen Zielen, in: Niedersächsische Verwaltungsblätter, 3. Jg., 1996, S. 174; Henneke, H.-G.: Jenseits von Bückeburg. Gesetzgeberische Gestaltungsspielräume für den kommunalen Finanzausgleich, in: Niedersächsische Verwaltungsblätter, 3. Jg., 1996, S. 13f.

6 Vgl: : Bundesverfassungsgericht: Urteil vom 27.05.1992, in: Entscheidungen des Bundesverfassungsgerichts, Bd. 86, Tübingen 1993, S. 225-229.

7 Nach dieser Regelung durften die KA zukünttig nur in den Kommunen erhoben werden, in denen dies damals schon der Fall gewesen war. Es bestand keine Möglichkeit der 
abgelöst worden, so daß 1992 noch für mehrere Jahre keine Möglichkeit einer bundeseinheitlichen Ermittlung eines Soll-Aufkommens bestand. Dieses Argument kann heute allerdings nicht mehr gelten, da aufgrund der gesetzlichen Neugestaltung der Konzessionsabgabenverordnung ${ }^{1}$ und der Aufhebung des Neuerhebungsverbots "die Abgaben mittlerweile mit Inkraftreten des gesamtdeutschen Finanzausgleichs ab 1995 in Form eines normierten (Durchschnitts-)Aufkommens ausgleichsfähig sind"2. Diese Meinung äußerten auch der Niedersächsische Landkreistag in seiner Stellungnahme zum NFAG und der Wissenschaftliche Beirat beim Bundesministerium der Finanzen, der die Konzessionsabgaben mit der schon - im LFA - berücksichtigten Bergrechtlichen Förderabgabe vergleicht. ${ }^{3}$

Auch Spielbank- und Troncabgabe ${ }^{4}$ sind in die Finanzkraftbemessung einzubeziehen, da ihr Aufkommen ebenfalls ausgleichsrelevant ist und zudem das Aufkommen sehr stark streut, da nur die vier hessischen Spielbankgemeinden von den Einnahmen profitieren und davon wiederum mit über 15 Mio. DM mehr als 50 v.H. auf die Stadt Bad Homburg entfallen. Im Vergleich zu den lokalen Aufwandund Verbrauchsteuern dürften die Kosten ihrer Berücksichtigung im Rahmen des KFA gering sein, da die Verwaltungshoheit der Abgaben beim Land liegt und es die Einnahmen an die empfangsberechtigten Gemeinden abführt, wobei es nur die geringe Anzahl hessischer Spielbankgemeinden zu berücksichtigen gilt. Als erstes Land erfaßt Niedersachsen seit 1999 die Spielbankabgabe im Rahmen der Steuerkraftberechnung des KFA, wobei das dortige kommunale Aufkommen mit etwas über 12,5 Mio. DM niedriger liegt als der in Hessen allein auf Bad Homburg entfallende Betrag. Als Begründung für eine Berücksichtigung kann das räumlich sehr ungleich anfallende Aufkommen angeführt werden. Weiterhin wird die

Neuerhebung.

1 Vgl.: Verordnung über Konzessionsabgaben für Strom und Gas (Konzessionsabgabenverordnung KAV) vom 09.01.1992, BGB1. I, S. 12-14. Für Wasser-Versorgungsunternehmen gilt noch immer die Konzessionsabgabenanordnung aus dem Jahr 1941, wodurch nicht die rechtlichen Voraussetzungen fur eine landesweite Erhebung dieser Konzessionsabgabe gegeben sind und diese auch nicht bei der Finanzkraftberechnung einzubeziehen ist.

2 Hidien, J. W.: Handbuch Länderfinanzausgleich, 1. Aufl., Baden-Baden 1999, S. 458.

3 Vgl.: Niedersächsischer Landkreistag: Gesonderte Stellungnahme des Niedersächsischen Landkreistages vom 15. Dezember 1998 zur Neuordnung des kommunalen Finanzausgleichs zum 1. Januar 1999, in: NLT-Information, Niedersächsischer Landkreistag, 22. Jg., 1999, S. 47; Wissenschaftlicher Beirat beim Bundesministerium der Finanzen: Gutachten zum Länderfinanzausgleich in der Bundesrepublik Deutschland, Schriftenreihe des Bundesministerium der Finanzen, Heft 47, Bonn 1992, S. 55.

4 Die Spielbanken in Deutschland unterliegen weder der Einkommen- bzw. der Körperschaft-, der Umsatz- noch der Gewerbesteuer. Dafür haben sie eine Abgabe an die Länder zu entrichten, die sich nach den jeweiligen Bruttospielerlösen richtet. Nach dem hessischen Spielbankgesetz beträgt der Abgabesatz 80 v.H. Die Spielbankgemeinden partizipieren mit 29,375 v.H. am örtlichen Aufkommen. Hinzu kommt noch die Hälfte der 4 v.H. betragenden Troncabgabe, welche auf die Trinkgelder der Croupiers (Tronc) erhoben wird. 
weitgehende Steuerbefreiung der Spielbanken genannt, so daß die Spielbankabgabe auch als teilweiser Ersatz für die Gewerbesteuerbefreiung angesehen werden kann und folglich wie die Gewerbesteuer bei der Steuerkraftberechnung einzubeziehen ist. ${ }^{1}$

\subsection{Krediteinnahmen}

Von den Kommunen aufgenommene Kredite, geben lediglich ihre Kreditfähigkeit, nicht aber eine eigene Leistungsfähigkeit wieder. ${ }^{2}$ Denn die Kreditaufnahme stellt nur ein temporäres, aber kein definitives Finanzierungsinstrument dar, der eine zukünftige Rückzahlungsverpflichtung gegenüber steht. Zudem ist sie selbst wiederum Ausdruck der Einnahmenkraft der Kommunen - vor allem in Form der Steuereinnahmen. Diese Steuerkraft wurde aber schon im KFA berücksichtigt. ${ }^{3}$ Folglich sollten auch die Krediteinnahmen nicht bei der Finanzkraftermittlung berücksichtigt werden.

Nach den bisherigen Ausführungen ist somit zu fordern, daß zum Zwecke der genaueren Finanzkraftermittlung neben den schon jetzt einbezogenen Realsteuern und den gemeindlichen Anteilen an der Einkommen- und Umsatzsteuer auch noch der kommunale Anteil an der Grunderwerbsteuer, der Spielbank- und der Troncabgabe sowie die Konzessionsabgaben einzubeziehen sind.

\subsection{Erfassungsgrad der ausgleichsrelevanten Einnahmen}

Als nächstes gilt es nun zu klären, in welchem Umfang die zu berücksichtigenden Einnahmenarten bei der Finanzkraftberechnung erfaßt werden sollen, da teilweise die Möglichkeit der direkten Beeinflussung der Einnahmenhöhe besteht. Ziel muß es sein, alle Einnahmenkategorien im gleichen Umfang zu berücksichtigen, um etwaige Verteilungswirkungen nicht schon bei der Finanzkraftberechnung zu induzieren. Da die Finanzkraft so genau wie möglich erfaßt werden soll, kommen bei den Einnahmenkategorien ohne Einnahmenautonomie nur die Ist-Einnahmen in Frage. Dies wäre dann bei der Einkommen-, der Grunderwerb-, der Umsatzsteuer und der Spielbankabgabe einschließlich Tronc der Fall. Bei den Realsteuern und den Konzessionsabgaben käme hingegen ein Normierungsverfahren zum Einsatz. ${ }^{4}$ Dabei kann für die Realsteuern das schon praktizierte Normierungsverfahren beibehalten werden, mit dem schon bisher vermieden wird, daß einzelne Gemeinden

1 Vgl.: Niedersächsischer Landtag: Entwurf eines Zweiten Gesetzes zur Änderung des Niedersächsischen Gesetzes über den Finanzausgleich vom 12.01.1998, Drucksache 14/440, S. 27.

2 Vgl:: Kirchhof, P.: Der Finanzausgleich als Grundlage kommunaler Selbstverwaltung, in: DVBl., 95. Jg., 1980, S. 716.

3 Vgl: Zimmermann, H.: Allgemeine Probleme des Finanzausgleichs, in: Neumark, F.; Andel, N. und Haller, $H$. (Hrsg.): Handbuch der Finanzwissenschaft, Bd. 4, 3. Aufl., Tübingen 1983, S. 52.

4 Vgl.: Hidien, J. W.: Handbuch Länderfinanzausgleich, 1. Aufl., Baden-Baden 1999, S. 205. 
über den Hebesatz Einfluß auf die Höhe der ihnen zufließenden Schlüsselzuweisungen nehmen können. Es ist aber zu untersuchen, ob die Bestimmung des Hebesatzes nicht modifiziert werden sollte. Dabei ist zu berücksichtigen, daß der Erfassungsgrad der Realsteuern im Vergleich zur Einkommensteuer in den Jahren seit 1980 immer niedriger lag (siehe Abschnitt 4.4.5.1.), obwohl eine gleichmäßige Erfassung aller bei der Finanzkraftermittlung berücksichtigten Einnahmekategorien angezeigt ist. Für die Konzessionsabgaben ist ein neues System zu entwickeln, da diese bislang im KFA nicht berücksichtigt wurden.

\subsection{Normierung des Realsteueraufkommens}

Bei der Darstellung des Normierungsverfahrens bei den Realsteuereinnahmen wurden einige Probleme aufgeführt. So gilt bisher ein landeseinheitlicher Normierungssatz für kreisfreie und kreisangehörige Städte und Gemeinden, der aber nur auf Datenbasis der kreisangehörigen Gemeinden ermittelt worden ist. Zudem wurde er über einen längeren Zeitraum konstant gehalten, so daß sich der Erfassungsgrad der Steuerkraft - Verhältnis des Normierungs- zum tatsächlichen Durchschnittshebesatz - verringerte. Dies führte zum unerwünschten Ergebnis, daß sowohl die Realsteuern untereinander als auch im Verhältnis zum Einkommensteueranteil ungleich im KFA erfaßt wurden. Aufgrund dieser Sachverhalte ergibt sich die Frage nach dem "richtigen" Nivellierungssatz, wobei es sich eigentlich um drei separate Überlegungen handelt:

- Sind für alle Gemeinden einheitliche Nivellierungssätze für die einzelnen Realsteuern zu verwenden?

-Wie soll das Niveau der Nivellierungssätze bestimmt werden?

-Wie können im Zeitablauf sinkende Erfassungsgrade vermieden werden?

\subsection{1. Einheitlicher oder differenzierter Nivellierungshebesatz}

In der (vor allem juristischen) Literatur wird häufig der Umstand kritisiert, daß nur ein Normierungssatz für alle Gemeinden Verwendung findet, obwohl doch aus empirischen Daten abzulesen ist, daß die tatsächlichen Hebesätze mit der Gemeindegröße, aber auch der zentralörtlichen Aufgabenerfüllung positiv korrelieren. ${ }^{1}$ Dieser gemeindegruppenspezifische Zusammenhang ist auch in Hessen bei den gewichteten Realsteuerhebesätzen feststellbar, wie aus der Abbildung $19 \mathrm{zu}$ entnehmen ist. Allgemein steigen die gewogenen Hebesätze der Gewerbe- und der Grundsteuer B mit der Gemeindegröße; klare Sprünge sind bei beiden Steuern sowohl bei der Gruppengrenzen 50.000 Einwohner als auch 100.000 Einwohner zu erkennen. Diese betragen bei der Grundsteuer B 63 bzw. 215 Prozentpunkte und bei der Gewerbesteuer um 45 bzw. 108 Prozentpunkte. Für die Grundsteuer

1 Vgl:: Grimme, L.: Neuberechnung der Steuerkraft im kommunalen Finanzausgleich - dargestellt am Beispiel des Landes Bayern, in: Räumliche Aspekte des kommunalen Finanzausgleichs (Veröffentlichungen der Akademie für Raumforschung und Landesplanung: Forschungs- und Sitzungsberichte, Bd. 159), Hannover 1985, S. 159-199. 
A ist wenn überhaupt nur ein umgekehrter Zusammenhang erkennbar, allerdings ohne auffallende Sprünge.

\section{Abbildung 19:Gemeindegruppenspezifische gewogene Realsteuerhebesätze} 1997

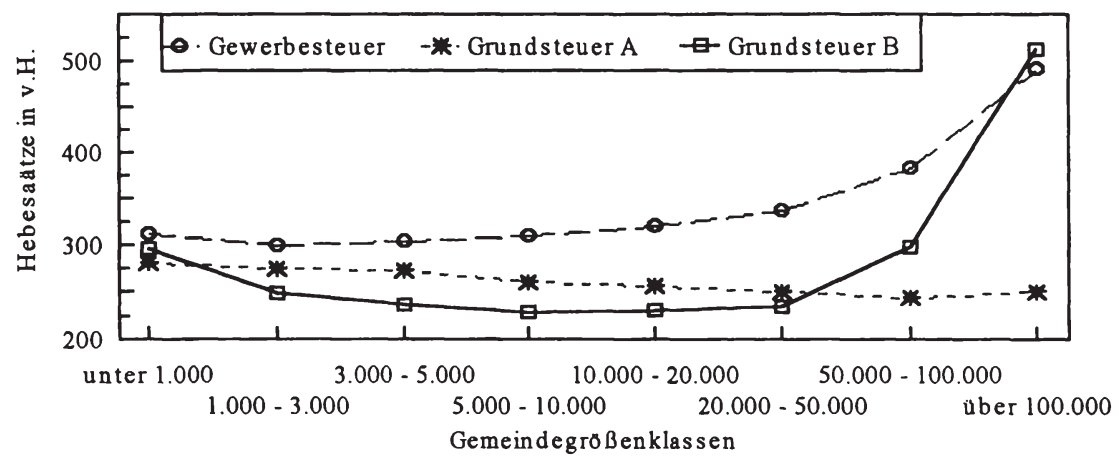

Quelle: Statistisches Bundesamt: Fachserie 14, Finanzen und Steuern, Reihe 10.1, Realsteuervergleich - Realsteuern und kommunale Einkommensteuerbeteiligung 1997, Stuttgart 1998, S. 60-62.

Es wird dann gefolgert, daß die auftretenden Differenzen auf die unterschiedlichen Möglichkeiten der Hebesatzanspannung bei den Gebietskörperschaften zurückgefuihrt werden können, wobei der Standortvorteil großen Gemeinden eine stärkere Steueranspannung ermöglicht. Dies wird dann auch mit dem Äquivalenzbezug der Gewerbesteuer begründet, da mit steigender Gemeindegröße mehr öffentliche Leistungen zur Attraktivitätssteigerung bereitgestellt werden und deshalb auch die Hebesätze stärker angespannt werden können. ${ }^{1}$

Würde in einer solchen Situation ein einheitlicher Hebesatz verwendet, so wäre dies ein Verstoß gegen das verfassungsrechtliche Willkürverbot, welches verbietet, "wesentlich (...) Ungleiches willkürlich gleich"2 zu behandeln. Als Folge eines landeseinheitlichen Hebesatzes wären nämlich die großen gegenüber den kleinen Gemeinden bevorzugt. Würde ein niedriger Hebesatz bestimmt, so würde ein groBer Anteil der Steuerkraft einwohnerstarker Gemeinden unberücksichtigt, und es gäbe trotz der hohen Steuereinnahmen noch Schlüsselzuweisungen; würde ein einheitlich hohes Niveau festgelegt, so könnten kleinere Gemeinden praktisch diesen Wert nicht erreichen, müßten aber trotzdem einen Verlust an Schlüsselzuweisungen aufgrund der relativ hohen fiktiven Steuerkraft erleiden. ${ }^{3}$

1 Vgl.: Kirchhof, P.: Der Finanzausgleich als Grundlage kommunaler Selbstverwaltung, in: DVBl., 95. Jg., 1980, S. $718 f$.

2 Bundesverfassungsgericht: Urteil vom 11.10.1977, in: Entscheidungen des Bundesverfassungsgerichts, Bd. 46, Tübingen 1978, S. 62.

3 Vgl.: Henneke, H.-G.: Der kommunale Finanzausgleich. Verfassungsrechtliche Vorgaben 
Aus diesen Überlegungen wird dann gefolgert, daß eine Normierung der Realsteuereinnahmen, mit Hilfe von nach der Gemeindegröße differenzierten Sätzen vorzunehmen ist. In der Praxis ist allerdings nur NRW bis $1997^{1}$ und seit 1999 Niedersachsen diesen Überlegungen gefolgt, wobei in NRW der höhere Normierungssatz ab 150.000 und in Niedersachsen ab 100.000 Einwohnern gilt.

Zwar ist der Überlegung prinzipiell zuzustimmen, daß einzelne Gemeinden aufgrund eines Standortvorteils relativ höhere Hebesätze bestimmen können, ohne deshalb gleich Abwanderungen befürchten zu müssen, doch fällt es schwer, für die Quantifizierung dieses Standortvorteils einen Indikator zu finden. Allein aus den Hebesätzen können solche Unterschiede nämlich nicht abgeleitet werden. Denn die Höhe des Hebesatzes kann auf zweierlei Begründungen fußen. So kann eine Gemeinde einen Standortvorteil haben und deshalb hohe Hebesätze festlegen, sie kann aber auch einen Standortnachteil haben und sich deshalb gezwungen sehen, die Hebesätze stark anzuspannen, um überhaupt Einnahmen zur Finanzierung der öffentlichen Leistungen zu erhalten. Daß in größeren Städten im Durchschnitt höhere Hebesätze gelten, kann im sonst nicht anders zu finanzierenden höheren Ausgabenbedarf begründet liegen, zumal auch die Ausgestaltung der Hauptansatzstaffel gerade diesen Sachverhalt unterstellt. Um möglicherweise Aussagen treffen zu können, sind nicht einfach nur die Hebesätze und die Einwohnergrößen zu vergleichen, sondern es ist noch die Standortqualität zu berücksichtigen. Als ein Indiz für diese Qualität einer Gemeinde könnte der Gewerbesteuergrund- oder der -meßbetrag pro Kopf angesehen werden, nicht aber die absolute Höhe des Grundbetrags. ${ }^{2}$

Bei einer graphischen Darstellung des Zusammenhangs zwischen dem Grundbetrag pro Kopf und der Gemeindegröße ergeben sich zwar Anzeichen darauf, daß dieser Wert allgemein mit der Einwohnergröße steigt, doch ist eine trennscharfe Separierung in Gemeindegrößenklassen nicht möglich (Abbildung 20).

und landesgesetzliche Ausgestaltung, in: Juristische Ausbildung (Jura), 1987, S. 401; Katz, A.: Der kommunale Finanzausgleich, in: Püttner, G. (Hrsg.): Handbuch der kommunalen Wissenschaft und Praxis, Bd. 6, 2. Aufl., Berlin u.a.O. 1985, S. 323.

1 Der Verfassungsgerichtshof erklärte die Staffelung für verfassungswidrig, da die Typisierung der Gemeindeklassen nicht nachvollziehbar gewesen ist. Vgl.: Verfassungsgerichtshof Nordrhein-Westfalen: Urteil vom 06.07.1993, in: OVGE, Bd. 43, Münster 1996, S. $262 f$.

2 Vgl.: Steinherr, M; Parsche, R.: Der Ausgleich zwischen Finanzbedarf und Finanzkraft im kommunalen Finanzausgleich des Landes Rheinland-Pfalz, ifo-Studien zur Finanzpolitik, Bd. 66, München 1998, S. 157. 
Abbildung 20:Gewerbesteuergrundbetrag pro Kopf ${ }^{1}$

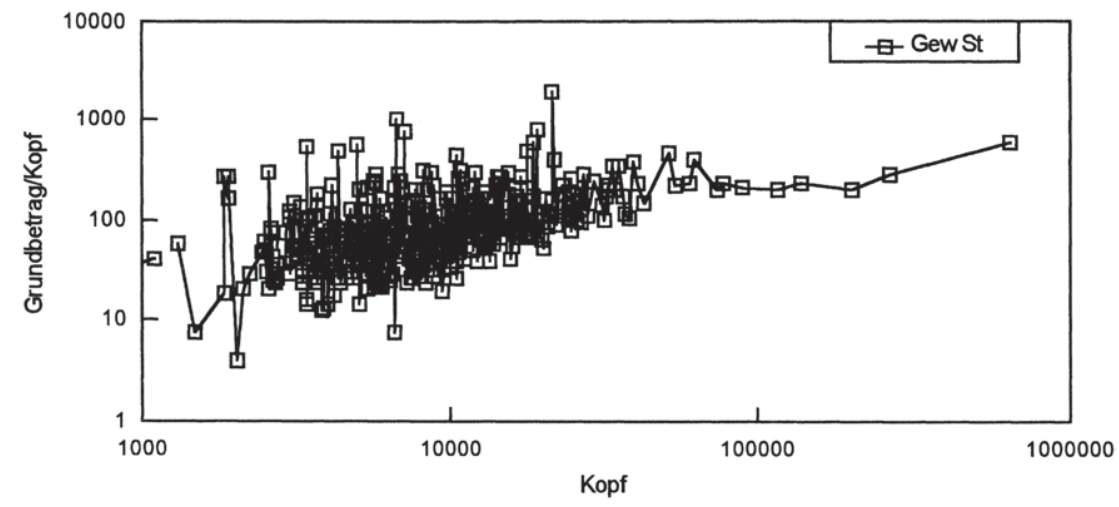

Quelle: Eigene Berechnung nach Daten des Finanzministeriums zum KFA 1998.

Denn es gibt sowohl große als auch kleine Städte, die einen hohen Gewerbesteuergrundbetrag aufweisen; so liegen beispielsweise die Grundbeträge der Gemeinden Stadtallendorf mit 1.879 und Eschborn mit 794 (beide haben etwa 20.000 Einwohner) über denen der Stadt Frankfurt mit $590 \mathrm{DM}$ pro Kopf bei etwa 650.000 Einwohnern; auch Gemeinden mit knapp 7.000 Einwohnern wie Dietzhölztal bzw. Großkrotzenburg weisen mit 1.022 bzw. 745 DM pro Kopf höhere Werte auf. Die kreisfreien Städte Kassel und Offenbach liegen mit ihrem Grundbeträgen an den Positionen 68 und 69 von 426 Gemeinden. Eine Regressionsanalyse zeigt weder eine signifikante Korrelation zwischen dem Grundbetrag pro Kopf und der Gemeindegröße, da das $r^{2}$ nur einen Wert von 0,065 annimmt, noch zwischen Hebesatz und Grundbetrag pro Kopf mit einem $r^{2}$ von $0,021{ }^{2}$ Somit kann gefolgert werden, daß es entgegen den allgemeinen Vermutungen keinen direkten statistischen Zusammenhang zwischen der jeweiligen Gemeindegröße und der Standortqualität, welche die Möglichkeiten der Hebesatzanspannung beeinflußt, gibt. Diese Schlußfolgerung kann noch durch zusätzliche Überlegungen gestützt werden, denn aufgrund der gut ausgebauten (Verkehrs-)Infrastruktur können auch kleinere Orte eine hohe Standortqualität aufweisen. ${ }^{3}$ Aufgrund dieser Ergebnisse und der fehlenden Trennschärfe der Hebesätze zwischen den Gemeindegrößenklassen sollte von einer gemeindegrößenspezifischen Differenzierung der Normierungssätze abgesehen werden.

1 Es handelt sich hierbei um eine logarithmische Darstellung, wobei auf den Ort Hesseneck (766 Einwohner) aufgrund darstellerischer Aspekte verzichtet wurde.

2 Auch für die Grundsteuer A und B kann keine signifikante Korrelation ermittelt werden. Die Werte liegen für zwischen 0,0004 und 0,110.

3 Vgl.: Parsche, R.; Steinherr, M.: Der kommunale Finanzausgleich des Landes NordrheinWestfalen, ifo-Studien zur Finanzpolitik, Bd. 59, 1995, S. 67f. 


\subsection{2. Niveaubestimmung des Nivellierungshebesatzes}

Weiterhin gilt es zu klären, wie das Niveau des fiktiven Hebesatzes für die einzelnen Realsteuern bestimmt werden soll. Zwar ist es richtig, daß unabhängig von der Höhe des Nivellierungssatzes, die Finanzkraft prozentual im gleichen Umfang erfaßt wird und somit auch die Variation eines einheitlichen fiktiven Hebesatzes zu prozentual gleichen Veränderungen bei der Erfassung der Finanzkraft führt, aber Gemeinden mit höheren Steuermeßzahlen bzw. -grundbeträgen realisieren absolut einen größeren Vorteil. ${ }^{1}$ Denn für die Verteilung der Schlüsselzuweisungen ist die absolute Höhe der Finanzkraft relevant. Dies soll im folgenden Beispiel (siehe Tabelle 24) anhand von zwei Gemeinden dargestellt werden. Die Bemessungsgrundlage und der Hebesatz betragen bei Ort A 1,8 Mio. DM und 400 v.H. bzw. bei Ort B 2 Mio. DM und 500 v.H. Es wird ein Ausgleichsgrad von 50 v.H., ein identischer Gesamtansatz von jeweils 10.000 bei einer Schlüsselmasse von 1 Mio. DM unterstellt; der Finanzbedarf beträgt in beiden Orten in Abhängigkeit vom Normierungssatz 5,75 oder 9,60 Mio. DM. Es kann festgestellt werden, $\mathrm{da}$ sich in Abhängigkeit vom Niveau des Normierungssatzes der Anteil der beiden Orte an den Schlüsselzuweisungen verändert. So beträgt dieser bei dem finanzstärkeren Ort A bei einem Satz von 250 v.H. 37,5 v.H. und verringert sich auf 27,4 v.H., wenn der gewichtete Durchschnittssatz zur Anwendung kommt. Umgekehrt verläuft die Entwicklung beim finanzschwachen Ort B.

Tabelle 24: Wirkungen des Normierungssatzes auf die Schlüsselzuweisun$\operatorname{gen}^{2}$ (in Mio. DM)

\begin{tabular}{|l|c|c|}
\hline & Ort A & Ort B \\
\hline Steuerkraft bei Normierungssatz 250 v.H. & 4,50 & 5,00 \\
\hline Zuweisungen bei Finanzbedarf von 5,75 Mio. DM & 0,38 & 0,63 \\
\hline Steuerkraft bei Normierungssatz 452,63 v.H. & 8,15 & 9,05 \\
\hline Zuweisungen bei Finanzbedarf von 9,60 Mio. DM & 0,27 & 0,73 \\
\hline
\end{tabular}

Weiterhin gilt es zu vermeiden, daß sich der Erfassungsgrad der verschiedenen kommunalen Einnahmenkategorien bei der Steuerkraftermittlung unterscheidet, da ansonsten implizit eine Gewichtung unter Berücksichtigung strukturpolitischer Ziele erfolgen kann. ${ }^{3}$ Denn Orte mit einem hohen Anteil einer gering gewichteten

1 Vgl.: Micosatt, G.: Raumwirksamkeit einer Reform des kommunalen Finanzausgleichs, Bochum 1990, S. 57. Siehe dazu auch PostLeP, der mit einzelnen Rechnungen die Auswirkungen der Hebesatzwahl dokumentiert. Postlep, R-D.: Räumliche Effekte der Steuerkraft- und Finanzbedarfsermittlung bei den Schlüsselzuweisungen, in: Räumliche Aspekte des kommunalen Finanzausgleichs (Veröffentlichungen der Akademie für Raumforschung und Landesplanung: Forschungs- und Sitzungsberichte, Bd. 159), Hannover 1985, S. 219-226.

2 Der unterschiedliche Finanzbedarf ergibt sich aufgrund der Änderung des Grundbetrags bei konstanter Schlüsselmasse.

3 Vgl: Hardt, U.: Kommunale Finanzkraft. Die Problematik einer objektiven Bestimmung 
Steuerart würden dann zusätzliche Schlüsselzuweisungen erhalten, obwohl eine Finanzschwäche nicht gegeben ist. Ein Beispiel dafür kann der geringe Erfassungsgrad der Grundsteuer A sein, der in den 70er Jahren zeitweise bei etwa 56 v.H. lag, und die damit verbundene finanzielle Förderung des ländlichen Raums (Abbildung 18). Sollen landwirtschaftlich geprägte Gemeinden gefördert werden, so sollte dies transparent über direkte Zuweisungen und nicht über eine Steuerkraftuntergewichtung im KFA erfolgen. Aus den genannten Gründen ist auf gewichtete durchschnittliche Hebesätze zurückzugreifen, um somit die tatsächliche im Mittel realisierte Hebesatzanspannung abzubilden. ${ }^{1}$

Diese Überlegung wird auch in Hessen der Bestimmung des Normierungssatzes zugrunde gelegt, auch wenn ihre Umsetzung inkonsistent ist. Denn einerseits wird trotz der getrennten Schlüsselmassen von kreisfreien Städten und kreisangehörigen Gemeinden ein identischer Hebesatz verwendet. Dieser wird aber ohne Begründung wiederum nur unter Berücksichtigung der Realsteuerhebesätze der kreisangehörigen Gemeinden ermittelt. ${ }^{2}$ Ein einheitlicher Gewerbesteuernormierungssatz hätte 1997 für alle Gemeinden 403 v.H. betragen müssen. Damit wären aber aufgrund der großen Streuung der tatsächlichen um den Normierungshebesatz Probleme verbunden gewesen, da sich die Steigerung der Gewerbesteuerbemessungsgrundlage für Orte mit sehr niedrigen Hebesätzen negativ auf die gesamte Finanzkraft ausgewirkt hätte. ${ }^{3}$ Zudem hätten von den 421 kreisangehörigen Gemeinden 418 und somit 99,29 v.H. diesen Wert unterschritten, so daß ihnen eine nicht erreichte Steuerkraft zugerechnet worden wäre. ${ }^{4}$ Aufgrund der separaten Schlüsselmassen der beiden Gemeindegruppen könnte aber auch - wie in Sachsen - der jeweilige gruppenspezifische gewichtete Durchschnittshebesatz verwendet werden; er würde im kreisfreien bzw. kreisangehörigen Raum bei etwa 492 bzw.

kommunaler Einnahmemöglichkeiten in der gemeindlichen Haushaltsplanung und im kommunalen Finanzausgleich, Frankfurt u.a.O. 1988, S. 49-51.

1 Vgl: Ehrlicher, W. u.a.: Kommunaler Finanzausgleich und Raumordnung, Hannover 1967, S. 80 .

2 Vgl.: Hessischer Landtag: Gesetzentwurf der Landesregierung für ein Gesetz zur Änderung des Finanzausgleichsgesetzes vom 21.10.1996, Drucksache 14/2259, S. 10; ders.: Änderung des Finanzausgleichsgesetzes, Art. 2 des Gesetzentwurf der Landesregierung für ein Gesetz zur Überleitung der Aufgaben der Hilfe zur Pflege vom 23.10.1992, S. 7; ders.: Gesetzentwurf der Landesregierung für ein Neuntes Gesetz zur Änderung des Finanzausgleichsgesetzes vom 05.10.1973, Drucksache 7/4135, S. 6.

3 Vgl.: Steinherr, M; Parsche, R.: Der Ausgleich zwischen Finanzbedarf und Finanzkraft im kommunalen Finanzausgleich des Landes Rheinland-Pfalz, ifo-Studien zur Finanzpolitik, Bd. 66, München 1998, S. 158.

4 Nur die drei Städte Gießen, Hanau und Maintal weisen über 403 v.H. liegende Hebesätze auf; bei der Grundsteuer B hätten 98,10 v.H. oder 413 Gemeinden einen geringeren Hebesatz gehabt. Für die Grundsteuer A hätte dieses Problem nicht bestanden, da das gewichtete Mittel landesweit mit dem der kreisangehörigen Gemeinden übereinstimmt. 
337 v.H. liegen. ${ }^{1}$ Durch diese Verfahrensweise könnte das angesprochene Problem gelöst werden. ${ }^{2}$

\subsubsection{2.1.3. Dynamisierung des Erfassungsgrades der Steuerkraft}

Als letztes sind nun noch Maßnahmen zu überlegen, die einer Fehlerfassung der Realsteuerkraft im Zeitablauf aufgrund der Veränderungen der tatsächlichen Hebesätze entgegenwirken, denn in Hessen wurden die Hebesätze der Grundsteuer A und B zwischen 1978 und 1998 nicht an die Entwicklung angepaßt. Zu geringe Nivellierungshebesätze führen aber zu einer Bevorzugung der steuerstarken Gemeinden, weshalb eine Anhebung der Nivellierungshebesätze das Verteilungsziel unterstützt. Auf die jährliche Neufestsetzung im Gesetz sollte verzichtet werden, da dieses dadurch eher einem jährlichen Gemeindefinanzierungsgesetz denn einem dauerhaften Finanzausgleichsgesetz entspräche.

Zur Lösung dieses Problems führte Niedersachsen eine regelgebundene Dynamisierung der Nivellierungshebesätze ein. Dabei wurden bis 1998 für das jeweilige Finanzausgleichsjahr der gewogene durchschnittliche Hebesatz der kreisangehörigen Gemeinden des vorvergangenen Jahres für alle Gemeinden - auch die kreisfreien - als Nivellierungssatz festgelegt. ${ }^{3}$ Die tatsächliche Ausgestaltung des Verfahrens ist aber nicht unkritisiert geblieben, da ein auf Basis der Daten der kreisangehörigen Gemeinden errechneter Nivellierungssatz die Einnahmen der kreisfreien Städte unterschätzt. ${ }^{4}$ Zudem ist in Niedersachsen die Gruppe der kreisangehörigen Gemeinden sehr heterogen. Es können bei einer Einteilung dieser Gemeinden nach der Aufgabenerfüllung drei Kategorien unterschieden werden, bei denen auch eine unterschiedliche Hebesatzanspannungsmöglichkeit unterstellt werden kann. ${ }^{5}$ Diese gerade aufgezeigten Probleme können aber bei separierten

1 Vgl.: Statistisches Bundesamt: Fachserie 14, Finanzen und Steuern, Reihe 3.3, Rechnungsergebnisse der kommunalen Haushalte 1997, Stuttgart 1999, S. 62. Für die vom Gesamtkreis und den kreisfreien Städten abzuführenden Landesumlagen sollte aber weiterhin ein einheitlicher Hebesatz zur Ermittlung der Finanzkraft verwendet werden.

2 Zu den Auswirkungen dieser Maßnahme vgl.: Broer, M.: Die Berechnung der Steuerkraft im Kommunalen Finanzausgleich - Verteilungswirkungen und Reformvorschläge am Beispiel der kreisfreien Städte in Hessen, in: Die Gemeindekasse, 1999, S. 336-338.

3 Vgl.: § 12 Abs. 2 des Niedersächsischen Gesetz über den Finanzausgleich (NFAG) vom 19.12.1995, Nds. GVBI., S. 463. Seit 1999 erfolgt diese Dynamisierung nach getrennten Gruppen. Vgl.: § 12 Abs. 2 des Gesetzes zur Änderung des Niedersächsischen Gesetzes über den Finanzausgleich und anderer Gesetze vom 12.03.1999, Nds. GVBl., S. 75.

4 Zum folgenden vgl.: Albers, H.: "Steuereinnahmen? Nein danke!"? Anmerkungen zur Nivellierungswirkung von Finanzausgleichssystemen, dargestellt am Beispiel des kommunalen Finanzausgleichs in Niedersachsen, in: ZKF, 44. Jg., 1994, S. 52; Henneke, H.-G.: Der kommunale Finanzausgleich in Niedersachsen im Spannungsfeld von verbesserter Ausgleichsgerechtigkeit und verfassungswidriger (Über-)Nivellierung, in: Der Gemeindehaushalt, 94. Jg., 1993, S. $86 f$.

5 Neben den großen selbständigen Städten gibt noch selbständige Gemeinden und sonstige 
Schlüsselmassen und der damit verbundenen größeren Homogenität der Gemeinden vermieden werden.

Zu klären ist noch, wie lang der Basiszeitraum zur Durchschnittsberechnung sein soll - ein Jahr oder mehrere Jahre. Für die Verwendung ausschließlich des aktuellen Hebesatzes spricht, da $\beta$ dieser die derzeitige Hebesatzanspannung genau widerspiegelt. Wird nur ein Jahr zu Berechnung des Nivellierungssatzes verwendet, so besteht aber die Gefahr, daß sich kurzfristige Schwankungen der Hebesätze z.B. zur Finanzierung einmalig auftretender Defizite - sehr stark auf die erfaßte Finanzkraft und somit wiederum auf die Planbarkeit der Schlüsselzuweisungen auswirken. So hätte z.B. die angestrebte Verringerung des Gewerbesteuerhebesatzes der Stadt Frankfurt von 515 auf 490 v.H. bei einem Basiszeitraum von einem Jahr starke Auswirkungen gezeigt. ${ }^{1}$ Der Nivellierungssatz würde sich von 492 (1997) auf 477 v.H. vermindern. ${ }^{2}$ Wird hingegen das gewogene Mittel der letzten fünf Jahre (1993-1997) verwendet, so kommt es durch die angeführte Minderung bei der Gewerbesteuer nur noch zu einer Veränderung des Normierungssatzes von 487,4 auf 484 v.H.

\subsection{Normierung des Konzessionsabgabenaufkommens}

$\mathrm{Zu}$ den $\mathrm{KA}$ ist vorab anzumerken, daß nur diejenigen einbezogen werden können, die auf Gas und Strom - nicht aber auf Wasser - erhoben werden. Denn nur für die erstgenannten gilt die neue Konzessionsabgabenverordnung, so da $\beta$ nur bei diesen das für alle Gemeinden gleiche Recht besteht. ${ }^{3}$ Die Anwendung eines für alle Gemeinden landeseinheitlichen Normierungssatz ist nicht möglich, da in der Verordnung nach Gemeindegrößen gestaffelte Maximalsätze festgelegt sind. ${ }^{4}$

Gemeinden. Auch die Größe streut sehr stark zwischen Städten mit mehr als 100.000 Einwohnern und solchen mit weniger als 1.000 Einwohnern.

1 Vgl.: o.V.: Frankfurt will Gewerbe- und Grundsteuer senken, in: Frankfurter Allgemeine Zeitung vom 22.04.1999, Nr. 93, S. 59. Der Anteil der Stadt Frankfurt an der Gesamtbemessungsgrundlage der füf kreisfreien Städte in Hessen schwankt 1998 zwischen 51,9 v.H. bei der Grundsteuer A und 69,5 v.H. bei der Gewerbesteuer, wodurch die Höhe der Gesamtsteuerkraft zum großen Teil durch die Stadt Frankfurt beeinflußt wird

2 Die längerfristig angestrebte Verminderung der Hebesätze bei der Grundsteuer B um 19,3 v.H. von derzeit 570 auf 460 v.H. und der Gewerbesteuer um ca. 4,85 v.H. von 515 auf 490 v.H. würde den Durchschnittshebesatz bei der Grundsteuer B um 12,5 v.H. und bei der Gewerbesteuer um ca. 3,4 v.H. vermindern.

3 Für Wasser-Versorgungsunternehmen gilt noch immer die Konzessionsabgabenanordnung aus dem Jahr 1941, wodurch nicht die rechtlichen Voraussetzungen für eine landesweite Erhebung dieser Konzessionsabgabe gegeben sind.

4 Vgl.: § 2 Verordnung über Konzessionsabgaben für Strom und Gas (Konzessionsabgabenverordnung - KAV) vom 09.01.1992, BGB1. I, S. 12. Dort beträgt z.B. der maximale Höchstbetrag je Kilowattstunde Strom nach dem Schwachlasttarif bei Gemeinden bis 25.000 Einwohnern 0,026 DM und bei über 500.000 Einwohnern 0,0469 DM. Die KA werden vollständigen in den Preis überwälzt, so daß sie letztlich vom Verbraucher getragen 
Dementsprechend sind die Normierungssätze für die einzelnen in der Verordnung angeführten Größenklassen zu bestimmen, wobei wie bei den Realsteuern das gewichtete Mittel zum Einsatz kommen sollte, da somit die Einnahmenkraft im Durchschnitt exakt erfaßt wird. Durch Verwendung eines gleitenden Durchschnittswertes mit einem mehrere Jahre umfassenden Basiszeitraum wird das Gewicht des aktuellen Wertes einer Gemeinde gemindert und gleichzeitig eine Dynamisierung erreicht, was zu einer Glättung im Zeitablauf führt.

Es ist aber noch auf eine Eigenart dieser Einnahmenart zu verweisen, die eine weitere Modifizierung des Normierungssystems notwendig macht. Grundsätzlich können zwei Arten von konzessionsabgabenpflichtigen Unternehmen unterschieden werden. Neben den rein privatwirtschaftlichen Unternehmen gibt es noch öffentliche Betriebe, die der Kommune ganz oder zum Teil gehören, wobei auch hier meist die Rechtsform einer Kapitalgesellschaft verwendet wird. Die KA sind nämlich nicht nur von den privatwirtschaftlichen Unternehmen zu zahlen, sondern auch von öffentlichen Betrieben. Somit kann bei öffentlichen Betrieben der körperschaftsteuerpflichtige Gewinn zugunsten der steuerfreien und als Betriebsausgabe steuerlich abzugsfähigen KA gemindert werden. ${ }^{1}$ Auf diese Art der verdeckten Gewinnausschüttung hat der Gesetzgeber allerdings mit einer Begrenzung der Abzugsfähigkeit der KA reagiert. ${ }^{2}$

Es wird nun unterstellt, daß ein Versorgungsunternehmen einen Gewerbeertrag von $100 \mathrm{DM}$ erwirtschaftet, daß dieser der Höhe nach dem körperschaftsteuerlichen Gewinn entspricht ${ }^{3}$ und $\mathrm{da} B$ für die Unternehmung die $\mathrm{KA}$ vollständig als Betriebsausgabe abzugsfähig ist. ${ }^{4}$ Bei Ausschüttung fallen Gewerbesteuer und -umlage, Körperschaftsteuer sowie Solidaritätszuschlag an. ${ }^{5}$ In der Ausgangslage wird die KA nicht im KFA berücksichtigt. Wie aus Tabelle $25 \mathrm{zu}$ entnehmen ist,

werden. Ihre Höhe wird von den Versorgungsunternehmen bei den Tarifpreisen ausgewiesen.

1 Vgl.: Stern, V.: Die Reform der Konzessionsabgabe, in: Wirtschaftsdienst, 72. Jg., 1992, S. 44.

2 Vgl.: Bekanntmachung der Neufassung der Körperschaftsteuer-Richtlinien 1985 (KStR 1985), in: Bundessteuerblatt vom 30.12.1985, Sonderheft Nr. 1/1986, S. 82f.

3 Da es keine allgemeinen Aussagen über die Relation dieser beiden Größen gibt, wird die gleiche Höhe unterstellt.

4 Tatsächlich ist die Konzessionsabgabe nur bei Unternehmen ohne Beteiligung von öffentlichen Körperschaften zu 100 v.H. abzugsfähig. Ansonsten dürfen bestimmte Höchstwerte nicht überschritten werden, weil sonst eine verdeckte Gewinnausschüttung unterstellt wird.

5 Als Gewerbesteuerhebesatz werden 410 v.H. angenommen (gewogenes Mittel in Hessen 1998414 v.H.) bei einer Meßzahl von 5 v.H. Weiterhin ist zu beachten, daß die Gewerbesteuer ihre eigene Bemessungsgrundlage und auch den körperschaftsteuerlichen Gewinn mindert, da sie als Betriebsausgabe abzugsfähig ist. Als Vervielfältiger für die Gewerbesteuerumlage werden die 84 v.H. des Jahres 1998 angenommen. 
erweist sich die Erhebung einer KA für die Gemeinde unabhängig vom Beteiligungsverhältnis als sinnvoll.

Tabelle 25: Nettoertragsvergleich zwischen Konzessionsabgabenerhebung und Gewinnausschüttung ohne KFA-EinfluB bei Gemeinden

\begin{tabular}{|rc|cc|}
\hline 100,00 & Ausschüttung & 100,00 & Konzessionsabgaben \\
$-17,01$ & Gewerbesteuer (brutto) & & \\
$-24,90$ & Körperschaftsteuer (30 v.H) & & \\
$-1,37$ & Solidaritätszuschlag (5,5 v.H) & & \\
\cline { 1 - 2 } 56,72 & Gewinnausschüttung & & \\
13,53 & Gewerbesteuer (netto) & & \\
\hline $\mathbf{7 0 , 2 5}$ & Gemeindeertrag & $\mathbf{1 0 0 , 0 0}$ & Gemeindeertrag \\
\hline
\end{tabular}

Hält die Kommune keine Anteile an diesem Betrieb, so würde sie bei Erhebung der KA 100 DM erhalten, bei Ausschüttung nur die Netto-Gewerbesteuer in Höhe von 13,53 DM, wobei die Netto-Gewerbesteuer noch die Steuerkraft erhöhen würde, was bei einem unterstellten Ausgleichsgrad von 50 v.H. zu einem Verlust an Schlüsselzuweisungen in Höhe von ca. 6,77 DM führt. ${ }^{1}$ Handelt es sich hingegen um ein öffentliches Unternehmen, so kann die Kommune zwischen 70,25 DM (bestehend aus 56,72 DM Gewinn und 13,53 DM Netto-Gewerbesteuer) bei Ausschüttung und KA in Höhe von $100 \mathrm{DM}$ wählen. Auch hier würden sich die Schlüsselzuweisungen um ca. 6,77 DM mindern, da nur die Netto-Gewerbesteuer die Steuerkraft erhöht.

Wird jetzt die KA auch im KFA angerechnet, so ändert sich das Entscheidungskalkül bei einem privaten Versorgungsunternehmen nicht. Bei Ausschüttung erhält die Kommune ca. 6,77 DM (Netto-Gewerbesteuer - Minderung der Schlüsselzuweisungen), bei KA hingegen 50 DM (100 DM KA - 50 DM Minderung der Schlüsselzuweisungen). Anders ist die Situation bei einem öffentlichen Unternehmen. Zwar unterliegt auch hier die Aussschüttung der Körperschaft- und der Gewerbesteuer, trotzdem kann eine solche Entscheidung für die Gemeinde von Vorteil sein. Denn die KA wirkt sich im vollen Umfang steuerkrafterhöhend aus, während für den Fall der Gewinnausschüttung nur die Netto-Gewerbesteuer in Höhe von 13,53 DM einzubeziehen ist, nicht aber der Gewinn von 56,72 DM. Bei KA ergeben sich somit ebenfalls $50 \mathrm{DM}$, bei Ausschüttung aber nun 63,48 DM (Gewinn in Höhe von 56,72 DM zuzüglich Netto-Gewerbesteuer von 13,53 DM abzüglich Minderung der Schlüsselzuweisungen um 6,77 DM). Bei einem 50

1 Da der KFA-Normierungssatz in diesem Beispiel mit dem individuellen Gewerbesteuerhebesatz der Gemeinde übereinstimmen soll, entfallt die sonst notwendige Modifikation der Steuerkraft für die Berechnung der Schlüsselzuweisungen. 
v.H.-Ausgleich zwischen Steuerkraft und Finanzbedarf erhält die Gemeinde mit Gewinnausschüttung immer um 43,24 DM höhere Schlüsselzuweisungen. ${ }^{1} \mathrm{Da}$ die Differenz zwischen dem jeweiligen Gemeindeertrag aus diesen zusätzlichen 100 DM bei Erhebung von Konzessionsabgaben geringer ist, wird die Gemeinde auf diese zugunsten der Gewinnausschüttung verzichten. ${ }^{2}$ Gemeinden, die schon heute auf die KA-Erhebung verzichten, stellen kein Problem dar, da für sie die Alternative der KA durch Berücksichtigung im KFA noch ungünstiger wird, anders solche, bei denen "finanzausgleichsmotivierte" Veränderungen auftreten. Deshalb soll nun untersucht werden, wie das Normierungsverfahren zu modifizieren ist, um diese Situation zu vermeiden.

Aus diesen Ausführungen ist ersichtlich, daß eine Gemeinde die ihr zufließenden Schlüsselzuweisungen bei öffentlichen Unternehmen beeinflussen kann. Dies kann auf zwei Wegen verhindert werden. So könnte das gemeindeindividuell ermittelte normierte Konzessionsabgabenaufkommen statt mit 100 mit einem geringeren Faktor angesetzt werden. Dieser müßte so gewählt werden, daß es sich für eine Gemeinde mit durchschnittlichem Hebesatz nicht lohnt, auf die Erhebung der KA zugunsten der Gewinnausschüttung zu verzichten. Dabei könnte dann in Hessen für die beiden Gemeindegruppen ein unterschiedlicher Anrechnungssatz verwendet werden, für kreisangehörige Gemeinden würde er bei 72 v.H., für kreisfreie Städte bei 74 v.H. liegen. ${ }^{3}$ Die unterschiedliche Höhe der Anrechnungssätze liegt in den verschiedenen Durchschnittshebesätzen der beiden Gemeindegruppen begründet. Denn der Vorteil der Gewinnausschüttung steigt mit sinkenden Gewerbesteuerhebesätzen, da dadurch der nicht im KFA berücksichtigte Gewinn erhöht wird, denn die steuerkrafterhöhende Gewerbesteuer ist niedriger. Weil der Hebesatz bei den kreisangehörigen Gemeinden niedriger ist, muß ihr Anrechnungssatz geringer sein.

Der maximalen Anrechnungsfaktor (y) ergibt sich nach der folgenden Formel (Abbildung 21), die sicherstellt, daß der Gemeindeertrag bei Gewinnausschüttung niemals größer ist als bei Erhebung von Konzessionsabgaben. Auf der linken Seite der Gleichung steht der Ertrag bei KA-Erhebung. Er ergibt sich aus der KA verringert um die Minderung der Schlüsselzuweisung. Diese wird berechnet

1 Der Wert ergibt sich aus der Differenz zwischen einberechneter Konzessionsabgabe und Gewerbesteuereinnahme bei identischem Finanzbedarf $(100-13,53) * 50$ v.H. $=43,235$.

2 Nur für den Fall, daß die Gemeinde abundant ist, wird sie sich immer für die Konzessionsabgabe entscheiden, da dann die Schlüsselzuweisung ein fixer Kopfbetrag ist und der Gemeindeertrag bei Gewinnausschüttung geringer ausfällt.

3 Damit sich für keine Gemeinde eine Umstellung auf Gewinnausschüttung rentiert, müßte sich der Anrechnungsfaktor an den höchsten tatsächlichen Hebesätzen der beiden Gruppen orientieren; er betrüge dann 74 v.H. für die kreisangehörigen und 73 v.H. für die kreisfreien Kommunen. Dies hätte zur Folge, da die Kommune mit dem höchsten Gewerbesteuerhebesatz nun den Anrechnungsfaktor beeinflussen kann. 
durch die Steuerkrafterhöhung yKA multipliziert mit dem Ausgleichsgrad (AG) in Höhe von 50 v.H. Auf der rechten Seite der Gleichung erhöhen der Gewinn und die Netto-Gewerbesteuer die Einnahmen, die sich um die normierte Gewerbesteuer multipliziert mit dem Ausgleichsgrad mindert, da um diesen Betrag die Schlüsselzuweisungen aufgrund der Steuerkrafterhöhung sinken. Wird die Gleichung nach y aufgelöst, so ergibt sich der Anrechnungsfaktor, bei dem die Kommune indifferent zwischen Ausschüttung und KA-Erhebung ist. Für die unterstellten Werte ergibt sich ein Anrechnungsfaktor von 73,03 v.H.

\section{Abbildung 21:Berechnung des KA-Anrechnungsfaktors zur Steuerkrafter- mittlung bei Gemeinden}

$$
\begin{aligned}
& \mathrm{KA}-\mathrm{y} \cdot \mathrm{KA} \cdot \mathrm{AG}=-\frac{\mathrm{GewSt} \cdot \text { Normierungssatz }}{\text { Ist-Hebesatz }} \cdot \mathrm{AG}+\mathrm{GewSt}+\text { Gewinn } \\
& \Leftrightarrow \mathrm{y}=\frac{1}{\text { Ausgleich }}+\frac{\text { GewSt } \bullet \text { Normierungssatz }}{\text { Ist-Hebesatz }} \cdot \frac{1}{\mathrm{KA}}-\left(\frac{\mathrm{GewSt}+\text { Gewinn }}{\mathrm{KA} \cdot \text { Ausgleich }}\right)
\end{aligned}
$$

Eine Alternative hierzu bestünde darin, den jeweils für eine Gemeindegrößenklasse hergeleiteten Normierungssatz auch bei den Gemeinden zur Ermittlung eines fiktiven KA-Aufkommens zu verwenden, die auf eine KA-Erhebung verzichten. Es muß dann aber das aus der Ausschüttung resultierende Gewerbesteueraufkommen unberücksichtigt bleiben, da diese Möglichkeit durch die fiktive KA ausgeschlossen wurde. Der Vorteil der ersten Methode besteht darin, daß für die Gemeinden ein Anreiz besteht, die Konzessionsabgabe zu erheben. Die Steuerkraft könnte dann relativ einfach ermittelt werden, auch wenn diese Einnahmenkategorie im geringeren Umfang als die übrigen einbezogen wird. Beim zweiten Ansatz müssen aufwendigere Überprüfungen und Bereinigungen vorgenommen werden, da das tatsächliche Gewerbesteueraufkommen so nicht zur Steuerkraftberechnung verwendet werden darf, dafür würden aber alle Einnahmenkategorien im gleichen Umfang berücksichtigt werden.

\subsubsection{Folgerungen für die zukünftige Steuerkraftberechnung}

Die Ausführungen zur bisherigen Verfahrensweise bei der Steuerkraftberechnung haben deutlich gemacht, daß sowohl die Eingrenzung der zu berücksichtigenden Einnahmen als auch der jeweilige Erfassungsgrad der Einnahmenkategorie zu reformieren sind. Die Begrenzung bei der Steuerkraftberechnung nur auf die Realsteuern und den kommunalen Anteil an der Einkommen- und Umsatzsteuer ist zu eng. Statt dessen sollten zusätzlich noch die Einnahmen aus der Grunderwerbsteuer, der Spielbank- und der Troncabgabe sowie den Konzessionsabgaben einbezogen werden. 
Bei allen Einnahmen ist der gleiche Erfassungsgrad anzustreben, wobei abgesehen von den Realsteuern und den Konzessionsabgaben jeweils das IstAufkommen anzusetzten ist. Bei den Abgaben mit Einnahmeautonomie ist eine Normierung vorzunehmen, wobei grundsätzlich die schon bei den Realsteuern verwendete Systematik genutzt werden kann. Diese ist aber zu modifizieren, um das Niveau der Berücksichtigung im intertemporalen Vergleich konstant zu halten. Zu diesem Zweck sollte der Wert des Normierungssatzes nicht explizit genannt, sondern nur die Art des Berechnungsverfahrens - gewichtetes Mittel der Ist-Hebesätze der letzten fünf Jahre - angeführt werden. Der jeweils geltende Normierungssatz könnte dann im Staatsanzeiger am Anfang eines Jahres zusammen mit der Abrechnung des KFA sowie den jeweils vorläufigen Grundbeträgen der drei Gruppen veröffentlicht werden. Eine Änderung des Normierungsverfahrens bei den Konzessionsabgaben - in der schon beschriebenen Weise - ist wegen deren Besonderheiten unumgänglich, um zu verhindern, daß Gemeinden selbst Einfluß auf die Höhe der ihnen zustehenden Schlüsselzuweisungen nehmen.

\subsubsection{Ermittlung der Umlagekraft der Landkreise}

\subsubsection{Darstellung der bisherigen Regelungen}

\subsection{Finanzkraft der Landkreise}

Den hessischen Landkreisen stehen als eigene Steuermittel neben der Jagd- und Fischerei- sowie der Gaststättenerlaubnissteuer noch Konzessionsabgaben sowie der Anteil am vorab an die kreisfreien Städte und Landkreise überwiesenen Drittel der Grunderwerbsteuer zu. ${ }^{1}$ Soweit diese Einnahmen und die Leistungsentgelte zuzüglich der Schlüsselzuweisungen nicht genügen, um die Aufgaben zu finanzieren, dürfen nach $\S 53 \mathrm{HKO}$ Umlagen von den kreisangehörigen Gemeinden erhoben werden.

Die Kreisumlage gilt als wichtigstes Finanzierungsinstrument dieser Gebietskörperschaftsgruppe. Die Höhe der von der einzelnen Gemeinde zu zahlenden Umlage wird von zwei Größen determiniert, den Umlagesätzen und der -bemessungsgrundlage. In die Bemessungsgrundlage gehen die zur Verteilung der gemeindlichen Schlüsselzuweisungen ermittelten Steuerkraftmeßzahlen der Gemeinden sowie deren Schlüsselzuweisungen ein. Im Zeitablauf änderten sich sowohl der Anrechnungsfaktor bei den Schlüsselzuweisungen als auch der Umfang der berücksichtigten gemeindlichen Steuerkraftmeßzahl. So bestand die Steuerkraft bis 1950 aus den normierten Realsteuereinnahmen, den sog. Bürgersteuerausgleichsbeträgen und den Schlüsselzuweisungen. Dabei wurde die Steuerkraft der Gewerbesteuer um 50 v.H. der gezahlten bzw. erhaltenen Ausgleichszuschüsse

Vgl: $\S 8$ des Gesetzes über kommunale Abgaben (KAG) vom 17.03.1970, Hess. GVBl. I, S. 225 und $\S 1$ Grunderwerbsteuerzuweisungsgesetz vom 25.03.1997, Hess. GVBl. I, S. 50 . 
modifiziert, auch die Schlüsselzuweisungen gingen nur hälftig in die Umlagegrundlagen ein.

Nachdem 1951 die in Form der Bürgersteuerausgleichsbeträge ausgezahlten Finanzmittel in voller Höhe in die Finanzausgleichsmasse eingestellt wurden und folglich die Schlüsselzuweisungen stiegen, erhöhte sich der Anrechnungssatz der Schlüsselzuweisungen für die Gemeinden belastungsneutral auf 75 v.H. ${ }^{1}$ Er stieg erst im Jahr 1997 auf 80 v.H. ${ }^{2}$ Ebenfalls 1951 wurde die Anrechnung der Gewerbesteuerausgleichsbeträge mit der nicht ganz nachvollziehbaren Begründung, daß sie bisher sehr niedrig festgelegt waren, nun aber etwa den Maximalwert ${ }^{3}$ erreichen, auf 100 v.H. erhöht. ${ }^{4}$ Sie wurden bei den Wohngemeinden auch dann noch mit 100 v.H. in die Bemessungsgrundlage für die abzuführende Umlage eingerechnet, als ihr Erfassungsgrad bei der gemeindlichen Finanzkraft zur Berechnung der Schlüsselzuweisungen nur bei 50 v.H. (1955) bzw. 1/3 v.H. (1959) lag, um die Haushaltswirtschaft der umlageerhebenden Gebietskörperschaften nicht zu beeinträchtigen. ${ }^{5}$ Zwischen 1953 und 1977 wurde von besonders finanzstarken Gemeinden die Steuerkraft, die über 150 v.H. der Bedarfsmeßzahl lag, ein zweites Mal in die Umlage einbezogen. ${ }^{6}$ Diese Größe wurde später auf 170 v.H. festgelegt.

Für die Sonderstatusstädte kommt nach $\S 37$ Abs. 2 FAG seit 1980 ein modifiziertes Verfahren zur Anwendung, durch das für diese Gemeindegruppe die Kreisumlagenbelastung verringert wird. So wird die Bemessungsgrundlage zwar

Vgl.: Begründung zu dem Gesetz zur Regelung des Finanzausgleichs vom 17. 07.1951, in: Staatsanzeiger für das Land Hessen, 1951, S. 522.

2 Für die Jahre 1997 bis 1999 gehen die Schlüsselzuweisungen mit 80, 85 und 90 v.H. in die Bemessungsgrundlage der Kreisumlage ein; ab 2000 zu 95 v.H. Vgl.: Gesetz zur Neuregelung des Finanzausgleichs (Finanzausgleichsgesetz - FAG -) vom 18.03.1997, Hess. GVBI. I, vom 10.04.1997, S. 67, § 37 und FN 13. Begründet wurde die höhere Anrechnung der Schlüsselzuweisungen mit der Anpassung bei den Steuerkraftmeßzahlen, die nun ebenfalls mit etwa 95 v.H. erfaßt werden und dies für alle kommunalen Einnahmen zu gelten hat. Vgl.: Hessischer Landtag: Gesetzentwurf der Landesregierung für ein Gesetz zur Änderung des Finanzausgleichsgesetzes vom 21.10.1996, Drucksache 14/2259, S. 12.

3 Die Höhe des Ausgleichszuschuß wurde durch die Regierung festgelegt, durfte aber nicht höher als die Hälfte des fiktiven auf einen Arbeitnehmer entfallenden Gewerbesteueraufkommens sein.

4 Vgl: : Hessischer Landtag: Vorlage der Landesregierung über ein Gesetz zur Regelung des Finanzausgleichs vom 31.05.1951, Drucksachen Abteilung I, 2/111, S.185.

5 Vgl: Ders.: Vorlage der Landesregierung für ein Gesetz zur Regelung des Finanzausgleichs vom 20.04.1955, Drucksachen Abteilung I, 3/107, S. 187; ders.: Vorlage der Landesregierung für ein Gesetz zur Regelung des Finanzausgleichs vom 08.12.1959, Drucksachen Abteilung I, 4/405, S. 1179.

6 Durch das Gesetz wurde die verstärkte Heranziehung abundanter Gemeinden verpflichtend. Vgl.: Ders.: Vorlage der Landesregierung für ein Gesetz zur Regelung des Finanzausgleichs vom 23.02.1953, Drucksachen Abteilung I, 2/588, S. 1146. 
auf identische Art wie bei den übrigen kreisangehörigen Gemeinden ermittelt, aber nur zu 50 v.H. zur Umlage herangezogen. Diese abweichende Behandlung der Städte mit mehr als 50.000 Einwohnern wird mit ihrer erweiterten Aufgabenbefugnis und den damit einhergehenden höheren Ausgaben erklärt. ${ }^{1}$ Dieses Verfahren führte aber bei den Kreisen zu finanziellen Problemen, da diesen bei den von ihnen selbst zu entrichtenden Umlagen die Finanzkraft der Sonderstatusstädte zu 100 v.H. angerechnet wurde. Als Lösung wurde für abundante Sonderstatusstädte 1992 wiederum eine Sonderbestimmung eingeführt. Die Einnahmen aus Schlüsselzuweisungen und Steuerkraftmeßzahl werden bis zur Höhe der Bedarfsmeßzahl zu 50 v.H., der darüber liegende Betrag wird zu 100 v.H. in die Umlagenbemessungsgrundlage einbezogen. ${ }^{2}$ Die Ermittlung der Umlagegrundlage verdeutlicht Tabelle 26 an einem Beispiel, wobei wie in Hessen seit dem Jahr 2000 die Schlüsselzuweisungen zu 95 v.H. und die Steuerkraftzahlen vollständig in die Kreisumlagebemessungsgrundlage eingehen.

Tabelle 26:Ermittlung der gemeindlichen Finanzkraft für die Kreisumlage

\begin{tabular}{|l|c|c|c|c|}
\hline \multicolumn{1}{|c|}{ Gemeindetyp } & Finanzbedarf & Steuerkraft & $\begin{array}{c}\text { Schlüssel- } \\
\text { zuweisung }\end{array}$ & $\begin{array}{c}\text { Umlage- } \\
\text { grundlage }\end{array}$ \\
\hline $\begin{array}{l}\text { kreisangehörige } \\
\text { Gemeinde }\end{array}$ & 100.000 & 80.000 & 10.000 & 89.500 \\
\hline $\begin{array}{l}\text { Sonderstatusstadt: } \\
\text { - nicht abundant } \\
\text { - abundant }\end{array}$ & 100.000 & 80.000 & 10.000 & 44.750 \\
\hline
\end{tabular}

Zur Ausgestaltung des Umlagesatzes ist anzumerken, daß in Hessen nach $\S 37$ FAG ein für alle Bestandteile der Umlagebemessungsgrundlage einheitlicher Umlagesatz vorgesehen ist. Eine Mehr- oder Minderbelastung der einzelnen Gemeinden durch differenzierte Umlagesätze nach $\S 53 \mathrm{Abs}$. $3 \mathrm{HKO}$ wurde zum 01.01.1992 abgeschafft. ${ }^{3}$ Dies wurde damit begründet, daß die Landkreise von dieser Differenzierung kaum noch Gebrauch gemacht haben, was einerseits an der Problematik der Ermittlung der Inanspruchnahme der Kreisleistungen durch jede einzelne Gemeinde gelegen hat und andererseits darin begründet war, daß der Zweck, nämlich einzelne Gemeinden für das Vorhalten von Einrichtungen der

1 Vgl:: Ders.: Gesetzentwurf der Landesregierung für ein Gesetz zur Änderung des Finanzausgleichsgesetzes und anderer Vorschriften vom 26.09.1979, Drucksache 9/1522, S. 10f., $15 \mathrm{f}$.

2 Vgl:: Ders.: Gesetzentwurf der Landesregierung für ein Gesetz zur Änderung des Finanzausgleichsgesetzes und anderer Rechtsvorschriften - Finanzausgleichsänderungsgesetz 1992 - vom 01.11.1991, Drucksache 13/841, S. 13f.

3 Vgl.: Änderung der Hessischen Landkreisordnung, Art 4 des Gesetzes zur Änderung des Finanzausgleichsgesetzes und anderer Rechtsvorschriften (Finanzausgleichsänderungsgesetz) vom 18.12.1991, Hess. GVB1. I, S. 423. 
Daseinsfürsorge zu entlasten, aufgnund der durch die Gebietsreform geschaffenen gleichmäßigen Infrastruktur im Kreis entfallen ist. ${ }^{1}$ Damit die Finanzkraft der kreisangehörigen Gemeinden nicht durch willkürliche Festlegungen des Umlagesatzes zu Gunsten der Kreise eingeschränkt wird, bedurften Sätze oberhalb von 36 v.H. der Genehmigung der Bezirksregierung. Da aber in den letzten Jahren in jedem Landkreis der Umlagesatz eine genehmigungspflichtige Höhe erreicht hat, 1996 lag die Spanne der Umlagesätze zwischen 38,5 und 44,5 v.H., ${ }^{2}$ wurde dieser Passus aus Gründen der Verwaltungsvereinfachung aus dem FAG gestrichen. ${ }^{3}$ Dies dürfte insofern unproblematisch sein, da einerseits bis dahin die Aufsichtsbehörden die Genehmigung gewährt haben und andererseits die Kreistagsabgeordneten meist die Interessen ihrer Gemeinden nicht aus den Augen verloren haben und auch dementsprechend handeln.

Zusätzlich zur Kreisumlage sind die Landkreise seit 1970 berechtigt, von den Gemeinden, die selbst nicht Schulträger sind, eine Schulumlage zu erheben. Hierunter fallen bis auf fünf Sonderstatusstädte, denen nach dem Hessischen Schulgesetz die Trägerschaft zugewiesen wurde ${ }^{4}$, und die Gemeinde Kelsterbach alle übrigen kreisangehörigen Gemeinden. Diese Umlage, deren Satz ursprünglich auf 6 v.H. der begrenzt war, wird in Form eines bis zu 8 v.H. betragenden Zuschlags auf die Summe aus Steuerkraftmeßzahl und Schlüsselzuweisungen erhoben; sie ist zweckgebunden, wobei jeder Landkreis den Höchstsatz erhebt. ${ }^{5}$ Die für die Sonderstatusstädte bei der Kreisumlage anzuwendende Minderung der Umlagegrundlagen gilt nach $\S 37$ Abs. 4 FAG nicht für die Schulumlage. Seit 1998 wird auch in diesem Bereich eine Differenzierung gegenüber den Sonderstatusstädten vorgenommen. Bei den nicht als Schulträger fungierenden Sonderstatusstädten, Wetzlar und Bad Homburg, darf nun nach $\S 37$ Abs. 4 Satz 2 der Zuschlag bis zu 10 v.H. betragen. ${ }^{6}$ Für beide Umlagen dürfen die Sätze innerhalb des laufenden

1 Vgl.: Hessischer Landtag: Gesetzentwurf der Landesregierung für ein Gesetz zur Änderung des Finanzausgleichsgesetzes und anderer Rechtsvorschriften - Finanzausgleichsänderungsgesetz 1992 vom 01.11.1991, Drucksache 13/841, S. 15.

2 Vgl: Hessischer Minister der Finanzen: Kreisumlagesätze nach kommunalen Haushaltsplänen einschließlich Nachträge - soweit diese vorliegen; unveröffentlichte Statistik, Stand 31.03.1999.

3 Vgl.: Hessischer Landtag: Art. 22 des Dritten Gesetzes zur Rechts- und Verwaltungsvereinfachung vom 28.04.1998, Drucksache 14/3849, S. 20.

4 Es handelt sich hierbei um Fulda, Gießen, Hanau, Marburg und Rüsselsheim. $§ 138$ des Gesetzes zur Änderung des Hessisches Schulgesetzes und anderer Gesetze und zur Neugliederung der Staatlichen Schulämter vom 15.05.1997, Hess. GVBI. I, S. 143.

5 Vgl: Hessisches Ministerium der Finanzen: Kreisumlagesätze nach kommunalen Haushaltsplänen einschließlich Nachträge - soweit diese vorliegen; unveröffentlichte Statistik, Stand 31.03.1999.

6 Vgl.: Art. 1 Nr. 5 des Finanzausgleichsänderungsgesetz 1998, vom 18.12.1997, Hess. GVB1. I, S. 442 
Jahres nur bis zum 31.08. verändert werden. Hierfür ist ein Beschluß des Kreistages notwendig.

\subsection{Berechnung der Umlagekraft der Landkreise}

Weil die Kreisumlage die wichtigste Einnahmenkategorie der Kreise darstellt, wird ausschließlich sie zur Berechnung der Umlagekraftmeßzahl herangezogen. Da die Landkreise durch Variation der Umlagesätze das Aufkommen beeinflussen können, kommt bei der Bestimmung der Umlagekraft ein normierter Umlagesatz zum Einsatz, mit der die potentiellen Einnahmen der Kreise "objektiviert" werden. Er beträgt seit 2000 nach $\S 18$ FAG für die Landkreise einheitlich 46 v.H. Die Bemessungsgrundlage ergibt sich aus der Summe von gemeindlicher Finanzkraft und 95 v.H. der gemeindlichen Schlüsselzuweisungen. ${ }^{1}$ Allerdings ist die identische Erfassung aller Einnahmenkategorien, also der Steuern und der Schlüsselzuweisungen, der Abschluß eines langen Prozesses. ${ }^{2}$ Bis 1959 wurde die Umlagekraftmeßzahl der Kreise nur durch die Steuerkraftmeßzahl gebildet, so $\mathrm{da} B$ die gemeindlichen Schlüsselzuweisungen keinerlei Auswirkungen auf die Verteilung der Kreisschlüsselzuweisungen ausübten. Die Steuerkraftmeßzahl wurde aber dadurch modifiziert, daß die Ausgleichszuschüsse ${ }^{3}$ an die Wohngemeinden zu 100 v.H. angerechnet wurden, obwohl sie bei der Bemessung der Steuerkraft seit $1955 \mathrm{zu}$ einem geringeren Anteil eingingen. Durch diese Veränderung sollten negative Auswirkungen auf die Kreise und den LWV als umlageberechtigte Gebietskörperschaften vermieden werden.

Bei der Änderung des Finanzausgleichsgesetzes für 1960 wurde die Reform der Kreisumlageerhebung auch bei der Umlagekraftermittlung berücksichtigt. So wurden die gemeindlichen Schlüsselzuweisungen nun mit 50 v.H. angerechnet und von besonders finanzstarken Gemeinden, die Steuerkraft, die über 170 v.H. der

Für die Jahre 1997 bis 1999 gehen die Schlüsselzuweisungen mit 80, 85 und 90 v.H. in die Bemessungsgrundlage der Kreisumlage ein; ab 2000 zu 95 v.H. Vgl.: Gesetz zur Neuregelung des Finanzausgleichs (Finanzausgleichsgesetz - FAG -) vom 18.03.1997, Hess. GVBl. I, vom 10.04.1997, S. 67, § 37 und FN 13. Für die Jahre 1998 und 1999 werden die Umlagegrundlagen mit 39 und 42,5 v.H. berücksichtigt; ab 2000 mit 46 v.H. Vgl.: Ebenda, S. 62, FN 9. Begründet wurde die höhere Umlagekraftmeßzahl mit der erfolgten Anpassung bei den Steuerkraftmeßzahlen. Durch die Anrechnung mit 46 v.H. werden die erzielten Einnahmen der Kreise ebenfalls zu 95 v.H. erfaßt werden. Vgl.: Hessischer Landtag: Gesetzentwurf der Landesregierung für ein Gesetz zur Änderung des Finanzausgleichsgesetzes vom 21.10.1996, Drucksache 14/2259, S. 12.

2 Der Erfassungsgrad der gemeindlichen Steuerkraft soll am Ende der Übergangszeit bei der Einkommensteuer und bei den Realsteuern der kreisangehörigen Gemeinden - auf dem Stand der Hebesätze von 1996 - 95 v.H. erreichen.

3 Es handelt sich um Zahlungen des Gewerbesteuerausgleichs. Diese wurden damit begründet, daß Arbeitnehmer, die nicht in der Betriebsgemeinde wohnen, ihrer Wohngemeinde Kosten verursachen, ohne das diese an den Gewerbesteuereinnahmen der Betriebsgemeinde parizipieren. 
Bedarfsmeßzahl lag, wie bei der Kreisumlage ein zweites Mal herangezogen. ${ }^{1}$ Durch diese Neuregelung sollte die Bevorzugung sowohl der Kreise mit Gemeinden, die überdurchschnittliche hohe Schlüsselzuweisungen erhalten, als auch der mit überdurchschnittlich vielen hochabundanten Gemeinden verringert werden, da bisher die aus der entsprechend höheren Kreisumlagegrundlage resultierenden Mehreinnahmen nicht berücksichtigt worden waren. ${ }^{2}$

Erst seit 1978 stimmen mit der damaligen Erhöhung der Anrechnung der gemeindlichen Schlüsselzuweisungen auf 75 v.H. die Bemessungsgrundlagen für die Kreisumlage und die Berechnung der Kreisschlüsselzuweisungen überein. Als Grund wurde angeführt, daß durch "die bisherige Regelung (...) vor allem Landkreise mit vielen kleinen und finanzschwachen Gemeinden begünstigt" wurden. "Dies ist nach der Gebietsreform entbehrlich, zumal die Begünstigung zur Übernivellierung führen kann"3. Zudem wurde durch diese Regelung die Finanzkraft der Kreise genauer erfaßt. Aber schon 1980 war mit der Hervorhebung der kreisangehörigen Gemeinden über 50.000 Einwohnern wiederum eine Modifikation notwendig geworden. Denn ihnen wurden Aufgaben des Kreises übertragen, die dieser mit Hilfe der Kreisumlage finanziert. Folglich mußte für die Sonderstatusstädte die Umlagebemessungsgrundlage gemindert werden. Dies wirkte sich auch mindernd auf die fiktive Umlagemeßzahl der Kreise aus, so daß es über die Kreisschlüsselzuweisungen einen relativen Ausgleich gab. ${ }^{4}$

Die vorerst letzte Änderung wurde 1991 notwendig, um einen finanziellen Ausgleich für diejenigen Kreise - Groß-Gerau und Hochtaunus - zu schaffen, deren Sonderstatusstädte - Rüsselsheim und Bad Homburg - abundant gewesen sind. Die hohe Steuerkraft konnte von den Kreisen nur teilweise abgeschöpft werden, da sie nur zu 50 v.H. in die Umlagekraftmeßzahl einging, während sie bei den von den Kreisen zu entrichtenden Umlagen für den Landeswohlfahrtsverband und die Krankenhausfinanzierung im vollen Umfang berücksichtigt werden. ${ }^{5}$ Als

1 Seit 1971 wurde auf das Verfahren zur Steuerkraftberücksichtigung der besonders finanzstarken Gemeinden ohne Begründung verzichtet.

2 Vgl.: Hessischer Landtag: Vorlage der Landesregierung für ein Gesetz zur Regelung des Finanzausgleichs vom 08.12.1959, Drucksachen Abteilung I, 4/405, S. 1179.

3 Ders.: Gesetzentwurf der Landesregierung für ein Gesetz zur Regelung des Finanzausgleichs und zur Änderung anderer Vorschriften vom 11.10.1977, Drucksache 8/4877, S. 39.

4 Vgl:: Ders.: Gesetzentwurf der Landesregierung für ein Gesetz zur Änderung des Finanzausgleichsgesetzes und anderer Vorschriften vom 26.09.1979, Drucksache 9/1522, S. 10f,, 15f. Dem von den Kreisen geäußerten Wunsch, auch die Bemessungsgrundlage der von ihnen zu entrichtenden Umlagen in gleicher Form zu verringern, wurde nicht gefolgt. Dies ist konsequent, denn zur Finanzierung der landesweiten Umlagen sind alle "Gesamtkreise" im gleichen Umfang heranzuziehen, wobei nur der Landkreis für die Abführung der Mittel zuständig ist. Die kreisinterne Aufgabenverteilung, die gegenüber Sonderstatusstädten eine andere ist, soll aber auf den Finanzierungsbeitrag keinen Einfluß haben.

5 Vgl.: Ders.: Gesetzentwurf der Landesregierung für ein Gesetz zur Änderung des Finanz- 
Lösung werden Schlüsselzuweisungen und Steuerkraft bis zur Bedarfsmeßzahl weiterhin zu 50 v.H. angerechnet, der darüber liegende Teil wird zu 100 v.H. berücksichtigt.

\subsubsection{Analyse und Reform}

\subsection{Abgrenzung der ausgleichrelevanten Einnahmen}

Einnahmen aus eigenen Abgaben fließen den Landkreisen nur aus dem kommunalen Grunderwerbsteueranteil, den Konzessionsabgaben und der Gaststättenerlaubnis- sowie der Jagd- und Fischereisteuer zu. Da die beiden erstgenannten bisher nicht als berücksichtigungsfähige Einnahmen angesehen wurden und das Aufkommen der übrigen Steuern als zu gering gilt, wird den Landkreisen keine originäre Finanzkraft zugewiesen. Statt dessen wird aus der Möglichkeit der Kreisund der Schulumlageererhebung eine Finanz- oder Umlagekraft abgeleitete. Da zumindest die Kreisumlagesätze selbst völlig frei von den Landkreisen bestimmt werden können, ${ }^{1}$ ist wie bei den Realsteuern eine Normierung notwendig; das Ergebnis wird als Umlagekraftmeßzahl bezeichnet. Vorgeschlagen wird nun, daß zusätzlich zur bisher aus der Kreisumlagegrundlage berechneten Umlagekraftmeßzahl - analog zur Vorgehensweise bei den Gemeinden - auch noch die kreiseigenen Einnahmen aus der Grunderwerbsteuer und den Konzessionsabgaben zu berücksichtigen sind. ${ }^{2}$ Dabei sind unterschiedliche Anrechnungsverfahren für Einnahmenkategorien $\mathrm{zu}$ verwenden.

Die Einnahmen aus der Grunderwerbsteuer sollen mit dem Ist-Aufkommen herangezogen werden, da den Landkreisen keine Möglichkeiten zur Beeinflussung des Steueraufkommens gegeben sind und somit auch keinerlei Normierungsbedarf besteht. Für die Umlagekraft sollten alle berücksichtigten gemeindlichen Einnahmen mit der gleichen Intensität erfaßt werden und darauf der landesdurchschnittliche Umlagesatz angewendet werden. Bei den Einnahmen aus KA ist zu beachten, daß auch den Landkreisen die Wahlmöglichkeit zwischen der Erhebung einer KA und der Gewinnausschüttung bleibt. Zwar fließen ihnen aus der Gewerbesteuer keine Einnahmen direkt zu, doch über die Kreisumlage sind auch sie an höheren Gewerbesteuereinnahmen beteiligt. Deshalb ist auch für die Kreise ein ähnliches Verfahren zur Einbeziehung der KA wie bei den Gemeinden zu verwenden.

ausgleichsgesetzes und anderer Rechtsvorschriften - Finanzausgleichsänderungsgesetz 1992 - vom 01.11.1991, Drucksache 13/841, S. 10, $13 f$.

1 Die Kreise können eine Schulumlage von bis zu 8 v.H. (10 v.H. bei Sonderstatusstädten) auf die Kreisumlagebemessungsgrundlage erheben. Da alle Kreise den Höchstsatz erreicht haben, besteht die Gestaltungsfreiheit nur noch in Form einer Umlagesatzsenkung.

2 Die übrigen Einnahmen sind wegen der schon vorgebrachten Argumente zu vernachlässigen. 


\subsection{Erfassungsgrad der ausgleichsrelevanten Einnahmen}

Derzeitig werden bei der Bemessung der Umlagekraftmeßzahl weder alle Einnahmen im gleichen Umfang erfaßt, noch wird der aktuelle landesdurchschnittliche Umlagesatz zur Normierung verwendet. ${ }^{1}$ Erst mit der 1997 beschlossenen Strukturreform des KFA, welche im Jahr 2000 abgeschlossen ist, wird erreicht, daß "die Umlagekraftmeßzahl die tatsächlichen Einnahmen aus Kreis- und Schulumlage 1995 zu 95 v.H. erfaßt, wie es das Ziel der vorgeschlagenen strukturellen Überarbeitung des Kommunalen Finanzausgleichs vorsieht"2. Allerdings dürfte der dann gültige fiktive Umlagesatz von 46 v.H. nicht mehr 95 v.H. des Durchschnittssatz der Kreis- und Schulumlage entsprechen. Dies führt wie bei den Gemeinden dazu, daß umlagestarke Kreise von der Untererfassung der Bemessungsgrundlage relativ stärker profitieren und ihren Anteil an den Kreisschlüsselzuweisungen auf Kosten umlageschwacher Kreise erhöhen können. Hinzu kommt noch der Vorteil, der sich aus der schon heute abzeichnenden Untererfassung der Realsteuern ab 2000 ergibt. Denn gerade die Gemeinden der umlagestarken Landkreise haben überdurchschnittliche Gewerbesteuergrundbeträge, die aber geringer erfaßt werden als die Einkommensteuer.

Ein weiterer zu modifizierender Aspekt ist die Berücksichtigung der Sonderstatusstädte. Nach der derzeitigen Regelung werden bei der Ermittlung der Umlagekraftmeßzahl die Schlüsselzuweisungen und die Steuerkraft bis zur Bedarfsmeßzahl zu 50 v.H. berücksichtigt, der darüber liegende Betrag zu 100 v.H. Die Umlagegrundlage zur Berechnung der Umlagekraftmeßzahl mindert sich für die sieben Sonderkreise dadurch um 345,55 Mio. DM, dies sind etwa 14,72 v.H. der tatsächlichen Umlagebemessungsgrundlage. ${ }^{3}$ Dies führt dazu, daß den Kreisen mit Sonderstatusstädten aufgrund der geringer erfaßten Finanzkraft vermehrt Schlüsselzuweisungen zufließen. Diese Bevorzugung ist abzulehnen, denn auch hier muß gelten, daß keine Außenwirkungen über die Kreisgrenzen durch die kreisinterne Aufgabenverteilung induziert werden; dies ist aber derzeitig der Fall. Sie wirkt sich vom Umfang her mit einem Volumen von 111,21 Mio. DM bei unverändertem Grundbetrag sehr viel stärker aus als die Hauptansatzstaffel und der Nebenansatz. Der Nettoverlust beläuft sich aufgrund der Erhöhung des Grundbetrags für die Kreisschlüsselzuweisungen nur auf 69,61 Mio. DM; dies macht bei den Sonderkreisen 16,79 v.H. der Schlüsselzuweisungen oder 37,56 DM pro Kopf aus (siehe Tabelle 27).

1998 lag der landesdurchschnittliche Kreisumlagesatz bei 42,49 v.H., unterstellt wurden im FAG 39 v.H.

2 Hessischer Landtag: Gesetzentwurf der Landesregierung für ein Gesetz zur Änderung des Finanzausgleichsgesetz vom 21.10.1996, Drucksache 14/2259, S. 12.

3 Bei einem landesdurchschnittlichen Kreisumlagesatz von 42,29 v.H. 1998 entgehen ihnen 146,13 Mio. DM, die sie den Sonderstatusstädten für die Aufgabenwahrnehmung überlassen. Dafür erhalten die Sonderkreise aber um 65,15 Mio. DM höhere Schlüsselzuweisungen wegen der geminderten Umlagekraftberücksichtigung. 
Tabelle 27:Umverteilung bei Abschaffung der ermäBigten Finanzkraftberücksichtigung der Sonderstatusstädte bei den Kreisschlüsselzuweisungen

\begin{tabular}{|c|r|r|r|}
\hline \multirow{2}{*}{ Landkreise } & \multicolumn{2}{|c|}{ Landkreise mit Ansatz } & \multirow{2}{*}{$\begin{array}{c}\text { ohne Ansatz } \\
\text { Gewinner }\end{array}$} \\
\cline { 2 - 3 } & \multicolumn{1}{|c|}{ Gewinner } & Verlierer & 14 \\
\hline Anzahl & 0 & 7 & 69.596 .320 \\
\hline Gewinn/Verlust in DM & 0 & -69.606 .926 & 11,94 \\
\hline Gewinn/Verlust in v.H. & 0,00 & $-16,79$ & 24,83 \\
\hline Gewinn/Verlust in pro DM/Kopf & 0,00 & $-37,56$ & 2 \\
\hline
\end{tabular}

Quelle: Eigene Berechnung nach Daten des Finanzministeriums zum KFA 1998.

Bei der Bemessung der Kreisumlage ist es adäquat, daß die Finanzkraft der Sonderstatusstädte nicht im vollen Umfang einbezogen wird, da der Kreis so indirekt Finanzmittel diesen Gemeinden für ihre Kreisaufgabenwahrnehmung zur Verfügung stellt. ${ }^{1}$ Die besondere Aufgabenteilung in den Sonderkreisen soll sich aber nicht außerhalb der Kreisgrenzen auswirken, also auch nicht auf die Verteilung der Kreisschlüsselzuweisungen. Folglich ist die Finanzkraft der Sonderstatusstädte vollständig in die Bemessungsgrundlage bei der Ermittlung der Kreisumlagekraft zur Verteilung der Kreisschlüsselzuweisungen einzubeziehen. Denn die tatsächliche finanzielle Situation der Sonderkreise hat sich nicht geändert. Statt selbst die Aufgaben zu erfüllen und sich in ausreichender Höhe bei den Sonderstatusstädten über die Kreisumlage zu finanzieren, haben sie diese Aufgaben und die zu ihrer Finanzierung notwendigen Mittel den Sonderstatusstädten übertragen.

Um Gestaltungsmöglichkeiten der Kreise bei der Konzessionsabgabe zu vermeiden, ist wie bei den Gemeinden vorzugehen, indem ein maximaler Anrechnungssatz für die KA ermittelt wird. Dazu wird das gewogene Mittel des landesdurchschnittlichen Gewerbesteuerhebesatzes der kreisangehörigen Gemeinden von 338 v.H. ${ }^{2}$ und der durchschnittliche Kreisumlagesatz (inklusive Schulumlage) von 50,49 v.H. - jeweils des Jahres 1998 - unterstellt. Dies führt bei Ausschüttung zu einem Gewinn von 58,47 DM (100 DM - 14,46 DM GewSt - 25,66 DM KSt 1,41 DM Solidaritätszuschlag). Über Kreis- und Schulumlage ist der Landkreis an den erhöhten Gewerbesteuereinnahmen der Gemeinden zu 50,49 v.H. beteiligt. Bei diesem Umlagesatz ergibt sich bei einer zusätzlichen gemeindlichen Gewerbesteuereinnahme von 10,87 DM (14,46 DM - [14,46 DM / 338 v.H. * 84 v.H. Gewerbesteuerumlage]) eine Erhöhung der Umlage um 5,49 DM und der Umlagekraft um 4,24 DM, da für letztere 1998 ein Umlagesatz von 39 v.H.

1 Die Regelung, daß die Steuerkraft oberhalb der Abundanzschwelle wiederum zu 100 v.H. zu berücksichtigen ist, wird im Abschnitt 4.4.6.2.3.2. angesprochen und kritisiert.

2 Dieser Hebesatz wird sowohl für die Gemeinden des Kreises (Durchschnittsbetrachtung) als tatsächlicher Hebesatz verwendet, als auch als Normierungssatz für die gemeindlichen Gewerbesteuereinnahmen. 
angenommen wird. Der Anrechnungsfaktor ergibt sich wie in Abbildung 22 dargelegt und nimmt für die vorgegebenen Größen den Wert 76,4 v.H. an.

\section{Abbildung 22:Berechnung des KA-Anrechnungsfaktors zur Umlagekrafter- mittlung bei Landkreisen}

$\mathrm{KA} \geq(\mathrm{y} \bullet \mathrm{KA}$ - Norm-Umlage $) \cdot$ Ausgleichsgrad + Ist-Umlage + Gewin

Norm-Umlage $=\frac{\text { GewSt } \bullet \text { Gemeindenormierungssatz }}{\text { Ist-Gemeindehebesatz }} \cdot$ Normierungsumlagesatz

Ist-Umlage $\left.=\frac{\text { GewSt } \bullet \text { Gemeindenormierungssatz }}{\text { Ist-Gemeindehebesatz }}\right) \cdot$ Ist-Umlagesatz

Auch hier könnte bei einer Umstellung von KA auf Gewinnausschüttung dem Landkreis für mehrere Jahre die KA-Erhebung unterstellt und der Finanzkraft zugerechnet werden. Die wegen der gestiegenen Gewerbesteuereinnahmen der Gemeinden realisierten Mehreinnahmen bei der Kreisumlage sind herauszurechnen.

\subsection{Probleme der Sonderstatusstädte - Interdependenzen zwischen Umla- gen und Gemeindeschlüsselzuweisungen}

4.4.6.2.3.1. Behandlung der Sonderstatusstädte bei an den Landkreis zu entrichtende Umlagen

Die Erhebung der Kreisumlage unterscheidet sich bei den Sonderstatusstädten von den übrigen kreisangehörigen Gemeinden, da ihre Schlüsselzuweisungen und ihre Steuerkraft bis zur Höhe der Bedarfsmeßzahl nur zu 50 v.H. zur Kreisumlage herangezogen werden. Dies wird damit begründet, daß so ein Ausgleich für die Übertragung von Kreisaufgaben auf diese Gemeinden erfolgt, was als angemessen betrachtet werden kann. Bei der Schulumlage gibt es für Sonderstatusstädte im Gegensatz zur Kreisumlage keine Ermäßigung, da der Kreis für die Sonderstatusstädte, die nicht Schulträger sind, die gleichen Aufgaben wahrnimmt wie für die übrigen kreisangehörigen Gemeinden, so daß eine Kürzung der Schulumlagegrundlage nicht begründbar ist. Wird dieser Argumentation gefolgt, ist aber nicht verständlich, daß die Umlage für die Sonderstatusstädte mit 10 v.H. zwei Prozentpunkte über der Schulumlage für die übrigen kreisangehörigen Gemeinden liegt. ${ }^{1}$ Als Begründung für diese erhöhte Umlage wurde eine Analyse

1 Die Landtagsfraktion der F.D.P. hat gegen diese Erhöhung Klage vor dem Verfassungsgerichtshof erhoben. In einem dazugehörigen Gutachten wird vorgebracht, daß die Begründung für die Erhöhung - die Umlage würde die Kosten nicht decken - nur für den Fall gelten könnte, daß die Minderung der Kreisumlagebemessungsgrundlage auch für die Schulumlage gelten würde. Da dies aber nicht der Fall ist, müßte bei tatsächlicher Unterdeckung der Umlagesatz für alle Gemeinden erhöht werden. Vgl.: Kisker, G.: Normenkontrollantrag gegen § 
der Haushaltsdaten der übrigen Sonderstatusstädte angeführt, die Schulträger sind. Die Untersuchung ergab, daß "im Durchschnitt über $10 \%$ ihrer Umlagegrundlagen zur Finanzierung der Unterdeckung im Einzelplan 2 (Schulen)"1 aufgewendet werden. An dieser Vorgehensweise ist zu kritisieren, daß die Anzahl der Sonderstatusstädte mit fünf zu gering ist, um statistisch abgesicherte Aussagen $\mathrm{zu}$ ermöglichen; weiterhin gibt es nur eine kreisangehörige Gemeinde mit Schulträgerschaft, so daß sich ein Vergleich mit deren Haushaltsdaten verbietet. Folglich ist eine differenzierte Behandlung der Sonderstatusstädte ohne Schulträgerschaft nicht begründbar, so daß der Umlagesatz wieder auf $8 \mathrm{v} . \mathrm{H}$. gemindert werden sollte.

\subsection{Behandlung der Sonderstatusstädte bei vom Landkreis zu entrichten- de Umlagen}

\subsection{1. Auswirkungen auf die vom Landkreis zu entrichtenden Umlagen}

$\mathrm{Zu}$ beachten sind noch die vom Kreis für den Gesamtkreis, der aus dem Kreis und den kreisangehörigen Gemeinden besteht, zu entrichtenden Umlagen. ${ }^{2}$ Es handelt sich dabei um Umlagen für den Landeswohlfahrtsverband und zur Krankenhausfinanzierung, also zur Finanzierung landesweiter Aufgaben (Landesumlagen). ${ }^{3}$ Bei der Bemessung der Umlagenhöhe wird derzeitig dem Sonderkreis auch die gesamte Finanzkraft der Sonderstatusstädte zugerechnet, also eine Umlagebemessungsgrundlage, die gar nicht zur Verfügung steht. Diese Vorgehensweise ist richtig, da auch hier wiederum der Gesamtkreis unabhängig von der kreisinternen Aufgabenverteilung umlagepflichtig ist. Sie benachteiligt aber die Sonderkreise, denn im Gegensatz zur Finanzierung der Kreisaufgaben, von der die Sonderstatusstädte richtigerweise teilweise freizustellen sind, müssen die Sonderstatusstädte zur Finanzierung landesweiter Aufgaben entsprechend ihrer Finanzkraft herangezogen werden, da dies nichts mit der kreisinternen Aufgabenverteilung zu tun hat. Es steht den Sonderkreisen aber nur die Kreisumlage mit der beschränkten Bemessungsgrundlage als Finanzierungsinstrument zur Verfügung. Andererseits

37 Abs. 4 Satz 2 des Hessischen Finanzausgleichsgesetzes in der Fassung des Finanzausgleichsänderungsgesetzes 1998 (Hess. GVBI. I 1997, S. 442) vom 18.12.1997, Gutachten im Auftrag der F.D.P.-Fraktion des Hessischen Landtags, Linden, o.J., S. 14-16.

1 Käss, W.: Der kommunale Finanzausgleich und finanzielle Zuweisungen des Landes zur Erfüllung öffentlicher Aufgaben, Erläuterungen zu § 37 FAG, in: Praxis der Gemeindeverwaltung, Hessen, Bd. E1, (Loseblattsammlung), Stand 1999, S. 86.

2 Die Schlußfolgerung, daß der Kreis für den Gesamtkreis die Umlage leistet, ist daraus zu ziehen, daß einmal die Finanzkraft des Gesamtkreises zur Umlagebemessung herangezogen wird und zudem die kreisfreien Städte, die als Institution dem Gesamtkreis entsprechen, ebenfalls mit ihrer gesamten Finanzkraft umlagepflichtig sind.

3 Nach dem Hessischen Krankenhausgesetz und dem Gesetz über die Mittelstufe der Verwaltung und den Landeswohlfahrtsverband Hessen werden die Landkreise und die kreisfreien Städte zur Finanzierung dieser Aufgaben zu einer Umlage herangezogen, deren Bemessungsgrundlage die Finanzkraft zuzüglich der Schlüsselzuweisungen ist ( $\S 38$ und 39 FAG). 
müssen noch die zusätzlichen Schlüsselzuweisungen berücksichtigt werden, die die Sonderkreise wegen ihrer geringeren Umlagekraftmeßzahl erhalten.

Trotz dieser offensichtlichen Inkonsistenz des Systems wurde erst reagiert, als die Situation für die Kreise aufgrund der hohen Steuerkraft zweier Gemeinden immer schwieriger wurde. ${ }^{1}$ Die dann beschlossene Reform löst die Schwierigkeit aber nur teilweise und dies auch nur im Hinblick auf abundante Gemeinden. Deren über der Bedarfsmeßzahl liegenden Einnahmen aus Schlüsselzuweisungen und Steuerkraft werden nach der Reform zu 100 v.H. zur Kreisumlage herangezogen; für die unter der Bedarfsmeßzahl liegenden Einnahmen hat sich nichts geändert; sie werden auch zukünftig zu 50 v.H. berücksichtigt. Dagegen werden den Kreisen aber weiterhin sowohl die Steuerkraft als auch die Schlüsselzuweisungen zu 100 v.H. zugerechnet. Die Differenz zwischen der tatsächlichen (Ist-) und der unterstellten (Soll-)Umlagegrundlage ist Tabelle $28 \mathrm{zu}$ entnehmen.

Tabelle 28:Die Behandlung der Sonderstatusstädte und ihre Auswirkungen auf die Umlagegrundlagen der Landesumlagen

\begin{tabular}{|c|c|c|c|c|}
\hline Landkreis & $\begin{array}{c}\text { Soll-Umlage- } \\
\text { grundlage }\end{array}$ & \multirow{2}{*}{$\begin{array}{c}\text { Ist-Umlage- } \\
\text { grundlage }\end{array}$} & \multicolumn{2}{|c|}{ Differenz } \\
\cline { 4 - 5 } & & & absolut (DM) & in v.H. \\
\hline Groß-Gerau & 323.448 .791 & 277.891 .967 & -45.556 .824 & $-14,08$ \\
\hline Hochtaunus & 343.765 .098 & 299.856 .104 & -43.908 .994 & $-12,77$ \\
\hline Main-Kinzig & 498.908 .514 & 434.471 .547 & -64.436 .967 & $-12,92$ \\
\hline Gießen & 304.654 .667 & 253.023 .224 & -51.631 .443 & $-16,95$ \\
\hline Lahn-Dill & 323.029 .143 & 287.124 .730 & -35.904 .413 & $-11,11$ \\
\hline Marburg-B. & 368.850 .260 & 315.230 .932 & -53.619 .328 & $-14,54$ \\
\hline Fulda & 258.638 .717 & 208.147 .274 & $\mathbf{- 5 0 . 4 9 1 . 4 4 3}$ & $-19,52$ \\
\hline insgesamt & $\mathbf{2 . 4 2 1 . 2 9 5 . 1 9 0}$ & $\mathbf{2 . 0 7 5 . 7 4 5 . 7 7 8}$ & $\mathbf{- 3 4 5 . 5 4 9 . 4 1 2}$ & $-\mathbf{- 1 4 , 2 7}$ \\
\hline
\end{tabular}

Quelle: Eigene Berechnung nach Daten des Finanzministeriums zum KFA 1998.

Dabei ist zu erkennen, daß der prozentuale Unterschied für den Landkreis Fulda mit 19,52 v.H. am größten ist, da dort die Stadt Fulda einen sehr hohen Anteil an der Bemessungsgrundlage des Kreises hat. Den absolut höchsten Verlust erleidet der Main-Kinzig-Kreis, da 50 v.H. der Bemessungsgrundlage der Stadt Hanau etwa 64,44 Mio. DM ausmachen. Insgesamt verringert sich die tatsächliche Bemessungsgrundlage für alle betroffenen Kreise um ca. 14,27 v.H. des theoretischen Wertes, was etwa 345,55 Mio. DM entspricht. Nach den Annahmen im FAG ergäbe sich eine Bemessungsgrundlage für Umlagen zur Finanzierung landesweiter

Vgl.: Hessischer Landtag: Gesetzentwurf der Landesregierung für ein Gesetz zur Änderung des Finanzausgleichsgesetzes und anderer Rechtsvorschriften - Finanzausgleichsänderungsgesetz 1992 - vom 01.11.1991, Drucksache 13/841, S. 10, 13f. 
Aufgaben in Höhe von 2.421,30 Mio. DM. Da sie tatsächlich aber nur ein Volumen von 2.075,75 Mio. DM annimmt, muß der Umlagesatz stärker angespannt werden. Davon werden aber wiederum die Sonderstatusstädte nur unterprortional belastet, so daß es hier zu unerwünschten Verteilungseffekten kommt.

Um die Folgen dieses Sachverhaltes zu verstehen, sind einige Vorüberlegungen notwendig. Dazu wird zuerst eine theoretische Aufspaltung des Kreisumlagesatzes in zwei Bestandteile vorgenommen. ${ }^{1}$ In den Kreisen dient ein Teil der Umlage der Finanzierung originärer Kreisaufgaben (Normalsatz). Der andere Teil wird zur Finanzierung der landesweiten Umlagen benötigt, die der Kreis für den Gesamtkreis entrichtet und auf die Gemeinden abwälzt (Landessatz). Der aggregierte Satz der beiden Landesumlagen betrug 1998 16,65 v.H. ${ }^{2}$ In den Kreisen beträgt der Anrechnungsfaktor der Schlüsselzuweisungen für die Kreis- und die Landesumlagen 199885 v.H. ${ }^{3}$ Für Sonderkreise gilt nun noch, daß die Kreisumlagegrundlagen um 50 v.H. der Summe aus Steuerkraftmeßzahl und Gemeindeschlüsselzuweisungen der Sonderstatusstädte bis zur Höhe der Bedarfsmeßzahl niedriger sind. Dies führt dazu, daß bei Anwendung des Landesumlagesatzes von 16,65 v.H. der Kreis die Soll-Höhe der Umlage nicht aufbringt, da seine tatsächliche Bemessungsgrundlage geringer ist. Den Betrag, der sich aus der Differenz der Bemessungsgrundlagen, multipliziert mit dem Landesumlagesatz, ergibt, gekürzt um die zusätzlichen Schlüsselzuweisungen, die der Kreis aufgrund der geringeren Umlagekraftmeßzahl erhält, muß der Sonderkreis über eine veränderte Normalumlage aufbringen.

In Tabelle 29 sind die finanziellen Auswirkungen dieser Regelung auf die Normalumlage und den Normalumlagesatz für das Jahr 1998 dargestellt, wobei für die Landesumlage der Satz von 16,65 v.H. gilt. Als Bezugsgröße für die zu erreichende Einnahmenhöhe werden die tatsächlich realisierten Einnahmen aus der Kreisumlage herangezogen. Dieser Wert wird nun in die theoretischen Größen Aufkommen für die Landes- und Aufkommen für die Normalumlage aufgeteilt. Die Soll-Größen ergeben sich, wenn die für die Landesumlage unterstellte Bemessungsgrundlage - sie ergibt sich durch die vollständige Berücksichtigung der Einnahmen der Sonderstatusstädte nach KFA - den Sonderkreisen für diese zur Verfügung stehen würde. Die Soll-Landesumlage wird dann ermittelt, indem der Umlagesatz von 16,65 v.H. mit der Soll-Umlagegrundlage (Tabelle 28) multipliziert wird. Die Soll-Normalumlage resultiert aus der Differenz zwischen tatsächlichem Kreisumlageaufkommen und dem Soll-Aufkommen aus der Landesumlage. Wird diese Größe auf die Ist-Umlagegrundlage (Tabelle 28) des Kreises bezogen, bei der also die Finanzkraft der Sonderstatusstädte nicht im vollen Umfang

1 Die Schulumlage wird nicht berücksichtigt, da sie für die Überlegungen keine Rolle spielt.

2 Von den 16,65 v.H. entfallen 14,79 Prozentpunkte auf die Umlage für den Landeswohlfahrtsverband und 1,86 Prozentpunkte auf die Krankenhausumlage.

3 Ab dem Jahr 2000 liegt dieser Wert bei 95 v.H. 
berücksichtigt wird, so ergibt sich der notwendige Normalsatz, um die Einnahmen aus der Normalumlage zu erreichen. Die Ist-Größen stellen die tatsächlichen Werte dar, wobei für die Ist-Landesumlage die derzeitige Bemessungsgrundlage mit dem für die Landesumlage geltenden Satz von 16,65 v.H. multipliziert wird. Der dann über die Normalumlage aufzubringende Betrag errechnet sich, indem die aufzubringende Umlagesumme um die Landesumlage gemindert wird. Wird der Wert der Ist-Normalumlage nun auf die derzeitige Bemessungsgrundlage bezogen, so resultiert daraus der Ist-Normalsatz.

Tabelle 29:Effekte der Behandlung der Sonderstatusstädte auf die Umlagenhöhe für das Jahr 1998; in Mio. DM und in v.H.

\begin{tabular}{|c|c|c|c|c|c|c|c|}
\hline \multirow[t]{2}{*}{ Landkreis } & \multirow{2}{*}{$\begin{array}{l}\text { tatsäch. } \\
\text { Kreis- } \\
\text { umlage }\end{array}$} & \multicolumn{3}{|c|}{ Soll-Größen (Mio. DM) } & \multicolumn{3}{|c|}{ Ist-Größen (Mio. DM) } \\
\hline & & $\begin{array}{l}\text { Landes- } \\
\text { umlage }\end{array}$ & $\begin{array}{l}\text { Normal- } \\
\text { umlage }\end{array}$ & $\begin{array}{l}\text { Normal- } \\
\text { satz }\end{array}$ & $\begin{array}{l}\text { Landes- } \\
\text { umlage }\end{array}$ & $\begin{array}{l}\text { Normal- } \\
\text { umlage }\end{array}$ & $\begin{array}{l}\text { Normal- } \\
\text { satz }\end{array}$ \\
\hline Groß-Gerau & 116,71 & 53,85 & 62,86 & 22,62 v.H. & 46,27 & 70,45 & 25,35 v.H. \\
\hline Hochtaunus & 130,44 & 57,24 & 73,20 & 24,41 v.H. & 49,93 & 80,51 & 26,85 v.H. \\
\hline Main-K. & 193,34 & 83,07 & 110,27 & 25,38 v.H. & 72,34 & 121,00 & 27,85 v.H. \\
\hline Gießen & 112,60 & 50,73 & 61,87 & 24,45 v.H. & 42,13 & 70,47 & 27,85 v.H. \\
\hline Lahn-Dill & 126,33 & 8 & 5 & 25,27 v.H. & 47,81 & 53 & 27,35 v.H. \\
\hline Marburg-B. & 138,70 & 61,41 & 77,29 & 24,52 v.H. & 52,49 & 86,22 & 27,35 v.H. \\
\hline Fulda & 81,18 & 43,06 & 38,11 & 18,31 v.H. & 34,66 & 46,52 & 22,35 v.H. \\
\hline insgesamt & 899,30 & 403,15 & 496,16 & - & 345,61 & 553,69 & - \\
\hline
\end{tabular}

Quelle: Eigene Berechnung nach Daten des Finanzministeriums zum KFA 1998.

Das Aufkommen aus der Soll-Landesumlage bei einem Satz vom 16,65 v.H. würde im Kreis Groß-Gerau 53,85 Mio. DM betragen. Die Normalumlage bei derzeitiger Bemessungsgrundlage läge bei 62,86 Mio. DM mit einem Normalsatz von 22,62 v.H. Da aber den Sonderkreisen auch für die Landesumlage die geringere Kreisumlagebemessungsgrundlage zur Verfügung steht, beträgt das Aufkommen der Ist-Landesumlage nur 46,27 Mio. DM. Weil die tatsächlich vom Kreis abzuführende Landesumlage aber der Höhe nach der Soll-Landesumlage entspricht, muß die Differenz durch eine erhöhte Ist-Normalumlage finanziert werden. Diese steigt um 7,59 Mio. DM und der Umlagesatz um 2,73 Prozentpunkte auf 25,35 v.H. Am stärksten ist mit 4,04 Prozentpunkten - dies sind etwa 8,41 Mio. DM im Kreis Fulda der durch die Sonderstatusstadtregelung induzierte Anstieg des Normalumlagesatzes. Denn in diesem Kreis ist der prozentuale Anteil von 19,52 v.H., den die Stadt Fulda an der Gesamtbemessungsgrundlage des Kreises aufweist, größer als bei allen übrigen Sonderstatusstädten. Der absolut höchste Wert ist im Main-Kinzig-Kreis mit 10,73 Mio. DM (121,00 - 110,27 Mio. DM) über die erhöhte Normalumlage zu finanzieren, da die Stadt Hanau die größte 
kommunale Finanzkraft der Sonderstatusstädte aufweist und somit auch dem Kreis der absolut höchste Betrag aus der Kreisumlage entgeht. Insgesamt müssen alle kreisangehörigen Gemeinden der Sonderkreise über eine erhöhte Normalumlage die 57,54 Mio. DM (403,15 - 345,61 Mio. DM), um welche die Sonderstatusstädte ungerechtfertigt von den Landesumlagen befreit sind, zusätzlich aufbringen. Dabei entspricht der von einer Gemeinde zu zahlende Betrag ihrem Anteil an der Kreisumlagebemessungsgrundlage ihres Kreises, so da $B$ auch hier die Sonderstatusstädte bevorzugt werden.

Insgesamt ergeben sich also aus der Behandlung der Sonderstatusstädte im KFA folgende externe Effekte bei der Verteilung der Schlüsselzuweisungen, die zu internalisieren sind:

- Die Sonderstatusstädte erhalten in ihrer Gesamtheit zusätzliche Schlüsselzuweisungen zu Lasten der übrigen kreisangehörigen Gemeinden in Höhe von 44,46 Mio. DM.

- Die Sonderkreise ihrerseits erhalten aus der Kreisschlüsselmasse durch die ermäßigte Umlagebemessungsgrundlage 69,61 Mio. DM an zusätzlichen Schlüsselzuweisungen; dem stehen zwar zusätzlich zu finanzierende Landesumlagen von 57,54 Mio. DM gegenüber, doch wird dieser Betrag über eine erhöhte Normalumlage an die kreisangehörigen Gemeinden des Sonderkreises überwälzt.

Folglich finanzieren die Normalkreise über geringere Kreisschlüsselzuweisungen und damit auch deren Gemeinden ${ }^{1}$ sowie die übrigen Gemeinden der Sonderkreise durch relativ höhere Normalumlagesätze und geringere Gemeindeschlüsselzuweisungen die Aufgabenverteilung innerhalb der Sonderkreise und deren finanzielle Besserstellung.

\subsection{2. Reformansätze der Sonderstatusstadtbehandlung}

Das Ziel ist es, mit geringen Änderungen des Systems das Problem zu lösen, daß die Sonderstatusstädte unbegründeter Weise zu den Landesumlagen nicht angemessen herangezogen werden. Da von der Idee her die gemeindliche Finanzkraft in Höhe der Landesumlage belastet werden soll, dies aber bei den Sonderstatusstädten nicht erreicht wird, ist eine Trennung dieser Umlagen vorzunehmen. Die Finanzkraft der Sonderstatusstädte ist dann bei der Kreisumlageerhebung nur zu 50 v.H. zu berücksichtigten, die Regelung für abundante Sonderstatusstädte wäre abzuschaffen, da sie nur eine Hilfskonstruktion darstellt, um diese Gemeinden zu den landesweiten Umlagen heranzuziehen, und bei den Landesumlagen ist die Finanzkraft dann zu 100 v.H. umlagepflichtig. Die Reform kann mit Hilfe zweier Verfahren erreicht werden.

1 Denn die Normalkreise könnten bei höheren Kreisschlüsselzuweisungen den Kreisumlagesatz mindern. 
Bei der ersten Lösung würde durch Gesetzesänderungen ${ }^{1}$ eingefügt werden, daß die Sonderstatusstädte zu den dort aufgeführten Umlagen zur Finanzierung landesweiter Aufgaben ebenfalls herangezogen werden. Die Bemessungsgrundlage für die von ihnen zu entrichtende Umlage betrüge dann 50 v.H. der Finanzkraft, da diese bisher noch nicht durch den in die Kreisumlage integrierten Landesanteil erfaßt wurde. Die Sonderkreise könnten dann den Normalsatz ihrer Kreisumlage und damit diese selbst mindern, da die Sonderstatusstädte den auf sie selbst entfallenden Betrag nun separat abführen und dies nicht mehr durch eine erhöhte Normalumlage geschehen muß. Dieses Verfahren führt zwar zum richtigen Ergebnis, doch wird dadurch weder die Komplexität des Systems gemindert noch dessen Transparenz erhöht.

Nach dem zweiten Ansatz sollte statt dessen im FAG festgelegt werden, daß eine separate Umlage durch die Kreise erhoben wird, vergleichbar der Schulumlage. Ihr Umlagesatz entspräche der Landesumlage; für die Sonderstatusstädte gäbe es keine Minderung dieser Umlagegrundlage, wohl aber bei der Kreisumlage. Im Gegenzug würde sich der Kreisumlagesatz aller Kreise vermindern, da diese nun nur noch der Finanzierung der internen Kreisaufgaben dienen muß. Für die Kreise und Gemeinden in Normalkreisen würde sich an den abzuführenden Beträgen nichts ändern, denn die Bemessungsgrundlage ist für alle Umlagen identisch. In den Sonderkreisen müßten nun die Gemeinden aber nicht mehr den Betrag der Sonderstatusstädte mitfinanzieren. Diese Trennung der Umlage hätte noch einen weiteren Vorteil, da so allen beteiligten Gemeinden aufgezeigt wird, welcher Anteil der von ihnen zu entrichtenden Gelder zur Finanzierung kreisinterner oder landesweiter Aufgaben verwendet wird. Dies könnte die derzeit noch vorhandenen Konflikte über die Festsetzung der Kreisumlage mindern. Denn wenn zur Finanzierung der landesweiten Aufgaben zusätzliche Mittel benötigt würden, würde dies auch offensichtlich, da nur die Landesumlage erhöht werden müßte. Im derzeitigen System ist aber für die Gemeinden der Auslöser der Erhöhung der Kreisumlage nicht eindeutig zu erkennen. Sie führen dies u.U. ungerechtfertigterweise auf ineffizientes Verhalten des Kreises zurück, was zu Widerständen führen kann.

\subsection{Interdependenzen mit den Gemeindeschlüsselzuweisungen}

Wie schon bei der Bedarfsmessung der Kreise und Gemeinden angesprochen wurde, bestehen Interdependenzen zwischen der Behandlung von Sonderstatusstädten bei den Gemeindeschlüsselzuweisungen und den Umlagen. Da die Sonderstatusstädte sowohl einen höheren Bedarf erhalten als auch geringere Umlagen

1 Finanzausgleichsänderungsgesetz 1999 vom 25.11.1998, Hess. GVBl. I, S. 496, Gesetz über die Mittelstufe der Verwaltung und den Landeswohlfahrtsverband Hessen vom 07.05.1953, Hess. GVBl. S. 93 und Gesetz zur Neuordnung des Krankenhauswesens in Hessen (Hessisches Krankenhausgesetz 1989 - HKHG -) vom 18.12.1989, Hess. GVB1. I, S. 452. 
leisten müssen, ist die Gesamtentlastung nicht genau zu erkennen. Deshalb wäre es sinnvoll, nur noch eine Entlastungsform zu wählen. Bei der anstehenden Reform sind zwei Aspekte zu berücksichtigen:

- Die Finanzkraft jedes Gesamtkreises ist für die vom Kreis zu entrichtenden landesweiten Umlagen und für die Verteilung der Kreisschlüsselzuweisungen vollständig zu erfassen; die kreisinterne Aufgabenverteilung darf keinerlei Auswirkungen auf die Umlagegrundlagen haben. Denn die vom Landkreis zu zahlenden landesweiten Umlagen beziehen sich auf den Gesamtkreis als solchen. Gleiches gilt für die Verteilung der Kreisschlüsselzuweisungen.

- Die Institution der Sonderstatusstädte darf keine Auswirkungen auf die von den übrigen Gemeinden des Sonderkreises abzuführenden Beträgen zu landesweiten Umlagen haben; zu diesen ist die Sonderstatusstadt mit ihrer gesamten Finanzkraft heranzuziehen. Denn die Aufgabenverteilung zwischen diesen besonderen Gemeinden und den Kreisen darf sich nur bei den Umlagen zur Finanzierung kreisinterner Aufgaben mindernd auswirken, nicht aber bei denen für landesweite Aufgaben.

Prinzipiell können zwei Ansätze verwendet werden, um diese Anforderungen zu erfüllen, wobei der erste auf bestehende Regelungen aufbaut, während beim zweiten im größeren Umfang Änderungen notwendig werden, dafür aber klarere Ergebnisse zu erreichen sind.

Beim ersten Ansatz bleibt der Nebenansatz für Sonderstatusstädte bei der Verteilung der Gemeindeschlüsselzuweisungen erhalten, so daß die übrigen Gemeinden diese Übertragung von Kreisaufgaben mitfinanzieren. Dafür müßten dann aber die Einwohner der Sonderstatusstädte bei der Bedarfsermittlung des Sonderkreises mit weniger als 100 v.H. gewichtet werden. Die Finanzkraft der Sonderstatusstadt wird sowohl zur tatsächlichen Kreisumlage als auch zur Umlagekraftmeßzahl des Landkreises, die der Verteilung der Kreisschlüsselzuweisungen dient, vollständig herangezogen. Normalkreise erhalten nun höhere Schlüsselzuweisungen und können deshalb den Normalsatz der Kreisumlage mindern, wodurch ihre Gemeinden für die Mitfinanzierung des erhöhten Anteils der Sonderstatusstädte an den Gemeindeschlüsselzuweisungen einen Ausgleich erhalten. Sonderkreise können ebenfalls ihre Normalumlage senken, da die Finanzkraft der Sonderstatusstadt vollständig bei der Kreisumlageerhebung berücksichtigt wird, so daß es auch zu Entlastungen der übrigen Gemeinden im Sonderkreis kommt. Für die Landesumlagen ergibt sich kein Problem mehr, da alle Umlagebemessungsgrundlagen identisch sind.

Der zweite Ansatz ist klarer, da sein Ziel direkt erreicht werden kann. Auf den Nebenansatz bei den Gemeindeschlüsselzuweisungen für Sonderstatusstädte wird verzichtet; es gibt nur noch eine direkte Entlastung für die Übernahme der 
Kreisaufgaben durch den betroffenen Kreis selbst in Form einer geminderten Umlagebemessungsgrundlage. Dadurch müssen die übrigen kreisangehörigen Gemeinden nicht mehr durch verringerte Gemeindeschlüsselzuweisungen die $\mathrm{Ar}$ beitsteilung zwischen den Sonderkreisen und den Sonderstatusstädten mitfinanzieren. Bei der Kreisbedarfsmessung müßten die Einwohner der Sonderstatusstädte weiterhin mit 100 v.H. gewichtet werden, da der Kreis in diesem Fall die entsprechenden Mittel nicht behält, sondern in Form einer geringeren Umlagebemessungsgrundlage und somit durch eine geminderte Kreisumlage an die Sonderstatusstädte weiterleitet. Die Umlagekraftmeßzahl berücksichtigt die vollständige Finanzkraft der Sonderstatusstädte, so daß die Sonderkreise insgesamt um 69,61 Mio. DM geringere Schlüsselzuweisungen bei ansonsten unveränderter Systematik erhalten würden. Denn die geringere tatsächliche Kreisumlage stellt nur einen Ausgleich für die aufgrund der Aufgabenübertragung auf die Sonderstatusstädte geminderten Kosten des Kreises dar. Durch eine separate Umlagebemessungsgrundlage zur Finanzierung landesweiter Aufgaben wird gesichert, daß hier die Sonderstatusstädte auch tatsächlich nach ihrer Finanzkraft zur Landesumlage herangezogen werden. ${ }^{1}$ Dadurch können die Sonderkreise die Normalumlage senken, da diese nun nicht mehr zur Finanzierung der 57,54 Mio. DM benötigt wird, um die bei der alten Regelung die tatsächlichen Einnahmen aus der Landesumlage aufgrund er geminderten Bemessungsgrundlage hinter den unterstellten zurückblieben.

\subsubsection{Allgemeine Grundlagen zum Ausgleich von Finanzbedarf und Finanzkraft}

Die einer einzelnen Gemeinde zustehenden jährlichen Schlüsselzuweisungen werden auf der Basis eines Vergleichs von Finanzbedarf und -kraft ermittelt. Nachdem zunächst der Finanzbedarf als Produkt von Grundbetrag und Gesamtansatz (letzterer ergibt sich als Summe aus Haupt- und Ergänzungsansätzen) errechnet worden ist, wird die Differenz zwischen Finanzbedarf und -kraft ermittelt. Ist sie negativ, gilt die Gemeinde als abundant; sie erhält keine Schlüsselzuweisungen. Allen übrigen Gemeinden werden Finanzmittel in Abhängigkeit von der Ausschüttungsquote zugewiesen. Mit der sog. Ausschüttungsquote wird festgelegt, zu welchem Teil die Differenz zwischen Bedarfs- und Steuerkraftmeßzahl mit Hilfe von Schlüsselzuweisungen ausgeglichen werden soll. Bei ihrer Bestimmung sind zwei sich widersprechende Ziele zu beachten.

Der Ausgleichsgrad ist so zu wählen, daß für die Kommunen ein direkter finanzieller Anreiz besteht, selbst etwas für die Stärkung der eigenen wirtschaftlichen

1 Genauso wird mit der Schulumlage bei den Sonderstatusstädten verfahren, die nicht Schulträger sind. Für diese Umlage wird die Bemessungsgrundlage der Städte Bad Homburg und Wetzlar nicht gemindert, sondern wie bei allen übrigen kreisangehörigen Gemeinden ermittelt. 
Kraft und somit ihrer Steuereinnahmen zu unternehmen. ${ }^{1}$ Denn wäre der Ausgleichsgrad $^{2}$ zwischen den ermittelten Größen 100 v.H., so würden die Schlüsselzuweisungen um den gleichen Betrag sinken, um den sich z.B. die Einkommensteuereinnahmen und somit die Steuerkraft erhöht hat. Aus diesem Zusammenhang heraus sollte der Ausgleichsgrad nicht zu hoch sein, um "die Anstrengungen für die örtliche Selbsthilfe anzuregen"3. Gegen einen zu weitgehenden Ausgleich kann weiterhin angeführt werden, da $B$ auch zwischen privaten Haushalten auf einen entsprechend starken Einkommensausgleich verzichtet wird. Folglich ist auch zwischen öffentlichen Haushalten keine Einnahmenidentität notwendig.

Andererseits ist zu beachten, daß alle Kommunen unabhängig von ihrer finanziellen Ausstattung einen Mindestbedarf haben, um die notwendigsten öffentlichen Leistungen anbieten zu können. Von einer geringen Ausschüttungsquote profitieren die finanzstarken Gemeinden in Form höherer Schlüsselzuweisungen, ${ }^{4}$ denn eine verminderte Ausschüttungsquote führt bei unveränderter Schlüsselmasse über einen steigenden Grundbetrag zur Erhöhung des Finanzbedarfs aller Kommunen, so daß auch finanzstarke Kommunen schlüsselzuweisungsberechtigt werden. Bei sehr finanzschwachen Kommunen kann zudem ein geringer Ausgleichsgrad dazu führen, daß die finanzielle Ausstattung nicht den Mindestbedarf deckt. Für solche Gemeinden wäre ein hoher Ausgleichsgrad notwendig. Dieser würde dann automatisch aufgrund des Zuweisungsplafonds über eine Verringerung des Grundbetrags den Finanzbedarf und damit die Zahl der ausgleichsfähigen Kommunen senken. ${ }^{5}$ Für die dann nicht abundanten Gemeinden würden sich die $\mathrm{Zu}$ weisungen erhöhen. Somit würde mit steigendem Ausgleichssatz die Einnahmeunterschiede der empfangenden Gemeinden stärker gemindert. Quoten bis zu 100 v.H. können deshalb mit der Gewährung einer finanziellen Mindestausstattung begründet werden.

1 Vgl:: Niedersächsischer Staatsgerichtshof: Beschluß vom 15.08.1995, in: DVBl., 110. Jg., 1995, S. 1178.

2 Der Ausgleichsgrad gibt an, in welchem Umfang die Differenz zwischen Finanzbedarf und Steuerkraft durch Zuweisungen ausgeglichen wird. Bei 100 v.H. erfolgt ein vollständiger Ausgleich.

3 Sachverständigenrat zur Begutachtung der gesamtwirtschaftlichen Entwicklung: "Vorrang für die Wachstumspolitik"; Jahresgutachten 1987/88, Stuttgart 1987, S. 158, Tz. 298.

4 Vgl.: Zimmermann, H.; Postlep, R.-D.: Probleme des kommunalen Finanzausgleichs - Überblick und Einordnung der Untersuchungsergebnisse, in: Räumliche Aspekte des kommunalen Finanzausgleichs (Veröffentlichungen der Akademie furr Raumforschung und Landesplanung: Forschungs- und Sitzungsberichte, Bd. 159), Hannover 1985, S. 8.

5 Der Finanzbedarf ist keine exogen vorgegebene Größe, sondern über den Grundbetrag von der Finanzausgleichsmasse abhängig; er ergibt sich aus der Multiplikation des meist einwohnerabhängigen Gesamtansatzes mit dem Grundbetrag. 


\subsubsection{Ausgleich von Finanzbedarf und Finanzkraft in Hessen}

\subsubsection{Entwicklung des Ausgleichsverfahrens im Zeitablauf}

Seit 1950 wird die Differenz zwischen Finanzkraft und -bedarf zu 50 v.H. durch Schlüsselzuweisungen ausgeglichen. ${ }^{1} \mathrm{Da}$ bei sehr einnahmeschwachen bzw. mit hohen Ausgabenbedarfen versehenen Kommunen dieser Ausgleichsgrad als nicht ausreichend gilt, wurden für die Kreise schon 1949 bei Unterschreitung einer Mindestfinanzkraft pro Einwohner zusätzliche Sonderzuweisungen gewährt. Diese Regelung wurde 1951 auf eine Mindestrelation zwischen eigener und durchschnittlicher Pro-Kopf-Umlagekraft erweitert, so daß auch die finanzschwachen Kreise 95 v.H. der Durchschnittseinnahmen aus Umlage und Schlüsselzuweisungen erhielten. ${ }^{2}$ Für Städte und Gemeinden wurde 1953 eine Mindestrelation zwischen Finanzkraft und -bedarf bestimmt (sog. Sockelgarantie), welche nicht unterschritten werden durfte. Sie betrug 75 v.H. der Bedarfsmeßzahl und konnte für ein Rechnungsjahr auf bis zu 80 v.H. erhöht werden, wobei die variable Handhabung mit dem komplizierten Berechnungsverfahren zur Verteilung der Schlüsselzuweisungen begründet wurde. ${ }^{3}$ Der Zweck der Sockelgarantie bestand darin, den finanzschwachen Gemeinden ein Minimum an Einnahmen zu sichern. ${ }^{4} 1956$ wurde für die Kreise das identische Verfahren eingeführt, da so die Umlagekraftgarantie verstärkt werden konnte. ${ }^{5}$ Seit 1993 gibt es eine nach Gemeindegruppen differenzierte Sockelgarantie. Sie beträgt für die kreisangehörigen Gemeinden 80 v.H., wodurch der Ausgleichsgrad verstärkt werden sollte. Für die kreisfreien Städte bzw. die Landkreise wurden 76 v.H. bzw. 78 v.H. festgelegt. ${ }^{6}$ Seit 01.01.2000 liegt die Sockelgarantie für die kreisfreien Städte bei 77 v.H. Von dieser Regelung profitiert allein die Stadt Kassel. Mit dieser Maßnahme wurde

1 Für das Jahr 1949 wurde eine Sonderregelung getroffen, indem die Differenz zwischen Finanzbedarf und -kraft bei den kreisfreien Städten zu 50 v.H., bei den kreisangehörigen Gemeinden aber nur zu 1/3 ausgeglichen wurde. Vgl.: § 4 des Zweiten Gesetz zur Änderung des Gesetzes zur Regelung des Finanzausgleichs vom 10.06.1948 vom 14.06.1949, Hess. GVB1. I, S. 47. Der niedrigere Ausgleichsgrad bei den kreisangehörigen Gemeinden wurde mit der Verringerung der Kreisumlage aufgrund der Schlüsselzuweisungen an die Landkreise begründet. Vgl.: Hessischer Landtag: Vorlage der Landesregierung betreffend ein Zweites Gesetz zu Änderung des Gesetzes zur Regelung des Finanzausgleichs vom 10.061948 (Hess. GVB1. S. 83) vom 20.05.1949, Drucksachen Abteilung I, 1/1124, Begründung zu Art. 4.

2 Vgl:: Ders.: Vorlage der Landesregierung über ein Gesetz zur Regelung des Finanzausgleichs vom 31.05.1951, Drucksachen Abteilung I, 2/111, S. 186.

3 Vgl.: Ders.: Vorlage der Landesregierung für ein Gesetz zur Regelung des Finanzausgleichs vom 23.02.1953, Drucksachen Abteilung I, 2/588, S. 1145.

4 Vgl:: Gesetz zur Regelung des Finanzausgleichs vom 11.05.1953, Hess. GVBl. I, S. 106 und Hessischer Landtag: Drucksachen Abteilung I vom 23.02.1953, 2/588, S. $1145 f$.

5 Vgl: Ders.: Vorlage der Landesregierung für ein Gesetz zur Regelung des Finanzausgleichs vom 15.02.1956, Drucksachen Abteilung I, 3/373, S. 1005.

6 Vgl.: Ders.: Art 2 des Gesetzentwurf der Landesregierung für ein Gesetz zur Überleitung der Aufgaben der Hilfe zur Pflege vom 23.10.1992, Drucksache 13/2984, S. 8, 11. 
ein Ausgleich für die gleichzeitig vorgenommene verstärkte Anrechnung der Schlüsselzuweisungen bei der Finanzkraftermittlung angestrebt. Eine Anhebung auf 80 v.H., wie sie gleichzeitig bei den Landkreisen vorgenommen wurde, um im kreisangehörigen Raum die gleiche Sockelgarantie zu erhalten, wurde aufgrund der damit verbundenen Umverteilungswirkungen nicht realisiert. ${ }^{1}$

\subsubsection{Regelungen für abundante Kommunen im Zeitablauf}

Abundante Kommunen erhielten ursprünglich keine Schlüsselzuweisungen. Später wurden für sie Mindestzuweisungen je Einwohner festgelegt. Dieser der Schlüsselmasse vorab entnommene Betrag soll sichern, daß "alle Lasten abgegolten sind, soweit nichts anderes bestimmt ist"2; damit sind die Leistungen der Kommunen für Aufgaben des übertragenen Wirkungskreises u.ä. gemeint. Abbbildung 23 zeigt die Entwicklung der Mindestschlüsselzuweisungen. ${ }^{3}$

\section{Abbildung 23:Entwicklung der Mindestzuweisungen im Zeitablauf}

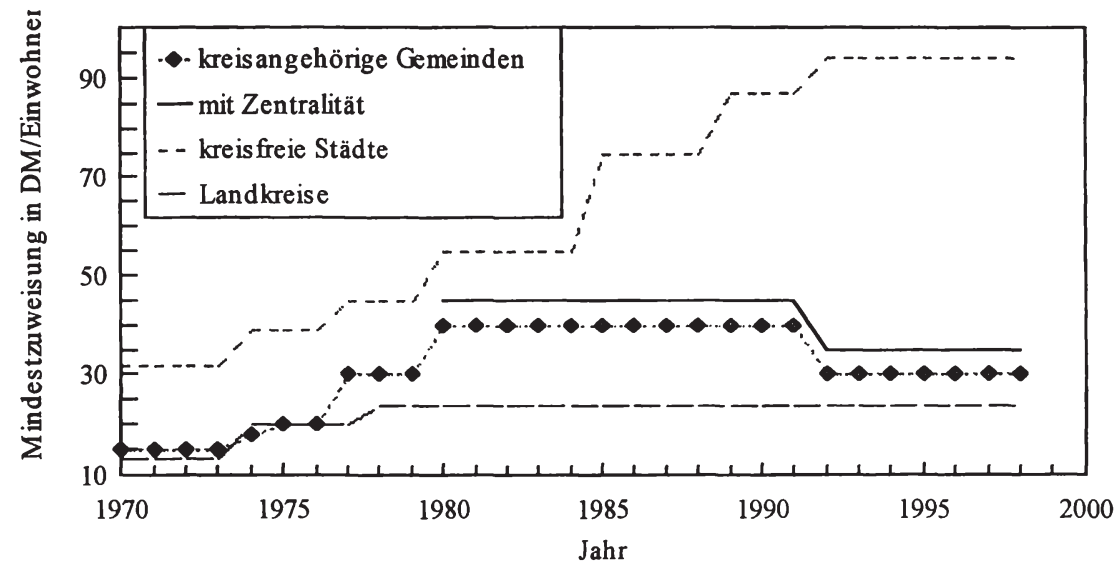

Quelle: Eigene Zusammenstellung aus verschiedenen Finanzausgleichsgesetzen.

Den Landkreisen wurde 1947 und den kreisfreien Städten 1950 eine Mindestzuweisung von 4 bzw. 2,5 DM je Einwohner gewährt. ${ }^{4}$ Mit dem FAG 1958 wurden dann auch Mindestschlüsselzuweisungen für die kreisangehörigen Gemeinden nach Größenklassen gestaffelt zwischen 0,75 und 2,5 DM je Einwohner -

1 Vgl.: Hessischer Landtag: Gesetzentwurf der Landesregierung für ein Gesetz zur Änderung des Finanzausgleichsgesetzes vom 21.10.1996, Drucksache 14/2259, S. $11 \mathrm{f}$.

$2 \S 5$ Abs. 2 des Hess. Finanzausgleichsgesetzes vom 18.03.1997, Hess. GVB1. I, S. 59.

3 Bei den kreisangehörigen Gemeinden ist wegen der Übersichtlichkeit nur die Entwicklung in der größten Gruppenklasse dargestellt. Aus dem gleichen Grund wurde auf die vor 1970 liegenden Werte verzichtet, da ansonsten der Maßstab hätte verändert werden müssen.

4 Vgl.: Gesetz zur Regelung des Finanzausgleichs vom 10.06.1948, Hess. GVBl. I, S. 83 und vom 11.05.1950, Hess. GVBl. I, S. 106. 
eingeführt. ${ }^{1}$ Diese sollten ebenfalls für die Ausgaben der Pflichtaufgaben nach Weisung bzw. Auftragsangelegenheiten entschädigt werden, zumal sich deren Umfang erhöht und somit auch die Kostens gestiegen waren. ${ }^{2}$ Dabei stiegen die Mindestzuweisungen mit dem Hauptansatz der Gemeinden. Nach der Strukturreform des KFA 1977 und der Einführung eines Ergänzungsansatzes für Zentralörtlichkeit wurde eine weitere Differenzierung bei den Mindestschlüsselzuweisungen als notwendig angesehen, damit auch abundante zentrale Orte für die Vorhaltung von Leistungen für das Umland besser entgolten werden konnten. Deshalb wurden für diese kreisangehörigen Orte - abweichend von der normalen Mindestzuweisung, die in Abhängigkeit vom Hauptansatz zwischen 12 und 30 DM lag - gemäß ihrer jeweiligen Zentralitätsstufe erhöhte Mindestzuweisungen zwischen 18 und $45 \mathrm{DM}$ zur Verfügung gestellt. ${ }^{3}$

Die nächste Veränderung bei den Mindestzuweisungen wurde im Zusammenhang mit der Finanzierung der deutschen Einheit vorgenommen, da die erhöhte Gewerbesteuerumlage als nicht ausreichend angesehen wurde. Auf eine separate Umlage - wie in Niedersachsen und Rheinland-Pfalz realisiert - wurde verzichtet. Dafür wurde der Anrechnungsfaktor des kommunalen Einkommensteueranteils erhöht, um so auch einkommensteuerstarke Gemeinden über verringerte Schlüsselzuweisungen zu belasten. Da diese Verfahrensweise bei abundanten kreisangehörigen Gemeinden nicht wirkt, wurde für diese die Mindestschlüsselzuweisung pro Einwohner auf 14 bis $35 \mathrm{DM}$ für zentralörtliche Gemeinden bzw. 10 bis $30 \mathrm{DM}$ für die sonstigen Gemeinden vermindert. ${ }^{4}$ Für kreisfreie Städte wurden hingegen die Pro-Kopf-Beträge sogar von 87 auf 94 DM pro Einwohner erhöht. Dabei ist zu beachten, da $B$ Frankfurt als einzige kreisfreie Stadt zeitweise den Status der Abundanz inne hatte und somit von dieser Regelung profitieren konnte. Entsprechend heißt es in der Gesetzesbegründung, daß mit dieser Maßnahme die Stadt Frankfurt für den Fall der Abundanz entlastet werden soll, da sie "aufgrund des hohen Gewerbesteueranteils über die Gewerbesteuerumlageerhöhung schon überproportional an der Finanzierung des Fonds "Deutsche Einheit" beteiligt"s ist.

1 Vgl.: Zweites Gesetz zur Änderung des Finanzausgleichsgesetzes vom 27.03.1958, Hess. GVB1. I, S. 33.

2 Vgl.: Hessischer Landtag: Vorlage der Landesregierung für ein Zweites Gesetz zur Änderung des Finanzausgleichsgesetzes vom 10.01.1958, Drucksachen Abteilung I, 3/974, S. 2642, 2647f.

3 Vgl:: Gesetz zur Änderung des Finanzausgleichsgesetzes und anderer Vorschriften vom 20.12.1979, Hess. GVB1. I, S. 12.

4 Bei weniger als 7.500 Einwohnern werden 10 DM pro Einwohner gezahlt. Dieser Betrag erhöht sich auf $14 \mathrm{DM}$ bis unter 30.000 Einwohner bzw. auf $18 \mathrm{DM}$ bis unter 50.000 Einwohner und nimmt ab 50.000 Einwohner den Wert 30 DM an. Sollte die abundante Gemeinde zentralörtliche Funktionen wahrnehmen, so betragen die Mindestzuweisungen bei einem Mittelzentrum 14 DM, 23 DM wenn zusätzlich noch Teilfunktionen eines Oberzentrums wahrgenommen werden und $35 \mathrm{DM}$ für ein Oberzentrum.

5 Hessischer Landtag: Gesetzentwurf der Landesregierung für ein Gesetz zur Änderung des 
Der Mindestbetrag bei Landkreisen liegt seit 1979 unverändert bei 24 DM je Einwohner.

\subsubsection{Funktionsweise des hessischen Ausgleichsmechanismus}

Bis jetzt wurde unterstellt, daß der einmal errechnete Grundbetrag den fiktiven Finanzbedarf pro gewichteten Einwohner darstellt und somit unter Berücksichtigung des Ausgleichsgrades die Schlüsselmasse möglichst vollständig auf die Anspruchsberechtigten verteilt wird. Dies ist nur der Fall, wenn weder abundante Gemeinden auftreten noch eine Finanzkraftgarantie für einnahmeschwache Gemeinden gewährt wird. Da bei Berechnung des Grundbetrags sowohl die Finanzkraft als auch der Gesamtansatz der abundanten Gemeinden berücksichtigt wurde, diesen aber nur Mindestzuweisungen zustehen, muß der Grundbetrag ohne diese Ansätze neu ermittelt und die Schlüsselmasse um die Summe der Mindestzuweisungen gekürzt werden. Zudem ist das Verfahren zur Sicherung der Sockelgarantie zu beachten, bei dem simultan der Grundbetrag gesenkt und Sonderzuweisungen gezahlt werden. Der verringerte Grundbetrag senkt allgemein den Finanzbedarf und erhöht somit c.p. die Relation von Finanzkraft und -bedarf.

Zum besseren Verständnis der Zusammenhänge sollen die Regelungen des KFA in Hessen an einem Beispiel erläutert werden. Es wird von drei Gemeinden (A, B und C) mit der jeweils angegebenen Finanzkraft und dem Gesamtansatz ausgegangen (siehe Tabelle 30); die Finanzausgleichsmasse beträgt 120.000, die Mindestzuweisung für abundante Gemeinden $10 \mathrm{DM}$ je Einwohner und die Ausschüttungsquote 50 v.H. bei einer Finanzkraftgarantie von 80 v.H.

Im ersten Schritt wird der Grundbetrag entsprechend der Formel in Abbildung 13 ermittelt, wodurch gesichert ist, daß die vorhandene Schlüsselmasse bei einer Ausschüttungsquote von 50 v.H. verbraucht wird. Bei dem so ermittelten Grundbetrag von 185 erweist sich die Stadt $C$ als abundant, da ihre Finanzkraft größer als ihr Finanzbedarf ist. Da im hessischen KFA auch abundante Gemeinden allgemeine Zuweisungen erhalten, muß die Finanzmasse um den entsprechenden Betrag verringert werden. Da die tatsächliche Einwohnerzahl des Ortes C 600 beträgt und die Fixzuweisung pro Einwohner mit $10 \mathrm{DM}$ angenommen wird, verringert sich die vorhandene Finanzmasse um 6.000 auf 114.000 DM. Mit dieser neuen Größe wird in Schritt 2 die Rechnung nur noch für die Städte A und B wiederholt. Bei einem Grundbetrag von 176 wird die Schlüsselmasse nun vollständig auf beide Orte verteilt; bei $\mathrm{C}$ wird aber nicht die Finanzkraftgarantie erreicht, da die Gesamteinnahmen nicht 80 v.H. der Bedarfsmeßzahl ausmachen. Deshalb wird in einem dritten Schritt dieser Stadt eine Sonderschlüsselzuweisung gewährt, deren Höhe 11.235 DM beträgt. Da sie der allgemeinen Schlüsselmasse entstammt,

Finanzausgleichsgesetzes und anderer Rechtsvorschriften - Finanzausgleichsänderungsgesetz 1992 - vom 01.11.1991, Drucksache 13/841, S. 8. 
verringert sich letztere und somit auch der Grundbetrag sowie als Folge davon der Finanzbedarf. Bei einem Grundbetrag von 168,51 wird der Gemeinde B die Finanzkraftgarantie von 80 v.H. der Bedarfsmeßzahl gewährt.

Tabelle 30:Grundbetragsermittlung unter Berücksichtigung von Abundanz und Sockelkraftgarantie einzelner Gemeinden

\begin{tabular}{|c|c|c|c|c|c|c|c|c|}
\hline \multirow[t]{2}{*}{ Ort } & \multirow{2}{*}{$\begin{array}{c}\text { Finanz- } \\
\text { kraft }\end{array}$} & \multirow{2}{*}{$\begin{array}{l}\text { Gesamt- } \\
\text { ansatz }\end{array}$} & \multirow{2}{*}{$\begin{array}{l}\text { Grund- } \\
\text { betrag }\end{array}$} & \multirow{2}{*}{$\begin{array}{l}\text { Finanz- } \\
\text { bedarf }\end{array}$} & \multicolumn{2}{|c|}{ Zuweisung } & \multirow{2}{*}{$\begin{array}{l}\text { Garantie- } \\
\text { summe }\end{array}$} & \multirow{2}{*}{$\begin{array}{l}\text { Gesamt- } \\
\text { einnahmen }\end{array}$} \\
\hline & & & & & Allgem. & Sonder. & & \\
\hline \multicolumn{9}{|c|}{ Schritt 1} \\
\hline $\mathrm{A}$ & 100.000 & 800 & 185,00 & 148.000 & & & & \\
\hline $\mathrm{B}$ & 200.000 & 2.200 & 185,00 & 407.000 & & & & \\
\hline $\mathrm{C}$ & 200.000 & 1.000 & 185,00 & 185.000 & & & & \\
\hline \multicolumn{9}{|c|}{ Schritt 2} \\
\hline A & 100.000 & 800 & 176,00 & 140.800 & 20.400 & & 112.640 & 120.400 \\
\hline B & 200.000 & 2.200 & 176,00 & 387.200 & 93.600 & & 309.760 & 293.600 \\
\hline $\mathrm{C}$ & 200.000 & & & & 6.000 & & & 206.000 \\
\hline \multicolumn{9}{|c|}{ Schritt 3} \\
\hline $\mathrm{A}$ & 100.000 & 800 & 168,52 & 134.808 & 17.404 & & 107.846 & 117.404 \\
\hline B & 200.000 & 2.200 & 168,52 & 370.722 & 85.361 & 11.235 & 296.578 & 296.596 \\
\hline $\mathrm{C}$ & 200.000 & 1.000 & & & 6.000 & & & 206.000 \\
\hline
\end{tabular}

Allgemein bestehen zwischen den relevanten Determinaten bei der Verteilung der Schlüsselzuweisungen folgende Interdependenzen. Es wird unterstellt, daß immer nur ein Parameter variiert wird:

- Ausschüttungsquote: Wird die Ausschüttungsquote gesenkt, so steigt Grundbetrag, da sich der Bedarf erhöhen muß, um die unveränderte Finanzmasse voll auszuschöpfen. Aufgrund des gestiegenen Bedarfs nimmt die Anzahl der abundanten Gemeinden (Finanzbedarf kleiner als Finanzkraft) $a b^{1}$, die Zahl der empfangsberechtigten Gemeinden steigt, während der durchschnittliche Zuweisungsbetrag sinkt und umgekehrt bei Erhöhung der Ausschüttungsquote.

- Nivellierungshebesatz: Wird der Nivellierungshebesatz vermindert, so sinkt sowohl die Summe der erfaßten Finanzkraft der Gemeinden als auch aufgrund eines verringerten Grundbetrags - Folge der konstanten Ausgleichsmasse - der Finanzbedarf. Da die letztgenannte Summe prozentual schwächer abnimmt ${ }^{2}$,

1 Vgl.: Kuhn, T.: Der kommunale Finanzausgleich - Argumente für eine Reform, in: AfK, 36. Jg., 1997, S. 218.

2 Der Finanzbedarf sinkt systembedingt c.p. um den gleichen absoluten Betrag wie die Finanzkraft; da letztere definitionsgemäß niedriger ist als der Bedarf, wirkt sich eine absolute Minderung prozentual stärker aus. 
erweitert sich der Kreis der empfangsberechtigten Gemeinden. ${ }^{1}$ Von einem niedrigen Nivellierungssatz profitieren Gemeinden mit hohen Bemessungsgrundlagen somit im stärkeren $\mathrm{Maße}$, da nun auch steuerstarke Gemeinden wegen des geringen Umfangs der erfaßten Steuermittel Schlüsselzuweisungen erhalten können. ${ }^{2}$

-Finanzmasse: Sinkt die Finanzmasse ${ }^{3}$, so bewirkt ein verringerter Grundbetrag eine parallele Entwicklung des Finanzbedarfs, da nur so die Differenz zwischen Finanzkraft und -bedarf bei vollständiger Verteilung der gesunkenen Finanzmittel möglich ist. Dies hat einen Anstieg der Anzahl abundanter Gemeinden zur Folge, so daß weniger Gemeinden Zuweisungen erhalten und gleichzeitig auch die Anzahl der Gemeinden sinkt, die unter die Sockelgarantie fällt ${ }^{4}$; bei einem Anstieg der Finanzmasse verläuft die Entwicklung in umgekehrter Richtung. ${ }^{5}$

- Finanzkraftgarantie: Durch Sonderschlüsselzuweisungen für Sockelgarantiegemeinden verringert sich die vorhandene Schlüsselmasse. Wird die Finanzkraftgarantie reduziert, steigt die Schlüsselmasse, da der Bedarf an Sonderschlüsselzuweisungen gesunken ist, und über den erhöhten Grundbetrag auch der Bedarfsansatz. Somit erhöht sich die Anzahl der Zuweisungsberechtigten, da bei unveränderter Finanzkraft der Finanzbedarf zunehmen muß, um die erhöhten Finanzmittel verteilen zu können. Wird die Finanzkraftgarantie erhöht, so kehrt sich die Entwicklung um. ${ }^{6}$

\subsubsection{Ausgleichsmechanismus bei Gemeinden und Städten}

\subsubsection{Wirkungen der bisherigen Regelungen}

Beim Ausgleichsmechanismus mit Sockelgarantie besteht die Gefahr einer verfassungswidrigen Nivellierung der Differenzen der ermittelten Steuerkraft. Zwar ist im aufgezeigten System keine Einebnung der zwischen abundanten und nicht abundanten Gemeinden bestehenden Finanzkraftunterschiede möglich, da der Differenzbetrag zwischen Steuerkraft und Finanzbedarf zu einem Wert unter 100 v.H. ausgeglichen wird, so daß die Summe aus ermittelten Zuweisungen und Steuerkraft, also die Finanzkraft, nicht gleich der Bedarfsmeßzahl sein kann und somit auch nicht die Finanzkraft abundanter Gemeinden erreichbar ist. Doch für

1 Vgl.: Ehrlicher, W. u.a.: Kommunaler Finanzausgleich und Raumordnung, Hannover 1967, S. $31 \mathrm{f}$.

2 Vgl.: Weber, M.: Die Schlüsselzuweisungen, Frankfurt 1981, S. 156.

3 Dies kann durch eine Variation der Verbundsätze oder aufgrund veränderter Steuereinnahmen geschehen.

4 Vgl.: Maas, B.: Kommunaler Finanzausgleich 1995 und 1996 auf neuer Rechtsgrundlage, in: Statistische Monatshefte Niedersachsen, Heft 6, 1996, hrsg. vom Niedersächsischen Landesamt für Statistik, S. 338f.

5 Vgl: Henneke, H.-G.: Probleme des kommunalen Finanzausgleichs, in: Ipsen, J. (Hrsg.): Kommunale Aufgabenerfullung im Zeichen der Finanzkrise, Baden-Baden 1995, S. 117.

6 Vgl:: Littmann, K. u.a.: Die Gestaltung des kommunalen Finanzsystems unter raumordnungspolitischen Gesichtspunkten, Hannover 1968, S. 16. 
die Kommunen, die unter die Sockelgarantie fallen, ist sehr wohl die Einebnung von originären Steuerkraftunterschieden durch den KFA möglich. In Hessen wird für alle drei kommunalen Gruppen, d.h. Landkreise, kreisfreie Städte und kreisangehörige Gemeinden, die Differenz zwischen Finanzbedarf und Steuerkraft zu 50 v.H. ausgeglichen, die Sockelgarantie liegt je nach Gruppe zwischen 77 und 80 v.H. Problematisch ist dabei die große Zahl der Kommunen, die unter die Sockelgarantie fällt, da ihre Finanzkraft vereinheitlicht wird. Dies sind bei bei den kreisangehörigen Gemeinden 49,17 v.H. (207 von 421) und bei den kreisfreien Städten 20,00 v.H. (1 von 5), so daß zumindest im kreisangehörigen Raum aus dem Ausnahme- der Regelfall geworden ist.

Im hessischen KFA tritt das Nivellierungsproblem dann auf, wenn die Steuerkraft einer Kommune unter 60 v.H. der Bedarfsmeßzahl liegt. ${ }^{1}$ Dies liegt darin begründet, daß alle Orte mit einer solch geringeren Steuerkraft auf eine identische Finanzkraft von 80 v.H. der Bedarfsmeßzahl angehoben werden, obwohl in der Ausgangssituation unterschiedliche Relationen gegeben waren. Diese Vereinheitlichung, die nur dann als hinnehmbar angesehen wird, wenn nur ein geringer Anteil der Kommunen - nämlich der finanzschwächste - davon betroffen ist, ist Tabelle $31 \mathrm{zu}$ entnehmen. ${ }^{2}$ Dort ist für drei Gemeinden das Verhältnis von Steuerund Finanzkraft zur Bedarfsmeßzahl abgebildet.

Tabelle 31: Nivellierungswirkung der Sockelgarantie

\begin{tabular}{|c|c|c|}
\hline Kommune & $\frac{\text { Steuerkraft }}{\text { Bedarfsmeßzahl }}$ & $\frac{\text { Finanzkraft }}{\text { Bedarfsmeßzahl }}$ \\
\hline Bad Sooden-Allendorf & 33,10 v.H. & 80,00 v.H. \\
\hline Marburg & 59,98 v.H. & 80,00 v.H. \\
\hline Friedewald & 60,03 v.H. & 80,02 v.H. \\
\hline
\end{tabular}

Quelle: Eigene Berechnung nach Daten des Finanzministeriums zum KFA 1998.

Bad Sooden-Allendorf und Marburg fallen beide unter die Sockelgarantie, wobei erstere die steuerschwächste, letztere die steuerstärkste kreisangehörige Sockelgarantiegemeinde in Hessen ist. Die Steuerkraft von Friedewald wiederum liegt mit 60,03 v.H. der Bedarfsmeßzahl gerade so hoch, daß die Sockelgarantie keine

1 Da die Differenz zu 50 v.H. ausgeglichen wird, ergibt sich bei einem Finanzbedarf von 100 und einer Steuerkraft von 60 eine Schlüsselzuweisung von 20. Steuerkraft und Schlüsselzuweisung ergeben zusammen die Sockelgarantie von 80 .

2 Vgl.: Mutius, A. v.; Henneke, H. G.: Grenzen der verfassungsrechtlichen Zulässigkeit bei der Festsetzung von Schlüsselzuweisungen, in: Der Gemeindehaushalt, 85. Jg., 1984, S. 152; Albers, H.: Die Ausgleichsregelungen des kommunalen Finanzausgleichs in Niedersachsen im Spannungsfeld von verfassungsrechtlichen Vorgaben und politischen Zielen, in: Niedersächsische Verwaltungsblätter, 3. Jg., 1996, S. 175. 
Wirkung entfaltet. ${ }^{1}$ Es zeigt sich nun, daß das derzeitige System den 26,88 Prozentpunkte betragenden Unterschied im Verhältnis von Steuerkraft und Bedarfsmeßzahl zwischen Bad Sooden-Allendorf und Marburg egalisiert, während die Differenz zwischen letzterer und Friedewald in Höhe von 0,05 Prozentpunkte nur gemindert wird. In Abbildung 24 ist die nivellierende Wirkung der Sockelgarantie am Beispiel der 300 finanzschwächsten kreisangehörigen Gemeinden dargestellt. ${ }^{2}$ Dabei ist zu erkennen, daß die vor KFA zum Teil starken Finanzkraftunterschiede völlig eingeebnet werden. So ist die 26,88 Prozentpunkte je Bedarfseinheit betragende Differenz zwischen der finanzschwächsten und der -stärksten Sockelgarantiegemeinde verschwunden. Eine mit dem beschriebenen Sachverhalt verbundene Schwierigkeit liegt darin, daß das derzeit in Hessen angewendete Ausgleichsverfahren mit Sockelgarantie zu einem wenig anreizfreundlichen Ergebnis führt, da sich für Gemeinden, die trotz Erhöhung ihrer Steuerkraftmeßzahl noch unter die Sockelgarantie fallen, die Finanzkraft nach Finanzausgleich nicht erhöht, da die Zuweisungen sich um die Steigerung der Steuerkraft mindern. ${ }^{3}$ Folglich haben die Kommunen keinerlei Anreiz zur Pflege der eigenen Steuerquellen. ${ }^{4}$

\section{Abbildung 24:Finanzkraft kreisangehöriger Gemeinden vor und nach KFA}

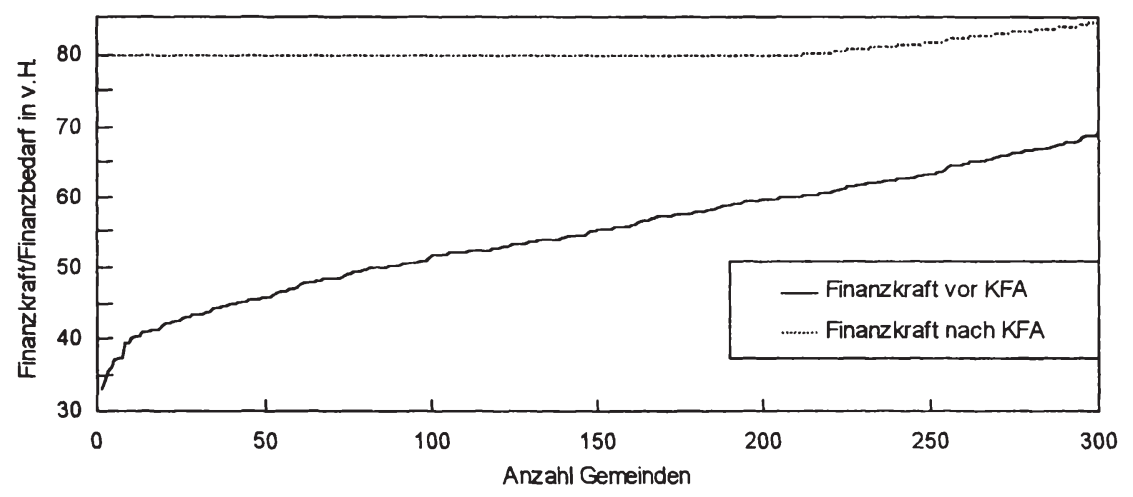

Quelle: Eigene Berechnung nach Daten des Finanzministeriums zum KFA 1998.

1 Bei einer Relation von Steuerkraft zu Gesamtansatz von 60,04 v.H. ergibt sich bei einem Ausgleichsgrad von 50 v.H. eine Finanzkraft von 80,02 bezogen auf den Gesamtansatz, so daß die Mindestrelation von 80 v.H. von Finanzkraft zum Gesamtansatz auch ohne Sockelgarantie erreicht ist.

2 Auf die Darstellung der übrigen 121 kreisangehörigen Gemeinden wurde verzichtet, da dies die Aussage der Abbildung nicht verändert. Für die kreisfreien Städte spielt das Nivellierungsproblem keine Rolle, da nur die Stadt Kassel unter die Sockelgarantie fällt.

3 Dies ist nicht der Fall, wenn nur die tatsächlichen Steuereinnahmen nicht aber die normierte Steuerkraft steigt, etwa als Folge einer stärkeren Hebesatzanspannung der Realsteuern.

4 Vernachlässigt wird hier, daß die Gemeinden auch aus anderen Gründen - etwa Schaffung neuer Arbeitsplätze - an der Pflege der eigenen Steuerquellen interessiert sein können, da es nur darum geht, ob Anreizeffekte in Form einer Einnahmenverbesserung gegeben sind. 
Allgemein muß die Steuerkraft mindestens folgende Höhe annehmen, damit bei beliebiger Sockelgröße und Ausgleichsquote ein positiver Nettoeffekt bei einer Finanzkraftsteigerung entsteht und somit ein entsprechender Anreiz verbleibt (Abbildung 25).

\section{Abbildung 25:Zusammenhang zwischen Finanzkraft und Sockelgarantie so- wie Ausgleichsquote bei einem Anreiz zur Einnahmensteigerung}

$$
\text { Steuerkraft }>\frac{\text { Finanzbedarf } \bullet \text { (Sockelgarantiequote }- \text { Ausgleichsquote })}{(1-\text { Ausgleichsquote })}
$$

Dieser Zusammenhang gilt allerdings nur, wenn der Anteil der betroffenen Kommune an der Summe des kommunalen Gesamtansatzes, der sich aus der Addition des Gesamtansatzes aller zuweisungsberechtigten Gemeinden ergibt, gering ist, was normalerweise auch zutrifft. Ansonsten führt der gestiegene Grundbetrag zu einem überdurchschnittlichen Anstieg des Finanzbedarfs, so daß es trotz Sockelgarantie nach einem Anstieg der Steuerkraft auch zu einer Erhöhung der Finanzkraft kommen kann.

Der sich bei einer Steuerkrafterhöhung um 0,5 Mio. DM ergebende Nettoeffekt ist beispielhaft für sechs kreisangehörige Gemeinden in der Tabelle 32 dargestellt. ${ }^{1}$ Dabei werden Gemeinden mit und ohne Sockelgarantie nach drei Gesamtansatzgrößenklassen unterteilt. Sie gehören zu den größten, den kleinsten oder entsprechen etwa dem Durchschnitt der hessischen Gemeinden. Der Anteil von Hanau, der größten hessischen kreisangehörigen Gemeinde, liegt bei 2,34 v.H. am Gesamtansatz aller kreisangehörigen Gemeinden, der von Gießen als größter Gemeinde mit Sockelgarantie bei 2,04 v.H. Erstere erhält bei einem Anstieg der Steuerkraft - aufgrund steigender Einkommensteuereinnahmen - um 0,5 Mio. DM eine um 254.911 DM höhere Finanzkraft nach KFA, der Nettoeffekt liegt bei 50,98 v.H.; den übrigen Gemeinden ohne Sockelgarantie verbleiben mindestens 50 v.H. der Einnahmensteigerung. ${ }^{2}$ Gießen behält von den zusätzlichen 0,5 Mio.

1 Es wird davon ausgegangen, daß diese Einnahmensteigerung nicht durch Umschichtung innerhalb der kreisangehörigen Gemeinden erfolgt. Würde dies unterstellt werden, so wäre zu berücksichtigen, ob die 0,5 Mio. DM von einer oder von den übrigen 420 aufzubringen ist. Für den ersten Fall wäre zu unterscheiden, ob es sich um eine abundante, eine Sockelgarantie- oder eine Gemeinde, auf die nur der Ausgleichsgrad von 50 v.H. Anwendung findet, handelt. Weiterhin wäre noch danach zu differenzieren, ob die vorher abundante Gemeinde nun ihre Abundanz verliert bzw. ob die Gemeinde nun unter die Sockelgarantie fällt. Für den zweiten Fall kommt es zu vergleichsweise geringen Abweichungen zur unterstellten Situation, weshalb auf eine eingehende Darstellung verzichtet wird

2 Da der kommunale Anteil an der Einkommensteuer bei 15 v.H. liegt, diese Steuerkrafterhöhung aber nur zu 95 v.H. berücksichtigt wird, müssen tatsächlich die Steuerzahler 3.508.772 Mio. DM zahlen. 
DM hingegen nur 13.673 DM oder 2,73 v.H. Bei allen anderen unter die Sockelgarantie fallenden Gemeinden ist die Steigerung noch geringer, da sie weniger vom Anstieg des Grundbetrags profitierten als Gießen, das einen überdurchschnittlich hohen Anteil am Gesamtansatz der Gemeinden hat. So erhöht sich bei der Stadt Bad Sooden-Allendorf die Finanzkraft nur um 1.576 DM, also um 0,32 v.H., bei Nüsttal sogar nur um 0,07 v.H. oder $326 \mathrm{DM}$.

Tabelle 32:Nettoeffekt einer Steuerkrafterhöhung kreisangehöriger Gemeinden um 0,5 Mio. DM bei einem Ausgleichsgrad von 50 v.H. und einer Sokkelgarantie von 80 v.H. (nach KFA); in DM und in v.H. der Steuerkrafterhöhung

\begin{tabular}{|c|c|c|c|c|}
\hline Sockelgarantie & Kommune & Gesamtansatz & \multicolumn{2}{|c|}{ Nettoeffekt } \\
\hline \multirow{3}{*}{ mit } & Gießen & 122.076 & $13.673 \mathrm{DM}$ & 2,73 v.H. \\
\cline { 2 - 5 } & Bad Sooden-Allendorf & 14.070 & $1.576 \mathrm{DM}$ & 0,32 v.H. \\
\cline { 2 - 5 } & Nüsttal & 2.909 & $326 \mathrm{DM}$ & 0,07 v.H. \\
\hline \multirow{3}{*}{ ohne } & Hanau & 140.314 & $254.911 \mathrm{DM}$ & 50,98 v.H. \\
\cline { 2 - 5 } & Geisenheim & 14.340 & $250.501 \mathrm{DM}$ & 50,10 v.H. \\
\cline { 2 - 5 } & Friedewald & 2.638 & $250.092 \mathrm{DM}$ & 50,02 v.H. \\
\hline
\end{tabular}

Quelle: Eigene Berechnung nach Daten des Finanzministeriums zum KFA 1998.

Wird noch berücksichtigt, daß die Steuerkraftsteigerung nur zu 95 v.H. in die Finanzkraftberechnung einbezogen wird, so ergibt sich für jede Stadt eine zusätzliche Erhöhung des ermittelten Nettoeffekts um 5,26 Prozentpunkte. ${ }^{1}$ Somit bleiben bei den Sockelgarantiegemeinden ein maximaler Nettogewinn einer Erhöhung der Steuerkraftzahl um 0,5 Mio. DM von 7,99 v.H. (2,73 + 5,26 v.H.), wobei dieser Betrag nur von Gießen erreicht werden kann. Für eine Gemeinde durchschnittlicher Größe liegt dieser Wert hingegen nur bei 5,58 v.H. $(0,32+5,26$ v.H.). Ganz anders die Werte für die Gemeinden, die weder unter die Sockelgarantie fallen noch abundant sind. Hanau kann aus der gleichen Steuerkrafterhöhung wie GieBen 56,24 v.H. (50,98 + 5,26 v.H.) der Einnahmen oder 281.227 DM als Nettogewinn verbuchen.

Aus diesen Ergebnissen ist zu ersehen, daß es für die der Sockelgarantie unterliegenden Gemeinden kaum einen ökonomischen Anreiz zur Erhöhung der Steuereinnahmen gibt. Ausschließlich qualitative Aspekte - Substitution von Zuweisungen durch Steuereinnahmen - können hier angeführt werden. Dies ist darüber hinaus zu kritisieren, da gerade die genannten Gemeinden als wirtschaftsschwach zu

1 Damit sich die im KFA berücksichtigte Steuerkraft um 0,5 Mio. DM erhöht, müssen die tatsächlichen Einkommensteuereinnahmen der Stadt um 0,53 Mio. DM gestiegen sein, da die Steuerkraft nur zu 95 v.H. angerechnet wird. Da somit 26.316 DM unberücksichtigt bleiben, kommt es zusätzlich zu einer Erhöhung um 5,26 v.H. Prozentpunkte. 
bezeichnen sind und deshalb eine Verbesserung der wirtschaftlichen Situation und somit auch der Steuerkraft anzustreben ist. Da die Möglichkeit besteht, daß der Staat durch zweckgebundene Zuweisungen diese Gemeinden fördert, sollte das Schlüsselzuweisungssystem das angestrebte Ziel unterstützen und nicht konterkarieren.

Die Situation bei den kreisfreien Städten weicht von jener der kreisangehörigen Gemeinden ab, da mit Kassel nur eine Stadt unter die Sockelgarantie fällt und somit die Finanzkraftunterschiede nur zum Teil stark gemindert werden, aber eine Nivellierung nicht eintritt (Abbildung 26).

\section{Abbildung 26:Finanzkraft kreisfreier Städte vor und nach KFA}

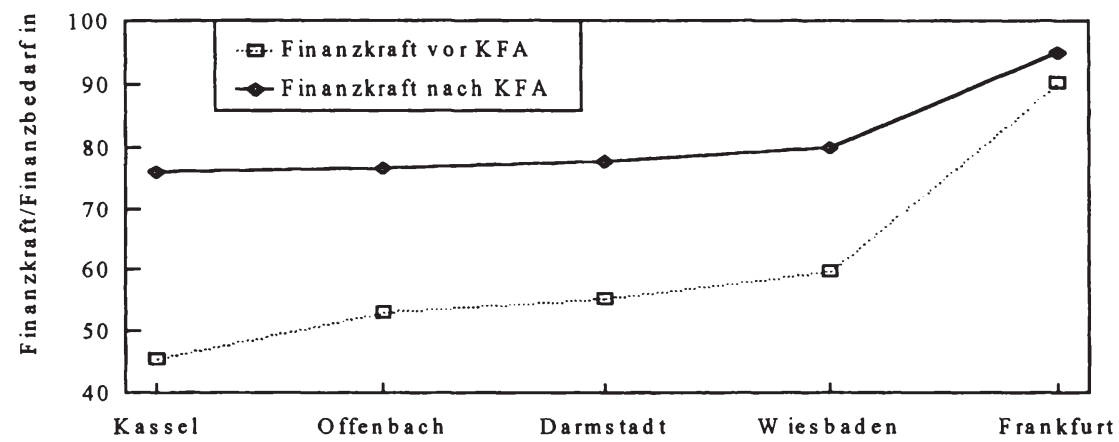

Quelle: Eigene Berechnung nach Daten des Finanzministeriums zum KFA 1998.

Zudem ergibt sich auch für die Stadt Kassel, die einzige mit Sockelgarantie, ein Anreiz zur Steigerung der Finanzkraft, da ihr Anteil am Gesamtansatz aller Städte bei 14,35 v.H. liegt und sie somit trotz Sockelgarantie über den gestiegenen Grundbetrag von einer Steuerkrafterhöhung profitiert. Wird eine Steigerung der Steuerkraft um 0,5 Mio. DM unterstellt, so ist zu beachten, daß der Anstieg der Steuerkraft den Grundbetrag und damit auch den Finanzbedarf erhöht, so daß sich auch für die übrigen Gemeinden ein Vorteil ergeben kann. Spaltenweise kann sowohl der sich für jede Stadt ergebende absolute Nettoeffekt nach KFA als auch der relative Vorteil der betroffenen Stadt (kursiv) abgelesen werden (Tabelle 33). ${ }^{1}$

1 Für die fünf kreisfreien Städte kann unterstellt werden, daß die Steuerkrafterhöhung von 0,5 Mio. DM zu Lasten der 421 kreisangehörigen Gemeinden Hessens oder des übrigen Bundesgebietes erfolgt, beides liefert das gleiche Resultat. Aufgrund der geringen Zahl kann eine Umschichtung innerhalb der Gruppe der kreisfreien Städte als unwahrscheinlich angesehen werden. Für diesen Fall treten folgende Werte auf: Darmstadt 48,73 v.H., Frankfurt 43,87 v.H., Offenbach 47,43 v.H., Wiesbaden 48,95 v.H. sowie Kassel 9,98 v.H. 
Tabelle 33:Nettoeffekt einer Steuerkrafterhöhung kreisfreier Städte um 0,5 Mio. DM bei einem Ausgleichsgrad von 50 v.H. und einer Sockelgarantie von 76 v.H. (nach KFA); in DM und in v.H. der Steuerkrafterhöhung

\begin{tabular}{|c|c|c|c|c|c|}
\hline Erhöhung in: & Darmstadt & Frankfurt & Offenbach & Wiesbaden & Kassel \\
\hline Vorteil & 54,60 v.H. & 72,17 v.H. & 53,80 v.H. & 59,29 v.H. & 20,27 v.H. \\
\hline Darmstadt & 272.995 & 22.995 & 22.995 & 22.995 & 45.926 \\
\hline Frankfurt & 110.860 & 360.860 & 110.860 & 110.860 & 221.412 \\
\hline Offenbach & 18.975 & 18.975 & 268.975 & 18.975 & 37.898 \\
\hline Wiesbaden & 46.426 & 46.426 & 46.426 & 296.426 & 92.724 \\
\hline Kassel & 50.745 & 50.745 & 50.745 & 50.745 & 101.349 \\
\hline
\end{tabular}

Quelle: Eigene Berechnung nach Daten des Finanzministeriums zum KFA 1998.

Es zeigt sich dabei, daß entgegen der Vermutung die Stadt Kassel trotz Sockelgarantie eine Erhöhung der Finanzkraft nach KFA um 20,27 v.H. realisiert. Dies ist auf die schon angesprochenen Erhöhung des Finanzbedarfs als Folge der Grundbetragssteigerung zurückzuführen. Da Kassel zu den größeren Städten zählt, bekommt sie über diesen Effekt zusätzliche Einnahmen. Es zeigt sich aber auch, daß für Frankfurt als größte und finanzstärkste Stadt der Nettoeffekt der Steuerkrafterhöhung bei rund 72,17 v.H. liegt. Wird noch berücksichtigt, daß die Steuerkraftsteigerung nur zu 95 v.H. in die Finanzkraftberechnung einbezogen wird, so ergibt sich für jede Stadt eine zusätzliche Erhöhung des Nettoeffekts um 5,26 Prozentpunkte. ${ }^{1}$ Kassel bleibt somit als Resultat der Erhöhung der Steuerkraft um 0,5 Mio. DM eine Verbesserung der Finanzkraft um 25,53 v.H.

\subsubsection{Problemlösungsvorschlag}

Aus den bisherigen Schilderungen wird klar, daß die der Sicherung einer finanziellen Mindestausstattung dienenden Sockelgarantieverfahrens zu einer Nivellierung der Finanzkraft führt und kaum einen Anreiz zur Pflege der eigenen Steuerquellen bietet. ${ }^{2}$ Es ergibt sich somit ein Zielkonflikt zwischen Allokation und Distribution. Aufgrund dieser Zieldichotomie sollte bei Verzicht auf eine Sockelgarantie der Ausgleichsgrad im Gegenzug erhöht werden, um den steuerschwächsten Kommunen ein zur vorherigen Situation vergleichbar hohes Schlüsselzuweisungsaufkommen zu gewähren. Da diese Reformmaßnahme dazu führt, daß sich bei Wachstum der Steuerkraftzahlen der Nettoeffekt erhöht, muß das alte Zuweisungsvolumen nicht vollständig erreicht werden. Im hier vorgestellten Ansatz

1 Damit sich die im KFA berücksichtigte Steuerkraft um 0,5 Mio. DM erhöht, müssen die tatsächlichen Einkommensteuereinnahmen der Stadt um 0,53 Mio. DM gestiegen sein, da die Steuerkraft nur zu 95 v.H. angerechnet wird. Da somit 26.316 DM unberücksichtigt bleiben, kommt es zusätzlich zu einer Erhöhung um 5,26 v.H. Prozentpunkte.

2 Vernachlässigt wird, daß nur 95 v.H. der Steuerkraft bzw. der -erhöhung einbezogen werden. 
wird der Ausgleichsgrad auf 75 v.H. festgelegt. Dadurch ergibt sich für die finanzschwachen Gemeinden, die ansonsten unter die Sockelgarantie fallen würden, eine Verbesserung der finanziellen Anreize, für die übrigen Gemeinden aber eine Verschlechterung, da sich für diese nun eine Minderung der Schlüsselzuweisungen um 75 statt um 50 v.H. des Steuerkraftanstiegs ergibt, was aber akzeptiert wird, da nun vor allem finanzschwache Gemeinden ein eigenes Interesse an der Pflege ihrer Steuerquellen haben.

$\mathrm{Zu}$ beachten ist, daß sich die Anzahl der Gemeinden, welche Schlüsselzuweisungen erhalten, mit steigender Ausgleichsquote verringert, obwohl sich deren Steuerkraft nicht erhöht hat. ${ }^{1}$ Denn aufgrund der Plafondienung der Schlüsselmasse verringert sich mit der Erhöhung des Ausgleichsgrades der Grundbetrag und somit der Finanzbedarf, so da $B$ mehr Gemeinden abundant werden und keine Schlüsselzuweisungen mehr erhalten. ${ }^{2}$ Beim angewendeten Verfahren ergibt sich dann zwar ein höherer Ausgleich bei den Gemeinden, welche noch Schlüsselzuweisungen erhalten, doch der Abstand innerhalb der Gruppe der abundanten Gemeinden vergrößert sich. ${ }^{3} \mathrm{Daß}$ nun neu-abundante Gemeinden sehr viel schlechter gestellt werden, gilt aber nicht für Hessen, da hier - im Gegensatz zu anderen Ländern - auch abundante Gemeinden eine Mindestschlüsselzuweisung erhalten, deren Höhe sich nach der Einwohnerzahl und der zentralörtlichen Aufgabenerfüllung richtet. Diese Mindestzuweisungen erhalten auch nicht abundante Gemeinden, wenn es für sie finanziell günstiger ist, sie also z.B. aufgrund der geringen Differenz zwischen Finanzbedarf und Steuerkraft knapp unter der Abundanzschwelle liegen und somit bei einem Ausgleichsgrad von 50 v.H. nur minimale Schlüsselzuweisungen bekommen würden.

Bei der Untersuchung der kreisangehörigen Gemeinden, die der Sockelgarantie unterlagen, erweist sich die Gemeinde Bad-Sooden-Allendorf als diejenige, die durch die Umstellung den prozentual größten Verlust mit $-8,42$ v.H. der Schlüsselzuweisungen hinnehmen muß; sie erreicht allerdings noch 91,58 v.H. der Sokkelgarantie, also 73,26 v.H. der alten Bedarfsmeßzahl. Dieser Verschlechterung ist die verbesserte Anreizstruktur entgegenzustellen, wie aus der Tabelle 34 ersichtlich ist. ${ }^{4}$

1 Vgl.: Kuhn, T.: Der kommunale Finanzausgleich - Argumente für eine Reform, in: AfK, 36. Jg., 1997, S. $218 \mathrm{f}$.

2 In den meisten Ländern erhalten abundante Gemeinden keinerlei Schlüsselzuweisungen.

3 Diese Gruppe setzt sich aus alt- und neu-abundanten Gemeinden zusammen. Die neuabundanten Gemeinden haben aber noch bei einer geringeren Ausgleichsquote Schlüsselzuweisungen erhalten, welche die Finanzkraftdifferenz zu den alt-abundanten gemindert haben.

4 Es wird davon ausgegangen, daß diese Einnahmensteigerung nicht durch Umschichtung innerhalb der kreisangehörigen Gemeinden erfolgt. 
Tabelle 34:Nettoeffekt einer Steuerkrafterhöhung kreisangehöriger Gemeinden um 0,5 Mio. DM (nach KFA) bei einem Ausgleichsgrad von 75 v.H.; in DM und in v.H. der Steuerkrafterhöhung

\begin{tabular}{|c|c|c|c|c|}
\hline Sockelgarantie & Kommune & Gesamtansatz & \multicolumn{2}{|c|}{ Nettoeffekt } \\
\hline \multirow{3}{*}{ mit } & Gießen & 122.076 & $134.156 \mathrm{DM}$ & 26,83 v.H. \\
\cline { 2 - 5 } & Bad Sooden-Allendorf & 14.070 & $126.055 \mathrm{DM}$ & 25,21 v.H. \\
\cline { 2 - 5 } & Nüsttal & 2.909 & $125.218 \mathrm{DM}$ & 25,04 v.H. \\
\hline \multirow{3}{*}{ ohne } & Hanau & 140.314 & $135.524 \mathrm{DM}$ & 27,10 v.H. \\
\cline { 2 - 5 } & Geisenheim & 14.340 & $126.067 \mathrm{DM}$ & 25,21 v.H. \\
\cline { 2 - 5 } & Friedewald & 2.638 & $125.198 \mathrm{DM}$ & 25,04 v.H. \\
\cline { 2 - 5 }
\end{tabular}

Quelle: Eigene Berechnung nach Daten des Finanzministeriums zum KFA 1998.

Nach diesen Ergebnissen erreicht jede Gemeinde bei Verzicht auf Sockelgarantie und Erhöhung der Ausgleichsquote auf 75 v.H. einen Nettoeffekt von mindestens 25 v.H., so daß von der Erhöhung der Steuerkraft um 500.000 DM zumindest eine Verbesserung der Nettoposition nach KFA um 125.000 DM bestehen bleibt. Diese Größe steigt in Abhängigkeit vom Gesamtansatz auf 135.524 DM oder 27,10 v.H. der Steuerkrafterhöhung. Für die untersuchten Beispielgemeinden würde sich bei Gießen als Nettoeffekt eine Erhöhung der Finanzkraft um 26,83 v.H. ergeben, für Nüsttal läge er noch bei 25,04 v.H. Da die Steuerkrafterhöhung nur zu 95 v.H. beim KFA berücksichtigt wird, liegen die tatsächlichen Steuereinnahmen für diesen Beispielfall um 26.316 DM höher, so daß sich bei jeder Gemeinde der Nettoeffekt noch einmal um 5,26 Prozentpunkte erhöht. ${ }^{1}$ Jeder Gemeinde verbleiben somit mindestens 30,26 v.H. der Steuerkrafterhöhung um 0,5 Mio. DM. Zwar ergibt sich für die vorher nicht unter die Sockelgarantie fallenden Gemeinden eine Verschlechterung, da sie von einer Steuerkrafterhöhung nur noch eine um 30,26 statt 55,26 v.H. erhöhte Steuerkraft nach KFA aufzeigen, doch dürfte diese Größenordnung noch ausreichen, um einen Anreiz zur Steuerkrafterhöhung zu bieten. Zudem besitzen nun alle der bei der jetzigen Regelung unter die Sockelgarantie fallenden Gemeinden einen Anreiz zur Steigerung der Steuerkraft und somit zur Pflege der eigenen Steuerquellen, was aus allokativen Gesichtspunkten sehr positiv zu bewerten ist.

$\mathrm{Zu}$ berücksichtigen ist noch, wie sich die tatsächliche Finanzkraft nach Modifikation des Ausgleichsgrades ändert. Diese verringert sich bei Nüsttal etwa um 2,49 v.H. oder 0,06 Mio. DM. Doch schon bei der unterstellten Steuerkrafterhöhung von 0,5 Mio. DM tritt eine Erhöhung der Finanzkraft um 2,36 v.H. oder 0,06 Mio. DM im Vergleich zur Sockelgarantiesituation ein. Für Bad SoodenAllendorf mindert sich der auf die Umstellung des Ausgleichsgrades folgende Verlust bei der unterstellten Steuerkrafterhöhung um 0,5 Mio. DM von 4,94 v.H.

1 Dies gilt aber auch für den Fall der Sockelgarantie. 
der Finanzkraft auf 3,94 v.H. bzw. von $-0,61$ auf $-0,49$ Mio. DM. Somit wird klar, daß bei der angesprochenen Veränderung des Ausgleichsmechanismus kurzfristige Einnahmenverluste nicht zu vermeiden sind. Diese können aber teilweise durch Leistungen aus dem zur Milderung außergewöhnlicher Belastungen gebildeten Landesausgleichsstock kompensiert werden.

Auch die Gefahr der Nivellierung der Finanzkraft ist bei dem vorgeschlagenen Verfahren nicht mehr gegeben, wie die Tabelle 35 wiederum für die Orte Bad Sooden-Allendorf, Marburg und Friedewald zeigt. Dabei ist zu erkennen, daß die vorhandenen Unterschiede gemindert, aber nicht völlig nivelliert werden, wie dies bei einer Sockelgarantie der Fall gewesen wäre. ${ }^{1}$

Tabelle 35:Keine Nivellierungswirkung bei erhöhtem Ausgleichsgrad

\begin{tabular}{|c|c|c|}
\hline Kommune & $\frac{\text { Steuerkraft }}{\text { Bedarfsmeßzahl }}$ & $\frac{\text { Finanzkraft }}{\text { Bedarfsmeßzahl }}$ \\
\hline Bad Sooden-Allendorf & 36,62 v.H. & 84,16 v.H. \\
\hline Marburg & 66,37 v.H. & 91,59 v.H. \\
\hline Friedewald & 66,43 v.H. & 91,61 v.H. \\
\hline
\end{tabular}

Quelle: Eigene Berechnung nach Daten des Finanzministeriums zum KFA 1998.

Bisher wurden nur ausgewählte Gemeinden betrachtet. Nun wird in der Abbildung 27 noch aufgezeigt, wie sich die Abschaffung der Sockelgarantie bei gleichzeitiger Erhöhung des Ausgleichsgrades auf 75 v.H. auf die finanzielle Situation der kreisangehörigen Gemeinden insgesamt auswirkt. Zur vereinfachten Darstellung sind aber ausschließlich die 370 finanzschwächsten Kommunen (87,89 v.H.) dargestellt. Dabei ist zu erkennen, daß beim derzeitigen System der Sockelgarantie über 200 Gemeinden unter die Sockelgarantieregelung fallen und somit keinen Anreiz zur Stärkung der eigenen Steuerkraft haben. Zudem werden die originären Steuerkraftunterschiede eingeebnet. Beim hier vorgeschlagenen System ohne Sockelgarantie zeigt sich, daß eine Nivellierung der originären Steuerkraft

1 Daß sich ohne Sockelgarantie der Grundbetrag und somit die Bedarfsmeßzahl ändern, ist zu beachten. Werden die Größen auf den unveränderten Gesamtansatz der einzelnen Gemeinde bezogen, so ergeben sich folgende DM-Beträge je veredelten Einwohner für die Steuerkraft (a), die Finanzkraft bei Sockelgarantie (b) und die Finanzkraft beim Ausgleichsgrad von 75 v.H. (c):

Bad Sooden-Allendorf: (a) $365,96 \mathrm{DM}$, (b) $884,60 \mathrm{DM}$ sowie (c) $840,92 \mathrm{DM}$; Marburg: (a) 663,21 DM, (b) 884,60 DM sowie (c) $915,23 \mathrm{DM}$; Friedewald: (a) $663,81 \mathrm{DM}$, (b) $884,78 \mathrm{DM}$ sowie (c) $915,38 \mathrm{DM}$.

Auch hier ist die nivellierende Wirkung der Sockelgarantie zu erkennen, da die maximalen Finanzkraftunterschiede zwischen Bad Sooden-Allendorf und Friedewald in Höhe von 297,85 DM je veredelten Einwohner auf 0,18 DM verringert werden. Beim Ausgleichsgrad von 75 v.H. mindert sich der Unterschied nur auf 74,46 DM je veredelten Einwohner. 
ausbleibt, die vorgefundenen Unterschiede werden nur verringert, und somit bleibt auch ein Interesse an der Pflege der eigenen Steuerkraft bestehen. Weiterhin verbessert sich für die finanzschwächsten Kommunen das Verhältnis von Finanzkraft zum ermittelten -bedarf. Erstere erreicht nun mindestens 84,16 v.H. der Bedarfsmeßzahl, während die Sockelgarantie nur ein Verhältnis von 80 v.H. sicherte.

\section{Abbildung 27:Vergleich der Relation von Finanzkraft zur BedarfsmeBzahl in Abhängigkeit vom gewählten Ausgleichsmechanismus}

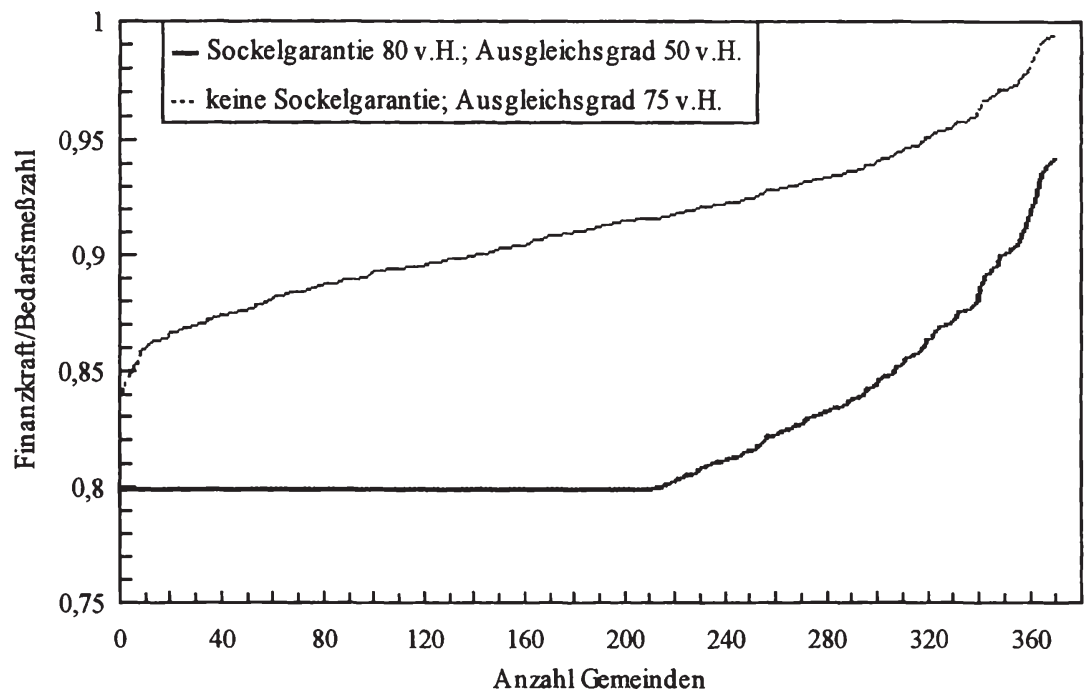

Quelle: Eigene Berechnung nach Daten des Finanzministeriums zum KFA 1998.

$\mathrm{Zu}$ beachten ist aber, daß der Finanzbedarf in Form der Finanzbedarfsmeßzahl keine konstante Größe ist. Aus dem System des kommunalen Finanzausgleichs und der Plafondierung der Finanzausgleichsmasse folgt, daß der in Form der Bedarfsmeßzahl ermittelte Finanzbedarf sich als Produkt aus dem Grundbetrag und dem gemeindeindividuellen Gesamtansatz ergibt. Ersterer wird aber durch den Ausgleichsmechanismus beeinflußt; er sinkt mit steigendem Ausgleichsgrad. Dies hat nun für das gewählte Beispiel zur Folge, daß sich die tatsächliche Finanzkraft nach KFA bei einem Teil der hier dargestellten 370 Gebietskörperschaften im Vergleich zur Situation vor der Reform verringert hat, trotz Verbesserung des Verhältnisses von Finanzkraft zur Finanzbedarfsmeßzahl, wie schon am Beispiel vom Nüsttal und Bad Sooden-Allendorf gezeigt wurde. Denn bei den finanzschwächsten Gemeinden wird die Verringerung der Finanzbedarfsmeßzahl nicht vollständig über den erhöhten Ausgleichsgrad kompensiert. Allerdings ist anzuführen, daß sich auch bei dem Ausgleichsmechanismus mit Sockelgarantie die 
Schlüsselzuweisungen im Zeitablauf in Abhängigkeit vom Volumen der Schlüsselmasse ändern, da dieses Verfahren sich ebenfalls auf den Grundbetrag auswirkt. Folglich wird auch bei Sockelgarantie keine absolute Mindestfinanzausstattung gesichert. Die daraus resultierenden Folgen für die Höhe der einnahmenwirksamen Finanzkraft ist der Abbildung 28 zu entnehmen.

\section{Abbildung 28:Vergleich der Relation von Finanzkraft zum Gesamtansatz in Abhängigkeit vom gewählten Ausgleichsmechanismus; in DM}

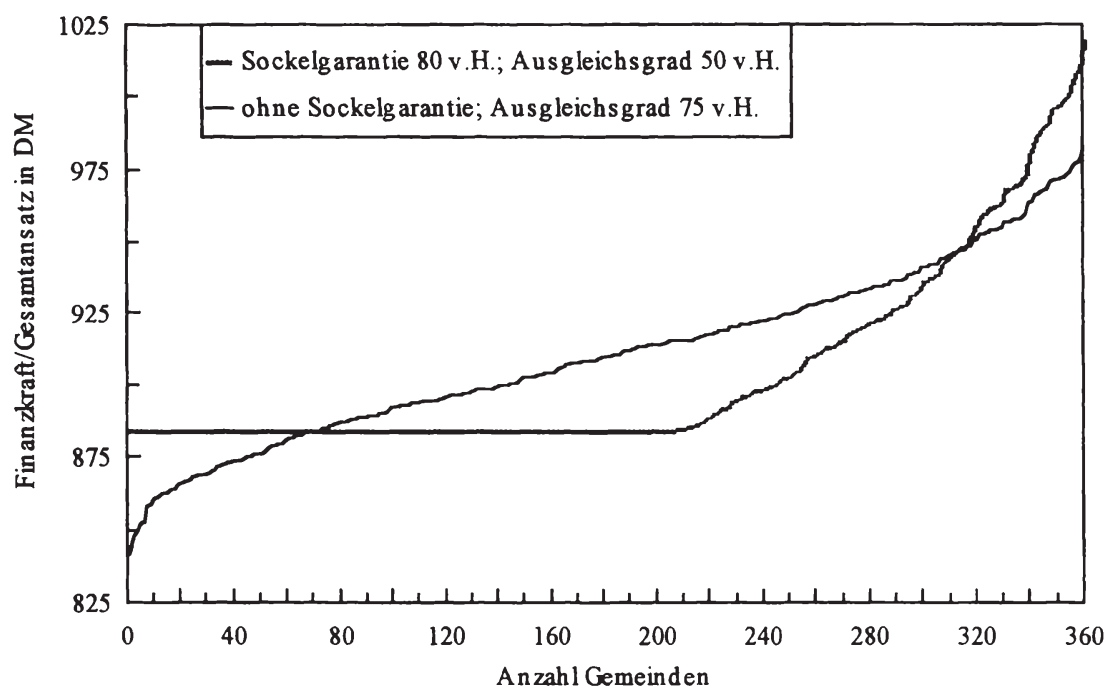

Quelle: Eigene Berechnung nach Daten des Finanzministeriums zum KFA 1998.

Als Ergebnis kann aus Abbildung 28 abgeleitet werden, daß sich die momentane finanzielle Situation bei 238 Gemeinden oder 56,53 v.H. verbessern würde. Davon fielen 135 Kommunen beim alten System unter die Sockelgarantie, dies sind 65,22 v.H. aller vormaligen Sockelgarantiegemeinden. Für 157 oder 37,39 v.H. vermindert sich die Finanzkraft. Von dieser Verschlechterung sind 72 oder 34,78 v.H. der vormals unter die Sockelgarantie fallenden Orte betroffen. Für $26 \mathrm{Kom}-$ munen oder 6,18 v.H. bleibt die Finanzkraft unverändert, da diese nur Mindestzuweisungen nach der Einwohnerzahl erhalten. ${ }^{1}$ Insgesamt käme man somit dem angestrebten Ziel näher, das Eigeninteresse an der Verbesserung der wirtschaftlichen Situation bei Sockelgarantiegemeinden zu steigern. Gleichzeitig würden sich für den größten Teil dieser Gemeinden durch die Systemumstellung

1 In Hessen erhalten abundante Gemeinden Mindestzuweisungen je Einwohner. Für nicht abundante Gemeinden ist teilweise diese Zuweisung höher, als bei Ermittlung der Zuweisung durch den Vergleich von Finanzkraft und -bedarf, was dann der Fall ist, wenn die Differenz zwischen diesen Größen sehr gering ist. Sie erhalten dann ebenfalls Mindestzuweisungen. 
finanzielle Vorteile ergeben, für den Rest könnten noch übergangsweise Zahlungen aus dem Landesausgleichsstock erfolgen.

Weiterhin ist noch zu klären, ob auch für die fünf kreisfreien Städte die Abschaffung der Sockelgarantie bei gleichzeitiger Erhöhung des Ausgleichsgrades gefordert werden soll. Denn im Vergleich zum kreisangehörigen Raum sieht die Situation bei den kreisfreien Städten etwas anders aus. Die Gefahr der Nivellierung besteht nicht, da nur die Stadt Kassel unter die Sockelgarantie fällt, für die sich schon bei bestehender Regelung ein finanzieller Anreiz zur Pflege der eigenen Steuerquellen ergibt. Beim hier vorgeschlagenen Ausgleichsgrad von 75 v.H. würde sich der Nettoeffekt einer Steuerkrafterhöhung, die nicht durch eine Umschichtung zwischen den kreisfreien Städte hervorgerufen wird, für Kassel auf insgesamt 50,88 v.H. $(45,62+5,26$ v.H. $)$ steigern und somit über dem bisherigen Nettoeffekt von insgesamt 25,53 v.H. liegen. Dafür würde er aber bei den kleineren Städten Darmstadt und Offenbach von insgesamt fast 60 v.H. auf 44,46 v.H. $\left(39,20+5,26\right.$ v.H.) und 41,98 v.H. (36,72 +5,26 v.H.) sinken. ${ }^{1} \mathrm{Da}$ für die kreisfreie Sockelgarantiestadt schon heute gilt, was bei den kreisangehörigen Gemeinden erst realisiert werden soll, daß nämlich ein positiver Netttoeffekt einer Steuerkrafterhöhung von etwa 25 v.H. erreicht wird - er beträgt derzeit für Kassel 25,53 v.H. -, kann die derzeitige Regelung mit Sockelgarantie beibehalten werden. Zumal dadurch die Nachteile für die kleineren kreisfreien Städte vermieden werden.

\subsubsection{Ausgleichsmechanismus bei Landkreisen}

\subsubsection{Wirkungen der bisherigen Regelungen}

Das schon angesprochene Nivellierungsproblem spielt bei den Landkreisen eine weitaus gewichtigere Rolle als bei den Gemeinden und Städten. Denn von den Landkreisen fallen 80,96 v.H. - dies sind $17^{2}$ von 21 Kommunen dieser Gebietskörperschaftsgruppe - unter die Sockelgarantie und weisen somit eine identische Finanzkraft nach KFA auf. Dieser Tatbestand impliziert die Gefahr der Verfassungswidrigkeit, da die vorgefundenen Unterschiede nicht völlig eingeebnet werden dürfen. ${ }^{3}$ Der starke Grad der Einebnung der Umlagekraftunterschiede ist in der Abbildung 29 dargestellt. $\mathrm{Th}$ ist $\mathrm{zu}$ entnehmen, daß bei den Sockelgarantiekreisen die Umlagekraftdifferenzen bezogen auf den Gesamtansatz von 88,59

1 Käme die Steuerkrafterhöhung durch eine Umschichtung innerhalb des kreisfreien Raums zustande, so lägen die Werte - abgesehen von Frankfurt - insgesamt zwischen 33,26 v.H. $(27,97+5,26$ v.H.) und 37,54 v.H. (32,28 + 5,26 v.H.). Für Frankfurt würde sich aufgrund der sich einstellenden Abundanz ein Wert von 105,26 v.H. (100 v.H. + 5,26 v.H.) ergeben.

2 Der 18. Kreis weist eine Umlagekraft von 652,55 DM auf im Vergleich zu 650,53 DM der 17 Sockelgarantiekreise, was in Abbildung 29 wegen des Maßstabs kaum zu erkennen ist.

3 Vgl: Bayerischer Verfassungsgerichtshof: Entscheidung vom 12.01.1998, in: DVBI., 113 Jg., 1998, S. 732, Leitsatz 2. 
DM zwischen dem finanzschwächsten Kreis Fulda mit 368,36 DM und dem Kreis Darmstadt-Dieburg mit 456,95 DM je Einheit Gesamtansatz (es handelt sich hier um die veredelten Einwohner), welches der finanzstärkste unter die Sokkelgarantie fallende Kreis ist, vollständig abgebaut werden. Alle Kreise mit Sokkelgarantie weisen nach KFA eine identische Umlagekraft je Einheit Gesamtansatz von $650,53 \mathrm{DM}$ auf.

\section{Abbildung 29:Kreisfinanzkraft vor und nach KFA}

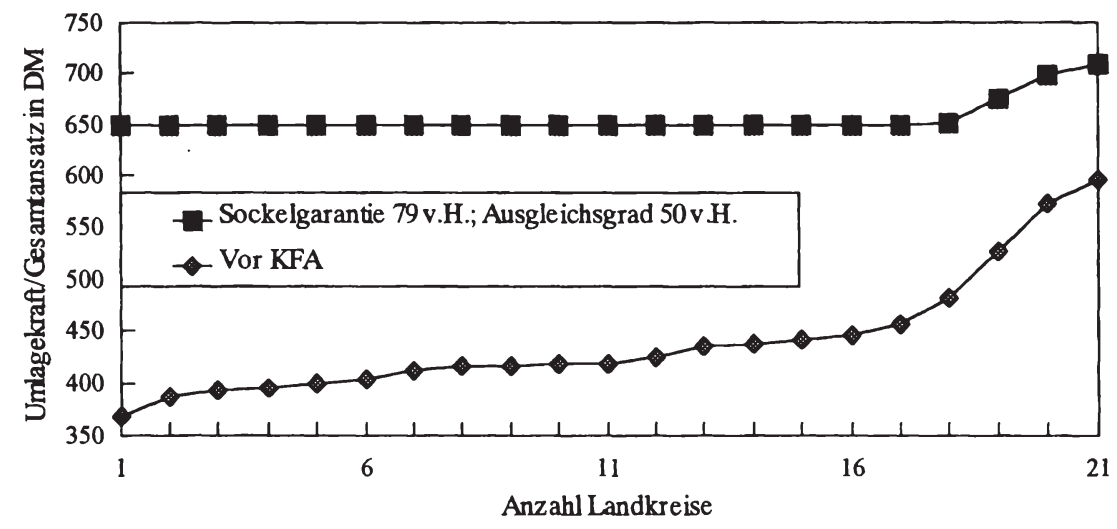

Quelle: Eigene Berechnung nach Daten des Finanzministeriums zum KFA 1998.

Für die Kreise spielen die Überlegungen der Erhöhung des Anreizes zur Pflege der eigenen Steuerquellen kaum eine Rolle, da bei ihnen keine den Gemeinden vergleichbare Möglichkeit der Einnahmensteigerung gegeben ist, denn ihnen stehen keine selbst beeinflußbaren Steuereinnahmen im größeren Umfang zur Verfügung. Bei der Grunderwerbsteuer ist auch indirekt - anders als bei den kreisfreien Städten - keine Beeinflussung der Steuerhöhe über eine Bemessungsgrundlagenpolitik möglich. Für die Kreise spielen die Konzessionsabgaben kaum noch eine Rolle, da sie seit der gesetzlichen Neuregelung nur noch in Ausnahmefällen erhebungberechtigt sind. Die Kreisumlage wiederum weist keine Rückkopplungen zu ihrer Bemessungsgrundlage auf, da nach ihrer Erhöhung die Verhaltensweisen der Gemeinden, die sich möglicherweise auf die Bemessungsgrundlage der Kreisumlage auswirken, unbekannt sind. $\mathrm{Zu}$ dem ist die parteipolitische Verbindung zwischen Kreistag und Stadträten zu beachten, so daß der Umlagesatz nur eingeschränkt variierbar ist. Insoweit besteht aus Anreizgesichtspunkten kein Grund, das Ausgleichssystem der Kreise zu reformieren.

\subsubsection{Problemlösungsvorschlag}

Die Problematik des derzeitigen Ausgleichssystems mit Sockelgarantie liegt bei den Landkreisen ausschließlich in der völligen Einebnung vorgefundener 
Einnahmenunterschiede. Um der Gefahr der verfassungswidrigen Nivellierung zu entgehen, kann deshalb ein anderes Verfahren als bei den kreisfreien Städten und kreisangehörigen Gemeinden genutzt werden. Dabei soll mit Hilfe der Sockelgarantie weiterhin den Landkreisen eine möglichst hohe Mindestausstattung gewährt werden, was auch aus Sicht der kreisangehörigen Gemeinden wegen der Interdependenzen zum Kreisumlagesatz positiv zu bewerten ist, und gleichzeitig die Nivellierung vermieden werden. $\mathrm{Zu}$ diesem $\mathrm{Zweck}$ wird die ermittelte Umlagekraft der Landkreise nicht zu 100 v.H., sondern zu einem geringeren Anteil für die Bestimmung der Schlüsselzuweisungen angerechnet. ${ }^{1}$ Denn die Anrechnung zu unter 100 v.H. verhindert, daß es bei einer Sockelgrenze zu einer Nivellierung der Einnahmen kommt. Es bleibt nämlich bei der Anpassung der Umlagekraft an das Sockelniveau für alle Gebietskörperschaften der gleiche Prozentsatz der Einnahmen, die aber unterschiedliche absolute Größen darstellen, unberücksichtigt. Dieser Umstand führt dazu, daß eine vollständige Nivellierung ausbleibt. ${ }^{2}$ Von der unvollständigen Umlagekrafterfassung profitieren wiederum die reichen Landkreise im größeren Umfang, weshalb der Anrechnungssatz nicht zu weit unter 100 v.H. liegen sollte.

Der geschilderte Sachverhalt ist in Tabelle 36 abgebildet, wobei für alle drei Landkreise ein Finanzbedarf von 100 angenommen wird. Die ermittelte Umlagekraft wird nur zu 95 v.H. bei der Berechnung der Schlüsselzuweisungen berücksichtigt (Spalte 3) und ergibt zusammen mit den Zuweisungen 79 v.H. des Finanzbedarfs (Sockelgarantieerfüllung). Bei der Betrachtung der Einnahmenkraft, Summe aus normativer Umlagekraft und Zuweisungen, ergibt sich aber keine Nivellierung (Spalte 5). Die ursprünglichen Unterschiede werden nur verringert, nicht aber aufgehoben.

Tabelle 36:Keine Nivellierung bei Umlagekraftberücksichtigung unter 100 v.H. bei einem Ausgleichsgrad von 50 v.H. und einer Sockelgarantie von 79 v.H.

\begin{tabular}{|c|c|c|c|c|}
\hline Ort & $\begin{array}{c}\text { ermittelte } \\
\text { Umlagekraft }\end{array}$ & $\begin{array}{c}\text { ermittelte Umla- } \\
\text { gekraft (95 v.H.) }\end{array}$ & $\begin{array}{c}\text { Zuweisung inkl. } \\
\text { Sockelgarantie }\end{array}$ & $\begin{array}{c}\text { Einnahmenkraft (ermittelte } \\
\text { Umlagekraft + Zuweisung) }\end{array}$ \\
\hline $\mathrm{X}$ & 50,00 & 47,50 & 31,50 & 81,50 \\
\hline $\mathrm{Y}$ & 40,00 & 38,00 & 41,00 & 81,00 \\
\hline $\mathrm{Z}$ & 41,00 & 38,95 & 40,05 & 81,05 \\
\hline
\end{tabular}

1 In Niedersachsen erfolgt z.B. eine Anrechnung zu 90 v.H. Vgl.: Gesetz zur Änderung des Niedersächsischen Gesetzes über den Finanzausgleich und anderer Gesetze vom 12.03.1999, Nds. GVBI., S. $75 f$.

2 Vgl.: Maas, B.: Kommunaler Finanzausgleich 1995 und 1996 auf neuer Rechtsgrundlage, in: Statistische Monatshefte Niedersachsen, Heft 6, 1996, hrsg. vom Niedersächsischen Landesamt fur Statistik, S. 341. 
Anhand von Tabelle 36 kann auch gezeigt werden, daß bei der Berücksichtigung der Umlagekraft unter 100 v.H. auch für Landkreise, die unter die Sockelgarantie fallen, ein geringer Anreiz zur Erhöhung ihrer Umlagekraft besteht. Wird nämlich unterstellt, daß es sich bei $Y$ und $Z$ um den identischen Landkreis zu unterschiedlichen Zeitpunkten handelt, nämlich $t_{0}=Y$ und $t_{1}=Z$, so zeigt sich, daß die Schlüsselzuweisungen um den gleichen Betrag sinken, um den die im KFA berücksichtigte Umlagekraft gestiegen ist. Da im Modellfall aber die Umlagekraft und somit auch deren Anstieg nur zu 95 v.H. erfaßt wird, nämlich von 38 auf 38,95 statt von 40 auf 41 , mindert sich die Schlüsselzuweisung nur um 0,95 statt um 1. Somit führt das im Beispiel aufgezeigte Wachstum der Umlagekraft um 2,5 v.H. schließlich trotz Sockelgarantie zu einer Erhöhung der Einnahmenkraft nach Finanzausgleich, wenn auch nur um 0,06 v.H. bzw. um 0,05 auf 81,05. Durch die Wahl des Erfassungsgrades der Umlagekraft ist also die negative Anreizwirkung der Sockelgarantie beeinflußbar.

Wird dieses Verfahren auf die Landkreise in Hessen angewendet, so wäre die Gefahr der verfassungswidrigen Nivellierung gebannt. Die Unterschiede zwischen der bisherigen vollständigen Berücksichtigung der Umlagekraft zur Verteilung der Schlüsselzuweisungen und der Anrechnung zu 95 v.H. sind aus Abbildung 30 ersichtlich.

Abbildung 30:Auswirkung einer unterschiedlichen Umlagekraftanrechnung bei der Verteilung der Schlüsselzuweisungen mit einem Ausgleichsgrad von 50 v.H. und einer Sockelgarantie von 79 v.H.

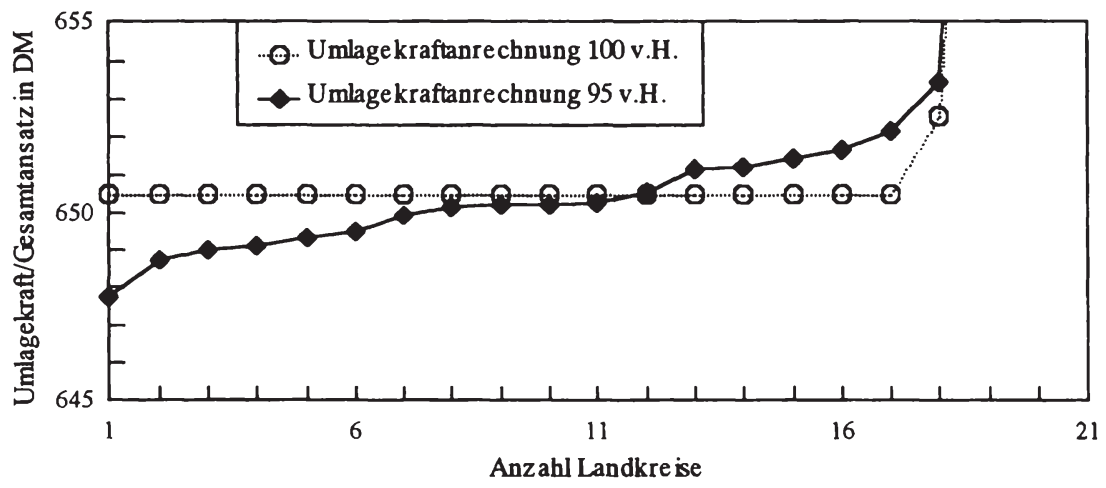

Quelle: Eigene Berechnung nach Daten des Finanzministeriums zum KFA 1998.

Da sich Grundbetrag und somit auch Finanzbedarf verändert haben, sind die GröBen auf den unveränderlichen Gesamtansatz bezogen. Auch hier wird die Umlagekraft der finanzschwächsten Kreise angehoben, allerdings kann die Nivellierung vermieden werden. Der tatsächliche Verlust des umlageschwächsten Kreises 
- Kreis Fulda - zur Ausgangssituation liegt bei 2,77 DM bezogen auf eine Einheit des Gesamtansatzes.

\subsubsection{Behandlung abundanter Kommunen}

\subsubsection{Höhe der Mindestzuweisungen}

Hessen gewährt auch den abundanten Kommunen eine Mindestschlüsselzuweisung, die als Zuschuß zu den Kosten der Ausführung von Pflichtaufgaben zur Erfüllung nach Weisung (Weisungsaufgaben) anzusehen ist. ${ }^{1}$ Dabei wird aber nur eine teilweise Abgeltung angestrebt, was mit einem kommunalen Eigeninteresse an der Aufgabenerfüllung (kommunale "Interessenquote") ${ }^{2} \mathrm{zu}$ begründen ist. Die für die drei kommunalen Gruppen vorgesehenen Mindestbeträge haben sich dabei im Zeitverlauf unterschiedlich entwickelt. ${ }^{3}$ Es ist festzustellen, daß in den letzten knapp 20 Jahren die Mindestzuweisungen für die Landkreise niemals und für die kreisfreien Städte dreimal erhöht wurden, zuletzt im Zusammenhang mit der Finanzierung der Deutschen Einheit, als sie gleichzeitig für die kreisangehörigen Gemeinden sogar verringert wurden.

Auch bei der Bestimmung der Höhe der Zuweisungen für die Weisungsaufgaben wird dem Land ein Gestaltungsspielraum zugebilligt. ${ }^{4}$ Bei der finanziellen Entgeltung der Wahrnehmung staatlicher Aufgaben sollten sich die Beträge zwischen dem kreisfreien und dem kreisangehörigen Raum nicht stark unterscheiden, da jeweils die gleichen Aufgaben übertragen wurden. Aufgrund der organisatorischen Zweiteilung des kreisangehörigen Raumes in Landkreise und deren Gemeinden spricht einiges dafür, da $\beta$ dort die Mindestbeträge in der Summe über denen der kreisfreien Städte liegen sollten. Bis Mitte der 80er Jahre ist auch eine solche Differenzierung feststellbar gewesen. Seitdem haben sich ausschließlich die Zuweisungen an die kreisfreien Städte erhöht. Derzeitig stehen dem kreisangehörigen Raum Mindestzuweisungen von maximal 59 DM pro Einwohner ${ }^{5}$ zur Verfügung, gegenüber $94 \mathrm{DM}$ bei den kreisfreien Städten.

\footnotetext{
Hessischer Landtag: Vorlage der Landesregierung furr ein Gesetz zur Regelung des Finanzausgleichs vom 02.05.1950, Drucksachen Abteilung I, 1/1482, S. 2023; ders.: Vorlage der Landesregierung für ein Zweites Gesetz zur Änderung des Finanzausgleichsgesetzes vom 10.01.1958, Drucksachen Abteilung I, 3/974, S. 2647f.

$2 \mathrm{Zu}$ diesen Weisungsaufgaben gehört z.B. das Meldewesen (Einwohnermeldekartei). Von den Daten, die daraus zu gewinnen sind, profitiert auch die Kommune.

3 Siehe Abschnitt 4.4.8.2.

4 Vgl.: Schneider, G; Dreßler, U.; Lüll, J.: Kommentar zu $\S 4$ in Verbindung mit $\S 3$ HGO, in: Schneider, G; Dreßler, U.; Lüll, J. (Hrsg.): Hessische Gemeindeordnung, Kommentar (Loseblattsammlung), Mainz, Stand 1999, § 4 Erl. 4 und § 3 Erl. 2.

5 In Hessen sind die Mindestzuweisungen der Gemeinden nach der Größe und der zentralörtlichen Aufgabenerfüllung differenziert. Der maximale Wert ergibt sich bei einem kreisangehörigen Oberzentrum (35 DM) und der Landkreiszuweisung (24 DM).
} 
Das Argument für die Erhöhung der Mindestzuweisungen bei den kreisfreien Städten, welche für die Differenz zwischen kreisfreiem und kreisangehörigem Raum verantwortlich ist, nämlich so eine finanzielle Entlastung der Stadt Frankfurt für erlittene Nachteile durch andere Maßnahmen - Verringerung der Einkommensteuereinnahmen - zu erreichen, ist zu kritisieren. ${ }^{1}$ Denn die Aufgabe der Mindestzuweisungen liegt darin, einen teilweisen Ausgleich für die Erfulllung staatlicher Aufgaben zu leisten. Hat sich deren finanzielle Belastung nicht verändert, so besteht auch kein Grund zu einer Veränderung. Erhält die Stadt Frankfurt trotz verminderter Einkommensteuereinnahmen keine zusätzlichen Schlüsselzuweisungen, so ist dies als eine Folge der immer noch vergleichsweise hohen Finanzkraft innerhalb der Gruppe der kreisfreien Städte anzusehen, die bei der Verteilung der Schlüsselzuweisungen zu berücksichtigen ist und weder zusätzliche Zuweisungen noch eine Erhöhung der Mindestbeträge erforderlich macht. Soll trotzdem eine finanzielle Kompensation durchgeführt werden, so ist dies explizit anzuführen und durch separate Regelungen anzustreben, um die Transparenz des Systems nicht weiter zu mindern. Anders wäre die Situation, wenn die Kosten der durch die Kommunen erfüllten Landesaufgaben so stark gestiegen sind, daß eine höhere Abgeltung vorzunehmen ist. Doch dann wären aufgrund der Aufgabenidentität auch die Mindestbeträge im kreisangehörigen Raum anzupassen.

Aus den angeführten Überlegungen folgt, daß die Höhe der Zuweisungen anzugleichen ist. Dies kann entweder durch Erhöhung der Mindestzuweisungen im kreisangehörigen Raum oder durch Minderung bei den kreisfreien Städten erfolgen. Für die zuerst genannte Maßnahme spricht, daß der Mindestbetrag im kreisangehörigen Raum trotz weiterer Aufgabenübertragungen in der Vergangenheit Kindergartenplatzgarantie - nicht erhöht wurde. Gegen eine Erhöhung der Mindestzuweisungen können die vorhandenen Interdependenzen zwischen deren Niveau und dem Ausgleichsziel des KFA vorgebracht werden. Denn diese Mittel fließen den abundanten Kommunen zu, und da sie der Schlüsselmasse entnommen werden, führt ihre Erhöhung automatisch zu geringeren Zuweisungen bei den nicht abundanten Gemeinden, wodurch die Angleichung der Finanzkraftunterschiede beeinträchtigt wird. ${ }^{2}$ Es wird eine Angleichung derart unterstellt, daß der

1 Von den kreisfreien Städten ist ausschließlich Frankfurt zeitweilig abundant gewesen, so daß die Regelung über die Mindestzuweisungen auch nur für sie Geltung besitzt. Vgl.: Hessischer Landtag: Gesetzentwurf der Landesregierung für ein Gesetz zur Änderung des Finanzausgleichsgesetzes - Finanzausgleichsänderungsgesetz 1985 vom 29.10.1984, Drucksache 11/2228, S. 7f.; ders.: Gesetzentwurf der Landesregierung für ein Gesetz zur Änderung des Finanzausgleichsgesetzes und anderer Rechtsvorschriften - Finanzausgleichsänderungsgesetz 1988 - vom 25.11.1987, Drucksache 12/1178, S. 18; ders.: Gesetzentwurf der Landesregierung für ein Gesetz zur Änderung des Finanzausgleichsgesetzes und anderer Rechtsvorschriften - Finanzausgleichsänderungsgesetz 1992 - vom 01.11.1991, Drucksache 13/841, S. 7, 12.

2 Vgl.: Niedersächsischer Staatsgerichtshof: Beschluß vom 15.08.1995, in: DVBl., 110. Jg., 1995, S. $1176 f$. 
- den kreisfreien Städten vor der letztmaligen Erhöhung zustehende - Betrag von $87 \mathrm{DM}$ je Einwohner nun sowohl für den kreisfreien als auch für den kreisangehörigen Raum gilt. ${ }^{1}$ Um einen genaueren Aufschluß über die notwendige Höhe dieser Zuweisungen zu erhalten, sollte in Hessen ähnliche wie in Niedersachsen vorgegangen werden. Dort wurde nach einer entsprechenden Untersuchung die Zuweisungshöhe für übertragene Aufgaben unter Berücksichtigung einer kommunalen Interessenquote für den kreisfreien auf 99,93 und den kreisangehörigen Raum auf 107,77 DM je Einwohner fixiert. ${ }^{2}$

Weiterhin ist bei der Differenzierung der Mindestzuweisungen bei den kreisangehörigen Gemeinden darauf $\mathrm{zu}$ achten, daß nur die Wahrnehmung von übertragenen Aufgaben gesondert entgolten wird. Diese Aufgaben, wie etwa das Meldewesen oder die Bauaufsicht, nimmt jede Kommune nur für die eigenen Einwohner wahr. Somit korrelieren die Ausgaben im hohe Maße mit der tatsächlichen Einwohnerzahl, so daß diese die richtige Maßgröße sein dürfte. Daraus folgt, daß eine zusätzliche Differenzierung dieser Zahlungen nach der zentralörtlichen Aufgabenerfüllung unsachgemäß ist. Denn zentrale Orte erfüllen zwar Leistungen für andere Kommunen, doch die hier abzugeltenden übertragenen Aufgaben korrelieren in keiner Weise mit der Zentralitätsstufe einer Gemeinde. Folglich ist auf diese zusätzliche Unterscheidung zu verzichten.

\subsubsection{Finanzausgleichsumlage}

\subsection{Zur Notwendigkeit einer Abschöpfungskomponente}

Ein grundsätzliches Problem des derzeitigen Systems ist, daß die Diskrepanzen zwischen der originären Steuerkraft nur durch Schlüsselzuweisungen ausgeglichen werden. Die Finanzkraft der abundanten Gemeinden bleibt hingegen unverändert. Dadurch hängt die Intensität des Finanzausgleichs - also der Abbau der Steuerkraftdifferenzen - vor allem vom Volumen der Schlüsselmasse $a b^{3}$, das aber aufgrund der exogenen Bestimmung gerade nicht erhöht werden kann, so daß der Angleichungsgrad der Finanzkraft beschränkt ist. Dies führt wiederum zu Regelungen, welche die simultane Zielerreichung im KFA erschweren. Denn das Distributionsziel kann nur durch einen hohen Ausgleichsgrad oder eine Sockelgarantie erreicht werden, wodurch aber die Eigeninitiative der Kommunen gehemmt wird. Deshalb sollte ein Ausgleichssystem neben einer Zuweisungs- auch noch

1 Denn die Erhöhung auf 94 DM wurde nicht mit einer Kostensteigerung bei den übertragenen Aufgaben begründet, sondern als Entlastung für die Belastung der Stadt Frankfurt durch die Gewerbesteuerumlage (siehe Abschnitt 4.4.8.2.).

2 Vgl:: Niedersächsisches Gesetz zur Regelung der Finanzverteilung zwischen Land und Kommunen (Niedersächsisches Finanzverteilungsgesetz - NFVG -) vom 12.03.1999, Nds. GVBl., S. 79.

3 Vgl.: Hardt, U.; Schmidt, J.: Neuordnung des kommunalen Finanzausgleichs in Niedersachsen. Eine Untersuchung im Auftrag des Niedersächsischen Innenministeriums, hrsg. vom Niedersächsischen Innenministerium, Hannover 1998, S. 12. 
eine Abschöpfungskomponente enthalten, mit deren Hilfe die Unterschiede in der Finanzkraft gemindert werden. ${ }^{1}$ Eine derartige Umlage wird in den Ländern Baden-Württemberg, Niedersachsen, Rheinland-Pfalz und Schleswig-Holstein schon praktiziert. Verfassungsrechtliche Bedenken gegen eine solche Umlage gibt es nicht, denn "Umlagen nach Art. 106 Abs. 6 Satz 6 GG dürfen für Zwecke des interkommunalen Finanzausgleichs erhoben werden"2. Auf Grundlage dieses Artikels basiert derzeit z.B. die Kreisumlageerhebung. ${ }^{3}$

Ein Umlagesystem wird aus mehreren Gründen positiv beurteilt, da ein bestehender Zielkonflikt - hier zwischen Allokation und Distribution - möglicherweise durch die Verwendung zusätzlicher Instrumente reduziert werden kann. Durch die in das Schlüsselzuweisungssystem integrierte Finanzausgleichsumlage wird einmal die interkommunale Steuerkraftdifferenz gemindert. Da zudem diese Mittel der Schlüsselmasse zufließen, verbessert sich gleichzeitig die Intensität des Finanzausgleichs. $\mathrm{Zu}$ berücksichtigen ist aber, da $\beta$ eine aufgabenadäquate Finanzausstattung auch nach Umlageerhebung bestehen bleiben muß, weshalb das Volumen der Umlage aufgrund ihres rein ergänzenden Charakters gering zu halten ist. ${ }^{4}$ Weiterhin ist darauf zu achten, daß die Eigeninitiative der umlagepflichtigen Gemeinden nicht gehemmt und keine (Über-)Nivellierung der Finanzkraft durch die Umlage induziert wird. Ebenso sind die Auswirkungen auf die Kreisumlage und somit auf die Finanzsituation der Kreise einzubeziehen. ${ }^{5}$

\subsection{Ausgestaltungsformen der Abschöpfungskomponente}

Die denkbaren Umlagetypen können nach der Umlageerhebung und der -verwendung kategorisiert werden. $\mathrm{Zu}$ beachten ist, daß das Gesamtergebnis erst bei Betrachtung der Erhebungs- und Verwendungsform bewertbar ist.

- Erhebung: Die Umlage kann von allen oder nur von den abundanten Gemeinden erhoben werden,

- Verwendung: Die erhöhte Schlüsselmasse kann nur den vormals nicht abundanten Gemeinden oder allen Gemeinden zugute kommen.

Für die Frage der möglichen Übernivellierung der Finanzkraft spielt der Kreis der umlagepflichtigen Gemeinden nur für den bisher nicht realisierten Fall eine Rolle,

1 Vgl.: Albers, H.: "Steuereinnahmen? Nein danke!"? Anmerkungen zur Nivellierungswirkung von Finanzausgleichssystemen, dargestellt am Beispiel des kommunalen Finanzausgleichs in Niedersachsen, in: ZKF, 44. Jg., 1994, S. 55.

2 Bundesverwaltungsgericht: Urteil vom 25.03.1998, in: DVBl., 113 Jg., 1998, S. 776.

3 Vgl: Maunz, T.: Kommentar zu Art. 106 GG, in: Maunz, T. u.a. (Hrsg.) : Kommentar zum Grundgesetz (Loseblattsammlung), Stand 1998, Rdnr. 93.

4 Vgl.: Bundesverwaltungsgericht: Urteil vom 25.03.1998, in: DVBl., 113 Jg., 1998, S. 779.

5 Derzeit gibt es nur unter den kreisangehörigen Gemeinden abundante Kommunen. Werden die angesprochenen Reformen bei der Steuerkraft- und der Bedarfsermittlung realisiert, kann die Abundanz auch bei den kreisfreien Städten und Landkreisen auftreten. 
daß ausschließlich die abundanten Gemeinden und diese dann auch mit ihrer gesamten und nicht nur mit der oberhalb der Bedarfsmeßzahl liegenden Steuerkraft umlagepflichtig sind. Denn dann könnten nach Umlageerhebung ehemals abundante Kommunen eine geringere Steuerkraft pro Bedarfseinheit aufweisen als ehemals nicht abundante Kommunen. Ansonsten wird den abundanten Gemeinden nur die oberhalb der Bedarfsmeßzahl liegende Steuerkraft teilweise abgeschöpft, wodurch selbst bei einem Umlagesatz von 100 v.H. keine Übernivellierung stattfindet. ${ }^{1}$ Allerdings sollte der Umlagesatz nicht $\mathrm{zu}$ hoch sein, um sich nicht negativ auf die kommunalen Aktivitäten zur Pflege der eigenen Steuerquellen auszuwirken. ${ }^{2}$ Eine solche Regelung wird in Niedersachsen und SchleswigHolstein praktiziert, wobei 20 v.H. der über der Bedarfsmeßzahl liegenden Steuerkraft abgeschöpft werden.

Werden hingegen alle Kommunen zur Umlage herangezogen, so kann bei einem geringeren Umlagesatz das gleiche Aufkommen erreicht werden, so daß die negativen Anreizwirkungen einer Umlage weniger spürbar bei der einzelnen Gebietskörperschaft werden, allerdings vermindert sich auch die Steuerkraftangleichung. Eine solche Umlage wird in Baden-Württemberg verwendet, wobei statt eines einheitlichen ein in Abhängigkeit von der Steuerkraft steigender Umlagesatz zum Einsatz kommt. Für Hessen würde bei einer Beschränkung nur auf die abundanten Gemeinden - es handelt sich dabei derzeit nur um kreisangehörige Gemeinden mit einem Umlagesatz von 20 v.H. ein Umlagevolumen von 59,14 Mio. DM erreicht. Dieser Satz würde etwa bei 1,247 v.H. liegen, wenn alle Gemeinden entsprechend ihrer Steuerkraft umlagepflichtig wären. Allerdings ist zu beachten, daß bei einer allgemeinen Umlage das Verhältnis zwischen Steuerkraft und Finanzbedarf nicht berücksichtigt wird, so daß Orte, die trotz einer hohen Steuerkraft aufgrund der Bedarfsmeßzahl als finanzschwach gelten, hohe Umlagen abzuführen haben. Dies ist als problematisch anzusehen.

Zur Verwendung der Einnahmen aus der Umlage gibt es nur theoretisch Alternativen. Denn für den Fall, daß die Auszahlung der Umlage den vormals abundanten Kommunen vorenthalten werden sollte, ist die Gefahr der Übernivellierung offensichtlich. Da die Umlage nur für die nicht abundanten Gemeinden ausgezahlt wird, erhöht sich bei diesen aufgrund des gestiegenen Grundbetrags der Finanzbedarf. Dies führt zu erhöhten Zuweisungen, so daß eine Übernivellierung zwischen vormals schwach abundanten Gemeinden und solchen, die sich knapp unterhalb der Abundanzschwelle befanden denkbar ist. ${ }^{3}$ Sind hingegen alle

1 Ein Umlagesatz von 100 v.H. führt zur Nivellierung aller abundanten Gemeinden auf ein Steuerkraftniveau statt, was abzulehnen ist; eine Übernivellierung ist aber nicht möglich.

2 Vgl.: Hardt, U.; Schmidt, J.: Neuordnung des kommunalen Finanzausgleichs in Niedersachsen. Eine Untersuchung im Auftrag des Niedersächsischen Innenministeriums, hrsg. vom Niedersächsischen Innenministerium, Hannover 1998, S. 88.

3 Dies liegt daran, daß bei der Verteilung der Schlüsselzuweisungen auf die nicht abundanten 
Kommunen potentielle Empfänger der Finanzausgleichsumlage, so ist eine Übernivellierung nicht möglich. Denn die nun auftretende Verteilung ist mit der ursprünglichen Zuweisung der Finanzmittel ohne Umlage vergleichbar, nur daß sich die Steuerkraftunterschiede zwischen den Kommunen gemindert haben und sich die Finanzausgleichsmasse erhöht hat. Auch dort war eine Übernivellierung aufgrund des unter 100 v.H. liegenden Ausgleichsgrads unmöglich. Für hoch abundante Gemeinden tritt keine Veränderung ein, da trotz der Erhöhung des Bedarfs dieser noch immer geringer ist als die geminderte Steuerkraft.

Da somit die Entscheidung für die Wahl der Umlageart nur von der Erhebungsseite abhängt, keine der beiden Möglichkeiten die Gefahr einer Übernivellierung birgt, ist die Umlage zu wählen, bei der sowohl Steuerkraft als auch Finanzbedarf berücksichtigt werden. Folglich werden nur die abundanten Gemeinden und auch nur mit der oberhalb der Bedarfsmeßzahl liegenden Steuerkraft umlagepflichtig. Bei dieser Umlage ist zudem der Ausgleichsumfang, also die Minderung der Steuerkraftunterschiede, größer, da nur die finanzstärksten Kommunen umlagepflichtig sind. Der Ausgleich tritt aufgrund des eingeschränkten Umlagevolumens stärker bei der Abschöpfung der sehr hohen Steuerkraft auf und weniger bei der Finanzkrafterhöhung der steuerschwachen Gemeinden. Zuletzt kann noch der Verwaltungsaufwand angeführt werden, der geringer ist, wenn nur die geringe Anzahl der abundanten Kommunen erfaßt werden muß. In Hessen sind 199846 von 421 Gemeinden abundant. Ansonsten müßten alle 421 Gemeinden in einem ersten Schritt die Umlage abführen, damit im zweiten Schritt 375 Kommunen zusätzliche Schlüsselzuweisungen erhalten können. ${ }^{1}$

Abbildung 31 zeigt die Finanzkraftmindenung der umlagepflichtigen Gemeinden und die damit verbundene Verringerung der Finanzkraftunterschiede. ${ }^{2}$ So mindert sich die Finanzkraft bezogen auf den jeweiligen Gesamtansatz bei der reichsten Gemeinde um 542,38 DM von 3.722,15 auf 3.179,77 DM, bei der nachfolgenden Kommune von 2.520,97 um 302,59 auf 2.218,37 DM. Die finanzschwächste abundante Gemeinde behält trotz der abzuführenden Umlage in Höhe von 1,17 DM mit 1.014,51 DM noch eine größere Finanzkraft als die reichste nicht abundante Gemeinde mit 1.010,97 DM, so daß tatsächlich keine Nivellierung auftritt.

Gemeinden ein höherer Grundbetrag verwendet wird, so daß auch bei einem Ausgleichsgrad unter 100 v.H. die Steuerkraft der abundanten Gemeinden übertroffen werden kann. Denn zu deren Bedarfsermittlung wurde der geringere Grundbetrag verwendet.

1 Die hier vorgeschlagene Veränderung des Ausgleichsmechanismus (ohne Sockelgarantie, Ausgleichsgrad von 75 v.H.) erhöht die Zahl der abundanten Gemeinden von 22 auf 46.

2 Der Umlagesatz beträgt 20 v.H. Aus Gründen der Übersichtlichkeit werden nur die Wirkungen einer Ausgleichsumlage bei dem hier propagierten System ohne Sockelgarantie und einem Ausgleichsgrad von 75 v.H. dargestellt. Umlagepflichtig sind die Gemeinden mit ihrer über der Bedarfsmeßzahl liegenden Steuerkraft. 
Abbildung 31:Wirkung einer Ausgleichsumlage beim Ausgleichsgrad von 75 v.H. auf die Finanzkraft der umlagepflichtigen abundanten Kommunen

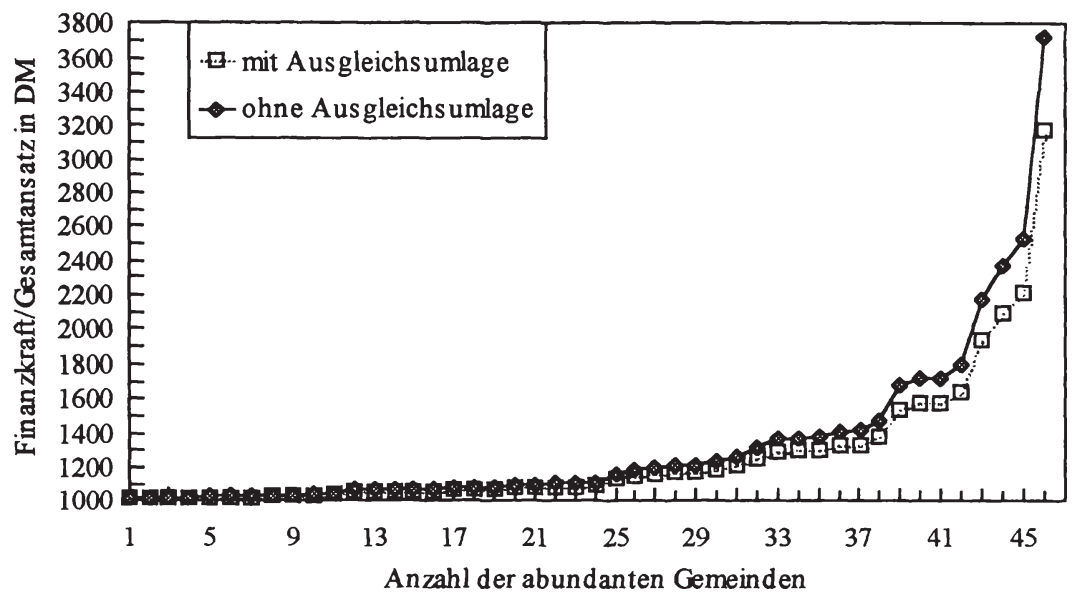

Quelle: Eigene Berechnung nach Daten des Finanzministeriums zum KFA 1998.

In Abbildung 32 ist die Auswirkung der Umlage auf die empfangenden Gemeinden dargestellt. Dabei ist zu erkennen, daß sich bei allen nicht abundanten Gemeinden durch die Umlageerhebung die Finanzkraft erhöht, dies sind $375 \mathrm{Kom}$ munen; für die übrigen Gemeinden vermindert sich hingegen die Finanzkraft aufgrund der zu leistenden Umlage. Die finanzschwächste Kommune erreicht durch die Umlage nun eine Finanzkraft je Einheit Gesamtansatz von 852,72 DM statt von 840,92 DM ohne Umlage. Insgesamt erhöht sich die Finanzkraft von $297 \mathrm{Ge}-$ meinden oder 70,55 v.H. durch die Kombination von erhöhtem Ausgleichsgrad ( 75 v.H.) und Abundanzumlage (20 v.H.) im Vergleich zur Ausgangssituation mit Sockelgarantie und einem Ausgleichsgrad von 50 v.H. Von den ehemals 207 Sockelgarantiegemeinden profitieren nun 170 oder 82,13 v.H. von der neuen Regelung. Für die übrigen 37 ehemaligen Sockelgarantiegemeinden tritt nur eine temporäre Verschlechterung auf. Denn einmal profitieren sie durch die Umlage von der Steuerkrafterhöhung der anderen Kommunen und zum zweiten ergibt sich für sie nun ein Anreiz zur Pflege der eigenen Steuerquellen. Die finanzielle Situation der 87 reichsten Gemeinden verschlechtert sich hingegen. Dies ist bei einem Teil von ihnen (41 Kommunen) auf den im Vergleich zur Ausgangssituation geminderten Grundbetrag zurückzuführen, so daß sie aufgrund des gesunkenen Bedarfs auch geringere Schlüsselzuweisungen erhalten, bei den 46 abundanten Kommunen liegt die Begründung in der zu leistenden Abundanzumlage. 
Abbildung 32:Wirkungen einer Ausgleichsumlage bei einem Ausgleichsgrad von 75 v.H. auf die Finanzkraft der umlageempfangenden Kommunen

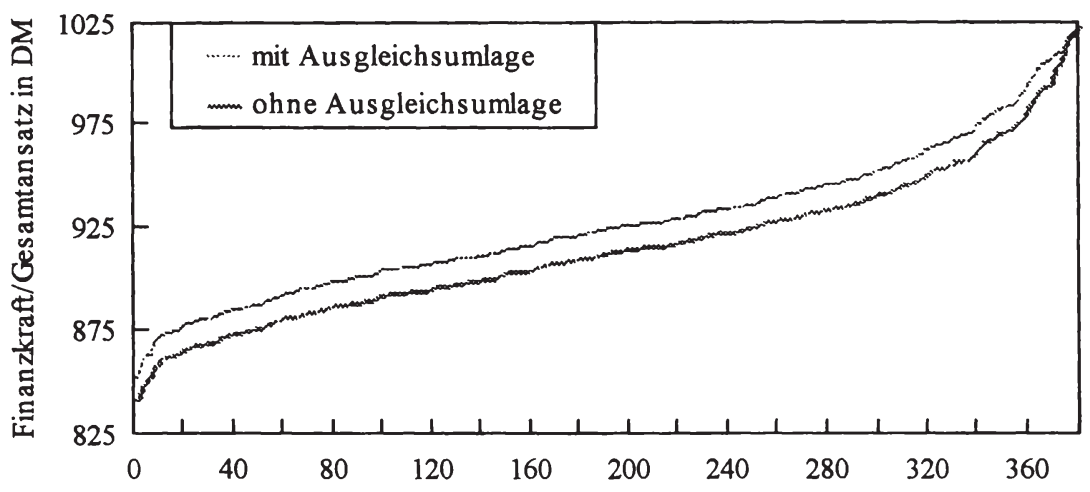

Anzahl Gemeinden

Quelle: Eigene Berechnung nach Daten des Finanzministeriums zum KFA 1998.

Die Verminderung der Finanzkraftdifferenzen bei den kreisangehörigen Gemeinden durch den reformierten kommunalen Finanzausgleich und eine zusätzliche Ausgleichsumlage sind in Tabelle 37 abgebildet. Es kann dabei differenziert werden zwischen der Verteilung der Schlüsselmasse nach der Einwohnerzahl, nach dem FAG sowie nach dem hier propagierten Reformansatz mit einem Ausgleichsgrad von 75 v.H. bei Verzicht auf eine Sockelgarantie und Einführung einer Umlage, wobei noch nach dem Kreis der Umlagepflichtigen - alle oder ausschließlich abundante Kommunen - unterschieden wird.

In der Ausgangssituation ohne Berücksichtigung der Relation von Finanzkraft und Finanzbedarf wird die Schlüsselmasse nur nach Einwohnern verteilt. Dabei zeigt sich eine Differenz von 3.370 DM je Bedarfseinheit zwischen maximalem und minimalem Wert, wobei der Mittelwert der absoluten Abweichungen bei $175 \mathrm{DM}$ je Bedarfseinheit liegt. Diese Unterschiede in der finanziellen Ausstattung verringern sich, wenn die Verteilung der Zuweisungen nach dem FAG bzw. dem Reformvorschlag erfolgt. Sie erreichen im Beispiel ihr Minimum, wenn bei einem Ausgleichsgrad von 75 v.H. und Verzicht auf eine Sockelgarantie von den abundanten Gemeinden eine Umlage auf die überschießende Steuerkraft erhoben wird, die dann wiederum unter Berücksichtigung des Verhältnisses von Steuerkraft und Finanzbedarf zu verteilen ist. Die Differenz zwischen maximalem und minimalem Betrag je Bedarfseinheit sinkt auf 2.327 DM je Bedarfseinheit bei einer absoluten Abweichung vom Mittelwert von nur noch 72 DM je Bedarfseinheit. Als weiteres Indiz für eine gleichmäßigere Finanzkraftverteilung durch die Einfuihrung einer Finanzausgleichsumlage kann das Konzentrationsma $\beta$ nach Gini herangezogen 
werden, wobei die zu ermittelnden Werte zwischen null und eins liegen können. ${ }^{1}$ Es zeigt sich, daß die Finanzkraftdifferenzen zwischen den Kommunen gemindert werden. Der Gini-Koeffizient sinkt von 0,135 bei einer ausschließlichen Verteilung der vorhandenen Mittel nach der Einwohnerzahl auf 0,0522 bei Realisation eines Ausgleichsgrads von 75 v.H. und einer Abundanzumlage.

Tabelle 37:Wirkung verschiedener Ausgleichsumlagen auf die Finanzkraftdifferenzen; in Finanzkraft pro Bedarfseinheit

\begin{tabular}{|c|c|c|c|c|}
\hline \multirow{2}{*}{} & \multicolumn{2}{|c|}{ Verteilung der Schlüsselzuweisungen nach: } \\
\cline { 2 - 5 } & Einwohner & FAG & \multicolumn{2}{c|}{ Reform + Umlage } \\
\cline { 3 - 5 } & & & $\begin{array}{c}\text { allgemeine } \\
\text { Umlage }\end{array}$ & $\begin{array}{c}\text { Abundanz- } \\
\text { umlage }\end{array}$ \\
\hline Mittelwert-Abweichung & $175 \mathrm{DM}$ & $95 \mathrm{DM}$ & $82 \mathrm{DM}$ & $72 \mathrm{DM}$ \\
\hline Minimum & $530 \mathrm{DM}$ & $885 \mathrm{DM}$ & $845 \mathrm{DM}$ & $853 \mathrm{DM}$ \\
\hline Maximum & $3.900 \mathrm{DM}$ & $3.722 \mathrm{DM}$ & $3.683 \mathrm{DM}$ & $3.180 \mathrm{DM}$ \\
\hline Konzentrationsmaß nach Gini & 0,1350 & 0,0624 & 0,0584 & 0,0522 \\
\hline
\end{tabular}

Quelle: Eigene Berechnung nach Daten des Finanzministeriums zum KFA 1998.

\subsection{Kompatibilität zwischen Abschöpfungskomponente und den übrigen Umlagen}

Zum Schluß ist noch die Kompatibilität der Abschöpfungskomponente zu den übrigen zu leistenden Umlagen zu betrachten. In diesem Zusammenhang stellt sich die Frage, ob die Steuerkraftminderung der abundanten Gemeinden bei anderen Umlagen zu berücksichtigen ist. Ist dies der Fall, mindert sich die Umlagebemessungsgrundlage der Kreise, so daß möglicherweise der Kreisumlagesatz zur Kompensation zu erhöhen ist. Geschieht dies hingegen nicht, so besteht die theoretische Gefahr, über die Kreisumlage zu einer Übernivellierung zu kommen. ${ }^{2}$ In der bisherigen Praxis bleibt die Minderung der Steuerkraft bei den übrigen Umlagen unberücksichtigt. Dies kann damit erklärt werden, daß durch diese Lösung nur der vergleichsweise kleine Kreis der abundanten Gemeinden betroffen ist, während sich bei der anderen Verfahrensweise eine größere Zahl von Gemeinden durch erhöhte Kreisumlagen belastet sehen würde. Rein systematisch sollte die gezahlte Finanzausgleichsumlage mindernd berücksichtigt werden, da ansonsten für eine nicht vorhandene Steuerkraft eine Umlage zu entrichten ist. Wenn aus (verteilungs-)politischen Gründen diesem Tatbestand keine Rechnung getragen

1 Da der Wert 0 der Gleichverteilung der Finanzkraft entspricht, vermindert sich mit sinkenden Werten die Ungleichverteilung.

2 Das gleiche Problem gilt auch für die kreisfreien Städte und die von ihnen zu entrichtende Landesumlagen, deren Umfang mit insgesamt 16,65 v.H. aber niedriger als die Belastung der kreisangehörigen Gemeinden ist, so daß auch die Gefahr einer Nivellierung geringer ist. 
werden soll, ist zumindest darauf zu achten, daß sich die Belastung der diversen Umlagen nicht so kumuliert, daß es zu einer Übernivellierung kommt.

Sollte entgegen den systematischen Erwägungen die Steuerkraftminderung der abundanten Gemeinden nicht bei den übrigen Umlagen berücksichtigt werden, kann trotzdem festgestellt werden, daß eine Übernivellierung bei einem Finanzausgleichsumlagesatz von 20 v.H. derzeitig nicht gegeben ist. Denn von der über der Bedarfsmeßzahl liegenden Steuerkraft bleiben der Gemeinde 80 v.H. erhalten. Da die maximale Abschöpfung der originären Steuerkraft durch Kreis- und Schulumlage bei 53,5 v.H. ${ }^{1}$ liegt, verbleiben den Gemeinden letztlich 26,5 v.H. ihrer über der Bedarfsmeßzahl liegenden Steuerkraft. ${ }^{2}$ Zudem haben nicht nur abundante Gemeinden die Kreisumlage zu entrichten. Deshalb kommt es bei einem Satz von 20 v.H. für die Abundanzumlage erst bei einem Kreisumlagesatz über 80 v.H. zur Übernivellierung. Ein solcher Satz würde aber die Finanzautonomie der Gemeinden einschränken und somit als verfassungswidrig gelten. Wird die Bemessungsgrundlage der Kreisumlage um die abzuführende Abundanzumlage gemindert, so ist eine Übernivellierung unmöglich. ${ }^{3}$ In diesem Fall verbleiben der Kommune 37,2 v.H. der über der Bedarfsmeßzahl liegenden Steuerkraft

Wenn den abundanten Gemeinden unter Berücksichtigung aller Umlagen nur 26,5 v.H. der über der Bedarfsmeßzahl liegenden Steuerkraft verbleiben, stellt sich die Frage, ob eine Abschöpfung von bis zu 73,5 v.H. eines Teils der Steuerkraft noch genügend Anreize zur Pflege der eigenen Steuerquellen bietet. Dies dürfte aber der Fall sein, denn bezogen auf die originäre Steuerkraft ist die zu zahlende Abundanzumlage sehr gering. Sie liegt zwischen 0,12 v.H. bei Königstein (Hochtaunuskreis) und 14,61 v.H. bei Stadtallendorf (Kreis Marburg-Biedenkopf) und beträgt im Durchschnitt 4,86 v.H. Durch die noch nicht berücksichtigten Mindestschlüsselzuweisungen vermindert sich noch der Anteil der Abundanzumlage an den allgemeinen Deckungsmittel.

In Tabelle 38 werden die Auswirkungen der Interdependenzen der verschiedenen Umlagen auf die Steuerkraft dargelegt. Der Ort A hat eine Steuerkraft von 120

1 Für 1999 beträgt der Kreisumlagesatz im Hochtaunuskreis 43,5 v.H. und die Schulumlage für Bad Homburg 10 v.H.

2 Liegt die Steuerkraft zweier Gemeinden bei 100 und 120 v.H. der Bedarfsmeßzahl, so werden bis zu deren Höhe jeweils 53,5 v.H. der Steuerkraft abgeschöpft, von den 20 v.H. über der Bedarfsmeßzahl 73,5 v.H. [20 + 53,5 v.H.]; $20-(20 * 0,2+20 * 0,535)=5,3 ; 5,3 / 20=$ 26,5 v.H.]. Erst eine Ausgleichsumlage von über 46,5 v.H. führt zur Übernivellierung. Allgemein tritt diese nur ein, wenn die Summe der jeweiligen Umlagesätze über $100 \mathrm{v} . \mathrm{H}$. liegt.

3 Wird beim gewählten Beispiel der zwei Gemeinden mit einer Steuerkraft von 100 bzw. 120 v.H. der Bedarfsmeßzahl die Abundanzumlage mindernd auf die Kreis- und Schulumlage angerechnet, so ist eine Nivellierung nur dann möglich, wenn der Satz der Abundanzumlage bei 100 v.H. liegen würde. Auch hier betragen Kreis- und Schulumlage zusammen 53,5 v.H. 
v.H. der Bedarfsmeßzahl. Kreis- und Schulumlage betragen 53,5 v.H. der Steuerkraft, die Abundanzumlage 20 v.H. der über der Bedarfsmeßzahl liegenden Steuerkraft. Es ist zu erkennen, daß sich die Steuerkraft mit und ohne Abundanzumlage nur um maximal 3,33 Prozentpunkte voneinander unterscheidet. Wird die Abundanzumlage noch mindernd auf die Kreisumlagebemessungsgrundlage angerechnet, ist die Differenz mit 1,55 Prozentpunkten noch geringer.

Tabelle 38:Auswirkungen verschiedener Umlagen auf die Steuerkraft nach Umlagezahlung in v.H. der originären Steuerkraft

\begin{tabular}{|l|c|}
\hline nur Kreisumlage & 46,50 \\
\hline $\begin{array}{l}\text { Kreis + Abundanzumlage; Minderung der Kreisumlagebemessungsgrund- } \\
\text { lage }\end{array}$ & 44,95 \\
\hline $\begin{array}{l}\text { Kreis+Abundanzumlage; ohne Minderung der Kreisumlagebemessungs- } \\
\text { grundlage }\end{array}$ & 43,17 \\
\hline
\end{tabular}

Die Kreise wären von einer Anrechnung der gezahlten Finanzausgleichsumlage in unterschiedlicher Weise betroffen, da sich die nun 46 abundanten Gemeinden nicht auf alle Landkreise verteilen. ${ }^{1}$ Für Kreise mit einer Vielzahl von abundanten Gemeinden - im Main-Taunus-Kreis befinden sich acht abundante Gemeinden, dies sind fast 66,7 v.H. seiner Gemeinden - ergäben sich geringere Einnahmen aus der Kreisumlage. Da aber die Minderung der Kreisumlagebemessungsgrundlage in diesem Kreis nur 0,79 Mio. DM oder 0,25 v.H. ausmacht, verringert sich das Aufkommen aus der Kreisumlage um ca. 0,39 Mio. DM. Diese Mindereinnahmen werden aber durch höhere Kreisschlüsselzuweisungen wieder teilweise ausgeglichen, denn die Umlagekraft der übrigen Kreise hat sich relativ erhöht. Da die Schlüsselzuweisungen zu keiner vollständigen Kompensation führen, muß möglicherweise zum Ausgleich der Mindereinnahmen der Kreisumlagesatz erhöht werden. Dieser müßte im Main-Taunus-Kreis von 49,5 um 0,12 auf 49,62 v.H. erhöht werden. Doch ist noch zu berücksichtigen, daß sich die Finanzkraft aller nicht abundanten Gemeinden um das Umlagevolumen erhöht hat und somit auch die Bemessungsgrundlage der Kreisumlage, wodurch sich wiederum ein den Kreisumlagesatz senkender Effekt ergibt.

Wichtig ist weiterhin, daß zukünftig im FAG bestimmt wird, daß die Umverteilung durch die Umlage nur innerhalb der jeweiligen Gruppe erfolgt. Denn die von den abundanten kreisangehörigen Gemeinden gezahlte Umlage soll nur den finanzschwachen kreisangehörigen Gemeinden zugute kommen. Nur so wird der Vorteil einer Umlage, Minderung der Steuerkraftdifferenz bei gleichzeitiger Erhöhung der Ausgleichsintensität über eine gestiegene Schlüsselmasse, vollständig erreicht.

1 Beim Ausgleichsgrad von 50 v.H. und Sockelgarantie waren nur 22 Kommunen abundant. 


\section{Zusammenfassung der Untersuchungsergebnisse}

In der vorliegenden Untersuchung wurde das System des kommunalen Finanzausgleichs in Hessen dargestellt und analysiert. Dies geschah vor dem Hintergrund, daß die Landeszuweisungen an die kommunale Ebene und hier vor allem die Schlüsselzuweisungen von herausragender Bedeutung für die Finanzausstattung der Selbstverwaltungsgebietskörperschaften sind. Daß das Verhältnis und die Aufteilung der finanziellen Ressourcen zwischen Land und Kommunen und den Kommunen untereinander nicht immer problemlos ist, zeigen die diversen Verfassungsklagen der Gemeinden und GV anderer Bundesländer gegen Regelungen des kommunalen Finanzausgleichs.

Der größte Streitpunkt zwischen staatlicher und kommunaler Ebene entzündet sich an der Frage, ob die vom Land zur Verfügung gestellte Zuweisungssumme angemessen ist, also an der vertikalen Mittelverteilung. Da weder aus der Verfassung noch aus der Wissenschaft eindeutige Kriterien zur Verteilung der vorhandenen Einnahmen abgeleitet werden können, kann ein Vergleich mit den Regelungen anderer Bundesländer als Indikator verwendet werden, wobei der unterschiedliche Kommunalisierungsgrad der Aufgaben zu beachten ist. Insgesamt konnte festgestellt werden, daß die vom Land Hessen der kommunalen Ebene zur Verfügung gestellte Finanzmasse jedenfalls nicht hinter dem zurückbleibt, was im Durchschnitt in den übrigen westdeutschen Flächenländern bereitgehalten wird. Bei einer solchen länderübergreifenden Betrachtung ist aber nur die Untergrenze der Verbundmasse zu ermitteln, es kann daraus nicht abgeleitet werden, daß eine Erhöhung der Verbundmasse ungerechtfertigt wäre. Durch die quotale Beteiligung der kommunalen Ebene an den Steuereinnahmen des Landes ist gesichert, daß beide Seiten am Wirtschaftswachstum und den damit verbundenen Steuermehreinnahmen beteiligt sind. Zwar ist dies in Hessen bisher im allgemeinen auch der Fall gewesen ist, doch griff das Land zeitweise disketionär ein und verringerte die Verbundquote mit der Begründung, daß sich auch die Kommunen an den Konsolidierungsbemühungen des Landes zu beteiligen haben, obwohl dies bereits automatisch über die verringerte Bemessungsgrundlage des Steuerverbundes der Fall ist. Um zu vermeiden, daß sich das Land zukünttig auf Kosten der kommunalen Ebene konsolidiert, könnte der in Sachsen realisierte "Milbradtsche Gleichmäßigkeitsgrundsatz" angewendet werden. Nach diesem Verfahren werden für die staatliche und die kommunale Ebene des Landes Anteile am gesamten beiden Gebietskörperschaftsebenen verbleibenden Steuer- und Zuweisungseinnahmen bestimmt. Die Verbundquote des KFA wird nur noch als Steuergröße verwendet, um die Einnahmen den Anteilen entsprechend zu verteilen. Bei unveränderten Aufgaben können somit Streitigkeiten bezüglich der Finanzausgleichsmasse beigelegt werden. 
Weiterhin ist zu prüfen, ob die Finanzausgleichsmasse zur Beeinflussung des konjunkturpolitischen Verhaltens der Kommunen genutzt werden kann, denn bisher ist das Ausgabeverhalten der Gemeinden und GV stark prozyklisch, da es durch die konjunkturreagiblen Einnahmen aus Steuern und Zuweisungen determiniert wird, wobei die Zuweisungen aus dem KFA noch stärker schwanken als die kommunalen Steuereinnahmen. Zu diesem Zweck bietet es sich an, zwischen zugewiesenen Anteilen am Gesamtaufkommen und tatsächlich ausgezahlten Beträgen zu unterscheiden und den Differenzbetrag in einen Fonds einzuzahlen bzw. diesem zu entnehmen. Dadurch könnte eine Glättung der kommunalen Einnahmen im Konjunkturverlauf und aufgrund der Abhängigkeit von den Einnahmen auch des Ausgabeverhaltens erreicht werden. Damit würde dann verhindert werden, daß die kommunale Ausgabenpolitik die staatlichen Konjunkturmaßnahmen konterkariert.

Neben dem Volumen ist noch die Aufteilung der Mittel auf die verschiedenen $\mathrm{Zu}-$ weisungsarten - freie und zweckgebundene - kritisch zu beleuchten. Dabei zeigt sich, daß in fast allen betrachteten Ländern der Anteil der zweckgebundenen zugunsten der freien Mittel vermindert wurde. Bei dieser als kommunalfreundlich zu bezeichnenden Gewichtsverlagerung nimmt Hessen allerdings keine Spitzenposition ein. Dies liegt an der relativ starken Dotierung der Sonderlastenausgleiche, mit denen die Kosten der Wahrnehmung ausgewählter Aufgaben steuerkraftunabhängig alimentiert werden. Es wurde aufgezeigt, daß die in den 80er Jahren unternommenen Maßnahmen, diese zweckgebundenen Mittel den allgemeinen Zuweisungen zuzuschlagen, unproblematisch weitergeführt werden könnten. Die Bedarfssteigerung durch die Aufgabenwahrnehmung würde über Nebenansätze wie den für Schulträger berücksichtigt bleiben. Deshalb ist zu überlegen, ob diese Mittel nicht der Schlüsselmasse zugeordnet werden sollen, was - wie dargestellt relativ einfach zu vollziehen ist.

Das Problem der horizontalen oder interkommunalen Einnahmenverteilung, welches sich nur bei den Schlüsselzuweisungen ergibt, wird in den meisten Ländern, in denen trotz unterschiedlicher Ausgabenstruktur kreisangehörige Gemeinden und kreisfreie Städte aus einer Schlüsselmasse dotiert werden, implizit über die starke Spreizung der Hauptansatzstaffel (Veredelungsfaktoren bis 180 v.H.) geregelt. Dadurch soll der aus der Wahrnehmung von Kreisaufgaben resultierende höhere Bedarf der kreisfreien Städte berücksichtigt werden. In Hessen wird diese Verteilung explizit vorgenommen, da alle drei kommunalen Gruppen Zuweisungen aus separaten Schlüsselmassen bekommen. Um Aussagen über die Verteilung zwischen dem kreisfreien und dem kreisangehörigen Raum treffen zu können, wurden die Landkreise und ihre Gemeinden zu Gesamtkreisen zusammengefaßt und den kreisfreien Städten gegenübergestellt. Dabei zeigte sich, daß die Städte aufgrund ihrer originären Einnahmenkraft zumindest nicht als unterfinanziert 
anzusehen sind und Forderungen nach einer Erhöhung ihres Anteils zu hinterfragen sind. Um auch hier den Verteilungskonflikt zu mindern, sollte ebenfalls ein Gleichmäßigkeitsgrundsatz eingeführt werden, zumal dies auf kommunaler Ebene leichter sein dürfte als zwischen dem Land und der Gesamtheit der Kommunen. Denn zwischen den kommunalen Gruppen dürfte in der Regel eine Konstanz im Verhältnis der Aufgabenwahrnehmung in der Art unterstellt werden, daß im kreisfreien wie im kreisangehörigen Raum die gleichen Aufgaben zu erfüllen sind. Anders die Situation zwischen Kommunen und Land, da letzteres Aufgaben überträgt. Die Ausgangsverteilung der Zuweisungen innerhalb des kreisangehörigen Raums spielt hingegen nur eine untergeordnete Rolle, da die Kreisumlage als regulierendes Instrument eingesetzt werden kann, so daß unabhängig von der Dotierung dieser beiden Schlüsselmassen eine gewünschte Verteilung zwischen beiden Gruppen erreichbar ist.

Bei der Bedarfsermittlung wird in vielen Ländern auf eine Hauptansatzstaffelung zurückgegriffen, die einen mit steigender Einwohnerzahl zunehmenden Finanzbedarf unterstellt, wobei dieses Instrument durch einen Ansatz für zentrale Orte und weitere Nebenansätze ergänzt wird. Für die kreisangehörigen Gemeinden konnte durch eine empirische Analyse des Zuschußbedarfs festgestellt werden, daß der Hauptansatz an sich als Indikator zur Bedarfsermittlung geeignet ist, daß die derzeitige Spreizung aber überarbeitet werden sollte. Auch an anderer Stelle hat sich Reformbedarf gezeigt. Denn entgegen der propagierten Zielrichtung, spielt der Ansatz für zentrale Orte bei den kreisangehörigen Gemeinden kaum eine Rolle. Den Bedarf determinieren somit neben der Einwohnerveredelung noch Nebenansätze, die - abgesehen vom derzeitigen Schulträgeransatz und vielleicht einem zukünftigen Demographieansatz - kaum einen Bedarf wiedergeben bzw. dem zentralörtlichen Ziel entgegenstehen. Der Ansatz für zentrale Orte sollte erhöht und die Anzahl der Gemeindegrößenklassen bei der Hauptansatzstaffel könnte gemindert werden, weiterhin ist die Anzahl der Nebenansätze zu mindern. Letzteres trägt auch dazu bei, die Transparenz und Ausführungsbilligkeit des Systems zu erhöhen.

Bei der Gruppe der kreisfreien Städte ist aufgrund der geringen Zahl und der damit verbundenen statistischen Abhängigkeiten keine Ermittlung der Hauptansatzstaffel mit Hilfe des kommunalen Zuschußbedarfs möglich. Deshalb wurden zu Vergleichszwecken die Regelungen anderer Bundesländer herangezogen. Dabei zeigte sich, daß die Spreizung der Hauptansatzwerte zwischen der kleinsten und der größten kreisfreien Stadt in Hessen zwar vergleichsweise gering ausfällt, aber eine offensichtlicher Reformbedarf nicht erkennbar ist. Für die kreisfreien Städte wird die zentralörtliche Aufgabenerfüllung über das Volumen der Schlüsselmasse abgegolten, da jede Stadt die Raumordnungsfunktion eines Oberzentrums ausübt, folglich ist ein solcher Ansatz hier nicht notwendig. 
Für die Bedarfsermittlung der Landkreise kann die bisherige Vorgehensweise beibehalten werden; allerdings sollte auf den bisher verwendeten Nebenansatz verzichtet werden. Auch die Regelungen für Sonderstatusstädte sollten grundlegend reformiert werden. Ziel muß es sein, daß sich die kreisinterne Aufgabenverteilung nicht finanziell auf die übrigen Kreise auswirkt, also keine Externalitäten auftreten. Gleichzeitig sollte die Transparenz des Systems erhöht werden, die derzeit sehr darunter leidet, daß der besondere Status der Gemeinden mit mehr als 50.000 Einwohnern auf verschiedene Arten berücksichtigt wird. Aus diesem Grund ist der Nebenansatz für Sonderstatusstädte bei der Bemessung der Gemeindeschlüsselzuweisungen abzuschaffen und gleichzeitig die Finanzkraft dieser Gemeindegruppe bei der Verteilung der Kreisschlüsselzuweisungen im vollen Umfang einzubeziehen. Die besondere Aufgabenteilung zwischen den Sonderstatusstädten und ihren Kreisen sollte nur im Rahmen der Kreisumlage berücksichtigt werden. Die Schulumlage, die zur Abgeltung der den Kreisen entstehenden Kosten der Schulträgerschaft von den kreisangehörigen Gemeinden zu entrichten ist, ist zu modifizieren. Für einen erhöhten Umlagesatz bei den Sonderstatusstädten gibt es keine Begründung, weshalb er auf das Niveau des für die übrigen kreisangehörigen Gemeinden geltenden Satzes zu senken ist. Für die vom Landkreis stellvertretend für den Gesamtkreis abzuführenden Landesumlagen sollte wie bei der Finanzierung der Schulträgerschaft durch die Kreise eine separate Umlage erhoben werden, ebenfalls mit einem einheitlichen Umlagesatz für alle Gemeinden. Dieses Verfahren verdeutlicht dann allen Beteiligten, welche Summe für die Kreisaufgaben und welche für die landesweiten Aufgaben über die Umlagen zu finanzieren sind.

Zur Steuerkrafterfassung sollten zusätzlich zu den bisher in Hessen berücksichtigten Realsteuern und dem Anteil an der Einkommensteuer noch die Konzessionsabgaben, die Spielbank- und Troncabgabe sowie der kommunale Grunderwerbsteueranteil herangezogen werden, da nur dann alle relevanten Einnahmenkategorien berücksichtigt werden. Weiter ist im Sinne der inneren Geschlossenheit darauf zu achten, daß diese Einnahmen im gleichen Umfang erfaßt werden. Damit im Zeitablauf bei allen Einnahmen der einmal erreichte identische Erfassungsgrad bestehen bleibt, ist bei denjenigen mit Autonomie der notwendige Normierungssatz zu dynamisieren. In die Umlagekraftermittlung der Kreise sind ebenfalls die Konzessionsabgaben und die Grunderwerbsteuer einzubeziehen. Ferner ist zu beachten, daß sich die Aufgabenaufteilung innerhalb eines Kreises mit Sonderstatusstädten nicht über die Kreisgrenzen hinweg finanziell auswirkt, was derzeit noch geschieht.

Um die beim derzeitigen Ausgleichsmechanismus auftretende Einebnung von originären Einnahmeunterschieden zu vermeiden sowie den unter die Sockelgarantie fallenden Kommunen einen Anreiz zur Steuerkrafterhöhung zu geben, sollte der 
Ausgleichssatz für die kreisangehörigen Gemeinden auf 75 v.H. erhöht und auf eine Sockelgarantie verzichtet werden; bei den Kreisen sollte die ermittelte Umlagekraft bei unverändertem Ausgleichsmechanismus nur zu 95 v.H. Berücksichtigung finden. Durch diese Maßnahme wird eine Nivellierung verhindert. Für die kreisfreien Städte erscheint keine Veränderung notwendig zu sein, da die Gefahr der Einebnung der Finanzkraft derzeit nicht besteht und, wie gezeigt werden konnte, schon jetzt ein genügend großer Anreiz zur Steuerkrafterhöhung besteht.

Die gewährte Mindestzuweisung für abundante Kommunen ist positiv zu bewerten, da mit Eintreten der Abundanz - z.B. nach einer geringen Steuerkrafterhöhung - nicht wie in anderen Ländern die Schlüsselzuweisungen in voller Höhe entfallen. Die Differenzen der Pro-Kopf-Beträge zwischen abundanten Kommunen im kreisfreiem und kreisangehörigen Raum sind abzubauen, da sie aufgrund der vergleichbaren Aufgabenerfüllung in der derzeitigen Form nicht zu begründen sind. Bei dieser Maßnahme sind die Mindestbeträge, die kreisangehörigen abundanten Gemeinden wegen ihrer zentralörtlichen Aufgabenerfüllung gewährt werden, abzuschaffen, da sie zukünftig nur nach der Einwohnerzahl bemessen sein sollen. Denn diese Zuweisungen sollen ausschließlich als Ausgleich für die von der staatlichen Ebene übertragenen Aufgaben dienen, die aber nur mit der tatsächlichen Einwohnerzahl und nicht mit der Zentralörtlichkeit korrelieren.

$\mathrm{Da}$ im derzeitigen Zuweisungssystem die Finanzkraftunterschiede nur durch die Differenzierung der Zuweisungen möglich ist und somit die Ausgleichsfunktion vor allem vom Volumen der Schlüsselmasse abhängt, sollte noch eine Abschöpfungskomponente installiert werden, mit der einerseits die Steuerkraftunterschiede verringert und andererseits die Schlüsselmasse erhöht werden könnte. Die Umlage sollte zu diesem Zweck auf die über der Bedarfsmeßzahl liegende Steuerkraft erhoben werden - somit wären ausschließlich abundante Kommunen umlagepflichtig - und der Schlüsselmasse zufließen. Durch diese Ausgestaltung wird die Gefahr einer durch die Umlage induzierten Übernivellierung der kommunalen Finanzkraft ausgeschlossen. Die gezahlte Abundanzumlage sollte aus systematischen Gründen mindernd auf die Bemessungsgnundlage der übrigen Umlagen angerechnet werden, denn den Kommunen stehen diese Mittel nicht mehr zur Verfügung. 



\section{Literaturverzeichnis}

\section{Aufsätze und Monographien:}

Achatz, M.: Finanzausgleichstheoretische Überlegungen zum Finanzbedarf, in: Smekal, C.; Theurl, E. (Hrsg.): Finanzkraft und Finanzbedarf von Gebietskörperschaften, Wien u.a. O. 1990, S. 176-182

Albers, H.: "Steuereinnahmen? Nein danke!"? Anmerkungen zur Nivellierungswirkung von Finanzausgleichssystemen, dargestellt am Beispiel des kommunalen Finanzausgleichs in Niedersachsen, in: ZKF, 44. Jg., 1994, S. 50-57

Ders.: Die Ausgleichsregelungen des kommunalen Finanzausgleichs in Niedersachsen im Spannungsfeld von verfassungsrechtlichen Vorgaben und politischen Zielen, in: 3. Jg., 1996, S. 169-180

Albers, W.: Möglichkeiten und Grenzen eines interkommunalen Finanzausgleichs, in: Friedrich-Ebert-Stiftung (Hrsg.): Kommunale Finanzreform, Schriftenreihe der Forschungsstelle der Friedrich-Ebert-Stiftung, Hannover 1962, S. 63-83

Andel, N.: Finanzwissenschaft, 4. Aufl., Tübingen 1998

Bayer, H.-W.; Elemenhorst, R.: Das System des gemeindlichen Fremdenverkehrsrechts, in: Kommunale Steuer-Zeitschrift, 44. Jg., 1995, S. 141-173

Bienert, F.: Zur Bestimmung der ökonomischen Auswirkungen des Fremdenverkehrs unter Berücksichtigung seines Einflusses auf den Haushalt einer Gemeinde - Eine empirische Fallstudie. Dargestellt am Beispiel der Stadt Westerland auf Sylt, Berlin 1974

Borchmann, M.: Stadt Lahn - Gebietsreform ohne Abschluß?, in: DVBl., 93. Jg., 1978, S. $785-789$

Brecht, A.: Internationaler Vergleich der öffentlichen Ausgaben, Leipzig - Berlin 1932

Broer, M.: Die Berechnung der Steuerkraft im Kommunalen Finanzausgleich Verteilungswirkungen und Reformvorschläge am Beispiel der kreisfreien Städte in Hessen, in: Die Gemeindekasse, 1999, S. 329-340

Brückmann, F.: Die Zentralität - Die vernachlässigte Bedarfsgröße im hessischen kommunalen Finanzausgleich, in: Kommunale Steuer-Zeitschrift, 39. Jg., 1990, S. 161-167 und 187-189

Ders.; Dette, W.: Stadt-Umland-Beziehungen und kommunaler Finanzausgleich nicht nur ein Problem der kreisfreien Städte, in: Kommunale SteuerZeitschrift, 45. Jg., 1996, S.41-48

Bund der Steuerzahler Rheinland-Pfalz: Abfallgebühren 1999, o.O., o.J.

Bundesministerium der Finanzen: Finanzbericht 1998, Bonn 1997 
Deubel, I., Münstermann, E.: Reiche Städte - Arme Landkreise? - Zur aktuellen Verteilungsproblematik im kommunalen Finanzausleich am Beispiel des Landes Nordrhein-Westfalen -, in: ZKF, 38. Jg., 1988, S. 242-251

Ders.: Der kommunale Finanzausgleich in Nordrhein-Westfalen, Köln u.a.O. 1984

Ders.: Mängel im Finanzausgleichssystem des Landes Nordrhein-Westfalen, in: Hoppe, W. (Hrsg.): Reform des kommunalen Finanzausgleichs, Köln u.a.O. 1985 , S. 61-84

Deutsche Bundesbank: Monatsbericht, April 1999

Deutscher Städtetag: Rückkehr zur verbundenen Steuerwirtschaft, Köln 1953

Ders.: Statistisches Jahrbuch Deutscher Gemeinden, 85. Jg., Köln 1998

Eckey, H.-F.: Möglichkeiten der Anpassung und Veränderungen des Kommunalen Finanzausgleichssystems für raumordnerische Zielsetzungen, in: Informationen zur Raumentwicklung: Die Reform der Kommunalfinanzen, Heft $8 / 9$, hrsg. von der Bundesforschungsanstalt für Landeskunde und Raumentwicklung, Bonn 1995, S. 583-596

Ehrlicher, W. u.a.: Kommunaler Finanzausgleich und Raumordnung, Hannover 1967

Ellwein, R; Hesse, J.J.: Staatsreform in Deutschland - das Beispiel Hessen, hrsg. vom Bund der Steuerzahler Hessen e.V., Wiesbaden 1997

Ellwein, T.: Perspektiven der kommunalen Selbstverwaltung in Deutschland, in: AfK, 36. Jg., 1997, S. 1-21

Emmel, W.: Die Entwicklung der Bruttowertschöpfung in den kreisfreien Städten und Landkreisen Hessens 1980 und 1995, in: Hessisches Statistisches Landesamt Wiesbaden: Staat und Wirtschaft in Hessen, 53. Jg., 1998, S. 234-238

Eschenbach, J.: Die Neukonzeption des kommunalen Finanzausgleichs in Niedersachsen - Neuanfang oder fortgesetzter Verfassungsbruch?, in: ZKF, 49. Jg., 1999, S. 53-60, 80-83

Fischer, H.: Finanzzuweisungen. Theoretische Grundlegung und praktische Ausgestaltung im bundesstaatlichen Finanzausgleich Australiens und der Bundesrepublik Deutschland, Berlin 1988

Fischer-Menshausen, H.: Finanzausgleich II - Grundzüge des Finanzausgleichsrechts, in: Albers, W. u.a. (Hrsg.): Handbuch der Wirtschaftswissenschaften, Bd. 2, Stuttgart - New York 1980, S. 636-662

Fuchs, M.: Zweckgebundene Zuweisungen - Hilfe oder Last der Gemeinden?, in: Der Gemeindehaushalt, 70. Jg., 1969, S. 145-148 und 169-172 
Gern, A.: Deutsches Kommunalrecht, 1. Aufl., Baden-Baden 1994

Geske, O.-E.: Gemeinden und Kreise im Finanzsystem der Bundesrepublik Deutschland, in: Püttner, G. (Hrsg.): Handbuch der kommunalen Wissenschaft und Praxis, Bd. 6, 2. Aufl., Berlin u.a.O. 1985, S. 29-49

Gläser, M.: Die staatlichen Finanzzuweisungen an die Gemeinden, Frankfurt Thun 1981

Gräf, H.: Investitionszuweisungen zwischen Schlüssel- und Zweckzuweisungen, in: Räumliche Aspekte des kommunalen Finanzausgleichs (Veröffentlichungen der Akademie für Raumforschung und Landesplanung: Forschungs- und Sitzungsberichte, Bd. 159), Hannover 1985, S. 271-292

Gretz-Roth, V.: Der kommunale Finanzausgleich und finanzielle Zuweisungen des Landes zur Erfüllung öffentlicher Aufgaben, Erläuterungen zu $\S \S 5$ bis 19, 37 bis 40 a und 46a FAG, in: Praxis der Gemeindeverwaltung, Hessen, Bd. E1, (Loseblattsammlung), Stand 1999

Grimme, L.: Neuberechnung der Steuerkraft im kommunalen Finanzausgleich dargestellt am Beispiel des Landes Bayern, in: Räumliche Aspekte des kommunalen Finanzausgleichs (Veröffentlichungen der Akademie für Raumforschung und Landesplanung: Forschungs- und Sitzungsberichte, Bd. 159), Hannover 1985, S. 159-199

Grossekettler, H.: Die Bestimmung der Schlüsselmasse im kommunalen Finanzausgleich. Eine ordnungspolitische Analyse der Entstehung von Finanzausgleichsbedarfen und der Möglichkeiten ihrer Quantifizierung, in: Finanzarchiv N.F., Bd. 45, 1987, S. 393-440

Häßler, G.: Die Kommunalwahlen in Hessen am 20. März 1977 - Teil 1: Gemeindewahlen in den kreisfreien Städten und Kreiswahlen, in: Hessisches Statistisches Landesamt Wiesbaden: Staat und Wirtschaft in Hessen, 32. Jg., 1977, S. $92-96$

Hansmeyer, K.-H.: Interkommunaler Finanzausgleich, in: AfK, 5. Jg., 1966, S. $261-284$

Ders.: Der kommunale Finanzausgleich als Instrument zur Förderung Zentraler Orte, in: Pohmer, D. (Hrsg.): Probleme des Finanzausgleichs II, Schriften des Vereins für Socialpolitik, N.F. Bd. 96/II, Berlin 1980, S. 83-150

Ders.; Kops, M.: Finanzwissenschaftliche Grundsätze für die Ausgestaltung des kommunalen Finanzausgleichs, in: Hoppe, W. (Hrsg.): Reform des kommunalen Finanzausgleichs, Köln u.a.O. 1985, S. 31-60

Hardt, U.: Kommunale Finanzkraft. Die Problematik einer objektiven Bestimmung kommunaler Einnahmemöglichkeiten in der gemeindlichen Haushaltsplanung und im kommunalen Finanzausgleich, Frankfurt u.a.O. 1988 
Dies.; Schmidt, J.: Neuordnung des kommunalen Finanzausgleichs in Niedersachsen. Eine Untersuchung im Auftrag des Niedersächsischen Innenministeriums, hrsg. vom Niedersächsischen Innenministerium, Hannover 1998

Haverkamp, F.: Die Finanzbeziehungen zwischen Ländern und Gemeinden, in: Arnold, V; Geske, O.-E. (Hrsg.): Öffentliche Finanzwirtschaft, München 1988, S. 55-120

Hendler, R.: Das Prinzip der Selbstverwaltung, in: Isensee, J.; Kirchhof, P.: Handbuch des Staatsrechts, Bd. 4, Heidelberg 1990, S.1133-1170

Henneke, H.-G.: Der kommunale Finanzausgleich. Verfassungsrechtliche Vorgaben und landesgesetzliche Ausgestaltung, in: Juristische Ausbildung (Jura), 1987, S. 393-403

Ders.: Die beabsichtigte Erweiterung des Art. 28 Abs. 2 GG - ein Danaergeschenk für Kreise und Gemeinden?, in: Der Landkreis, 63, Jg., 1993, S. 212-218

Ders.: Der kommunale Finanzausgleich in Niedersachsen im Spannungsfeld von verbesserter Ausgleichsgerechtigkeit und verfassungswidriger (Über-)Nivellierung, in: Der Gemeindehaushalt, 94. Jg., 1993, S. 83-89

Ders.: Der kommunale Finanzausgleich. Einer der verfassungsgerichtlich am sorgfältigsten behauenen Ecksteine der Grundlagen kommunaler Selbstverwaltung, in: DÖV, 47. Jg., 1994, S. 1-12

Ders.: Der Finanzbedarf kommunaler Gebietskörperschaften, in: Niedersächsische Verwaltungsblätter, 1. Jg., 1994, S. 49-60

Ders.: Möglichkeiten zur Stärkung der kommunalen Selbstverwaltung, in: DÖV, 47. Jg., 1994, S. 705-715

Ders.: Finanzausgleich: Die Verteilung der Schlüsselzuweisungen als DreiKomponenten-System, in: Die Gemeindekasse, 1995, S. 1-8, 33-37

Ders.: Probleme des kommunalen Finanzausgleichs, in: Ipsen, J. (Hrsg.): Kommunale Aufgabenerfüllung im Zeichen der Finanzkrise, Baden-Baden 1995, S. 81-123

Ders.: Jenseits von Bückeburg. Gesetzgeberische Gestaltungsspielräume für den kommunalen Finanzausgleich, in: Niedersächsische Verwaltungsblätter, 3. Jg., 1996, S. 9-14

Ders.: Die Landkreise im kommunalen Finanzausgleich 1997, in: Der Landkreis, 67. Jg., 1997, S. 123-135

Herbel, A.: Verwaltungs- und Gebietsreform in Hessen, in: Kommunalwirtschaft, 1971, S. 322-325

Hessischer Minister des Innern (Hrsg.): Zur Planung der gebietlichen Neuordnung auf Gemeindeebene in Hessen, o.O. 1969 
Ders.: Vorschläge zur gebietlichen Neugliederung auf der Kreisebene in Hessen, o.O. 1971

Ders.: Verwaltungsreform in Hessen. Bestandsaufnahme, Maßnahmen, Überlegungen, Vorausschau, Wiesbaden 1968

Ders.: Hessen - Gemeinden und Landkreise nach der Gebietsreform, Melsungen 1977

Hessischer Minister für Landesentwicklung, Umwelt, Landwirtschaft und Forsten: Regionaler Raumordnungsplan für die Planregion Nordhessen - Sachlicher Teilplan -, in: Staatsanzeiger für das Land Hessen, 1979, S. 734-776

Ders.: Regionaler Raumordnungsplan für die Planregion Mittelhessen - Sachlicher Teilplan -, in: Staatsanzeiger für das Land Hessen, 1979, S. 819-845

Ders.: Regionaler Raumordnungsplan für die Planregion Osthessen - Sachlicher Teilplan -, in: Staatsanzeiger für das Land Hessen, 1979, S. 397-414

Ders.: Regionaler Raumordnungsplan für die Planregion Rhein-Main-Taunus Sachlicher Teilplan -, in: Staatsanzeiger für das Land Hessen, 1979, S. 667-704

Ders.: Regionaler Raumordnungsplan für die Planregion Starkenburg - Sachlicher Teilplan -, in: Staatsanzeiger für das Land Hessen, 1979, S. 444-466

Ders.: Regionaler Raumordnungsplan für die Planregion Untermain - Sachlicher Teilplan -, in: Staatsanzeiger für das Land Hessen, 1979, S. 1286-1316

Hessischer Minister für Wirtschaft, Verkehr und Landesentwicklung: Vorlage zum Landesentwicklungsplan 2000, 0.O., o.J.

Hessischer Minister der Finanzen (Hrsg.): Kommunaler Finanzausgleich 1956-1988, Wiesbaden 1989

Hessischer Ministerium der Finanzen: Kreisumlagesätze nach kommunalen Haushaltsplänen einschließlich Nachträge - soweit diese vorliegen; unveröffentlichte Statistik, Stand 31.03.1999

Dass.: Abrechnung über den KFA 1998 (Exceldatei)

Hessisches Statistisches Landesamt: Hessische Gemeindestatistik 1996, 17. Aufl. 1997

Hidien, J. W.: Handbuch Länderfinanzausgleich, 1. Aufl., Baden-Baden 1999

Hoppe, W.: Der Anspruch der Kommunen auf aufgabengerechte Finanzausstattung, in: DVB1., 107. Jg., 1992, S. 117-124

Junkernheinrich, M.: Sonderbedarfe im kommunalen Finanzausgleich, Berlin 1992 
Kabinetts-Kommission zur Vorbereitung der Verwaltungsreform: Die Verwaltungsreform in Hessen, Bd. I, Wiesbaden 1947

Käss, W.: Der kommunale Finanzausgleich und finanzielle Zuweisungen des Landes zur Erfüllung öffentlicher Aufgaben, Erläuterungen zu $\S \S 1$ bis 4, 20 bis 36, 41 bis 46, 47 bis 49 FAG, in: Praxis der Gemeindeverwaltung, Hessen, Bd. E1, (Loseblattsammlung), Stand 1999

Karrenberg, H.; Münstermann, E.: Der Gemeindefinanzbericht 1993, in: Der Städtetag, 46. Jg., 1993, S. 60-153

Dies.: Der Gemeindefinanzbericht 1997, in: Der Städtetag, 50. Jg., 1997, S. 129-209

Dies.: Der Gemeindefinanzbericht 1998, in: Der Städtetag, 51. Jg., 1998, S. 143-233

Dies.: Der Gemeindefinanzbericht 1999, in: Der Städtetag, 52. Jg., 1999, S. $151-240$

Katz, A.: Der kommunale Finanzausgleich, in: Püttner, G. (Hrsg.): Handbuch der kommunalen Wissenschaft und Praxis, Bd. 6, 2. Aufl., Berlin u.a.O. 1985, S. $301-330$

Kirchhof, P.: Der Finanzausgleich als Grundlage kommunaler Selbstverwaltung, in: DVB1., 95. Jg., 1980, S. 711-719

Ders.: Rechtliche Rahmenbedingungen des kommunalen Finanzausgleichs, in: Hoppe, W. (Hrsg.): Reform des kommunalen Finanzausgleichs, Köln u.a.O. 1985 , S. 1-29

Ders.: Die kommunale Finanzhoheit, in: Püttner, G. (Hrsg.): Handbuch der kommunalen Wissenschaft und Praxis, Bd. 6, 2. Aufl., Berlin u.a.O. 1985, S. 3-28

Kisker, G.: Normenkontrollantrag gegen $\S 37$ Abs. 4 Satz 2 des Hessischen Finanzausgleichsgesetzes in der Fassung des Finanzausgleichsänderungsgesetzes 1998 (Hess. GVBl. I 1997, S. 442) vom 18.12.1997, Gutachten im Auftrag der F.D.P.-Fraktion des Hessischen Landtags, Linden, o.J.

Kisseler, W.; Gretz, W.: Hauptansatzstaffel im Kommunalen Finanzausgleich. Eine empirische Untersuchung für Hessen, in: HLT Gesellschaft für Forschung, Planung und Entwicklung: HLT-Report Nr. 574, Wiesbaden 1999

Klein, R. R.; Münstermann, E.: Kommunen und Konjunkturpolitik, in: AfK, 17. Jg., 1978, S. 213-233

Knemeyer, F.-L.: Das verfassungsrechtliche Verhältnis der Kommunen zueinander und zum Staat, DVB1., 99. Jg., 1984, S. 23-29.

Kock, H.: Vorschläge zu einer Verstetigung der Gemeindefinanzen, in: Konjunkturpolitik, 21. Jg., 1975, S. 309-336 
Kuhn, T.: Der kommunale Finanzausgleich - Argumente für eine Reform, in: AfK, 36. Jg., 1997, S. 211-232

Leibfritz, W.; Teschner, S.: Der Einfluß des Steuersystems und des kommunalen Finanzausgleichssystems auf die Landesentwicklung, Schriftenreihe des IfoInstituts für Wirtschaftsforschung, Nr. 108, Berlin 1981

Littmann, K. u.a.: Die Gestaltung des kommunalen Finanzsystems unter raumordnungspolitischen Gesichtspunkten, Hannover 1968

Ders.: Ergebnisse und Empfehlungen des Gutachtens des Wissenschaftlichen Beirats beim Bundesministerium der Finanzen zur Reform der Gemeindesteuern - Sollte die Gewerbesteuer durch eine Wertschöpfungsteuer ersetzt werden?, in: Der Gemeindehaushalt, 84. Jg., 1983, S. 178-184

Maas, B.: Kommunaler Finanzausgleich 1995 und 1996 auf neuer Rechtsgrundlage, in: Statistische Monatshefte Niedersachsen, Heft 6, 1996, hrsg. vom Niedersächsischen Landesamt für Statistik, S. 329-344

Mäding, H.: Überlegungen zur Eignung des kommunalen Finanzausgleichs zur Förderung raumordnungspolitischer Konzepte, in: Informationen zur Raumentwicklung: Die Reform der Kommunalfinanzen, Heft 8/9. 1995, hrsg. von der Bundesforschungsanstalt für Landeskunde und Raumentwicklung, Bonn 1995, S. 605-618

Massat, D.: Die ökonomische Problematik von Zuweisungen an Kommunen, Thun - Frankfurt 1984

Mattenklodt, H.-F.: Territoriale Gliederung - Gemeinden und Kreise vor und nach der Gebietsreform, in: Püttner, G. (Hrsg.): Handbuch der kommunalen Wissenschaft und Praxis Bd. 2, 2. Aufl., Berlin u.a.O. 1981, S.154-182

Maunz, T.: Kommentar zu Art. 106 GG, in: Maunz, T. u.a. (Hrsg.) : Kommentar zum Grundgesetz (Loseblattsammlung), Stand 1998

Ders.: Kommentar zu Art. 28 GG, in: Maunz, T. u.a. (Hrsg.) : Kommentar zum Grundgesetz (Loseblattsammlung), Stand 1998.

Ders.; Scholz, R.: Kommentar zu Art. 28 GG, in: Maunz, T. u.a. (Hrsg.): Kommentar zum Grundgesetz (Loseblattsammlung), Stand 1997

Maurer, H.: Verfassungsrechtliche Grundlagen der kommunalen Selbstverwaltung, in: DVB1., 110. Jg., 1995, S. 1037-1046

Michalk, J.: Die Garantieklauseln im Länderfinanzausgleich, in: Wirtschaftsdienst, 69. Jg., 1989, S. 446-453

Micosatt, G.: Raumwirksamkeit einer Reform des kommunalen Finanzausgleichs, Bochum 1990

Münstermann, E.: Die Berücksichtigung zentralörtlicher Funktionen im kommunalen Finanzausgleich, Opladen 1975 
Ders.: Der kommunale Finanzausgleich in den Bundesländern - Teil III -, in: ZKF, 30. Jg., 1980, S. 120-122

Ders.: Der kommunale Finanzausgleich in den Bundesländern - Teil V-, in: ZKF, 30. Jg., 1980, S. 152-157

Ders.: Möglichkeiten und Grenzen einer "Kommunalisierung" von Zweckzuweisungen, in: Räumliche Aspekte des kommunalen Finanzausgleichs (Veröffentlichungen der Akademie für Raumforschung und Landesplanung: Forschungs- und Sitzungsberichte, Bd. 159), Hannover 1985, S.235-270

Ders.; Becker, H.: Finanzausgleichsleistungen an Kommunen. Ein Vergleich der Finanzausgleichssysteme in den Bundesländern (Reihe G; DST-Beiträge zur Finanzpolitik, Heft 7), Köln 1978

Muntzke, H.: Wie soll die Verwaltungsreform auf der kommunalen Ebene in Hessen fortgeführt werden?, in: Der Gemeindetag, 23. Jg., 1970, S. 392-394

Mutius, A. v.; Henneke, H. G.: Grenzen der verfassungsrechtlichen Zulässigkeit bei der Festsetzung von Schlüsselzuweisungen, in: Der Gemeindehaushalt, 85. Jg., 1984, S. 149-155

Dies.: Verfassungsrechtliche Anforderungen an die Durchführung des kommunalen Finanzausgleichs - dargestellt am Beispiel Nordrhein-Westfalens, in: AfK, 24. Jg., 1985, S. 261-284

Neumark, F.: Steuern I: Grundlagen, in: Albers, W. (Hrsg.): Handbuch der Wirtschaftswissenschaft, Bd. 2, Stuttgart-New York 1977, S. 295-309

Ders.: Bemerkungen zu einigen ökonomischen Aspekten der grundgesetzlichen Vorschriften über die Einheitlichkeit der Lebensverhältnisse in der Bundesrepublik Deutschland, in: Dreißig, W. (Hrsg.): Probleme des Finanzausgleichs I, Schriften des Vereins für Socialpolitik, N.F. Bd. 96/I, Berlin 1978, S. $165-175$

Niedersächsischer Landkreistag: Gesonderte Stellungnahme des Niedersächsischen Landkreistages vom 15. Dezember 1998 zur Neuordnung des kommunalen Finanzausgleichs zum 1. Januar 1999, in: NLT-Information, Niedersächsischer Landkreistag, 22. Jg., 1999, S. 45-48

o.V.: Rückkehr zur verbundenen Steuerwirtschaft, in: Der Städtetag, 6. Jg., 1953, S. $185-186$

o.V.: Vereinbarung zur Bildung einer Koalitionsregierung im Land Hessen, in: Der Gemeindetag, 23. Jg., 1970, S. 394-397

o.V.: Bekanntmachung der Neufassung der Körperschaftsteuer-Richtlinien 1985 vom 30.12.1985, in: Körperschaftsteuerrecht, Sonderheft Nr. 1/1986, S. 82-84 
o.V.: Das Land benachteiligt die Kommunen, in: Hannoversche Allgemeine Zeitung vom 26.11.1997, S. 1

o.V.: Frankfurt will Gewerbe- und Grundsteuer senken, in: Frankfurter Allgemeine Zeitung vom 22.04.1999, Nr. 93, S. 59-60

Pagenkopf, H.: Kommunalrecht, Köln u.a.O. 1971

Parsche, R.; Steinherr, M.: Der kommunale Finanzausgleich des Landes Nordrhein-Westfalen, ifo-Studien zur Finanzpolitik, Bd. 59, München 1995

Dies.: Reformvorschläge zum kommunalen Finanzausgleich des Landes Nordrhein-Westfalen, in: ifo-Schnelldienst, 48. Jg., Nr. 28, 1995, S. 8-17

Patzig, W.: Der kommunale Finanzausgleich im Zeichen des Steuerverbundes, in: DVBl., 74. Jg., 1959, S. 1-7

Ders.: Der kommunale Finanzausgleich im Zeichen der Konsolidierung der Länderhaushalte, in: DVBl., 100. Jg., 1985, S. 137-143

Peffekoven, R.: Finanzausgleich I, in: Albers, W., u.a. (Hrsg.): Handbuch der Wirtschaftswissenschaft, Bd. 2, Stuttgart u.a.O. 1980, S.608-636

Ders.: Zur Problematik der Umsatzsteuerverteilung, in: Cansier, D.; Kath, D.: Öffentliche Finanzen, Kredit und Kapital, Berlin 1985, S. 53-79

Petri, W.: Die staatlichen Zweckzuweisungen im kommunalen Finanzsystem. Dargestellt am Beispiel des Landes Niedersachsen, Berlin 1977

Pittermann, W.: Entwicklung in der Organisation der Landesverwaltung - Grundlinien, Schnittstellen, Funktionalreform, in: Stein, E. (Hrsg.): 30 Jahre Hessische Verfassung 1946-1976, Wiesbaden 1976

Popitz, J.: Der künftige Finanzausgleich zwischen Reich, Ländern und Gemeinden, Berlin 1932

Postlep, R-D.: Räumliche Effekte der Steuerkraft- und Finanzbedarfsermittlung bei den Schlüsselzuweisungen, in: Räumliche Aspekte des kommunalen Finanzausgleichs (Veröffentlichungen der Akademie für Raumforschung und Landesplanung: Forschungs- und Sitzungsberichte, Bd. 159), Hannover 1985, S. 201-234

Ders.: Zur Berücksichtigung raumordnerischer Belange im Kommunalen Finanzausgleich, in: Informationen zur Raumentwicklung: Die Reform der Kommunalfinanzen, Heft 8/9. 1995, hrsg. von der Bundesforschungsanstalt für Landeskunde und Raumentwicklung, Bonn 1995, S. 597-603

Pünder, T.: Der Landeswohlfahrtsverband Hessen und seine Probleme, Hessische Städte- und Gemeinde-Zeitung, 35. Jg., 1985, S. 462-465.

Püttner, G.: Kommunale Selbstverwaltung, in: Isensee, J.; Kirchhof, P. (Hrsg.): Handbuch des Staatsrechts, Bd. 4, Heidelberg 1990, S. 1171-1194. 
Ders.: Stufenweise Reform. Stand und Methode der Verwaltungsreform in Hessen, in: AfK, 8. Jg., 1969, S. 266-277

Recker, E.: Räumliche Verteilung des Gemeindeanteils an der Einkommensteuer, in: Räumliche Aspekte des kommunalen Finanzausgleichs (Veröffentlichungen der Akademie für Raumforschung und Landesplanung: Forschungsund Sitzungsberichte, Bd. 159), Hannover 1985, S. 313-352

Sachverständigenkommission für Verwaltungsreform und Verwaltungsvereinfachung in Hessen: Zur Stärkung der Verwaltungskraft der Gemeinden, Veröffentlichungen 2, Wiesbaden 1968

Sachverständigenkommission zur Vorklärung finanzverfassungsrechtlicher Fragen für künftige Neufestlegungen der Umsatzsteueranteile: Verteilung der Umsatzsteuer - Maßstäbe und Verfahren, in: Schriftenreihe des Bundesministeriums der Finanzen, Heft 30, Bonn 1981

Sachverständigenrat zur Begutachtung der gesamtwirtschaftlichen Entwicklung: "Unter Anpassungszwang"; Jahresgutachten 1980/81, Stuttgart 1980

Ders.: "Weiter auf Wachstumskurs" Jahresgutachten 1986/87, Stuttgart 1986

Ders.: "Arbeitsplätze im Wettbewerb"; Jahresgutachten 1988/89, Stuttgart 1988

Ders.: "Wachstum, Beschäftigung, Währungsunion - Orientierungen für die $\mathrm{Zu}$ kunft"; Jahresgutachten 1997/98, Stuttgart 1997

Ders.: "Wachstum, Beschäftigung, Währungsunion - Orientierungen für die $\mathrm{Zu}$ kunft" Jahresgutachten 1998/99, Stuttgart 1998

Schelpmeier, H.: Finanzausgleich für zentrale Orte?, in: Raumforschung und Raumordnung, 56. Jg., 1998, S. 299-306

Scherf, W.: Schlüsselzuweisungen und Kreisumlage, Frankfurt u.a.O. 1998

Schlempp, H.; Schlempp, D.: Kommentar zur Hessischen Gemeindeordnung (HGO) (Loseblattsammlung), Stand 1996

Schmidt-Jortzig, E.: Gemeinde- und Kreisaufgaben. Funktionsordnung des Kommunalbereiches nach "Rastede", in: DÖV, 46. Jg., 1993, S. 973-984

Schneider, G; Dreßler, U.; Lüll, J. (Hrsg.): Hessische Gemeindeordnung; Kommentar (Loseblattsammlung), Mainz, Stand 1999

Schnur, R.: Zur Abgrenzung der gemeindlichen Aufgaben von de Aufgaben der Kreise gemäss Art. 28 Abs. 2 GG, in: Die Verwaltung 1986, Bd. 19, S. $39-64$

Schoch, F.: Aufgaben und Funktionen der Landkreise, in: DVB1., 110. Jg., 1995, S. 1047-1056

Ders.: "...Wer anschaffen will, der soll gefälligst bezahlen...", in: Der Landkreis, 64. Jg., 1994, S. 531-536 
Ders.: Finanzierungsverantwortung für gesetzgeberisch veranlaßte Ausgaben. in: Der Landkreis, 64. Jg., 1994, S. 253-258

Schwarting, G.: Grundsätze für die Kreditaufnahme der Gemeinden, in: Püttner, G. (Hrsg.): Handbuch der kommunalen Wissenschaft und Praxis, Bd. 6, 2. Aufl., Berlin u.a.O. 1985, S. 621-634

Seiler, G.: Ziele und Mittel des kommunalen Finanzausgleichs - Ein Rahmenkonzept für einen aufgabenbezogenen kommunalen Finanzausgleich, in: Pohmer, D. (Hrsg.): Probleme des Finanzausgleichs II, Schriften des Vereins für Socialpolitik, N.F. Bd.96/II, Berlin 1980, S. 11-82

Smekal, C.: Transfers zwischen Gebietskörperschaften (TG) - Ziele und Ausgestaltungsprobleme, in: Pohmer, D. (Hrsg.): Probleme des Finanzausgleichs II, Schriften des Vereins für Socialpolitik, N.F. Bd. 96/II, Berlin 1980, S. $151-220$

Spahn, P. B.: Dezentralisierte Haushaltspolitik und makroökonomische Steuerung, in: Galler, H. P.; Wagner, G. (Hrsg.): Empirische Forschung und wirtschaftspolitische Beratung, Empirische Forschung und wirtschaftspolitische Beratung. Festschrift für Hans-Jürgen Krupp zum 65. Geburtstag, Frankfurt, New York 1998, S. 322-344

Statistisches Bundesamt: Finanzen und Steuern, Reihe 9, Realsteuern, I. Realsteuervergleich 1970, Stuttgart - Mainz 1971

Dass.: Finanzen und Steuern, Reihe 9, Realsteuern, I. Realsteuervergleich 1972, Stuttgart - Mainz 1974

Dass.: Finanzen und Steuern, Reihe 9, Realsteuern, I. Realsteuervergleich 1974, Stuttgart - Mainz 1975

Dass.: Fachserie 14, Finanzen und Steuern: Reihe 3.1, Rechnungsergebnisse des öffentlichen Gesamthaushalts 1996, Stuttgart 1998

Dass.: Fachserie 14, Finanzen und Steuern, Reihe 3.3, Rechnungslegung der kommunalen Haushalte 1996, Stuttgart 1997

Dass.: Fachserie 14, Finanzen und Steuern, Reihe 3.3, Rechnungsergebnisse der kommunalen Haushalte 1997, Stuttgart 1999

Dass.: Fachserie 14, Finanzen und Steuern, Reihe 4, Steuerhaushalt - 4. Vierteljahr und Jahr 1996, Stuttgart 1997

Dass.: Fachserie 14, Finanzen und Steuern, Reihe 4, Steuerhaushalt - 4. Vierteljahr und Jahr 1997, Stuttgart 1998

Dass.: Fachserie 14, Finanzen und Steuern. Reihe 4.S.1, Kassenmäßige Steuereinnahmen 1977 bis 1987, Stuttgart 1989

Dass.: Arbeitsunterlage über die Rechnungsergebnisse der staatlichen Haushalte von 1970-1989 
Dass.: Fachserie 14, Finanzen und Steuern, Reihe 10.1, Realsteuervergleich 1978, Stuttgart - Mainz 1980

Dass.: Fachserie 14, Finanzen und Steuern, Reihe 10.1, Realsteuervergleich 1980, Stuttgart - Mainz 1982

Dass.: Fachserie 14, Finanzen und Steuern, Reihe 10.1, Realsteuervergleich und kommunale Einkommensteuerbeteiligung 1992, Stuttgart 1994

Dass.: Fachserie 14, Finanzen und Steuern, Reihe 10.1, Realsteuervergleich und kommunale Einkommensteuerbeteiligung 1993, Stuttgart 1995

Dass.: Fachserie 14, Finanzen und Steuern, Reihe 10.1, Realsteuervergleich und kommunale Einkommensteuerbeteiligung 1995, Stuttgart 1997

Dass.: Fachserie 14, Finanzen und Steuern, Reihe 10.1, Realsteuervergleich und kommunale Einkommensteuerbeteiligung 1997, Stuttgart 1998

Dass.: Konzessionsabgaben der Gemeinden, Gemeindeverbände und Zweckverbände (unveröffentlichte Arbeitsunterlage; o.J.).

Dass.: Statistisches Jahrbuch der Bundesrepublik Deutschland 1969, Stuttgart Mainz 1969

Dass.: Statistisches Jahrbuch der Bundesrepublik Deutschland 1970, Stuttgart Mainz 1970

Dass.: Statistisches Jahrbuch der Bundesrepublik Deutschland 1980, Stuttgart Mainz 1980

Dass.: Statistisches Jahrbuch der Bundesrepublik Deutschland 1997, Stuttgart 1997

Dass.: Statistisches Jahrbuch der Bundesrepublik Deutschland 1998, Stuttgart 1998

Steinherr, M.; u.a.: Das Schlüsselzuweisungssystem im kommunalen Finanzausgleich des Freistaates Sachsen, ifo-Studien zur Finanzpolitik, Bd. 63, München 1997

Ders.: Kommunaler Finanzausgleich: Balanceakt zwischen Verteilungs- und Allokationszielen - Das Beispiel Rheinland-Pfalz, in: ifo-Schnelldienst, 51. Jg., Nr. 8, 1998, S. 15-24

Ders.; Parsche, R.: Der Ausgleich zwischen Finanzbedarf und Finanzkraft im kommunalen Finanzausgleich des Landes Rheinland-Pfalz, ifo-Studien zur Finanzpolitik, Bd. 66, München 1998

Stern, K.: Gemeinden I: Rechtsstellung in der Bundesrepublik Deutschland, in: Albers, W. u.a. (Hrsg.): Handbuch der Wirtschaftswissenschaft, Bd. 3, Stuttgart - New York 1981, S. 486-495 
Ders.: Das Staatsrecht der Bundesrepublik Deutschland, Bd. 1, 2. Aufl., München 1984

Ders.: Die Verfassungsgarantie der kommunalen Selbstverwaltung, in: Püttner, G. (Hrsg.): Handbuch der kommunalen Wissenschaft und Praxis, Bd. 1, 2. Aufl., Berlin u.a.O. 1981, S. 204-228

Storck, F.: Der Schüleransatz im Finanzausgleich des Landes NW, in: Städte- und Gemeinderat, 38. Jg., 1984, S. 13-17

Storck, H.: Der Krebsschaden der Finanzzuweisungen, in: Der Städtetag, 2. Jg., 1949, S. 217-220

Strauß, W.: Probleme und Möglichkeiten einer Substituierung der Gewerbesteuer, Opladen 1984.

Ders.: Ökonomische Wirkungen einer Substitution der Gewerbesteuern (Realsteuern) durch eine Wertschöpfungsteuer, Frankfurt u.a. 1988

Stüer, B.; Ehebrecht-Stüer, E.-M.: Normenkontrollantrag des Vorsitzenden der CDU-Fraktion im Niedersächsischen Landtag, Christian Wulff, sowie der weiteren Mitglieder der CDU-Fraktion im Niedersächsischen Landtag, Gutachten im Auftrag der CDU-Fraktion des Niedersächsischen Landtags, Münster, o.J.

Tettinger, P. J.: Konjunkturpolitik und Gemeinden, Finanzplanung, in: Püttner, G. (Hrsg.): Handbuch der kommunalen Wissenschaft und Praxis, Bd. 6, 2. Aufl., Berlin u.a.O. 1985, S.441-450

Thieme, W.; Prillwitz, G.: Durchführung und Ergebnisse der kommunalen Gebietsreform, Baden-Baden 1981

Voigt, R.: Das System des kommunalen Finanzausgleichs in der Bundesrepublik Deutschland, Stuttgart u.a.O. 1980

Ders.: Finanzierung kommunaler Aufgaben in Nordrhein-Westfalen, Diskussionsbeiträge der Universität/Gesamthochschule Siegen, HiMoN DB 88/86, Siegen 1986

Voigtländer, H.: Die Finanzzuweisungen an die Gemeinden und Gemeindeverbände in konjunkturpolitischer Sicht, in: AfK, 9. Jg., 1970, S. 303-313

Voit, H.: Die kommunale Gebietsreform in Hessen, in: 30 Jahre Hessische Verfassung, hrsg. von Stein, E., Wiesbaden 1976, S. 366-387

Weber, M.: Die Schlüsselzuweisungen, Frankfurt 1981

Wissenschaftlicher Beirat beim Bundesministerium der Finanzen: Gutachten zum Gemeindesteuersystem und zur Gemeindesteuerreform in der Bundesrepublik Deutschland, in: Schriftenreihe des Bundesministeriums der Finanzen, Heft 10, Bonn 1968 
Ders.: Gutachten zur Reform der Gemeindesteuern, Schriftenreihe des Bundesministerium der Finanzen, Heft 31, Bonn 1982

Ders.: Gutachten zum Länderfinanzausgleich in der Bundesrepublik Deutschland, Schriftenreihe des Bundesministerium der Finanzen, Heft 47, Bonn 1992

Ders.: Einnahmenverteilung zwischen Bund und Ländern, Schriftenreihe des Bundesministerium der Finanzen, Heft 56, Bonn 1995

Ders.: Stellungnahme zum Finanzausgleichsurteil des Bundesverfassungsgerichts vom 11. November 1999, Schriftenreihe des Bundesministerium der Finanzen, Heft 68, Bonn 2000

Wöhe, G.: Die Steuern des Unternehmens, 6. Aufl., München 1991

Wolff, H. J.; Bachof, O.; Stober, R.: Verwaltungsrecht II, 5. Aufl., München 1987

Wolf, G.: Zur Umsatzsteuerverteilung zwischen Bund und Ländern, in: Bundesministerium der Finanzen (Hrsg.): Die Finanzbeziehungen zwischen Bund, Ländern und Gemeinden aus finanzverfassungsrechtlicher und finanzwissenschaftlicher Sicht, Bonn 1982, S. 254-306

Zimmermann, H.; Postlep, R.-D.: Probleme des kommunalen Finanzausgleichs Überblick und Einordnung der Untersuchungsergebnisse, in: Räumliche Aspekte des kommunalen Finanzausgleichs (Veröffentlichungen der Akademie für Raumforschung und Landesplanung: Forschungs- und Sitzungsberichte, Bd. 159), Hannover 1985, S. 1-13

Zimmermann, H.: Allgemeine Probleme des Finanzausgleichs, in: Neumark, F.; Andel, N. und Haller, H. (Hrsg.): Handbuch der Finanzwissenschaft, Bd. 4, 3. Aufl., Tübingen 1983, S. 3-52

Ders.: Stärkung der kommunalen Finanzautonomie, in: Staatswissenschaft und Staatspraxis, 6. Jg., 1995, S. 659-674

Zezschwitz, F. v.: Kommentar zu Art. 137 HV, in: Zinn, G. A.; Stein, E.: Verfassung des Landes Hessen - Kommentar (Loseblattsammlung), Bad Homburg v. d. Höhe 1990

Zwilling, E.: Untersuchungen zu einem rationalen Steuersystem der Gemeinden, Meisenheim am Glan 1971

\section{Gerichtsentscheidungen:}

Bayerischer Verfassungsgerichtshof: Entscheidung vom 12.01.1998, in: DVBl., 113. Jg., 1998, S. 732

Bundesverfassungsgericht: Urteil 07.05.1957, in: Entscheidungen des Bundesverfassungsgerichts, Bd. 6, Tübingen 1957, S. $376-385$ 
Dass.: Urteil vom 21.05.1968, in: Entscheidungen des Bundesverfassungsgerichts, Bd. 23, Tübingen 1968, S. 353 - 373

Dass.: Urteil vom 10.06.1969, in: Entscheidungen des Bundesverfassungsgerichts, Bd. 26, Tübingen 1970, S. 172 - 186

Dass.: Urteil vom 24.06.1969, in: Entscheidungen des Bundesverfassungsgerichts, Bd. 26, Tübingen 1970, S. 228 - 246

Dass.: Urteil vom 11.10.1977, in: Entscheidungen des Bundesverfassungsgerichts, Bd. 46, Tübingen 1978, S. 55 - 66

Dass.: Urteil vom 24.07.1979, in: Entscheidungen des Bundesverfassungsgerichts, Bd. 52, Tübingen 1980, S. 95 - 131

Dass.: Beschluß vom 15.10.1985, in: Entscheidungen des Bundesverfassungsgerichts, Bd. 71, Tübingen 1986, S. 25 - 38

Dass.: Urteil vom 23.11.1988, in: Entscheidungen des Bundesverfassungsgerichts, Bd. 79, Tübingen 1989, S. 127 - 161

Dass.: Urteil vom 27.05.1992, in: Entscheidungen des Bundesverfassungsgerichts, Bd. 86, Tübingen 1993, S. 148 - 279

Bundesverwaltungsgericht: Urteil vom 09.07.1964, in: Entscheidungen des Bundesverwaltungsgerichts, Bd. 19, Berlin 1965, S. 121 - 125

Dass.: Urteil vom 25.03.1998, in: DVBl., 113 Jg., 1998, S. 776-780

Hessischer Verwaltungsgerichtshof: Urteil vom 23.09.1970, in: Entscheidungen des Hessischen Verwaltungsgerichtshofs und des Verwaltungsgerichtshofs Baden-Württemberg mit Entscheidungen der Staatsgerichtshöfe beider Länder - ESVGH -, Bd. 21, Karlsruhe 1971, S. 74 - 80

Niedersächsischer Staatsgerichtshof: Urteil vom 27.11.1997, in: DVBl., 113. Jg., 1998, S. 185-189

Ders.: Beschluß vom 15.08.1995, in: DVBl., 110. Jg., 1995, S. 1175-1179

Verfassungsgerichtshof für das Land Nordrhein-Westfalen: Urteil vom 19.07.1985, in: Entscheidungen der Oberverwaltungsgerichte für das Land Nordrhein-Westfalen in Münster und für das Land Niedersachsen in Lüneburg mit Entscheidungen des Verfassungsgerichtshofes Nordrhein-Westfalen und des Niedersächsischen Staatsgerichtshofes (OVGE), Bd. 38, Münster 1989 , S. 312 - 320

Ders.: Urteil vom 06.07.1993, in: OVGE, Bd. 43, Münster 1996, S. 252 -266

Verfassungsgerichtshof Rheinland-Pfalz: Entscheidung vom 30.01.1998, in: DÖV, 51. Jg., 1998, S. 505-510 


\section{Gesetze und Verordnungen des Landes Hessen:}

Begründung zu dem Gesetz zur Regelung des Finanzausgleichs vom 17.07.1951, in: Staatsanzeiger für das Land Hessen, 1951, S. 521

Gesetz über die Mittelstufe der Verwaltung und den Landeswohlfahrtsverband Hessen vom 07.05.1953, Hess. GVB1. S. 93

Hessisches Ausführungsgesetz zum Bundesozialhilfegesetz (HA/BSHG) vom 28.05.1962 in der Fassung vom 16.09.1970, Hess. GVB1. I, S. 573

Gesetz über kommunale Abgaben (KAG) vom 17.03.1970, Hess. GVBl. I, S. 225

Sechstes Gesetz zur Änderung des Finanzausgleichsgesetzes vom 15.06.1971, Hess. GVBl., S. 154

Achtes Gesetz zur Änderung des Finanzausgleichsgesetzes vom 18.12.1972, Hess. GVB1. I, S. 432

Verordnung über die Aufstellung und Ausführung des Haushaltsplans der Gemeinden (Gemeindehaushaltsverordnung - GemHVO) vom 13.07.1973, Hess. GVB1. I, S. 275 und ÄndVO vom 08.07.1996, Hess. GVBI. I, S. 334

Gesetz zur Neugliederung der Landkreise Biedenkopf und Marburg und der Stadt Marburg (Lahn) vom 12.03.1974, Hess. GVBl. I, S. 154

Gesetz über den Umlandverband Frankfurt vom 11.09.1974, Hess. GVBI. I, S. 427

Verordnung zur Übertragung von Aufgaben auf Gemeinden mit 7500 und mehr Einwohnern vom 24.10.1974, Hess. GVB1. I, S. 551

Elftes Gesetz zur Änderung des Finanzausgleichsgesetzes vom 15.12.1975, Hess. GVBl. I, S. 299

Gesetz zur Regelung des Finanzausgleichs und zur Änderung anderer Vorschriften vom 28.12.1977, Hess. GVBl. I, S. 481

Gesetz zur Neugliederung des Lahn-Dill-Gebiets und zur Übertragung von weiteren Aufgaben auf kreisangehörigen Gemeinden mit mehr als 50000 Einwohnern sowie zur Regelung sonstiger Fragen der Verwaltungsreform vom 10.07.1979, Hess. GVBl. I, S. 179

Gesetz über die Zuweisung von Grunderwerbsteueranteilen an die Landkreise und kreisfreien Städte (Grunderwerbsteuerzuweisungsgesetz) vom 24.03.1983, Hess. GVBI. I, S. 31

Zweites Gesetz zur Änderung des Gesetzes über die Aufhebung von Bagatellsteuergesetzen vom 24.06.1983, Hess. GVB1. I, S. 97

Verordnung zur Durchführung des Grunderwerbsteuerzuweisungsgesetzes vom 10.10.1983, Hess. GVB1. I, S. 141 
Verordnung über den Anteil der Spielbankgemeinden an der Spielbankabgabe und die Verwendung des Troncs der öffentlichen Spielbanken in Hessen vom 15.11.1989, Hess. GVB1. I, S. 431

Hessisches Spielbankgesetz (Hess. SpielbG) vom 21.12.1988, Hess. GVB1. I, S. 1

Gesetz zur Neuordnung des Krankenhauswesens in Hessen (Hessisches Krankenhausgesetz 1989 - HKHG -) vom 18.12.1989, Hess. GVBl. I, S. 452

Gesetz über den Staatsgerichtshof vom 30.11.1994, Hess. GVBl. S. 684

Hessisches Ministerium der Finanzen: Ausführungsbestimmungen zum Finanzausgleichsgesetz vom 17.04.1995, in: Staatsanzeiger für das Land Hessen, 1995, S. 1239

Gesetz zur Regelung des Finanzausgleichs (Finanzausgleichsgesetz - FAG -) vom 18.03.1997, Hess. GVBI. I, S. 58

Gesetz über die Zuweisung von Grunderwerbsteueranteilen an die Landkreise und kreisfreien Städte (Grunderwerbsteuerzuweisungsgesetz) vom 25.03.1997, Hess. GVBl. I, S. 50

Gesetz zur Änderung des Hessisches Schulgesetzes und anderer Gesetze und zur Neugliederung der Staatlichen Schulämter vom 15.05.1997, Hess. GVBl. I, S. 143

Finanzausgleichsänderungsgesetz 1999 vom 25.11.1998, Hess. GVBl. I, S. 496

Gesetz zur Aufgabenänderung des Umlandverbandes Frankfurt vom 17.12.1998, Hess. GVBl. I, S. 584

\section{Gesetze anderer Bundesländer:}

Niedersächsischen Gesetz über den Finanzausgleich (NFAG) vom 19.12.1995, Nds. GVBl., S. 463

Gesetz zur Änderung des Niedersächsischen Gesetzes über den Finanzausgleich und anderer Gesetze vom 12.03.1999, Nds. GVBl., S. 74

Niedersächsisches Gesetz zur Regelung der Finanzverteilung zwischen Land und Kommunen (Niedersächsisches Finanzverteilungsgesetz - NFVG -) vom 12.03.1999, Nds. GVBl., S. 79

Gesetz zur Regelung der Zuweisungen des Landes Nordrhein-Westfalen an die Gemeinden und Gemeindeverbände im Haushaltsjahr 1999 und zur Regelung des interkommunalen Ausgleichs der finanziellen Beteiligung der Gemeinden am Solidarbeitrag zur Deutschen Einheit im Haushaltsjahr 1999 und zur Änderung anderer Vorschriften vom 17.12.1998, GVBl. für das Land Nordrhein-Westfalen, S. 765 
Gesetz über einen Finanzausgleich mit den Gemeinden und Landkreisen 1998 im

Freistaat Sachsen (Finanzausgleichsgesetz 1998 - FAG 1998) vom 09.12.1997, Sächsisches GVBI., S. 662

\section{Bundesgesetze:}

Verordnung über die einstweilige Regelung des Finanz- und Lastenausgleichs (Finanzausgleichs-Verordnung) vom 30.10.1944, RGB1. I, S. 282

Gesetz zur Änderung und Ergänzung des Artikels 106 des Grundgesetzes vom 24.12.1956, BGBl. I, S. 1077

Einundzwanzigstes Gesetz zur Änderung des Grundgesetz (Finanzreformgesetz) vom 12.05.1969, BGBl. I, S. 359

Gesetz zur Änderung des Grundgesetzes (Artikel 3, 20a, 28, 29, 72, 74, 75, 76, $77,80,87,93,118$ a und 125a) vom 27.10.1994, BGBl. I, S. 3146

Gesetz zur Änderung des Grundgesetzes (Artikel 28 und 106) vom 24.10.1997, BGB1. I, S. 2470

\section{Drucksachen des Hessischen Landtags:}

Hessischer Landtag: Entwurf des Ministers der Finanzen für ein Gesetz zur Regelung des Finanzausgleichs für das Haushaltsjahr 1946 vom 09.03.1947, Drucksachen Abteilung I, 1/122

Ders.: Entwurf eines Gesetz zur Regelung des Finanzausgleichs für das Haushaltsjahr 1947 vom 12.07.1947, Drucksachen Abteilung I, 1/340

Ders.: Vorlage der Landesregierung für ein Zweites Gesetz zu Änderung des Gesetzes zur Regelung des Finanzausgleichs vom 10.061948 (GVBl. S. 83) vom 20.05.1949, Drucksachen Abteilung I, 1/1124

Ders.: Vorlage der Landesregierung über ein Gesetz zur Regelung des Finanzausgleichs vom 02.05.1950, Drucksachen Abteilung I, 1/1482

Ders.: Vorlage der Landesregierung über ein Gesetz zur Regelung des Finanzausgleichs vom 31.05.1951, Drucksachen Abteilung I, 2/111

Ders.: Vorlage der Landesregierung über den Entwurf eines Gesetzes zur Regelung des Finanzausgleichs vom 23.02.1953, Drucksachen Abteilung I, 2/588

Ders.: Regierungserklärung des Hessischen Ministerpräsidenten Dr. Zinn, 3. Wahlperiode, Stenographische Berichte, 3. Sitzung vom 19.01.1955

Ders.: Vorlage der Landesregierung für ein Gesetz zur Regelung des Finanzausgleichs vom 20.04.1955, Drucksachen Abteilung I, 3/107

Ders.: Vorlage der Landesregierung für ein Gesetz zur Regelung des Finanzausgleichs vom 15.02.1956, Drucksachen Abteilung I, 3/373. 
Ders.: Vorlage der Landesregierung für ein Zweites Gesetz zur Änderung des Finanzausgleichsgesetzes vom 10.01.1958, Drucksachen Abteilung I, 3/974

Ders.: Vorlage der Landesregierung für ein Gesetz zur Regelung des Finanzausgleichs vom 08.12.1959, Drucksachen Abteilung I, 4/405

Ders.: Vorlage der Landesregierung für ein Gesetz über die Beteiligung der Gemeinden und Landkreise am Aufkommen der Kraftfahrzeugsteuer (Kraftfahrzeugsteuerverbundgesetz) vom 31.08.1960, Drucksachen Abteilung I, $4 / 698$

Ders.: Zweites Gesetz zur Änderung des Finanzausgleichsgesetzes vom 17.08.1961, Drucksachen Abteilung I, 4/1131

Ders.: Vorlage der Landesregierung für ein Gesetz über die Verwendung der Vermögensteuer zugunsten der Gemeinden und Gemeindeverbände (Vermögensteuerverbundgesetz) vom 12.02.1963, Drucksachen Abteilung I, 5/44

Ders.: Vorlage der Landesregierung für ein Drittes Gesetz zu Änderung des Finanzausgleichs vom 12.02.1963, Drucksachen Abteilung I, 5/45

Ders.: Vorlage der Landesregierung für ein Gesetz zur Regelung des Finanzausgleichs (Finanzausgleichsgesetz - FAG -) vom 31.08.1964, Drucksachen Abteilung I, 5/989

Ders.: Haushaltsrede des Hessischen Finanzministers Dr. Conrad, 5. Wahlperiode, Stenographische Berichte, 29. Sitzung vom 09.09.1964

Ders.: Vorlage der Landesregierung für ein Erstes Gesetz zur Änderung des Gesetzes zur Regelung des Finanzausgleichs (Finanzausgleichsgesetz - FAG -) vom 26.08.1965, Drucksachen Abteilung I, 5/1464

Ders.: Antrag der Fraktion der CDU betreffend Verwaltungsreform in Hessen vom 04.05.1965, Drucksachen Abteilung I, 5/1361, gleichlautend vom 21.01.1966 Drucksache 6/681

Ders.: Initiativantrag der Fraktion der FDP betreffend den Entwurf eines Gesetzes über die Bildung von Regionen vom 24.01.1966, Drucksachen Abteilung I, 5/1681, gleichlautend vom 30.01.1968 Drucksache 6/939

Ders.: Regierungserklärung des Hessischen Ministerpräsidenten Dr. Zinn, 6. Wahlperiode, Stenographische Berichte, 3. Sitzung vom 27.01.1967

Ders.: Gesetz über die Grenzen der Regierungsbezirke und den Dienstsitz der Regierungspräsidenten vom 23.11.1967, Drucksache 6/857

Ders.: Vorlage der Landesregierung für ein Fünftes Gesetz zur Änderung des Finanzausgleichsgesetzes vom 07.07.1969, Drucksache 6/2194

Ders.: Vorlage der Landesregierung betreffend den Entwurf für ein Sechstes Gesetz zur Änderung des Finanzausgleichsgesetzes vom 09.03.1971, Drucksache $7 / 151$ 
Ders.: Vorlage der Landesregierung betreffend den Entwurf für ein Siebentes Gesetz zur Änderung des Finanzausgleichsgesetzes vom 14.02.1972, Drucksache $7 / 1322$

Ders.: Vorlage der Landesregierung betreffend den Entwurf für ein Gesetz zur Neugliederung der Landkreise Alsfeld und Lauterbach vom 01.03.1972, Drucksache $7 / 1370$

Ders.: Vorlage der Landesregierung betreffend den Entwurf für ein Gesetz zur Neugliederung der Landkreise Fulda und Hünfeld und der Stadt Fulda vom 30.03.1972, Drucksache 7/1499

Ders.: Vorlage der Landesregierung betreffend den Entwurf für ein Achtes Gesetz zur Änderung des Finanzausgleichsgesetzes vom 07.08.1972, Drucksache $7 / 2030$

Ders.: Gesetzentwurf der Landesregierung für ein Neuntes Gesetz zur Änderung des Finanzausgleichsgesetzes vom 05.10.1973, Drucksache 7/4135

Ders.: Gesetzentwurf der Landesregierung für ein Gesetz zur Neugliederung des Dillkreises, der Landkreise Gießen und Wetzlar und der Stadt Gießen vom 15.01.1974, Drucksache 7/4685

Ders.: Gesetzentwurf der Landesregierung für ein Gesetz über die Bildung des Umlandverbandes Frankfurt vom 20.05.1974, Drucksache 7/5321

Ders.: Gesetzentwurf der Landesregierung für ein Zehntes Gesetz zur Änderung des Finanzausgleichsgesetzes vom 27.03.1975, Drucksache 8/457

Ders.: Gesetzentwurf der Landesregierung für ein Elftes Gesetz zur Änderung des Finanzausgleichsgesetzes vom 04.09.1975, Drucksache 8/1439

Ders.: Gesetzentwurf der Landesregierung für ein Zwölftes Gesetz zur Änderung des Finanzausgleichsgesetzes vom 13.09.1976, Drucksache 8/3054

Ders.: Gesetzentwurf der Landesregierung für ein Gesetz zur Regelung des Finanzausgleichs und zur Änderung anderer Vorschriften vom 11.10.1977, Drucksache 8/4877

Ders.: Regierungserklärung des Hessischen Ministerpräsidenten Börner, 9. Wahlperiode, Plenarprotokolle, 2. Sitzung vom 13.12.1978

Ders.: Ausführungen des Abgeordneten Wilke (F.D.P.), 9. Wahlperiode, Stenographische Berichte, 6. Sitzung vom 21.03.1979

Ders.: Gesetzentwurf der Landesregierung für ein Gesetz zur Änderung des Finanzausgleichsgesetzes und anderer Vorschriften vom 26.09.1979, Drucksache $9 / 1522$

Ders.: Gesetzentwurf der Landesregierung für ein Gesetz zur Neuorganisation der Regierungsbezirke und der Landesplanung vom 23.06.1980, Drucksache 9/3135 
Ders.: Gesetzentwurf der Landesregierung für ein Gesetz zur Änderung des Finanzausgleichsgesetzes vom 29.09.1980, Drucksachen 9/3593

Ders.: Antwort der Landesregierung auf die Große Anfrage der Fraktion der CDU betreffend Finanzielle Abhängigkeit der hessischen Kommunen von Land und Bund Drucksache 9/3470 vom 01.07.1981, Drucksache 9/4951

Ders.: Begründung zu Art. 5 des Gesetzentwurfs der Landesregierung für ein Gesetz zur Verbesserung der Haushaltsstruktur (Haushaltsstrukturgesetz) vom 12.11.1981, Drucksache 9/5583

Ders.: Gesetzentwurf der Landesregierung für ein Gesetz über die Zuweisung von Grunderwerbsteueranteilen und über die Verwendung der Gesamtschlüsselmasse 1983 vom 20.01.1983, Drucksache 10/169

Ders.: Gesetzentwurf der Landesregierung für ein Gesetz zur Änderung des Finanzausgleichsgesetzes - Finanzausgleichsänderungsgesetz 1985 vom 29.10.1984, Drucksache 11/2228

Ders.: Gesetzentwurf der Landesregierung für ein Gesetz zur Änderung des Finanzausgleichsgesetzes und anderer Rechtsvorschriften - Finanzausgleichsänderungsgesetz 1988 - vom 25.11.1987, Drucksache 12/1178

Ders.: Gesetzentwurf der Landesregierung für ein Gesetz über die Feststellung des Haushaltsplans des Landes Hessen für das Haushaltsjahr 1989 und zur Änderung des Finanzausgleichsgesetzes und anderer Rechtsvorschriften vom 19.09.1988, Drucksache 12/3032

Ders.: Gesetzentwurf der Landesregierung zur Änderung des Gesetzes über die Feststellung des Haushaltsplans des Landes Hessen für die Jahre 1990 und 1991 (Drittes Nachtragshaushaltsgesetz 1990/91) und zur Änderung des Finanzausgleichsgesetzes vom 11.10.1990, Drucksache 12/7470

Ders.: Gesetzentwurf der Landesregierung für ein Gesetz zur Änderung des Finanzausgleichsgesetzes und anderer Rechtsvorschriften - Finanzausgleichsänderungsgesetz 1992 - vom 01.11.1991, Drucksache 13/841

Ders.: Art 2 des Gesetzentwurf der Landesregierung für ein Gesetz zur Überleitung der Aufgaben der Hilfe zur Pflege vom 23.10.1992, Drucksache $13 / 2984$

Ders.: Gesetzentwurf der Landesregierung für ein Gesetz zur Änderung des Finanzausgleichsgesetzes vom 25.07.1994, Drucksache 13/6401

Ders.: Änderungsantrag der Landesregierung zu dem Gesetzentwurf der Landesregierung für ein Gesetz zur Änderung des Finanzausgleichsgesetzes Drucksache 14/312 vom 22.12.1995, Drucksache 14/946

Ders.: Gesetzentwurf der Landesregierung für ein Gesetz zur Änderung des Finanzausgleichsgesetz vom 21.10.1996, Drucksache 14/2259 
Ders.: Art. 22 des Dritten Gesetzes zur Rechts- und Verwaltungsvereinfachung vom 28.04.1998, Drucksache 14/3849

Ders.: Gesetzentwurf der Landesregierung für ein Finanzausgleichsänderungsgesetz 1999 vom 23.06.1998, Drucksache 14/4013

Ders.: Gesetzentwurf der Fraktionen der CDU, der SPD, BÜNDNIS 90/ DIE GRÜNEN und der F.D.P. für ein Gesetz zur Aufgabenänderung des Umlandverbandes Frankfurt vom 28.04.1998, Drucksache 14/3850 sowie Änderungsantrag vom 10.12.1998, Drucksache 14/4421

\section{Drucksachen anderer Bundesländer:}

Niedersächsischer Landtag: Entwurf eines Zweiten Gesetzes zur Änderung des Niedersächsischen Gesetzes über den Finanzausgleich vom 12.01.1998, Drucksache 14/440

Ders.: Entwurf eines Gesetzes zur Regelung der Niedersächsischen Finanzverteilung (Niedersächsisches Finanzverteilungsgesetz - NFVG -) vom 02.02.1999, Drucksache 14/500

Landtag Rheinland-Pfalz: Gesetzentwurf der Landesregierung für ein Landesgesetz über den Finanzausgleich in Rheinland-Pfalz (Finanzausgleichsgesetz FAG -) vom 16.05.1977, Drucksache 8/2106

Sächsischer Landtag: Gesetzentwurf der Staatsregierung über ein Gesetz über einen Finanzausgleich mit den Gemeinden und Landkreisen 1998 im Freistaat Sachsen (Finanzausgleichsgesetz 1998 - FAG 1998) vom 04.08.1997, Drucksache 2/6535

\section{Bundestagsdrucksachen:}

Deutscher Bundestag: Entwurf eines Gesetzes zur Änderung und Ergänzung der Finanzverfassung (Finanzverfassungsgesetz) vom 29.04.1954, Drucksache II/480

Ders.: Entwurf eines ... Gesetzes zur Änderung und Ergänzung des Grundgesetzes (Finanzreformgesetz) vom 30.04.1968, Drucksache V/2861 


\section{FINANZWISSENSCHAFTLICHE SCHRIFTEN}

Band 1 Werner Steden: Finanzpolitik und Einkommensverteilung. Ein Wachstums- und Konjunkturmodell der Bundesrepublik Deutschland. 1979.

Band 2 Rainer Hagemann: Kommunale Finanzplanung im föderativen Staat. 1976.

Band 3 Klaus Scherer: Maßstäbe zur Beurteilung von konjunkturellen Wirkungen des öffentlichen Haushalts. 1977.

Band 4 Brita Steinbach: "Formula Flexibility" - Kritische Analyse und Vergleich mit diskretionärer Konjunkturpolitik. 1977.

Band 5 Hans-Georg Petersen: Personelle Einkommensbesteuerung und Inflation. Eine theoretisch-empirische Analyse der Lohn- und veranlagten Einkommensteuer in der Bundesrepublik Deutschland. 1977.

Band 6 Friedemann Tetsch: Raumwirkungen des Finanzsystems der Bundesrepublik Deutschland. Eine Untersuchung der Auswirkungen der Finanzreform von 1969 auf die Einnahmenposition der untergeordneten Gebietskörperschaften und ihrer regionalpolitischen Zieladäquanz. 1978.

Band 7 Wilhelm Pfähler: Normative Theorie der fiskalischen Besteuerung. Ein methodologischer und theoretischer Beitrag zur Integration der normativen Besteuerungstheorie in der Wohlfahrtstheorie. 1978.

Band 8 Wolfgang Wiegard: Optimale Schattenpreise und Produktionsprogramme für öffentliche Unternehmen. Second-Best Modelle im finanzwirtschaftlichen Staatsbereich. 1978.

Band 9 Hans P. Fischer: Die Finanzierung des Umweltschutzes im Rahmen einer rationalen Umweltpolitik. 1978.

Band 10 Rainer Paulenz: Der Einsatz finanzpolitischer Instrumente in der Forschungs- und Entwicklungspolitik. 1978.

Band 11 Hans-Joachim Hauser: Verteilungswirkungen der Staatsverschuldung. Eine kreislauftheoretische Inzidenzbetrachtung. 1979.

Band 12 Gunnar Schwarting: Kommunale Investitionen. Theoretische und empirische Untersuchungen der Bestimmungsgründe kommunaler Investitionstätigkeit in NordrheinWesttalen 1965-1972. 1979.

Band 13 Hans-Joachim Conrad: Stadt-Umland-Wanderung und Finanzwirtschaft der Kernstädte. Amerikanische Erfahrungen, grundsätzliche Zusammenhänge und eine Fallstudie für das Ballungsgebiet Frankfurt am Main. 1980.

Band 14 Cay Folkers: Vermögensverteilung und staatliche Aktivität. Zur Theorie distributiver Prozesse im Interventionsstaat. 1981.

Band 15 Helmut Fischer: US-amerikanische Exporttörderung durch die DISC-Gesetzgebung. 1981.

Band 16 Günter Ott: Einkommensumverteilungen in der gesetzlichen Krankenversicherung. Eine quantitative Analyse. 1981.

Band 17 Johann Hermann von Oehsen: Optimale Besteuerung. (Optimal Taxation). 1982.

Band 18 Richard Kössler: Sozialversicherungsprinzip und Staatszuschüsse in der gesetzlichen Rentenversicherung. 1982.

Band 19 Hinrich Steffen: Zum Handlungs- und Entscheidungsspielraum der kommunalen Investitionspolitik in der Bundesrepublik Deutschland. 1983.

Band 20 Manfred Scheuer: Wirkungen einer Auslandsverschuldung des Staates bei flexiblen Wechselkursen. 1983. 
Band 21 Christian Schiller: Staatsausgaben und crowding-out-Effekte. Zur Effizienz einer Finanzpolitik keynesianischer Provenienz. 1983.

Band 22 Hannelore Weck: Schattenwirtschaft: Eine Möglichkeit zur Einschränkung der öffentlichen Verwaltung? Eine ökonomische Analyse. 1983.

Band 23 Wolfgang Schmitt: Steuern als Mittel der Einkommenspolitik. Eine Ergänzung der Stabilitätspolitik? 1984.

Band 24 Wolfgang Laux: Erhöhung staatswirtschaftlicher Effizienz durch budgetäre Selbstbeschränkung? Zur Idee einer verfassungsmäßig verankerten Ausgabengrenze. 1984.

Band 25 Brita Steinbach-van der Veen: Steuerinzidenz. Methodologische Grundlagen und empirisch-statistische Probleme von Länderstudien. 1985.

Band 26 Albert Peters: Ökonomische Kriterien für eine Aufgabenverteilung in der Marktwirtschaft. Eine deskriptive und normative Betrachtung für den Allokationsbereich. 1985.

Band 27 Achim Zeidler: Möglichkeiten zur Fortsetzung der Gemeindefinanzreform. Eine theoretische und empirische Analyse. 1985.

Band 28 Peter Bartsch: Zur Theorie der längerfristigen Wirkungen 'expansiver' Fiskalpolitik. Eine dynamische Analyse unter besonderer Berücksichtigung der staatlichen Budgetbeschränkung und ausgewählter Möglichkeiten der öffentlichen Defizitfinanzierung. 1986.

Band 29 Konrad Beiwinkel: Wehrgerechtigkeit als finanzpolitisches Verteilungsproblem. Möglichkeiten einer Kompensation von Wehrungerechtigkeit durch monetäre Transfers. 1986.

Band 30 Wolfgang Kitterer: Effizienz- und Verteilungswirkungen des Steuersystems. 1986.

Band 31 Heinz Dieter Hessler: Theorie und Politik der Personalsteuern. Eine Kritik ihrer Einkommens- und Vermögensbegriffe mit Blick auf die Leistungsfähigkeitstheorie. 1994.

Band 32 Wolfgang Scherf: Die beschäftigungspolitische und fiskalische Problematik der Arbeitgeberbeiträge zur Rentenversicherung. Eine Auseinandersetzung mit der Kritik an der lohnbezogenen Beitragsbemessung. 1987.

Band 33 Andreas Mästle: Die Steuerunion. Probleme der Harmonisierung spezifischer Gütersteuern. 1987.

Band 34 Günter Ott: Internationale Verteilungswirkungen im Finanzausgleich der Europäischen Gemeinschaften. 1987.

Band 35 Heinz Haller: Zur Frage der zweckmäßigen Gestalt gemeindlicher Steuern. Ein Diskussionsbeitrag zur Gemeindesteuerreform. 1987.

Band 36 Thomas Kuhn: Schlüsselzuweisungen und fiskalische Ungleichheit. Eine theoretische Analyse der Verteilung von Schlüsselzuweisungen an Kommunen. 1988.

Band 37 Walter Hahn: Steuerpolitische Willensbildungsprozesse in der Europäischen Gemeinschaft. Das Beispiel der Umsatzssteuer-Harmonisierung. 1988.

Band 38 Ulrike Hardt: Kommunale Finanzkraft. Die Problematik einer objektiven Bestimmung kommunaler Einnahmemöglichkeiten in der gemeindlichen Haushaltsplanung und im kommunalen Finanzausgleich. 1988.

Band 39 Jochen Michaelis: Optimale Finanzpolitik im Modell überlappender Generationen. 1989.

Band 40 Bernd Raffelhüschen: Anreizwirkungen der sozialen Alterssicherung. Eine dynamische Simulationsanalyse. 1989.

Band 41 Berend Diekmann: Die Anleihe- und Darlehenstransaktionen der Europäischen Gemeinschaften. 1990.

Band 42 Helmut Kaiser: Konsumnachfrage, Arbeitsangebot und optimale Haushaltsbesteuerung. Theoretische Ergebnisse und mikroökonometrische Simulation für die Bundesrepublik Deutschland. 1990. 
Band 43 Rũdiger von Kleist: Das Gramm-Rudman-Hollings-Gesetz. Ein gescheiterter Versuch der Haushaltskonsolidierung. 1991.

Band 44 Rolf Hagedorn: Steuerhinterziehung und Finanzpolitik. Ein theoretischer Beitrag unter besonderer Berücksichtigung der Hinterziehung von Zinserträgen. 1991.

Band 45 Cornelia S. Behrens: Intertemporale Verteilungswirkungen in der gesetzlichen Krankenversicherung der Bundesrepublik Deutschland. 1991.

Band 46 Peter Saile: Ein ökonomischer Ansatz der Theorie der intermediären Finanzgewalten Die Kirchen als Parafisci. 1992.

Band 47 Peter Gottried: Die verdeckten Effizienzwirkungen der Umsatzsteuer. Eine empirische allgemeine Gleichgewichtsanalyse. 1992.

Band 48 Andreas Burger: Umweltorientierte Beschäftigungsprogramme. Eine Effizienzanalyse am Beispiel des "Sondervermögens Arbeit und Umwelt". 1992.

Band 49 Jeanette Malchow: Die Zuordnung verteilungspolitischer Kompetenzen in der Europäischen Gemeinschaft. Eine Untersuchung aufgrund einer Fortentwicklung der ökonomischen Theorie des Föderalismus. 1992.

Band 50 Barbara Seidel: Die Einbindung der Bundesrepublik Deutschland in die Europäischen Gemeinschaften als Problem des Finanzausgleichs. 1992.

Band 51 Ralph Wiechers: Markt und Macht im Rundfunk. Zur Stellung der öffentlich-rechtlichen Rundfunkanstalten im dualen Rundfunksystem der Bundesrepublik Deutschland. 1992.

Band 52 Klaus Eckhardt: Probleme einer Umweltpolitik mit Abgaben. 1993.

Band 53 Oliver Schwarkopf: Die Problematik unterschiedlicher Körperschaftsteuersysteme innerhalb der EG. 1993.

Band 54 Thorsten Giersch: Bergson-Wohlfahrtsfunktion und normative Ökonomie. 1993.

Band 55 Li-Fang Chou: Selbstbeteiligung bei Arzneimitteln aus ordnungspolitischer Sicht. Das Beispiel der Bundesrepublik Deutschland. 1993.

Band 56 Harald Schlee: Einkommensteuerliche Behandlung von Transferzahlungen. Zur Neuordnung der Familienbesteuerung sowie der Besteuerung von Versicherungsleistungen und Sozialtransfers. 1994.

Band 57 Alexander Spermann: Kommunales Krisenmanagement. Reaktionen baden-württembergischer Stadtkreise auf steigende Sozialhilfekosten und Einnahmenausfälle (198092). 1993.

Band 58 Otto Roloff / Sibylle Brander / Ingo Barens / Claudia Wesselbaum: Direktinvestitionen und internationale Steuerkonkurrenz. 1994.

Band 59 Claudia Wesselbaum-Neugebauer: Internationale Steuerbelastungsvergleiche. 1994.

Band 60 Stephanie Miera: Kommunales Finanzsystem und Bevölkerungsentwicklung. Eine Analyse des kommunalen Finanzsystems vor dem Hintergrund der sich abzeichnenden Bevölkerungsentwicklung am Beispiel Niedersachsens unter besonderer Berücksichtigung des Landkreises Wolfenbüttel und seiner Gemeinden. 1994.

Band 61 Wolfgang Schert: Die Bedeutung des kaldorianischen Verteilungsmechanismus für die gesamtwirtschaftlichen Wirkungen der staatlichen Neuverschuldung. 1994.

Band 62 Rainer Volk: Vergleich der Vergünstigungseffekte der verschiedenen investitionsfördernden Maßnahmen. 1994.

Band 63 Hans-Georg Napp: Kommunale Finanzautonomie und ihre Bedeutung für eine effiziente lokale Finanzwirtschaft. 1994. 2., unveränderte Auflage 1994.

Band 64 Bernd Rahmann / Uwe Steinborn / Günter Vornholz: Empirische Analyse der Autonomie lokaler Finanzwirtschaften in der Europäischen Gemeinschaft. 1994. 
Band 65 Carsten Kühl: Strategien zur Finanzierung der Altlastensanierung. 1994.

Band 66 Stephan Boll: Intergenerationale Umverteilungswirkungen der Fiskalpolitik in der Bundesrepublik Deutschland. Ein Ansatz mit Hilfe des Generational Accounting. 1994.

Band 67 Karl Justus Bernhard Neumärker: Finanzverfassung und Staatsgewalt in der Demokratie. Ein Beitrag zur konstitutionellen Finanztheorie. 1995.

Band 68 Christian Haslbeck: Zentrale versus dezentrale Internalisierung externer Effekte bei unvollständiger Information. 1995.

Band 69 Regina Müller: Horizontale oder vertikale Transfers zur Durchsetzung eines horizontalen Finanzausgleichs. 1995.

Band 70 Christian Hockenjos: Öffentliche Sportförderung in der Bundesrepublik Deutschland. Darstellung und finanztheoretische Analyse. 1995.

Band 71 Manfred Rosenstock: Die Kontrolle und Harmonisierung nationaler Beihilfen durch die Kommission der Europäischen Gemeinschaften. 1995.

Band 72 Christian Rüsch: Wohnungsbau- und Wohneigentumspolitik im Rahmen der Einkommensteuer. Eine Analyse unter steuersystematischen, verteilungspolitischen und fiskalischen Aspekten. 1996.

Band 73 Stephan Winters: Die kollektive Vorsorge für den Pflegefall im Alter. Eine Untersuchung am Beispiel der gesetzlichen Pflegeversicherung in den Niederlanden. 1996.

Band 74 Knut Blind: Allokationsineffizienzen auf Sicherheitsmärkten: Ursachen und Lösungsmöglichkeiten. Fallstudie: Informationssicherheit in Kommunikationssystemen. 1996.

Band 75 Barbara Petrick-Rump: Ökonomische Wirkungen von Steueramnestien. Untersuchung konkreter Erfahrungen ausgewählter Länder mit dem Einsatz von Steueramnestien anhand eines effizienten Steueramnestieprogramms. 1996.

Band 76 Georg Hirte: Effizienzwirkungen von Finanzausgleichsregelungen. Eine Empirische Allgemeine Gleichgewichtsanalyse für die Bundesrepublik Deutschland. 1996.

Band 77 Ulrike Kirchhoff: Die rheinland-pfälzischen Gemeinden im System des Finanzausgleichs. 1996.

Band 78 Kerstin Keil: Der soziale Mietwohnungsbau: Mängel und Alternativen. 1996.

Band 79 Bernhard Manzke: Kinderlastenausgleich versus verstärkte Einwanderung. Alternative Ansätze zur langfristigen Sicherung der Gesetzlichen Rentenversicherung. 1997.

Band 80 Hariolf $M$. Wenzler: Institutionenökonomik und öffentliche Finanzkontrolle. Eine Analyse am Beispiel der Europäischen Union. 1997.

Band 81 Joachim Nagel: Supply-Side Policy in den USA. Eine theoretische und empirische Analyse der angebotsorientierten Wirtschaftspolitik Reagans unter besonderer Berücksichtigung steuerlicher Aspekte. 1997.

Band 82 Heinz Lampert: Krise und Reform des Sozialstaates. 1997.

Band 83 Monika Hanswillemenke / Bernd Rahmann: Zwischen Reformen und Verantwortung für Vollbeschäftigung. Die Finanz- und Haushaltspolitik der sozial-liberalen Koalition von 1969 bis 1982. 1997

Band 84 Berthold Fürst: Die Maastrichter Budgetkriterien im Konflikt mit der Verschuldungsautonomie der deutschen Gebietskörperschaften. 1997.

Band 85 Burkhard Pahnke: Einkommensorientierte Förderung des sozialen Mietwohnungsbaues. Bestandsaufnahme und Kritik. 1998.

Band 86 Judith Safford: Staatsverschuldung im Vereinigten Königreich. Die öffentliche Verschuldung unter der Konservativen Regierung von 1979-1994. Ursachen und Auswirkungen. 1998. 
Band 87 Ralf Oberheide: Die Bekämpfung der Steuerumgehung. 1998.

Band 88 Achim Truger: Die neue Finanzwissenschaft zwischen Realitätsferne und Irrelevanz der Annahmen. Eine methodologische Analyse potentieller Verteidigungsstrategien der neuen Finanzwissenschaft gegen den Vorwurf der Realitätsferne ihres entscheidungstheoretischen Fundamentes. 1998.

Band 89 Karin Bickel: Familienbezogene Elemente im System der gesetzlichen Rentenversicherung. Unter besonderer Berücksichtigung von Ein-Eltern-Familien. 1999.

Band 90 Wolfgang Schert: Schlüsselzuweisungen und Kreisumlage. Die Problematik der Finanzierung der Landkreise am Beispiel des kommunalen Finanzausgleichs von RheinlandPfalz. 1998.

Band 91 Sandra Ehmann: Familienpolitik in Frankreich und Deutschland - ein Vergleich. 1999.

Band 92 Hendrik Suermann: Einkommensteuerliche Behandlung von Währungsgewinnen und -verlusten. Eine finanzwissenschaftliche Analyse des Steuerrechts in den USA und in Deutschland. 1999.

Band 93 Rolf Bösinger: Die Neuordnung des bundesstaatlichen Finanzausgleichs 1995. Eine theoretische und empirische Analyse unter Berücksichtigung von allokationstheoretischen und polit-ökonomischen Gesichtspunkten. 1999.

Band 94 Ulrich Ermschel: Finanzwirtschaftliche Konsequenzen beim Übergang auf das Ursprungslandprinzip im Europäischen Binnenmarkt. Eine Untersuchung am Beispiel des unvollkommenen oligopolistischen Neufahrzeugmarktes. 1999.

Band 95 Ute Hansen: Überwälzte Leistungen der Administration. Eine empirische und theoretische Analyse. 2000.

Band 96 Hans-Werner Seiler: Zur Durchsetzung der Einmalbesteuerung deutscher Körperschaftsgewinne. Strategien zur Vermeidung der im deutschen Körperschaftsteuersystem angelegten Benachteiligung ausländischer Anteilseigner. Eine finanzwissenschaftliche Analyse. 2000.

Band 97 Steffen Meyer: Zwischenstaatliche Finanzzuweisungen im zusammenwachsenden Europa. Zur Gestaltung eines Finanzausgleichs für die Europäische Union. 2000.

Band 98 Marion Hübner: Ökodumping? Umweltpolitik in internationalen Oligopolmärkten. 2000.

Band 99 Christhart Bork: Steuern, Transfers und private Haushalte. Eine mikroanalytische Simulationsstudie der Aufkommens- und Verteilungswirkungen. 2000.

Band 100 Norbert Eichler: Die Probleme des Gemeindefinanzausgleichs im Kooperativen Föderalismus. Eine ökonomische Analyse am Beispiel des Bundeslandes Nordrhein-Westfalen. 2000.

Band 101 Wolfgang Scherf: Der Länderfinanzausgleich in Deutschland. Ungelöste Probleme und Ansatzpunkte einer Reform. 2000.

Band 102 Stefan Dietrich Josten: Staatsverschuldung, intertemporale Allokation und Wirtschaftswachstum. Eine theoretische Analyse staatlicher Verschuldungspolitik in Modellen exogenen und endogenen Wachstums. 2000.

Band 103 Axel Breitbach: Steuerhinterziehung und Schattenwirtschaft aus gesamtwirtschaftlicher Sicht. 2000.

Band 105 Michael Broer: Der kommunale Finanzausgleich in Hessen. Historische Darstellung und ökonomische Analyse unter besonderer Berücksichtigung der Schlüsselzuweisungen. 2001. 
Wolfgang Scherf

\section{Der Länderfinanzausgleich in Deutschland}

\section{Ungelöste Probleme und Ansatzpunkte einer Reform}

Frankfurt/M., Berlin, Bern, Bruxelles, New York, Oxford, Wien, 2000. 238 S., 16 Abb., 15 Tab.

Finanzwissenschaftliche Schriften. Bd. 101

Verantwortlicher Herausgeber: Alois Oberhauser

ISBN 3-631-36866-6 · br. DM 68.-*

Der Länderfinanzausgleich in Deutschland steht vor einer grundlegenden Neuordnung. Das Gutachten für das Land Hessen analysiert den heutigen Länderfinanzausgleich einschließlich der Bundesergänzungszuweisungen aus finanzwissenschaftlicher Sicht. Ausgehend von den Defekten des bestehenden Systems werden die Möglichkeiten einer zielgerichteten Reform erörtert. Der Verfasser plädiert für die Beseitigung aller Sonderregelungen und für die Abschaffung der Bundesergänzungszuweisungen mit Ausnahme der Hilfen für die neuen Länder. Darüber hinaus empfiehlt er eine deutliche Reduktion der Nivellierungsintensität des horizontalen Länderfinanzausgleichs durch ein flexibles Tarifmodell, das positive Leistungsanreize für Zahler- wie Empfängerländer sicherstellt.

Aus dem Inhalt: Föderalismus und Finanzausgleich - Finanzausgleich zwischen Effizienz und Verteilung · Ökonomische Wirkungen des Länderfinanzausgleichs - Vertikale und horizontale Steuerverteilung - Der horizontale Länderfinanzausgleich - Ein Vorschlag zur Reform des Ausgleichstarifs - Die Bundesergänzungszuweisungen

Frankfurt/M - Berlin - Bern - Bruxelles - New York · Oxford · Wien

Auslieferung: Verlag Peter Lang AG

Jupiterstr. 15, CH-3000 Bern 15

Telefax (004131) 9402131

*inklusive Mehrwertsteuer

Preisänderungen vorbehalten

Homepage http://wuw.peterlang.de 